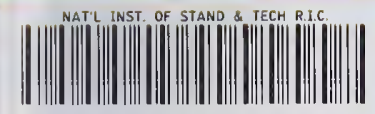

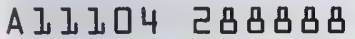

NBS

PUBLICATIONS

\title{
Standard X-ray Diffraction \\ Powder Patterns
}

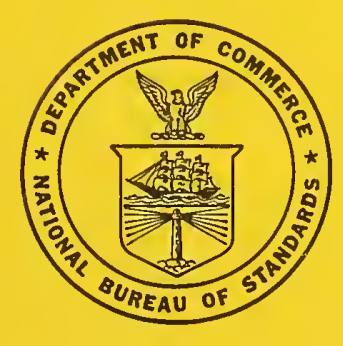

$2 \mathrm{C}$ 100 4556 No.25, Sec. 1 1969 Copy 2.
NBS MONOGRAPH 25-SECTION 7

Ci 11969 


\section{NATIONAL BUREAU OF STANDARDS}

The National Bureau of Standards ' was established by an act of Congress March 3, 1901. Today, in addition to serving as the Nation's central measurement laboratory, the Bureau is a principal focal point in the Federal Government for assuring maximum application of the physical and engineering sciences to the advancement of technology in industry and commerce. To this end the Bureau conducts research and provides central national services in four broad program areas. These are: (1) basic measurements and standards, (2) materials measurements and standards, (3) technological measurements and standards, and (4) transfer of technology.

The Bureau comprises the Institute for Basic Standards, the Institute for Materials Research, the Institute for Applied Technology, the Center for Radiation Research, the Center for Computer Sciences and Technology, and the Office for Information Programs.

THE INSTITUTE FOR BASIC STANDARDS provides the central basis within the United States of a complete and consistent system of physical measurement; coordinates that system with measurement systems of other nations; and furnishes essential services leading to accurate and uniform physical measurements throughout the Nation's scientific community, industry, and commerce. The Institute consists of an Office of Measurement Services and the following technical divisions:

Applied Mathematics-Electricity-Metrology-Mechanics-Heat-Atomic and Molecular Physics-Radio Physics "-Radio Engineering "-Time and Frequency ״-Astrophysics "-Cryogenics."

THE INSTITUTE FOR MATERIALS RESEARCH conducts materials research leading to improved methods of measurement standards, and data on the properties of well-characterized materials needed by industry, commerce, educational institutions, and Government; develops, produces, and distributes standard reference materials; relates the physical and chemical properties of materials to their behavior and their interaction with their environments; and provides advisory and research services to other Government agencies. The Institute consists of an Office of Standard Reference Materials and the following divisions:

Analytical Chemistry-Polymers-Metallurgy_Inorganic Materials_Physical Chemistry.

THE INSTITUTE FOR APPLIED TECHNOLOGY provides technical services to promote the use of available technology and to facilitate technological innovation in industry and Government; cooperates with public and private organizations in the development of technological standards, and test methodologies; and provides advisory and research services for Federal, state, and local government agencies. The Institute consists of the following technical divisions and offices:

Engineering Standards-Weights and Measures - Invention and Innovation - Vehicle Systems Research-Product Evaluation-Building Research-Instrument Shops-Measurement Engineering-Electronic Technology-Technical Analysis.

THE CENTER FOR RADIATION RESEARCH engages in research, measurement, and application of radiation to the solution of Bureau mission problems and the problems of other agencies and institutions. The Center consists of the following divisions:

Reactor Radiation-Linac Radiation-Nuclear Radiation-Applied Radiation.

THE CENTER FOR COMPUTER SCIENCES AND TECHNOLOGY conducts research and provides technical services designed to aid Government agencies in the selection, acquisition, and effective use of automatic data processing equipment; and serves as the principal focus for the development of Federal standards for automatic data processing equipment, techniques, and computer languages. The Center consists of the following offices and divisions:

Information Processing Standards-Computer Information - Computer Services - Systems Development-Information Processing Technology.

THE OFFICE FOR INFORMATION PROGRAMS promotes optimum dissemination and accessibility of scientific information generated within NBS and other agencies of the Federal government; promotes the development of the National Standard Reference Data System and a system of information analysis centers dealing with the broader aspects of the National Measurement System, and provides appropriate services to ensure that the NBS staff has optimum accessibility to the scientific information of the world. The Office consists of the following organizational units:

Office of Standard Reference Data-Clearinghouse for Federal Scientific and Technical Information "-Office of Technical Information and Publications-Library-Office of Public Information-Office of International Relations.

1 Headquarters and Laboratories at Gaithersburg, Maryland, unless otherwise noted: mailing address Washington, D.C. 20234.

3 Located at 5285 Port Royal Road, Springfield, Virginia 22151. 
MATONAL BUREAS OF STANDARAS

UNITED STATES DEPARTMENT OF COMMERCE

Maurice H. Stans, Secretary

National Bureau of Standards

\title{
Standard X-ray Diffraction
}

\section{Powder Patterns}

\author{
H. E. Swanson, H. F. McMurdie, M. C. Morris, \\ and E. H. Evans
}

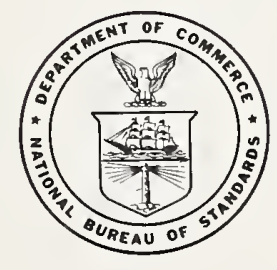

L,

U.S. National Bureau of Standards, Monograph 25-Section 7

' Nat. Bur. Stand. (U.S.), Monogr. 25 Section 7, 188 pages (Sept. 1969)

CODEN: NBSMA

Issued September 1969

For sale by the Superintendent of Documents, U.S. Government Printing Office Washington, D.C. 20402 - Price $\$ 1.50$ 
Library of Congress Catalog Card Number: 53-61386 
Experimental patterns:

Ammonium Cadmium Sulfate,

$\left(\mathrm{NH}_{4}\right)_{2} \mathrm{Cd}_{2}\left(\mathrm{SO}_{4}\right)_{3} \ldots \ldots \ldots \ldots \ldots \ldots \ldots \ldots \ldots$

Ammonium Copper Chloride, $\mathrm{NH}_{4} \mathrm{CuCl}_{3} \ldots$.

Ammonium Manganese Sulfate,

$\left(\mathrm{NH}_{4}\right)_{2} \mathrm{Mn}_{2}\left(\mathrm{SO}_{4}\right)_{3}$

Barium Borate, $\mathrm{BaB}_{8} \mathrm{O}_{13} \ldots \ldots \ldots \ldots \ldots \ldots \ldots \ldots . . . . . .$.

Cesium Calcium Sulfate, $\mathrm{Cs}_{2} \mathrm{Ca}_{2}\left(\mathrm{SO}_{4}\right)_{3} \ldots$

Cesium Copper Sulfate Hexahydrate,

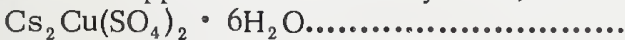

Cesium Iron Sulfate Hexahydrate,

$\mathrm{Cs}_{2} \mathrm{Fe}\left(\mathrm{SO}_{4}\right)_{2} \cdot 6 \mathrm{H}_{2} \mathrm{O} . \ldots \ldots \ldots \ldots \ldots \ldots \ldots \ldots . . . . .$.

Cesium Magnesium Sulfate Hexahydrate,

$\mathrm{Cs}_{2} \mathrm{Mg}\left(\mathrm{SO}_{4}\right)_{2} \cdot 6 \mathrm{H}_{2} \mathrm{O} \ldots \ldots \ldots \ldots \ldots \ldots \ldots \ldots . . . . .$.

Cesium Manganese Sulfate Hexahydrate,

$\mathrm{Cs}_{2} \mathrm{Mn}\left(\mathrm{SO}_{4}\right)_{2} \cdot 6 \mathrm{H}_{2} \mathrm{O} \ldots \ldots \ldots \ldots \ldots \ldots \ldots \ldots . . . . . . . .$.

Cesium Mercury Chloride, $\mathrm{CsHgCl}_{3}$.........

Cesium Nickel Sulfate Hexahydrate,

$\mathrm{Cs}_{2} \mathrm{Ni}\left(\mathrm{SO}_{4}\right)_{2} \cdot 6 \mathrm{H}_{2} \mathrm{O}$.

Cesium Zinc Sulfate Hexahydrate,

$\mathrm{Cs}_{2} \mathrm{Zn}\left(\mathrm{SO}_{4}\right)_{2} \cdot 6 \mathrm{H}_{2} \mathrm{O}$

Imidazole Nickel Nitrate,

$\left(\mathrm{C}_{3} \mathrm{H}_{4} \mathrm{~N}_{2}\right)_{6} \mathrm{Ni}\left(\mathrm{NO}_{3}\right)$

Magnesium Molybdate, MgMoO.............

Magnesium Perchlorate Hexahydrate,

$\mathrm{Mg}\left(\mathrm{ClO}_{4}\right)_{2} \cdot 6 \mathrm{H}_{2} \mathrm{O}$.

Mercuric Iodide, $\mathrm{HgI}_{2}$ (tetragonal) (re-

vised)

Potassium Cadmium Sulfate, $\mathrm{K}_{2} \mathrm{Cd}_{2}\left(\mathrm{SO}_{4}\right)_{3}$

Potassium Calcium Chloride (chlorocal-

cite), $\mathrm{KCaCl}_{3}$

Potassium Calcium Magnesium Sulfate,

$\mathrm{K}_{2} \mathrm{CaMg}\left(\mathrm{SO}_{4}\right)_{3}$

Potassium Calcium Sulfate $\mathrm{K}_{2} \mathrm{Ca}_{2}$

Potassium Copper Chloride, $\mathrm{KCuCl}_{3} \ldots \ldots \ldots$

Potassium Nickel Fluoride, $\mathrm{KNiF}_{3} \ldots . . . . . .$.

Potassium Zinc Sulfate Hexahydrate,

$\mathrm{K}_{2} \mathrm{Zn}\left(\mathrm{SO}_{4}\right)_{2} \cdot 6 \mathrm{H}_{2} \mathrm{O} \ldots \ldots \ldots \ldots \ldots \ldots \ldots \ldots \ldots . . .$.

Rubidium Cadmium Sulfate, $\mathrm{Rb}_{2} \mathrm{Cd}_{2}\left(\mathrm{SO}_{4}\right)_{3}$

Rubidium Calcium Chloride, $\mathrm{RbCaCl}_{3} \ldots \ldots .$.

Rubidium Calcium Sulfate, $\mathrm{Rb}_{2} \mathrm{Ca}_{2}\left(\mathrm{SO}_{4}\right)_{3} \ldots$

Rubidium Magnesium Sulfate,

$\mathrm{Rb}_{2} \mathrm{Mg}_{2}\left(\mathrm{SO}_{4}\right)_{3}$

Rubidium Manganese Sulfate,

$\mathrm{Rb}_{2} \mathrm{Mn}_{2}\left(\mathrm{SO}_{4}\right)_{3}$

Rubidium Strontium Chloride, $\mathrm{RbSrCl} \ldots \ldots$

Rubidium Zinc Sulfate Hexahydrate,

$\mathrm{Rb}_{2} \mathrm{Zn}\left(\mathrm{SO}_{4}\right)_{2} \cdot 6 \mathrm{H}_{2} \mathrm{O}$

Rubidium Zinc Fluoride, $\mathrm{RbZnF}$.............

Scandium Silicate (thortveitite), $\mathrm{Sc}_{2} \mathrm{Si}_{2} \mathrm{O}_{7} \ldots$

Selenium Oxide (selenolite), $\mathrm{SeO}_{2}$ (re-

vised)

Sodium Dichromate Dihydrate,

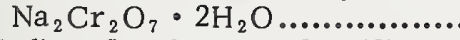

Sodium Lanthanum Fluosilicate,

$\mathrm{Na}_{2} \mathrm{La}_{8} \quad\left(\mathrm{SiO}_{4}\right)_{6} \mathrm{~F}$

Sodium Neodymium Fluosilicate, .

$\mathrm{Na}_{7} \mathrm{Nd}_{8} \quad\left(\mathrm{SiO}_{4}\right)_{6} \mathrm{~F}_{2}$

Sodium Praseodymium Fluosilicate,

$\mathrm{Na}_{2} \mathrm{Pr}_{8}\left(\mathrm{SiO}_{4}\right)_{6} \mathrm{~F}_{2}$

Thallium Cobalt Sulfate Hexahydrate,

$\mathrm{Tl}_{2} \mathrm{Co}\left(\mathrm{SO}_{4}\right)_{2} \cdot 6 \mathrm{H}_{2} \mathrm{O}$
Page

5

8

10

12

14

18

20

22

23

25

27

28

30

32

34

36

37

39

41

42

43

45

47

48

50

52

54

55

57

58

60

62

64

66

68

Thallium Copper Sulfate Hexahydrate,

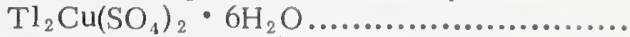

Thallium Magnesium Sulfate Hexahydrate,

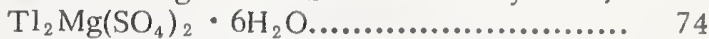

Thallium Manganese Sulfate, $\mathrm{Tl}_{2} \mathrm{Mn}_{2}\left(\mathrm{SO}_{4}\right)_{3}$

Thallium Nickel Sulfate Hexahydrate,

$\mathrm{Tl}_{2} \mathrm{Ni}\left(\mathrm{SO}_{4}\right)_{2} \cdot 6 \mathrm{H}_{2} \mathrm{O} \ldots \ldots \ldots \ldots \ldots \ldots \ldots \ldots . . . . . .$.

Thallium Zinc Sulfate Hexahydrate,

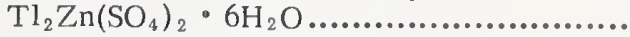

Titanium Oxide (anatase), $\mathrm{TiO}_{2}$ (revised)...

Titanium Oxide (rutile), $\mathrm{TiO}_{2}$ (revised).....

Calculated patterns:

Arsenic Acid, $\mathrm{H}_{5} \mathrm{As}_{3} \mathrm{O}_{10}$

Azobenzene, $\mathrm{C}, \mathrm{H}, \mathrm{N}$

Beryllium Calcium Oxide, $\mathrm{Be}_{17} \mathrm{Ca}_{12} \mathrm{O}_{29} \ldots .$.

Beryllium Niobium, $\mathrm{Be}_{2} \mathrm{Nb} . . . . . . . . . . . . . . . . . .$.

Cadmium Nitrate Tetrahydrate,

$\mathrm{Cd}\left(\mathrm{NO}_{3}\right)_{2} \cdot 4 \mathrm{H}_{2} \mathrm{O}$

Calcium Phosphate, beta-pyro-, $\mathrm{Ca}_{2} \mathrm{P}_{2} \mathrm{O}_{7} \ldots$

Cerium Copper, $\mathrm{CeCu}_{6} \ldots \ldots \ldots \ldots . . . . . . . . . . . .$.

Cesium Cerium Chloride, $\mathrm{Cs}_{2} \mathrm{CeCl}_{6} \ldots \ldots \ldots$.

Cesium Iodine Bromide, $\mathrm{CsI}_{2} \mathrm{Br}$...............

Cesium Lithium Fluoride, $\mathrm{CsLiF}_{2} \ldots \ldots . . . .$.

Chromium Fluoride, $\mathrm{Cr}_{2} \mathrm{~F}_{5}$

Copper Glutamate Dihydrate,

$\mathrm{CuC}_{5} \mathrm{H}_{7} \mathrm{NO}_{4} \cdot 2 \mathrm{H}_{2} \mathrm{O}$

Copper Phosphate, alpha-pyro-, $\mathrm{Cu}_{2} \mathrm{P}_{2} \mathrm{O}_{7} \ldots$

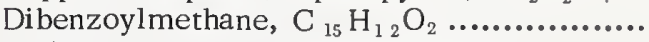

Gadolinium Chloride Hexahydrate,

$\mathrm{GdCl}_{3} \cdot 6 \mathrm{H}_{2} \mathrm{O}$

Hexamethylenediammonium Adipate,

$\mathrm{C}_{12} \mathrm{H}_{26} \mathrm{~N}_{2} \mathrm{O}_{4}$

Imidazole Zinc Chloride, $\left(\mathrm{C}_{3} \mathrm{H}_{4} \mathrm{~N}_{2}\right){ }_{2} \mathrm{ZnCl}_{2}$

Lithium Beryllium Fluoride, $\mathrm{Li}_{2} \mathrm{BeF}_{4} \ldots \ldots$..

Lithium Rubidium Fluoride, $\mathrm{LiRbF}_{2}$........

Lithium Uranium Fluoride, $\operatorname{LiUF}_{5} \ldots . . . . .$. .

Magnesium Chloride Dodecahydrate,

$\mathrm{MgCl}_{2} \cdot 12 \mathrm{H}_{2} \mathrm{O}$

Magnesium Hydrogen Phosphate Trihy -

drate, newberyite, $\mathrm{MgHPO}_{4} \cdot 3 \mathrm{H}_{2} \mathrm{O} \ldots . . .$. .

Manganese, alpha, Mn ........................

bis-(N-isopropyl-3-ethylsalicylaldiminato)

Palladium, $\left(\mathrm{C}_{12} \mathrm{H}_{16} \mathrm{NO}\right)_{2} \mathrm{Pd}$...

$\mathrm{N}$-methylphenazinium Tetracyanoquinodi-

methanide, $\mathrm{C}_{25} \mathrm{H}_{15} \mathrm{~N}_{6} \ldots \ldots . \ldots . \ldots \ldots . . . . . . . . . .$.

Niobium Oxychloride, $\mathrm{NbOCl}_{3} \ldots \ldots \ldots \ldots . . . . .$.

Phosphorus Bromide, $\mathrm{PBr}$...................

Pimelic Acid, $\mathrm{C}_{7} \mathrm{H}_{12} \mathrm{O}_{4} \ldots \ldots \ldots \ldots \ldots \ldots \ldots \ldots . . . .$.

Silver Permanganate, $\mathrm{AgMnO}_{4} \ldots . . . . . . . . . .$.
Sodium Aluminum Chloride Silicate, soda-

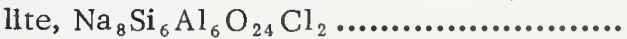

Sodium Borate, $\mathrm{Na}_{2} \mathrm{~B}_{8} \mathrm{O}_{13} \ldots \ldots \ldots \ldots \ldots \ldots$........

Sodium Hydrogen Silicate Tetrahydrate,

$\mathrm{Na}_{2} \mathrm{H}_{2} \mathrm{SiO}_{4} \cdot 4 \mathrm{H}_{2} \mathrm{O}$..........................

Sodium Tin Fluoride, $\mathrm{NaSn}_{2} \mathrm{~F}_{5} \ldots . . . . . . . . . .$.

d-Tartaric Acid, $\mathrm{C}_{4} \mathrm{H}_{6} \mathrm{O}_{6} \ldots \ldots \ldots \ldots \ldots \ldots . . . .$.

Zinc Glutamate Dihydrate,

$\mathrm{ZnC}_{5} \mathrm{H}_{7} \mathrm{NO}_{4} \cdot 2 \mathrm{H}_{2} \mathrm{O}$

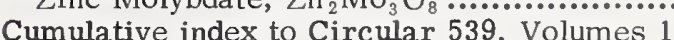

through 10, and Monograph 25, Sections 1

through 7

Cumulative mineral index 
Monograph 25

Sec. 4, p. 4, column 2; at line 4 in the table, for $h k l(211)$, the $2 \theta$ value for Tungsten (W) should be 73.184

Sec. 4, p. 23, hkl's for $2.036,1.815$, and 1.3505 should be $\overline{1} 04, \overline{2} 21$, and $\overline{4} 01$ respectively.

Sec. 6, p. 4; in the 13th line from the end, the formula should be $\mathrm{MgF}_{2}$

Sec. 6, p. 22; the space group symbol should be $C_{3}^{6} v-R 3 c$

Sec. 6, p. 42; the error for the NBS lattice constant in the table should be \pm .0001

Sec. 6, p. 63; the second word of the title should be Magnesium

\section{STANDARD X-RAY DIFFRACTION POWDER PATTERNS}

lnformation on ten volumes in this series listed as follows is available from Mr. Howard E. Swanson, Room A221, Materials Building, National Bureau of Standards, Washington, D. C., 20234:

NBS Circular 539, Volume 1, Standard X-ray Diffraction Powder Patterns (Data for 54 substances).

NBS Circular 539, Volume 2, Standard X-ray Diffraction Powder Patterns (Data for 30 substances).

NBS Circular 539, Volume 3, Standard X-ray Diffraction Powder Patterns (Data for 34 substances).

NBS Circular 539, Volume 4, Standard X-ray Diffraction Powder Patterns (Data for 42 sunstances).

NBS Circular 539, Volume 5, Standard X-ray Diffraction Powder Patterns (Data for 45 substances).

NBS Circular 539, Volume 6, Standard X-ray Diffraction Powder Patterns (Data for 44 substances).

NBS Circular 539, Volume 7, Standard X-ray Diffraction Powder Patterns (Data for 53 substances).

NBS Circular 539, Volume 8, Standard X-ray Diffraction Powder Patterns (Data for 61 substances).

NBS Circular 539, Volume 9, Standard X-ray Diffraction Powder Patterns (Data for 43 substances).

NBS Circular 539, Volume 10, Standard X-ray Diffraction Powder Patterns (Data for 40 substances).

The following five volumes in this series are available from the Superintendent of Documents, U.S. Government Printing Office, Washington, D. C., 20402, as follows:

NBS Monograph 25, Section 1, Standard X-ray Diffraction Powder Patterns (Data for 46 substances) 40 cents.

NBS Monograph 25, Section 2, Standard X-ray Diffraction Powder Patterns (Data for 37 substances) 35 cents.

NBS Monograph 25, Section 3, Standard X-ray Diffraction Powder Patterns (Data for 51 substances) 40 cents.

NBS Monograph 25, Section 4, Standard X-ray Diffraction Powder Patterns (Data for 103 substances) 55 cents.

NBS Monograph 25, Section 5, Standard X-ray Diffraction Powder Patterns (Data for 60 substances) 55 cents.

NBS Monograph 25, Section 6, Standard X-ray Diffraction Powder Patterns (Date for 60 substances) 50 cents.

Send orders with remittance for the above six Monographs to Superintendent of Documents, U.S. Government Printing Office, Washington, D.C., 20402. Remittance from foreign countries should include an additional one-fourth of the purchase price for postage.

Those wishing to be notified of future issues should send mailing address to the Government Printing Office. 


\title{
STANDARD X-RAY DIFFRACTION POWDER PATTERNS
}

\author{
Section 7._Data for 81 substances
}

Howard E. Swanson, Howard F. McMurdie, ${ }^{1}$ Marlene C. Morris, ${ }^{1}$ and Eloise H. Evans ${ }^{1}$

\begin{abstract}
Standard $x$-ray diffraction powder patterns are presented for 81 substances. Forty-five of these patterns represent experimental data and 36 are calculated. The experimental x-ray powder diffraction patterns were obtained with a Geiger or proportional counter x-ray diffractometer, using samples of high purity. All d-values were assigned Miller indices determined by comparison with computed interplanar spacings and from consideration of space group extinctions. The densities and lattice constants were calculated, and the refractive indices were measured whenever possible. The calculated $x$-ray powder diffraction patterns were computed from published crystal structure data. Both peak height and integrated intensities are reported for the calculated patterns.

Key words: Crystal structure; integrated intensities; peak intensities; reference intensities; lattice constants; powder patterns; standard; x-ray diffraction.
\end{abstract}

\section{INTRODUCTION}

The Powder Diffraction File $[1968]^{2}$ is a compilation of diffraction patterns, gathered from many sources and produced under the auspices of the Joint Committee on Powder Diffraction Standards. ${ }^{3}$ The File is used for identification of crystalline materials by matching d-spacings and diffraction intensity measurements. Under the partial sponsorship of the Joint Committee, our program at the National Bureau of Standards contributes new data for this File. Our work also aids in the evaluation and revision of published $\mathrm{x}$-ray data and in the development of diffraction techniques. This report presents information for 81 compounds (45 experimental and 36 calculated patterns), and is the seventeenth of the series of "Standard X-ray Diffraction Powder Patterns." ${ }^{4}$

\section{EXPERIMENTAL POWDER PATTERNS}

Sample. The samples used to make NBS patterns were special preparations of high purity obtained from a variety of sources or prepared in small quantities in our laboratory. Treating the sample by appropriate annealing, recrystallizing, or heating in hydrothermal bombs improved the definition of most of the patterns. A check of phase purity was usually provided by the $x$-ray pattern itself, when it was indexed by comparison with computed d-values.

\footnotetext{
I Research Associate, at the National Bureau of Standards sponsored by the Joint Committee on Powder Diffraction Standards.

${ }^{2}$ Dates in brackets indicate the literature references at the end of each section of this paper.

${ }^{3}$ This committee is sponsored jointly by the American Society for Testing and Materials, the American Crystallographic Association, the (British) Institute of Physics, and The National Association of Corrosion Engineers.
}

${ }^{4}$ See previous page for listing of other published volumes.
Optical data, color. A microscopic inspection for phase purity was made on the nonopaque materials during the refractive index determination. The latter was done by grain-immersion methods in white light, with oils standardized in sodium light, in the range 1.40 to 2.1 .

The names of the sample colors were selected from the ISCC-NBS Centroid Color Charts [1965].

Structure, lattice constants. The space groups are listed with short Hermann-Mauguin symbols as well as the space group numbers given in the International Tables for X-ray Crystallography Vol. 1 [1952].

Orthorhombic cell dimensions are presented according to the Dana convention $b>a>c$ [Palache et al., 1944].

A computer program [Evans et al., 1963] assigned $h k l$ 's and refined the lattice constants. Cell refinement was based only upon $2 \theta$ values which could be indexed without ambiguity. The number of significant figures reported for d-values varies slightly with the symmetry and crystallinity of each sample. Unit cell constants and their standard errors are based on least squares refinement of the variance-covariance matrix derived from the unweighted $\Delta \theta$ residuals.

Published unit cell data in $\mathrm{kX}$ units and data given in angstrom units prior to 1947 were converted to angstrom units using the factor 1.00206 reported by Bearden [1964].

Density. The densities calculated from the NBS lattice constants are expressed in grams per cubic centimeter and are computed using the Avogadro number $\left(6.02252 \times 10^{23}\right)$ and using atomic weights based on carbon 12 [International Union, 1961].

Interplanar spacings. Specimens for the interplanar spacing patterns were prepared by packing into a shallow holder a sample containing approximately $5 \mathrm{wt}$. percent tungsten powder that served as an internal standard. When tungsten lines were found to interfere, 25 percent silver was used in 
place of tungsten. If the internal standard correction varied along the length of the pattern, linear interpolations were used. To avoid aberrations at the top of the peak, the reading for low values of $2 \theta$ was taken at a position about $25 \%$ of the way down from the top, and in the center of the peak width. For higher values of $2 \theta$, where $\alpha_{1}$ and $\alpha_{2}$ peaks were resolved, the $\alpha_{1}$ peak was measured in the same way. The internal standard correction appropriate to each region was then applied to the measurement of $2 \theta$. The internal standard lattice constants used were $3.16516 \AA$ for tungsten and $4.08641 \mathrm{~A}$ for silver at $25^{\circ} \mathrm{C}$, as determined by Swanson, Morris, and Evans [1966] and modified to correspond with the Bearden [1964] wavelength. (Prior to this, the internal standard constants used were $3.1648 \AA$ and $4.0861 \AA$, through June 1966, and then 3.16504 $\AA$ and 4.08625 $\AA$ until June 1968.) All of the NBS patterns, unless otherwise noted, were made on a diffractometer at $25{ }^{\circ} \mathrm{C}$ using copper radiation with a monochromator having a curved lithium fluoride crystal. The wavelength of $\mathrm{CuK} \alpha_{1}$ was assumed to be $1.54056 \AA$ [Bearden, 1964].

Intensity measurements. At least three patterns for intensity measurements were prepared for each sample to check reproducibility. It was found that samples which gave satisfactory intensity patterns usually had an average particle size smaller than $10 \mu$ as recommended by Alexander et al. [1948] . In order to avoid the orientation effects which occur when powdered samples are packed or pressed, a sample holder was made that had in its top face a rectangular cavity which extended to one end of the holder. To prepare the sample, a glass slide was clamped over the top face to form a temporary cavity wall (see Fig. 1), and the powdered sample was drifted into the end opening while the holder was held in a vertical position. With the sample holder returned to a horizontal position, the glass slide was carefully removed so that the sample could be exposed to the $\mathrm{x}$-ray beam (as shown in Fig 2). If the sample powder did not flow readily, or was prone to orient excessively, approximately 50 volume percent of finely ground silica-gel was added as a diluent. The intensities of the diffraction lines were measured as peak heights above background and were expressed in percentages of the intensity of the strongest line.

Reference intensity. For reference intensity measurements, $\alpha \mathrm{Al}_{2} \mathrm{O}_{3}$ (corundum) was chosen as an internal standard to be mixed $1: 1$ by weight with the sample. This mixture is mounted in our regular intensity sample holder (illust. pg. 3). Only the portion of the $x$-ray pattern that includes the strongest line of each component is run; for the standard, the (113) reflection with $d=2.085 \AA$ is used. The direct ratio of the heights of the two lines is then reported as $1 / I_{\text {corundum }}$. In a few instances the strongest line of one of the materials may fall on a line of the other. In this case, the second strongest line is measured, and based upon previous knowledge of the relative peak heights, a correction is made, thus enabling one to calculate the value for the strongest line.

\section{CALCULATED POWDER PATTERNS}

Since some substances are not readily available for experimental work, calculated powder patterns were made. These were based on published crystal structure data using a FORTRAN program developed by Smith [1967].

Lattice parameters. Before the computations of the patterns, corrections were made as necessary in the published lattice parameters so that they would correspond to the Bearden [1964] value of the copper wavelength; specifically, the published parameter in $\AA$ was multiplied by 1.00004 . Both the ailtered parameter and the original published value are given.

Scattering factors. Whenever possible, the same scattering factors were used which the author of the reference article specified. Otherwise, they were used directly from the International Tables [1962]. We have referred to this source by table number $3.3 .1 \mathrm{~A}$ or $3.3 .1 \mathrm{~B}$, and $3.3 .2 \mathrm{~A}$ or $3.3 .2 \mathrm{~B}$; they are found respectively on pages 202, 210,213, and 214 of the International Tables, Vol. III [1962]. Corrections were made for dispersion if the authors had done so.

Thermal parameters. When these parameters were used directly as given by the authors, the reference is specified. When, for reasons necessitated by the computer program, the parameters were modified here, no reference is given.

Scale factors. For each compound, this conversion factor when multiplied by the scaled integrated intensities will reproduce the unscaled intensities derived from the structure factors for a single unit cell for the copper $\mathrm{K}_{\alpha_{1}}$ wavelength. The scale factors are not usable for comparisons between compounds since they have not been standardized for the effects of volume and absorption.

Integrated intensities. Intensity calculations were based on the copper $K a_{1}$ wavelength, 1.54056 $\AA$, determined by Bearden [1964]. The integrated intensities were computed from the formula:

$$
1=\mathrm{F}^{2}(\mathrm{Lp})(\mathrm{FAC})
$$

where $F$ is the standard structure factor FAC is the powder multiplicity

$$
\text { and } \quad L p=\frac{1+2 \cos ^{2} 2 \theta}{\sin ^{2} \theta \cos \theta}
$$


The intensities are scaled to the strongest line as 100. Reflections with intensities equal to or less than 0.7 are not reported.

Peak intensities. In the Smith program, the integrated intensities can be assigned a Cauchy profile, and a half-width can be designated so as to simulate a trace from diffractometers in current use. The value of the half-width used here was $0.075^{\circ}$ at $40^{\circ}(2 \theta)$. The program then sums the intensities from the overlapping peak profiles and scales the resulting peak intensities to the strongest peak height. Reflections are not reported which had peak heights equal to or less than 0.7 . When adjacent peaks have nearly equal $2 \theta$ values, resolution of individual peaks in the powder pattern would be unlikely; therefore, one composite peak is given. The angle of this peak is assigned the $h \mathrm{kl}$ of the reflection with the greatest integrated intensity.

The authors are indebted to J. H. deGroot for the preparation of many samples used, and to S. J. Carmel for his assistance with the work, particularly in performing intensity measurements.

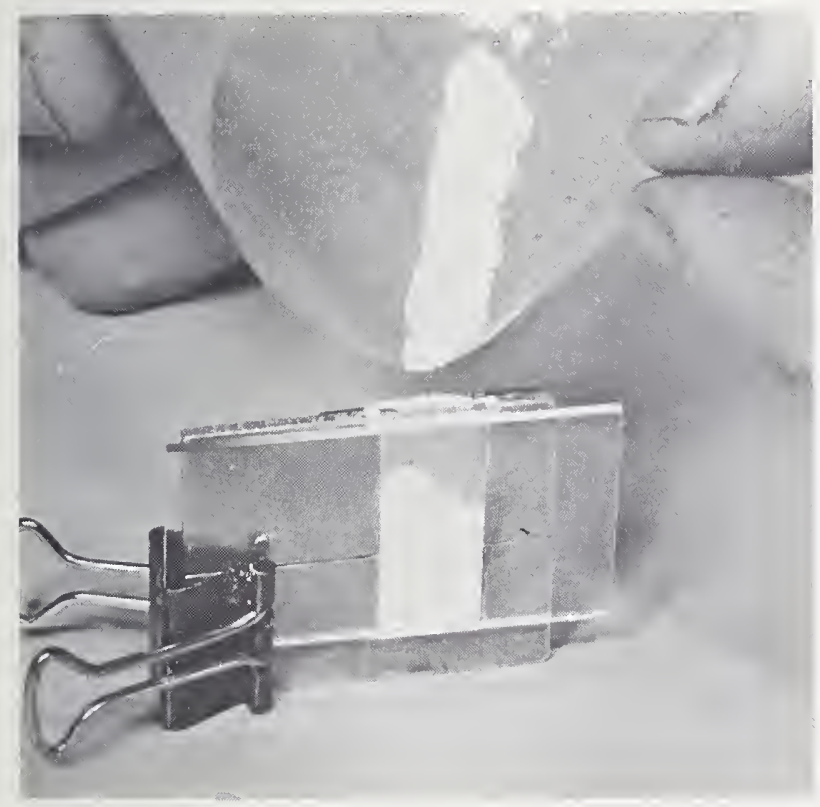

Figure 1

\section{REFERENCES}

Alexander, L., H. P. Klug, and E. Kummer (1948). Statistical factors affecting the intensity of $x$-rays diffracted by crystalline powders, J. Appl. Phvs. 19, No. 8, 742-753.

Bearden, J. A. (1964). X-ray Wavelengths, U.S. A.t. Energy Comm. NYO-10586.

Evans, H. T. Jr., D. E. Appleman, and D. S. Handwerker (1963). The least-squares refinement of crystal unit cells with powder diffraction data by an automatic computer indexing method, (abs.) Am. Crystal. Assoc. Annual Meeting, Cambridge, Mass. Program 42-43.

International Tables for X-ray Crystallography, I (1952). (The Kynoch Press, Birmingham, England.) Ibid. III (1962). Pgs. 202, 210,213, 214

International Union of Pure and Applied Chemistry, (1961). Chem. Eng. News. 39(4), Nov. 20, 42.

ISCC-NBS Centroid Color Charts, Standard Sample No. 2106, obtained from the Office of StandardReference Materials, Room B328, Chemistry Building, National Bureau of Standards, Washington, D.C. 20234. \$3.00 per set.

Palache, C., H. Berman, and C. Frondel (1944). Dana's Sys tem of Mineralogy (John Wiley \& Sons, New York, 7th Ed.), I, 6

Powder Diffraction File (1968). American Society for Testing and Materials, 1916 Race St., Philadelphia, Pa, 19103.

Smith, D. K. (1967). A revised program for calculating x-ray powder diffraction patterns, UCRL 50264, University of California, Lawrence Radiation Laboratory, Livermore, Calif. 94551.

Swanson, H. E., M. C. Morris, and E. H. Evans (1966). Standard X-ray Diffraction Powder Patterns, Natl. Bur. Std. U.S. Monograph 25, Sec. 4, 3.

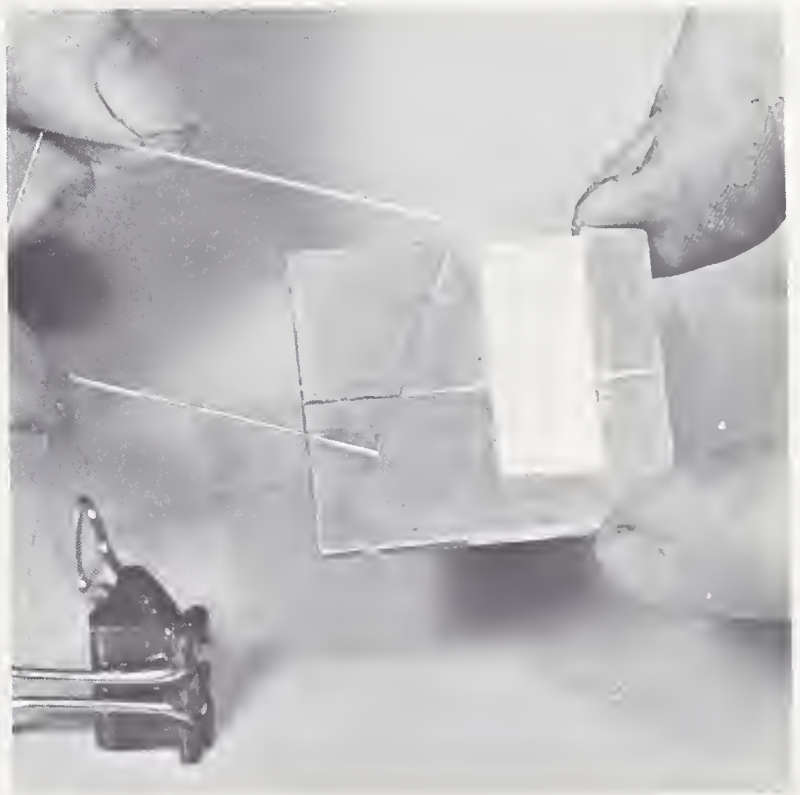

Fisure 2 



\section{Sample}

The sample was prepared at NBS by partial evaporation at $90^{\circ} \mathrm{C}$ of a water solution of $\left(\mathrm{NH}_{4}\right)_{2} \mathrm{SO}_{4}$ and $\mathrm{CdSO}_{4}$, in a $2: 1$ weight ratio. The resulting double salt was washed with water and alcohol.

\section{Major impurities}

0.001-0.01\% each: $\mathrm{Ca}, \mathrm{Mg}$, and $\mathrm{Mn}$

\section{Color}

Colorless

\section{Optical data}

Isotropic, $\mathrm{N}=1.603$

\section{Structure}

Cubic, $P 2_{1} 3$ (198) $\mathrm{Z}=4$, langbeinite type, [Gattow and Zemann, 1958].

\section{Lattice constants}

\begin{tabular}{|c|c|}
\hline & $a(\AA)$ \\
\hline $\begin{array}{l}\text { Jona and Pepinsky [1956] } \\
\text { Gattow and Zemann [1958] } \\
\text { NBS, sample at } 25{ }^{\circ} \mathrm{C}\end{array}$ & $\begin{array}{r}10.35 \\
\pm .005 \\
10.350 \\
\pm .003 \\
10.3511 \\
\pm .0001\end{array}$ \\
\hline
\end{tabular}

Density

(calculated) $3.288 \mathrm{~g} / \mathrm{cm}^{3}$ at $25^{\circ} \mathrm{C}$.

\section{Reference intensity}

$$
I / I_{\text {corundum }}=3.8
$$

\section{Polymorphism}

Inverts below $-186^{\circ} \mathrm{C}$ to a ferroelectric form [Jona and Pepinsky, 1956].

\section{References}

Gattow, G.and J.Zemann(1958). Über Doppelsulfate vom Langbeinit-Typ, $\mathrm{A}_{2}^{+} \mathrm{B}_{2}^{2+}\left(\mathrm{SO}_{4}\right)_{3}$, Z. Anorg. Allgem. Chem. 293, 233-240.

Jona, F. and R. Pepinsky (1956). Ferroelectricity in the langbeinite system, Phy. Rev. 103, 1126.

\begin{tabular}{|c|c|c|c|}
\hline \multicolumn{4}{|c|}{$\begin{array}{l}\text { Internal standard } \mathrm{W}, \mathrm{a}=3.16516 \AA \\
\mathrm{CuK} \alpha_{1} \quad \lambda=1.54056 \AA \text {; temp. } 25{ }^{\circ} \mathrm{C}\end{array}$} \\
\hline$d(\AA)$ & $I$ & $h k l$ & $2 \theta(\circ)$ \\
\hline 5.973 & 50 & 111 & 14.82 \\
\hline 4.628 & 63 & 210 & 19.16 \\
\hline 4.225 & 11 & 211 & 21.01 \\
\hline 3.449 & 20 & 221 & 25.81 \\
\hline 3.271 & 100 & 310 & 27.24 \\
\hline 3.121 & 11 & 311 & 28.58 \\
\hline 2.870 & 9 & 320 & 31.14 \\
\hline 2.765 & 65 & 321 & 32.35 \\
\hline 2.587 & 1 & 400 & 34.65 \\
\hline 2.511 & 9 & 410 & 35.73 \\
\hline 2.441 & 1 & 411 & 36.79 \\
\hline 2.375 & 5 & 331 & 37.85 \\
\hline 2.259 & 6 & 421 & 39.88 \\
\hline 2.207 & 1 & 332 & 40.86 \\
\hline 2.113 & 20 & 422 & 42.76 \\
\hline 2.071 & 10 & 430 & 43.68 \\
\hline 2.030 & 20 & 510 & 44.59 \\
\hline 1.993 & 13 & 511 & 45.48 \\
\hline 1.922 & 18 & 520 & 47.26 \\
\hline 1.890 & 1 & 521 & 48.10 \\
\hline 1.831 & 1 & 440 & 49.77 \\
\hline 1.801 & 10 & 522 & 50.64 \\
\hline 1.774 & 6 & 530 & 51.46 \\
\hline 1.750 & 1 & 531 & 52.22 \\
\hline 1.703 & 1 & 610 & 53.80 \\
\hline 1.6788 & 17 & 611 & 54.62 \\
\hline 1.6364 & 7 & 620 & 56.16 \\
\hline 1.6166 & 8 & 621 & 56.91 \\
\hline 1.5974 & 6 & 541 & 57.66 \\
\hline 1.5786 & 2 & 533 & 58.41 \\
\hline 1.5606 & 1 & 622 & 59.15 \\
\hline 1.5433 & 3 & 630 & 59.88 \\
\hline 1.5260 & 7 & 631 & 60.63 \\
\hline 1.4938 & 2 & 444 & 62.08 \\
\hline 1.4 .784 & 5 & 632 & 62.80 \\
\hline 1.4640 & 5 & 710 & 63.49 \\
\hline 1.4497 & 3 & 711 & 64.19 \\
\hline 1.4360 & 1 & 640 & 64.88 \\
\hline 1.4219 & 5 & 720 & 65.60 \\
\hline 1.4086 & 3 & 721 & 66.30 \\
\hline
\end{tabular}




\begin{tabular}{|c|c|c|c|c|c|c|c|}
\hline \multicolumn{4}{|c|}{$\begin{array}{l}\text { Internal standard } W, a=3.16516 \AA \\
\text { CuK } a_{1} \lambda=1.54056 \AA \text {; temp. } 25^{\circ} \mathrm{C}\end{array}$} & \multicolumn{4}{|c|}{$\begin{array}{l}\text { Internal standard } \mathrm{W}, \mathrm{a}=3.16516 \AA \\
\text { CuKa }{ }_{1} \quad \lambda=1.54056 \AA \text {; temp. } 25{ }^{\circ} \mathrm{C}\end{array}$} \\
\hline$d(\AA)$ & $I$ & $h k l$ & $2 \theta(0)$ & $d(\AA)$ & $I$ & $h k l$ & $2 \theta\left({ }^{\circ}\right)$ \\
\hline 1.3832 & 2 & 642 & 67.68 & .9654 & $<1$ & 953 & 105.86 \\
\hline 1.3709 & 2 & 722 & 68.37 & .9611 & 1 & $10 \cdot 4 \cdot 0$ & 105.54 \\
\hline 1.3591 & 1 & 730 & 69.05 & .9569 & 1 & $10 \cdot 4 \cdot 1$ & 107.21 \\
\hline 1.3478 & 1 & 731 & 69.71 & .9528 & 1 & $10 \cdot 3 \cdot 3$ & 107.88 \\
\hline 1.3252 & 2 & 650 & 71.08 & .9449 & 1 & $10 \cdot 4 \cdot 2$ & 109.21 \\
\hline 1.3144 & 2 & 732 & 71.75 & .9410 & 1 & 962 & 109.89 \\
\hline 1.2841 & 3 & 810 & 73.72 & .9371 & 1 & $11^{\circ} 1 \cdot 0^{\circ}$ & 110.57 \\
\hline 1.2742 & 2 & 811 & 74.39 & .9333 & 1 & $11 \cdot 1 \cdot 1$ & 111.25 \\
\hline 1.2649 & 1 & 733 & 75.03 & .9257 & 2 & $11 \cdot 2 \cdot 0$ & 112.63 \\
\hline 1.2556 & 1 & 820 & 75.68 & .9221 & 1 & $11 \cdot 2 \cdot 1$ & 113.30 \\
\hline 1.2463 & 1 & 821 & 76.35 & .9150 & $<1$ & 880 & 114.67 \\
\hline 1.2374 & 1 & 653 & 77.00 & .9113 & 2 & $11 \cdot 2 \cdot 2$ & 115.40 \\
\hline 1.2203 & 3 & 822 & 78.28 & .9078 & 1 & $11 \cdot 3 \cdot 0$ & 116.11 \\
\hline 1.2114 & 1 & 830 & 78.96 & .9042 & 1 & $11 \cdot 3 \cdot 1$ & 116.83 \\
\hline 1.2034 & 4 & 831 & 79.60 & .9009 & 1 & $10 \cdot 4 \cdot 4$ & 117.53 \\
\hline 1.1952 & 3 & 751 & 80.25 & .8976 & 1 & 964 & 118.23 \\
\hline 1.1796 & 2 & 832 & 81.54 & .8943 & 2 & $11 \cdot 3 \cdot 2$. & 118.94 \\
\hline 1.1721 & 2 & 752 & 82.17 & .8876 & 1 & $10 \cdot 6 \cdot 0$ & 120.41 \\
\hline 1.1573 & 1 & 840 & 83.45 & .8844 & 1 & $11 \cdot 4 \cdot 0$ & 121.14 \\
\hline 1.1502 & 1 & 841 & 84.09 & .8813 & $<1$ & $11 \cdot 4 \cdot 1$ & 121.87 \\
\hline 1.1428 & $<1$ & 910 & 84.76 & .8780 & 1 & $11 \cdot 3 \cdot 3$ & 122.64 \\
\hline 1.1362 & 1 & 911 & 85.37 & .8716 & 1 & $11 \cdot 4 \cdot 2$ & 124.19 \\
\hline 1.1294 & 1 & 842 & 86.00 & .8687 & $<1$ & 965 & 124.92 \\
\hline 1.1225 & 1 & 920 & 86.66 & .8626 & 1 & $12 \cdot 0 \cdot 0$ & 126.49 \\
\hline 1.1160 & 3 & 921 & 87.29 & .8596 & 2 & $12 \cdot 1 \cdot 0$ & 127.29 \\
\hline 1.1034 & 1 & 664 & 88.55 & .8567 & 2 & $12 \cdot 1 \cdot 1$ & 128.09 \\
\hline 1.0971 & 4 & 922 & 89.19 & .8538 & 2 & $11 \cdot 5 \cdot 1$ & 128.90 \\
\hline 1.0910 & 2 & 930 & 89.83 & .8480 & 2 & $12 \cdot 2 \cdot 1$ & 130.55 \\
\hline 1.0849 & 1 & 931 & 90.47 & .8451 & 1 & $11 \cdot 5 \cdot 2$ & 131.41 \\
\hline 1.0732 & 1 & 852 & 91.74 & .8395 & 1 & $12 \cdot 2 \cdot 2$ & 133.13 \\
\hline 1.0676 & 1 & 932 & 92.36 & .8369 & 2 & $12 \cdot 3 \cdot 0$ & 133.97 \\
\hline 1.0565 & 1 & 844 & 93.62 & .8341 & 1 & $12 \cdot 3 \cdot 1$ & 134.87 \\
\hline 1.0509 & $<1$ & 940 & 94.27 & .8315 & 1 & $11 \cdot 5 \cdot 3$ & 135.76 \\
\hline 1.0455 & 2 & 941 & 94.91 & .8261 & 1 & $12 \cdot 3 \cdot 2$ & 137.64 \\
\hline 1.0404 & 2 & 933 & 95.53 & .8235 & 2 & $11 \cdot 6 \cdot 1$ & 138.59 \\
\hline 1.0298 & 3 & $10 \cdot 1 \cdot 0$ & 96.83 & .8183 & 1 & $12 \cdot 4 \cdot 0$ & 140.53 \\
\hline 1.0249 & 2 & $10 \cdot 1 \cdot 1$ & $97: 45$ & .8158 & 2 & $12 \cdot 4 \cdot 1$ & 141.54 \\
\hline 1.0150 & $<1$ & $10 \cdot 2 \cdot 0$ & 98.73 & .8133 & 1 & $12 \cdot 3 \cdot 3$ & .142 .58 \\
\hline 1.0101 & 1 & $10 \cdot 2 \cdot 1$ & 99.38 & .8108 & 1 & 991 & 143.62 \\
\hline 1.0054 & 1 & 950 & 100.02 & .8083 & $<1$ & $12 \cdot 4 \cdot 2$ & 144.73 \\
\hline 1.0006 & 1 & 951 & 100.67 & .8059 & $<1$ & $10 \cdot 8 \cdot 1$ & 145.82 \\
\hline 0.9915 & $<1$ & $10 \cdot 3 \cdot 0$ & 101.95 & .8034 & 2 & $11 \cdot 6 \cdot 3$ & 146.98 \\
\hline .9869 & 1 & $10 \cdot 3 \cdot 1$ & 102.61 & .7986 & 1 & $10 \cdot 8 \cdot 2$ & 149.38 \\
\hline .9739 & 1 & $10 \cdot 3 \cdot 2$ & 104.55 & .7963 & 1 & $12 \cdot 5 \cdot 0$ & 150.65 \\
\hline .9694 & 1 & 871 & 105.24 & .7939 & 1 & $13 \cdot 1 \cdot 0$ & 152.00 \\
\hline
\end{tabular}


Sample

Needle shaped crystals were obtained after partial evaporation of a solution of equimolar amounts $\mathrm{NH}_{4} \mathrm{Cl}$ and $\mathrm{CuCl}_{2}$ in anhydrous ethyl alcohol. X-ray patterns were produced from samples in a dry-air mounting to prevent hydration.

Major impurities

0.001-0.01\% each: $\mathrm{Ca}, \mathrm{Cr}, \mathrm{Fe}$, and $\mathrm{Mg}$

$0.1-1.0 \%$ each: $\mathrm{Ni}$

\section{Color}

Moderate reddish brown

\section{Optical data}

Anisotropic, $\mathrm{N}_{\alpha}=1.660, \mathrm{~N}_{\gamma}=1.850$. Strongly pleochroic. Crystals were very fine.

\section{Structure}

Monoclinic, P2 /C (14) $\mathrm{z}=4$.Structure was determined by willett et al.,[1963]

\section{Lattice constants}

\begin{tabular}{|c|c|c|c|c|}
\hline & $a(\AA)$ & $b(\stackrel{\cap}{)}$ & $c(\stackrel{\circ}{A})$ & $\beta\left({ }^{\circ}\right)$ \\
\hline $\begin{array}{l}\text { Willett } \\
\text { et al.- } \\
{[1963]}\end{array}$ & $\begin{array}{l}4.066 \\
\pm .005\end{array}$ & $\begin{array}{r}14.189 \\
\pm .003\end{array}$ & $\begin{array}{l}9.003 \\
\pm .004\end{array}$ & $\begin{array}{r}97^{\circ} 30^{\prime} \\
\pm \quad 5^{\prime}\end{array}$ \\
\hline $\begin{array}{l}\text { NBS } \\
\text { sample } \\
\text { at } 25{ }^{\circ} \mathrm{C}\end{array}$ & $\begin{array}{l}4.030 \\
\pm .001\end{array}$ & $\begin{array}{r}14.187 \\
\pm .002\end{array}$ & $\begin{array}{r}8.978 \\
\pm .002\end{array}$ & $\begin{array}{r}96^{\circ} 28^{\prime} \\
\quad \pm I^{\prime}\end{array}$ \\
\hline
\end{tabular}

\section{Density}

(calculated) $2.447 \mathrm{~g} / \mathrm{cm}^{3}$ at $25^{\circ} \mathrm{C}$.

\section{Reference intensity}

$$
\mathrm{I} / \mathrm{I}_{\text {corundum }}=0.6
$$

\section{References}

Willett,R.D., C.Dwiggens Jr.,R.F. Kruh, and R. E. Rundle (1963). Crystal structures of $\mathrm{KCuCl}_{3}$ and $\mathrm{NH}_{4} \mathrm{CuCl}_{3}, \mathrm{~J} . \mathrm{Chem}$. Phys.38, 2429-2436.

\begin{tabular}{|c|c|c|c|}
\hline \multicolumn{4}{|c|}{$\begin{array}{l}\text { Internal standard } \mathrm{W}, \mathrm{a}=3.16516 \AA \\
\mathrm{CuK} a_{1} \quad \lambda=1.54056 \AA \text {; temp. } 25{ }^{\circ} \mathrm{C}\end{array}$} \\
\hline$d(\AA)$ & $I$ & $h k l$ & $2 \theta\left({ }^{\circ}\right)$ \\
\hline 7.54 & 88 & 011 & 11.73 \\
\hline 7.08 & 100 & 020 & 12.50 \\
\hline 5.552 & 58 & 021 & 15.95 \\
\hline 4.462 & 17 & 002 & 19.88 \\
\hline 4.257 & 10 & 012 & 20.85 \\
\hline 4.178 & 18 & 031 & 21.25 \\
\hline 3.688 & 47 & $\overline{1} 11$ & 24.11 \\
\hline 3.546 & 10 & 040 & 25.09 \\
\hline 3.244 & 17 & 032 & 27.47 \\
\hline 3.161 & 52 & $\overline{1} 02$ & 28.21 \\
\hline 3.088 & 35 & $\overline{1} 12$ & 28.89 \\
\hline 2.967 & 10 & $\underline{1} 31$ & 30.09 \\
\hline 2.888 & 49 & $\overline{1} 22$ & 30.94 \\
\hline 2.818 & 25 & 131 & 31.73 \\
\hline 2.777 & 58 & 042 & 32.21 \\
\hline 2.743 & 18 & 023 & 32.62 \\
\hline 2.704 & 22 & 051 & 33.10 \\
\hline 2.656 & 12 & 140 & 33.72 \\
\hline 2.626 & 28 & $\overline{1} 32,122$ & 34.11 \\
\hline 2.395 & 18 & 052 & 37.52 \\
\hline 2.363 & 8 & 060 & 38.05 \\
\hline 2.315 & 19 & 151 & 38.87 \\
\hline 2.286 & 25 & 061 & 39.39 \\
\hline 2.209 & 6 & 142 & 40.82 \\
\hline 2.127 & 11 & 024 & 42.47 \\
\hline 2.089 & 12 & 062 & 43.27 \\
\hline 2.058 & 16 & $\bar{I}_{43}$ & 43.95 \\
\hline 2.017 & 16 & 034 & 44.89 \\
\hline 1.976 & 10 & 071 & 45.88 \\
\hline 1.961 & 9 & 161 & 46.25 \\
\hline 1.911 & 15 & 143 & 47.53 \\
\hline 1.887 & 17 & $044, \overline{1} 53$ & 48.17 \\
\hline 1.846 & 13 & 114,072 & 49.32 \\
\hline 1.808 & 19 & 170 & 50.42 \\
\hline 1.791 & 11 & $\overline{1} 71$ & 50.94 \\
\hline 1.7739 & 11 & $\overline{1} 44,080$ & 51.47 \\
\hline 1.7549 & 11 & 171,202 & 52.07 \\
\hline 1.6746 & 5 & 073 & 54.77 \\
\hline
\end{tabular}




\section{Sample}

The material was crystallized at $100{ }^{\circ} \mathrm{C}$ from an aqueous solution of stoichiometric amounts of $\left(\mathrm{NH}_{4}\right)_{2} \mathrm{SO}_{4}$ and $\mathrm{MnSO}_{4}$.

\section{Color}

Colorless

\section{Optical data}

Isotropic, $\mathrm{N}=1.602$

\section{Structure}

Cubic, P2 3 (198), Z=4, langbeinite type, [Gattow and Zemann, 1958].The langbeinite structure was described by Zemann and Zemann [1957].

\section{Lattice constants}

\begin{tabular}{|l|c|}
\hline \multirow{2}{*}{ Gattow and Zemann [1958]------- } & $a(\AA)$ \\
\cline { 2 - 2 } NBS, sample at $25{ }^{\circ} \mathrm{C}-----------$ & $\begin{array}{c}10.003 \\
10.1892 \\
\end{array}$ \\
\hline
\end{tabular}

\section{Density}

(calculated) $2.726 \mathrm{~g} / \mathrm{cm}^{3}$ at $25^{\circ} \mathrm{C}$.

\section{Reference intensity}

$\mathrm{I} / \mathrm{I}_{\text {corundum }}=2.4$

\section{References}

Gattow, G. and J. Zemann (1958). Über Doppelsulfate vom Langbeinit-Typ, $\mathrm{A}_{2}^{+} \mathrm{B}_{2}^{2+}-$ $\left(\mathrm{SO}_{4}\right)_{3}, \mathrm{Z}$. Anorg. Allgem. Chem.293, 233240 .

Zemann,A. and J. Zemann (1957). Die' Kristallstruktur vom Langbeinit, $\mathrm{K}_{2} \mathrm{Mg}_{2}\left(\mathrm{SO}_{4}\right)_{3}$ Acta Cryst. 10, 409-413.

\begin{tabular}{|c|c|c|c|}
\hline \multicolumn{4}{|c|}{$\begin{array}{l}\text { Internal standard } \mathrm{W}, \mathrm{a}=3.16516 \AA \\
\mathrm{CuKa} a_{1} \quad \lambda=1.54056 \AA \text {; temp. } 25^{\circ} \mathrm{C}\end{array}$} \\
\hline$d(\AA)$ & $I$ & $h k l$ & $2 \theta\left({ }^{\circ}\right)$ \\
\hline 5.87 & 33 & 111 & 15.07 \\
\hline 4.55 & 23 & 210 & 19.49 \\
\hline 4.156 & 25 & 211 & 21.36 \\
\hline 3.600 & 5 & 220 & 24.71 \\
\hline 3.396 & 14 & 221 & 26.22 \\
\hline 3.220 & 100 & 310 & 27.68 \\
\hline 3.071 & 13 & 311 & 29.05 \\
\hline 2.825 & 6 & 320 & 31.65 \\
\hline 2.722 & 55 & 321 & 32.88 \\
\hline 2.473 & 4 & 410 & 36.30 \\
\hline 2.402 & 2 & 411 & 37.41 \\
\hline 2.338 & 3 & 331 & 38.48 \\
\hline 2.223 & 4 & 421 & 40.54 \\
\hline 2.172 & 2 & 332 & 41.54 \\
\hline 2.080 & 13 & 422 & 43.48 \\
\hline 2.038 & 7 & 430 & 44.41 \\
\hline 1.999 & 9 & 510 & 45.34 \\
\hline 1.961 & 7 & 511 & 46.25 \\
\hline 1.892 & 17 & 520 & 48.05 \\
\hline 1.861 & 4 & 521 & 48.91 \\
\hline 1.774 & 14 & 522 & 51.47 \\
\hline 1.748 & 5 & 530 & 52.30 \\
\hline 1.722 & $<1$ & 531 & 53.15 \\
\hline 1.699 & $<1$ & 600 & 53.91 \\
\hline 1.6749 & 3 & 610 & 54.76 \\
\hline 1.6527 & 17 & 611 & 55.56 \\
\hline 1.6109 & 5 & 620 & 57.13 \\
\hline 1.5916 & 4 & 621 & 57.89 \\
\hline 1.5728 & 3 & 541 & 58.65 \\
\hline 1.5540 & 1 & 533 & 59.43 \\
\hline 1.5362 & $<1$ & 622 & 60.19 \\
\hline 1.5193 & 7 & 630 & 60.93 \\
\hline 1.5023 & 7 & 631 & 61.69 \\
\hline 1.4711 & 2 & 444 & 63.15 \\
\hline 1.4560 & 5 & 632 & 63.88 \\
\hline 1.4413 & 4 & 710 & 64.61 \\
\hline 1.4272 & 2 & 711 & 65.33 \\
\hline 1.4128 & 2 & 640 & 66.08 \\
\hline 1.3997 & 5 & 720 & 66.78 \\
\hline 1.3866 & 4 & 721 & 67.49 \\
\hline
\end{tabular}




\begin{tabular}{|c|c|c|c|}
\hline \multicolumn{4}{|c|}{$\begin{array}{l}\text { Internal standard } \mathrm{W}, \mathrm{a}=3.16516 \AA \\
\mathrm{CuK} a_{1} \lambda=1.54056 \AA \text {; temp. } 25^{\circ} \mathrm{C}\end{array}$} \\
\hline$d(\AA ̊)$ & $I$ & $h k l$ & $2 \theta\left({ }^{\circ}\right)$ \\
\hline 1.3617 & 3 & 642 & 68.90 \\
\hline 1.3498 & 2 & 722 & 69.59 \\
\hline 1.3378 & 1 & 730 & 70.31 \\
\hline 1.3266 & 4 & 731 & 70.99 \\
\hline 1.3047 & 2 & 650 & 72.37 \\
\hline 1.2942 & 2 & 732 & 73.05 \\
\hline 1.2733 & $<1$ & 800 & 74.45 \\
\hline 1.2639 & 3 & 810 & 75.10 \\
\hline 1.2544 & 2 & 811 & 75.77 \\
\hline 1.2446 & 1 & 733 & 76.47 \\
\hline 1.2356 & 1 & 820 & 77.13 \\
\hline 1.2268 & 1 & 821 & 77.79 \\
\hline 1.2180 & 1 & 653 & 78.46 \\
\hline 1.2008 & 2 & 822 & 79.80 \\
\hline 1.1926 & 1 & 830 & 80.46 \\
\hline 1.1844 & 4 & 831 & 81.14 \\
\hline 1.1765 & 3 & 751 & 81.80 \\
\hline 1.1611 & 2 & 832 & 83.12 \\
\hline 1.1536 & 2 & 752 & 83.78 \\
\hline 1.1392 & $<1$ & 840 & 85.09 \\
\hline 1.1321 & 1 & 841 & 85.75 \\
\hline 1.1250 & $<1$ & 910 & 86.42 \\
\hline 1.1184 & 1 & 911 & 87.06 \\
\hline 1.1118 & 1 & 842 & 87.71 \\
\hline 1.1053 & 1 & 920 & 88.36 \\
\hline 1.0987 & 3 & 921 & 89.03 \\
\hline 1.0860 & 1 & 664 & 90.35 \\
\hline 1.0800 & 3 & 922 & 91.00 \\
\hline 1.0740 & 3 & 930 & 91.65 \\
\hline 1.0681 & 2 & 931 & 92.30 \\
\hline 1.0567 & 1 & 852 & 93.60 \\
\hline 1.0510 & 2 & 932 & 94.26 \\
\hline 1.0399 & 1 & 844 & 95.59 \\
\hline 1.0345 & 1 & 940 & 96.25 \\
\hline 1.0293 & 2 & 941 & 96.90 \\
\hline 1.0241 & 1 & 932 & 97.56 \\
\hline 1.0189 & 1 & $10 \cdot 0 \cdot 0$ & 98.23 \\
\hline 1.0138 & 4 & $10 \cdot 1 \cdot 0$ & 98.89 \\
\hline 1.0089 & 2 & $10 \cdot 1 \cdot 1$ & 99.55 \\
\hline 0.9993 & 1 & $10 \cdot 2 \cdot 0$ & 100.86 \\
\hline
\end{tabular}

\begin{tabular}{|c|c|c|c|}
\hline \multicolumn{4}{|c|}{$\begin{array}{l}\text { Internal standard } W, a=3.16516 \AA \\
\text { CuKa } a_{1} \lambda=1.54056 \AA \text {; temp. } 25{ }^{\circ} \mathrm{C}\end{array}$} \\
\hline$d(\stackrel{\AA}{A})$ & $I$ & $h k l$ & $2 \theta\left({ }^{\circ}\right)$ \\
\hline .9943 & 3 & $10 \cdot 2 \cdot 1$ & 101.55 \\
\hline .9897 & 2 & 950 & 102.21 \\
\hline .9851 & 1 & 951 & 102.87 \\
\hline .9805 & $<1$ & $10 \cdot 2 \cdot 2$ & 103.55 \\
\hline .9760 & 2 & $10 \cdot 3 \cdot 0$ & 104.23 \\
\hline .9714 & 2 & $10 \cdot 3 \cdot 1$ & 104.92 \\
\hline .9585 & 2 & $10 \cdot 3 \cdot 2$ & 106.95 \\
\hline .9544 & 2 & 871 & 107.63 \\
\hline .9503 & 2 & 953 & 108.31 \\
\hline .9460 & 1 & $10 \cdot 4 \cdot 0$ & 109.02 \\
\hline .9420 & 2 & 960 & 109.72 \\
\hline .9380 & 1 & $10 \cdot 3 \cdot 3$ & 110.41 \\
\hline .9302 & 1 & $10 \cdot 4 \cdot 2$ & 111.80 \\
\hline .9264 & 1 & 962 & 112.50 \\
\hline .9224 & 1 & $11 \cdot 1 \cdot 0$ & 113.25 \\
\hline .9188 & $<1$ & $11 \cdot 1 \cdot 1$ & 113.93 \\
\hline .9114 & 1 & $11 \cdot 2 \cdot 0$ & 115.38 \\
\hline .9078 & 1 & $11 \cdot 2 \cdot 1$ & 116.10 \\
\hline .9006 & 1 & 880 & 117.59 \\
\hline .8971 & 2 & $11 \cdot 2 \cdot 2$ & 118.32 \\
\hline .8938 & 1 & $11 \cdot 3 \cdot 0$ & 119.05 \\
\hline .8902 & $<1$ & $11 \cdot 3 \cdot 1$ & 119.82 \\
\hline .8868 & 1 & $10 \cdot 4 \cdot 4$ & 120.59 \\
\hline .8835 & 2 & 964 & 121.35 \\
\hline .8802 & 3 & $11 \cdot 3 \cdot 2$ & 122.11 \\
\hline .8737 & 1 & $10 \cdot 6 \cdot 0$ & 123.68 \\
\hline .8705 & 1 & $11 \cdot 4 \cdot 0$ & 124.48 \\
\hline .8673 & 2 & $11 \cdot 4 \cdot 1$ & 125.28 \\
\hline .8642 & 2 & $11 \cdot 3 \cdot 3$ & 126.09 \\
\hline .8581 & 1 & $11 \cdot 4 \cdot 2$ & 127.71 \\
\hline .8550 & 1 & 965 & 128.55 \\
\hline .8491 & 1 & $12 \cdot 0 \cdot 0$ & 130.24 \\
\hline
\end{tabular}




\section{Sample}

The sample was prepared at NBS by $E$. Levin. Stoichiometric amounts of barium nitrate and boric acid were ground together and heated near $800^{\circ} \mathrm{C}$.

\section{Color}

Colorless

\section{Optical data}

Biaxial (-) $\mathrm{N}_{\alpha}=1.558, \mathrm{~N}_{\gamma}=1.590, \quad 2 \mathrm{~V}$ is small.

\section{Structure}

Orthorhombic, $\mathrm{Z}=8$ [Krogh-Moe, 1960].

\section{Lattice constants}

\begin{tabular}{|c|c|c|c|}
\hline & $a(\stackrel{\circ}{A})$ & $b(\stackrel{\circ}{A})$ & $c(\stackrel{\circ}{A})$ \\
\hline $\begin{array}{l}\text { Krogh-Moe }[1960]-- \\
\text { NBS, } \\
\text { sample at } 25^{\circ} \mathrm{C}--\end{array}$ & $\begin{array}{l}8.56 \\
8.550 \\
\pm .001\end{array}$ & $\begin{array}{r}17.38 \\
17.352 \\
\pm .002\end{array}$ & $\begin{array}{r}13.20 \\
13.211 \\
\pm .003\end{array}$ \\
\hline
\end{tabular}

Density

(calculated) $2.927 \mathrm{~g} / \mathrm{cm}^{3}$ at $25^{\circ} \mathrm{C}$.

\section{Reference intensity}

$$
1 / I_{\text {corundum }}=1.4
$$

\section{Polymorphism}

A high temperature tetragonal form is being studied by Levin and Robbins [private communication]

\section{Additional patterns}

1. PDF card 6-0277 [McMurdie and Levin, 1949].

\begin{tabular}{|c|c|c|c|}
\hline \multicolumn{4}{|c|}{$\begin{array}{l}\text { Internal standard } \mathrm{Ag}, a=4.08641 \AA \\
\mathrm{CuK} a_{1} \quad \lambda=1.54056 \AA \text {; temp. } 25{ }^{\circ} \mathrm{C}\end{array}$} \\
\hline$d(\stackrel{\cap}{)}$ & $I$ & $h k l$ & $2 \theta\left({ }^{\circ}\right)$ \\
\hline 7.25 & 10 & 021 & 12.20 \\
\hline 6.09 & 55 & 120 & 14.54 \\
\hline 5.24 & 100 & 102 & 16.92 \\
\hline 4.35 & 6 & 032,040 & 20.42 \\
\hline 4.277 & 5 & 200,013 & 20.75 \\
\hline 4.120 & 13 & 041 & 21.55 \\
\hline 4.068 & 8 & 201 & 21.83 \\
\hline 3.916 & 6 & 103 & 22.69 \\
\hline 3.872 & 13 & 132,140 & 22.95 \\
\hline 3.836 & 6 & 220 & 23.17 \\
\hline 3.714 & 13 & 141 & 23.94 \\
\hline 3.682 & 5 & 221 & 24.15 \\
\hline 3.625 & 5 & 042 & 24.54 \\
\hline 3.590 & 7 & 202 & 24.78 \\
\hline 3.572 & 9 & 123 & 24.91 \\
\hline 3.515 & 2 & 212 & 25.32 \\
\hline 3.337 & 95 & 142 & 26.69 \\
\hline 3.316 & 100 & 222 & 26.86 \\
\hline 3.124 & 3 & 151 & 28.55 \\
\hline 3.081 & 14 & 104 & 28.96 \\
\hline 3.047 & 30 & 232,240 & 29.29 \\
\hline 2.965 & 2 & 241 & 30.12 \\
\hline 2.904 & 805 & 124 & 30.77 \\
\hline 2.895 & 80 & 060,223 & 30.86 \\
\hline 2.826 & 4 & 061 & 31.64 \\
\hline 2.740 & 25 & 160 & 32.65 \\
\hline 2.709 & 25 & 233,320 & 33.04 \\
\hline 2.683 & II & 161 & 33.37 \\
\hline 2.650 & 16 & 321,062 & 33.80 \\
\hline 2.617 & 25 & 302 & 34.24 \\
\hline 2.531 & 5 & 162 & 35.43 \\
\hline 2.524 & 6 & 105 & 35.54 \\
\hline 2.510 & 7 & 144,331 & 35.75 \\
\hline 2.502 & 9 & 243,224 & 35.86 \\
\hline 2.418 & 5 & 063 & 37.15 \\
\hline 2.393 & 4 & 303,054 & 37.56 \\
\hline 2.380 & 3 & $234,170,+$ & 37.76 \\
\hline 2.346 & 3 & 341,171 & 38.34 \\
\hline 2.326 & 4 & 163 & 38.68 \\
\hline 2.306 & 4 & 323 & 39.02 \\
\hline
\end{tabular}


Barium Borate, $\mathrm{BaB}_{8} \mathrm{O}_{13}$ (orthorhombic) - continued

\begin{tabular}{|c|c|c|c|}
\hline \multicolumn{4}{|c|}{$\begin{array}{l}\text { Internal standard } \mathrm{Ag}, \mathrm{a}=4.08641 \AA \\
\mathrm{CuK} a_{1} \quad \lambda=1.54056 \AA \text { 的 }\end{array}$} \\
\hline$d(\AA)$ & $I$ & $h k l$ & $2 \theta\left({ }^{\circ}\right)$ \\
\hline 2.251 & 30 & 262 & 40.02 \\
\hline 2.241 & 50 & 342,172 & 40.20 \\
\hline 2.238 & 50 & 244 & 40.27 \\
\hline 2.169 & 14 & 080 & 41.60 \\
\hline 2.138 & 20 & 400 & 42.23 \\
\hline 2.132 & 25 & 026,106 & 42.35 \\
\hline 2.122 & 8 & 410 & 42.56 \\
\hline 2.111 & 11 & 401,164 & 42.81 \\
\hline 2.105 & 17 & $263,180,+$ & 42.93 \\
\hline 2.094 & 19 & $173,324,+$ & 43.17 \\
\hline 2.076 & 7 & 181,420 & 43.57 \\
\hline 2.050 & 6 & 421 & 44.13 \\
\hline 2.033 & 3 & 402 & 44.52 \\
\hline 2.003 & 10 & 182,136 & 45.24 \\
\hline 1.980 & 12 & 422 & 45.79 \\
\hline 1.945 & 5 & 083,216 & 46.67 \\
\hline 1.938 & 6 & 264,305 & 46.83 \\
\hline 1.935 & 7 & 280 & 46.91 \\
\hline 1.922 & 5 & 403 & 47.25 \\
\hline 1.918 & 7 & 432,440 & 47.37 \\
\hline 1.913 & 11 & $281,146,+$ & 47.48 \\
\hline 1.907 & 13 & 091 & 47.64 \\
\hline 1.898 & 6 & 441,183 & 47.88 \\
\hline 1.878 & 2 & 423,017 & 48.43 \\
\hline 1.856 & 3 & 282 & 49.04 \\
\hline 1.841 & 2 & 442 & 49.46 \\
\hline 1.813 & 3 & 084 & 50.28 \\
\hline 1.794 & 4 & 404,037 & 50.85 \\
\hline 1.774 & 5 & 265,184 & 51.46 \\
\hline 1.769 & 5 & 345,175 & 51.61 \\
\hline 1.741 & 5 & 306,291 & 52.51 \\
\hline 1.729 & 6 & 193,364 & 52.92 \\
\hline 1.719 & 3 & 460,217 & 53.25 \\
\hline 1.712 & 3 & 381 & 53.49 \\
\hline 1.702 & 3 & $510,1 \cdot 10 \cdot 0$ & 53.83 \\
\hline 1.687 & 5 & $511,1 \cdot 10 \cdot 1$ & 54.34 \\
\hline 1.679 & 7 & $0 \cdot 10 \cdot 2,520$ & 54.61 \\
\hline 1.670 & 6 & $382,284,+$ & 54.95 \\
\hline 1.665 & 9 & $094,275,+$ & 55.11 \\
\hline 1.657 & 7 & 502 & 55.42 \\
\hline
\end{tabular}

\section{References}

Krogh-Moe, J. (1960). A note on the structure of barium tetraborate, Acta Chem. Scand. 14, No. 5, 1229-1230.

Levin, E.M. and H.F. McMurdie (1949). The system $\mathrm{BaO}-\mathrm{B}_{2} \mathrm{O}_{3}, \mathrm{~J}$. Res. Natl. Bur. Std. 42, (RPI956) 13I-138. 


\section{Sample}

The sample was prepared by melting a stoichiometric mixture of $\mathrm{CsaSO}_{4}$ and $\mathrm{CaSO}_{4}$ followed by annealing for 18 hours at $700{ }^{\circ} \mathrm{C}$.

\section{Color}

Colorless

\section{Optical data}

Isotropic, $\mathrm{N}=1.549$

\section{Structure}

Cubic, P2 3 (198), Z=4, langbeinite type [Gattow and Zemann, 1958]. The langbeinite structure was determined by Zemann and Zemann [1957].

\section{Lattice constants}

\begin{tabular}{|c|c|}
\hline & $a(\AA)$ \\
\hline 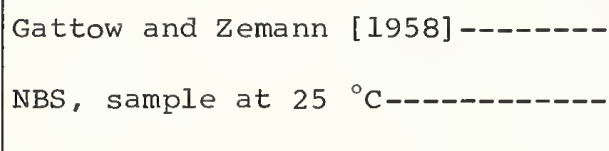 & $\begin{array}{r}10.724 \\
\pm .005 \\
10.7213 \\
\pm .0001\end{array}$ \\
\hline
\end{tabular}

\section{Density}

(calculated) $3.417 \mathrm{~g} / \mathrm{cm}^{3}$ at $25^{\circ} \mathrm{C}$.

Reference intensity

$\mathrm{I} / \mathrm{I}_{\text {corundum }}=4.8$

\section{References}

Gattow, G. and J. Zemann (1958). Über Doppelsulfate vom Langbeinit-typ, $\mathrm{A}_{2}^{+} \mathrm{B}_{2}^{2+}-$ $\left(\mathrm{SO}_{4}\right)_{3}, \mathrm{Z}$. Anorg. Allgem. Chem. 293, 233240 .

Zemann, A. and J. Zemann (1957). Die Kristallstructur vom Langbeinit, $\mathrm{K}_{2} \mathrm{Mg}_{2}\left(\mathrm{SO}_{4}\right)_{3}$, Acta Cryst. 10, 409-413.

\begin{tabular}{|c|c|c|c|}
\hline \multicolumn{4}{|c|}{$\begin{array}{l}\text { Internal standard Ag, } a=4.08641 \AA \\
\text { CuK } \alpha_{1} \quad \lambda=1.54056 \AA \text {; temp. } 25{ }^{\circ} \mathrm{C}\end{array}$} \\
\hline$d(\AA)$ & $I$ & $h k l$ & $2 \theta\left({ }^{\circ}\right)$ \\
\hline 6.18 & 16 & 111 & 14.32 \\
\hline 4.79 & 9 & 210 & 18.51 \\
\hline 4.373 & 7 & 211 & 20.29 \\
\hline 3.792 & 12 & 220 & 23.44 \\
\hline 3.574 & 8 & 221 & 24.89 \\
\hline 3.391 & 100 & 310 & 26.26 \\
\hline 3.234 & 18 & 311 & 27.56 \\
\hline 3.095 & 1 & 222 & 28.82 \\
\hline 2.973 & 21 & 320 & 30.03 \\
\hline 2.865 & 53 & 321 & 31.19 \\
\hline 2.599 & 23 & 410 & 34.48 \\
\hline 2.525 & 1 & 411 & 35.52 \\
\hline 2.460 & 8 & 331 & 36.50 \\
\hline 2.397 & 3 & 420 & 37.49 \\
\hline 2.339 & 7 & 421 & 38.45 \\
\hline 2.286 & 9 & 332 & 39.38 \\
\hline 2.188 & 16 & 422 & 41.22 \\
\hline 2.145 & 2 & 430 & 42.10 \\
\hline 2.102 & 30 & 510 & 43.00 \\
\hline 2.063 & 2 & 511 & 43.84 \\
\hline 1.991 & 8 & 520 & 45.52 \\
\hline 1.958 & 2 & 521 & 46.33 \\
\hline 1.897 & $<1$ & 440 & 47.92 \\
\hline 1.866 & 4 & 522 & 48.75 \\
\hline 1.839 & 1 & 530 & 49.52 \\
\hline 1.812 & 2 & 531 & 50.30 \\
\hline 1.788 & 1 & 600 & 51.05 \\
\hline 1.762 & 4 & 610 & 51.84 \\
\hline 1.739 & 17 & 611 & 52.59 \\
\hline 1.695 & 6 & 620 & 54.05 \\
\hline 1.675 & 9 & 621 & 54.77 \\
\hline 1.654 & 8 & 541 & 55.50 \\
\hline 1.635 & 2 & 533 & 56.23 \\
\hline 1.617 & 1 & 622 & 56.89 \\
\hline 1.598 & 7 & 630 & 57.64 \\
\hline 1.5806 & 5 & 631 & 58.33 \\
\hline 1.5471 & 4 & 444 & 59.72 \\
\hline 1.5318 & 2 & 632 & 60.38 \\
\hline 1.5163 & $I$ & 550 & 61.06 \\
\hline 1.5010 & 1 & 711 & 61.75 \\
\hline 1.4867 & $I$ & 640 & 62.41 \\
\hline 1.4723 & 2 & 720 & 63.09 \\
\hline 1.4589 & 6 & 721 & 63.74 \\
\hline 1.4328 & 4 & 642 & 65.04 \\
\hline 1.4208 & 1 & 722 & 65.66 \\
\hline
\end{tabular}


Cesium Calcium Sulfate, $\mathrm{Cs}_{2} \mathrm{Ca}_{2}\left(\mathrm{SO}_{4}\right)_{3}($ cubic $)$ - continued

\begin{tabular}{|c|c|c|c|}
\hline \multicolumn{4}{|c|}{$\begin{array}{l}\text { Internal standard Ag, } a=4.08641 \AA \\
\mathrm{CuK}_{a_{1}} \quad \lambda=1.54056 \AA \text { A; temp. } 25{ }^{\circ} \mathrm{C}\end{array}$} \\
\hline$d(\AA)$ & $I$ & $h k l$ & $2 \theta(\%)$ \\
\hline 1.4079 & 3 & 730 & 66.34 \\
\hline 1.3958 & 3 & 731 & 66.99 \\
\hline 1.3729 & 2 & 650 & 68.26 \\
\hline 1.3612 & 3 & 732 & 68.93 \\
\hline 1.3403 & 1 & 800 & 70.16 \\
\hline 1.3299 & 2 & 810 & 70.79 \\
\hline 1.3198 & 1 & 811 & 71.41 \\
\hline 1.3097 & 1 & 733 & 72.05 \\
\hline 1.3002 & 1 & 820 & 72.66 \\
\hline 1.2909 & 4 & 821 & 73.27 \\
\hline 1.2814 & 2 & 653 & 73.90 \\
\hline 1.2636 & 2 & 822 & 75.12 \\
\hline 1.2548 & 1 & 830 & 75.74 \\
\hline 1.2464 & 5 & 831 & 76.34 \\
\hline 1.2382 & 2 & 751 & 76.94 \\
\hline 1.2220 & $<1$ & 832 & 78.15 \\
\hline 1.2139 & 2 & 752 & 78.77 \\
\hline 1.1913 & 1 & 841 & 80.57 \\
\hline 1.1838 & 1 & 910 & 81.19 \\
\hline 1.1767 & 2 & 911 & 81.78 \\
\hline 1.1696 & 1 & 842 & 82.38 \\
\hline 1.1628 & 1 & 920 & 82.97 \\
\hline 1.1560 & 3 & 921 & 83.57 \\
\hline 1.1431 & 1 & 664 & 84.73 \\
\hline 1.1365 & 3 & 922 & $85 \cdot 34$ \\
\hline 1.1302 & 3 & 930 & 85.93 \\
\hline 1.1241 & $<1$ & 931 & 86.51 \\
\hline 1.1119 & 1 & 852 & 87.70 \\
\hline 1.1059 & 2 & 932 & 88.30 \\
\hline 1.0886 & 1 & 940 & 90.08 \\
\hline 1.0831 & 1 & 941 & 90.66 \\
\hline 1.0778 & 1 & 933 & 91.23 \\
\hline 1.0722 & 1 & $10 \cdot 0 \cdot 0$ & 91.85 \\
\hline 1.0668 & 1 & $10 \cdot 1 \cdot 0$ & 92.45 \\
\hline 1.0618 & $<1$ & $10 \cdot 1 \cdot 1$ & 93.01 \\
\hline 1.0514 & 1 & $10 \cdot 2 \cdot 0$ & 94.21 \\
\hline 1.0464 & 2 & $10 \cdot 2 \cdot 1$ & 94.81 \\
\hline 1.0415 & 2 & 950 & 95.39 \\
\hline 1.0365 & 1 & 951 & 96.00 \\
\hline 1.0317 & 1 & $10 \cdot 2 \cdot 2$ & 96.59 \\
\hline 1.0270 & 1 & $10 \cdot 3 \cdot 0$ & 97.19 \\
\hline 1.0221 & 2 & $10 \cdot 3 \cdot 1$ & 97.81 \\
\hline 1.0086 & 1 & $10 \cdot 3 \cdot 2$ & 99.59 \\
\hline 1.0041 & $<1$ & 871 & 100.19 \\
\hline 0.9998 & $<1$ & 953 & 100.79 \\
\hline
\end{tabular}

\begin{tabular}{|c|c|c|c|}
\hline \multicolumn{4}{|c|}{$\begin{array}{l}\text { Internal standard } \mathrm{Ag}, \mathrm{a}=4.08641 \AA \\
\mathrm{CuK} a_{1} \quad \lambda=1.54056 \AA \text { A; temp. } 25{ }^{\circ} \mathrm{C}\end{array}$} \\
\hline$d(\AA)$ & $I$ & $h k l$ & $2 \theta\left({ }^{\circ}\right)$ \\
\hline .9955 & 1 & $10 \cdot 4 \cdot 0$ & 101.39 \\
\hline .9912 & 1 & 960 & 102.00 \\
\hline .9871 & 1 & $10 \cdot 3 \cdot 3$ & 102.59 \\
\hline .9788 & 1 & $10 \cdot 4 \cdot 2$ & 103.81 \\
\hline .9746 & 1 & 962 & 104.44 \\
\hline .9707 & 2 & $11 \cdot 1 \cdot 0$ & 105.04 \\
\hline .9669 & 1 & $11 \cdot 1 \cdot 1$ & 105.63 \\
\hline .9590 & 1 & $11 \cdot 2 \cdot 0$ & 106.88 \\
\hline .9551 & 2 & $11 \cdot 2 \cdot 1$ & 107.51 \\
\hline .9475 & $<1$ & 880 & 108.78 \\
\hline .9439 & 1 & $11 \cdot 2 \cdot 2$ & 109.38 \\
\hline .9368 & 1 & $11 \cdot 3 \cdot 1$ & 110.62 \\
\hline .9332 & 1 & $10 \cdot 4 \cdot 4$ & 111.26 \\
\hline .9296 & $<1$ & 964 & 111.91 \\
\hline .9262 & 2 & $11 \cdot 3 \cdot 2$ & 112.54 \\
\hline .9192 & 1 & $10 \cdot 6 \cdot 0$ & 113.85 \\
\hline .9159 & 1 & $11 \cdot 4 \cdot 0$ & 114.49 \\
\hline .9127 & 1 & $11 \cdot 4 \cdot 1$ & 115.12 \\
\hline .9029 & 1 & $11 \cdot 4 \cdot 2$ & 117.11 \\
\hline .8998 & 1 & 965 & 117.76 \\
\hline .8934 & $<1$ & $12 \cdot 0 \cdot 0$ & 119.12 \\
\hline .8904 & 1 & $12 \cdot 1 \cdot 0$ & 119.79 \\
\hline .8873 & 1 & $12 \cdot 1 \cdot 1$ & 120.47 \\
\hline .8844 & 1 & $11 \cdot 5 \cdot 1$ & 121.15 \\
\hline .8814 & $<1$ & $12 \cdot 2 \cdot 0$ & 121.84 \\
\hline .8782 & 2 & $12 \cdot 2 \cdot 1$ & 122.58 \\
\hline .8754 & 2 & $11 \cdot 5 \cdot 2$ & 123.27 \\
\hline .8696 & 1 & $12 \cdot 2 \cdot 2$ & 124.70 \\
\hline .8668 & 1 & $12 \cdot 3 \cdot 0$ & 125.41 \\
\hline .8639 & 1 & $12 \cdot 3 \cdot 1$ & 126.17 \\
\hline .8612 & 1 & $11 \cdot 5 \cdot 3$ & 126.86 \\
\hline .8556 & $<1$ & $12 \cdot 3 \cdot 2$ & 128.40 \\
\hline .8529 & 1 & $11 \cdot 6 \cdot 1$ & 129.15 \\
\hline .8475 & 1 & $12 \cdot 4 \cdot 0$ & 130.70 \\
\hline .8449 & 1 & $12 \cdot 4 \cdot 1$ & 131.48 \\
\hline .8423 & 1 & $12 \cdot 3 \cdot 3$ & 132.25 \\
\hline .8398 & 1 & 991 & 133.04 \\
\hline .8371 & 1 & $12 \cdot 4 \cdot 2$ & 133.89 \\
\hline .8347 & $<1$ & $10 \cdot 8 \cdot 1$ & 134.70 \\
\hline .8321 & 2 & $11 \cdot 6 \cdot 3$ & 135.54 \\
\hline
\end{tabular}




\section{Sample}

The material was made by slow evaporation at room temperature of an equimolar solution of $\mathrm{Cs}_{2}\left(\mathrm{SO}_{4}\right)$ and $\mathrm{CuSO}_{4}$.

\section{Color}

Unground: brilliant greenish blue Ground: very pale greenish blue

\section{Optical data}

Biaxial (+) $\mathrm{N}_{\alpha}=1.504, \mathrm{~N}_{\beta}=1.506, \mathrm{~N}_{\gamma}=1.514$ $2 \mathrm{~V}$ is medium

\section{Structure}

Monoclinic, $\mathrm{P} 21 / \mathrm{a}$ (14), $\mathrm{Z}=2$.

$\mathrm{Cs}_{2} \mathrm{Cu}\left(\mathrm{SO}_{4}\right)_{2} \cdot 6 \mathrm{H}_{2} \mathrm{O}$ is a "Tutton Salt"[Tutton, 1893]. The structure of a "Tutton Salt", $\left(\mathrm{NH}_{4}\right)_{a} \mathrm{Mg}\left(\mathrm{SO}_{4}\right)_{2} \cdot 6 \mathrm{H}_{2} \mathrm{O}$ was determined by Margulis and Templeton, [1962] .

\section{Lattice constants}

\begin{tabular}{|l|c|r|c|c|}
\hline & $a(\AA)$ & $b(\AA)$ & $c(\AA)$ & $\beta\left({ }^{\circ}\right)$ \\
\cline { 2 - 5 } NBS, & & & & \\
sample at & & & & $106^{\circ} 1 I^{\prime}$ \\
$25^{\circ} \mathrm{C}----$ & 9.439 & $\begin{array}{r}12.762 \\
\pm .002\end{array}$ & $\begin{array}{l}6.310 \\
\pm .001\end{array}$ & $\pm I^{\prime}$ \\
\hline
\end{tabular}

\section{Density}

(calculated) $2.864 \mathrm{~g} / \mathrm{cm}^{3}$ at $25^{\circ} \mathrm{C}$.

\section{Reference intensity}

$$
1 / 1_{\text {corundum }}=2.2
$$

\begin{tabular}{|c|c|c|c|}
\hline \multicolumn{4}{|c|}{$\begin{array}{l}\text { Internal standard } \mathrm{Ag}, \mathrm{a}=4.08641 \AA \\
\mathrm{CuK} a_{1} \quad \lambda=1.54056 \AA \\
\AA\end{array}$} \\
\hline$d(\AA)$ & $I$ & $h k l$ & $2 \theta\left({ }^{\circ}\right)$ \\
\hline 7.37 & 11 & 110 & 11.98 \\
\hline 6.06 & 4 & 001 & 14.60 \\
\hline 5.47 & 4 & IIII & 16.18 \\
\hline 5.21 & 8 & 120 & 16.99 \\
\hline 4.54 & 6 & 200 & 19.55 \\
\hline 4.39 & 13 & 021 & 20.19 \\
\hline 4.31 & 40 & $\bar{I} 21$ & 20.61 \\
\hline 4.25 & 100 & $\overline{2} 01,111$ & 20.90 \\
\hline 4.026 & 14 & $\overline{2} 11$ & 22.06 \\
\hline 3.852 & 60 & 130 & 23.07 \\
\hline 3.696 & 5 & 220 & 24.06 \\
\hline 3.439 & 8 & $\overline{1} 31$ & 25.89 \\
\hline 3.227 & 25 & 201 & 27.62 \\
\hline 3.192 & 25 & 040 & 27.93 \\
\hline 3.126 & 16 & 211 & 28.53 \\
\hline 3.089 & 25 & 131 & 28.88 \\
\hline 3.030 & 25 & Ī12 & 29.16 \\
\hline 3.027 & 4 & 002 & 29.48 \\
\hline 2.984 & 12 & $\overline{3} 11$ & 29.92 \\
\hline 2.948 & 20 & 012 & 30.29 \\
\hline 2.939 & 20 & 310 & 30.39 \\
\hline 2.926 & 25 & $\overline{2} 02$ & 30.53 \\
\hline 2.878 & 15 & 221 & 31.05 \\
\hline 2.825 & 25 & $\overline{\mathrm{I}} 22,041$ & 31.65 \\
\hline 2.799 & 9 & $\overline{1} 41$ & 31.95 \\
\hline 2.765 & 10 & $\overline{3} 21$ & 32.35 \\
\hline 2.732 & 9 & 320 & 32.75 \\
\hline 2.658 & 5 & $\overline{2} 22$ & 33.69 \\
\hline 2.609 & 3 & 240 & 34.35 \\
\hline 2.603 & 4 & 112,141 & 34.43 \\
\hline 2.569 & 6 & 231 & 34.90 \\
\hline 2.553 & 6 & $\overline{2} 41$ & 35.16 \\
\hline 2.531 & 10 & İ32 & 35.43 \\
\hline 2.488 & 25 & $\overline{3} 31$ & 36.07 \\
\hline 2.456 & 13 & 150,122 & 36.56 \\
\hline 2.403 & 2 & 311 & 37.40 \\
\hline 2.348 & 17 & $\overline{4} 01$ & 38.30 \\
\hline 2.311 & 5 & $\overline{4} 11$ & 38.94 \\
\hline 2.284 & 12 & 321 & 39.41 \\
\hline 2.266 & 20 & 241,400 & 39.74 \\
\hline
\end{tabular}




\begin{tabular}{|c|c|c|c|}
\hline \multicolumn{4}{|c|}{$\begin{array}{l}\text { Internal standard Ag, } a=4.08641 \AA \\
\text { CuK } a_{1} \lambda=1.54056 \AA \text {; temp. } 25^{\circ} \mathrm{C}\end{array}$} \\
\hline$d(\AA)$ & $I$ & $h k l$ & $2 \theta\left({ }^{\circ}\right)$ \\
\hline 2.247 & 8 & 202 & 40.10 \\
\hline 2.225 & 8 & 250 & 40.54 \\
\hline 2.198 & 9 & 042 & 41.03 \\
\hline 2.187 & 12 & $\overline{2} 51$ & 41.25 \\
\hline 2.156 & 11 & $\overline{2} 42$ & 41.87 \\
\hline 2.135 & 10 & 420 & 42.30 \\
\hline 2.129 & 5 & $\underline{0} 60$ & 42.43 \\
\hline 2.092 & 5 & $\overline{4} 12$ & 43.21 \\
\hline 2.072 & 6 & $\overline{2} 03, \overline{1} 13,+$ & 43.65 \\
\hline 2.055 & 4 & $\overline{4} 31$ & 44.02 \\
\hline 2.020 & 6 & 003 & 44.84 \\
\hline 2.014 & 3 & $\overline{4} 22$ & 44.97 \\
\hline 2.002 & 5 & 251,430 & 45.26 \\
\hline 1.996 & 6 & $013, \overline{1} 23$ & 45.39 \\
\hline 1.961 & 4 & $\overline{3} 51$ & 46.25 \\
\hline 1.951 & 12 & 401,350 & 46.50 \\
\hline 1.927 & 6 & $\overline{3} 13,023,+$ & 47.13 \\
\hline 1.898 & 4 & $\overline{4} 32$ & 47.89 \\
\hline 1.882 & 8 & $\overline{1} 33$ & 48.32 \\
\hline 1.871 & 5 & 312 & 48.63 \\
\hline 1.865 & 7 & $\overline{3} 23, \overline{2} 33$ & 48.80 \\
\hline 1.847 & 9 & 440 & 49.30 \\
\hline 1.842 & 5 & 152 & 49.44 \\
\hline 1.813 & 3 & 322 & 50.27 \\
\hline 1.809 & 6 & $\overline{5} 21$ & 50.41 \\
\hline 1.789 & 10 & 123,170 & 51.02 \\
\hline 1.775 & 5 & $261, \overline{5} 12$ & 51.44 \\
\hline 1.763 & 5 & $\overline{1} 62$ & 51.80 \\
\hline 1.7562 & 5 & $\overline{4} 13$ & 52.03 \\
\hline 1.7281 & 9 & $\overline{4} 51,332$ & 52.94 \\
\hline 1.7072 & 6 & 133,043 & 53.64 \\
\hline 1.6890 & 9 & 171 & 54.26 \\
\hline 1.6634 & 5 & $\overline{3} 43$ & 55.17 \\
\hline 1.6505 & 4 & $\overline{5} 32$ & 55.64 \\
\hline 1.6362 & 3 & $\overline{4} 33$ & 56.17 \\
\hline 1.6308 & 4 & $\overline{4} 52$ & 56.37 \\
\hline 1.6090 & 6 & $143, \overline{2} 53$ & 57.20 \\
\hline
\end{tabular}

\section{References}

Margulis, T.N. and D. H. Templeton (1962). Crystal structure and hydrogen bonding of magnesium ammonium sulfate hexahydrate, Z. Krist. 117, 334-357.

Tutton, A. E. (1893). Connection between the atomic weight of contained metals and the magnitude of the angles of crystals of isomorphous series. A study of the potassium, rubidium and cesium salts of the monoclinic series of double sulphates $\mathrm{R}_{2} \mathrm{M}\left(\mathrm{SO}_{4}\right)_{2} \cdot 6 \mathrm{H}_{2} \mathrm{O}$, J. Chem. Soc. 63, $337-423$. 


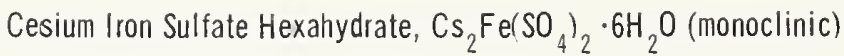

Sample

The sample was made by slow evaporation at room temperature of an equimolar solution of $\mathrm{Cs}_{2} \mathrm{SO}_{4}$ and $\mathrm{FeSO}_{4}$.

\section{Color}

Unground: very pale green

Ground: colorless

\section{Optical data}

Biaxial ( + ) $\mathrm{N}_{\alpha}=1.501, \mathrm{~N}_{\beta}=1.504, \mathrm{~N}_{\gamma}=1.516$. $2 \mathrm{~V}$ is medium

\section{Structure}

Monoclinic, $\mathrm{P} 2_{1} / \mathrm{a}(14), \mathrm{Z}=2$, Isostructural with other "Tutton Salts" [Tutton, 1893]. The structure of a "Tutton Salt", $\left(\mathrm{NH}_{4}\right)_{2} \mathrm{Mg}\left(\mathrm{SO}_{4}\right)_{2} \cdot 6 \mathrm{H}_{2} \mathrm{O}$, was determined by Margulis and Templeton [1962].

\section{Lattice constants}

\begin{tabular}{|c|c|c|c|c|}
\hline & $a(\AA)$ & $b(\stackrel{\circ}{)}$ & $c(\AA)$ & $\beta\left({ }^{\circ}\right)$ \\
\hline $\begin{array}{l}\text { NBS, } \\
\text { sample } \\
\text { at } 25^{\circ} \mathrm{C}\end{array}$ & $\begin{array}{l}9.355 \\
\pm .001\end{array}$ & $\begin{array}{r}12.893 \\
\pm .002\end{array}$ & $\begin{array}{r}6.378 \\
\pm .001\end{array}$ & $\mid \begin{array}{r}106^{\circ} 53^{\prime} \\
\pm I^{\prime}\end{array}$ \\
\hline
\end{tabular}

\section{Density}

(calculated) $2.805 \mathrm{~g} / \mathrm{cm}^{3}$ at $25^{\circ} \mathrm{C}$.

\section{Reference intensity}

$\mathrm{I} / \mathrm{I}_{\text {corundum }}=2.8$

\begin{tabular}{|c|c|c|c|}
\hline \multicolumn{4}{|c|}{$\begin{array}{l}\text { Internal standard } W, a=3.16516 \AA \\
\text { CuK } a_{1} \lambda=1.54056 \AA \text {; temp. } 25{ }^{\circ} \mathrm{C}\end{array}$} \\
\hline$d(\AA)$ & $I$ & $h k l$ & $2 \theta\left({ }^{\circ}\right)$ \\
\hline 7.34 & 12 & 110 & 12.04 \\
\hline 6.10 & 5 & 001 & 14.52 \\
\hline 5.518 & 3 & 011 & 16.05 \\
\hline 5.233 & 12 & 120 & 16.93 \\
\hline 4.478 & 5 & 200 & 19.81 \\
\hline 4.438 & 11 & 021 & 19.99 \\
\hline 4.356 & 19 & $\bar{I}_{21}$ & 20.37 \\
\hline 4.247 & 300 & $\overline{2} 01$ & 20.90 \\
\hline 4.227 & 100 & 210,111 & 21.00 \\
\hline 4.033 & 6 & $\overline{2} 11$ & 22.02 \\
\hline 3.877 & 75 & 130 & 22.92 \\
\hline 3.681 & 4 & 220,121 & 24.16 \\
\hline 3.475 & 10 & $\overline{1} 31$ & 25.61 \\
\hline 3.221 & 30 & 040 & 27.67 \\
\hline 3.194 & 20 & 201 & 27.91 \\
\hline 3.101 & & $211,230,+$ & 28.77 \\
\hline 3.091 & 10\{ & 112 & 28.86 \\
\hline 3.033 & 2 & -140 & 29.43 \\
\hline 2.970 & 25 & $\overline{3} I I, 012$ & 30.06 \\
\hline 2.954 & 19 & $\overline{2} 02$ & 30.23 \\
\hline 2.907 & 13 & 310 & 30.73 \\
\hline 2.860 & 30\{ & $22 I$ & 31.25 \\
\hline 2.851 & $30 ?$ & $04 I$ & 31.35 \\
\hline 2.830 & 6 & $-\bar{I} 41$ & 31.59 \\
\hline 2.761 & 2 & $\overline{3} 21,022$ & 32.40 \\
\hline 2.707 & 6 & 320 & 33.07 \\
\hline 2.683 & 3 & $\overline{2} 22$ & 33.37 \\
\hline 2.614 & 6 & 240,141 & 34.27 \\
\hline 2.567 & 17 & $\overline{2} 41$ & 34.93 \\
\hline 2.557 & 17 & $\overline{1} 32$ & 35.06 \\
\hline 2.489 & 35 & $\overline{3} 31,032$ & 36.06 \\
\hline 2.461 & 9 & 122 & 36.48 \\
\hline 2.453 & 8 & 330 & 36.61 \\
\hline 2.374 & 12 & 051 & 37.87 \\
\hline 2.358 & 8 & $\overline{3} 22$ & 38.14 \\
\hline 2.330 & 2 & $\underline{4} \underline{1} O I$ & 38.61 \\
\hline 2.296 & 7 & $\overline{4} I I$ & 39.21 \\
\hline 2.267 & 20 & $241, \bar{I} 42$ & 39.73 \\
\hline 2.264 & 17 & 321,132 & 39.79 \\
\hline 2.235 & 14 & $250,15 I$ & 40.32 \\
\hline
\end{tabular}




\begin{tabular}{|c|c|c|c|}
\hline \multicolumn{4}{|c|}{$\begin{array}{l}\text { Internal standard } W, a=3.16516 \AA \\
\text { CuK } a_{1} \lambda=1.54056 \AA \text {; temp. } 25{ }^{\circ} \mathrm{C}\end{array}$} \\
\hline$d(\AA)$ & $I$ & $h k l$ & $2 \theta\left({ }^{\circ}\right)$ \\
\hline 2.215 & 5 & $\overline{3} 41,042$ & 40.69 \\
\hline 2.204 & 9 & $212, \overline{2} 51,+$ & 40.92 \\
\hline 2.176 & 13 & $\overline{2} 42$ & 41.47 \\
\hline 2.149 & 3 & 060 & 42.02 \\
\hline 2.114 & 8 & 420,222 & 42.74 \\
\hline 2.094 & 6 & $\overline{4} 12, \overline{1} 13,+$ & 43.16 \\
\hline 2.050 & 3 & $\overline{4} 31$ & 44.14 \\
\hline 2.035 & 7 & 003 & 44.48 \\
\hline 2.017 & 3 & $\overline{4} 22, \overline{1} 23$ & 44.91 \\
\hline 2.006 & 6 & 251 & 45.16 \\
\hline 1.992 & 2 & $\overline{2} 23, \overline{3} 42$ & 45.50 \\
\hline 1.970 & 12 & $\overline{3} 51,052$ & 46.04 \\
\hline 1.946 & 6 & $\overline{3} 13$ & 46.64 \\
\hline 1.918 & 6 & $\overline{2} 6 \underline{1}$ & 47.36 \\
\hline 1.903 & 8 & $\overline{4} 32, \overline{1} 33$ & 47.75 \\
\hline 1.882 & 5 & $\overline{2} 33, \overline{3} 23$ & 48.32 \\
\hline 1.853 & 3 & $152, \overline{5} 11$ & 49.14 \\
\hline 1.838 & 11 & 440,242 & 49.56 \\
\hline 1.803 & 5 & 170,322 & 50.57 \\
\hline 1.796 & 10 & $\overline{5} 21,123$ & 50.78 \\
\hline 1.785 & 4 & $\overline{4} 03$ & 51.13 \\
\hline 1.781 & 5 & $\overline{1} 62$ & 51.25 \\
\hline 1.773 & 6 & $510, \overline{4} 42,+$ & 51.50 \\
\hline 1.759 & 3 & $431, \overline{1} 71$ & 51.93 \\
\hline 1.725 & 5 & 520 & 53.05 \\
\hline 1.721 & 9 & $332, \overline{4} 23,+$ & 53.17 \\
\hline 1.703 & 8 & 270,171 & 53.78 \\
\hline 1.664 & 2 & 213 & 55.15 \\
\hline 1.650 & 7 & $\overline{5} 32$ & 55.65 \\
\hline 1.638 & 2 & $\overline{3} 62, \overline{1} 53$ & 56.11 \\
\hline 1.626 & 2 & $\overline{2} 53$ & 56.56 \\
\hline 1.606 & 1 & 361 & 57.32 \\
\hline
\end{tabular}

\section{References}

Margulis, T.N. and D. H. Templeton (1962). Crystal structure and hydrogen bonding of magnesium ammonium sulfate hexahydrate, Z. Krist. 117, 334-357.

Tutton, A. E. (1893). Connection between the atomic weight of contained metals and the magnitude of the angles of crystals of isomorphous series. A study of the potassium, rubidium and cesium salts of the monoclinic series of double sulphates $\mathrm{Re} M\left(\mathrm{SO}_{4}\right)_{2} \cdot 6 \mathrm{H}_{2} \mathrm{O}$, J.Chem. Soc. 63, $337-423$. 


\section{Sample}

The sample was made by slow evaporation at room temperature of an equimolar solution of $\mathrm{Cs}_{2} \mathrm{SO}_{4}$ and $\mathrm{MgSO}_{4}$.

\section{Color}

Colorless

\section{Optical data}

Biaxial (t) $\mathrm{N}_{\alpha}=1.481, \mathrm{~N}_{\beta}=1.485, \mathrm{~N}_{\gamma}=1.492$ $2 \mathrm{~V}$ is medium.

\section{Structure}

Monoclinic, $\mathrm{P}_{1} / \mathrm{a}$ (14), $\mathrm{z}=2$.

$\mathrm{Cs}_{2} \mathrm{Mg}\left(\mathrm{SO}_{4}\right)_{2} \cdot 6 \mathrm{H}_{2} \mathrm{O}$ is a "Tutton Salt "[Tutton, 1893]. The structure of a "Tutton Salt", $\left(\mathrm{NH}_{4}\right)_{2} \mathrm{Mg}\left(\mathrm{SO}_{4}\right)_{2} \cdot 6 \mathrm{H}_{2} \mathrm{O}$ was determined by Margulis and Templeton, [1962] .

\section{Lattice constants}

\begin{tabular}{|c|c|c|c|c|}
\hline & $a(\stackrel{\circ}{)}$ & $b(\AA)$ & $c(\AA)$ & $\beta\left({ }^{\circ}\right)$ \\
\hline $\begin{array}{l}\text { NBS, } \\
\text { sample } \\
\text { at } 25^{\circ} \mathrm{C}\end{array}$ & $\begin{array}{l}9.330 \\
\pm .001\end{array}$ & $\begin{array}{r}12.848 \\
\pm .003\end{array}$ & $\begin{array}{l}6.360 \\
\pm .001\end{array}$ & $\begin{array}{r}107^{\circ} 2^{\prime} \\
\pm 1\end{array}$ \\
\hline
\end{tabular}

\section{Density}

(calculated) $2.689 \mathrm{~g} / \mathrm{cm}^{3}$ at $25^{\circ} \mathrm{C}$.

\section{Reference intensity}

$$
\mathrm{I} / \mathrm{I}_{\text {corundurn }}=1.8
$$

\begin{tabular}{|c|c|c|c|}
\hline \multicolumn{4}{|c|}{$\begin{array}{l}\text { Internal standard } \mathrm{W}, \mathrm{a}=3.16516 \AA \\
\mathrm{CuK} a_{1} \quad \lambda=1.54056 \AA \text {; temp. } 25{ }^{\circ} \mathrm{C}\end{array}$} \\
\hline$d(\AA)$ & $I$ & $h k l$ & $2 \theta\left({ }^{\circ}\right)$ \\
\hline 7.31 & 45 & 110 & 12.09 \\
\hline 6.42 & 5 & 020 & 13.78 \\
\hline 6.08 & 15 & 001 & 14.55 \\
\hline 5.50 & 6 & 011 & 16.11 \\
\hline 5.21 & 19 & 120 & 16.99 \\
\hline 4.42 & 10 & 021 & 20.09 \\
\hline 4.34 & 31 & 121 & 20.44 \\
\hline 4.23 & 100 & 201 & 20.98 \\
\hline 4.026 & 8 & 211 & 22.06 \\
\hline 3.860 & 83 & 130 & 23.02 \\
\hline 3.537 & 4 & $\overline{2} 21$ & 25.16 \\
\hline 3.463 & 9 & $\overline{1} 31$ & 25.70 \\
\hline 3.210 & 46 & 040 & 27.77 \\
\hline 3.181 & 36 & 201 & 28.03 \\
\hline 3.087 & 97 & 131,211 & 28.90 \\
\hline 3.041 & 7 & 002 & 29.35 \\
\hline 3.010 & 5 & 231 & 29.65 \\
\hline 2.965 & 39 & $\overline{3} 11$ & 30.12 \\
\hline 2.948 & 38 & $\overline{2} 02$ & 30.29 \\
\hline 2.899 & 11 & 310 & 30.82 \\
\hline 2.846 & 38 & $\overline{1} 22$ & 31.41 \\
\hline 2.822 & 10 & 141 & 31.68 \\
\hline 2.753 & 3 & $\overline{3} 21$ & 32.50 \\
\hline 2.699 & 8 & 320 & 33.16 \\
\hline 2.607 & 6 & 240,141 & 34.37 \\
\hline 2.559 & 29 & $\overline{2} 41$ & 35.03 \\
\hline 2.553 & 31 & $23 \underline{1}$ & 35.12 \\
\hline 2.485 & 33 & $\overline{3} 31, \overline{3} 12$ & 36.12 \\
\hline 2.450 & 16 & 122 & 36.66 \\
\hline 2.443 & 18 & 330 & 36.75 \\
\hline 2.367 & 13 & 051,311 & 37.98 \\
\hline 2.352 & 10 & 322 & 38.23 \\
\hline 2.289 & 9 & $\overline{4} 1 \underline{1}$ & 39.33 \\
\hline 2.259 & 22 & $241, \overline{1} 42$ & 39.88 \\
\hline 2.230 & 17 & 400 & 40.42 \\
\hline 2.197 & 11 & $410, \overline{2} 51$ & 41.04 \\
\hline 2.172 & 13 & $\overline{2} 42$ & 41.55 \\
\hline 2.107 & 6 & 420 & 42.88 \\
\hline 2.092 & 6 & $\overline{2} 03, \overline{4} 12$ & 43.22 \\
\hline 2.086 & 7 & $\overline{1} 13$ & 43.33 \\
\hline
\end{tabular}




\begin{tabular}{|c|c|c|c|}
\hline \multicolumn{4}{|c|}{ Internal standard $\mathrm{W}, \mathrm{a}=3.16516 \AA$} \\
\hline \multicolumn{3}{|c|}{$\mathrm{CuK} a_{1} \lambda=1.54056 \AA$; temp. $25{ }^{\circ} \mathrm{C}$} \\
\hline$d(\AA)$ & $I$ & $h k l$ & $2 \theta\left(^{\circ}\right)$ \\
\hline 2.063 & 1 & $\overline{2} 13$ & 43.83 \\
2.046 & 5 & $142, \overline{4} 31$ & 44.24 \\
2.027 & 8 & 003 & 44.67 \\
1.998 & 7 & $\overline{1} 52,251$ & 45.35 \\
1.963 & 10 & $\overline{3} 51,052$ & 46.21 \\
& & & \\
1.943 & 6 & 350 & 46.71 \\
1.912 & 6 & $\overline{2} 61$ & 47.51 \\
1.879 & 5 & $\overline{2} 33, \overline{3} 23$ & 48.39 \\
1.8326 & 12 & 033,440 & 49.71 \\
1.7918 & 12 & $\overline{5} 21$ & 50.92 \\
& & & \\
1.7886 & 11 & 123 & 51.02 \\
1.7847 & 11 & $\overline{3} 33$ & 51.14 \\
1.7689 & 7 & $\overline{4} 42,510$ & 51.63 \\
1.7382 & 2 & 360 & 52.61 \\
1.7203 & 6 & $520, \overline{5} 22$ & 53.20 \\
& & & \\
1.7143 & 7 & 043,332 & 53.40 \\
1.6970 & 6 & 270,171 & 53.99 \\
1.6480 & 8 & 441,530 & 55.73 \\
1.6354 & 3 & $\overline{4} 52, \overline{3} 62$ & 56.20 \\
1.6213 & 3 & $\overline{2} 53$ & 56.73 \\
\hline
\end{tabular}

\section{References}

Margulis, T.N. and D. H. Templeton (1962). Crystal structure and hydrogen bonding of magnesium ammonium sulfate hexahydrate, Z. Krist. 117, 334-357.

Tutton, A. E. (1893). Connection between the atomic weight of contained metals and the magnitude of the angles of crystals of isomorphous series. A study of the potassium, rubidium and cesium salts of the monoclinic series of double sulphates $\mathrm{R}_{2} \mathrm{M}\left(\mathrm{SO}_{4}\right)_{a} \cdot 6 \mathrm{H}_{2} \mathrm{O}, \mathrm{J}$. Chem. Soc. 63, $337-423$. 
Sample

The sample was made by slow evaporation at room temperature of an equimolar solution of $\mathrm{Cs}_{2} \mathrm{SO}_{4}$ and $\mathrm{MnSO}_{4}$.

\section{Color}

Unground: purplish white

Ground: colorless

\section{Optical data}

Biaxial (+) $\mathrm{N}_{\alpha}=1.495, \mathrm{~N}_{\beta}=1.497, \mathrm{~N}_{\gamma}=1.502$ $2 \mathrm{~V}$ is large

\section{Structure}

Monoclinic, P21/a (14), $\mathrm{Z}=2$ Isostructural with other"Tutton Salts"[Tutton, 1893] The structure of a "Tutton SaIt", $\left(\mathrm{NH}_{4}\right)_{2}$ $\mathrm{Mg}\left(\mathrm{SO}_{4}\right)_{2} \cdot 6 \mathrm{H}_{2} \mathrm{O}$, was determined by Margulis and Templeton [1962].

\section{Lattice constants}

\begin{tabular}{|c|c|c|c|c|}
\hline & $a(\stackrel{\circ}{)}$ & $b(\AA)$ & $c(\stackrel{\circ}{A})$ & $\beta\left({ }^{\circ}\right)$ \\
\hline $\begin{array}{l}\text { NBS, } \\
\text { sample } \\
\text { at } 25{ }^{\circ} \mathrm{C}\end{array}$ & $\begin{array}{l}9.425 \\
\pm .001\end{array}$ & $\begin{array}{r}12.976 \\
\pm .002\end{array}$ & $\begin{array}{l}6.389 \\
\pm .001\end{array}$ & $\begin{array}{r}107^{\circ} 10^{\prime} \\
\pm 1^{\prime}\end{array}$ \\
\hline
\end{tabular}

\section{Density}

(calculated) $2.763 \mathrm{~g} / \mathrm{cm}^{3}$ at $25^{\circ} \mathrm{C}$.

\section{Reference intensity}

$I / I_{\text {corundum }}=2.2$

\begin{tabular}{|c|c|c|c|}
\hline \multicolumn{4}{|c|}{$\begin{array}{l}\text { Internal standard } \mathrm{Ag}, \mathrm{a}=4.08641 \AA \\
\mathrm{CuK} \alpha_{1} \quad \lambda=1.54056 \AA \text {; temp. } 25^{\circ} \mathrm{C}\end{array}$} \\
\hline$d(\AA)$ & $I$ & $h k l$ & $2 \theta(\circ)$ \\
\hline 7.41 & 17 & 110 & 11.94 \\
\hline 6.11 & 7 & 001 & 14.48 \\
\hline 5.524 & 4 & 011 & 16.03 \\
\hline 5.273 & 15 & 120 & 16.80 \\
\hline 4.510 & 5 & 200 & 19.67 \\
\hline 4.442 & II & 021 & 19.97 \\
\hline 4.384 & 22 & $\overline{1} 21$ & 20.24 \\
\hline 4.281 & 100 & $\overline{2} 01$ & 20.73 \\
\hline 4.261 & 59 & 210 & 20.83 \\
\hline 4.241 & 61 & 111 & 20.93 \\
\hline 4.064 & 5 & $\overline{2} 11$ & 21.85 \\
\hline 3.899 & 76 & 130 & 22.79 \\
\hline 3.693 & 3 & 220 & 24.08 \\
\hline 3.497 & 10 & 131 & 25.45 \\
\hline 3.244 & 33 & 040 & 27.47 \\
\hline 3.201 & 23 & 201 & 27.85 \\
\hline 3.111 & 79 & 131,211 & 28.67 \\
\hline 3.103 & 72 & $\overline{1} 12$ & 28.75 \\
\hline 3.049 & 3 & 002,140 & 29.27 \\
\hline 2.993 & 20 & 311 & 29.83 \\
\hline 2.965 & 29 & $\overline{2} 02$ & 30.12 \\
\hline 2.927 & 15 & 310 & 30.52 \\
\hline 2.894 & $I$ & $\overline{2} 12$ & 30.87 \\
\hline 2.869 & 42 & 221 & 31.15 \\
\hline 2.861 & 11 & $041, \overline{1} 22$ & 31.24 \\
\hline 2.778 & 2 & $\overline{3} 21$ & 32.19 \\
\hline 2.723 & 6 & 320 & 32.86 \\
\hline 2.699 & 5 & $\overline{2} 22$ & 33.17 \\
\hline 2.632 & 7 & 240 & 34.04 \\
\hline 2.624 & 3 & 141 & 34.14 \\
\hline 2.583 & 15 & $\overline{2} 41$ & 34.70 \\
\hline 2.568 & 17 & 132 & 34.91 \\
\hline 2.507 & 41 & $\overline{3} 31$ & 35.79 \\
\hline 2.501 & 30 & 312 & 35.88 \\
\hline 2.464 & 14 & 330,122 & 36.44 \\
\hline 2.388 & 12 & 051 & 37.63 \\
\hline 2.374 & 9 & 322 & 37.87 \\
\hline 2.349 & 2 & 401 & 38.28 \\
\hline 2.311 & 11 & $\overline{4} 11$ & 38.94 \\
\hline 2.277 & 22 & 241 & 39.54 \\
\hline
\end{tabular}


Cesium Manganese Sulfate Hexahydrate, $\mathrm{Cs}_{2} \mathrm{Mn}\left(\mathrm{SO}_{4}\right)_{2} \cdot 6 \mathrm{H}_{2} \mathrm{O}$ (monoclinic) - continued

\begin{tabular}{|l|r|c|c|}
\hline \multicolumn{4}{|c|}{ Internal standard Ag, a $=4.08641 \AA$} \\
\hline \multicolumn{3}{|c|}{ CuKa $a_{1} \lambda=1.54056 \AA ;$ temp. $25{ }^{\circ} \mathrm{C}$} \\
\hline$d(\AA)$ & $I$ & $h k l$ & $2 \theta\left({ }^{\circ}\right)$ \\
\hline 2.271 & 20 & 321 & 39.65 \\
2.250 & 12 & 400 & 40.04 \\
2.239 & 11 & 202 & 40.25 \\
2.232 & 3 & $\overline{3} 41$ & 40.38 \\
2.218 & 13 & $410, \overline{2} 51$ & 40.64 \\
2.187 & 15 & $\overline{2} 42$ & 41.24 \\
2.162 & 2 & 060 & 41.74 \\
2.127 & 8 & 420 & 42.47 \\
2.110 & 6 & $\overline{4} 12$ & 42.82 \\
2.103 & 7 & $160, \overline{2} 03$ & 42.98 \\
& & $\overline{1}$ & \\
2.075 & 2 & $\overline{2} 13$ & 43.59 \\
2.065 & 3 & $\overline{4} 31$ & 43.81 \\
2.035 & 10 & 003 & 44.49 \\
2.016 & 7 & 251 & 44.93 \\
2.005 & 6 & $\overline{3} 42$ & 45.19 \\
1.984 & 6 & $\overline{3} 51$ & 45.69 \\
1.977 & 12 & 052 & 45.86 \\
1.953 & 8 & $\overline{3} 13, \overline{2} 52$ & 46.45 \\
1.929 & 7 & $\overline{2} 61$ & 47.08 \\
1.907 & 10 & $\overline{1} 33$ & 47.66 \\
1.891 & 5 & $\overline{3} 23, \overline{2} 33$ & 48.07 \\
1.866 & 3 & $\overline{5} 11$ & 48.75 \\
1.849 & 11 & 113,440 & 49.24 \\
1.843 & 6 & 242,033 & 49.40 \\
1.817 & 3 & $\overline{3} 52$ & 50.16 \\
1.810 & 8 & $\overline{5} 21$ & 50.37 \\
1.796 & 11 & $123, \overline{4} 03$ & 50.78 \\
1.783 & 8 & 512,510 & 51.19 \\
1.771 & 3 & 351 & 51.57 \\
1.740 & 2 & $\overline{4} 51$ & 52.55 \\
1.735 & 5 & $\overline{5} 22,520$ & 52.72 \\
1.730 & 5 & $\overline{4} 23$ & 52.87 \\
1.725 & 6 & 332,043 & 53.06 \\
1.712 & 9 & 171 & 53.49 \\
\hline
\end{tabular}

\section{References}

Margulis, T.N. and D. H. Templeton (1962). Crystal structure and hydrogen bonding of magnesium ammonium sulfate hexahydrate, Z. Krist. 117, 334-357.

Tutton, A.E.(1893). Connection between the atomic weight of contained metals and the angles of crystals of isomorphous series. A study of the potassium, rubidium and cesium salts of the monoclinic series of double sulphates $\mathrm{R}_{2} \mathrm{M}\left(\mathrm{SO}_{4}\right)_{2} \cdot 6 \mathrm{H}_{2} \mathrm{O}$ J. Chem. Soc. 63, 337-423. 


\section{Sample}

The sample was prepared by mixing saturated solutions of $\mathrm{HgCl}_{2}$ and $\mathrm{CsCl}$ at room temperature.

\section{Color}

Colorless

\section{Optical data}

Very low birefringence, $\quad \mathrm{N}=1.790$

\section{Structure}

$\mathrm{CsHgCl}_{3}$ has been reported as cubic [Natta and Passerini, 1928]. Náray -Szabó [1947], found it to be monoclinic with $\mathrm{a}=\mathrm{b}=\mathrm{c}$ and $\beta \sim 90^{\circ}$. In this work it is considered as a distorted perovskite and has been tentatively indexed as orthorhombic, isostructural with $\mathrm{NaMnF}_{3}$ and $\mathrm{RbCaCl}_{3}$. Very weak lines at 26.82, 27.94 and $29.50^{\circ}(2 \theta)$ suggest that a small amount of a second phase may be present, or that the material has a larger supercell. The assumed space group is Pnma (62) with $\mathrm{Z}$ equal to 4.

\section{Lattice constants}

\begin{tabular}{|l|r|r|r|}
\hline & $a(\AA)$ & $b(\AA)$ & $c(\AA)$ \\
\cline { 2 - 4 } $\begin{array}{l}\text { Natta and } \\
\text { Passerini [1928] }\end{array}$ & $5.45 *$ & & \\
Náray-Szabó & & & \\
$\begin{array}{l}\text { [1947] } \\
\text { NBS, sample }\end{array}$ & $10.92 *$ & $10.92 *$ & $10.92 *$ \\
at 25 C & 7.688 & 10.878 & 7.669 \\
& \pm .002 & \pm .002 & \pm .001 \\
\hline
\end{tabular}

* from $\mathrm{kX}$

${ }^{1}$ indexed as cubic

${ }^{2}$ indexed as monoclinic with $\beta \sim 90^{\circ}$.

\section{Density}

(calculated) $4.555 \mathrm{~g} / \mathrm{cm}^{3}$ at $25^{\circ} \mathrm{C}$.

\section{Reference intensity}

$$
I_{\text {corundum }}=3.0
$$

\begin{tabular}{|c|r|c|c|}
\hline \multicolumn{4}{|c|}{ Internal standard Ag, a $=4.08641 \AA$} \\
CuKa $\alpha_{1} \lambda=1.54056 \AA$ А; temp. $25{ }^{\circ} \mathrm{C}$ \\
\hline$d(\AA)$ & $I$ & $h k l$ & $2 \theta\left({ }^{\circ}\right)$ \\
\hline 5.42 & 15 & 101 & 16.34 \\
3.834 & 100 & 002 & 23.18 \\
3.132 & 1 & 022 & 28.47 \\
2.715 & 45 & 202 & 32.96 \\
2.428 & 11 & $301,222,+$ & 37.00 \\
& & & \\
2.217 & 45 & 042,123 & 40.67 \\
1.920 & 25 & 242 & 47.30 \\
1.894 & 1 & 250,410 & 48.00 \\
1.810 & 5 & $303,143,+$ & 50.38 \\
1.717 & 25 & $402,323,+$ & \\
& & & \\
1.570 & 6 & 440 & 58.75 \\
1.567 & 6 & 044 & 58.90 \\
1.506 & 2 & 501,343 & 61.51 \\
1.453 & 15 & $442,521,+$ & 64.03 \\
1.451 & 17 & 244 & 64.14 \\
1.357 & 3 & 404 & 69.16 \\
1.319 & 2 & 460,181 & 71.45 \\
1.317 & 2 & 064,424 & 71.59 \\
1.279 & 5 & 325 & 74.05 \\
1.214 & 6 & 444 & 78.74 \\
1.187 & 1 & 381 & 80.94 \\
1.184 & 1 & 226 & 81.16 \\
1.159 & 2 & $640,561,+$ & 83.27 \\
1.157 & 2 & 046 & 83.50 \\
1.110 & 3 & 480,642 & 87.89 \\
1.108 & 3 & 264 & 88.10 \\
1.087 & 1 & 464 & 90.30 \\
1.065 & 5 & 604 & 92.59 \\
1.064 & 4 & 406 & 92.73 \\
1.010 & 1 & $662,741,+$ & 99.43 \\
1.008 & 1 & 266 & 99.65 \\
.9921 & 2 & 644,723 & 101.86 \\
.9911 & 2 & 446 & 102.01 \\
\hline
\end{tabular}

\section{References}

Natta, G. and L. Passerini (1928). Isomorfismo, polimorfismo e morfotropia I.Composti del tipo $\mathrm{ABX}_{3}$. Gazz.Chim.Ital. 58, 472-484.

Náray-Szabó, S. (1947). The perovskite structure family, Müegyetemi. Kozlemen. No. 1, 30-41. 


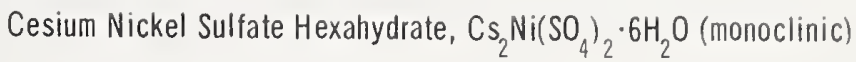

\section{Sample}

The sample was prepared by slow evaporation at room temperature of an equimolar solution of $\mathrm{Cs}_{2} \mathrm{SO}_{4}$ and $\mathrm{NiSO}_{4}$.

\section{Color}

Unground: strong bluish green

Ground: very pale green

\section{Optical data}

Biaxial (-) $N_{\alpha}=1.507, N_{\beta}=1.512, N_{Y}=1.516$ $2 \mathrm{~V}$ is very large

\section{Structure}

Monoclinic, P2 $/ \mathrm{a}$ (14), $\mathrm{Z}=2$.

Cs2 Ni $\left(\mathrm{SO}_{4}\right)_{2} \cdot 6 \mathrm{H}_{2} \mathrm{O}$ is a "Tutton Salt"[Tutton, 1893]. The structure of a "Tutton Salt", $\left(\mathrm{NH}_{4}\right)_{2} \mathrm{Mg}\left(\mathrm{SO}_{4}\right)_{2} \cdot 6 \mathrm{H}_{2} \mathrm{O}$ was determined by Margulis and Templeton, [1962] .

\section{Lattice constants}

\begin{tabular}{|l|c|c|c|c|}
\hline & $a(\AA)$ & $b(\AA)$ & $c(\AA)$ & $\beta\left({ }^{\circ}\right)$ \\
\cline { 2 - 5 } $\begin{array}{l}\text { NBS, } \\
\text { sample at } \\
25^{\circ} \mathrm{C}----\end{array}$ & $\begin{array}{r}9.264 \\
\pm .001\end{array}$ & $\begin{array}{r}12.773 \\
\pm .002\end{array}$ & $\begin{array}{r}6.359 \\
\pm .001\end{array}$ & $\begin{array}{r}106^{\circ} 59^{\prime} \\
\pm 1^{\prime}\end{array}$ \\
\hline
\end{tabular}

Density

(calculated) $2.883 \mathrm{~g} / \mathrm{cm}^{3}$ at $25^{\circ} \mathrm{C}$.

\section{Reference intensity}

$I / I_{\text {corundum }}=2.7$

\begin{tabular}{|c|c|c|c|}
\hline \multicolumn{4}{|c|}{$\begin{array}{l}\text { Internal standard } \mathrm{Ag}, \mathrm{a}=4.08641 \AA \\
\mathrm{CuK} a_{1} \quad \lambda=1.54056 \AA \text {; temp. } 25{ }^{\circ} \mathrm{C}\end{array}$} \\
\hline$d(\AA)$ & $I$ & $h k l$ & $2 \theta(0)$ \\
\hline 7.284 & 10 & 110 & 12.14 \\
\hline 6.083 & 6 & 001 & 14.55 \\
\hline 5.487 & 6 & 011 & 16.14 \\
\hline 5.184 & 12 & 120 & 17.09 \\
\hline 4.405 & 12 & 021 & 20.14 \\
\hline 4.327 & 18 & $\overline{1} 21$ & 20.51 \\
\hline 4.215 & 700 & $\overline{2} 01$ & 21.06 \\
\hline 4.201 & 100\{ & III & 21.13 \\
\hline 4.001 & 6 & $\overline{2} 11$ & 22.20 \\
\hline 3.837 & 70 & 130 & 23.16 \\
\hline $3.64 I$ & 4 & 220 & 24.43 \\
\hline 3.448 & 10 & İ31 & 25.82 \\
\hline 3.193 & 25 & 040 & 27.92 \\
\hline 3.168 & 20 & 201 & 28.14 \\
\hline 3.074 & 70 & $131,211,+$ & 29.02 \\
\hline 3.005 & 2 & 140 & 29.71 \\
\hline 2.961 & 12 & 012 & 30.16 \\
\hline 2.943 & 30 & $\overline{3} 11, \overline{2} 02$ & 30.35 \\
\hline 2.877 & 14 & 310 & 31.06 \\
\hline 2.839 & 30 & 221 & 31.49 \\
\hline 2.806 & 7 & İ4I & 31.87 \\
\hline 2.738 & 1 & $\overline{3} 21$ & 32.68 \\
\hline 2.681 & 8 & 320 & 33.40 \\
\hline 2.673 & 6 & $\overline{2} 22$ & 33.50 \\
\hline 2.591 & 6 & $112,240,+$ & 34.59 \\
\hline 2.545 & 25 & $\overline{1} 32, \overline{2} 41,+$ & 35.24 \\
\hline 2.468 & 30 & $\overline{3} 12, \overline{3} 31$ & 36.37 \\
\hline 2.447 & 11 & 122 & 36.69 \\
\hline 2.428 & 4 & 330 & 37.00 \\
\hline 2.354 & 12 & 051,311 & 38.20 \\
\hline 2.342 & 10 & $\overline{1} 51, \overline{3} 22$ & 38.41 \\
\hline 2.310 & 2 & 401 & 38.96 \\
\hline 2.272 & 9 & $\overline{4} 11$ & 39.64 \\
\hline 2.249 & 20 & 132,241 & 40.06 \\
\hline 2.243 & 20 & 321 & 40.17 \\
\hline 2.222 & 7 & 202 & 40.57 \\
\hline 2.213 & 13 & $151,250,+$ & 40.73 \\
\hline 2.201 & 5 & 042 & 40.97 \\
\hline 2.184 & 9 & $\overline{2} 5 \underline{1}, 410$ & 41.30 \\
\hline 2.163 & 13 & 242 & 41.73 \\
\hline
\end{tabular}




\begin{tabular}{|c|c|c|c|}
\hline \multicolumn{4}{|c|}{$\begin{array}{l}\text { Internal standard } \mathrm{Ag}, \mathrm{a}=4.08641 \AA \\
\mathrm{CuK} a_{1} \quad \lambda=1.54056 \AA \text {; temp. } 25{ }^{\circ} \mathrm{C}\end{array}$} \\
\hline$d(\AA)$ & $I$ & $h k l$ & $2 \theta\left({ }^{\circ}\right)$ \\
\hline 2.130 & 2 & 060 & 42.41 \\
\hline 2.106 & 2 & $\overline{4} 02$ & 42.91 \\
\hline 2.093 & 9 & 420 & 43.19 \\
\hline 2.079 & 6 & $\overline{4} 12$ & 43.49 \\
\hline 2.070 & 4 & 160 & 43.69 \\
\hline 2.062 & 2 & $\overline{2} 13$ & 43.86 \\
\hline 2.029 & 10 & 431,003 & 44.63 \\
\hline 2.007 & 4 & $061, \bar{I} 23$ & 45.14 \\
\hline 2.001 & 6 & $\overline{1} 61, \overline{4} 22$ & 45.29 \\
\hline 1.989 & 4 & 251 & 45.58 \\
\hline 1.977 & 3 & $\overline{3} 42$ & 45.87 \\
\hline 1.955 & 12 & 052 & 46.42 \\
\hline 1.952 & 12 & $\overline{3} 51$ & 46.49 \\
\hline 1.937 & 7 & $\overline{3} 13$ & 46.87 \\
\hline 1.916 & 2 & 341 & 47.40 \\
\hline 1.910 & 3 & 401 & 47.58 \\
\hline 1.898 & 8 & $\overline{2} 61$ & 47.89 \\
\hline 1.894 & 11 & 133 & 48.00 \\
\hline 1.873 & 6 & $\overline{3} 23$ & 48.56 \\
\hline 1.839 & 1 & 152 & 49.53 \\
\hline 1.830 & 4 & 033,421 & 49.79 \\
\hline 1.819 & 10 & 440 & 50.10 \\
\hline 1.787 & 12 & 170,123 & 51.07 \\
\hline 1.767 & 5 & $\overline{1} 62,261$ & 51.69 \\
\hline 1.755 & 6 & 510 & 52.08 \\
\hline 1.743 & 5 & $171,431,+$ & 52.47 \\
\hline 1.728 & 1 & 360 & 52.96 \\
\hline 1.710 & 6 & $043, \overline{4} 23$ & 53.53 \\
\hline 1.707 & 12 & 520,332 & 53.65 \\
\hline 1.688 & 8 & 171,270 & 54.31 \\
\hline 1.659 & 2 & 162 & 55.32 \\
\hline 1.654 & 2 & 213 & 55.50 \\
\hline 1.636 & 6 & $\overline{5} 32,530$ & 56.16 \\
\hline 1.625 & 3 & $\overline{3} 62, \overline{4} 52$ & 56.58 \\
\hline 1.616 & 4 & $\overline{2} 53$ & 56.92 \\
\hline 1.591 & 4 & $36 \underline{1}$ & 57.92 \\
\hline 1.587 & 3 & $053, \overline{2} 04$ & 58.06 \\
\hline 1.582 & 3 & $\overline{1} 72,271$ & 58.27 \\
\hline 1.571 & 4 & $180, \overline{5} 13,+$ & 58.74 \\
\hline 1.563 & 3 & $\overline{3} 71$ & 59.04 \\
\hline 1.552 & 7 & $370, \overline{4} 43$ & 59.52 \\
\hline
\end{tabular}

\section{References}

Margulis, T.N. and D. H. Templeton (1962). Crystal structure and hydrogen bonding of magnesium ammonium sulfate hexahydrate, $\mathrm{z}$. Krist. 117, 334-357.

Tutton, A. E. (1893). Connection between the atomic weight of contained metals and the magnitude of the angles of crystals of isomorphous series. A study of the potassium, rubidium and cesium salts of the monoclinic series of double sulphates $\mathrm{R}_{2} \mathrm{M}\left(\mathrm{SO}_{4}\right)_{2 .} 6 \mathrm{He}, \mathrm{J}$. Chem. Soc. 63, $337-423$. 
Cesium Zinc Sulfate Hexahydrate, $\mathrm{Cs}_{2} \mathrm{Zn}\left(\mathrm{SO}_{4}\right)_{2} \cdot 6 \mathrm{H}_{2} \mathrm{O}$ (monoclinic)

\section{Sample}

The sample was prepared by slow evaporation at room temperature of an equimolar solution of $\mathrm{Cs}_{2} \mathrm{SO}_{4}$ and $\mathrm{ZnSO}_{4}$.

\section{Color}

Colorless

\section{Optical data}

Biaxial (-) $\mathrm{N}_{\alpha}=1.594, \mathrm{~N}_{\beta}=1.610, \mathrm{~N}_{\gamma}=1.615$ $2 \mathrm{~V}$ is large.

\section{Structure}

Monoclinic, $\mathrm{P}_{1} / \mathrm{a}$ (14), $\mathrm{Z}=2$.

$\mathrm{Cs}_{2} \mathrm{Zn}\left(\mathrm{SO}_{4}\right)_{2} \cdot 6 \mathrm{H}_{2} \mathrm{O}$ is a "Tutton Salt"[Tut-

ton, 1893]. The structure of a "Tutton Salt", $\left(\mathrm{NH}_{4}\right)_{2} \mathrm{Mg}\left(\mathrm{SO}_{4}\right)_{2} \cdot 6 \mathrm{H}_{2} \mathrm{O}$ was determined by Margulis and Templeton, [1962].

\section{Lattice constants}

\begin{tabular}{|l|c|c|c|c|}
\hline & $a(\AA)$ & $b(\AA)$ & $c(\AA)$ & $\beta\left({ }^{\circ}\right)$ \\
\cline { 2 - 5 } NBS, \\
$\begin{array}{l}\text { sample } \\
\text { at } 25^{\circ} \mathrm{C}\end{array}$ & $\begin{array}{c}9.316 \\
\pm .001\end{array}$ & $\begin{array}{c}12.815 \\
\pm 002\end{array}$ & $\begin{array}{r}6.373 \\
\pm .001\end{array}$ & $\begin{array}{r}106^{\circ} 57^{\prime} \\
\pm I^{\prime}\end{array}$ \\
\hline
\end{tabular}

\section{Density}

(calculated) $2.881 \mathrm{~g} / \mathrm{cm}^{3}$ at $25^{\circ} \mathrm{C}$.

Reference intensity

$$
I / I_{\text {corundum }}=2.3
$$

\begin{tabular}{|c|c|c|c|}
\hline \multicolumn{4}{|c|}{$\begin{array}{l}\text { Internal standard } \mathrm{Ag}, \mathrm{a}=4.08641 \AA \\
\mathrm{CuK} a_{1} \quad \lambda=1.54056 \AA \text {; temp. } 25^{\circ} \mathrm{C}\end{array}$} \\
\hline$d(\AA)$ & $I$ & $h k l$ & $2 \theta\left({ }^{\circ}\right)$ \\
\hline 7.31 & 8 & 110 & 12.10 \\
\hline 6.095 & 3 & 001 & 14.52 \\
\hline 5.504 & 3 & 011 & 16.09 \\
\hline 5.202 & 9 & 120 & 17.03 \\
\hline 4.451 & 7 & 200 & 19.93 \\
\hline 4.416 & 10 & 021 & 20.09 \\
\hline 4.337 & 16 & $\overline{1} 21$ & 20.46 \\
\hline 4.227 & 100 & $\overline{2} 01$ & 21.00 \\
\hline 4.019 & 5 & $\overline{2} 11$ & 22.10 \\
\hline 3.854 & 60 & 130 & 23.06 \\
\hline 3.660 & 4 & 121,220 & 24.30 \\
\hline 3.461 & 9 & $\overline{1} 31$ & 25.72 \\
\hline 3.204 & 25 & 040 & 27.82 \\
\hline 3.185 & 15 & 201 & 27.99 \\
\hline 3.087 & 65 & $131, \overline{1} 12,+$ & 28.90 \\
\hline 2.962 & 20 & $\overline{3} 11$ & 30.15 \\
\hline 2.951 & 20 & $\overline{2} 02$ & 30.26 \\
\hline 2.896 & 1.1 & 310 & 30.85 \\
\hline 2.850 & 30 & $\overline{1} 22,221$ & 31.36 \\
\hline 2.816 & 6 & $\overline{1} 41$ & 31.75 \\
\hline 2.749 & 2 & $\overline{3} 21,022$ & 32.54 \\
\hline 2.695 & 5 & 320 & 33.21 \\
\hline 2.678 & 4 & $\overline{2} 22$ & 33.43 \\
\hline 2.601 & 6 & $240,112,+$ & 34.45 \\
\hline 2.552 & 18 & $231, \overline{1} 32$ & 35.13 \\
\hline 2.479 & 30 & $\overline{3} 31, \overline{3} 12$ & 36.21 \\
\hline 2.452 & 8 & 122 & 36.62 \\
\hline 2.439 & 4 & 330 & 36.82 \\
\hline 2.362 & 9 & $05 \underline{1}$ & 38.07 \\
\hline 2.351 & 8 & $\overline{3} 22, \overline{15} 1$ & 38.25 \\
\hline 2.321 & 2 & $\underline{4} 01$ & 38.76 \\
\hline 2.284 & 7 & $\overline{4} 11$ & 39.41 \\
\hline 2.256 & 19 & $132, \overline{1} 42,+$ & 39.92 \\
\hline 2.229 & 8 & 400 & 40.43 \\
\hline 2.222 & 9 & 151,250 & 40.56 \\
\hline 2.209 & 4 & 042 & 40.81 \\
\hline 2.194 & 7 & 410,251 & 41.11 \\
\hline 2.170 & 11 & $\overline{2} 42$ & 41.59 \\
\hline 2.135 & 2 & 060 & 42.30 \\
\hline 2.105 & 8 & 420 & 42.94 \\
\hline
\end{tabular}


Cesium Zinc Sulfate Hexahydrate, $\mathrm{Cs}_{2} \mathrm{Zn}\left(\mathrm{SO}_{4}\right)_{2} \cdot 6 \mathrm{H}_{2} \mathrm{O}$ (monoclinic) - continued

\begin{tabular}{|c|c|c|c|}
\hline \multicolumn{4}{|c|}{$\begin{array}{l}\text { Internal standard } \mathrm{Ag}, \mathrm{a}=4.08641 \AA \\
\mathrm{CuK} a_{1} \quad \lambda=1.54056 \AA \text { 的 temp. } 25{ }^{\circ} \mathrm{C}\end{array}$} \\
\hline$d(\AA)$ & $I$ & $h k l$ & $2 \theta\left({ }^{\circ}\right)$ \\
\hline 2.092 & 4 & $\underline{I} 13$ & 43.22 \\
\hline 2.088 & 3 & 412 & 43.30 \\
\hline 2.040 & 3 & 431 & 44.37 \\
\hline 2.032 & 7 & 003 & 44.55 \\
\hline 2.009 & 3 & $\overline{4} 22, \overline{1} 61$ & 45.09 \\
\hline 1.996 & 3 & 251,152 & 45.39 \\
\hline 1.978 & 1 & 232 & 45.83 \\
\hline 1.962 & 8 & 052 & 46.24 \\
\hline 1.941 & 6 & 350 & 46.75 \\
\hline 1.907 & 5 & $\overline{2} 61$ & 47.64 \\
\hline 1.900 & 8 & $\overline{1} 3 \underline{3}, 411$ & 47.84 \\
\hline 1.878 & 4 & 323 & 48.42 \\
\hline 1.844 & 2 & 511,152 & 49.38 \\
\hline 1.839 & 2 & 421 & 49.53 \\
\hline 1.829 & 9 & 440 & 49.81 \\
\hline 1.792 & 9 & 123,170 & 50.93 \\
\hline 1.773 & 2 & 162,261 & 51.49 \\
\hline 1.765 & 5 & $512,413,+$ & 51.76 \\
\hline 1.755 & 3 & 351 & 52.06 \\
\hline 1.751 & 3 & 431 & 52.21 \\
\hline 1.7161 & 8 & $043, \overline{5} 22,+$ & 53.34 \\
\hline 1.6938 & 5 & 171,270 & 54.10 \\
\hline 1.6596 & 1 & 212 & 55.31 \\
\hline 1.6442 & 7 & $\overline{4} 33, \overline{5} 32,+$ & 55.87 \\
\hline 1.6319 & 3 & $\overline{4} 52, \overline{3} 62$ & 56.33 \\
\hline 1.6208 & 2 & $\overline{2} 53$ & 56.75 \\
\hline 1.6161 & 2 & 342 & 56.93 \\
\hline 1.5981 & 2 & 361 & 57.63 \\
\hline 1.5871 & 5 & $271, \bar{I} 72$ & 58.07 \\
\hline 1.5779 & 7 & $\overline{5} 13,511$ & 58.44 \\
\hline 1.5583 & 6 & 370,233 & 59.25 \\
\hline 1.5422 & 5 & $523,460,+$ & 59.93 \\
\hline
\end{tabular}

\section{References}

Margulis, T.N. and D. H. Templeton (1962). Crystal structure and hydrogen bonding of magnesium ammonium sulfate hexahydrate, Z. Krist. 117, 334-357.

Tutton, A. E. (1893). Connection between the atomic weight of contained metals and the magnitude of the angles of crystals of isomorphous series. A study of the potassium, rubidium and cesium salts of the monoclinic series of double sulphates $\mathrm{R}_{2} \mathrm{M}\left(\mathrm{SO}_{4}\right)_{2} \cdot 6 \mathrm{H}_{2} \mathrm{O}, \mathrm{J}$. Chem. Soc. 63, $337-423$. 
Imidazole Nickel Nitrate, $\left(\mathrm{C}_{3} \mathrm{H}_{4} \mathrm{~N}_{2}\right) \mathrm{Ni}\left(\mathrm{NO}_{3}\right)_{2}$ (hexagonal)

Sample

The sample was prepared at NBS by C.W. Reimann. It was precipitated from water solutions of imidazole and $\mathrm{Ni}\left(\mathrm{NO}_{3}\right)_{2}$.

\section{Color}

Unground - very purplish blue

\section{Optical data}

Uniaxial (-) $\quad \mathrm{N}_{\mathrm{e}}=1.582, \mathrm{~N}_{\mathrm{o}}=1.594$

\section{Structure}

Hexagonal, $\mathrm{R} \overline{3}$ (148), $\mathrm{z}=3$, structure de-termined by Santoro et al..,[1969].

\section{Lattice constants}

\begin{tabular}{|l|c|c|}
\hline \multirow{2}{*}{ NBS, sample at $25^{\circ} \mathrm{C}-----$} & $a(\AA)$ & $c(\AA)$ \\
\cline { 2 - 3 } & $\begin{array}{r}12.353 \\
\pm .001\end{array}$ & $\begin{array}{r}14.804 \\
\pm .002\end{array}$ \\
\hline
\end{tabular}

\section{Density}

(calculated) $1.505 \mathrm{~g} / \mathrm{cm}^{3}$ at $25^{\circ} \mathrm{C}$.

\section{Reference intensity}

$$
I / I_{\text {corundum }}=3.0
$$

\section{References}

Santoro,A., A.D. Mighell, M.Zocchi and C. W. Reimann (1969). The crystal and molecular structure of hexakis (imidazole) nickel (II) nitrate, $\left(\mathrm{C}_{3} \mathrm{H}_{4} \mathrm{~N}_{2}\right)_{6} \mathrm{Ni}\left(\mathrm{NO}_{3}\right)_{2}$, Acta Cryst. B25, 842-847.

\begin{tabular}{|c|c|c|c|}
\hline \multicolumn{4}{|c|}{$\begin{array}{l}\text { Internal standard } W, a=3.16516 \AA \\
\text { CuK } a_{1} \quad \lambda=1.54056 \AA \text {; temp. } 25{ }^{\circ} \mathrm{C}\end{array}$} \\
\hline$d(\AA)$ & $I$ & $h k l$ & $2 \theta\left({ }^{\circ}\right)$ \\
\hline 8.67 & 20 & 101 & 10.19 \\
\hline 6.17 & 50 & 110 & 14.34 \\
\hline 6.08 & 100 & 012 & 14.56 \\
\hline 5.03 & 3 & 021 & 17.60 \\
\hline 4.94 & 3 & 003 & 17.94 \\
\hline 4.333 & 6 & 202 & 20.48 \\
\hline 3.899 & 35 & 211 & 22.79 \\
\hline 3.854 & 40 & 113 & 23.06 \\
\hline 3.549 & 30 & 122 & 25.07 \\
\hline 3.497 & 25 & 104 & 25.45 \\
\hline 3.090 & 3 & 220 & 28.87 \\
\hline 3.047 & 2 & 024 & 29.29 \\
\hline 2.910 & 6 & 131 & 30.69 \\
\hline 2.892 & 8 & 303 & 30.89 \\
\hline 2.855 & 9 & 015 & 31.30 \\
\hline 2.754 & 5 & 312 & 32.48 \\
\hline 2.732 & 4 & 214 & 32.75 \\
\hline 2.619 & 8 & 223 & 34.21 \\
\hline 2.592 & 6 & 205 & 34.57 \\
\hline 2.514 & 1 & 042 & 35.68 \\
\hline 2.467 & 1 & 006 & 36.39 \\
\hline 2.422 & 3 & 321 & 37.08 \\
\hline 2.389 & 6 & 125 & 37.61 \\
\hline 2.330 & 8 & 232 & 38.60 \\
\hline 2.313 & 5 & 134 & 38.90 \\
\hline 2.168 & 2 & 404 & 41.63 \\
\hline 2.119 & 4 & 051 & 42.64 \\
\hline 2.111 & 5 & 413 & 42.80 \\
\hline 2.095 & 2 & 315 & 43.15 \\
\hline 2.046 & 2 & 324 & 44.23 \\
\hline 2.030 & 2 & 306 & 44.59 \\
\hline 2.003 & 3 & 241 & 45.24 \\
\hline 1.985 & 2 & 045 & 45.67 \\
\hline 1.967 & 1 & 027 & 46.11 \\
\hline 1.950 & 3 & 422 & 46.54 \\
\hline 1.900 & 3 & 333 & 47.83 \\
\hline 1.889 & 2 & 235 & 48.13 \\
\hline 1.874 & 1 & 217 & 48.54 \\
\hline 1.782 & 2 & 600 & 51.22 \\
\hline 1.774 & 2 & 244 & 51.46 \\
\hline 1.748 & 1 & 208,431 & 52.29 \\
\hline 1.734 & 2 & 505 & 52.75 \\
\hline 1.722 & 2 & 137 & 53.15 \\
\hline 1.713 & 2 & 520 & 53.45 \\
\hline 1.696 & 2 & 416 & 54.02 \\
\hline
\end{tabular}




\section{Sample}

The sample was prepared by W. S. Brower as a single crystal pulled from a melt. After grinding, the effect of very strong cleavage, $\{110\}$, was noted in some sample mountings.

\section{Color}

Colorless

\section{Optical data}

Biaxial (-) $\quad N_{\alpha}=1.82, \quad N_{\beta}=1.83, \quad N_{\gamma}=1.84$ $2 \mathrm{~V}$ is medium large

\section{Structure}

Monoclinic, $\mathrm{C} 2 / \mathrm{m}$ (12), $\mathrm{z}=8$, isostructural with $\mathrm{MnMOO}_{4}$, structure of $\mathrm{MnMoO}_{4}$ determined by Abrahams and Reddy [1965].

\section{Density}

(calculated) $3.809 \mathrm{~g} / \mathrm{cm}^{3}$ at $25^{\circ} \mathrm{C}$.

\section{Reference intensity}

$$
I / I_{\text {corundum }}=2.6
$$

\section{Polymorphism}

Another monoclinic form is described as the high pressure modification, (wolframite type), PDF card 16-308. [Young and Schwartz, 1963].

\begin{tabular}{|c|c|c|c|}
\hline \multicolumn{4}{|c|}{$\begin{array}{l}\text { Internal standard Ag, a }=4.08641 \AA \\
\mathrm{CuK} a_{1} \quad \lambda=1.54056 \AA \text {; temp. } 25{ }^{\circ} \mathrm{C}\end{array}$} \\
\hline$d(\AA)$ & $I$ & $h k l$ & $2 \theta\left({ }^{\circ}\right)$ \\
\hline 6.727 & 4 & 110,001 & 13.15 \\
\hline 5.323 & 7 & III & 16.64 \\
\hline 4.667 & 29 & $\overline{2} 01$ & 19.00 \\
\hline 4.354 & 3 & 111 & 20.38 \\
\hline 3.823 & 46 & 021 & 23.25 \\
\hline 3.513 & 25 & 201 & 25.33 \\
\hline 3.374 & 100 & 220 & 26.39 \\
\hline 3.283 & 32 & $\bar{I} 12$ & 27.14 \\
\hline 3.252 & 15 & $\overline{2} 02$ & 27.40 \\
\hline 3.156 & 20 & $\overline{3} \perp 1$ & 28.25 \\
\hline 3.091 & 4 & 310 & 28.86 \\
\hline 2.795 & 14 & $112, \overline{1} 31$ & 31.99 \\
\hline 2.724 & 6 & 022 & 32.85 \\
\hline 2.669 & 13 & $\overline{3} 12$ & 33.55 \\
\hline 2.663 & 10 & $\overline{2} 22$ & 33.62 \\
\hline 2.623 & 3 & 131 & 34.16 \\
\hline 2.556 & $<1$ & 311 & 35.08 \\
\hline 2.460 & 8 & 400 & 36.50 \\
\hline 2.322 & 8 & $040, \overline{1} 32$ & 38.74 \\
\hline 2.275 & 2 & $\overline{3} 31$ & 39.58 \\
\hline 2.252 & 10 & 330 & 40.00 \\
\hline 2.176 & 9 & 222 & 41.47 \\
\hline 2.128 & 4 & 132 & 42.44 \\
\hline 2.112 & 4 & $\overline{3} 13$ & 42.79 \\
\hline $2.100^{\circ}$ & 3 & 240 & 43.04 \\
\hline 2.086 & 8 & $\overline{4} 22$ & 43.35 \\
\hline 2.080 & 10 & 241 & 43.48 \\
\hline 2.068 & 8 & $\overline{2} 23$ & 43.73 \\
\hline 2.018 & 5 & $312,331,+$ & 44.88 \\
\hline 2.011 & 8 & 113 & 45.05 \\
\hline
\end{tabular}

\section{Lattice constants}

\begin{tabular}{|c|c|c|c|c|}
\hline & $a(\stackrel{0}{A})$ & $b(\stackrel{\circ}{A})$ & $c(\stackrel{\circ}{A})$ & $\beta\left({ }^{\circ}\right)$ \\
\hline $\begin{array}{l}\text { Pakhomov and Medvedev [I'968]--- } \\
\text { NBS, sample at } 25{ }^{\circ} \mathrm{C}-\end{array}$ & $\begin{array}{r}10.35 \\
10.281 \\
\pm .001\end{array}$ & $\begin{array}{l}9.23 \\
9.291 \\
\pm .001\end{array}$ & $\begin{array}{l}7.12 \\
7.030 \\
\pm .001\end{array}$ & $\begin{array}{r}106^{\circ} 30^{\prime} \\
106^{\circ} 54^{\prime} \\
\pm 1^{\prime}\end{array}$ \\
\hline
\end{tabular}




\begin{tabular}{|c|c|c|c|}
\hline \multicolumn{4}{|c|}{$\begin{array}{l}\text { Internal standard } \mathrm{Ag}, \mathrm{a}=4.08641 \AA \\
\mathrm{CuK} a_{1} \quad \lambda=1.54056 \stackrel{\circ}{\mathrm{A}} \text {; temp. } 25^{\circ} \mathrm{C}\end{array}$} \\
\hline$d(\AA)$ & $I$ & $h k l$ & $2 \theta\left({ }^{\circ}\right)$ \\
\hline 1.966 & 5 & $\overline{403}$ & 46.14 \\
\hline 1.938 & 11 & 241 & 46.84 \\
\hline 1.928 & 12 & 421 & 47.09 \\
\hline 1.924 & 10 & $510, \overline{5} 12$ & 47.21 \\
\hline 1.911 & 4 & 042 & 47.53 \\
\hline 1.890 & 2 & $\overline{2} 42$ & 48.09 \\
\hline 1.867 & 2 & $\overline{1} 33$ & 48.74 \\
\hline 1.848 & 2 & 203 & 49.27 \\
\hline 1.825 & 2 & 150 & 49.94 \\
\hline 1.787 & 2 & $\overline{1} 51$ & 51.06 \\
\hline 1.755 & 10 & 204 & 52.07 \\
\hline 1.739 & 2 & 151 & 52.58 \\
\hline 1.720 & 6 & $\overline{4} 41, \underline{3} 32$ & 53.21 \\
\hline 1.714 & 8 & $\overline{1} 14, \overline{5} 31$ & 53.42 \\
\hline 1.689 & 10 & 242,440 & 54.28 \\
\hline 1.678 & 2 & $\overline{6} 02$ & 54.66 \\
\hline 1.660 & 4 & $530, \overline{5} 32$ & 55.31 \\
\hline 1.646 & 4 & $\overline{4} 4 \underline{2}$ & 55.79 \\
\hline 1.642 & 3 & $\overline{1} 52, \overline{2} 24$ & 55.95 \\
\hline 1.638 & 3 & $\overline{2} 43$ & 56.09 \\
\hline 1.625 & 2 & $\overline{3} 51, \overline{4} 04$ & 56.59 \\
\hline 1.616 & 4 & 313,350 & 56.94 \\
\hline 1.612 & 4 & $\underline{0} 43$ & 57.08 \\
\hline 1.607 & 2 & $\overline{6} 21$ & $57 \cdot 30$ \\
\hline 1.5811 & 6 & 024 & 58.31 \\
\hline 1.5696 & 2 & 152 & 58.78 \\
\hline 1.5602 & 2 & 114 & 59.17 \\
\hline 1.5457 & 6 & $\overline{3} 5 \underline{2}, 620$ & 59.78 \\
\hline 1.5341 & 6 & $\overline{4} 24$ & 60.28 \\
\hline 1.5092 & 4 & 061 & 61.38 \\
\hline 1.5006 & 4 & $\overline{4} 43$ & 61.77 \\
\hline 1.4960 & 4 & 601 & 61.98 \\
\hline 1.4767 & 6 & 260 & 62.88 \\
\hline 1.4661 & 2 & 204 & 63.39 \\
\hline 1.4505 & 4 & 333 & 64.15 \\
\hline 1.4457 & 6 & $243, \overline{7} 11$ & 64.39 \\
\hline
\end{tabular}

\section{References}

Abrahams, S.C. and J.M. Reddy(1965). Crystal structure of the transition-metal molybdates. I.paramagnetic alpha-MnMOO , J. Chem. Phys. 43, No.7, 2533-2543.

Pakhomov, V. I. and A. V. Medvedev (1968). Preliminary data on the crystal structure of magnesium molybdate, soviet Phys. Cryst. (English Transl.) 12, No.6, 925. Young, A.P. and C.M. Schwartz (1963).Highpressure synthesis of molybdates with the wolframite structure, Science 141 , 348-349. 
Sample

Crystals of the hexahydrate were formed very slowly when anhydrous magnesium perchlorate hydrated in a loosely stoppered bottle.

Major impurities

$0.001-0.01 \%$ each: $\mathrm{ca}$

\section{Color}

Coloriess

Optical data

Uniaxial (-) $\mathrm{N}_{\mathrm{O}}=1.484, \mathrm{~N}_{\mathrm{e}}=1.468$

\section{Structure}

Hexagona 1, $\mathrm{P} 6 / \mathrm{mmm}$ (I9I), $\mathrm{Z}=4$ or orthorhombic, Pmn $2_{1}$ (3I), $\mathrm{Z}=2$

Structure determined by West, [1935]

\section{Lattice constants}

\begin{tabular}{|c|c|c|}
\hline & $a(\stackrel{\circ}{)}$ & $c(\AA)$ \\
\hline 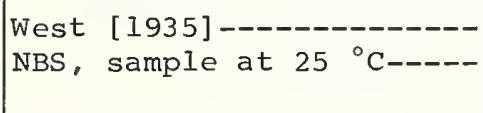 & $\begin{array}{r}15.55 \\
15.606 \\
\pm .001\end{array}$ & $\begin{array}{l}5.27 \\
5.2788 \\
\pm .0005\end{array}$ \\
\hline
\end{tabular}

\section{Density}

(calculated) $1.976 \mathrm{~g} / \mathrm{cm}^{3}$ at $25^{\circ} \mathrm{C}$.

\section{Reference intensity}

$$
I_{\text {corundum }}=1.7
$$

\section{Additional patterns}

I. PDF card 14-22 [Hanawalt et aI., 1938]

\begin{tabular}{|c|c|c|c|}
\hline \multicolumn{4}{|c|}{$\begin{array}{l}\text { Internal standard } \mathrm{W}, \mathrm{a}=3.16516 \AA \\
\mathrm{CuK} a_{1} \quad \lambda=1.54056 \AA \text {; temp. } 25^{\circ} \mathrm{C}\end{array}$} \\
\hline$d(\AA)$ & $I$ & $h k l$ & $2 \theta\left({ }^{\circ}\right)$ \\
\hline 6.75 & 6 & 200 & 13.11 \\
\hline 4.92 & 4 & 101 & 18.03 \\
\hline 4.37 & 6 & 111 & 20.29 \\
\hline 4.15 & 90 & 201 & 21.37 \\
\hline 3.90 & 100 & 220 & 22.80 \\
\hline 3.670 & 2 & $2 I I$ & 24.23 \\
\hline 3.424 & 2 & 301 & 26.00 \\
\hline 3.377 & 4 & 400 & 26.37 \\
\hline 3.054 & $I$ & 311 & 29.22 \\
\hline 2.843 & 85 & 401 & 31.44 \\
\hline $2.67 I$ & $<I$ & $32 I$ & 33.52 \\
\hline 2.638 & 11 & 002 & 33.95 \\
\hline 2.573 & $I$ & 411 & 34.83 \\
\hline 2.552 & 20 & 420 & 35.13 \\
\hline 2.458 & 3 & 202 & 36.52 \\
\hline 2.332 & $I$ & $33 I$ & 38.58 \\
\hline 2.297 & 13 & $42 I$ & 39.18 \\
\hline 2.292 & 2 & 600 & 40.01 \\
\hline 2.205 & $<I$ & 511 & 40.90 \\
\hline 2.1862 & $<I$ & 222 & 41.26 \\
\hline 2.0796 & 4 & 402 & 43.48 \\
\hline 2.0715 & 4 & 601 & 43.66 \\
\hline 2.0474 & $<I$ & 431 & 44.20 \\
\hline 2.0023 & $I$ & 521 & 45.25 \\
\hline 1.9501 & 10 & 440 & 46.53 \\
\hline 1.9202 & $I$ & 611 & 47.30 \\
\hline 1.8740 & 2 & 620 & 48.54 \\
\hline 1.8354 & 9 & 422 & 49.63 \\
\hline 1.8135 & $<I$ & $53 I$ & 50.27 \\
\hline 1.7872 & $<I$ & 512 & 51.06 \\
\hline 1.7660 & 11 & 621 & 51.72 \\
\hline 1.7134 & $I$ & 602 & 53.43 \\
\hline 1.7025 & 2 & 203,630 & 53.80 \\
\hline 1.6897 & $I$ & 800 & 54.24 \\
\hline I. 6440 & $<I$ & 541 & 55.88 \\
\hline 1.6208 & $<1$ & 631 & 56.75 \\
\hline 1.6089 & $I$ & 801 & 57.21 \\
\hline 1.5931 & $<I$ & 313 & 57.83 \\
\hline 1.5684 & $<I$ & 442 & 58.83 \\
\hline 1.5609 & 3 & 403,550 & 59.14 \\
\hline 1.5499 & $<I$ & 640 & 59.60 \\
\hline
\end{tabular}




\begin{tabular}{|c|c|c|c|}
\hline \multicolumn{4}{|c|}{$\begin{array}{l}\text { Internal standard } W, a=3.16516 \AA \\
\text { CuKa } a_{1} \lambda=1.54056 \AA \text {; temp. } 25{ }^{\circ} \mathrm{C}\end{array}$} \\
\hline$d(\AA)$ & $I$ & $h k l$ & $2 \theta\left({ }^{\circ}\right)$ \\
\hline 1.5283 & 2 & 622 & 60.53 \\
\hline 1.4874 & 4 & 641 & 62.38 \\
\hline 1.4746 & 4 & 503,820 & 62.98 \\
\hline 1.4493 & $<1$ & 423 & 64.21 \\
\hline 1.4451 & $<1$ & 901 & 64.42 \\
\hline 1.4229 & 2 & 802 & 65.55 \\
\hline 1.4200 & 2 & 821 & 65.70 \\
\hline 1.3866 & 1 & 603 & 67.49 \\
\hline 1.3686 & $<1$ & 651 & 68.50 \\
\hline 1.3550 & 1 & 741 & 69.29 \\
\hline 1.3517 & 1 & $10 \cdot 0 \cdot 0$ & 69.48 \\
\hline 1.3378 & $<1$ & 613 & 70.31 \\
\hline 1.3005 & 4 & 533,660 & 72.64 \\
\hline 1.2829 & 1 & $623,10 \cdot 1 \cdot 0$ & 73.80 \\
\hline 1.2503 & 1 & 224 & 76.06 \\
\hline 1.2412 & 1 & 841 & 76.72 \\
\hline 1.2184 & $<1$ & 803 & 78.43 \\
\hline 1.2161 & $<1$ & 931 & 78.60 \\
\hline 1.2032 & $<1$ & $10 \cdot 0 \cdot 2$ & 79.61 \\
\hline 1.1829 & 2 & $10 \cdot 2 \cdot 1$ & 81.26 \\
\hline 1.1665 & 1 & 662 & 82.65 \\
\hline 1.1634 & 1 & 643 & 82.92 \\
\hline 1.1496 & $<1$ & 842 & 84.14 \\
\hline 1.1388 & $<1$ & 604 & 85.13 \\
\hline 1.1263 & $<1$ & $524,12 \cdot 0 \cdot 0$ & 86.30 \\
\hline 1.1027 & $<1$ & $10 \cdot 2 \cdot 2$ & 88.62 \\
\hline 1.0931 & $<1$ & 444 & 89.61 \\
\hline 1.0872 & $<1$ & 861 & 90.22 \\
\hline 1.0820 & 1 & $833,10 \cdot 4 \cdot 0$ & 90.78 \\
\hline
\end{tabular}

\section{References}

Hanawalt, J.D., H.W. Rinn, and L.K. Frevel (1938). Chemical analysis by x-ray difEraction, Ind. Eng. Chem. Anal. Ed. 10, 457-513.

West, C.D. (1935). Crystal structures of hydrated compounds. II structure type $\mathrm{Mg}\left(\mathrm{ClO}_{4}\right)_{2} \cdot 6 \mathrm{H}_{2} \mathrm{O}$, Z.Krist. (A) $91,480-493$ 


\section{Sample}

The sample was obtained from Mallinckrodt Chemical Works.

Major impurities

trace amounts of $\mathrm{Fe}, \mathrm{Ca}, \mathrm{Cr}$, and $\mathrm{Mg}$.

Color

Orange-red

\section{Structure}

Tetragonal, $\mathrm{P} 4 \mathrm{a} / \mathrm{nmc}$ (137), $\mathrm{Z}=2$, structure determined by Bijvoet et aI.,[1926].

\section{Lattice constants}

\begin{tabular}{|c|c|c|}
\hline & $a(\AA)$ & $c(\stackrel{\AA}{A})$ \\
\hline $\begin{array}{l}\text { Havighurst* [1925] } \\
\text { Bijvoet et al.* [1926] } \\
\text { Huggins and Magill*[1927] } \\
\text { Swanson and Tatge [1953]- } \\
\text { Vlasse [1963]- } \\
\text { NBS, sample at } 25{ }^{\circ} \mathrm{C}\end{array}$ & $\begin{array}{l}4.356 \\
4.357 \\
4.34 \\
4.390 \\
4.361 \\
4.3693 \\
\pm .0001\end{array}$ & $\begin{array}{l}12.34 \\
12.36 \\
12.34 \\
12.38 \\
12.450 \\
12.4399 \\
\pm .0004\end{array}$ \\
\hline
\end{tabular}

*values as published

\section{Density}

(calculated) $6.354 \mathrm{~g} / \mathrm{cm}^{3}$ at $25^{\circ} \mathrm{C}$.

\section{Reference intensity}

$\mathrm{I}_{\text {corundum }}=3.8$

\section{Polymorphism}

Goya et al.[1962] reported a yellow, orthorhombic form stable above $127{ }^{\circ} \mathrm{C}$. Vlasse [1963] also notes a metastable orange, cubic or pseudo cubic form.

\section{Additional patterns}

1. Havighurst [1925]

2. Hanawalt, Rinn, and Frevel [1938]

3. PDF card 4-454 [Swanson and Tatge,1953]

\begin{tabular}{|c|c|c|c|}
\hline \multicolumn{4}{|c|}{$\begin{array}{l}\text { Internal standard } W, a=3.16516 \AA \\
\text { CuK } a_{1} \quad \lambda=1.54056 \AA \text {; temp. } 25{ }^{\circ} \mathrm{C}\end{array}$} \\
\hline$d(\AA)$ & $I$ & $h k l$ & $2 \theta(\circ)$ \\
\hline 6.223 & 55 & 002 & 14.22 \\
\hline 4.122 & 70 & 101 & 21.54 \\
\hline 3.577 & 100 & 102 & 24.87 \\
\hline 3.113 & 3 & 004 & 28.65 \\
\hline 3.092 & 2 & 110 & 28.85 \\
\hline 3.009 & 40 & 103 & 29.66 \\
\hline 2.768 & 30 & 112 & 32.32 \\
\hline 2.534 & 7 & 104 & 35.39 \\
\hline 2.192 & 60 & 114 & 41.14 \\
\hline 2.186 & 55 & 200 & 41.27 \\
\hline 2.163 & 17 & 105 & 41.73 \\
\hline 2.074 & 14 & 006 & 43.60 \\
\hline 2.062 & 6 & 202 & 43.87 \\
\hline 1.931 & 9 & 211 & 47.02 \\
\hline 1.874 & 15 & 106 & 48.55 \\
\hline 1.865 & 14 & $21 \overline{2}$ & 48.79 \\
\hline 1.789 & 1 & 204 & 51.02 \\
\hline 1.768 & 6 & 213 & 51.65 \\
\hline 1.722 & 1 & 116 & 53.14 \\
\hline 1.6543 & 3 & 214 & 55.50 \\
\hline 1. 6464 & 5 & 107 & 55.79 \\
\hline 1.5554 & 5 & 008 & 59.37 \\
\hline I. 5450 & 4 & 220 & 59.81 \\
\hline 1.5371 & 5 & 215 & 60.15 \\
\hline 1.5039 & 6 & 206 & 61.62 \\
\hline 1.4655 & 2 & 108 & 63.42 \\
\hline 1.4469 & 2 & 301 & 64.33 \\
\hline 1.4221 & 4 & 216 & 65.59 \\
\hline 1.4181 & 5 & 302 & 65.80 \\
\hline 1.3745 & 2 & 303 & 68.17 \\
\hline 1.3490 & 2 & 312 & 69.64 \\
\hline 1.3176 & 3 & 109 & 71.55 \\
\hline 1. 3144 & 4 & 217 & 71.75 \\
\hline 1.2669 & 5 & 208 & 74.89 \\
\hline 1.2629 & 7 & 314 & 75.17 \\
\hline 1.2570 & 3 & 305 & 75.58 \\
\hline 1.2389 & 2 & 226 & 76.89 \\
\hline 1.2168 & 1 & 218 & 78.55 \\
\hline 1.2061 & 1 & 321 & 79.38 \\
\hline 1.1966 & 1 & $1 \cdot 0 \cdot 10$ & 80.14 \\
\hline
\end{tabular}




\begin{tabular}{|c|c|c|c|}
\hline \multicolumn{4}{|c|}{$\begin{array}{l}\text { Internal standard } \mathrm{W}, \mathrm{a}=3.16516 \AA \\
\mathrm{CuKa}_{1} \quad \lambda=1.54056 \AA \text { 作 temp. } 25{ }^{\circ} \mathrm{C}\end{array}$} \\
\hline$d(\AA)$ & $I$ & $h k l$ & $2 \theta\left({ }^{\circ}\right)$ \\
\hline 1.1917 & 2 & $3 \cdot 0 \cdot 6$ & 80.54 \\
\hline 1.1895 & 2 & 322 & 80.72 \\
\hline 1.1631 & 1 & 323 & 82.95 \\
\hline 1.1542 & 3 & $1 \cdot 1 \cdot 10$ & 83.73 \\
\hline 1.1285 & 1 & 219 & 86.09 \\
\hline 1.0958 & 2 & 228 & 89.33 \\
\hline 1.0926 & 2 & 400 & 89.66 \\
\hline 1.0893 & 1 & 325 & 90.00 \\
\hline 1.0759 & 1 & 402 & 91.44 \\
\hline 1.0630 & $I$ & 308 & 92.88 \\
\hline 1.0559 & 1 & 411 & 93.69 \\
\hline 1.0494 & 1 & $2 \cdot 1 \cdot 10$ & 94.45 \\
\hline 1.0462 & 1 & 326 & 94.83 \\
\hline 1.0448 & 2 & 412 & 95.00 \\
\hline 1.0266 & 1 & 413 & 97.24 \\
\hline 1.0160 & $<1$ & 332 & 98.60 \\
\hline 1.0087 & 1 & $1 \cdot 0 \cdot 12$ & 99.57 \\
\hline 1.0027 & $<1$ & 309 & 100.38 \\
\hline 0.9828 & 2 & $1 \cdot 1 \cdot 12$ & 103.21 \\
\hline .9777 & 1 & 334 & 103.97 \\
\hline .9770 & 2 & 319,420 & 104.08 \\
\hline .9664 & 1 & 406 & 105.70 \\
\hline .9435 & 1 & 416 & 109.45 \\
\hline .9244 & 2 & $3 \cdot 1 \cdot 10$ & 112.87 \\
\hline .9157 & 2 & $2 \cdot 1 \cdot 12$ & 114.54 \\
\hline .9101 & 2 & 417 & 115.63 \\
\hline .8938 & 2 & 408 & 119.03 \\
\hline .8838 & 1 & 426 & 121.27 \\
\hline .8653 & $<1$ & 432 & 125.79 \\
\hline .8550 & 1 & 433,511 & 128.56 \\
\hline .8489 & 2 & 512 & 130.29 \\
\hline .8291 & 1 & $3 \cdot 1 \cdot 12$ & 136.56 \\
\hline .8272 & 2 & 428 & 137.25 \\
\hline .8261 & 1 & 514 & 137.63 \\
\hline .8067 & $<1$ & $4 \cdot 1 \cdot 10$ & 145.44 \\
\hline .8052 & $<1$ & 436 & 146.12 \\
\hline .8045 & $<1$ & 522 & 146.43 \\
\hline .7963 & $<1$ & 523 & 150.63 \\
\hline .7933 & 1 & $3 \cdot 3 \cdot 10$ & 152.32 \\
\hline .7878 & 2 & $3 \cdot 2 \cdot 12$ & 155.82 \\
\hline
\end{tabular}

\section{References}

Bijvoet, J. M., A. Claassen, and A. Karssen (1926). The crystal structure of red mercuric iodide, Koninkl. Ned. Akad. Wetenschap. Proc. B 29, 529-546.

Goya H.,J.L.T. Waugh and H. Zeitlin (1962) The color of mercuric iodide on alumina, J. Phys. Chem. 66, 1906-1907.

Hanawalt J.D., H.W. Rinn, and L. K. Frevel (1938). Chemical analysis by x-ray diffraction, Ind. Eng. Chem., Anal. Ed. 10, 457-512.

Havighurst R.J. (1925). X-ray reflections from mercuric iodide, Am. J. Sci. 10, 556-558.

Huggins, M. L. and P. L. Magill(1927). The crystal structures of mercuric and mercurous iodides, J. Am. Chem. Soc. 49, $2357-2367$.

Swanson, H.E. and E. Tatge (1953). Standard x-ray diffraction powder patterns, Natl. Bur. Std. U.S. Circ. 539, Vol. I, 74-76.

Vlasse, Marcus (1963) The structure of the crystalline phases in the mercuric iodide system, 2lst Annual Pittsburgh Diffraction Conference, (Abstracts). 
Potassium Cadmium Sulfate $\mathrm{K}_{2} \mathrm{Cd}_{2}\left(\mathrm{SO}_{4}\right)_{3}$ (orthorhombic)

Sample

The sample was prepared at NBS by melting a stoichiometric mixture of $\mathrm{K}_{2} \mathrm{SO}_{4}$ and $\mathrm{CdSO}_{4}$ and annealing for 18 hours at $300{ }^{\circ} \mathrm{C}$ and then for 3 days at $150{ }^{\circ} \mathrm{C}$.

Major impurities

0.001-0.01\% each: $\mathrm{Na}$

$0.01-0.1 \%$ each: $\mathrm{Ca}$ and $\mathrm{Al}$

Color

Yellowish white

\section{Optical data}

Very low double refraction. $N_{\alpha}=1.588$ and $\mathrm{N}_{\gamma}=1.592$ (data limited by small grain size of the sample)。

\section{Structure}

Orthorhombic, probably P $2_{1} 2_{1} 2_{1}$ (19), $\mathrm{Z}=4$ Distorted langbeinite-type. A cubic cell was reported by Gattow and Zemann [1958]

\section{Lattice constants}

\begin{tabular}{|c|c|c|c|}
\hline & $a(\AA)$ & $b(\AA)$ & $c(\AA)$ \\
\cline { 2 - 4 } $\begin{array}{c}\text { Gattow and } \\
\text { Zemann [1958]-- }\end{array}$ & $\begin{array}{r}10.28 \\
\pm .05\end{array}$ & & \\
NBS, sample at & & & \\
$25{ }^{\circ} \mathrm{C}--------$ & $\begin{array}{r}10.212 \\
\pm .001\end{array}$ & $\begin{array}{r}10.280 \\
\pm .001\end{array}$ & $\begin{array}{r}10.171 \\
\pm .001\end{array}$ \\
\hline
\end{tabular}

\section{Density}

(calculated) $3.677 \mathrm{~g} / \mathrm{cm}^{3}$ at $25{ }^{\circ} \mathrm{C}$.

Reference intensity

$I_{\text {corundum }}=2.7$

\section{Polymorphism}

DTA measurements show a reversible inversion at $166{ }^{\circ} \mathrm{C}$. This is interpreted as a change to the undistorted langbeinite structure on heating.

\begin{tabular}{|c|c|c|c|}
\hline \multicolumn{4}{|c|}{$\begin{array}{l}\text { Internal standard } W, a=3.16516 \AA \\
\text { CuK } a_{1} \lambda=1.54056 \AA \text {; temp. } 25^{\circ} \mathrm{C}\end{array}$} \\
\hline$d(\AA)$ & $I$ & $h k l$ & $2 \theta(\circ)$ \\
\hline 7.23 & $<I$ & 110,011 & 12.23 \\
\hline 5.90 & 33 & - $\quad$ III & 15.00 \\
\hline 5.11 & 3 & 200 & 17.34 \\
\hline 4.572 & 35 & 210,201 & 19.40 \\
\hline 4.184 & 22 & 121 & 21.22 \\
\hline 3.607 & 4 & 202 & 24.66 \\
\hline 3.413 & 27 & 221,122 & 26.09 \\
\hline 3.245 & 80 & 130,031 & 27.46 \\
\hline 3.227 & 100\{ & 310,301 & 27.62 \\
\hline 3.216 & & 013,103 & 27.72 \\
\hline 3.094 & 15 & 131 & 28.83 \\
\hline 3.077 & 22 & 311 & 28.99 \\
\hline 2.841 & 8 & 032,320 & 31.46 \\
\hline 2.830 & 12 & 023,302 & 31.59 \\
\hline 2.737 & 82\{ & $231,132,+$ & 32.69 \\
\hline 2.727 & & $312,123,+$ & 32.81 \\
\hline 2.568 & 4 & 040 & 34.91 \\
\hline 2.551 & $<1$ & 400 & 35.15 \\
\hline 2.483 & 8 & 232 & 36.15 \\
\hline 2.475 & 12 & 401,223 & 36.27 \\
\hline 2.468 & 8 & 014,104 & 36.37 \\
\hline 2.404 & 3 & 303 & 37.38 \\
\hline 2.346 & 10 & 133 & 38.33 \\
\hline 2.340 & 8 & 313 & 38.44 \\
\hline 2.295 & 3 & 240,042 & 39.22 \\
\hline 2.276 & $<1$ & 024,204 & 39.57 \\
\hline 2.237 & 11 & 241,142 & 40.28 \\
\hline 2.231 & $8\} 1$ & 421 & 40.40 \\
\hline 2.226 & 1 & 412,124 & 40.49 \\
\hline 2.176 & 12 & 323 & 41.46 \\
\hline 2.092 & 15 & 242 & 43.21 \\
\hline 2.084 & $28\} 1$ & 422 & 43.38 \\
\hline 2.081 & ${ }^{20} y$ & 224 & 43.46 \\
\hline 2.051 & 5 & 340 & 44.12 \\
\hline 2.039 & 11 & 403 & 44.39 \\
\hline 2.016 & 10 & 150,051 & 44.92 \\
\hline 2.009 & 28 & 143,431 & 45.10 \\
\hline 2.001 & 32 & 413 & 45.29 \\
\hline 1.977 & 6 & 151 & 45.85 \\
\hline 1.966 & 8\{ & 333,511 & 46.13 \\
\hline 1.959 & 4 & 115 & 46.31 \\
\hline 1.906 & 5 & 250,052 & 47.68 \\
\hline 1.896 & 22\{ & $234,423,+$ & 47.93 \\
\hline 1.890 & 22\{ & 025,205 & 48.09 \\
\hline 1.875 & 1 & 251,152 & 48.52 \\
\hline 1.865 & 2 & 521,512 & 48.79 \\
\hline
\end{tabular}


Potassium Cadmium Sulfate $\mathrm{K}_{2} \mathrm{Cd}_{2}\left(\mathrm{SO}_{4}\right)_{3}$ (orthorhombic) - continued

\begin{tabular}{|c|c|c|c|}
\hline \multicolumn{4}{|c|}{$\begin{array}{l}\text { Internal standard } W, a=3.16516 \AA \\
\mathrm{CuKa}, \quad \lambda=1.54056 \AA \text {; temp. } 25^{\circ} \mathrm{C}\end{array}$} \\
\hline$d(\stackrel{\AA}{)}$ & $I$ & $h k l$ & $2 \theta(0)$ \\
\hline 1.802 & 1 & 404 & 50.62 \\
\hline 1.783 & 8\{ & 441 & 51.20 \\
\hline 1.778 & 8\{ & 522 & 51.33 \\
\hline 1.775 & 8 & 414,225 & 51.45 \\
\hline 1.760 & 4 & 350 & 51.91 \\
\hline 1.755 & 5 & 343,530 & 52.07 \\
\hline 1.749 & 5 & 503,035 & 52.26 \\
\hline 1.734 & 3 & 351,153 & 52.75 \\
\hline 1.729 & 2 & 531 & 52.90 \\
\hline 1.725 & 1 & 513,135 & 53.05 \\
\hline 1.706 & 1 & 442 & 53.67 \\
\hline 1.690 & 2 & 160,061 & 54.24 \\
\hline 1.678 & 3 & 610,601 & 54.65 \\
\hline 1.666 & 9 & 161 & 55.06 \\
\hline 1.663 & 17 & 352,253 & 55.20 \\
\hline 1.658 & 12 & 532,611 & 55.38 \\
\hline 1.650 & 13 & 116 & 55.64 \\
\hline 1.624 & 6 & 260,062 & 56.61 \\
\hline 1.614 & $<1$ & 602 & 57.01 \\
\hline 1.609 & 6 & 026,206 & 57.20 \\
\hline 1.605 & 6 & 261,162 & 57.37 \\
\hline 1.597 & 6 & $443,344,+$ & 57.69 \\
\hline 1.591 & $<1$ & $405,126,+$ & 57.93 \\
\hline 1.579 & 8 & 541,154 & 58.38 \\
\hline 1.575 & 8 & 145 & 58.55 \\
\hline 1.573 & 7 & 514,415 & 58.63 \\
\hline 1.558 & 3 & 533 & 59.26 \\
\hline 1.539 & 3 & 622 & 60.05 \\
\hline 1.535 & 2 & 226 & 60.23 \\
\hline 1.527 & 3 & 452 & 60.57 \\
\hline 1.524 & 3 & 630 & 60.71 \\
\hline 1.520 & $<1$ & 425,036 & 60.91 \\
\hline 1.514 & 2 & 361 & 61.16 \\
\hline 1.508 & 2 & 631 & 61.45 \\
\hline 1.504 & 5 & 136 & 61.63 \\
\hline 1.475 & 5 & 444 & 62.95 \\
\hline 1.465 & 4 & 362,263 & 63.42 \\
\hline 1.459 & $<1$ & 632,623 & 63.72 \\
\hline 1.455 & 4 & 326 & 63.92 \\
\hline 1.444 & 5 & $701,534,+$ & 64.48 \\
\hline
\end{tabular}

\section{References}

Gattow, G.and J.Zemann (1958). Über Doppelsulphate vom Langbeinit-Typ, $\mathrm{A}_{2}^{+} \mathrm{B}_{2}^{2+}\left(\mathrm{SO}_{4}\right)_{3}$ Z. Anorg. Allgem. Chem. 293, 233-240. 
Potassium Calcium Chloride (chlorocalcite), $\mathrm{KCaCl}_{3}$ (orthorhombic)

\section{Sample}

The sample was prepared by melting a mixture of $\mathrm{KCl}$ and anhydrous $\mathrm{CaCl}_{z}$ at $750{ }^{\circ} \mathrm{C}$. The material is hygroscopic and the patterns were made with the sample enclosed in a dry-mount.

\section{Color}

\section{Colorless}

\section{Optical data}

Very low birefringence, $\mathrm{N}=1.568$, shows polysynthetic twinning.

Structure

Orthorhombic, Pnma (62), $\mathrm{Z}=4$, by analogy with $\mathrm{NaZnF}_{3}$ and similar distorted perovskites.

\section{Lattice constants}

\begin{tabular}{|l|c|c|c|}
\hline & $a(\AA)$ & $b(\AA)$ & $c(\AA)$ \\
\cline { 2 - 4 } NBS, sample & & & \\
at 25 ${ }^{\circ} \mathrm{C}-------$ & 7.551 & 10.442 & 7.251 \\
& \pm .001 & \pm .001 & \pm .001 \\
\hline
\end{tabular}

\section{Density}

(calculated) $2.155 \mathrm{~g} / \mathrm{cm}^{3}$ at $25^{\circ} \mathrm{C}$.

\section{Reference intensity}

$\mathrm{I} / \mathrm{I}_{\text {corundu. }}=1.0$

\begin{tabular}{|c|c|c|c|}
\hline \multicolumn{4}{|c|}{$\begin{array}{l}\text { Internal standard } \mathrm{W}, \mathrm{a}=3.16516 \AA \\
\mathrm{CuK} a_{1} \quad \lambda=1.54056 \AA \text {; temp. } 25{ }^{\circ} \mathrm{C}\end{array}$} \\
\hline$d(\AA)$ & $I$ & $h k l$ & $2 \theta\left({ }^{\circ}\right)$ \\
\hline 5.227 & 25 & 101,020 & 16.95 \\
\hline 4.679 & 6 & 111 & 18.95 \\
\hline 3.776 & 9 & 200 & 23.54 \\
\hline 3.697 & 30 & 121 & 24.05 \\
\hline 3.622 & 5 & 002 & 24.56 \\
\hline 3.348 & 7 & 201 & 26.60 \\
\hline 3.270 & 12 & 102 & 27.25 \\
\hline 3.188 & 14 & 211 & 27.96 \\
\hline 3.137 & 35 & 031 & 28.43 \\
\hline 3.119 & 30 & 112 & 28.60 \\
\hline 3.058 & 18 & 220 & 29.18 \\
\hline 2.978 & 12 & 022 & 29.98 \\
\hline 2.896 & 14 & 131 & 30.85 \\
\hline 2.819 & 13 & 221 & 31.72 \\
\hline 2.770 & 8 & 122 & 32.29 \\
\hline
\end{tabular}

\begin{tabular}{|c|c|c|c|}
\hline$d(\AA)$ & $I$ & $h k l$ & $2 \theta\left({ }^{\circ}\right)$ \\
\hline 2.614 & 100 & 202 & 34.27 \\
\hline 2.610 & $+\infty$ & 040 & 34.33 \\
\hline 2.559 & 7 & 230 & 35.03 \\
\hline 2.537 & 10 & 212 & 35.35 \\
\hline 2.414 & 4 & 231 & 37.21 \\
\hline 2.377 & 15 & 301 & 37.82 \\
\hline 2.338 & 13 & 222,141 & 38.48 \\
\hline 2.318 & 13 & 311 & 38.81 \\
\hline 2.248 & 4 & 113 & 40.07 \\
\hline 2.163 & 8 & 321 & 41.72 \\
\hline 2.146 & 13 & 240 & 42.06 \\
\hline 2.119 & 14 & 042 & 42.64 \\
\hline 2.107 & 25 & 123 & 42.89 \\
\hline 2.037 & 5 & 203 & 44.45 \\
\hline 2.006 & 3 & 051 & 45.16 \\
\hline 1.963 & 3 & 331 & 46.21 \\
\hline 1.920 & 4 & 133 & 47.31 \\
\hline 1.887 & 5 & 400 & 48.19 \\
\hline 1.857 & 2 & 410 & 49.00 \\
\hline 1.847 & 12 & 242 & 49.30 \\
\hline 1.827 & 6 & 250,401 & 49.87 \\
\hline 1.812 & 1 & 004 & 50.31 \\
\hline 1.778 & 7 & 332 & 51.35 \\
\hline 1.772 & 10 & 251 & 51.52 \\
\hline 1.758 & 10 & 341,237 & 51.97 \\
\hline 1.744 & 1 & 303 & 52.43 \\
\hline 1.740 & $<1$ & 060 & 52.55 \\
\hline 1.720 & $<1$ & 313 & 53.21 \\
\hline 1.674 & $<1$ & 402 & 54.81 \\
\hline 1.670 & 2 & 124 & 54.94 \\
\hline 1.652 & 3 & 161 & 55.59 \\
\hline 1.632 & 4 & 252 & 56.32 \\
\hline 1.615 & $<1$ & 214 & 56.96 \\
\hline 1.606 & $<1$ & 243 & 57.34 \\
\hline 1.569 & 1 & 351,062 & 58.80 \\
\hline 1.559 & 2 & 224,333 & 59.22 \\
\hline 1.547 & 2 & 153 & 59.74 \\
\hline 1.530 & 2 & 440 & 60.45 \\
\hline 1.509 & 3 & 432 & 61.40 \\
\hline 1.489 & 1 & 044 & 62.30 \\
\hline 1.479 & 2 & 234,501 & 62.78 \\
\hline 1.474 & $<1$ & 413 & 63.03 \\
\hline 1.464 & 1 & 511 & 63.49 \\
\hline 1.461 & 1 & 071,144 & 63.64 \\
\hline 1.434 & $<1$ & 171 & 64.96 \\
\hline 1.4311 & $I$ & 423 & 65.13 \\
\hline 1.4164 & $<I$ & 324 & 65.89 \\
\hline 1.4103 & $<1$ & 115 & 66.21 \\
\hline 1.3998 & $<1$ & 450 & 66.77 \\
\hline
\end{tabular}


Potassium Calcium Magnesium Sulfate, $\mathrm{K}_{2} \mathrm{CaMg}\left(\mathrm{SO}_{4}\right)_{3}$ (cubic)

\section{Sample}

The $\mathrm{K}_{2} \mathrm{CaMg}\left(\mathrm{SO}_{4}\right)_{3}$ was prepared by melting a stoichiometric mixture of $\mathrm{K}_{2} \mathrm{SO}_{4}$, $\mathrm{CaSO}_{4}$, and $\mathrm{MgSO}_{4}$. The sample was annealed for 20 hours at $800^{\circ} \mathrm{C}$ and 17 hours at $400^{\circ} \mathrm{C}$.

\section{Major impurities}

$0.01-0.1 \%$ each: $\mathrm{Cs}, \mathrm{Cu}, \mathrm{Na}$, and $\mathrm{Rb}$

$0.1-1.0 \%$ each: $\mathrm{Fe}$

\section{Color}

Yellowish white

\section{Optical data}

Isotropic, $\mathrm{N}=1.525$

\section{Structure}

Cubic, P2 3 (198), $\mathrm{Z}=4$ by analogy with langbeinite, $\mathrm{K}_{2} \mathrm{Mg}_{2}\left(\mathrm{SO}_{4}\right)_{3}$. The langbeinite structure was determined by Zemann and Zemann [1957].

\section{Lattice constants}

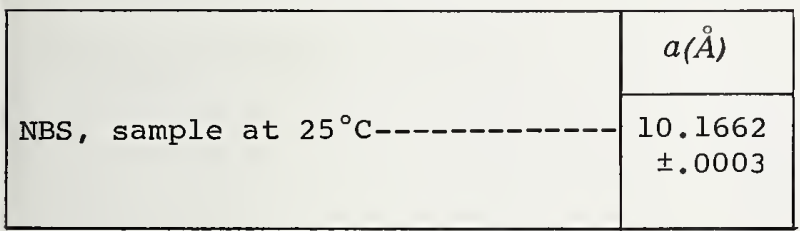

Density

(calculated) $2.723 \mathrm{~g} / \mathrm{cm}^{3}$ at $25^{\circ} \mathrm{C}$.

\section{Reference intensity}

$$
I / I_{\text {corundum }}=2.6
$$

\begin{tabular}{|c|c|c|c|}
\hline \multicolumn{4}{|c|}{$\begin{array}{l}\text { Internal standard } \mathrm{W}, \mathrm{a}=3.16516 \AA \\
\mathrm{CuK} \alpha_{1} \quad \lambda=1.54056 \AA \text {; temp. } 25{ }^{\circ} \mathrm{C}\end{array}$} \\
\hline$d(\AA)$ & $I$ & $h k l$ & $2 \theta\left({ }^{\circ}\right)$ \\
\hline 5.86 & 4 & 111 & 15.11 \\
\hline 4.544 & 1 & 210 & 19.52 \\
\hline 4.149 & 21 & 211 & 21.40 \\
\hline 3.596 & $<1$ & 220 & 24.74 \\
\hline 3.388 & 2 & 221 & 26.28 \\
\hline 3.212 & 100 & 310 & 27.75 \\
\hline 3.066 & 9 & 311 & 29.10 \\
\hline 2.819 & 7 & 320 & 31.72 \\
\hline 2.717 & 34 & 321 & 32.94 \\
\hline 2.540 & $<1$ & 400 & 35.30 \\
\hline 2.466 & 4 & 322 & 36.40 \\
\hline 2.333 & 2 & 331 & 38.56 \\
\hline 2.273 & 1 & 420 & 39.61 \\
\hline 2.219 & 2 & 421 & 40.63 \\
\hline 2.168 & 4 & 332 & 41.63 \\
\hline 2.075 & 7 & 422 & 43.59 \\
\hline 2.033 & 1 & 430 & 44.54 \\
\hline 1.994 & 10 & 510 & 45.45 \\
\hline 1.957 & 1 & 511 & 46.35 \\
\hline 1.888 & 4 & 520 & 48.16 \\
\hline 1.856 & 1 & 521 & 49.04 \\
\hline 1.769 & 3 & 522 & 51.61 \\
\hline 1.743 & 1 & 530 & 52.44 \\
\hline 1.718 & $<1$ & 531 & 53.27 \\
\hline 1.695 & $<1$ & 600 & 54.06 \\
\hline 1.671 & 1 & 610 & 54.90 \\
\hline 1.649 & 8 & 611 & 55.69 \\
\hline 1.608 & 2 & 620 & 57.26 \\
\hline 1.588 & 2 & 621 & 58.04 \\
\hline 1.569 & 2 & 541 & 58.82 \\
\hline
\end{tabular}


Potassium Calcium Magnesium Sulfate, $\mathrm{K}_{2} \mathrm{CaMg}\left(\mathrm{SO}_{4}\right)_{3}$ (cubic) - continued

\begin{tabular}{|c|c|c|c|}
\hline \multicolumn{4}{|c|}{$\begin{array}{l}\text { Internal standard } \mathrm{W}, \mathrm{a}=3.16516 \AA \\
\mathrm{CuK} a_{1} \lambda=1.54056 \AA \text { А; temp. } 25{ }^{\circ} \mathrm{C}\end{array}$} \\
\hline$d(\AA)$ & $I$ & $h k l$ & $2 \theta\left({ }^{\circ}\right)$ \\
\hline 1.550 & $<1$ & 533 & 59.58 \\
\hline 1.532 & $<1$ & 622 & 60.37 \\
\hline 1.5154 & 2 & 630 & 61.10 \\
\hline 1.4986 & 2 & 631 & 61.86 \\
\hline 1.4678 & 1 & 444 & 63.31 \\
\hline 1.4522 & 1 & 632 & 64.07 \\
\hline 1.4380 & $<1$ & 550 & 64.78 \\
\hline 1.4096 & 1 & 640 & 66.25 \\
\hline 1.3967 & 1 & 720 & 66.94 \\
\hline 1.3834 & 2 & 721 & 67.67 \\
\hline 1.3586 & 1 & 642 & 69.08 \\
\hline 1.3468 & $<1$ & 722 & 69.77 \\
\hline 1.3348 & $<1$ & 730 & 70.49 \\
\hline 1.3234 & 1 & 731 & 71.19 \\
\hline 1.3014 & 1 & 650 & 72.58 \\
\hline 1.2909 & 1 & 732 & 73.27 \\
\hline 1.2612 & 1 & 810 & 75.29 \\
\hline 1.2517 & $<1$ & 811 & 75.96 \\
\hline 1.2423 & $<1$ & 733 & 76.64 \\
\hline 1.2328 & $<1$ & 820 & 77.34 \\
\hline 1.2240 & $<1$ & 821 & 78.00 \\
\hline 1.2154 & $<1$ & 653 & 78.66 \\
\hline 1.1985 & $<1$ & 822 & 79.99 \\
\hline 1.1817 & 1 & 831 & 81.36 \\
\hline 1.1740 & $<1$ & 751 & 82.01 \\
\hline 1.1513 & $<1$ & 752 & 83.99 \\
\hline 1.1290 & $<1$ & 841 & 86.04 \\
\hline 1.1223 & $<1$ & 910 & 86.68 \\
\hline 1.1158 & $<1$ & 911 & 87.31 \\
\hline 1.1090 & $<1$ & 842 & 87.99 \\
\hline 1.0961 & 1 & 921 & 89.30 \\
\hline 1.0836 & $<1$ & 664 & 90.61 \\
\hline 1.0774 & $<1$ & 922 & 91.28 \\
\hline 1.0717 & $<1$ & 930 & 91.90 \\
\hline 1.0545 & $<1$ & 852 & 93.85 \\
\hline
\end{tabular}

\section{References}

Zemann,A. and J. Zemann (1957). Die Kristallstruktur vom Langbeinit, $\mathrm{K}_{2} \mathrm{Mg}_{2}\left(\mathrm{SO}_{4}\right)_{3}$, Acta Cryst. 10, 409-413. 
Potassium Calcium Sulfate, $\mathrm{K}_{2} \mathrm{Ca}_{2}\left(\mathrm{SO}_{4}\right)_{3}$ (orthorhombic)

Sample

The sample was prepared at NBS by melting a stoichiometric mixture of $\mathrm{K}_{2} \mathrm{SO}_{4}$ and $\mathrm{CaSO}_{4}$. This was then annealed for 18 hours at $700{ }^{\circ} \mathrm{C}$.

\section{Major impurities}

$0.01-0.1 \%$ each: $\mathrm{Ag}$ and $\mathrm{Cu}$.

$0.1-1.0 \%$ each: $\mathrm{Cs}$ and $\mathrm{Na}$.

\section{Color}

Colorless.

Optical data

Biaxial (-) $\mathrm{N}_{\alpha}=1.522, \mathrm{~N}_{\beta}=1.526, \mathrm{~N}_{\gamma}=1.527$, $2 \mathrm{~V}$ is small.

\section{Structure}

Orthorhombic, probably $\mathrm{P} 2_{1} 2_{1} 2_{1}$ (19), $\mathrm{Z}=4$. Distorted langbeinite type. $\mathrm{K}_{2} \mathrm{Ca}_{2}\left(\mathrm{SO}_{4}\right)_{3}$ has been reported as cubic [Ramsdell, 1935].

\section{Lattice constants}

\begin{tabular}{|c|c|c|c|}
\hline & $a(\AA)$ & $b(\AA)$ & \multicolumn{1}{c|}{$c(\AA)$} \\
\cline { 2 - 4 } Ramsdell [1935] -- & 10.35 & & \\
NBS, sample at & & & \\
$25^{\circ}$ C------- & $\begin{array}{rlr}10.334 \\
\pm .001\end{array}$ & $\begin{aligned} 10.501 \\
\pm .001\end{aligned}$ & $\begin{array}{r}10.186 \\
\pm .001\end{array}$ \\
\hline
\end{tabular}

\section{Density}

(calculated) $2.683 \mathrm{~g} / \mathrm{cm}^{3}$ at $25^{\circ} \mathrm{C}$.

\section{Reference intensity}

$$
I / I_{\text {corundum }}=0.9
$$

\section{Polymorphism}

Inverts to a cubic langbeinite form at $200{ }^{\circ} \mathrm{C}$ [Morey et al,,1964]. An inversion at $940{ }^{\circ} \mathrm{C}$ has also been reported [Bellanca, 1942] .

\begin{tabular}{|c|c|c|c|}
\hline \multicolumn{4}{|c|}{$\begin{array}{l}\text { Internal standard } \mathrm{W}, \mathrm{a}=3.16516 \AA \\
\mathrm{CuK} \alpha_{1} \quad \lambda=1.54056 \AA \text {; temp. } 25{ }^{\circ} \mathrm{C}\end{array}$} \\
\hline$d(\AA)$ & $I$ & $h k l$ & $2 \theta\left({ }^{\circ}\right)$ \\
\hline 7.32 & 4 & 011 & 12.08 \\
\hline 5.969 & 8 & 111 & 14.83 \\
\hline 4.665 & 3 & 021 & 19.01 \\
\hline 4.574 & 6 & 012,102 & 19.39 \\
\hline 4.255 & 28 & 121 & 20.86 \\
\hline 4.221 & 23 & 211 & 21.03 \\
\hline 4.189 & 16 & 112 & 21.19 \\
\hline 3.462 & 12 & 221 & 25.71 \\
\hline 3.315 & 93 & 130,031 & 26.87 \\
\hline 3.272 & 79 d & 310 & 27.23 \\
\hline 3.263 & 19 & 301 & 27.31 \\
\hline 3.225 & 100 & 013,103 & 27.64 \\
\hline 3.152 & 18 & 131 & 28.29 \\
\hline 3.116 & 18 & 311 & 28.62 \\
\hline 3.082 & 3 & 113 & 28.95 \\
\hline 2.987 & 2 & 222 & 29.89 \\
\hline 2.881 & 18 & 032,320 & 31.02 \\
\hline 2.853 & 6 & 302,023 & 31.33 \\
\hline 2.786 & 43 & 231 & 32.10 \\
\hline 2.776 & 49 & 132 & 32.22 \\
\hline 2.750 & & 312,123 & 32.53 \\
\hline 2.743 & 43 & 213 & 32.62 \\
\hline 2.543 & 6 & 140,041 & 35.26 \\
\hline 2.510 & 4 & 410,322 & 35.75 \\
\hline 2.497 & 4 & 223 & 35.93 \\
\hline 2.471 & 4 & 104,141 & 36.32 \\
\hline 2.436 & 2 & 033,411 & 36.86 \\
\hline 2.406 & 2 & 114 & 37.35 \\
\hline 2.387 & 3 & 331 & 37.65 \\
\hline 2.372 & 7 & 133 & 37.90 \\
\hline 2.357 & 2 & 313 & 38.15 \\
\hline 2.341 & 2 & 240 & 38.42 \\
\hline 2.333 & 2 & 042 & 38.55 \\
\hline 2.276 & 5 & 142 & 39.56 \\
\hline 2.250 & 2 & 412 & 40.04 \\
\hline 2.232 & 3 & 214 & 40.38 \\
\hline 2.213 & 4 & 332 & 40.74 \\
\hline 2.204 & 8 & 233 & 40.91 \\
\hline 2.196 & 11 & 323 & 41.07 \\
\hline 2.125 & 4 & 242 & 42.50 \\
\hline 2.109 & 18 & 422 & 42.84 \\
\hline
\end{tabular}


Potassium Calcium Sulfate, $\mathrm{K}_{2} \mathrm{Ca}_{2}\left(\mathrm{SO}_{4}\right)_{3}$ (orthorhombic) - continued

\begin{tabular}{|c|c|c|c|}
\hline \multicolumn{4}{|c|}{$\begin{array}{l}\text { Internal standard } \mathrm{W}, \mathrm{a}=3.16516 \AA \\
\mathrm{CuK} a_{1} \lambda=1.54056 \AA \text {; temp. } 25{ }^{\circ} \mathrm{C}\end{array}$} \\
\hline$d(\AA)$ & $I$ & $h k l$ & $2 \theta\left({ }^{\circ}\right)$ \\
\hline 2.093 & 22 & 224 & 43.18 \\
\hline 2.058 & 8 & $150,051,+$ & 43.97 \\
\hline 2.044 & 6 & 341 & 44.27 \\
\hline 2.037 & 14 & 431,143 & 44.44 \\
\hline 2.018 & 19 & $413,151,+$ & 44.88 \\
\hline 2.010 & 14 & 314 & 45.06 \\
\hline 1.990 & 4 & 333,511 & 45.55 \\
\hline 1.941 & 2 & 052 & 46.75 \\
\hline 1.932 & 5 & 342 & 47.00 \\
\hline 1.924 & 6 & 432,520 & 47.21 \\
\hline 1.914 & 6 & $502,423,+$ & 47.46 \\
\hline 1.908 & 4 & 152,324 & 47.62 \\
\hline 1.891 & 3 & 521 & 48.08 \\
\hline 1.884 & 1 & 512 & 48.26 \\
\hline 1.818 & 2 & 252 & 50.14 \\
\hline 1.811 & 6 & 441 & 50.33 \\
\hline 1.799 & 2 & 144,522 & 50.71 \\
\hline 1.793 & 3 & 350 & 50.88 \\
\hline 1.786 & 4 & 414.053 & 51.09 \\
\hline 1.780 & 4 & 530,343 & 51.27 \\
\hline 1.765 & 2 & 503,035 & 51.75 \\
\hline 1.753 & 2 & 305,531 & 52.13 \\
\hline 1.730 & 2 & 315 & 52.89 \\
\hline 1.725 & 2 & 160,061 & 53.04 \\
\hline 1.700 & 6 & 161,610 & 53.87 \\
\hline 1.697 & 6 & 601,006 & 53.98 \\
\hline 1.692 & 8 & 352 & 54.17 \\
\hline 1.688 & 8 & 253 & 54.30 \\
\hline 1.675 & 5 & $016,106,+$ & 54.74 \\
\hline 1.664 & 4 & 325 & 55.16 \\
\hline 1.654 & 5 & 062,116 & 55.51 \\
\hline 1.635 & 2 & 162 & 56.22 \\
\hline 1.631 & 2 & 602 & 56.35 \\
\hline 1.610 & 7 & 434,045 & 57.15 \\
\hline 1.604 & 6 & 504,541 & 57.40 \\
\hline 1.600 & 4 & 154,405 & 57.55 \\
\hline 1.594 & 2 & 216 & 57.78 \\
\hline
\end{tabular}

Additional patterns

1.PDF card 17-0741 [Morey et al.,1964]

\section{References}

Bellanca,A. (1942) L'aftitalite nel sistema ternario $\mathrm{K}_{2} \mathrm{SO}_{4}-\mathrm{Na}_{2} \mathrm{SO}_{4}-\mathrm{CaSO}_{4}$, Periodico Mineral. (Rome) 13, 21-85.

Morey, G.W., J.J. Rowe, and R.O. Fournier (1964). The system $\mathrm{K}_{2} \mathrm{Mg}_{2}\left(\mathrm{SO}_{4}\right)_{3}$ (langbeinite) - $\mathrm{K}_{0} \mathrm{Ca}\left(\mathrm{SO}_{4}\right)_{3}$ (calcium-langbeinite), J. Inorg. Nucl. Chem. 26, 53-58.

Ramsdell, L。S. (1935). An x-ray study of the system $\mathrm{K}_{2} \mathrm{SO}_{4}-\mathrm{MgSO}_{4}-\mathrm{CaSO}_{4}$, Am. Mineralogist. 20, 569-574. 
Sample

The sample was crystallized from a mixture of concentrated hydrochloric acid, $\mathrm{KCl}$ and $\mathrm{CuCl}_{2}$ by dehydration in a desiccator.

\section{Major impurities}

less than $0.001 \%$ each of $\mathrm{Al}, \mathrm{Ba}, \mathrm{Ca}, \mathrm{Mg}$, and $\mathrm{si}$

Color

strong brown

Optical data

Anisotropic, $N_{\alpha}=1.670, N_{\gamma}=1.890$. Crystals were very fine and needle shaped.

\section{Structure}

Monoclinic, $\mathrm{P} 2{ }_{1} / \mathrm{C}(14), \mathrm{Z}=4$, Structure determined by Willett et al. [1963]

\section{Lattice constants}

\begin{tabular}{|l|c|c|c|c|}
\hline & $a(\AA)$ & $b(\AA)$ & $c(\AA)$ & $\beta\left({ }^{\circ}\right)$ \\
\cline { 2 - 5 } $\begin{array}{l}\text { Willett } \\
\text { et al.- } \\
\text { [1963] }\end{array}$ & $\begin{array}{l}4.029 \\
\pm .005\end{array}$ & $\begin{array}{r}13.785 \\
\pm .003\end{array}$ & $\begin{array}{l}8.736 \\
\pm .004\end{array}$ & $\begin{array}{r}97^{\circ} 20^{\prime} \\
\pm 5^{\prime}\end{array}$ \\
$\begin{array}{l}\text { NBS } \\
\text { sample } \\
\text { at 25 }{ }^{\circ} \mathrm{C}\end{array}$ & $\begin{array}{l}4.031 \\
\pm .001\end{array}$ & $\begin{array}{r}13.788 \\
\pm .002\end{array}$ & $\begin{array}{l}8.732 \\
\pm .001\end{array}$ & $\begin{array}{r}97^{\circ} 10^{\prime} \\
\pm 1^{\prime}\end{array}$ \\
& & & & \\
\hline
\end{tabular}

\section{Density}

(calculated) $2.883 \mathrm{~g} / \mathrm{cm}^{3}$ at $25^{\circ} \mathrm{C}$.

\section{Reference intensity}

$$
\mathrm{I} / \mathrm{I}_{\text {corundum }}=1.0
$$

\section{References}

Willett, R. D., C. Dwiggins,Jr.,R. F. Kruh and R. E. Rundle (1963). Crystal structures of $\mathrm{KCuCl}_{3}$ and $\mathrm{NH}_{4} \mathrm{CuCl}_{3}$. J. Chem. Phys. 38, 2429-2436.

\begin{tabular}{|c|c|c|c|}
\hline \multicolumn{4}{|c|}{$\begin{array}{l}\text { Internal standard } W, a=3.16516 \AA \\
\text { CuK } a_{1} \lambda=1.54056 \AA \text {; temp. } 25{ }^{\circ} \mathrm{C}\end{array}$} \\
\hline$d(\AA)$ & $I$ & $h k l$ & $2 \theta\left({ }^{\circ}\right)$ \\
\hline 7.33 & 65 & 011 & 12.06 \\
\hline 6.89 & 100 & 020 & 12.84 \\
\hline 5.40 & 55 & 021 & 16.41 \\
\hline 4.33 & 14 & 002 & 20.47 \\
\hline 4.13 & 30 & 012 & 21.48 \\
\hline 4.06 & 40 & 031 & 21.87 \\
\hline 3.67 & 75 & 022 & 24.20 \\
\hline 3.45 & 8 & 040 & 25.81 \\
\hline 3.366 & 15 & 111 & 26.46 \\
\hline 3.342 & 12 & $\overline{1} 21$ & 26.65 \\
\hline 3.178 & 80 & 1002 & 28.42 \\
\hline 3.061 & 95 & $\overline{1} 12$ & 29.15 \\
\hline 2.937 & 15 & $\overline{1} 31$ & 30.41 \\
\hline 2.856 & 90 & $\overline{1} 22$ & 31.29 \\
\hline 2.772 & 40 & 102,131 & 32.27 \\
\hline 2.714 & 90 & 112 & 32.98 \\
\hline 2.698 & 75 & 042 & 33.18 \\
\hline 2.665 & 40 & 023 & 33.60 \\
\hline 2.629 & 60 & 051 & 34.07 \\
\hline 2.572 & 60 & 122 & 34.85 \\
\hline 2.454 & 6 & $\overline{1} 13$ & 36.58 \\
\hline 2.374 & 25 & 132 & 37.87 \\
\hline 2.346 & 30 & $\overline{1} 23$ & 38.34 \\
\hline 2.326 & 17 & 052 & 38.67 \\
\hline 2.271 & 45 & 150 & 39.66 \\
\hline 2.222 & 45 & 061 & 40.57 \\
\hline 2.215 & 45 & 043 & 40.69 \\
\hline 2.186 & 11 & 113 & 41.26 \\
\hline 2.158 & 13 & 142,151 & 41.83 \\
\hline 2.142 & 4 & 014 & 42.16 \\
\hline 2.110 & 12 & 123 & 42.83 \\
\hline 2.066 & 20 & 024 & 43.77 \\
\hline 2.020 & 17 & $\overline{1} 43$ & 44.83 \\
\hline 2.001 & 40 & 200 & 45.29 \\
\hline 1.959 & 25 & 034 & 46.30 \\
\hline 1.932 & 12 & $\overline{1} 24$ & 46.99 \\
\hline 1.921 & 20 & 071 & 47.28 \\
\hline 1.863 & 30 & 143 & 48.84 \\
\hline 1.850 & 17 & $\overline{1} 53$ & 49.22 \\
\hline 1.844 & 16 & $\overline{1} 34$ & 49.38 \\
\hline 1.792 & 18 & 072 & 50.90 \\
\hline 1.767 & 17 & 170 & 51.69 \\
\hline 1.750 & 19 & 171 & 52.21 \\
\hline 1.703 & 9 & 054 & 53.78 \\
\hline
\end{tabular}




\section{Sample}

The sample was prepared by adding hydrofluoric acid to a mixture of $\mathrm{K}_{2} \mathrm{CO}_{3}$ and $\mathrm{NiCO}_{3}$. The material was then heated to about $200{ }^{\circ} \mathrm{C}$.

\section{Color}

Pale yellow green

\section{Structure}

Cubic, perovskite type, Pm3m (221) $\quad \mathrm{Z}=1$ [Rïdorff et al., 1958]. $\mathrm{KNiF}_{3}$ was reported by Martin et al., [1956] as pseudocubic.

\section{Lattice constants}

\begin{tabular}{|c|c|}
\hline & $a(\AA)$ \\
\hline Martin et al.[1956]------------ & $4.01 *$ \\
\hline Ruidorff et al.[1959]--n--n-n & 4.009 \\
\hline Hirakawa et al.[1960]---------- & 4.015 \\
\hline Okazaki and Suemune[196I]------- & $\begin{array}{l} \pm .001 \\
4.014 \\
\pm .001\end{array}$ \\
\hline 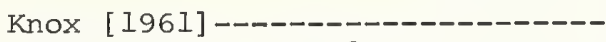 & 4.012 \\
\hline NBS, sample at $25{ }^{\circ} \mathrm{C}-$ & $\begin{array}{r}4.0127 \\
\pm .0001\end{array}$ \\
\hline
\end{tabular}

*pseudocubic

Density

(calculated) $3.978 \mathrm{~g} / \mathrm{cm}^{3}$ at $25^{\circ} \mathrm{C}$.

\section{Reference intensity}

$$
1 / I_{\text {corundum }}=3.0
$$

\section{Additional patterns}

1.PDF card 1-0985 Dow Cilemical Co., Midland, Michigan.

\begin{tabular}{|c|c|c|c|}
\hline \multicolumn{4}{|c|}{$\begin{array}{l}\text { Internal standard } W, a=3.16516 \AA \\
\text { CuK } a_{1} \lambda=1.54056 \AA \text {; temp. } 25{ }^{\circ} \mathrm{C}\end{array}$} \\
\hline$d(\stackrel{\circ}{A})$ & $I$ & $h k l$ & $2 \theta\left({ }^{\circ}\right)$ \\
\hline 4.02 & 30 & 100 & 22.12 \\
\hline 2.84 & 100 & 110 & 31.48 \\
\hline 2.317 & 12 & III & 38.83 \\
\hline 2.006 & 65 & 200 & 45.15 \\
\hline 1.795 & 11 & 210 & 50.83 \\
\hline 1.639 & 30 & $2 I I$ & 56.07 \\
\hline 1.418 & 25 & 220 & 65.80 \\
\hline 1.3376 & 4 & 300 & 70.32 \\
\hline 1.2686 & 10 & 310 & 74.77 \\
\hline $1 \cdot .2096$ & 2 & 311 & 79. II \\
\hline 1.1581 & 8 & 222 & 83.38 \\
\hline 1.1129 & 2 & 320 & 87.60 \\
\hline 1.0726 & 9 & 321 & 91.80 \\
\hline 1.0032 & 4 & 400 & 100.32 \\
\hline .9732 & 2 & 410 & 104.65 \\
\hline .9457 & 6 & 411 & 109.08 \\
\hline .9206 & 2 & 331 & 113.59 \\
\hline .8973 & 8 & 420 & 118.29 \\
\hline .8757 & 2 & 421 & 123.20 \\
\hline .8554 & 4 & 332 & 128.45 \\
\hline .8190 & 5 & 422 & 140.27 \\
\hline .7870 & 3 & 510 & 156.35 \\
\hline
\end{tabular}

\section{References}

Hirakawa, K., K.Hirakawa and T. Hashimoto (1960). Magnetic properties of potassium iron group fluorides, $\mathrm{KMF}_{3}, \mathrm{~J} . \mathrm{Phy}$. Soc. Jäpan 15, 2063-2068.

Knox, K.(196I). Perovskite-like fluorides, I. Structures of $\mathrm{KMnF}_{3}, \mathrm{KFeF}_{3}, \mathrm{KCoF}_{3}$, $\mathrm{KNiF}_{3}$, and $\mathrm{KZnF}_{3}$. Crystal field effects in the series and in $\mathrm{KCrF}_{3}$ and $\mathrm{KCuF}_{3}$, Acta Cryst. 14, 583-585.

Martin, R.I., R.S. Nyholm and N. C. Stephenson (1956). Antiferromagnetism in complex fluorides with perovskite structure Chem. Ind. (London) 1956, 83-85.

Okazaki,A. and Y. Suemune(1961). The crystal structures of $\mathrm{KMnF}_{3}, \mathrm{KFeF}_{3}, \mathrm{KCOF}_{3}$, $\mathrm{KNiF}_{3}$ and $\mathrm{KCuF}_{3}$ above and below their Néel temperatures, J. Phys. Soc. Japan $16,671-675$.

Riidorff, W., J. Kändler, G. Lincke and D. Babel (1959). Über Doppelfluoride von Nickel und Kobalt, Angew. Chem. 71, 672. 
Potassium Zinc Sulfate Hexahydrate, $\mathrm{K}_{2} \mathrm{Zn}\left(\mathrm{SO}_{4}\right)_{2} \cdot 6 \mathrm{H}_{2} \mathrm{O}$ (monoclinic)

\section{Sample}

The sample was prepared at NBS by slow evaporation of an equimolar solution of $\mathrm{K}_{2} \mathrm{SO}_{4}$ and $\mathrm{ZnSO}_{4}$.

\section{Major impurities}

less than $0.001 \%$ each: $\mathrm{Ca}, \mathrm{Cu}, \mathrm{Li}, \mathrm{Mg}$, $\mathrm{Mn}, \mathrm{Rb}$, and $\mathrm{Si}$

Color

Colorless

\section{Optical data}

Biaxial (+) $\mathrm{N}_{\alpha}=1.478, \mathrm{~N}_{\beta}=1.481, \mathrm{~N}_{\gamma}=1.496$ $2 \mathrm{~V}$ is large

\section{Structure}

Monoclinic, $\mathrm{P} 2_{1} / \mathrm{a}(14), \mathrm{Z}=2$. Isostructural with other"Tutton's salts"[Tutton, 1893]. The structure of a "Tutton Salt", $\left(\mathrm{NH}_{4}\right)_{2} \mathrm{Mg}\left(\mathrm{SO}_{4}\right)_{2} \cdot 6 \mathrm{H}_{2} \mathrm{O}$, was determined by Margulis and Templeton [1962].

\section{Lattice constants}

\begin{tabular}{|l|c|c|c|c|}
\hline & $a(\AA)$ & $b(\AA)$ & $c(\AA)$ & $\beta\left({ }^{\circ}\right)$ \\
\cline { 2 - 5 } $\begin{array}{l}\text { Kohler, } \\
\text { Franke* } \\
{[1965]}\end{array}$ & 9.04 & 12.20 & 6.15 & $104^{\circ} 48^{\prime}$ \\
$\begin{array}{l}\text { NBS, } \\
\text { sample } \\
\text { at } 25{ }^{\circ} \mathrm{C}\end{array}$ & $\begin{array}{r}9.041 \\
\pm .001\end{array}$ & $\begin{array}{r}12.215 \\
\pm .001\end{array}$ & $\begin{array}{r}6.156 \\
\pm .001\end{array}$ & $\begin{array}{r}104^{\circ} 49^{\prime} \\
\pm 1^{\prime}\end{array}$ \\
\hline
\end{tabular}

*PDF card 18-1074

\section{Density}

(calculated) $2.242 \mathrm{~g} / \mathrm{cm}^{3}$ at $25^{\circ} \mathrm{C}$.

\section{Reference intensity}

$$
\mathrm{I} / \mathrm{I}_{\text {corundum }}=1.3
$$

\begin{tabular}{|c|c|c|c|}
\hline \multicolumn{4}{|c|}{$\begin{array}{l}\text { Internal standard } W, a=3.16516 \AA \\
\mathrm{CuK}_{a_{1}} \lambda=1.54056 \AA \text {; temp. } 25{ }^{\circ} \mathrm{C}\end{array}$} \\
\hline$d(\AA)$ & $I$ & $h k l$ & $2 \theta(0)$ \\
\hline 6.12 & 14 & 020 & 14.46 \\
\hline 5.96 & 6 & 001 & 14.86 \\
\hline 5.35 & 12 & 011 & 16.57 \\
\hline 5.13 & 12 & $\overline{1} 11$ & 17.28 \\
\hline 5.00 & 4 & 120 & 17.72 \\
\hline 4.38 & 25 & 200 & 20.27 \\
\hline 4.265 & 28 & 021 & 20.81 \\
\hline 4.154 & 87 & 111 & 21.37 \\
\hline 4.051 & 82 & $\underline{2} 01$ & 21.92 \\
\hline 3.845 & 4 & $\overline{2} 11$ & 23.11 \\
\hline 3.691 & 100 & 130 & 24.09 \\
\hline 3.581 & 10 & 121 & 24.84 \\
\hline 3.552 & 10 & 220 & 25.05 \\
\hline 3.374 & 4 & $\overline{2} 21$ & 26.39 \\
\hline 3.362 & 12 & 031 & 26.49 \\
\hline 3.303 & 25 & $\overline{1} 31$ & 26.97 \\
\hline 3.159 & 18 & 201 & 28.23 \\
\hline 3.058 & 41 & 211,040 & 29.18 \\
\hline 2.975 & 64 & $002, \overline{1} 12$ & 30.01 \\
\hline 2.872 & 4 & $\overline{2} 31$ & 31.11 \\
\hline 2.846 & 8 & $\overline{3} 11$ & 31.40 \\
\hline 2.832 & 17 & 310 & 31.56 \\
\hline 2.806 & 29 & 221 & 31.86 \\
\hline 2.742 & 21 & $\overline{1} 22$ & 32.63 \\
\hline 2.684 & 7 & $\overline{1} 41$ & 33.35 \\
\hline 2.640 & 7 & $\overline{3} 21$ & 33.93 \\
\hline 2.557 & 6 & $\overline{2} 22$ & 35.06 \\
\hline 2.513 & 5 & 141 & 35.70 \\
\hline 2.502 & 10 & 240 & 35.86 \\
\hline 2.495 & 13 & 231 & 35.97 \\
\hline 2.448 & $<2$ & $\overline{1} 32$ & 36.68 \\
\hline 2.406 & & 122 & 37.34 \\
\hline 2.401 & 5\{ & 032 & 37.43 \\
\hline 2.380 & 46 & $\overline{3} 31$ & 37.77 \\
\hline 2.260 & 7 & 051 & 39.86 \\
\hline 2.244 & 6 & $\overline{4} 01,322,+$ & 40.15 \\
\hline 2.203 & 9 & 132 & 40.93 \\
\hline 2.195 & 23 & 241 & 41.09 \\
\hline 2.176 & $<2$ & 212 & 41.46 \\
\hline 2.150 & 3 & 410 & 41.98 \\
\hline
\end{tabular}


Potassium Zinc Sulfate Hexahydrate, $\mathrm{K}_{2} \mathrm{Zn}\left(\mathrm{SO}_{4}\right)_{2} \cdot 6 \mathrm{H}_{2} \mathrm{O}$ (monoclinic) - continued

\begin{tabular}{|c|c|c|c|}
\hline \multicolumn{4}{|c|}{$\begin{array}{l}\text { Internal standard } W, a=3.16516 \AA \\
\mathrm{CuK} a_{1} \quad \lambda=1.54056 \AA \text {; temp. } 25{ }^{\circ} \mathrm{C}\end{array}$} \\
\hline$d(\AA)$ & $I$ & $h k l$ & $2 \theta(0)$ \\
\hline 2.137 & & 151 & 42.26 \\
\hline 2.131 & 16\{ & 250.042 & 42.37 \\
\hline 2.114 & $<2$ & $\overline{3} 4 \underline{1}$. & 42.73 \\
\hline 2.107 & 4 & $340, \overline{4} 21$ & 42.88 \\
\hline 2.093 & 3 & $\overline{2} 51$ & 43.19 \\
\hline 2.071 & 12 & $\overline{2} 42$ & 43.66 \\
\hline 2.058 & 14 & 331,420 & 43.95 \\
\hline 2.037 & $<2\{$ & -060 & 44.44 \\
\hline 2.024 & 2\{ & $\overline{4} 02, \overline{1} 13$ & 44.74 \\
\hline 2.010 & 2 & $\overline{2} 03$ & 45.07 \\
\hline 1.984 & 13 & $003, \overline{2} 13$ & 45.69 \\
\hline 1.945 & $<2$ & $\overline{1} 23,232$ & 46.67 \\
\hline 1.932 & 2 & 251 & 47.00 \\
\hline 1.926 & 5 & $\underline{0} 61, \underline{4} 30$ & 47.16 \\
\hline 1.910 & $<2$ & $\overline{1} 52, \overline{2} 23$ & 47.57 \\
\hline 1.893 & 6 & $\overline{3} 42$ & 48.03 \\
\hline 1.889 & 8 & $05 \underline{2}$ & 48.14 \\
\hline 1.876 & 8 & $411, \overline{3} 51$ & 48.49 \\
\hline 1.871 & 8 & 350 & 48.61 \\
\hline 1.856 & 8 & $\overline{3} 13$ & 49.03 \\
\hline 1.849 & 3 & 161 & 49.23 \\
\hline 1.831 & 6 & 1 & 49.75 \\
\hline 1.820 & 8 & $\overline{2} 61$ & 50.09 \\
\hline 1.815 & 4 & $113,421,+$ & 50.23 \\
\hline 1.803 & 5 & $\overline{2} 33$ & 50.59 \\
\hline 1.783 & 7 & 033 & 51.18 \\
\hline 1.776 & 8 & 322,440 & 51.41 \\
\hline 1.758 & 2 & 123 & 51.96 \\
\hline 1.731 & 6 & 510 & 52.85 \\
\hline 1.722 & $<2$ & 431 & 53.15 \\
\hline 1.707 & 3 & $35 \underline{1}, \overline{3} 33$ & 53.66 \\
\hline 1.696 & 2 & İ62 & 54.02 \\
\hline 1.691 & 4 & 332 & 54.21 \\
\hline 1.680 & 4 & $520,062,+$ & 54.58 \\
\hline 1.674 & 4 & 071,133 & 54.79 \\
\hline 1.654 & $<2$ & $20 \underline{3}, \overline{4} 51$ & 55.51 \\
\hline 1.650 & $<2$ & $\overline{2} 62$ & .55 .66 \\
\hline 1.639 & $<2$ & $213, \overline{4} 23,+$ & 56.07 \\
\hline 1.628 & 2 & 450 & 56.46 \\
\hline 1.623 & 4 & 171 & 56.68 \\
\hline
\end{tabular}

\begin{tabular}{|c|c|c|c|}
\hline \multicolumn{4}{|c|}{$\begin{array}{l}\text { Internal standard } \mathrm{W}, \mathrm{a}=3.16516 \AA \\
\mathrm{CuK} a_{1} \quad \lambda=1.54056 \AA \text {; temp. } 25{ }^{\circ} \mathrm{C}\end{array}$} \\
\hline$d(\AA)$ & $I$ & $h k l$ & $2 \theta\left({ }^{\circ}\right)$ \\
\hline 1.608 & 2 & 162 & 57.23 \\
\hline 1.597 & $<2$ & 223 & 57.68 \\
\hline 1.577 & 3 & $\overline{5} 32$ & 58.47 \\
\hline 1.566 & $<2$ & 412 & 58.93 \\
\hline 1.559 & $<2$ & $\overline{4} 52,511$ & 59.20 \\
\hline 1.555 & $<2$ & $\overline{5} 41$ & 59.39 \\
\hline 1.539 & $<2$ & 053 & 60.05 \\
\hline 1.533 & 2 & $\overline{2} 04,233$ & 60.32 \\
\hline 1.522 & $<2$ & 214, I1 14 & 60.83 \\
\hline 1.517 & 2 & $540, \overline{1} 72$ & 61.05 \\
\hline 1.505 & 2 & 072,180 & 61.59 \\
\hline 1.496 & $<2$ & $370, \overline{6} 11$ & 62.00 \\
\hline 1.486 & 2 & $\overline{1} 24, \overline{4} 43$ & 62.46 \\
\hline 1.4 .74 & $<2$ & $\overline{1} 81, \overline{5} 23$ & 63.02 \\
\hline 1.472 & $<2$ & 432 & 63.09 \\
\hline 1.467 & $<2$ & 153,531 & 63.35 \\
\hline 1.463 & $<2$ & $313, \overline{6} 21$ & 63.52 \\
\hline 1.452 & $<2$ & $\overline{5} 51,172$ & 64.06 \\
\hline 1.445 & $<2$ & $024, \overline{1} 63,+$ & 64.42 \\
\hline
\end{tabular}

\section{Additional patterns}

1. PDF card 1-421, [Hanawalt et al., 1938]

2. PDF card 18-1074, [Kohler and Franke. 1965]

\section{References}

Hanawalt, J.D., H.D. Rinn, and L.K. Frevel (1938). Chemical analyses by x-ray diffraction, Ind. Eng. Chem. Anal. Ed. 10, 457-512。

Kohler, K. and W. Franke (1965). Mineralogisches Institut Freie Universität, Berlin, Germany。

Margulis, T. N. and D.H. Templeton (1962). Crystal structure and hydrogen bonding of magnesium ammonium sulfate hexahydrate, Z Krist. 117, 344-357.

Tutton, A. E. (1893). Connection between the atomic weight of contained metals and the magnitude of the angles of crystals of isomorphous series.A study of the potassium, rubidium, and cesium salts of the monoclinic series of double sulphates $\mathrm{R}_{2} \mathrm{M}\left(\mathrm{SO}_{4}\right) \cdot 6 \mathrm{H}_{2} \mathrm{O}$, J. Chem. Soc.63, $337-423$. 
Rubidium Cadmium Sulfate, $\mathrm{Rb}_{2} \mathrm{Cd}_{2}\left(\mathrm{SO}_{4}\right)_{3}$ (cubic)

\section{Sample}

The sample was prepared by melting a stoichiometric mixture of $\mathrm{Rb}_{2} \mathrm{SO}_{4}$ and $3 \mathrm{CaSO}_{4} \cdot 8 \mathrm{H}_{2} \mathrm{O}$, which was then air quenched and annealed at $700{ }^{\circ} \mathrm{C}$ for 18 hours.

\section{Color}

Colorless

\section{Optical data}

Isotropic, $\mathrm{N}=1.590$

\section{Structure}

Cubic, P2, 3 (198), Z=4, langbeinite type [Gattow and Zemann, 1958]. The langbeinite structure was determined by Zemann and Zemann, [1957].

\section{Lattice constants}

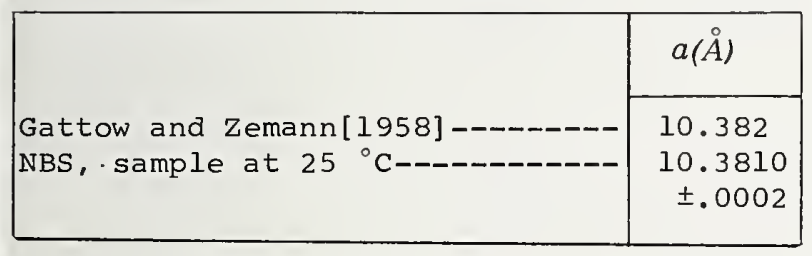

\section{Density}

(calculated) $4.060 \mathrm{~g} / \mathrm{cm}^{3}$ at $25^{\circ} \mathrm{C}$.

\section{Reference intensity}

$$
\mathrm{I} / \mathrm{I}_{\text {corundum }}=5.3
$$

\begin{tabular}{|c|c|c|c|}
\hline \multicolumn{4}{|c|}{$\begin{array}{l}\text { Internal standard } \mathrm{Ag}, \mathrm{a}=4.08641 \AA \\
\mathrm{CuK} a_{1} \quad \lambda=1.54056 \AA \text {; temp. } 25{ }^{\circ} \mathrm{C}\end{array}$} \\
\hline$d(\AA)$ & $I$ & $h k l$ & $2 \theta\left({ }^{\circ}\right)$ \\
\hline 5.98 & 4 & 111 & 14.80 \\
\hline 4.64 & 9 & 210 & 19.12 \\
\hline 4.23 & 15 & 211 & 20.96 \\
\hline 3.669 & 5 & 220 & 24.24 \\
\hline 3.460 & 2 & 221 & 25.73 \\
\hline 3.283 & 100 & 310 & 27.14 \\
\hline 3.130 & 11 & 311 & 28.50 \\
\hline 2.881 & 5 & 320 & 31.02 \\
\hline 2.773 & 65 & 321 & 32.25 \\
\hline 2.593 & 1 & 400 & 34.56 \\
\hline 2.517 & 3 & 410 & 35.64 \\
\hline 2.380 & 2 & 331 & 37.76 \\
\hline 2.321 & 1 & 420 & 38.77 \\
\hline 2.265 & 1 & 421 & 39.77 \\
\hline 2.214 & 4 & 332 & 40.72 \\
\hline 2.119 & 24 & 422 & 42.63 \\
\hline 2.076 & 3 & 430 & 43.57 \\
\hline 2.037 & 31 & 510 & 44.44 \\
\hline 1.997 & 4 & 511 & 45.38 \\
\hline 1.927 & 7 & 520 & 47.11 \\
\hline 1.895 & 1 & 521 & 47.97 \\
\hline 1.806 & 5 & 522 & 50.48 \\
\hline 1.779 & 3 & 530 & 51.30 \\
\hline 1.706 & 2 & 610 & 53.68 \\
\hline 1.6840 & 19 & 611 & 54.44 \\
\hline 1.6413 & 8 & 620 & 55.98 \\
\hline 1.6208 & 6 & 621 & 56.75 \\
\hline 1.6017 & 8 & 541 & 57.49 \\
\hline 1.5476 & 3 & 630 & 59.70 \\
\hline 1.5304 & 6 & 631 & 60.44 \\
\hline 1.4978 & 3 & 444 & 61.90 \\
\hline 1.4829 & 3 & 632 & 62.59 \\
\hline 1.4680 & 3 & 710 & 63.30 \\
\hline 1.4538 & 1 & 711 & 63.99 \\
\hline 1.4397 & 1 & 640 & 64.69 \\
\hline 1.4260 & 2 & 720 & 65.39 \\
\hline 1.4126 & 5 & 721 & 66.09 \\
\hline 1.3874 & 2 & 642 & 67.45 \\
\hline 1.3748 & 1 & 722 & 68.15 \\
\hline 1.3631 & 2 & 730 & 68.82 \\
\hline
\end{tabular}


Rubidium Cadmium Sulfate, $\mathrm{Rb}_{2} \mathrm{Cd}_{2}\left(\mathrm{SO}_{4}\right)_{3}$ (cubic) - continued

\begin{tabular}{|c|c|c|c|}
\hline \multicolumn{4}{|c|}{$\begin{array}{l}\text { Internal standard } \mathrm{Ag}, \mathrm{a}=4.08641 \AA \\
\mathrm{CuK} a_{1} \quad \lambda=1.54056 \AA \text { A; temp. } 25{ }^{\circ} \mathrm{C}\end{array}$} \\
\hline$d(\AA)$ & $I$ & $h k l$ & $2 \theta\left({ }^{\circ}\right)$ \\
\hline 1.3514 & 2 & 731 & 69.50 \\
\hline 1.3291 & 1 & 650 & 70.84 \\
\hline 1.3182 & 3 & 732 & 71.51 \\
\hline 1.2971 & 1 & 800 & 72.86 \\
\hline 1.2877 & 2 & 810 & 73.48 \\
\hline 1.2779 & 1 & 811 & 74.14 \\
\hline 1.2684 & 2 & 733 & 74.79 \\
\hline 1.2598 & $<1$ & 820 & 75.43 \\
\hline 1.2499 & 1 & 821 & 76.09 \\
\hline 1.2409 & 1 & 653 & 76.74 \\
\hline 1.2236 & 4 & 822 & 78.03 \\
\hline 1.2148 & 1 & 830 & 78.70 \\
\hline 1.2068 & 5 & 831 & 79.33. \\
\hline 1.1990 & 2 & 751 & 79.95 \\
\hline 1.1827 & 1 & 832 & 81.28 \\
\hline 1.1754 & 3 & 752 & 81.89 \\
\hline 1.1534 & 1 & 841 & 83.80 \\
\hline 1.1465 & $<1$ & 910 & 84.42 \\
\hline 1.1395 & 1 & 911 & 85.06 \\
\hline 1.1261 & 1 & 920 & 86.32 \\
\hline 1.1196 & 2 & 921 & 86.94 \\
\hline 1.1068 & 1 & 664 & 88.21 \\
\hline 1.1003 & 2 & 922 & 88.86 \\
\hline 1.0943 & 3 & 930 & 89.48 \\
\hline 1.0882 & 1 & 931 & 90.12 \\
\hline 1.0767 & 1 & 852 & 91.35 \\
\hline 1.0706 & 1 & 932 & 92.02 \\
\hline 1.0596 & 1 & 844 & 93.26 \\
\hline 1.0541 & $<1$ & 940 & 93.90 \\
\hline 1.0486 & 1 & 941 & 94.54 \\
\hline 1.0434 & 1 & 933 & 95.16 \\
\hline 1.0331 & 2 & $10 \cdot 1 \cdot 0$ & 96.42 \\
\hline 1.0278 & 1 & $10 \cdot 1 \cdot 1$ & 97.09 \\
\hline 1.0179 & 1 & $10 \cdot 2 \cdot 0$ & 98.35 \\
\hline 1.0130 & 1 & $10 \cdot 2 \cdot 1$ & 99.00 \\
\hline 1.0083 & 1 & 950 & 99.62 \\
\hline
\end{tabular}

\section{References}

Gattow,G.and J.Zemann (1958). Über Doppelsulfate vom Langbeinit-typ, $\mathrm{A}_{2}^{+} \mathrm{B}_{2}^{2+}\left(\mathrm{SO}_{4}\right)_{3}$, Z. Anorg. Allgem. Chem. 293, 233-240.

Zemann, A. and J. Zemann (1957). Die Kristallstruktur vom Langbeinit, $\mathrm{K}_{2} \mathrm{Mg}_{2}\left(\mathrm{SO}_{4}\right)_{3}$, Acta Cryst. 10, 409-413. 
Rubidium Calcium Chloride, $\mathrm{RbCaCl}_{3}$ (orthorhombic)

\section{Sample}

The material was made by melting a stoichiometric mixture of $\mathrm{RbCl}$ and $\mathrm{CaCl}_{2}$. The sample was very hygroscopic.

\section{Color}

Colorless

\section{Optical data}

Very low birefringence, $\mathrm{N} \cong 1.576$, polysynthetic twinning was noted.

\section{Structure}

Orthorhombic, distorted perovskite, Pnma (62), $Z=4$, by analogy with $\mathrm{NaZnF}_{\mathrm{a}}$.

\section{Lattice constants}

\begin{tabular}{|c|c|c|c|}
\hline & $a(\AA)$ & $b(\AA)$ & $c(\AA)$ \\
\hline $\begin{array}{l}\text { NBS, } \\
\text { sample at } 25{ }^{\circ} \mathrm{C}\end{array}$ & $\begin{array}{l}7.541 \\
\pm .001\end{array}$ & $\begin{array}{r}10.667 \\
\pm .001\end{array}$ & $\begin{array}{l}7.469 \\
\pm .001\end{array}$ \\
\hline
\end{tabular}

\section{Density}

(calculated) $2.564 \mathrm{~g} / \mathrm{cm}^{3}$ at $25^{\circ} \mathrm{C}$.

\begin{tabular}{|c|c|c|c|}
\hline \multicolumn{4}{|c|}{$\begin{array}{l}\text { Internal standard } \mathrm{W}, \mathrm{a}=3.16516 \AA \\
\mathrm{CuK} \alpha_{1} \lambda=1.54056 \AA \text {; temp. } 25{ }^{\circ} \mathrm{C}\end{array}$} \\
\hline$d(\AA)$ & $I$ & $h k l$ & $2 \theta\left({ }^{\circ}\right)$ \\
\hline 2.588 & 5 & 230 & 34.63 \\
\hline 2.577 & 7 & 212 & 34.79 \\
\hline 2.443 & 5 & 231 & 36.76 \\
\hline 2.438 & 4 & 132 & 36.84 \\
\hline 2.382 & 3 & 141,301 & 37.73 \\
\hline 2.326 & 9 & 311 & 38.68 \\
\hline 2.307 & 3 & 113 & 39.01 \\
\hline 2.174 & 30 & 321 & 41.50 \\
\hline 2.163 & 45 & 123 & 41.73 \\
\hline 2.086 & 3 & 142,302 & 43.34 \\
\hline 2.078 & 3 & 203 & 43.52 \\
\hline 2.052 & 3 & 051 & 44.10 \\
\hline 2.039 & 2 & 033,213 & 44.39 \\
\hline 1.978 & 4 & 151,331 & 45.83 \\
\hline 1.882 & 35 & 242 & 48.33 \\
\hline 1.866 & 8 & 004 & 48.75 \\
\hline 1.827 & 1 & 401 & 49.86 \\
\hline 1.812 & 2 & 104 & 50.32 \\
\hline 1.800 & 4 & 152,332 & 50.68 \\
\hline 1.745 & 1 & 313 & 52.40 \\
\hline 1.730 & 2 & 421 & 52.88 \\
\hline 1.686 & 8 & 161 & 54.38 \\
\hline 1.683 & 7 & 402 & 54.47 \\
\hline 1.663 & 2 & 252,412 & 55.20 \\
\hline 1.615 & 2 & 134 & 56.98 \\
\hline 1.605 & 4 & 062,422 & 57.38 \\
\hline 1.585 & 4 & 153,333 & 58.16 \\
\hline 1.539 & 3 & 440 & 60.05 \\
\hline 1.529 & 3 & 044 & 60.50 \\
\hline 1.521 & 4 & 432 & 60.86 \\
\hline 1.465 & 2 & 511,171 & 63.45 \\
\hline .1 .447 & 2 & 423 & 64.31 \\
\hline 1.4429 & 2 & 324 & 64.53 \\
\hline 1.4235 & 4 & 442 & 65.52 \\
\hline 1.4208 & 4 & 163 & 65.66 \\
\hline 1.4133 & 6 & $270,125,+$ & 66.05 \\
\hline 1.3865 & $<1$ & 172,512 & 67.50 \\
\hline 1.3646 & 2 & 531 & 68.73 \\
\hline 1.3333 & 2 & 080 & 70.58 \\
\hline 1.2569 & 5 & 280,600 & 75.59 \\
\hline 1.2539 & 5 & 363,523 & 75.80 \\
\hline 1.2482 & 4 & 325,610 & 76.21 \\
\hline 1.1917 & 3 & 282,602 & 80.54 \\
\hline 1.1878 & 3 & 444 & 80.86 \\
\hline 1.1310 & 2 & 290,165 & 85.85 \\
\hline
\end{tabular}

Internal standard $\mathrm{W}, \mathrm{a}=3.16516 \AA$ $\mathrm{CuK} a_{1} \quad \lambda=1.54056 \AA$; temp. $25{ }^{\circ} \mathrm{C}$

\begin{tabular}{|c|r|c|c|}
\hline$d(\AA)$ & $I$ & $h k l$ & $2 \theta\left({ }^{\circ}\right)$ \\
\hline 4.751 & 5 & 111 & 18.66 \\
3.765 & 85 & 200,121 & 23.61 \\
3.740 & 25 & 002 & 23.77 \\
3.557 & 3 & 210 & 25.01 \\
3.366 & 6 & 201 & 26.46 \\
& & & \\
3.351 & 7 & 102 & 26.58 \\
3.208 & 12 & 031,211 & 27.79 \\
3.194 & 11 & 112 & 27.91 \\
3.077 & 45 & 220 & 28.99 \\
3.059 & 45 & 022 & 29.17 \\
& & & \\
2.952 & 7 & 131 & 30.25 \\
2.846 & 9 & 221 & 31.41 \\
2.837 & 13 & 122 & 31.51 \\
2.667 & 65 & 040 & 33.58 \\
2.653 & 100 & 202 & 33.75 \\
& & &
\end{tabular}

$16516 \AA$ CuK $a_{1} \lambda=1.54056 \AA$; temp. $25{ }^{\circ} \mathrm{C}$ 
Rubidium Calcium Sulfate, $\mathrm{Rb}_{2} \mathrm{Ca}_{2}\left(\mathrm{SO}_{4}\right)_{3}$ (cubic)

Sample

The material was prepared by melting a 1:2 mixture of $\mathrm{Rb}_{2} \mathrm{SO}_{4}$ and $\mathrm{CaSO}_{4}$. This was followed by quenching in air, grinding, and then annealing at $650{ }^{\circ} \mathrm{C}$ for several days.

\section{Color}

Colorless

Optical data

Isotropic, $\mathrm{N}=1.520$

\section{Structure}

Cubic, $\mathrm{P} 2{ }_{1} 3$ (198), $\mathrm{Z}=4$, langbeinite type [Gattow and Zemann, 1958]. The langbeinite structure was described by Zemann and Zemann, [1957].

\section{Lattice constants}

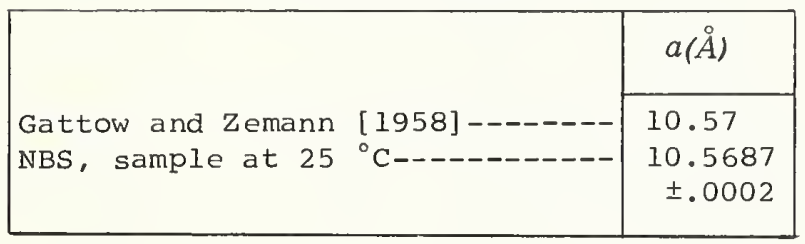

\section{Density}

(calculated) $3.034 \mathrm{~g} / \mathrm{cm}^{3}$ at $25^{\circ} \mathrm{C}$.

\section{Reference intensity}

$I / I_{\text {corundum }}=4.6$

\section{References}

Gattow, G. and J. Zemann (1958). Über Doppelsulfate vom Langbeinit-Typ, $\mathrm{A}_{2}^{+} \mathrm{B}_{2}^{2+}-$ $\left(\mathrm{SO}_{4}\right)_{3}, \mathrm{Z}$.Anorg. Allgem. Chem. 293, 233240 .

Zemann, A. and J. Zemann (1957). Die Kristallstruktur vom Langbeinit, $\mathrm{K}_{2} \mathrm{Mg}_{2}\left(\mathrm{SO}_{4}\right)_{3}$, Acta Cryst. 10, 409-413.

\begin{tabular}{|c|c|c|c|}
\hline \multicolumn{4}{|c|}{$\begin{array}{l}\text { Internal standard } \mathrm{Ag}, \mathrm{a}=4.08641 \AA \\
\mathrm{CuK} a_{1} \quad \lambda=1.54056 \AA \text {; temp. } 25^{\circ} \mathrm{C}\end{array}$} \\
\hline$d(\AA)$ & $I$ & $h k l$ & $2 \theta\left({ }^{\circ}\right)$ \\
\hline 6.09 & 4 & 111 & 14.54 \\
\hline 4.726 & 2 & 210 & 18.76 \\
\hline 4.312 & 6 & 211 & 20.58 \\
\hline 3.734 & 5 & 220 & 23.81 \\
\hline 3.519 & 3 & 221 & 25.29 \\
\hline 3.340 & 100 & 310 & 26.67 \\
\hline 3.185 & 11 & 311 & 27.99 \\
\hline 2.928 & 12 & 320 & 30.50 \\
\hline 2.821 & 45 & 321 & 31.69 \\
\hline 2.641 & 2 & 400 & 33.91 \\
\hline 2.564 & 12 & 410 & 34.97 \\
\hline 2.424 & 4 & 331 & 37.05 \\
\hline 2.364 & 1 & 420 & 38.03 \\
\hline 2.306 & 3 & 421 & 39.03 \\
\hline 2.252 & 7 & 332 & 40.00 \\
\hline 2.157 & 12 & 422 & 41.85 \\
\hline 2.113 & 2 & 430 & 42.75 \\
\hline 2.073 & 20 & 510 & 43.63 \\
\hline 2.033 & 1 & 511 & 44.52 \\
\hline 1.963 & 5 & 520 & 46.22 \\
\hline 1.929 & 1 & 521 & 47.06 \\
\hline 1.840 & 3 & 522 & 49.51 \\
\hline 1.813 & 1 & 530 & 50.28 \\
\hline 1.787 & - 1 & 531 & 51.06 \\
\hline 1.760 & 1 & 600 & 51.90 \\
\hline 1.738 & 2 & 610 & 52.63 \\
\hline 1.714 & 12 & 611 & 53.42 \\
\hline 1.6710 & 5 & 620 & 54.90 \\
\hline 1.6502 & 5 & 621 & 55.65 \\
\hline 1.6311 & 4 & 541 & 56.36 \\
\hline 1.6120 & 1 & 533 & 57.09 \\
\hline 1.5931 & 1 & 622 & 57.83 \\
\hline 1.5752 & 4 & 630 & 58.55 \\
\hline 1.5580 & 4 & 631 & 59.26 \\
\hline 1.5251 & 2 & 444 & 60.67 \\
\hline 1.5096 & 2 & 632 & 61.36 \\
\hline 1.4943 & 1 & 710 & 62.06 \\
\hline 1.4799 & 1 & 711 & 62.73 \\
\hline 1.4651 & 1 & 640 & 63.44 \\
\hline 1.4516 & 1 & 720 & 64.10 \\
\hline 1.4380 & 4 & 721 & 64.78 \\
\hline
\end{tabular}


Rubidium Calcium Sulfate, $\mathrm{Rb}_{2} \mathrm{Ca}_{2}\left(\mathrm{SO}_{4}\right)_{3}$ (cubic) - continued

\begin{tabular}{|c|c|c|c|}
\hline \multicolumn{4}{|c|}{$\begin{array}{l}\text { Internal standard } \mathrm{Ag}, \mathrm{a}=4.08641 \AA \\
\mathrm{CuK} \alpha_{1} \quad \lambda=1.54056 \AA \text { 作 }\end{array}$} \\
\hline$d(\AA)$ & $I$ & $h k l$ & $2 \theta\left({ }^{\circ}\right)$ \\
\hline 1.4122 & 2 & 642 & 66.11 \\
\hline 1.3997 & 1 & 722 & 66.78 \\
\hline 1.3879 & 1 & 730 & 67.42 \\
\hline 1.3755 & 2 & 731 & 68.11 \\
\hline 1.3531 & 1 & 650 & 69.40 \\
\hline 1.3421 & 2 & 732 & 70.05 \\
\hline 1.3211 & $<1$ & 800 & 71.35 \\
\hline 1.3106 & 1 & 810 & 71.99 \\
\hline 1.3010 & 1 & 811 & 72.61 \\
\hline 1.2912 & 1 & 733 & 73.25 \\
\hline 1.2820 & 1 & 820 & 73.86 \\
\hline 1.2724 & 2 & 821 & 74.51 \\
\hline 1.2633 & 1 & 653 & 75.14 \\
\hline 1.2454 & 1 & 822 & 76.41 \\
\hline 1.2370 & 1 & 830 & 77.03 \\
\hline 1.2288 & 2 & 831 & 77.64 \\
\hline 1.2203 & 1 & 751 & 78.28 \\
\hline 1.2047 & 1 & 832 & 79.49 \\
\hline 1.1966 & 1 & 752 & 80.14 \\
\hline 1.1741 & 1 & 663 & 82.00 \\
\hline 1.1601 & 1 & 911 & 83.21 \\
\hline 1.1533 & 1 & 842 & 83.81 \\
\hline 1.1465 & 1 & 920 & 84.42 \\
\hline 1.1397 & 1 & 921 & 85.04 \\
\hline 1.1270 & 1 & 664 & 86.23 \\
\hline 1.1202 & 2 & 922 & 86.89 \\
\hline 1.1140 & 2 & 930 & 87.49 \\
\hline 1.1080 & 1 & 931 & 88.09 \\
\hline 1.0958 & 1 & 852 & 89.33 \\
\hline 1.0900 & 1 & 932 & 89.93 \\
\hline 1.0732 & 1 & 940 & 91.74 \\
\hline 1.0674 & 1 & 941 & 92.38 \\
\hline 1.0624 & 1 & 933 & 92.94 \\
\hline 1.0568 & 1 & $10 \cdot 0 \cdot 0$ & 93.58 \\
\hline 1.0516 & 1 & $10 \cdot 0 \cdot 1$ & 94.19 \\
\hline 1.0466 & 1 & $10 \cdot 1 \cdot 1$ & 94.78 \\
\hline 1.0364 & 1 & $10 \cdot 2 \cdot 0$ & 96.01 \\
\hline 1.0316 & 1 & $10 \cdot 2 \cdot 1$ & 96.61 \\
\hline 1.0267 & 1 & 950 & 97.22 \\
\hline
\end{tabular}


Rubidium Magnesium Sulfate, $\mathrm{Rb}_{2} \mathrm{Mg}_{2}\left(\mathrm{SO}_{4}\right)_{3}$ (cubic)

Sample

The sample was prepared by melting a stoichiometric mixture of $\mathrm{Rb}_{2} \mathrm{SO}_{4}$ and $\mathrm{MgSO}_{4} \cdot 7 \mathrm{H}_{2} \mathrm{O}$. The melt was cooled quickly and annealed at $800{ }^{\circ} \mathrm{C}$ for 18 hours.

\section{Color}

Colorless

\section{Optical data}

Isotropic, $\mathrm{N}=1.556$

\section{Structure}

Cubic, $\mathrm{P} 22_{1} 3$ (198), Z=4, langbeinite type [Gattow and Zemann, 1958]. The langbeinite structure was determined by Zemann and Zemann, [1957].

\section{Lattice constants}

\begin{tabular}{|l|c|}
\hline & $a(\AA)$ \\
\cline { 2 - 2 } Gattow and Zemann [1958]-------- & 10.005 \\
NBS, sample at 25 ${ }^{\circ} \mathrm{C}----------$ & 10.0051 \\
& \pm .0003 \\
\hline
\end{tabular}

Density

(calculated) $3.367 \mathrm{~g} / \mathrm{cm}^{3}$ at $25^{\circ} \mathrm{C}$.

\section{Reference intensity}

$1 / 1_{\text {corundum }}=3 \cdot 3$

\section{References}

Gattow, G.and J.Zemann (1958). Über Doppelsulfate vom Langbeinit-typ, $\mathrm{A}_{2}^{+} \mathrm{B}_{2}^{2+}\left(\mathrm{SO}_{4}\right)_{3}$, Z. Anorg. Allgem. Chem. 293, 233-240.

Zemann,A. and J. Zemann (1957). Die Kristallstruktur vom Langbeinit, $\mathrm{K}_{2} \mathrm{Mg} 2\left(\mathrm{SO}_{4}\right)_{3}$, Acta Cryst. 10, 409-413.

\begin{tabular}{|c|c|c|c|}
\hline$d(\stackrel{\circ}{)}$ & $I$ & $h k l$ & $2 \theta\left({ }^{\circ}\right)$ \\
\hline 5.76 & 6 & 111 & 15.36 \\
\hline 4.47 & 7 & 210 & 19.86 \\
\hline 4.086 & 6 & 211 & 21.73 \\
\hline 3.537 & 5 & 220 & 25.16 \\
\hline 3.336 & 6 & 221 & 26.70 \\
\hline 3.162 & 100 & 310 & 28.20 \\
\hline 3.015 & 22 & 311 & 29.60 \\
\hline 2.890 & 3 & 222 & 30.92 \\
\hline 2.772 & 25 & 320 & 32.27 \\
\hline 2.673 & 41 & 321 & 33.50 \\
\hline 2.500 & 2 & 400 & 35.89 \\
\hline 2.424 & 24 & 410 & 37.05 \\
\hline 2.356 & 2 & 411 & 38.15 \\
\hline 2.294 & 10 & 331 & 39.24 \\
\hline 2.237 & 4 & 420 & 40.29 \\
\hline 2.184 & 8 & 421 & 41.31 \\
\hline 2.133 & 9 & 332 & 42.33 \\
\hline 2.043 & 10 & 422 & 44.30 \\
\hline 2.001 & 2 & 430 & 45.27 \\
\hline 1.961 & 19 & 510 & 46.25 \\
\hline 1.926 & 2 & 511 & 47.15 \\
\hline 1.857 & 7 & 520 & 49.00 \\
\hline 1.827 & 2 & 521 & 49.88 \\
\hline 1.741 & 6 & 522 & 52.52 \\
\hline 1.716 & 2 & 530 & 53.34 \\
\hline 1.691 & 2 & 531 & 54.18 \\
\hline 1.668 & 2 & 600 & 55.01 \\
\hline 1.645 & 6 & 610 & 55.84 \\
\hline 1.623 & 15 & 611 & 56.66 \\
\hline 1.582 & 4 & 620 & 58.26 \\
\hline 1.563 & 10 & 621 & 59.06 \\
\hline 1.544 & 7 & 541 & 59.86 \\
\hline 1.526 & 3 & 533 & 60.62 \\
\hline 1.508 & 2 & 622 & 61.40 \\
\hline 1.492 & 8 & 630 & 62.17 \\
\hline 1.475 & 5 & 631 & 62.96 \\
\hline 1.444 & 4 & 444 & 64.50 \\
\hline 1.429 & 3 & 632 & 65.24 \\
\hline 1.415 & 2 & 710 & 65.97 \\
\hline 1.400 & 2 & 711 & 66.74 \\
\hline
\end{tabular}


Rubidium Magnesium Sulfate, $\mathrm{Rb}_{2} \mathrm{Mg}_{2}\left(\mathrm{SO}_{4}\right)_{3}$ (cubic) - continued

\begin{tabular}{|c|c|c|c|}
\hline \multicolumn{4}{|c|}{$\begin{array}{l}\text { Internal standard } W, a=3.16516 \AA \\
\mathrm{CuK} a_{1} \quad \lambda=1.54056 \AA \text {; temp. } 25^{\circ} \mathrm{C}\end{array}$} \\
\hline$d(\AA)$ & $I$ & $h k l$ & $2 \theta\left({ }^{\circ}\right)$ \\
\hline 1.387 & 1 & 640 & 67.47 \\
\hline 1.374 & 2 & 720 & 68.21 \\
\hline 1.3613 & 6 & $72 I$ & 68.92 \\
\hline 1.3366 & 3 & 642 & 70.38 \\
\hline 1.3250 & 2 & 722 & 71.09 \\
\hline 1.3136 & 3 & 730 & 71.80 \\
\hline 1.3025 & 4 & 731 & 72.51 \\
\hline 1. 2807 & 3 & 650 & 73.95 \\
\hline 1.2705 & 4 & 732 & 74.64 \\
\hline 1.2413 & 3 & 810 & 76.71 \\
\hline 1.2313 & 2 & 811 & 77.45 \\
\hline 1.2224 & $I$ & 733 & 78.12 \\
\hline 1.2137 & 2 & 820 & 78.79 \\
\hline 1.2049 & 3 & 821 & 79.48 \\
\hline 1.1956 & 2 & 653 & 80.22 \\
\hline 1.1790 & 3 & 822 & 81.59 \\
\hline 1.1709 & 1 & 830 & 82.26 \\
\hline 1.1630 & 4 & 831 & 82.95 \\
\hline 1. 1551 & 3 & 751 & 83.65 \\
\hline 1.1473 & 1 & 662 & 84.33 \\
\hline 1.1327 & 2 & 752 & 85.69 \\
\hline 1.1117 & 2 & 841 & 87.72 \\
\hline 1.1049 & 1 & 910 & 88.40 \\
\hline 1.0982 & 2 & 911 & 89.08 \\
\hline 1.0915 & 2 & 842 & 89.77 \\
\hline 1.0853 & 1 & 920 & 90.43 \\
\hline 1.0788 & 2 & 921 & 91.13 \\
\hline 1.0666 & 2 & 664 & 92.47 \\
\hline 1.0606 & 3 & 922 & 93.15 \\
\hline 1.0548 & 2 & 930 & 93.82 \\
\hline 1.0491 & 1 & 931 & 94.49 \\
\hline 1.0376 & 1 & 852 & 95.87 \\
\hline 1.0321 & 2 & 932 & 96.55 \\
\hline 1.0161 & 2 & 940 & 98.59 \\
\hline 1.0108 & 3 & 941 & 99.29 \\
\hline 1.0057 & $I$ & 933 & 99.98 \\
\hline
\end{tabular}


Rubidium Manganese Sulfate, $\mathrm{Rb}_{2} \mathrm{Mn}_{2}\left(\mathrm{SO}_{4}\right)_{3}$ (cubic)

\section{Sample}

The sample was prepared at NBS by melting an equimolar mixture of $\mathrm{Rb}_{2} \mathrm{SO}_{4}$ and $\mathrm{MnSO}_{4}$.

\section{Major impurities}

$0.01-0.1 \%$ each: $\mathrm{Ag}, \mathrm{Al}, \mathrm{Cu}, \mathrm{Na}$, and $\mathrm{Sr}$

$0.1-1.0 \%$ each: cs

\section{Color}

Colorless

\section{Optical data}

Isotropic, $\mathrm{N}=1.590$

\section{Structure}

Cubic, $P 2_{1} 3$ (198), $\mathrm{Z}=4$, langbeinite-type [Gattow and Zemann, 1958]. The langbeinite structure was determined by Zemann and Zemann [1957]。

\section{Lattice constants}

\begin{tabular}{|l|c|}
\hline \multirow{2}{*}{ Gattow and Zemann [1958]------- } & $a(\AA)$ \\
\cline { 2 - 2 } NBS, sample at $25{ }^{\circ} \mathrm{C}----------$ & 10.218 \\
& \pm .004 \\
& \pm .0001 \\
\hline
\end{tabular}

Density

(calculated) $3.546 \mathrm{~g} / \mathrm{cm}^{3}$ at $25^{\circ} \mathrm{C}$.

\section{Reference intensity}

$I / I_{\text {corundum }}=3.6$

\section{References}

Gattow, G. and J.Zemann (1958). Über Doppelsulfate vom Langbeinit-typ, $\mathrm{A}_{2}^{+} \mathrm{B}_{2}^{2+}\left(\mathrm{SO}_{4}\right)_{3}$, Z. Anorg. Allgem. Chem. 293, 233-240.

Zemann, A。 and J.Zemann (1957)。 Die Kristallstruktur vom Langbeinit, $\mathrm{K}_{2} \mathrm{Mg} 2\left(\mathrm{SO}_{4}\right)_{3}$, Acta Cryst. 10, 409-413.

\begin{tabular}{|c|c|c|c|}
\hline \multicolumn{4}{|c|}{$\begin{array}{l}\text { Internal standard } W, a=3.16516 \AA \\
\text { CuK } a_{1} \lambda=1.54056 \AA \text {; temp. } 25^{\circ} \mathrm{C}\end{array}$} \\
\hline$d(\stackrel{\circ}{A})$ & $I$ & $h k l$ & $2 \theta\left({ }^{\circ}\right)$ \\
\hline 5.901 & 2 & 111 & 15.00 \\
\hline 4.170 & 6 & 211 & 21.29 \\
\hline 3.613 & 6 & 220 & 24.62 \\
\hline 3.405 & 2 & 221 & 26.15 \\
\hline 3.230 & 100 & 310 & 27.59 \\
\hline 3.081 & 17 & 311 & 28.96 \\
\hline 2.950 & 1 & 222 & 30.27 \\
\hline 2.833 & 3 & 320 & 31.55 \\
\hline 2.731 & 50 & 321 & 32.76 \\
\hline 2.554 & 2 & 400 & 35.11 \\
\hline 2.479 & 12 & 410 & 36.21 \\
\hline 2.344 & 4 & 331 & 38.37 \\
\hline 2.284 & 2 & 420 & 39.41 \\
\hline 2.228 & 3 & 421 & 40.45 \\
\hline 2.179 & 7 & 332 & 41.40 \\
\hline 2.086 & 15 & 422 & 43.34 \\
\hline 2.043 & 2 & 430 & 44.30 \\
\hline 2.004 & 22 & 510 & 45.21 \\
\hline 1.967 & 2 & 511 & 46.11 \\
\hline 1.898 & 7 & 520 & 47.89 \\
\hline 1.866 & 2 & 521 & 48.76 \\
\hline 1.805 & 1 & 440 & 50.52 \\
\hline 1.778 & 5 & 522 & 51.33 \\
\hline 1.752 & 2 & 530 & 52.16 \\
\hline 1.727 & 2 & 531 & 52.99 \\
\hline 1.703 & 1 & 600 & 53.79 \\
\hline 1.679 & 4 & 610 & 54.60 \\
\hline 1.658 & 15 & 611 & 55.37 \\
\hline 1.615 & 5 & 620 & 56.96 \\
\hline 1.595 & 7 & 621 & 57.74 \\
\hline 1.576 & 6 & 541 & 58.50 \\
\hline 1.557 & 2 & 533 & 59.29 \\
\hline 1.540 & 2 & 622 & 60.02 \\
\hline 1.523 & 6 & 630 & 60.76 \\
\hline 1.506 & 5 & 631 & 61.52 \\
\hline 1.474 & 3 & 444 & 62.99 \\
\hline 1.459 & 3 & 632 & 63.72 \\
\hline 1.445 & 2 & 710 & 64.43 \\
\hline 1.431 & 1 & 711 & 65.15 \\
\hline 1.417 & 2 & 640 & 65.85 \\
\hline 1.403 & 2 & 720 & 66.60 \\
\hline 1.390 & 6 & 721 & 67.30 \\
\hline 1.365 & 3 & 642 & 68.71 \\
\hline 1.353 & 2 & 722 & 69.43 \\
\hline 1.341 & 2 & 730 & 70.13 \\
\hline
\end{tabular}


Rubidium Manganese Sulfate, $\mathrm{Rb}_{2} \mathrm{Mn}_{2}\left(\mathrm{SO}_{4}\right)_{3}$ (cubic) - continued

\begin{tabular}{|c|c|c|c|}
\hline \multicolumn{4}{|c|}{$\begin{array}{l}\text { Internal standard } W, a=3.16516 \AA \\
\text { CuK } \alpha_{1} \quad \lambda=1.54056 \AA \text {; temp. } 25{ }^{\circ} \mathrm{C}\end{array}$} \\
\hline$d(\AA)$ & $I$ & $h k l$ & $2 \theta\left({ }^{\circ}\right)$ \\
\hline 1.3297 & 3 & 731 & 70.80 \\
\hline 1.3080 & 2 & 650 & 72.16 \\
\hline 1.2973 & 2 & 732 & 72.85 \\
\hline 1.2771 & 1 & 800 & 74.19 \\
\hline 1.2670 & 2 & 810 & 74.88 \\
\hline 1.2572 & 2 & 811 & 75.57 \\
\hline 1.2477 & 2 & 733 & 76.25 \\
\hline 1.2389 & 1 & 820 & 76.89 \\
\hline 1.2297 & 2 & 821 & 77.57 \\
\hline 1.2208 & 2 & 653 & 78.24 \\
\hline 1.2040 & 2 & 822 & 79.55 \\
\hline 1.1954 & 1 & 830 & 80.24 \\
\hline 1.1875 & 4 & 831 & 80.88 \\
\hline 1.1793 & 2 & 751 & 81.56 \\
\hline 1.1642 & 1 & 832 & 82.85 \\
\hline 1.1563 & 2 & 752 & 83.54 \\
\hline 1.1352 & 2 & 841 & 85.46 \\
\hline 1.1278 & 1 & 910 & 86.15 \\
\hline 1.1211 & 2 & 911 & 86.80 \\
\hline 1.1143 & 2 & 842 & 87.46 \\
\hline 1.1081 & 2 & 920 & 88.08 \\
\hline 1.1013 & 3 & 921 & 88.76 \\
\hline 1.0891 & 4 & 664 & 90.03 \\
\hline 1.0826 & 4 & 922 & 90.72 \\
\hline 1.0766 & 2 & 930 & 91.36 \\
\hline 1.0708 & 2 & 931 & 92.00 \\
\hline 1.0592 & 1 & 852 & 93.31 \\
\hline 1.0536 & 3 & 932 & 93.96 \\
\hline 1.0374 & 2 & 940 & 95.89 \\
\hline 1.0319 & 2 & 941 & 96.57 \\
\hline 1.0267 & 1 & 933 & 97.22 \\
\hline 1.0216 & 2 & $10 \cdot 0 \cdot 0$ & 97.88 \\
\hline 1.0166 & 2 & $10 \cdot 1 \cdot 0$ & 98.53 \\
\hline 1.0116 & $<1$ & $10 \cdot 1 \cdot 1$ & 99.19 \\
\hline 1.0017 & 1 & $10 \cdot 2 \cdot 0$ & 100.53 \\
\hline 0.9969 & 2 & $10 \cdot 2 \cdot 1$ & 101.19 \\
\hline .9923 & 3 & 950 & 101.84 \\
\hline .9873 & 1 & 951 & 102.55 \\
\hline .9784 & 2 & $10 \cdot 3 \cdot 0$ & 103.87 \\
\hline .9738 & 2 & $10 \cdot 3 \cdot 1$ & 104.54 \\
\hline .9608 & 1 & $10 \cdot 3 \cdot 2$ & 106.58 \\
\hline .9568 & 2 & 871 & 107.23 \\
\hline .9525 & 1 & 953 & 107.93 \\
\hline .9485 & 2 & $10 \cdot 4 \cdot 0$ & 108.61 \\
\hline .9445 & 3 & $10 \cdot 4 \cdot 1$ & 109.28 \\
\hline Plus 15 & lines & to 0.8369 & \\
\hline
\end{tabular}


Rubidium Strontium Chloride, $\mathrm{RbSrCl}_{3}$ (orthorhombic)

\section{Sample}

The sample was prepared by fusion of RbCl and $\mathrm{SrCl}_{2}$. The material was hygroscopic.

\section{Color}

Colorless

\section{Optical data}

Very low birefringence, $N \cong 1.550$; polysynthetic twinning.

\section{Structure}

Orthorhombic, Pnma (62), $\mathrm{Z}=4$, distorted perovskite, by analogy with $\mathrm{RbCaCl}_{3}$ and other $\mathrm{ABX}_{3}$ compounds.

\section{Lattrce constants}

\begin{tabular}{|c|c|c|c|}
\hline \multirow{2}{*}{ NBS, sample } & $a(\AA)$ & \multicolumn{1}{|c|}{$b(\AA)$} & $c(\AA)$ \\
\cline { 2 - 4 } at 25 ${ }^{\circ} \mathrm{C}-1----$ & 7.924 & 10.973 & 7.631 \\
& \pm .001 & \pm .002 & \pm .001 \\
\hline
\end{tabular}

\section{Density}

(calculated) $2.797 \mathrm{~g} / \mathrm{cm}^{3}$ at $25^{\circ} \mathrm{C}$.

\begin{tabular}{|c|c|c|c|}
\hline \multicolumn{4}{|c|}{$\begin{array}{l}\text { Internal standard } \mathrm{Ag} \text {, } \mathrm{a}=4.08641 \AA \\
\mathrm{CuK} a_{1} \lambda=1.54056 \AA \text {; temp. } 25^{\circ} \mathrm{C}\end{array}$} \\
\hline$d(\AA)$ & $I$ & $h k l$ & $2 \theta\left({ }^{\circ}\right)$ \\
\hline 5.49 & 11 & 101,020 & 16.14 \\
\hline 4.91 & 10 & 111 & 18.06 \\
\hline 3.962 & 30 & 200 & 22.42 \\
\hline 3.885 & 100 & 121 & 22.87 \\
\hline 3.818 & 35 & 002 & 23.28 \\
\hline 3.521 & 4 & 201 & 25.27 \\
\hline 3.435 & 6 & 102 & 25.92 \\
\hline 3.350 & 8 & 211 & 26.59 \\
\hline 3.296 & 10 & 031 & 27.03 \\
\hline 3.282 & 12 & 112 & 27.15 \\
\hline 3.211 & 7 & 220 & 27.76 \\
\hline 3.134 & 6 & 022 & 28.46 \\
\hline 3.043 & 12 & 131 & 29.33 \\
\hline 2.960 & 11 & 221 & 30.17 \\
\hline 2.910 & 9 & 122 & 30.70 \\
\hline 2.744 & 80 & 040 & 32.60 \\
\hline 2.686 & 10 & 230 & 33.33 \\
\hline 2.666 & 10 & 212 & 33.59 \\
\hline 2.534 & 4 & 231 & 35.40 \\
\hline 2.497 & 11 & 301 & 35.94 \\
\hline 2.455 & 12 & 222,141 & 36.57 \\
\hline 2.435 & 15 & 311 & 36.89 \\
\hline 2.273 & 20 & 321 & 39.62 \\
\hline 2.256 & 20 & 240 & 39.92 \\
\hline 2.227 & 25 & 042 & 40.48 \\
\hline 2.217 & 40 & 123 & 40.67 \\
\hline 2.141 & 4 & 203 & 42.17 \\
\hline 2.109 & 3 & 051 & 42.84 \\
\hline 2.062 & 6 & 331 & 43.88 \\
\hline 1.980 & 9 & 400 & 45.78 \\
\hline 1.941 & 20 & 242 & 46.75 \\
\hline 1.907 & 11 & 004 & 47.65 \\
\hline 1.863 & 6 & 420,251 & 48.85 \\
\hline 1.848 & 7 & 233,341 & 49.28 \\
\hline 1.758 & 4 & 402 & 51.96 \\
\hline 1.737 & 12 & 323,412 & 52.65 \\
\hline 1.715 & 5 & 252 & 53.37 \\
\hline
\end{tabular}


Rubidium Zinc Sulfate Hexahydrate, $\mathrm{Rb}_{2} \mathrm{Zn}\left(\mathrm{SO}_{4}\right)_{2} \cdot 6 \mathrm{H}_{2} \mathrm{O}$ (monoclinic)

\section{Sample}

The sample was prepared by slow evaporation at room temperature of an equimolar solution of $\mathrm{Rb}_{2} \mathrm{SO}_{4}$ and $\mathrm{ZnSO}_{4}$.

\section{Color}

Colorless

\section{Optical data}

Biaxial (t) $\mathrm{N}_{\alpha}=1.483, \mathrm{~N}_{\beta}=1.489, \mathrm{~N}_{\gamma}=1.497$ $2 \mathrm{~V}$ is large.

\section{Structure}

Monoclinic, $\mathrm{P} 2_{1} / \mathrm{a}$ (14), $\mathrm{Z}=2$.

$\mathrm{Rb}_{2} \mathrm{Zn}\left(\mathrm{SO}_{4}\right)_{2} \cdot 6 \mathrm{H}_{2} \mathrm{O}$ is a "Tutton Salt"[Tutton, 1893]. The structure of a "Tutton Salt", $\left(\mathrm{NH}_{4}\right)_{2} \mathrm{Mg}\left(\mathrm{SO}_{4}\right)_{2} \cdot 6 \mathrm{H}_{2} \mathrm{O}$ was determined by Margulis and Templeton, [1962].

\section{Lattice constants}

\begin{tabular}{|l|c|c|c|c|}
\hline \multirow{2}{*}{$\begin{array}{l}\text { NBS } \\
\text { sample }\end{array}$} & $a(\AA)$ & $b(\AA)$ & $c(\AA)$ & $\beta\left({ }^{\circ}\right)$ \\
\cline { 2 - 5 } at $25^{\circ} \mathrm{C}$ & $\begin{array}{r}9.185 \\
\pm .001\end{array}$ & $\begin{array}{r}12.450 \\
\pm .002\end{array}$ & $\begin{array}{r}6.242 \\
\pm .001\end{array}$ & $105^{\circ} 54.6$ \\
& & & $\pm .5^{\prime}$ \\
\hline
\end{tabular}

Density

(calculated) $2.596 \mathrm{~g} / \mathrm{cm}^{3}$ at $25^{\circ} \mathrm{C}$.

\section{Reference intensity}

$$
\mathrm{I} / \mathrm{I}_{\text {corundum }}=1.6
$$

\begin{tabular}{|c|c|c|c|}
\hline \multicolumn{4}{|c|}{$\begin{array}{l}\text { Internal standard } \mathrm{Ag}, \mathrm{a}=4.08641 \AA \\
\mathrm{CuK} \alpha_{1} \quad \lambda=1.54056 \AA \text {; temp. } 25^{\circ} \mathrm{C}\end{array}$} \\
\hline$d(\AA)$ & $I$ & $h k l$ & $2 \theta\left({ }^{\circ}\right)$ \\
\hline 7.20 & 6 & 110 & 12.28 \\
\hline 6.23 & 3 & 020 & 14.20 \\
\hline 6.00 & 2 & 001 & 14.74 \\
\hline 5.23 & 5 & I11 & 16.94 \\
\hline 5.084 & 4 & 120 & 17.43 \\
\hline 4.416 & 11 & 200 & 20.09 \\
\hline 4.318 & 20 & 021 & 20.55 \\
\hline 4.176 & 90 & 111 & 21.26 \\
\hline 4.139 & 100 & $\overline{2} 01$ & 21.45 \\
\hline 3.754 & 95 & 130 & 23.68 \\
\hline 3.607 & 8 & 121,220 & 24.66 \\
\hline 3.411 & 3 & 031 & 26.10 \\
\hline 3.367 & 17 & 131 & 26.45 \\
\hline 3.170 & 20 & 201 & 28.13 \\
\hline 3.114 & 25 & 040 & 28.64 \\
\hline 3.070 & 25 & 211 & 29.06 \\
\hline 3.021 & 55 & $\overline{1} 12,230$ & 29.54 \\
\hline 2.933 & 5 & $\overline{2} 31,140$ & 30.45 \\
\hline 2.905 & 10 & $\underline{3} 11$ & 30.75 \\
\hline 2.876 & 20 & $\overline{2} 02$ & 31.07 \\
\hline 2.868 & 20 & 310 & 31.16 \\
\hline 2.822 & 20 & 221 & 31.68 \\
\hline 2.801 & 8 & $\overline{2} 12$ & 31.93 \\
\hline 2.785 & 15 & $\overline{1} 22$ & 32.11 \\
\hline 2.763 & 4 & 041 & 32.38 \\
\hline 2.738 & 7 & $\overline{1}_{41}$ & 32.68 \\
\hline 2.694 & 3 & $\overline{3} 21$ & 33.23 \\
\hline 2.662 & 2 & 320 & 33.64 \\
\hline 2.611 & 4 & $\overline{2} 22$ & 34.31 \\
\hline 2.546 & 7 & 141,240 & 35.22 \\
\hline 2.518 & 5 & 231 & 35.63 \\
\hline 2.491 & 9 & $\overline{\mathbf{I}} 32, \overline{2} 41$ & 36.03 \\
\hline 2.424 & 40 & $\overline{3} 31,122$ & 37.05 \\
\hline 2.400 & 4 & 330 & 37.44 \\
\hline 2.300 & 10 & 051 & 39.14 \\
\hline 2.293 & 10 & $\overline{3} 22$ & 39.25 \\
\hline 2.248 & 6 & $\overline{4} 11$ & 40.07 \\
\hline 2.237 & 6 & 321 & 40.29 \\
\hline 2.221 & 20 & 132,241 & 40.58 \\
\hline 2.217 & 7 & 202 & 40.67 \\
\hline
\end{tabular}


Rubidium Zinc Sulfate Hexahydrate, $\mathrm{Rb}_{2} \mathrm{Zn}\left(\mathrm{SO}_{4}\right)_{2} \cdot 6 \mathrm{H}_{2} \mathrm{O}$ (monoclinic) - continued

\begin{tabular}{|c|c|c|c|}
\hline \multicolumn{4}{|c|}{$\begin{array}{l}\text { Internal standard } \mathrm{Ag}, \mathrm{a}=4.08641 \AA \\
\mathrm{CuK} a_{1} \quad \lambda=1.54056 \AA \text {; temp. } 25{ }^{\circ} \mathrm{C}\end{array}$} \\
\hline$d(\AA)$ & $I$ & $h k l$ & $2 \theta\left({ }^{\circ}\right)$ \\
\hline 2.182 & 3 & 212 & 41.35 \\
\hline 2.168 & 9 & 250 & 41.62 \\
\hline 2.160 & 8 & 042 & 41.78 \\
\hline 2.134 & 6 & $\overline{2} 51$ & 42.32 \\
\hline 2.112 & 11 & $\overline{2} 42$ & 42.78 \\
\hline 2.081 & 10 & 420 & 43.46 \\
\hline 2.076 & 6 & 331,050 & 43.56 \\
\hline 2.070 & 3 & $\underline{4} 02$ & 43.69 \\
\hline 2.045 & 4 & $\overline{2} 03$ & 44.26 \\
\hline 2.020 & 3 & $160, \overline{2} 13$ & 44.84 \\
\hline 2.001 & 9 & $\overline{4} 31,003$ & 45.28 \\
\hline 1.962 & 5 & 061 & 46.23 \\
\hline 1.958 & 5 & 251 & 46.34 \\
\hline 1.934 & 3 & $\overline{3} 42$ & 46.95 \\
\hline 1.916 & 8 & 052 & 47.42 \\
\hline 1.911 & 4 & 401 & 47.55 \\
\hline 1.899 & 9 & 341 & 47.85 \\
\hline 1.896 & 6 & $\overline{3} 13$ & 47.95 \\
\hline 1.857 & 10 & 133 & 49.00 \\
\hline 1.854 & 5 & $\overline{2} 61$ & 49.09 \\
\hline 1.843 & 2 & $\overline{2} 33$ & 49.66 \\
\hline 1.817 & 3 & $\overline{5} 11$ & 50.16 \\
\hline 1.801 & 8 & 440 & 50.65 \\
\hline 1.784 & 2 & 322 & 51.17 \\
\hline 1.769 & 3 & 123 & 51.63 \\
\hline 1.762 & 2 & $\overline{5} 21$ & 51.86 \\
\hline 1.749 & 5 & 510 & 52.27 \\
\hline 1.735 & 2 & 261,431 & 52.73 \\
\hline 1.727 & 4 & $351, \overline{1} 62$ & 52.98 \\
\hline 1.710 & 2 & $\overline{2} 43$ & 53.56 \\
\hline 1.699 & 5 & $\overline{1} 71,332,+$ & 53.93 \\
\hline 1.686 & 5 & 133 & 54.37 \\
\hline 1.682 & 3 & $043, \overline{2} 62,+$ & 54.50 \\
\hline 1.675 & 3 & $\overline{4} 23$ & 54.77 \\
\hline 1.650 & 6 & 171,270 & 55.66 \\
\hline 1.633 & 1 & $\overline{3} 43, \overline{2} 71$ & 56.28 \\
\hline 1.625 & 2 & 530 & 56.60 \\
\hline 1.6091 & 5 & 532 & 57.20 \\
\hline 1.5979 & 2 & 342 & 57.64 \\
\hline
\end{tabular}

\section{References}

Margulis, T.N. and D. H. Templeton (1962). Crystal structure and hydrogen bonding of magnesium ammonium sulfate hexahydrate, Z. Krist. 117, 334-357.

Tutton, A. E. (1893). Connection between the atomic weight of contained metals and the magnitude of the angles of crystals of isomorphous series. A study of the potassium, rubidium and cesium salts of the monoclinic series of double sulphates $\mathrm{R}_{2} \mathrm{M}\left(\mathrm{SO}_{4}\right)_{2} \cdot 6 \mathrm{H}_{2} \mathrm{O}$, J. Chem. Soc. 63, 337-423. 


\section{Sample}

The sample was prepared at NBS by adding a solution of $\mathrm{ZnCl}_{2}$ to one of $\mathrm{RbF}$ in $\mathrm{HF}$. The precipitate was washed in water and alcohol.

\section{Major impurities}

$0.01-0.1 \%$ each: $\mathrm{Al}, \mathrm{Ca}, \mathrm{Cr}, \mathrm{Cu}, \mathrm{Fe}$, and $\mathrm{Pt}$

$0.1-1.0 \%$ each: $\mathrm{Ba}$ and $\mathrm{Mg}$

\section{Color}

Colorless

\section{Optical data}

Isotropic, $\mathrm{N}=1.508$

\section{Structure}

Cubic, Pm3m (221), $\mathrm{z}=1$. Perovskite-type. Various distortions of the perovskite structure have been reported as shown in the lattice constant table. The NBS pattern was indexed with the smaller cubic cell; however diffraction peaks were not sharp which may indicate that there was a slight degree of distortion.

\section{Lattice constants}

\begin{tabular}{|c|c|c|}
\hline & $a(\AA)$ & $c(\AA)$ \\
\hline $\begin{array}{l}\text { Ludekens and Welch [1952] } \\
\text { Klasens et al. [1953]-- } \\
\text { Schmitz-DuMont and } \\
\text { Bornefeld [1956] } \\
\text { Crocket and Haendler } \\
\text { [1960]-- } \\
\text { NBS, sample at } 25{ }^{\circ} \mathrm{C}\end{array}$ & $\begin{array}{l}8.71 * \\
4.10 * * \\
8.25 * \\
4.116 \\
4.1215 \\
\pm .0001\end{array}$ & $8.03 *$ \\
\hline
\end{tabular}

* from $\mathrm{kX}$

**pseudocubic

\section{Density}

(calculated) $4.929 \mathrm{~g} / \mathrm{cm}^{3}$ at $25^{\circ} \mathrm{C}$.

\section{Reference intensity}

$I_{\text {corundum }}=5.6$

\begin{tabular}{|l|r|r|r|}
\hline \multicolumn{4}{|c|}{ Internal standard $\mathrm{W}, \mathrm{a}=3.16516 \AA$} \\
\hline \multicolumn{3}{|c|}{$\mathrm{CuK}_{1} \lambda=1.54056 \AA$; temp. $25{ }^{\circ} \mathrm{C}$} \\
\hline$d(\AA)$ & $I$ & $h k l$ & $2 \theta\left(^{\circ}\right)$ \\
\hline 4.13 & $<1$ & 100 & 21.52 \\
2.914 & 100 & 110 & 30.66 \\
2.380 & 17 & 111 & 37.77 \\
2.062 & 53 & 200 & 43.88 \\
1.6829 & 38 & 211 & 54.48 \\
& & & \\
1.4574 & 25 & 220 & 63.81 \\
1.3033 & 13 & 310 & 72.46 \\
1.2427 & 3 & 311 & 76.61 \\
1.1899 & 6 & 222 & 80.68 \\
1.1014 & 12 & 321 & 88.75 \\
& & & \\
1.0307 & 2 & 400 & 96.76 \\
0.9717 & 6 & 330 & 104.89 \\
.9457 & 1 & 331 & 109.08 \\
.9217 & 6 & 420 & 113.39 \\
.8788 & 3 & 332 & 122.44 \\
.8413 & 4 & 422 & 132.58 \\
.8084 & 7 & 510 & 144.69 \\
\hline
\end{tabular}

\section{Additional patterns}

1. PDF card 12-0039 [Schmitz-DuMont and Bornefeld]

\section{References}

Crocket,D.S. and H.M. Haendler(1960). Synthesis of fluorometallates in methanol. Some structure relationships, J.Am.Chem. Soc., 82,4158-4162.

Klasens, H. A., P. Zalm, and F. O. Huysman (1953). The manganese emission in $\mathrm{ABF}_{3}$ compounds, Philips Res. Rept. 8,441-451.

Ludekens, W.L.W., and A.J.E. Welch (1952). Reactions between metal oxides and fluorides: some new double-fluoride structures of type $\mathrm{ABF}_{3}$, Acta Cryst., 5, 841 . Schmitz-DuMont, $O$. and $H$. Bornefeld (1956). Die Systemreihe Alkalifluorid/Zinkfluorid, Z.Anorg.Al J.gem.Chem., 287, 120-137. 


\section{Sample}

The sample of chortveitite was synthesized hydrothermally by Jun Ito. A stoichiometric mixture of $\mathrm{Sc}_{2} \mathrm{O}_{3}$ and $\mathrm{SiO}_{2}$ was heated to $700{ }^{\circ} \mathrm{C}$ at a pressure of $2 \mathrm{ki}-$ lobars for 20 hours.

\section{Color}

Yellowish white

\section{Optical data}

Birefringent, $\mathbb{N}_{\alpha}=1.745, N_{\gamma}=1.760$

\section{Structure}

Monoclinic, $\mathrm{c} 2 / \mathrm{m}$ (12), $\mathrm{z}=2$ [Gossner and Mussgnug, 1929]. Structure determined by [Zachariasen, 1930].

\section{Density}

(calculated) $3.394 \mathrm{~g} / \mathrm{cm}^{3}$ at $25^{\circ} \mathrm{C}$.

\section{Additional patterns}

1. PDF card 15-383 [Sakurai et al. 1962]

2. PDF card 15-798 [Toporov et al. 1962]

3. PDF card 19-1125 [Horne, 1966]

4. Sabina and Traill, [1960]

5. Sakurai et al. [1962] 2nd pattern

\section{References}

Gossner, B. and F. Mussgnug (1929). Beitrag zur Kenntnis des Thortveitites, Centr. Mineral., Geol. A, 1-5.

Horne,J.E.T. (1966). X-ray diffraction data for thortveitite, Bull. Geol. Surv. Gt. Brit. No.25, 97-99.

Sabina, A.P.and R.J.Traill (1960). Catalog of X-ray Diffraction Patterns and Specimen Mounts on File at the Geol. Surv. of Canada, Geol. Surv. Paper 60-4, 104.

Sakurai,K.,K.Nagashima, and A.Kato (1962). Thortveitite from Kobe, Omiya, Kyoto, Japan, Bull.Chem.Soc. Japan 35, 1776-1779.

Toporov, N. A. and V. A. Vasil eva (1962). Equilibrium diagram of the scandium oxide-silica binary system, Russ.J.Inorg. Chem. 7, 1001-1005.

Zachariasen, W. H. (1930). The structure of thortveitite, $\mathrm{Sc}_{2} \mathrm{Si}_{2} \mathrm{O}_{7}, \mathrm{Z} . \mathrm{Krist} .73,1-6$.

Lattice constants

\begin{tabular}{|c|c|c|c|c|}
\hline & $a(\stackrel{\circ}{A})$ & $b(\AA)$ & $c(A)$ & $\beta\left({ }^{\circ}\right)$ \\
\hline $\begin{array}{l}\text { Gossner et al. [1929]**-- } \\
\text { Horne }[1966] * * \\
\text { NBS, sample at } 25{ }^{\circ} \mathrm{C}-----\end{array}$ & $\begin{array}{l}* 6.57 \\
6.65 \\
\pm .01 \\
6.508 \\
\pm .001\end{array}$ & $\begin{array}{l}8.60 \\
8.62 \\
\pm .01 \\
8.506 \\
\pm .001\end{array}$ & $\begin{array}{l}4.75 \\
4.68 \\
\pm .01 \\
4.677 \\
\pm .001\end{array}$ & $\begin{array}{r}103^{\circ} 8^{\prime} \\
102^{\circ} 12^{\prime} \\
\pm 30^{\prime} \\
102^{\circ} 43^{\prime} \\
\pm 1^{\prime}\end{array}$ \\
\hline
\end{tabular}

*as published

**natural mineral 


\begin{tabular}{|c|c|c|c|}
\hline \multicolumn{4}{|c|}{$\begin{array}{l}\text { Internal standard } \mathrm{W}, \mathrm{a}=3.16516 \AA \\
\mathrm{CuK} a_{1} \quad \lambda=1.54056 \AA \text {; temp. } 25{ }^{\circ} \mathrm{C}\end{array}$} \\
\hline$d(\AA)$ & $I$ & $h k l$ & $2 \theta\left({ }^{\circ}\right)$ \\
\hline 5.09 & 15 & 110 & 17.40 \\
\hline 4.57 & 7 & 001 & 19.41 \\
\hline 4.257 & 3 & 020 & 20.85 \\
\hline 3.131 & 100 & 111 & 28.48 \\
\hline 3.114 & $100\}$ & 021 & 28.64 \\
\hline 2.926 & 47 & 201 & 30.53 \\
\hline 2.588 & 15 & 130 & 34.63 \\
\hline 2.543 & 13 & 220 & 35.26 \\
\hline 2.373 & 3 & 201 & 37.89 \\
\hline 2.279 & 2 & 002 & 39.50 \\
\hline 2.236 & 2 & Ī12 & 40.29 \\
\hline 2.169 & 18 & 131 & 41.60 \\
\hline 2.126 & 3 & $\underline{0} 40$ & 42.48 \\
\hline 2.084 & 10 & $\overline{2} 02$ & 43.39 \\
\hline 2.073 & 8 & 221 & 43.62 \\
\hline 2.043 & 16 & $\overline{3} 11$ & 44.29 \\
\hline 2.010 & 1 & 022 & 45.06 \\
\hline 1.957 & 1 & 112 & 46.35 \\
\hline 1.927 & 5 & $\underline{0} 41$ & 47.12 \\
\hline 1.870 & 14 & $\overline{2} 22$ & 48.64 \\
\hline 1.794 & 2 & $\overline{1} 32$ & 50.85 \\
\hline 1.737 & 3 & $31 \underline{1}$ & 52.62 \\
\hline 1.720 & 4 & $\overline{2} 41, \overline{3} 12$ & 53.21 \\
\hline 1.696 & 7 & 330 & 54.01 \\
\hline 1.690 & 10 & $\overline{3} 31$ & 54.22 \\
\hline 1.685 & 9 & 202 & 54.40 \\
\hline 1.640 & 19 & 132 & 56.02 \\
\hline 1.587 & 5 & 400 & 58.08 \\
\hline I.5:19 & 6 & I5I & 60.96 \\
\hline 1.507 & 9 & $\overline{2} 03$ & 61.49 \\
\hline 1.493 & 6 & $\overline{3} 32$ & 62.14 \\
\hline 1.489 & 5 & $\overline{2} 42$ & 62.32 \\
\hline 1.462 & 2 & $\overline{4} 02$ & 63.59 \\
\hline 1.418 & 7 & 060 & 65.83 \\
\hline 1.391 & 3 & 113 & 67.24 \\
\hline 1.366 & 7 & $\overline{1} 33$ & 68.64 \\
\hline 1.335 & 3 & 421 & 70.46 \\
\hline 1.323 & 5 & $\overline{3} 51$ & 71.20 \\
\hline
\end{tabular}


Sample

A sample of $\mathrm{SeO}_{2}$ from the Mallinckrodt Chemical Works was dried at $220{ }^{\circ} \mathrm{C}$. The material was hygroscopic and the patterns were made with the sample enclosed in a dry mount.

\section{Color}

Colorless

\section{Structure}

Tetragonal, $\mathrm{P} 4 / \mathrm{mbc}$ (135), $\mathrm{Z}=8$, structure determined by McCullough [1937].

\section{Lattice constants}

\begin{tabular}{|l|l|l|}
\hline & \multicolumn{1}{|c|}{$a(\AA)$} & \multicolumn{1}{|c|}{$c(\AA)$} \\
\cline { 2 - 3 } McCullough [1937] -_-_-_- & 8.370 & 5.061 \\
Swanson and Tatge [1953]- & 8.35 & 5.08 \\
NBS, sample at 25 ${ }^{\circ} \mathrm{C}-\ldots--$ & 8.3635 & 5.0635 \\
& \pm .0001 & \pm .0002 \\
\hline
\end{tabular}

Density

(calculated) $4.161 \mathrm{~g} / \mathrm{cm}^{3}$ at $25^{\circ} \mathrm{C}$.

\section{Reference intensity}

$$
\mathrm{I} / \mathrm{I}_{\text {corundum }}=2.6
$$

\section{Additional patterns}

1. PDF card 4-429 [Swanson and Tatge, 1953]

\section{References}

McCullough, J.D. (1937). The crystal structure of selenium dioxide, J.Am. Chem. Soc. 59, 789-794.

Swanson, H.E. and E. Tatge (1953). Standard X-ray Diffraction Powder Patterns, Natl.Bur.Std.U.S. Circ.539.Vol.I, 53-54.

\begin{tabular}{|c|c|c|c|}
\hline \multicolumn{4}{|c|}{$\begin{array}{l}\text { Internal standard } \mathrm{W}, \mathrm{a}=3.16516 \AA \\
\mathrm{CuK} \alpha_{1} \quad \lambda=1.54056 \AA \text {; temp. } 25{ }^{\circ} \mathrm{C}\end{array}$} \\
\hline$d(\AA)$ & $I$ & $h k l$ & $2 \theta\left({ }^{\circ}\right)$ \\
\hline 5.909 & 12 & 110 & 14.98 \\
\hline 4.180 & 55 & 200 & 21.24 \\
\hline 3.742 & 60 & 210 & 23.76 \\
\hline 3.227 & 55 & 201 & 27.62 \\
\hline 3.008 & 100 & 211 & 29.67 \\
\hline 2.644 & 9 & 310 & 33.87 \\
\hline 2.531 & 25 & 002 & 35.43 \\
\hline 2.345 & 10 & 311 & 38.36 \\
\hline 2.327 & 10 & 112 & 38.66 \\
\hline $2.32 I$ & 10 & 320 & 38.77 \\
\hline 2.167 & 11 & 202 & 41.65 \\
\hline 2.110 & 6 & 321 & 42.83 \\
\hline 2.092 & 5 & 400 & 43.21 \\
\hline 2.029 & $<\mathrm{I}$ & 410 & 44.63 \\
\hline 1.972 & 2 & 330 & 45.98 \\
\hline 1.933 & 18 & 401 & 46.98 \\
\hline 1.883 & 14 & 411 & 48.30 \\
\hline 1.870 & 5 & 420 & 48.65 \\
\hline 1.829 & 14 & 312 & 49.82 \\
\hline 1.754 & 10 & 421 & 52.09 \\
\hline 1.710 & 11 & 322 & 53.53 \\
\hline 1.673 & 1 & 430 & 54.83 \\
\hline 1.640 & 2 & 510 & 56.03 \\
\hline 1.589 & 1 & 431 & 58.00 \\
\hline 1.566 & 5 & 203 & 58.94 \\
\hline I.5599 & 8 & 511 & 59.18 \\
\hline 1.5528 & 8 & 520 & 59.48 \\
\hline 1.5387 & 11 & 213 & 60.08 \\
\hline 1.5041 & 3 & 422 & 61.61 \\
\hline 1.4850 & $<\mathrm{I}$ & 521 & 62.49 \\
\hline 1.4784 & $I$ & 440 & 62.80 \\
\hline 1.4340 & $I$ & 530 & 64.98 \\
\hline 1.4225 & I & 313 & 65.57 \\
\hline 1.3956 & 2 & 432 & 67.00 \\
\hline 1.3800 & 6 & 531 & 67.86 \\
\hline I. 3748 & 9 & 610 & 68.15 \\
\hline 1.3268 & 2 & 611 & 70.98 \\
\hline 1.3237 & 5 & 522 & 71.17 \\
\hline 1.3135 & 4 & 403 & 71.81 \\
\hline 1.3064 & 1 & 540 & 72.26 \\
\hline
\end{tabular}




\begin{tabular}{|c|c|c|c|}
\hline \multicolumn{4}{|c|}{$\begin{array}{l}\text { Internal standard } \mathrm{W}, \mathrm{a}=3.16516 \AA \\
\mathrm{CuK} a_{1} \quad \lambda=1.54056 \AA \text {; temp. } 25{ }^{\circ} \mathrm{C}\end{array}$} \\
\hline$d(\AA)$ & $I$ & $h k l$ & $2 \theta\left({ }^{\circ}\right)$ \\
\hline 1.2974 & 2 & 413 & 72.84 \\
\hline 1.2798 & 1 & 621 & 74.01 \\
\hline 1.2768 & 3 & 442 & 74.21 \\
\hline 1.2646 & 4 & 541 & 75.05 \\
\hline 1.2528 & 2 & 423 & 75.88 \\
\hline 1.2105 & 5 & 631 & 79.04 \\
\hline 1.2083 & 7 & 612 & 79.21 \\
\hline 1.1826 & 1 & 550 & 81.29 \\
\hline 1.1760 & 2 & 513 & 81.84 \\
\hline 1. 1608 & 2 & 542 & 83.15 \\
\hline 1.1516 & 1 & 711 & 83.96 \\
\hline 1. 1112 & 2 & 324 & 87.77 \\
\hline 1.0982 & $I$ & 730 & 89.08 \\
\hline 1.0929 & 2 & 533 & 89.63 \\
\hline 1.0732 & 3 & 731 & 91.74 \\
\hline 1.0709 & 2 & 650 & 91.99 \\
\hline 1.0651 & 1 & 334 & 92.64 \\
\hline 1.0546 & $<1$ & 642 & 93.84 \\
\hline 1.0475 & 2 & 651 & 94.67 \\
\hline 1.0375 & I & 740 & 95.88 \\
\hline 1.0331 & $<1$ & 543 & 96.42 \\
\hline 1.0238 & $<1$ & 801 & 97.59 \\
\hline 1.0162 & $<1$ & 741 & 98.58 \\
\hline 1.0143 & 1 & 820 & 98.83 \\
\hline 1.0027 & 2 & 633 & 100.38 \\
\hline . 9945 & 1 & 821 & 101.52 \\
\hline .9862 & 1 & 652 & 102.72 \\
\hline .9687 & 1 & 713 & 105.35 \\
\hline .9611 & 1 & 831 & 106.54 \\
\hline . 9598 & 1 & 742 & 106.75 \\
\hline .9550 & $<1$ & 751 & 107.53 \\
\hline .9457 & 1 & 315 & 109.07 \\
\hline .9415 & 1 & 822 & 109.79 \\
\hline .9351 & 1 & 840 & 110.92 \\
\hline .9314 & 3 & 614 & 111.59 \\
\hline
\end{tabular}

\begin{tabular}{|c|c|c|c|}
\hline \multicolumn{4}{|c|}{$\begin{array}{l}\text { Internal standard } W, a=3.16516 \AA \\
\text { CuK } a_{1} \quad \lambda=1.54056 \AA \text {; temp. } 25{ }^{\circ} \mathrm{C}\end{array}$} \\
\hline$d(\AA)$ & $I$ & $h k l$ & $2 \theta\left({ }^{\circ}\right)$ \\
\hline .9236 & 1 & 910 & 113.02 \\
\hline .9204 & 2 & 733 & 113.62 \\
\hline .9113 & 1 & 405 & 115.40 \\
\hline .9089 & 2 & 544 & 115.88 \\
\hline .9073 & 2 & 760 & 116.20 \\
\hline $.904 I$ & 1 & 653 & 116.85 \\
\hline .8930 & 1 & 921 & 119.22 \\
\hline .8815 & 1 & 930 & 121.82 \\
\hline .8772 & 1 & 842 & 122.83 \\
\hline .8732 & 1 & 851 & 123.81 \\
\hline .8684 & $<1$ & 931 & 124.99 \\
\hline .8643 & $<1$ & 554 & 126.06 \\
\hline .8539 & 1 & 762 & 128.85 \\
\hline .8492 & 1 & 940 & 130.20 \\
\hline .8466 & $<1$ & 833 & 130.95 \\
\hline .8447 & 1 & 770 & 131.53 \\
\hline .8374 & 1 & 941 & 133.80 \\
\hline .8325 & 1 & 932 & 135.42 \\
\hline .8251 & 2 & 861 & 137.98 \\
\hline .8176 & 1 & 654 & 140.84 \\
\hline .8095 & 2 & $10 \cdot 2 \cdot 1$ & 144.17 \\
\hline .8051 & 1 & 942 & 146.18 \\
\hline .8024 & 2 & 744 & 147.45 \\
\hline .8021 & 2 & 951 & 147.60 \\
\hline .8011 & 2 & $10 \cdot 3 \cdot 0$ & 148.10 \\
\hline .7991 & 1 & 763 & 149.15 \\
\hline
\end{tabular}


Sodium Dichromate Dihydrate, $\mathrm{Na}_{2} \mathrm{Cr}_{2} \mathrm{O}_{7} \cdot 2 \mathrm{H}_{2} \mathrm{O}$ (monoclinic)

\section{Sample}

The sample was recrystallized from a water solution of reagent grade material from J.T.Baker Chemical Co.,Phillipsburg, N.J.

\section{Color}

Unground: deep orange

Ground: vivid orange

\section{Optical data}

Biaxial (+) $\mathrm{N}_{\alpha}=1.660, \mathrm{~N}_{\beta}=1.698, \mathrm{~N}_{\gamma}=1.743$ $2 \mathrm{~V} \cong 90$

\section{Structure}

Monoclinic, $\mathrm{P} 2{ }_{1} / \mathrm{m}$ (11), $\mathrm{Z}=4$, [Campbell, 1956]

\section{Lattice constants}

\begin{tabular}{|c|c|c|c|c|}
\hline & $a(\AA)$ & $b(\AA)$ & $c(\AA)$ & $\beta\left({ }^{\circ}\right)$ \\
\hline $\begin{array}{r}\text { Campbell } \\
\text { [1956] }\end{array}$ & 12.6 & 10.5 & 6.05 & $94^{\circ} 54^{\prime}$ \\
\hline $\begin{array}{l}\text { NBS, } \\
\text { sample } \\
\text { at } 25^{\circ} \mathrm{C}\end{array}$ & $\begin{array}{r}12.740 \\
\pm .001\end{array}$ & $\begin{array}{r}10.778 \\
\pm .001\end{array}$ & $\begin{array}{l}6.132 \\
\pm .001\end{array}$ & $\begin{array}{r}95^{\circ} 7^{\prime} \\
\pm 1^{\prime}\end{array}$ \\
\hline
\end{tabular}

\section{Density}

(calculated) $2.360 \mathrm{~g} / \mathrm{cm}^{3}$ at $25^{\circ} \mathrm{C}$.

Reference intensity

$$
\mathrm{I} / \mathrm{I}_{\text {corundum }}=0.8
$$

\begin{tabular}{|c|c|c|c|}
\hline \multicolumn{4}{|c|}{$\begin{array}{l}\text { Internal standard } \mathrm{W}, \mathrm{a}=3.16516 \AA \\
\mathrm{CuK} a_{1} \quad \lambda=1.54056 \AA \text {; temp. } 25{ }^{\circ} \mathrm{C}\end{array}$} \\
\hline$d(\AA)$ & $I$ & $h k l$ & $2 \theta\left({ }^{\circ}\right)$ \\
\hline 8.20 & 5 & 110 & 10.78 \\
\hline 6.34 & 5 & 200 & 13.95 \\
\hline 5.70 & 20 & İol & 15.54 \\
\hline 5.47 & 25 & 210 & 16.19 \\
\hline 5.40 & 80 & 020 & 16.40 \\
\hline 5.31 & 20 & 101,011 & 16.69 \\
\hline 5.041 & 20 & İl1 & 17.58 \\
\hline 4.957 & 10 & 120 & 17.88 \\
\hline 4.772 & 20 & ll1 & 18.58 \\
\hline 4.607 & 10 & $\overline{2} 01$ & 19.25 \\
\hline 4.239 & 40 & $\overline{2} 11$ & 20.94 \\
\hline 4.105 & 10 & 220 & 21.63 \\
\hline 4.042 & 10 & $02 \underline{1}$ & 21.97 \\
\hline 3.924 & 85 & $211, \overline{1} 21$ & 22.64 \\
\hline 3.786 & 25 & 121 & 23.48 \\
\hline 3.632 & 5 & $\overline{3} 01$ & 24.49 \\
\hline 3.502 & 30 & $\overline{2} 21$ & 25.41 \\
\hline 3.444 & 5 & $\overline{3} 11$ & 25.85 \\
\hline 3.321 & 20 & 221 & 26.82 \\
\hline 3.175 & 20 & 400 & 28.08 \\
\hline 3.041 & 100 & $410, \overline{1} 31$ & 29.35 \\
\hline 3.013 & 15 & $\overline{3} 21$ & 29.62 \\
\hline 2.976 & 10 & 131 & 30.00 \\
\hline 2.937 & 5 & 012 & 30.41 \\
\hline 2.927 & 10 & $\overline{4} 01$ & 30.52 \\
\hline 2.911 & 25 & 102 & 30.69 \\
\hline 2.852 & 10 & $\overline{2} 02$ & 31.34 \\
\hline 2.834 & 40 & $\overline{2} 31$ & 31.54 \\
\hline 2.821 & 30 & $\overline{4} 11$ & 31.69 \\
\hline 2.812 & 40 & 112 & 31.80 \\
\hline 2.760 & 10 & $\overline{2} 12$ & 32.41 \\
\hline 2.735 & 25 & 231,420 & 32.72 \\
\hline 2.716 & 10 & 401 & 32.95 \\
\hline 2.696 & 20 & 040 & 33.20 \\
\hline 2.642 & 3 & $\overline{1} 22$ & 33.90 \\
\hline 2.635 & 5 & $140,41.1$ & 34.00 \\
\hline 2.589 & 10 & $\overline{3} 02$ & 34.62 \\
\hline 2.582 & 10 & 212 & 34.71 \\
\hline 2.560 & 5 & 122 & 35.02 \\
\hline 2.516 & 5 & $\overline{3} 12$ & 35.65 \\
\hline
\end{tabular}




\begin{tabular}{|c|c|c|c|}
\hline \multicolumn{4}{|c|}{$\begin{array}{l}\text { Internal standard } \mathrm{W}, \mathrm{a}=3.16516 \AA \\
\mathrm{CuK} \alpha_{1} \lambda=1.54056 \AA \text {; temp. } 25{ }^{\circ} \mathrm{C}\end{array}$} \\
\hline$d(\AA)$ & $I$ & $h k l$ & $2 \theta\left({ }^{\circ}\right)$ \\
\hline 2.471 & 5 & 510 & 36.32 \\
\hline 2.447 & 3 & 331 & 36.69 \\
\hline 2.438 & 5 & $\overline{1} 41$ & 36.84 \\
\hline 2.428 & 5 & 421 & 37.00 \\
\hline 2.403 & 5 & 141 & 37.39 \\
\hline 2.384 & 5 & 222 & 37.70 \\
\hline 2.362 & 5 & $\overline{5} 11$ & 38.07 \\
\hline 2.332 & 3 & $\overline{3} 22$ & 38.57 \\
\hline 2.320 & 2 & 312 & 38.78 \\
\hline 2.305 & 2 & $\overline{4} 02$ & 39.05 \\
\hline 2.271 & 10 & 340,241 & 39.66 \\
\hline 2.263 & 5 & 132 & 39.80 \\
\hline 2.233 & 3 & $\overline{2} 32$ & 40.35 \\
\hline 2.223 & 3 & 511 & 40.55 \\
\hline 2.209 & 3 & 521 & 40.81 \\
\hline 2.168 & 2 & 431 & 41.63 \\
\hline 2.115 & 5 & 600 & 42.72 \\
\hline 2.100 & 5 & $\overline{3} 32$ & 43.04 \\
\hline 2.095 & 5 & 521 & 43.14 \\
\hline 2.074 & $<1$ & 610,530 & 43.60 \\
\hline 2.053 & 5 & 440 & 44.07 \\
\hline 2.041 & 3 & 250 & 44.35 \\
\hline 2.017 & 3 & $\overline{1} 51$ & 44.91 \\
\hline 2.009 & 3 & $\overline{5} 31, \overline{5} 12$ & 45.08 \\
\hline 2.001 & 3 & 013 & 45.27 \\
\hline 1.977 & 5 & 142 & 45.85 \\
\hline 1.969 & 5 & 620 & 46.06 \\
\hline 1.959 & 2 & $\overline{2} 42, \overline{2} 13$ & 46.31 \\
\hline 1.953 & 2 & $\overline{2} 51$ & 46.47 \\
\hline 1.920 & 10 & $531,350,+$ & 47.30 \\
\hline 1.914 & 10 & 611,441 & 47.47 \\
\hline 1.907 & 15 & 123 & 47.65 \\
\hline 1.902 & 15 & $\overline{3} 03$ & 47.77 \\
\hline 1.871 & 10 & 502 & 48.61 \\
\hline 1.867 & 10 & $\overline{2} 23, \overline{3} 42$ & 48.74 \\
\hline 1.861 & 5 & 213,123 & 48.91 \\
\hline 1.847 & 5 & 540 & 49.31 \\
\hline 1.843 & 3 & 512 & 49.40 \\
\hline 1.830 & 4 & 621 & 49.79 \\
\hline 1.811 & 3 & 700,351 & 50.33 \\
\hline
\end{tabular}

\begin{tabular}{|c|r|c|c|}
\hline \multicolumn{4}{|c|}{ Internal standard $\mathrm{W}, \mathrm{a}=3.16516 \AA$} \\
\hline \multicolumn{3}{|c|}{$\mathrm{CuK}_{1} \quad \lambda=1.54056 \mathrm{\AA}$; temp. $25{ }^{\circ} \mathrm{C}$} \\
\hline$d(\AA)$ & $I$ & $h k l$ & $2 \theta\left({ }^{\circ}\right)$ \\
\hline 1.800 & 5 & $\overline{5} 41$ & 50.66 \\
1.796 & 5 & 060 & 50.80 \\
1.783 & 5 & $342, \overline{7} 01$ & 51.20 \\
1.775 & 5 & $\overline{5} 32$ & 51.43 \\
1.763 & 3 & $\overline{4} 13$ & 51.82 \\
& & & \\
1.758 & 5 & $\overline{7} 11, \overline{1} 52$ & 51.96 \\
1.752 & 3 & $\overline{4} 42$ & 52.15 \\
1.732 & 10 & $\overline{1} 52$ & 52.81 \\
1.720 & 10 & $\overline{6} 2 \frac{2}{2} 52$ & 53.22 \\
1.692 & 2 & $\overline{7} 21$ & 54.17 \\
& & & \\
1.673 & 10 & $\overline{2} 61,233$ & 54.83 \\
1.663 & 5 & $640, \overline{5} 03$ & 55.20 \\
1.657 & 10 & $\overline{3} 52$ & 55.41 \\
1.642 & 5 & $550, \overline{5} 13$ & 55.94 \\
\hline
\end{tabular}

\section{Additional patterns}

1. PDF card 1-0460 [Hanawalt et al.,1938].

\section{References}

Campbell, J.A. (1956). Note on the crystal structure of sodium dichromate dihydrate Acta Cryst. 9, 192.

Hanawalt, J.D., H.W. Rinn, and L.K. Frevel (1938). Chemical analysis by x-ray diffraction, Ind. Eng. Chem. Anal. Ed. 10, 457-512. 
Sample

J. Ito prepared the sample by heating lanthanum oxide and silicic acid with excess NaF. The crystals were removed from the molten NaF bath after several days.

* Exact analysis of percentages of $\mathrm{Na}$ and La is not known.

Color

Colorless

Optical data

Uniaxial (-) $\mathrm{N}_{\mathrm{O}}=1.838, \mathrm{~N}_{\mathrm{e}}=1.816$

Structure

Hexagonal, $P 6_{3}$ (173), $\mathrm{Z}=1$, closely analogous to hydroxyapatite [Bowen and Dickens, 1968].

\section{Lattice constants}

\begin{tabular}{|l|l|l|}
\hline & $a(\AA)$ & $c(\AA)$ \\
\cline { 2 - 3 } Bowen and Dickens [1968] -- & 9.72 & 7.16 \\
Ito [1968]--------- & 9.68 & 7.19 \\
NBS, Sample at 25 ${ }^{\circ} \mathrm{C}------$ & 9.6890 & 7.1805 \\
& \pm .0002 & \pm .0002 \\
\hline
\end{tabular}

Density

(calculated) $4.97 I^{\dagger} \mathrm{g} / \mathrm{cm}^{3}$ at $25^{\circ} \mathrm{C}$.

$\dagger$ assuming $\mathrm{Na}_{2} \mathrm{La}_{8}\left(\mathrm{SiO}_{4}\right)_{6} \mathrm{~F}_{8}$

\section{References}

Bowen, J. S. and B. Dickens (1968). Private communication.

Ito,J. (1968). Private communication.

\begin{tabular}{|c|c|c|c|}
\hline \multicolumn{4}{|c|}{$\begin{array}{l}\text { Internal standard } W, a=3.16516 \AA \\
\text { CuKa } a_{1} \lambda=1.54056 \AA \text {; temp. } 25^{\circ} \mathrm{C}\end{array}$} \\
\hline$d(\AA)$ & $I$ & $h k l$ & $2 \theta\left({ }^{\circ}\right)$ \\
\hline 4.195 & 24 & 200 & 21.16 \\
\hline 4.013 & 25 & 111 & 22.13 \\
\hline 3.622 & 4 & 201 & 24.56 \\
\hline 3.594 & 32 & 002 & 24.75 \\
\hline 3.300 & 41 & 102 & 27.00 \\
\hline 3.172 & $3 I$ & 210 & 28.11 \\
\hline 2.901 & 100 & 211 & 30.80 \\
\hline 2.883 & 70 & 112 & 30.99 \\
\hline 2.798 & 28 & 300 & 31.96 \\
\hline 2.727 & 3 & 202 & 32.81 \\
\hline 2.378 & 2 & 212 & 37.80 \\
\hline 2.328 & 9 & 310 & 38.64 \\
\hline 2.296 & 3 & 221 & 39.20 \\
\hline 2.207 & 4 & 302 & 40.85 \\
\hline 2.146 & 15 & 113 & 42.08 \\
\hline 2.098 & 7 & 400 & 43.08 \\
\hline 2.080 & 3 & 203 & 43.47 \\
\hline 2.009 & 21 & 222 & 45.10 \\
\hline 1.953 & 16 & 312 & 46.45 \\
\hline 1.926 & 6 & 320 & 47.16 \\
\hline 1.910 & 36 & 213 & 47.56 \\
\hline 1.860 & 17 & 321 & 48.93 \\
\hline 1.831 & 17 & 410 & 49.76 \\
\hline 1.811 & 22 & 402 & 50.33 \\
\hline 1.795 & 19 & 004 & 50.82 \\
\hline 1.774 & 2 & 411 & 51.47 \\
\hline 1.697 & 1 & 322 & 53.98 \\
\hline 1.683 & 1 & 114 & 54.48 \\
\hline 1.679 & 1 & 500 & 54.63 \\
\hline 1.668 & 1 & 313 & 55.01 \\
\hline 1.651 & 3 & 204 & 55.62 \\
\hline 1.6314 & 2 & 412 & 56.35 \\
\hline 1.5858 & 5 & 420 & 58.12 \\
\hline 1.5755 & 7 & 331 & 58.54 \\
\hline I. 5626 & 10 & 214 & 59.07 \\
\hline 1.5488 & 1 & 421 & 59.65 \\
\hline 1.5206 & 8 & 502 & 60.87 \\
\hline 1.5112 & 8 & 304 & 61.29 \\
\hline 1.4997 & 8 & 323 & 61.81 \\
\hline 1.4744 & 8 & 511 & 62.99 \\
\hline
\end{tabular}


Sodium Lanthanum Fluosilicate, $\left(\mathrm{Na}_{2} \mathrm{La}_{8}\right)\left(\mathrm{SiO}_{4}\right)_{6} \mathrm{~F}_{2}$ (hexagonal) - continued

\begin{tabular}{|c|c|c|c|}
\hline \multicolumn{4}{|c|}{$\begin{array}{l}\text { Internal standard } \mathrm{W}, \mathrm{a}=3.16516 \AA \\
\mathrm{CuK} \alpha_{1} \quad \lambda=1.54056 \AA \text {; temp. } 25^{\circ} \mathrm{C}\end{array}$} \\
\hline$d(\AA)$ & $I$ & $h k l$ & $2 \theta\left({ }^{\circ}\right)$ \\
\hline 1.4728 & 9 & 332 & 63.07 \\
\hline 1.4213 & 1 & 314 & 65.64 \\
\hline 1.3896 & 1 & 512 & 67.33 \\
\hline 1.3768 & 2 & 115 & 68.04 \\
\hline 1.3732 & 2 & 601 & 68.25 \\
\hline 1.3638 & 3 & 404 & 68.78 \\
\hline 1.3546 & 1 & 431 & 69.31 \\
\hline 1.3431 & 2 & 520 & 69.99 \\
\hline 1.3389 & 3 & 333 & 70.24 \\
\hline 1.3207 & 5 & 521 & 71.36 \\
\hline 1.3128 & 3 & 324 & 71.85 \\
\hline 1.3081 & 9 & 215 & 72.15 \\
\hline 1.3031 & 6 & 602 & 72.47 \\
\hline I. 2874 & 3 & 432 & 73.49 \\
\hline 1.2817 & 10 & 414 & 73.88 \\
\hline 1. 2755 & 5 & 513 & 74.30 \\
\hline 1.2597 & 8 & 611 & 75.39 \\
\hline I. 2582 & 9 & 522 & 75.50 \\
\hline 1.2356 & $I$ & 225 & 77.13 \\
\hline 1.2261 & 1 & 504 & 77.84 \\
\hline 1.2112 & 4 & 440 & 78.98 \\
\hline 1.1985 & 1 & 530 & 79.99 \\
\hline I. 1967 & 2 & 006 & 80.13 \\
\hline I. 1954 & 3 & 433 & 80.24 \\
\hline I. 1888 & 3 & 424 & 80.77 \\
\hline 1.1853 & 4 & 405,106 & 81.06 \\
\hline 1.1824 & $I$ & 531 & 81.30 \\
\hline 1.1718 & 2 & 523 & 82.20 \\
\hline 1.1635 & 1 & 620 & 82.91 \\
\hline I. 1620 & 4 & 116 & 83.04 \\
\hline 1.1541 & 2 & 514 & 83.74 \\
\hline 1.1509 & 5 & 325,206 & 84.02 \\
\hline 1.1473 & 2 & 442 & 84.35 \\
\hline 1.1369 & 4 & 532 & 85.30 \\
\hline 1.1286 & 2 & 613 & 86.08 \\
\hline
\end{tabular}

\begin{tabular}{|c|c|c|c|}
\hline \multicolumn{4}{|c|}{$\begin{array}{l}\text { Internal standard } \mathrm{W}, \mathrm{a}=3.16516 \AA \\
\mathrm{CuK} \alpha_{1} \lambda=1.54056 \AA \text {; temp. } 25{ }^{\circ} \mathrm{C}\end{array}$} \\
\hline$d(\AA)$ & $I$ & $h k l$ & $2 \theta\left({ }^{\circ}\right)$ \\
\hline 1.1198 & $<1$ & 216 & 86.92 \\
\hline 1.1115 & 2 & 710 & 87.74 \\
\hline 1.0758 & 1 & 524 & 91.45 \\
\hline 1.0730 & 4 & 335,226 & 91.76 \\
\hline 1.0644 & 4 & 425,316 & 92.72 \\
\hline 1.0619 & 2 & 712 & 93.00 \\
\hline 1.0489 & 1 & 800 & 94.51 \\
\hline 1.0460 & 2 & 631 & 94.85 \\
\hline 1.0420 & 3 & 614 & 95.33 \\
\hline 1.0393 & 6 & 515,406 & 95.66 \\
\hline 1.0290 & 3 & 542 & 96.93 \\
\hline 1.0250 & 1 & 720 & 97.44 \\
\hline 1.0141 & 3 & 632 & 98.85 \\
\hline 1.0068 & 3 & 802 & 99.83 \\
\hline 1.0040 & 5 & 444 & 100.21 \\
\hline 0.9968 & $I$ & 534 & 101.20 \\
\hline .9949 & $I$ & 435 & 101.47 \\
\hline .9811 & 2 & 525 & 103.47 \\
\hline .9759 & 5 & 217 & 104.24 \\
\hline .9743 & 3 & 506 & 104.49 \\
\hline .9688 & 1 & 550 & 105.33 \\
\hline .9670 & 1 & 633 & 105.61 \\
\hline .9623 & 2 & 640 & 106.34 \\
\hline .9613 & 2 & 336 & 106.50 \\
\hline .9551 & 2 & 615,426 & 107.51 \\
\hline .9539 & 1 & 641 & 107.70 \\
\hline .9472 & 3 & 812 & 108.83 \\
\hline .9446 & 5 & 227 & 109.26 \\
\hline
\end{tabular}


Sample source

Ito prepared the sample by heating neodymium oxide and silicic acid with an excess of NaF. The crystals were removed from the molten NaF bath after several days.

*Exact analysis of percentages of $\mathrm{Na}$ and Nd is not known.

\section{Color}

Unground- moderate purplish blue

Ground- pale purplish blue

\section{Optical data}

Uniaxial (-) $\mathrm{N}_{\mathrm{O}}=1.884, \mathrm{~N}_{\mathrm{e}}=1.860$

\section{Structure}

Hexagonal, $\mathrm{PG}_{3}$ (173), $\mathrm{Z}=1$. Closely analogous to hydroxyapatite. [Bowen and Dickens, 1968]

\section{Lattice constants}

\begin{tabular}{|c|c|c|}
\hline & $a(\stackrel{\circ}{)}$ & $c(\stackrel{\AA}{)})$ \\
\hline $\begin{array}{l}\text { Bowen and Dickens [1968]-- } \\
\text { Ito [1968]-- } \\
\text { NBS, sample at } 25{ }^{\circ} \mathrm{C}-\end{array}$ & $\begin{array}{l}9.51 \\
9.55 \\
9.5411 \\
\pm .0001\end{array}$ & $\begin{array}{l}7.02 \\
7.03 \\
7.0331 \\
\pm .0002\end{array}$ \\
\hline
\end{tabular}

Density

(calculated) $5.362^{\neq} \mathrm{g} / \mathrm{cm}^{3}$ at $25^{\circ} \mathrm{C}$.

assuming $\mathrm{Na}_{2} \mathrm{Nd}_{8}\left(\mathrm{SiO}_{4}\right)_{6} \mathrm{~F}_{2}$

Reference intensity

$\mathrm{I} / 1_{\text {corundum }}=3.2$

\section{References}

Bowen,J.S. and B. Dickens (1968). Private communication.

Ito,J. (1968). Private communication.

\begin{tabular}{|c|c|c|c|}
\hline \multicolumn{4}{|c|}{$\begin{array}{l}\text { Internal standard } W, a=3.16516 \AA \\
\text { CuKa } a_{1} \lambda=1.54056 \AA \text {; temp. } 25{ }^{\circ} \mathrm{C}\end{array}$} \\
\hline$d(\AA)$ & $I$ & $h k l$ & $2 \theta\left({ }^{\circ}\right)$ \\
\hline 4.769 & 3 & 110 & 18.59 \\
\hline 4.130 & 26 & 200 & 21.50 \\
\hline 3.948 & 24 & 111 & 22.50 \\
\hline 3.513 & 22 & 002 & 25.33 \\
\hline 3.235 & 42 & 102 & 27.55 \\
\hline 3.122 & 33 & 210 & 28.57 \\
\hline 2.855 & 100 & 211 & 31.30 \\
\hline 2.831 & 52 & 112 & 31.58 \\
\hline 2.754 & 29 & 300 & 32.48 \\
\hline 2.677 & 2 & 202 & 33.45 \\
\hline 2.336 & 2 & 212 & 38.51 \\
\hline 2.293 & 8 & 310 & 39.26 \\
\hline 2.258 & 5 & 221 & 39.89 \\
\hline 2.180 & 2 & 311 & 41.39 \\
\hline 2.169 & 4 & 302 & 41.60 \\
\hline 2.105 & 12 & 113 & 42.94 \\
\hline 2.067 & 6 & 400 & 43.77 \\
\hline 2.039 & 2 & 203 & 44.40 \\
\hline 1.974 & 23 & 222 & 45.93 \\
\hline 1.921 & 16 & 312 & 47.28 \\
\hline 1.896 & 6 & 320 & 47.94 \\
\hline 1.875 & 31 & 213 & 48.52 \\
\hline 1.831 & 16 & 321 & 49.77 \\
\hline 1.803 & 20 & 410 & 50.59 \\
\hline 1.781 & 23 & 402 & 51.26 \\
\hline 1.757 & 14 & 004 & 51.99 \\
\hline 1.6690 & 1 & 322 & 54.97 \\
\hline 1.6500 & 1 & 114 & 55.66 \\
\hline 1.6177 & 3 & 204 & 56.87 \\
\hline 1.6048 & 1 & 412 & 57.37 \\
\hline 1.5614 & 4 & 420 & 59.12 \\
\hline 1.5511 & 8 & 331 & 59.55 \\
\hline 1.5322 & 8 & 214 & 60.36 \\
\hline 1.5240 & 2 & 421 & 60.72 \\
\hline 1.4960 & 10 & 502 & 61.98 \\
\hline 1.4820 & 7 & 304 & 62.63 \\
\hline 1.4743 & 8 & 323 & 63.00 \\
\hline 1.4518 & 6 & 511 & 64.09 \\
\hline 1.4487 & 8 & 332 & 64.24 \\
\hline 1.4291 & 2 & 413 & 65.23 \\
\hline 1.3948 & 2 & 314 & 67.04 \\
\hline 1.3674 & 1 & 512 & 68.57 \\
\hline 1.3490 & 2 & 115 & 69.64 \\
\hline 1.3389 & 3 & 404 & 70.24 \\
\hline 1.3338 & 3 & 431 & 70.55 \\
\hline
\end{tabular}


Sodium Neodymium Fluosilicate $\left(\mathrm{Na}_{2} \mathrm{Nd}_{8}\right)\left(\mathrm{SiO}_{4}\right)_{6} \mathrm{~F}_{2}$ (hexagonal) - continued

\begin{tabular}{|c|c|c|c|}
\hline \multicolumn{4}{|c|}{$\begin{array}{l}\text { Internal standard } \mathrm{W}, \mathrm{a}=3.16516 \AA \\
\mathrm{CuK} a_{1} \quad \lambda=1.54056 \AA \text {; temp. } 25{ }^{\circ} \mathrm{C}\end{array}$} \\
\hline$d(\AA)$ & $I$ & $h k l$ & $2 \theta\left({ }^{\circ}\right)$ \\
\hline 1.3234 & 1 & 520 & 71.19 \\
\hline 1.3162 & 3 & 333 & 71.64 \\
\hline 1.3003 & 4 & 521,423 & 72.65 \\
\hline 1.2893 & 2 & 324 & 73.37 \\
\hline 1.2829 & 10 & 215,602 & 73.80 \\
\hline 1.2673 & 2 & 432 & 74.86 \\
\hline 1.2605 & 12 & 610 & 75.34 \\
\hline 1.2586 & 12 & 414 & 75.47 \\
\hline 1.2541 & 6 & 513 & 75.79 \\
\hline 1.2401 & 6 & 611 & 76.80 \\
\hline 1.2385 & 9 & 522 & 76.92 \\
\hline 1.2117 & 1 & 225 & 78.94 \\
\hline 1.2041 & 2 & 504 & 79.54 \\
\hline 1.1991 & 1 & 315 & 79.94 \\
\hline 1.1930 & 4 & 440 & 80.43 \\
\hline 1.1862 & 2 & 612 & 80.99 \\
\hline 1.1804 & 2 & 530 & 81.47 \\
\hline 1.1756 & 2 & 441,433 & 81.87 \\
\hline 1.1723 & 2 & 006 & 82.15 \\
\hline 1.1676 & 3 & 424 & 82.56 \\
\hline 1.1608 & 2 & 106 & 83.15 \\
\hline 1.1522 & 3 & 523 & 83.91 \\
\hline 1.1458 & 2 & 620 & 84.48 \\
\hline 1.1381 & 4 & 116 & 85.19 \\
\hline 1.1339 & 3 & 514 & 85.58 \\
\hline 1.1307 & 4 & 621 & 85.88 \\
\hline 1.1293 & 4 & 325,442 & 86.01 \\
\hline 1.1190 & 4 & 532 & 87.00 \\
\hline 1.1100 & 3 & 613 & 87.89 \\
\hline 1.0974 & 1 & 216 & 89.16 \\
\hline 1.0944 & 2 & 710 & 89.47 \\
\hline 1.0894 & 1 & 622 & 89.99 \\
\hline 1.0841 & $<1$ & 604 & 90.56 \\
\hline 1.0813 & $<1$ & 711 & 90.86 \\
\hline 1.0785 & 1 & 306 & 91.16 \\
\hline 1.0751 & 1 & 434 & 91.53 \\
\hline 1.0579 & 1 & 540 & 93.46 \\
\hline 1.0543 & 3 & 533 & 93.87 \\
\hline 1.0519 & 3 & 226 & 94.15 \\
\hline 1.0453 & 3 & 425,712 & 94.94 \\
\hline 1.0435 & 3 & 316 & 95.15 \\
\hline 1.0409 & 1 & 630 & 95.47 \\
\hline 1.0329 & $<1$ & 800 & 96.45 \\
\hline 1.0299 & 3 & 631 & 96.82 \\
\hline 1.0244 & 4 & 614 & 97.52 \\
\hline
\end{tabular}

\begin{tabular}{|c|c|c|c|}
\hline \multicolumn{4}{|c|}{$\begin{array}{l}\text { Internal standard } W, a=3.16516 \AA \\
\text { CuK } a_{1} \quad \lambda=1.54056 \AA \text {; temp. } 25{ }^{\circ} \mathrm{C}\end{array}$} \\
\hline$d(\AA)$ & $I$ & $h k l$ & $2 \theta\left({ }^{\circ}\right)$ \\
\hline 1.0210 & 4 & 515 & 97.95 \\
\hline 1.0195 & 3 & 406 & 98.14 \\
\hline 1.0131 & 3 & 542 & 98.99 \\
\hline 1.0095 & 3 & 720 & 99.47 \\
\hline 0.9983 & 4 & 632 & 101.00 \\
\hline .9910 & 4 & 802 & 102.03 \\
\hline .9871 & 5 & 444 & 102.59 \\
\hline .9830 & 4 & 117,416 & 103.19 \\
\hline .9701 & 1 & 722 & 105.12 \\
\hline .9672 & 2 & 810 & 105.58 \\
\hline .9638 & 2 & 525 & 106.11 \\
\hline .9600 & 2 & 624 & 106.72 \\
\hline .9564 & 6 & 217 & 107.29 \\
\hline .9514 & 1 & 633 & 108.12 \\
\hline .9477 & 1 & 640 & 108.74 \\
\hline .9437 & 1 & 307,336 & 109.42 \\
\hline .9394 & 1 & 641 & 110.16 \\
\hline .9387 & 3 & 615 & 110.29 \\
\hline .9325 & 2 & 812 & 111.39 \\
\hline .9297 & 2 & 730 & 111.89 \\
\hline .9291 & 2 & 714 & 112.01 \\
\hline .9272 & 1 & 723 & 112.34 \\
\hline .9215 & 2 & 731 & 113.41 \\
\hline .9181 & 1 & 900 & 114.07 \\
\hline .9096 & 1 & 445 & 115.74 \\
\hline .9065 & 1 & 544 & 116.37 \\
\hline .9042 & 1 & 535 & 116.84 \\
\hline .8958 & $<1$ & 634 & 118.61 \\
\hline .8942 & 3 & 821 & 118.95 \\
\hline .8926 & 2 & 606 & 119.30 \\
\hline .8905 & 1 & 804 & 119.76 \\
\hline .8877 & 3 & 327 & 120.40 \\
\hline .8838 & $<1$ & 553 & 121.29 \\
\hline .8792 & 3 & 008 & 122.36 \\
\hline .8774 & 3 & 526 & 122.79 \\
\hline .8733 & 2 & 822 & 123.78 \\
\hline .8662 & 1 & 650 & 125.57 \\
\hline .8641 & 2 & 733 & 126.11 \\
\hline .8597 & 2 & 651 & 127.27 \\
\hline .8550 & 1 & 903 & 128.57 \\
\hline .8505 & 1 & 741 & 129.83 \\
\hline .8494 & 1 & 337 & 130.15 \\
\hline
\end{tabular}


Sodium Praseodymium Fluosilicate $\left(\mathrm{Na}_{2} \mathrm{Pr}_{8}\right) *\left(\mathrm{SiO}_{4}\right)_{6} \mathrm{~F}_{2}$ (hexagonal)

Sample source

Ito prepared the sample by heating praseodymium oxide and silicic acid with an excess of NaF. The crystals were removed from the molten NaF bath after several days.

*Exact analysis of percentages of $\mathrm{Na}$ and $\operatorname{Pr}$ is not known.

\section{Color}

Unground- brilliant yellow green

Ground- very light yellow green

\section{Optical data}

Uniaxial (-) $\mathrm{N}_{\mathrm{O}}=1.874, \mathrm{~N}_{\mathrm{e}}=1.855$

\section{Structure}

Hexagonal, $\mathrm{P}_{3}$ (173) $\mathrm{Z}=1$. Closely analogous to hydroxyapatite, [Bowen and Dickens, 1968] .

\section{Lattice constants}

\begin{tabular}{|l|l|l|}
\hline & $a(\AA)$ & $c(\AA)$ \\
\cline { 2 - 3 } Bowen and Dickens [1968]-- & 9.58 & 7.05 \\
Ito[1968]--19 & 9.60 & 7.09 \\
NBS, sample at 25 ${ }^{\circ} \mathrm{C}------$ & 9.5828 & 7.0728 \\
& \pm .0002 & \pm .0002 \\
\hline
\end{tabular}

Density

(calculated) $5.207^{\ddagger} \mathrm{g} / \mathrm{cm}^{3}$ at $25^{\circ} \mathrm{C}$.

† assuming $\mathrm{Na}_{2} \mathrm{Pr}_{8}\left(\mathrm{SiO}_{4}\right)_{6} \mathrm{~F}_{2}$

\section{Reference intensity}

$$
I / I_{\text {corundum }}=1.9
$$

\begin{tabular}{|c|c|c|c|}
\hline \multicolumn{4}{|c|}{$\begin{array}{l}\text { Internal standard } \mathrm{Ag}, a=4.08641 \AA \\
\mathrm{CuK} \alpha_{1} \lambda=1.54056 \AA \text {; temp. } 25{ }^{\circ} \mathrm{C}\end{array}$} \\
\hline$d(\stackrel{\AA}{A})$ & $I$ & $h k l$ & $2 \theta\left({ }^{\circ}\right)$ \\
\hline 4.80 & 3 & 110 & 18.48 \\
\hline 4.149 & 19 & 200 & 21.40 \\
\hline 3.969 & 20 & 111 & 22.38 \\
\hline 3.531 & 14 & 002 & 25.20 \\
\hline 3.252 & 29 & 1.02 & 27.40 \\
\hline 3.136 & 27 & 210 & 28.44 \\
\hline 2.866 & 100 & 211 & 31.18 \\
\hline 2.846 & 46 & 112 & 31.41 \\
\hline 2.764 & 26 & 300 & 32.36 \\
\hline 2.693 & 3 & 202 & 33.24 \\
\hline 2.395 & 2 & 220 & 37.52 \\
\hline 2.302 & 8 & 310 & 39.09 \\
\hline 2.268 & 3 & 221,103 & 39.70 \\
\hline 2.188 & 4 & 311 & 41.23 \\
\hline 2.179 & 4 & 302 & 41.41 \\
\hline 2.115 & 14 & 113 & 42.71 \\
\hline 2.075 & 6 & 400 & 43.59 \\
\hline 2.051 & 2 & 203 & 44.12 \\
\hline 1.983 & 28 & 222 & 45.72 \\
\hline 1.929 & 17 & 312 & 47.08 \\
\hline 1.904 & 7 & 320 & 47.73 \\
\hline 1.885 & 40 & 213 & 48.25 \\
\hline 1.838 & 20 & 321 & 49.54 \\
\hline 1.811 & 20 & 410 & 50.34 \\
\hline 1.789 & 32 & 402 & 51.00 \\
\hline 1.768 & 14 & 004 & 51.65 \\
\hline 1.680 & I. & 223 & 54.57 \\
\hline 1.677 & 2 & 322 & 54.70 \\
\hline 1.660 & 2 & 500,114 & 55.30 \\
\hline 1.626 & 3 & 204 & 56.55 \\
\hline 1.613 & 2 & 412 & 57.06 \\
\hline 1.5681 & 6 & 420 & 58.84 \\
\hline 1.5575 & 9 & 331,403 & 59.28 \\
\hline 1.5403 & 10 & 214 & 60.01 \\
\hline 1.5315 & 6 & 421 & 60.39 \\
\hline 1.5023 & 12 & 502 & 61.69 \\
\hline 1.4895 & 11 & 510,304 & 62.28 \\
\hline 1.4810 & 10 & 323 & 62.68 \\
\hline 1.4581 & 11 & 511 & 63.78 \\
\hline 1.4556 & 11 & 332 & 63.90 \\
\hline
\end{tabular}


Sodium Praseodymium Fluosilicate $\left(\mathrm{Na}_{2} \mathrm{Pr}_{8}\right)\left(\mathrm{SiO}_{4}\right)_{6} \mathrm{~F}_{2}$ (hexagonal) - continued

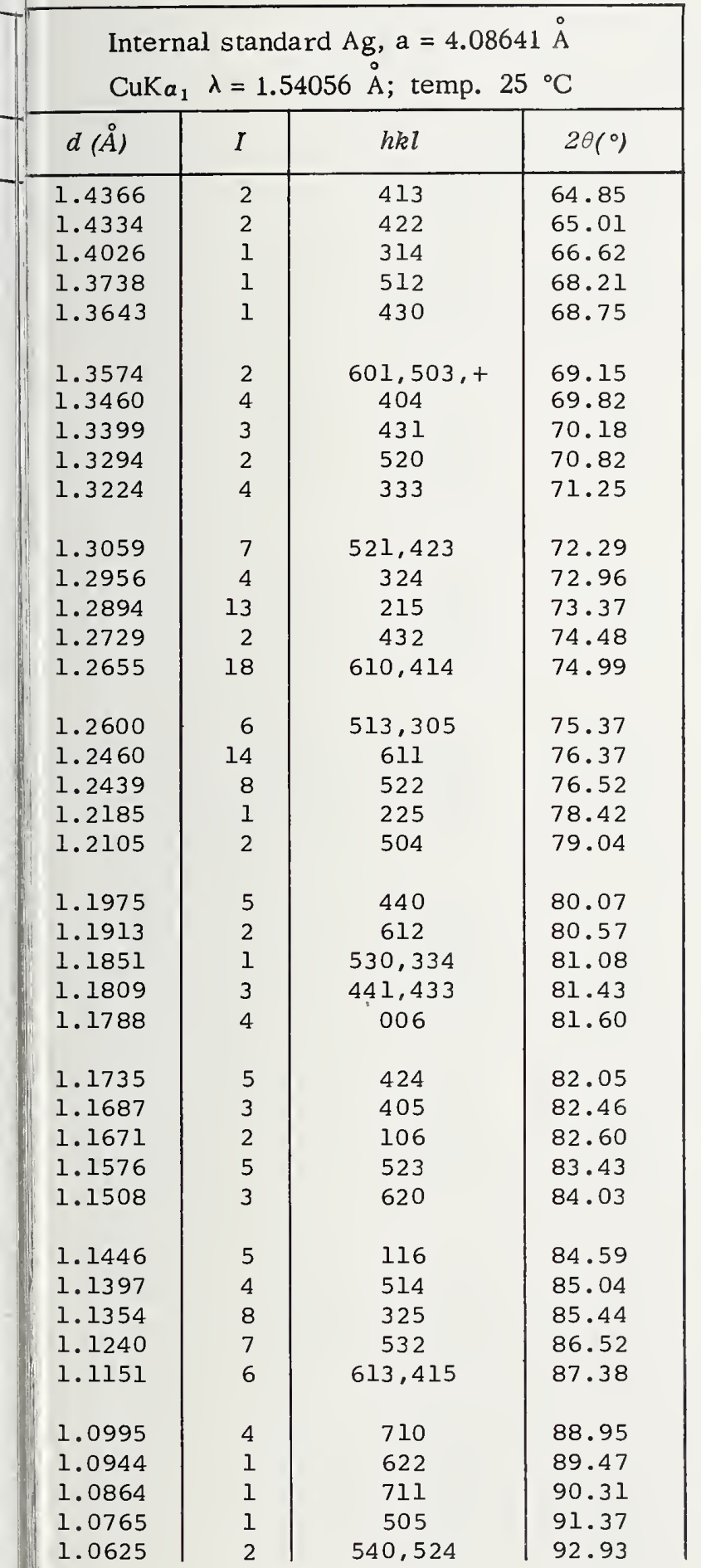

\begin{tabular}{|c|c|c|c|}
\hline \multicolumn{4}{|c|}{$\begin{array}{l}\text { Internal standard } \mathrm{Ag}, \mathrm{a}=4.08641 \AA \\
\mathrm{CuK} a_{1} \quad \lambda=1.54056 \AA \mathrm{A} ; \text { temp. } 25{ }^{\circ} \mathrm{C}\end{array}$} \\
\hline$d(\AA)$ & $I$ & $h k l$ & $2 \theta\left({ }^{\circ}\right)$ \\
\hline 1.0591 & 7 & 533,335 & 93.32 \\
\hline 1.0575 & 6 & 226 & 93.51 \\
\hline 1.0495 & 5 & 712,316 & 94.44 \\
\hline 1.0375 & 2 & 800 & 95.88 \\
\hline 1.0340 & 3 & 631,623 & 96.31 \\
\hline 1.0290 & 5 & 614 & 96.93 \\
\hline 1.0261 & 6 & 801,515 & 97.30 \\
\hline 1.0250 & 6 & 406 & 97.44 \\
\hline 1.0176 & 5 & 542 & 98.40 \\
\hline 1.0139 & 1 & 720 & 98.88 \\
\hline 1.0036 & 3 & 721 & 100.26 \\
\hline 1.0027 & 5 & 632 & 100.39 \\
\hline 0.9954 & 5 & 802 & 101.40 \\
\hline .9916 & 5 & 444 & 101.94 \\
\hline .9847 & 2 & 534 & 102.93 \\
\hline .9819 & 2 & 435,207 & 103.35 \\
\hline .9746 & 1 & 722 & 104.44 \\
\hline .9686 & 3 & 543,525 & 105.36 \\
\hline .9644 & 2 & 624 & 106.01 \\
\hline .9617 & 5 & 217 & 106.44 \\
\hline .9584 & 2 & 550 & 106.98 \\
\hline .9520 & $<1$ & 640 & 108.02 \\
\hline .9485 & 3 & 336 & 108.61 \\
\hline .9432 & 3 & 615 & 109.51 \\
\hline .9365 & 3 & 812 & 110.67 \\
\hline .9334 & 4 & 730,714 & 111.23 \\
\hline .9310 & 3 & 227 & 111.65 \\
\hline .9258 & 2 & 731 & 112.62 \\
\hline .9220 & 1 & 900 & 113.32 \\
\hline .9193 & 1 & 642 & 113.84 \\
\hline .9085 & 2 & 535,407 & 115.97 \\
\hline .8980 & 2 & 821,813 & 118.13 \\
\hline .8971 & 2 & 606 & 118.32 \\
\hline .8947 & 3 & 804 & 118.84 \\
\hline .8925 & 5 & 327 & 119.32 \\
\hline
\end{tabular}

\section{References}

Bowen,J.S.and B.Dickens(1968) Private communication.

Ito, Jun (1968). Private communication 
Thallium Cobalt Sulfate Hexahydrate $\mathrm{TI}_{2} \mathrm{Co}\left(\mathrm{SO}_{4}\right)_{2} \cdot 6 \mathrm{H}_{2} \mathrm{O}$ (monoclinic)

\section{Sample}

The sample was prepared by slow evaporation at room temperature of an aqueous solution of $\mathrm{Tl}_{2} \mathrm{SO}_{4}$ and $\mathrm{CoSO}_{4}$ in a $\mathrm{I}: 8$ molar proportion. The first crystals formed were used.

\section{Color}

Unground: dark yellowish pink.

Ground: light pink

\section{Optical data}

Biaxial (-) $N_{\alpha}=1.599, N_{\beta}=1.613, N_{\gamma}=1.624$ $2 \mathrm{~V}$ is medium large

\section{Structure}

Monoclinic, $\mathrm{P} 21 / \mathrm{a}$ (14), $\mathrm{Z}=2$. Isostructural with other "Tutton Salts" [Tutton, 1925]. The structure of a "Tutton Salt", $\left(\mathrm{NH}_{4}\right)_{2} \mathrm{Mg}\left(\mathrm{SO}_{4}\right)_{2} \cdot 6 \mathrm{H}_{2} \mathrm{O}$ was determined by Margulis and Templeton, [1962].

\section{Lattice constants}

\begin{tabular}{|c|c|c|c|c|}
\hline & $a(\stackrel{\AA}{)}$ & $b(\AA)$ & $c(\stackrel{\circ}{)})$ & $\beta\left({ }^{\circ}\right)$ \\
\hline $\begin{array}{l}\text { NBS, } \\
\text { sample } \\
\text { at } 25{ }^{\circ} \mathrm{C}\end{array}$ & $\begin{array}{l}9.235 \\
\pm .001\end{array}$ & $\begin{array}{r}12.442 \\
\pm .002\end{array}$ & $\begin{array}{l}6.227 \\
\pm .001\end{array}$ & $\begin{array}{r}106^{\circ} 24^{\prime} \\
\pm I^{\prime}\end{array}$ \\
\hline
\end{tabular}

Density

(calculated) $4.180 \mathrm{~g} / \mathrm{cm}^{3}$ at $25^{\circ} \mathrm{C}$.

\section{Reference intensity}

$$
I I_{\text {corundum }}=3.7
$$

\section{References}

Margulis, T.N. and D. H. Templeton (1962). Crystal structure and hydrogen bonding of magnesium ammonium sulfate hexahydrate, Z. Krist. 117, 334-357.

Tutton, A.E. (1925). The monoclinic double sulphates containing thallium, thallium nickel and thallium cobalt sulphate, Proc.Roy.Soc. London, Ser.A 108, 240-261.

\begin{tabular}{|c|c|c|c|}
\hline \multicolumn{4}{|c|}{$\begin{array}{l}\text { Internal standard Ag, } a=4.08641 \AA \\
\mathrm{CuK} a_{1} \quad \lambda=1.54056 \AA \text {; temp. } 25{ }^{\circ} \mathrm{C}\end{array}$} \\
\hline$d(\AA)$ & $I$ & $h k l$ & $2 \theta\left({ }^{\circ}\right)$ \\
\hline 7.213 & 22 & 110 & 12.26 \\
\hline 6.219 & 1 & 020 & 14.23 \\
\hline 5.977 & 11 & 001 & 14.81 \\
\hline 5.388 & 16 & 011 & 16.44 \\
\hline 5.089 & 26 & 120 & 17.41 \\
\hline 4.429 & 2 & 200 & 20.03 \\
\hline 4.310 & 9 & $\underline{0} 21$ & 20.59 \\
\hline 4.225 & 35 & $\overline{1} 21$ & 21.01 \\
\hline 4.154 & 100 & 111 & 21.37 \\
\hline 3.952 & 5 & $\overline{2} I I$ & 22.48 \\
\hline 3.757 & 55 & 130 & 23.66 \\
\hline 3.611 & 2 & $\underline{2} 20$ & 24.63 \\
\hline 3.463 & $I$ & $\underline{2} 21$ & 25.70 \\
\hline 3.368 & 6 & $\bar{I} 31$ & 26.44 \\
\hline 3.159 & 16 & 201 & 28.23 \\
\hline 3.112 & 25 & 040 & 28.66 \\
\hline 3.059 & 15 & 211 & 29.17 \\
\hline 3.019 & 46 & $131, \bar{I} 12$ & 29.56 \\
\hline 2.991 & 3 & 002 & 29.85 \\
\hline 2.935 & 8 & 140 & 30.43 \\
\hline 2.922 & 14 & $\overline{3} 11$ & 30.57 \\
\hline 2.907 & 14 & OI2 & 30.73 \\
\hline 2.881 & 23 & $\overline{2} 02$ & 31.01 \\
\hline 2.817 & 10 & 221 & 31.74 \\
\hline 2.781 & 12 & $\bar{I} 22$ & 32.16 \\
\hline 2.756 & 7 & 041 & 32.44 \\
\hline 2.737 & 8 & $\bar{I} 41$ & 32.69 \\
\hline 2.669 & 6 & 320 & 33.55 \\
\hline 2.617 & 2 & $\overline{2} 22$ & 34.24 \\
\hline 2.560 & 1 & 112 & 35.03 \\
\hline 2.542 & 1 & 141 & 35.28 \\
\hline 2.513 & 5 & $23 I$ & 35.70 \\
\hline 2.490 & 15 & $\overline{2} 41, \bar{I} 32$ & 36.04 \\
\hline 2.433 & 27 & $\overline{3} 31, \overline{3} 12$ & 36.91 \\
\hline 2.412 & 11 & 122 & 37.25 \\
\hline 2.406 & 10 & 330 & 37.34 \\
\hline 2.302 & 16 & $\overline{3} 22$ & 39.10 \\
\hline 2.298 & 15 & $\overline{4} 0 \underline{1}, 051$ & 39.17 \\
\hline 2.261 & 7 & $\overline{4} I I$ & 39.84 \\
\hline 2.232 & 10 & 321 & 40.37 \\
\hline
\end{tabular}




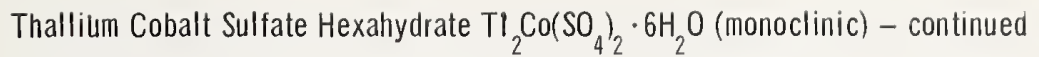

\begin{tabular}{|c|c|c|c|}
\hline \multicolumn{4}{|c|}{$\begin{array}{l}\text { Internal standard Ag, } a=4.08641 \AA \\
\text { CuK } \alpha_{1} \lambda=1.54056 \AA \text {; temp. } 25^{\circ} \mathrm{C}\end{array}$} \\
\hline$d(\AA)$ & $I$ & $h k l$ & $2 \theta\left({ }^{\circ}\right)$ \\
\hline 2.214 & 19 & 400,132 & 40.72 \\
\hline 2.170 & 8 & 212,250 & 41.58 \\
\hline 2.155 & 5 & 042,340 & 41.88 \\
\hline 2.136 & 5 & $\overline{2} 51$ & 42.27 \\
\hline 2.114 & 10 & 242 & 42.73 \\
\hline 2.086 & 5 & 420 & 43.34 \\
\hline 2.075 & 5 & 060 & 43.59 \\
\hline 2.053 & 5 & $\overline{4} 12$ & 44.07 \\
\hline 2.043 & 3 & $\overline{1} 13, \overline{2} 03$ & 44.29 \\
\hline 2.019 & 5 & $160, \overline{2} 13$ & 44.86 \\
\hline 1.991 & 7 & 003 & 45.52 \\
\hline 1.965 & 3 & $013, \overline{1} 23$ & 46.14 \\
\hline 1.954 & 5 & 251,430 & 46.43 \\
\hline 1.939 & 3 & $\overline{3} 42$ & 46.81 \\
\hline 1.912 & 10 & 052 & 47.52 \\
\hline 1.899 & 5 & $\overline{3} 13$ & 47.85 \\
\hline 1.886 & 3 & 411 & 48.21 \\
\hline 1.856 & 8 & $\overline{2} 61$ & 49.05 \\
\hline 1.853 & 8 & $\overline{1} 33$ & 49.13 \\
\hline 1.835 & 4 & $\overline{3} 23,312$ & 49.63 \\
\hline 1.804 & 7 & 440 & 50.54 \\
\hline 1.776 & 3 & 322 & 51.38 \\
\hline 1.771 & 5 & $\overline{5} 21$ & 51.56 \\
\hline 1.759 & 4 & 123 & 51.93 \\
\hline 1.743 & 4 & $\overline{3} 33,170$ & 52.44 \\
\hline 1.725 & 1 & $\overline{1} 62,351+$ & 53.03 \\
\hline 1.704 & 3 & 520,071 & 53.74 \\
\hline 1.692 & 5 & $332, \overline{5} 22$ & 54.16 \\
\hline 1.6772 & 4 & 133,043 & 54.68 \\
\hline 1. 6489 & 5 & 270,171 & 55.70 \\
\hline 1.6290 & 3 & 530 & 56.44 \\
\hline 1.6179 & 4 & $\overline{5} 32$ & 56.86 \\
\hline 1.5969 & 2 & $\overline{4} 52$ & 57.68 \\
\hline 1.5801 & 3 & $143, \overline{2} 53$ & 58.35 \\
\hline 1.5660 & 3 & 412 & 58.93 \\
\hline 1.5554 & 3 & 080,053 & 59.37 \\
\hline 1.5422 & 5 & $\overline{2} 14$ & 59.93 \\
\hline 1.5299 & 4 & $\overline{3} 71$ & 60.46 \\
\hline
\end{tabular}


Thallium Copper Sulfate Hexahydrate, $\mathrm{TI}_{2} \mathrm{Cu}\left(\mathrm{SO}_{4}\right)_{2} \cdot 6 \mathrm{H}_{2} \mathrm{O}$ (monoclinic)

Sample

The sample was prepared by slow evaporation at room temperature of an aqueous solution of $\mathrm{TI}_{2} \mathrm{SO}_{4}$ and $\mathrm{CuSO}_{4}$ in a $1: 8$ molar proportion. The first crystals formed were used.

\section{Color}

Unground: brilliant greenish blue

Ground: greenish white

\section{Optical data}

Biaxial, $N_{\alpha}=1.600, \quad N_{\beta}=1.610, \quad N_{\gamma}=1.620$ $2 \mathrm{~V}$ is very large.

\section{Structure}

Monoclinic, $\mathrm{P} 21 / \mathrm{a}(14), \mathrm{Z}=2$. Isostructural.with other "Tutton" salts [Tutton, 1928]. The structure of a "Tutton" salt, $\left(\mathrm{NH}_{4}\right)_{2} \mathrm{Mg}\left(\mathrm{SO}_{4}\right)_{2} \cdot 6 \mathrm{H}_{2} \mathrm{O}$ was determined by Margulis and Templeton, [1962] .

\section{Lattice constants}

\begin{tabular}{|l|c|c|c|c|}
\hline & $a(\AA)$ & $b(\AA)$ & $c(\AA)$ & $\beta\left({ }^{\circ}\right)$ \\
\cline { 2 - 5 } & & & & \\
NBS, & & & \\
sample $25^{\circ} \mathrm{C}$ & 9.268 & 12.364 & $\begin{array}{r}6.216 \\
\pm .001\end{array}$ & $\pm 05^{\circ} 33.3^{\prime}$ \\
& \pm .001 & \pm .001 & $\pm .5^{\prime}$ \\
\hline
\end{tabular}

\section{Density}

(calculated) $3.740 \mathrm{~g} / \mathrm{cm}^{3}$ at $25^{\circ} \mathrm{C}$.

\section{Reference intensity}

$\mathrm{I} / \mathrm{I}_{\text {corundum }}=4.5$

\begin{tabular}{|c|c|c|c|}
\hline \multicolumn{4}{|c|}{$\begin{array}{l}\text { Internal standard } \mathrm{Ag}, \mathrm{a}=4.08641 \AA \\
\mathrm{CuK} \alpha_{1} \quad \lambda=1.54056 \AA \text {; temp. } 25^{\circ} \mathrm{C}\end{array}$} \\
\hline$d(\AA)$ & $I$ & $h k l$ & $2 \theta\left({ }^{\circ}\right)$ \\
\hline 7.22 & 26 & 110 & 12.24 \\
\hline 5.98 & 13 & 001 & 14.79 \\
\hline 5.394 & 20 & 011 & 16.42 \\
\hline 5.084 & 22 & 120 & 17.43 \\
\hline 4.467 & 4 & 200 & 19.86 \\
\hline 4.302 & 10 & 021 & 20.63 \\
\hline 4.197 & 100 & $111,210,+$ & 21.15 \\
\hline 4.153 & 70 & $\overline{2} 01$ & 21.38 \\
\hline 3.940 & 13 & $\overline{2} 11$ & 22.55 \\
\hline 3.742 & 64 & 130 & 23.76 \\
\hline 3.623 & 3 & 220 & 24.55 \\
\hline 3.450 & 1 & $\overline{2} 21$ & 25.80 \\
\hline 3.399 & 2 & 031 & 26.20 \\
\hline 3.350 & 8 & $\overline{1} 31$ & 26.59 \\
\hline 3.195 & 28 & 201 & 27.90 \\
\hline 3.091 & 38 & 040,211 & 28.86 \\
\hline 3.024 & 36 & 230,131 & 29.51 \\
\hline 3.008 & 37 & Ī12 & 29.67 \\
\hline 2.920 & 22 & $140, \overline{3} 11$ & 30.59 \\
\hline 2.910 & 19 & 012 & 30.70 \\
\hline 2.894 & 13 & 310 & 30.87 \\
\hline 2.868 & 26 & $\overline{2} 02$ & 31.16 \\
\hline 2.837 & 11 & 221 & 31.51 \\
\hline 2.795 & 3 & $\overline{2} 12$ & 32.00 \\
\hline 2.773 & 16 & $\overline{1} 22$ & 32.25 \\
\hline 2.746 & 7 & 041 & 32.58 \\
\hline 2.721 & 9 & 141 & 32.89 \\
\hline 2.682 & 10 & 320 & 33.38 \\
\hline 2.603 & 4 & $\overline{2} 22$ & 34.43 \\
\hline 2.523 & 4 & 231 & 35.55 \\
\hline 2.477 & 18 & $\overline{2} 41, \overline{1} 32$ & 36.23 \\
\hline 2.428 & 37 & $\overline{3} 31$ & 36.99 \\
\hline 2.421 & 35 & $032, \overline{3} 12$ & 37.11 \\
\hline 2.412 & 15 & 330 & 37.24 \\
\hline 2.375 & 2 & 311 & 37.85 \\
\hline 2.304 & 7 & $\overline{4} 01$ & 39.06 \\
\hline 2.291 & 16 & $\overline{3} 22$ & 39.29 \\
\hline 2.286 & 17 & 051 & 39.39 \\
\hline 2.265 & 8 & $\overline{4} 11$ & 39.77 \\
\hline 2.254 & 11 & 321 & 39.96 \\
\hline
\end{tabular}


Thallium Copper Sulfate Hexahydrate, $\mathrm{TI}_{2} \mathrm{Cu}\left(\mathrm{SO}_{4}\right)_{2} \cdot 6 \mathrm{H}_{2} \mathrm{O}$ (monoclinic) - continued

\begin{tabular}{|c|c|c|c|}
\hline \multicolumn{4}{|c|}{$\begin{array}{l}\text { Internal standard } \mathrm{Ag}, \mathrm{a}=4.08641 \AA \\
\mathrm{CuK} a_{1} \lambda=1.54056 \AA \text {; temp. } 25^{\circ} \mathrm{C}\end{array}$} \\
\hline$d(\AA)$ & $I$ & $h k l$ & $2 \theta\left({ }^{\circ}\right)$ \\
\hline 2.232 & 8 & 400 & 40.38 \\
\hline 2.221 & 23 & 241,132 & 40.58 \\
\hline 2.191 & 2 & $212, \bar{I} 42$ & 41.17 \\
\hline 2.162 & 9 & 250,151 & 41.74 \\
\hline 2.154 & 5 & $\overline{3} 41$ & 41.91 \\
\hline 2.124 & 8 & $\overline{2} 51$ & 42.53 \\
\hline 2.117 & 6 & $\overline{3} 32$ & 42.67 \\
\hline 2.102 & 16 & $\overline{2} 42$ & 43.00 \\
\hline 2.061 & 4 & 060 & 43.89 \\
\hline 2.047 & 6 & $\overline{4} 12$ & 44.20 \\
\hline 2.041 & 6 & $\overline{1} 13$ & 44.34 \\
\hline 2.009 & 6 & $\overline{4} 31, \overline{2} 13,+$ & 45.08 \\
\hline 2.005 & 6 & 142 & 45.19 \\
\hline 1.996 & 10 & 003 & 45.40 \\
\hline 1.964 & 2 & $\overline{1} 23,430$ & 46.19 \\
\hline 1.959 & 4 & 232 & 46.32 \\
\hline 1.939 & 3 & $\bar{I} 6 \underline{1}$ & 46.81 \\
\hline 1.928 & 4 & $401, \overline{3} 42$ & 47.10 \\
\hline 1.907 & 12 & 052,411 & 47.64 \\
\hline 1.890 & 5 & $\overline{3} 13$ & 48.11 \\
\hline 1.850 & 10 & I $3 \underline{3}$ & 49.21 \\
\hline 1.846 & 9 & $\overline{4} 41, \overline{2} 61$ & 49.33 \\
\hline 1.827 & 5 & $\overline{3} 23, \overline{2} 33$ & 49.86 \\
\hline 1.809 & 10 & 440 & 50.40 \\
\hline 1.794 & 2 & 322 & 50.86 \\
\hline 1.775 & 6 & $\overline{5} 21$ & 51.45 \\
\hline 1.769 & 10 & 123 & 51.64 \\
\hline 1.766 & 6 & 510 & 51.71 \\
\hline 1.747 & 2 & $431, \overline{3} 52$ & 52.33 \\
\hline 1.737 & 4 & $\overline{4} 03, \overline{5} 12$ & 52.65 \\
\hline 1.733 & 5 & 170 & 52.79 \\
\hline 1.715 & 4 & $\overline{1} 62,520$ & 53.38 \\
\hline 1.705 & 7 & 332 & 53.70 \\
\hline 1.687 & 4 & $\overline{1} 71, \overline{5} 22$ & 54.34 \\
\hline 1.684 & 6 & $\overline{4} 51,133$ & 54.43 \\
\hline 1.674 & 4 & $\overline{2} 62, \overline{4} 23$ & 54.80 \\
\hline 1.649 & 2 & 213 & 55.71 \\
\hline 1.644 & 4 & 270 & 55.89 \\
\hline 1.641 & 6 & 171 & 56.00 \\
\hline 1.638 & 6 & 530 & 56.10 \\
\hline
\end{tabular}

\begin{tabular}{|c|c|c|c|}
\hline \multicolumn{4}{|c|}{$\begin{array}{l}\text { Internal standard } \mathrm{Ag}, \mathrm{a}=4.08641 \AA \\
\mathrm{CuKa_{1 }} \lambda=1.54056 \AA \text { 的 temp. } 25^{\circ} \mathrm{C}\end{array}$} \\
\hline$d(\AA)$ & $I$ & $h k l$ & $2 \theta\left({ }^{\circ}\right)$ \\
\hline 1.626 & $\overrightarrow{2}$ & $\overline{3} 43, \overline{2} 71$ & 56.55 \\
\hline 1.614 & 4 & $\overline{5} 32$ & 57.01 \\
\hline 1.590 & 3 & $\overline{4} 52, \overline{5} 41$ & 57.95 \\
\hline 1.582 & 4 & $412, \overline{3} 62$ & 58.27 \\
\hline 1.5725 & 4 & $\overline{2} 53$ & 58.66 \\
\hline 1.5502 & 2 & $\overline{2} 04$ & 59.59 \\
\hline 1.5455 & 3 & $271,080,+$ & 59.79 \\
\hline 1.5378 & 4 & $\overline{2} 14$ & 60.12 \\
\hline 1.5338 & 6 & $\bar{I} 72, \bar{I} 14$ & 60.29 \\
\hline 1.5229 & 5 & $180, \overline{3} 71$ & 60.77 \\
\hline 1.5143 & 6 & $\overline{4} 43,460$ & 61.15 \\
\hline 1.4995 & 5 & $\overline{1} 24, \overline{3} 14$ & 61.82 \\
\hline 1.4889 & 4 & 432,600 & 62.31 \\
\hline 1.4732 & 2 & 313 & 63.05 \\
\hline 1.4538 & 2 & $\overline{5} 33$ & 63.99 \\
\hline 1.4344 & 4 & $\overline{4} 04,063$ & 64.96 \\
\hline 1.4264 & 2 & 371 & 65.37 \\
\hline 1.4179 & 3 & $442, \overline{3} 34$ & 65.81 \\
\hline 1.4067 & 1 & 034,114 & 66.40 \\
\hline
\end{tabular}

\section{References}

Margulis, T.N. and D. H. Templeton (1962). Crystal structure and hydrogen bonding of magnesium ammonium sulfate hexahydrate, Z. Krist. 117, 334-357.

Tutton, A. E. (1928). The hexahydrated double sulphates containing thallium, Proc. Roy. Soc. London, Ser. A 118, 367-392. 


\section{Sample}

The sample was prepared by slowly evaporating a 1:8 mixture of molar solutions of $\mathrm{Tl}_{2} \mathrm{SO}_{4}$ and $\mathrm{MgSO}_{4}$, and using the first crystals formed.

\section{Color \\ Colorless}

\section{Optical data}

Biaxial, $N_{\alpha}=1.570, N_{\beta}=1.588, \quad N_{\gamma}=1.595$, $2 \mathrm{~V}$ is very large.

\section{Structure}

Monoclinic, $\mathrm{P} 21 / \mathrm{a}(14), \mathrm{Z}=2$, isostructural with other "Tutton Salts" [Tutton, 1928]. The structure of a Tutton salt, $\left(\mathrm{NH}_{4}\right)_{2} \mathrm{Mg}\left(\mathrm{SO}_{4}\right)_{2} \cdot 6 \mathrm{H}_{2} \mathrm{O}$, was determined by Margulis and Templeton [1962].

\section{Lattice constants}

\begin{tabular}{|c|c|c|c|c|}
\hline \multirow{3}{*}{$\begin{array}{l}\text { Hoffman } \\
\text { [1932] } \\
\text { NBS, } \\
\text { sample } \\
\text { at } 25^{\circ} \mathrm{C}\end{array}$} & $a(\AA)$ & $b(\AA)$ & $c(\AA)$ & $\beta\left({ }^{\circ}\right)$ \\
\hline & 9.24 & 12.44 & 6.197 & $106^{\circ} 30^{\prime}$ \\
\hline & $\begin{array}{l}9.273 \\
\pm .001\end{array}$ & $\begin{array}{r}12.472 \\
\pm .002\end{array}$ & $\begin{array}{l}6.214 \\
\pm .001\end{array}$ & $\begin{array}{rr}106^{\circ} & 23^{\prime} \\
\pm 1\end{array}$ \\
\hline
\end{tabular}

\section{Density}

(calculated) $3.532 \mathrm{~g} / \mathrm{cm}^{3}$ at $25^{\circ} \mathrm{C}$.

\section{Reference intensity}

$$
I_{\text {corundum }}=2.6
$$

\begin{tabular}{|c|c|c|c|}
\hline \multicolumn{4}{|c|}{$\begin{array}{l}\text { Internal standard Ag, } a=4.08641 \AA \\
\text { CuK } a_{1} \lambda=1.54056 \AA \text {; temp. } 25{ }^{\circ} \mathrm{C}\end{array}$} \\
\hline$d(\AA)$ & $I$ & $h k l$ & $2 \theta\left({ }^{\circ}\right)$ \\
\hline 7.24 & 50 & 110 & 12.22 \\
\hline 6.24 & 2 & 020 & 14.19 \\
\hline 5.95 & 19 & 001 & 14.87 \\
\hline 5.37 & 19 & 011 & 16.48 \\
\hline 5.10 & 33 & 120 & 17.37 \\
\hline 4.44 & 2 & 200 & 19.97 \\
\hline 4.30 & 8 & 021 & 20.62 \\
\hline 4.227 & 53 & $\overline{1} 21$ & 21.00 \\
\hline 4.162 & 100 & 111 & 21.33 \\
\hline 3.957 & 8 & $\overline{2} 11$ & 22.45 \\
\hline 3.764 & 86 & 130 & 23.62 \\
\hline 3.467 & 2 & $\overline{2} 21$ & 25.67 \\
\hline 3.369 & 6 & $\overline{1} 31$ & 26.43 \\
\hline 3.164 & 25 & 201 & 28.18 \\
\hline 3.117 & 40 & 040 & 28.61 \\
\hline 3.065 & 21 & 211 & 29.11 \\
\hline 3.025 & 47 & 230,131 & 29.50 \\
\hline 3.010 & 39 & I112 & 29.65 \\
\hline 2.982 & 5 & 002 & 29.94 \\
\hline 2.931 & 26 & $\overline{3} 11$ & 30.47 \\
\hline 2.897 & 21 & 012 & 30.84 \\
\hline 2.882 & 35 & $\overline{2} 02,310$ & 31.00 \\
\hline 2.820 & 8 & 221 & 31.70 \\
\hline 2.808 & 7 & $\overline{2} 12$ & 31.84 \\
\hline 2.777 & 18 & $\overline{1} 22$ & 32.21 \\
\hline 2.742 & 11 & $\overline{1}_{41}$ & 32.63 \\
\hline 2.678 & 13 & 320 & 33.43 \\
\hline 2.614 & 1 & $\overline{2} 22$ & 34.27 \\
\hline 2.555 & 4 & 112,240 & 35.09 \\
\hline 2.517 & 7 & 231 & 35.64 \\
\hline 2.499 & 21 & $\overline{2} 41$ & 35.91 \\
\hline 2.488 & 20 & Iิ32 & 36.07 \\
\hline 2.442 & 35 & $\overline{3} 31$ & 36.77 \\
\hline 2.412 & 18 & 330,122 & 37.25 \\
\hline 2.302 & 24 & 051 & 39.10 \\
\hline 2.270 & 10 & $\overline{4} 11$ & 39.68 \\
\hline 2.239 & 11 & 321 & 40.24 \\
\hline 2.222 & 25 & 241 & 40.57 \\
\hline 2.205 & 9 & 202 & 40.86 \\
\hline 2.171 & 9 & 212,151 & 41.57 \\
\hline 2.155 & 3 & 042 & 41.88 \\
\hline
\end{tabular}


Thallium Magnesium Sulfate Hexahydrate, $\left.\mathrm{Tl}_{2} \mathrm{Mg}_{\left(\mathrm{SO}_{4}\right.}\right)_{2} \cdot 6 \mathrm{H}_{2} \mathrm{O}$ (monoclinic) - continued

\begin{tabular}{|c|c|c|c|}
\hline \multicolumn{4}{|c|}{$\begin{array}{l}\text { Internal standard } \mathrm{Ag}, \mathrm{a}=4.08641 \AA \\
\mathrm{CuK} a_{1} \quad \lambda=1.54056 \AA \text {; temp. } 25{ }^{\circ} \mathrm{C}\end{array}$} \\
\hline$d(\AA)$ & $I$ & $h k l$ & $2 \theta\left({ }^{\circ}\right)$ \\
\hline 2.141 & 8 & $\overline{2} 51$ & 42.18 \\
\hline 2.116 & 10 & $\overline{2} 42$ & 42.70 \\
\hline 2.096 & 3 & 420 & 43.12 \\
\hline 2.079 & 2 & 222,060 & 43.50 \\
\hline 2.058 & 5 & $\overline{4} 12$ & 43.97 \\
\hline 2.039 & 3 & I13 & 44.39 \\
\hline 2.024 & 3 & 160 & 44.75 \\
\hline 2.018 & 4 & $\overline{4} 31$ & 44.89 \\
\hline 1.987 & 6 & 003 & 45.62 \\
\hline 1.960 & 7 & 251,430 & 46.29 \\
\hline 1.955 & 6 & $\overline{1} 61$ & 46.40 \\
\hline 1.942 & 3 & $\overline{3} 42$ & 46.74 \\
\hline 1.913 & 9 & 401,052 & 47.48 \\
\hline 1.898 & 4 & $\overline{3} 13$ & 47.90 \\
\hline 1.862 & 4 & $\overline{2} 61$ & 48.88 \\
\hline 1.851 & 5 & $\overline{1} 33$ & 49.17 \\
\hline 1.835 & 5 & $\overline{3} 23$ & 49.63 \\
\hline 1.811 & 8 & 440 & 50.35 \\
\hline 1.801 & 2 & 242 & 50.64 \\
\hline 1.778 & 5 & $\overline{5} 21$ & 51.35 \\
\hline 1.760 & 5 & $\overline{3} 52$ & 51.91 \\
\hline 1.757 & 5 & 123 & 51.99 \\
\hline 1.746 & 7 & $\overline{4} 03,170$ & 52.35 \\
\hline 1.724 & 1 & I 62 & 53.00 \\
\hline 1.711 & 1 & $\overline{5} 20, \overline{3} 61$ & 53.52 \\
\hline 1.695 & 5 & 332,451 & 54.07 \\
\hline 1.676 & 3 & 133,043 & 54.72 \\
\hline 1.651 & 6 & 252,171 & 55.61 \\
\hline 1.635 & 4 & 213,343 & 56.20 \\
\hline 1.622 & 3 & $\overline{5} 32$ & 56.72 \\
\hline
\end{tabular}

\section{References}

Hoffman,W. (1931). Die Struktur der Tuttonschen Salze, Z. Krist, 78, 279-333.

Margulis, T.N. and D。H. Templeton (1962). Crystal structure and hydrogen bonding of magnesium and ammonium suflate hexahydrate, Z. Krist, 117, 344-357.

Tutton, A.E. (1928). The hexahydrated double sulphates containing thallium, Proc.

Roy. Soc. London Ser. A 118 367-392。 
Thallium Manganese Sulfate, $\mathrm{Tl}_{2} \mathrm{Mn}_{2}\left(\mathrm{SO}_{4}\right)_{3}$ (cubic)

Sample

The $\mathrm{Tl}_{2} \mathrm{Mn}\left(\mathrm{SO}_{4}\right)_{3}$ was crystallized by evaporation at $90^{\circ} \mathrm{C}$ from a stoichiometric aqueous solution of $\mathrm{Tl}_{2} \mathrm{SO}_{4}$ and $\mathrm{MnSO}_{4}$.

Major impurities

$0.01-0.1 \%$ each: $\mathrm{Al}, \mathrm{Cu}$, and $\mathrm{K}$

$0.1-1.0 \%$ each: Mg

\section{Color}

Colorless

\section{Optical data}

Isotropic, $\mathrm{N}=1.722$

\section{Structure}

Cubic, $\mathrm{P} 2_{1} / 3$ (198), $\mathrm{z}=4$, langbeinite type, structure of langbeinite, $\mathrm{K}_{2} \mathrm{Mg}_{2}\left(\mathrm{SO}_{4}\right)_{3}$, determined by Zemann and Zemann [1957].

\section{Lattice constants}

\begin{tabular}{|l|c|}
\hline & $a(\AA)$ \\
\cline { 2 - 2 } Zemann and Zemann [1957]------- & 10.223 \\
Gattow and Zemann [1958]-------- & \pm .006 \\
& 10.229 \\
NBS, sample at $25{ }^{\circ} \mathrm{C}----0.004$ \\
& 10.2236 \\
& \pm .0002 \\
\hline
\end{tabular}

Density

(calculated) $5.015 \mathrm{~g} / \mathrm{cm}^{3}$ at $25^{\circ} \mathrm{C}$.

\section{Reference intensity}

$$
I / I_{\text {corundum }}=4.3
$$

\begin{tabular}{|c|c|c|c|}
\hline \multicolumn{4}{|c|}{$\begin{array}{l}\text { Internal standard } \mathrm{W}, \mathrm{a}=3.16516 \AA \\
\mathrm{CuK} \alpha_{1} \quad \lambda=1.54056 \AA \text {; temp. } 25{ }^{\circ} \mathrm{C}\end{array}$} \\
\hline$d(\AA)$ & $I$ & $h k l$ & $2 \theta\left({ }^{\circ}\right)$ \\
\hline 5.90 & 27 & 111 & 15.01 \\
\hline 5.110 & 2 & 200 & 17.34 \\
\hline 4.574 & 12 & 210 & 19.39 \\
\hline 4.172 & 18 & 211 & 21.28 \\
\hline 3.613 & 22 & 220 & 24.62 \\
\hline 3.407 & 12 & 221 & 26.13 \\
\hline 3.232 & 100 & 310 & 27.58 \\
\hline 3.082 & 18 & 311 & 28.95 \\
\hline 2.951 & 1 & 222 & 30.26 \\
\hline 2.834 & 22 & 320 & 31.54 \\
\hline 2.731 & 58 & 321 & 32.76 \\
\hline 2.479 & 24 & 410 & 36.20 \\
\hline 2.409 & 1 & 411 & 37.29 \\
\hline 2.356 & 7 & 331 & 38.34 \\
\hline 2.286 & 2 & 420 & 39.38 \\
\hline 2.231 & 8 & 421 & 40.39 \\
\hline 2.180 & 8 & 332 & 41.39 \\
\hline 2.087 & 17 & 422 & 43.31 \\
\hline 2.045 & 3 & 430 & 44.26 \\
\hline 2.005 & 33 & 510 & 45.18 \\
\hline 1.968 & 2 & 511 & 46.09 \\
\hline 1.899 & 9 & 520 & 47.87 \\
\hline 1.867 & 3 & 521 & 48.74 \\
\hline 1.807 & $I$ & 440 & 50.46 \\
\hline 1.779 & 4 & 522 & 51.31 \\
\hline 1.754 & 1 & 530 & 52.10 \\
\hline 1.729 & 1 & 531 & 52.92 \\
\hline 1.704 & 1 & 600 & 53.76 \\
\hline 1.681 & 4 & 610 & 54.56 \\
\hline 1.659 & 17 & 611 & 55.34 \\
\hline 1.617 & 5 & 620 & 56.91 \\
\hline 1.596 & 12 & 621 & 57.70 \\
\hline 1.577 & 9 & 541 & 58.46 \\
\hline 1.559 & 3 & 533 & 59.21 \\
\hline 1.541 & $<1$ & 622 & 59.96 \\
\hline 1.524 & 7 & 630 & 60.73 \\
\hline 1.507 & 5 & 631 & 61.46 \\
\hline 1.476 & 4 & 444 & 62.91 \\
\hline 1.461 & 2 & 632 & 63.63 \\
\hline 1.446 & 2 & 550 & 64.37 \\
\hline
\end{tabular}




\begin{tabular}{|c|c|c|c|}
\hline \multicolumn{4}{|c|}{$\begin{array}{l}\text { Internal standard } \mathrm{W}, \mathrm{a}=3.16516 \AA \\
\mathrm{CuK} a_{1} \lambda=1.54056 \AA \text {; temp. } 25^{\circ} \mathrm{C}\end{array}$} \\
\hline$d(\AA)$ & $I$ & $h k l$ & $2 \theta\left({ }^{\circ}\right)$ \\
\hline 1.432 & 2 & 711 & 65.07 \\
\hline 1.4179 & 1 & 640 & 65.81 \\
\hline 1.4045 & 2 & 720 & 66.52 \\
\hline 1.3912 & 7 & 721 & 67.24 \\
\hline 1.3664 & 2 & 642 & 68.63 \\
\hline 1.3541 & 1 & 722 & 69.34 \\
\hline 1.3428 & 3 & 730 & 70.01 \\
\hline 1.3310 & 3 & 731 & 70.72 \\
\hline 1.3092 & 2 & 650 & 72.08 \\
\hline 1.2983 & 3 & 732 & 72.78 \\
\hline 1.2783 & 1 & 800 & 74.11 \\
\hline 1.2682 & 3 & 810 & 74.80 \\
\hline 1.2586 & 1 & 811 & 75.47 \\
\hline 1.2493 & 2 & 733 & 76.13 \\
\hline 1.2401 & 1 & 820 & 76.80 \\
\hline 1.2308 & 4 & 821 & 77.49 \\
\hline 1.2223 & 2 & 653 & 78.13 \\
\hline 1.2050 & 2 & 822 & 79.47 \\
\hline 1.1966 & 1 & 830 & 80.14 \\
\hline 1.1885 & 5 & 831 & 80.80 \\
\hline 1.1809 & 1 & 751 & 81.43 \\
\hline 1.1729 & 1 & 662 & 82.10 \\
\hline 1.1652 & 1 & 832 & 82.76 \\
\hline 1.1576 & 2 & 752 & 83.43 \\
\hline 1.1359 & 1 & 841 & 85.39 \\
\hline 1.1290 & 1 & 910 & 86.04 \\
\hline 1.1221 & 2 & 911 & 86.70 \\
\hline 1.1158 & 1 & 842 & 87.31 \\
\hline 1.1089 & 1 & 920 & 88.00 \\
\hline 1.1025 & 2 & 921 & 88.64 \\
\hline 1.0898 & 1 & 664 & 89.95 \\
\hline 1.0837 & 3 & 922 & 90.60 \\
\hline 1.0776 & 2 & 930 & 91.25 \\
\hline 1.0717 & $<1$ & 931 & 91.90 \\
\hline 1.0601 & 1 & 852 & 93.21 \\
\hline 1.0545 & 2 & 932 & 93.85 \\
\hline 1.0381 & 1 & 940 & 95.80 \\
\hline 1.0326 & 1 & 941 & 96.48 \\
\hline 1.0275 & 1 & 933 & 97.12 \\
\hline 1.0223 & $<1$ & $10 \cdot 0 \cdot 0$ & 97.78 \\
\hline
\end{tabular}

\begin{tabular}{|c|c|c|c|}
\hline \multicolumn{4}{|c|}{$\begin{array}{l}\text { Internal standard } \mathrm{W}, \mathrm{a}=3.16516 \AA \\
\mathrm{CuK} a_{1} \quad \lambda=1.54056 \AA \text {; temp. } 25^{\circ} \mathrm{C}\end{array}$} \\
\hline$d(\AA)$ & $I$ & $h k l$ & $2 \theta\left({ }^{\circ}\right)$ \\
\hline 1.0172 & 1 & $10 \cdot 1 \cdot 0$ & 98.45 \\
\hline 1.0122 & $<1$ & $10 \cdot 1 \cdot 1$ & 99.11 \\
\hline 1.0025 & 1 & $10 \cdot 2 \cdot 0$ & 100.42 \\
\hline 0.9976 & 1 & $10 \cdot 2 \cdot 1$ & 101.09 \\
\hline . 9930 & 1 & 950 & 101.74 \\
\hline .9884 & $<1$ & 951 & 102.40 \\
\hline .9838 & 1 & $10 \cdot 2 \cdot 2$ & 103.06 \\
\hline .9792 & 1 & $10 \cdot 3 \cdot 0$ & 103.74 \\
\hline .9747 & 1 & $10 \cdot 3 \cdot 1$ & 104.42 \\
\hline .9617 & 1 & $10 \cdot 3 \cdot 2$ & 106.44 \\
\hline .9576 & $<1$ & 871 & 107.10 \\
\hline .9535 & 1 & 953 & 107.77 \\
\hline .9493 & 1 & $10 \cdot 4 \cdot 0$ & 108.47 \\
\hline .9451 & 1 & 960 & 109.18 \\
\hline • 9411 & $<1$ & $10 \cdot 3 \cdot 3$ & 109.86 \\
\hline .9333 & 1 & $10 \cdot 4 \cdot 2$ & 111.25 \\
\hline . 9294 & $<1$ & 962 & 111.95 \\
\hline .9255 & 2 & $11 \cdot 1 \cdot 0$ & 112.67 \\
\hline .9219 & 1 & $11 \cdot 1 \cdot 1$ & 113.34 \\
\hline .9145 & 1 & $11 \cdot 2 \cdot 0$ & 114.77 \\
\hline .9108 & 2 & $11 \cdot 2 \cdot 1$ & 115.50 \\
\hline .9036 & $<1$ & 880 & 116.95 \\
\hline .9001 & 2 & $11 \cdot 2 \cdot 2$ & 117.69 \\
\hline .8932 & 1 & $11 \cdot 3 \cdot 1$ & 119.16 \\
\hline .8898 & 1 & $10 \cdot 4 \cdot 4$ & 119.91 \\
\hline .8864 & 1 & 964 & 120.69 \\
\hline .8831 & 2 & $11 \cdot 3 \cdot 2$ & 121.44 \\
\hline .8766 & 2 & $10 \cdot 6 \cdot 0$ & 122.98 \\
\hline .8735 & $<1$ & $11 \cdot 4 \cdot 0$ & 123.74 \\
\hline .8702 & 1 & $11 \cdot 4 \cdot 1$ & 124.54 \\
\hline .8671 & 1 & $11 \cdot 3 \cdot 3$ & 125.32 \\
\hline .8609 & 1 & $11 \cdot 4 \cdot 2$ & 126.94 \\
\hline .8580 & 1 & 965 & 127.74 \\
\hline .8520 & 1 & $12 \cdot 0 \cdot 0$ & 129.41 \\
\hline .8490 & 1 & $12 \cdot 1 \cdot 0$ & 130.26 \\
\hline
\end{tabular}

\section{References}

Gattow, G. and J. Zemann (1958). Über Doppelsulfate vom Langbeinit-Typ, $A_{2}^{+} B_{2}^{2+}-$ $\left(\mathrm{SO}_{4}\right)_{3}, \mathrm{Z}$. Anorg. Allgem. Chem. 293,233240 .

Zemann,A. and J. Zemann (1957). Die Kristallstruktur vom Langbeinit, $\mathrm{K}_{2} \mathrm{Mg}_{2}\left(\mathrm{SO}_{4}\right)_{3}$ Acta Cryst. 10, 409-413. 
Thallium Nickel Sulfate Hexahydrate, $\mathrm{TI}_{2} \mathrm{Ni}\left(\mathrm{SO}_{4}\right)_{2} \cdot 6 \mathrm{H}_{2} \mathrm{O}$ (monoclinic)

Sample

The sample was prepared by slowly evaporating an equimolar solution of $\mathrm{TI}_{2} \mathrm{SO}_{4}$ and $\mathrm{NiSO}_{4}$.

\section{Color}

Unground: strong bluish green

Ground: very pale green

\section{Optical data}

Biaxial (-) $\mathrm{N}_{\alpha}=1.602, \mathrm{~N}_{\beta}=1.615, \mathrm{~N}_{\gamma}=1.620$ $2 \mathrm{~V}$ is large

\section{Structure}

Monoclinic, $\mathrm{P} 21 / \mathrm{a}$ (14), $\mathrm{Z}=2$, isostructural with other "Tutton's salts" [Tutton, 1925]. The structure of a Tutton salt, $\left(\mathrm{NH}_{4}\right)_{2} \mathrm{Mg}\left(\mathrm{SO}_{4}\right)_{2} \cdot 6 \mathrm{H}_{2} \mathrm{O}$, was determined by Margulis and Templeton [1962].

\section{Lattice constants}

\begin{tabular}{|c|c|c|c|c|}
\hline & $a(\AA)$ & $b(\AA)$ & $c(\AA)$ & $\beta\left({ }^{\circ}\right)$ \\
\hline $\begin{array}{l}\text { NBS, } \\
\text { sample } \\
\text { at } 25{ }^{\circ} \mathrm{C}\end{array}$ & $\begin{array}{l}9.166 \\
\pm .001\end{array}$ & $\begin{array}{r}12.392 \\
\pm .002\end{array}$ & $\begin{array}{l}6.216 \\
\pm .001\end{array}$ & $\begin{array}{r}106^{\circ} 20^{\prime} \\
\pm 1^{\prime}\end{array}$ \\
\hline
\end{tabular}

Density

(calculated) $3.763 \mathrm{~g} / \mathrm{cm}^{3}$ at $25^{\circ} \mathrm{C}$.

\section{Reference intensity}

$\mathrm{I} / \mathrm{I}_{\text {corundum }}=3.8$

\section{References}

Margulis, T. N. and D.H. Templeton (1962). Crystal structure and hydrogen bonding of magnesium ammonium sulfate hexahydrate, Z. Krist. 117, 344-357.

Tutton, A.E.H. (1925). The monoclinic double sulfates containing thallium - thalIium nickel and thallium cobalt sulphates. Proc. Roy. Soc.London Ser.A 118, 240-26l.

\begin{tabular}{|c|c|c|c|}
\hline \multicolumn{4}{|c|}{$\begin{array}{l}\text { Internal standard Ag, } a=4.08641 \AA \\
\text { CuKa } a_{1} \quad \lambda=1.54056 \AA \text { A; temp. } 25^{\circ} \mathrm{C}\end{array}$} \\
\hline$d(\AA)$ & $I$ & $h k l$ & $2 \theta\left({ }^{\circ}\right)$ \\
\hline 7.18 & 18 & 110 & 12.31 \\
\hline 5.95 & 8 & 001 & 14.88 \\
\hline 5.37 & 13 & 011 & 16.49 \\
\hline 5.06 & 22 & 120 & 17.50 \\
\hline 4.39 & 2 & 200 & 20.20 \\
\hline 4.294 & 8 & 021 & 20.67 \\
\hline 4.209 & 30 & $\bar{I} 2 I$ & 21.09 \\
\hline 4.139 & 100 & $111, \overline{2} 01,+$ & 21.45 \\
\hline 3.926 & 5 & $\overline{2} I I$ & 22.63 \\
\hline 3.740 & 51 & 130 & 23.77 \\
\hline 3.587 & $I$ & 220,121 & 24.80 \\
\hline 3.396 & $I$ & 031 & 26.22 \\
\hline 3.353 & 6 & $\overline{1} 31$ & 26.56 \\
\hline 3.142 & 15 & 201 & 28.38 \\
\hline 3.097 & 22 & 040 & 28.80 \\
\hline 3.046 & 16 & $2 I I$ & 29.30 \\
\hline 3.007 & 47 & $131, \overline{1} 12,+$ & 29.68 \\
\hline 2.982 & 5 & 002 & 29.94 \\
\hline 2.923 & 6 & $\overline{2} 31,140$ & 30.56 \\
\hline 2.901 & 18 & $\overline{3} I 1,012$ & 30.80 \\
\hline 2.872 & 16 & $\overline{2} 02$ & 31.11 \\
\hline 2.853 & 11 & 310 & 31.33 \\
\hline 2.802 & 9 & 221 & 31.91 \\
\hline 2.774 & 10 & $\overline{1} 22$ & 32.24 \\
\hline 2.749 & 6 & 041 & 32.54 \\
\hline 2.727 & 7 & $\bar{I} 41$ & 32.81 \\
\hline 2.651 & 5 & 320 & 33.78 \\
\hline 2.607 & 2 & $\overline{2} 22$ & 34.37 \\
\hline 2.554 & 1 & 112 & 35.11 \\
\hline 2.534 & 2 & 240,141 & 35.40 \\
\hline 2.501 & 5 & 231 & 35.87 \\
\hline 2.480 & 16 & $\overline{1} 32, \overline{2} 41$ & 36.19 \\
\hline 2.421 & 24 & $\overline{3} 31, \overline{3} 12,+$ & 37.11 \\
\hline 2.406 & 12 & 122 & 37.35 \\
\hline 2.391 & 6 & 330 & 37.58 \\
\hline 2.290 & 16 & $\overline{3} 22,051$ & 39.31 \\
\hline 2.244 & 6 & $\overline{4} 11$ & 40.15 \\
\hline 2.221 & 8 & 321 & 40.59 \\
\hline 2.205 & 15 & 132,241 & 40.90 \\
\hline 2.199 & 14 & 400,202 & 41.00 \\
\hline 2.160 & 8 & 250,151 & 41.79 \\
\hline 2.148 & 6 & $\overline{3} 41,042$ & 42.02 \\
\hline 2.126 & 4 & $\overline{2} 51$ & 42.48 \\
\hline 2.106 & 8 & $\overline{2} 42$ & 42.91 \\
\hline 2.071 & 5 & $420,222,+$ & 43.66 \\
\hline
\end{tabular}


Thallium Nickel Sulfate Hexahydrate, $\mathrm{TI}_{2} \mathrm{Ni}\left(\mathrm{SO}_{4}\right)_{2} \cdot 6 \mathrm{H}_{2} \mathrm{O}$ (monoclinic) - continued

\begin{tabular}{|c|c|c|c|}
\hline \multicolumn{4}{|c|}{$\begin{array}{l}\text { Internal standard } \mathrm{Ag}, \mathrm{a}=4.08641 \AA \\
\mathrm{CuK} a_{1} \quad \lambda=1.54056 \AA{ }^{\circ} \text {; temp. } 25{ }^{\circ} \mathrm{C}\end{array}$} \\
\hline$d(\AA)$ & $I$ & $h k l$ & $2 \theta\left({ }^{\circ}\right)$ \\
\hline 2.065 & 5 & 060 & 43.80 \\
\hline 2.042 & 5 & $412, \overline{1} 13,+$ & 44.33 \\
\hline 2.012 & 4 & $\underline{2} 13,160$ & 45.02 \\
\hline 1.997 & 3 & $\overline{4} 31,142$ & 45.37 \\
\hline 1.988 & 5 & 003 & 45.60 \\
\hline 1.963 & 2 & $013, \overline{1} 23,+$ & 46.22 \\
\hline 1.945 & 5 & $25 \underline{1}, \overline{1} 61$ & 46.66 \\
\hline 1.929 & 2 & $\overline{3} 42$ & 47.07 \\
\hline 1.906 & 7 & $\overline{3} 51,052$ & 47.68 \\
\hline 1.893 & 4 & 023,350 & 48.02 \\
\hline 1.887 & 4 & $34 \underline{1}$ & 48.18 \\
\hline 1.875 & 2 & $411, \overline{2} 52$ & 48.50 \\
\hline 1.849 & 9 & $\overline{1} 33, \overline{2} 61$ & 49.24 \\
\hline 1.829 & 3 & $\overline{3} 23, \overline{2} 33$ & 49.81 \\
\hline 1.793 & 8 & $440,242,+$ & 50.89 \\
\hline 1.770 & 2 & 322 & 51.59 \\
\hline 1.757 & 5 & $\overline{5} 21,123$ & 52.00 \\
\hline 1.742 & 4 & 510 & 52.50 \\
\hline 1.737 & 5 & $\overline{3} 33,170$ & 52.66 \\
\hline 1.724 & 3 & 431 & 53.09 \\
\hline 1.720 & 3 & $\overline{1} 43, \overline{1} 62$ & 53.22 \\
\hline 1.692 & 3 & $520, \overline{1} 71$ & 54.15 \\
\hline 1.687 & 5 & 332 & 54.34 \\
\hline 1.680 & 4 & $\overline{5} 22, \overline{4} 51$ & 54.59 \\
\hline 1.673 & 5 & $\overline{4} 23,043,+$ & 54.82 \\
\hline 1.642 & 5 & 270,171 & 55.95 \\
\hline 1.618 & 2 & 530,441 & 56.84 \\
\hline 1.608 & 4 & $\overline{5} 32$ & 57.25 \\
\hline 1.589 & 2 & $\overline{4} 52, \overline{1} 53$ & 58.01 \\
\hline 1.576 & 3 & $143, \overline{2} 53$ & 58.53 \\
\hline 1.558 & 3 & 511,412 & 59.24 \\
\hline 1.551 & 3 & $\overline{2} 04,053$ & 59.57 \\
\hline 1.538 & 5 & $\overline{2} 14, \overline{1} 72$ & 60.10 \\
\hline 1.526 & 3 & $\overline{6} 01,180$ & 60.64 \\
\hline 1.523 & 4 & $\overline{3} 71,072,+$ & 60.78 \\
\hline 1.516 & 5 & $\overline{4} 43,370,+$ & 61.07 \\
\hline 1.505 & 4 & $\overline{5} 23,262,+$ & 61.56 \\
\hline
\end{tabular}


Thallium Zinc Sulfate Hexahydrate, $\mathrm{TI}_{2} \mathrm{Zn}\left(\mathrm{SO}_{4}\right)_{2} \cdot 6 \mathrm{H}_{2} \mathrm{O}$ (monoclinic)

Sample

The sample was prepared by slowly evaporating a $1: 8$ mixture of molar solutions of $\mathrm{Tl}_{2} \mathrm{SO}_{4}$ and $\mathrm{ZnSO}_{4}$, and using the first crystals formed.

Color

Colorless

\section{Optical data}

Biaxial (-) $N_{\alpha}=1.592, N_{\beta}=1.610, N_{\gamma}=1.615$ $2 \mathrm{~V}$ is large.

\section{Structure}

Monoclinic, $\mathrm{P} 21 / \mathrm{a}$ (14), $\mathrm{Z}=2$, isostructural with other "Tutton's salts" [Tutton, 1910]. The structure of a Tutton salt, $\left(\mathrm{NH}_{4}\right)_{2} \mathrm{Mg}\left(\mathrm{SO}_{4}\right)_{2} \cdot 6 \mathrm{H}_{2} \mathrm{O}$, was determined by Margulis and Templeton [1962].

\section{Lattice constants}

\begin{tabular}{|l|c|c|c|r|}
\hline & $a(\AA)$ & $b(\AA)$ & $c(\AA)$ & $\beta\left({ }^{\circ}\right)$ \\
\cline { 2 - 5 } $\begin{array}{l}\text { NBS, } \\
\text { sample } \\
\text { at } 25^{\circ} \mathrm{C}\end{array}$ & $\begin{array}{r}9.219 \\
\pm .001\end{array}$ & $\begin{array}{r}12.433 \\
\pm .002\end{array}$ & $\begin{array}{l}6.2317 \\
\pm .0005\end{array}$ & $106^{\circ} 17.6$ \\
& & \pm .4 \\
\hline
\end{tabular}

\section{Density}

(calculated) $3.763 \mathrm{~g} / \mathrm{cm}^{3}$ at $25^{\circ} \mathrm{C}$.

\section{Reference intensity}

$$
I_{\text {corundum }}=3.8
$$

\section{References}

Margulis, T.N. and D.H. Templeton (1962). Crystal structure and hydrogen bonding of magnesium and ammonium sulfate hexahydrate, Z. Krist, 117, 344-357.

Tutton, A. E. H. (1910). The relation of thallium to the alkali metals: a study of thallium-zinc sulphate and selenate, Proc. Roy. Soc. London Ser.A 83 221-226.

\begin{tabular}{|c|c|c|c|}
\hline \multicolumn{4}{|c|}{$\begin{array}{l}\text { Internal standard } \mathrm{Ag}, \mathrm{a}=4.08641 \AA \\
\mathrm{CuK} a_{1} \quad \lambda=1.54056 \AA \text {; temp. } 25^{\circ} \mathrm{C}\end{array}$} \\
\hline$d(\stackrel{\circ}{)})$ & $I$ & $h k l$ & $2 \theta\left({ }^{\circ}\right)$ \\
\hline 7.20 & 22 & 110 & 12.28 \\
\hline 5.98 & 8 & 001 & 14.79 \\
\hline 5.39 & 15 & 011 & 16.42 \\
\hline 5.09 & 23 & 120 & 17.40 \\
\hline 4.421 & 3 & 200 & 20.07 \\
\hline 4.310 & 10 & 021 & 20.59 \\
\hline 4.229 & 32 & $\overline{1} 21$ & 20.99 \\
\hline 4.164 & 100 & $210,111,+$ & 21.32 \\
\hline 3.943 & 5 & $\overline{2} 11$ & 22.53 \\
\hline 3.751 & 52 & 130 & 23.70 \\
\hline 3.606 & 2 & 220,121 & 24.67 \\
\hline 3.363 & 6 & $\overline{1} 31$ & 26.48 \\
\hline 3.161 & 14 & 201 & 28.21 \\
\hline 3.109 & 17 & 040 & 28.69 \\
\hline 3.062 & 14 & 211 & 29.14 \\
\hline 3.018 & 40 & $131, \overline{1} 12$ & 29.57 \\
\hline 2.991 & 3 & 002 & 29.85 \\
\hline 2.932 & 6 & $\overline{2} 31,140$ & 30.46 \\
\hline 2.916 & 10 & $\overline{3} 11$ & 30.63 \\
\hline 2.909 & 15 & 012 & 30.71 \\
\hline 2.880 & 16 & $\overline{2} 02$ & 31.03 \\
\hline 2.872 & 15 & 310 & 31.12 \\
\hline 2.816 & 8 & 221 & 31.75 \\
\hline 2.809 & 6 & $\overline{2} 12$ & 31.83 \\
\hline 2.782 & 9 & $\overline{1} 22$ & 32.15 \\
\hline 2.758 & 6 & 041 & 32.44 \\
\hline 2.737 & 6 & 141 & 32.69 \\
\hline 2.666 & 4 & 320 & 33.59 \\
\hline 2.614 & 2 & $\overline{2} 22$ & 34.27 \\
\hline 2.543 & 2 & 240,141 & 35.26 \\
\hline 2.512 & 4 & 231 & 35.71 \\
\hline 2.488 & 12 & $\overline{2} 41, \overline{1} 32$ & 36.07 \\
\hline 2.429 & 24 & $\overline{3} 31, \overline{3} 12$ & 36.98 \\
\hline 2.424 & 15 & 032 & 37.06 \\
\hline 2.414 & 10 & 122 & 37.22 \\
\hline 2.404 & 8 & 330 & 37.38 \\
\hline 2.300 & 14 & $\overline{3} 22$ & 39.13 \\
\hline 2.296 & 16 & $051, \overline{4} 01$ & 39.20 \\
\hline 2.256 & 6 & $\overline{4} 11$ & 39.92 \\
\hline 2.233 & 8 & 321 & 40.35 \\
\hline
\end{tabular}




\begin{tabular}{|c|c|c|c|}
\hline \multicolumn{4}{|c|}{$\begin{array}{l}\text { Internal standard } \mathrm{Ag}, \mathrm{a}=4.08641 \AA \\
\mathrm{CuK} a_{1} \lambda=1.54056 \AA \text { 的 }\end{array}$} \\
\hline$d(\AA)$ & $I$ & $h k l$ & $2 \theta\left({ }^{\circ}\right)$ \\
\hline 2.215 & 18 & 241,132 & 40.70 \\
\hline 2.208 & 14 & 202,400 & 40.83 \\
\hline 2.179 & 2 & 410 & 41.41 \\
\hline 2.173 & 4 & 212 & 41.53 \\
\hline 2.168 & 7 & 250,151 & 41.62 \\
\hline 2.155 & 4 & 042 & 41.88 \\
\hline 2.133 & 4 & $\overline{2} 51$ & 42.33 \\
\hline 2.112 & 8 & $\overline{2} 42$ & 42.77 \\
\hline 2.084 & 4 & 420 & 43.39 \\
\hline 2.079 & 4 & $222, \overline{4} 02$ & 43.49 \\
\hline 2.072 & 3 & 060,331 & 43.64 \\
\hline 2.050 & 4 & $\overline{4} 12$ & 44.13 \\
\hline 2.046 & 4 & $\overline{1} 13, \overline{2} 03$ & 44.23 \\
\hline 2.018 & 4 & $\overline{2} 13,160$ & 44.88 \\
\hline 2.007 & 3 & $\overline{4} 31$ & 45.14 \\
\hline 2.003 & 4 & 142 & 45.23 \\
\hline 1.994 & 5 & 003 & 45.46 \\
\hline 1.967 & 2 & $\overline{1}_{23}$ & 46.12 \\
\hline 1.954 & 4 & 251 & 46.43 \\
\hline 1.950 & 4 & $430, \overline{1} 61$ & 46.53 \\
\hline 1.937 & 2 & $\overline{3} 42$ & 46.87 \\
\hline 1.912 & 6 & 052 & 47.52 \\
\hline 1.908 & 5 & 401 & 47.62 \\
\hline 1.897 & 4 & $341, \overline{3} 13,+$ & 47.92 \\
\hline 1.885 & 2 & 411 & 48.23 \\
\hline 1.854 & 8 & $\overline{2} 61, \overline{1} 33$ & 49.09 \\
\hline 1.835 & 3 & $312, \overline{3} 23,+$ & 49.65 \\
\hline 1.802 & 6 & 152,440 & 50.61 \\
\hline 1.799 & 4 & 242 & 50.71 \\
\hline 1.778 & 2 & 322 & 51.33 \\
\hline 1.767 & 4 & $\overline{5} 21$ & 51.68 \\
\hline 1.762 & 4 & 123 & 51.83 \\
\hline 1.752 & 3 & 510 & 52.15 \\
\hline 1.742 & 3 & $\overline{3} 33,170$ & 52.47 \\
\hline 1.738 & 3 & $\overline{5} 12$ & 52.63 \\
\hline 1.733 & 2 & 431,261 & 52.78 \\
\hline 1.724 & 2 & $\overline{1} 62,351,+$ & 53.06 \\
\hline 1.702 & 3 & 071,520 & 53.81 \\
\hline 1.694 & 4 & 332 & 54.08 \\
\hline 1.687 & 3 & $\overline{4} 51$ & 54.35 \\
\hline
\end{tabular}

\begin{tabular}{|c|c|c|c|}
\hline \multicolumn{4}{|c|}{$\begin{array}{l}\text { Internal standard } \mathrm{Ag}, \mathrm{a}=4.08641 \AA \\
\mathrm{CuK} a_{1} \quad \lambda=1.54056 \AA \text {; temp. } 25{ }^{\circ} \mathrm{C}\end{array}$} \\
\hline$d(\stackrel{\AA}{)})$ & $I$ & $h k l$ & $2 \theta\left({ }^{\circ}\right)$ \\
\hline 1.733 & 2 & 431,261 & 52.78 \\
\hline 1.724 & 2 & $\overline{1} 62,351,+$ & 53.06 \\
\hline 1.702 & 3 & 071,520 & 53.81 \\
\hline 1.694 & 4 & 332 & 54.08 \\
\hline 1.687 & 3 & $\overline{4} 51$ & 54.35 \\
\hline 1.679 & 4 & $\overline{4} 23,043$ & 54.63 \\
\hline 1.648 & 4 & 270,171 & 55.74 \\
\hline 1.638 & 2 & 213 & 56.12 \\
\hline 1.633 & 2 & $\overline{3} 43, \overline{2} 71$ & 56.28 \\
\hline 1.627 & 2 & 530 & 56.50 \\
\hline 1.615 & 3 & $\overline{5} 32$ & 56.98 \\
\hline 1.595 & 2 & $\overline{4} 52$ & 57.74 \\
\hline 1.593 & 1 & $342, \overline{1} 53$ & 57.85 \\
\hline 1.586 & 2 & $\overline{5} 41$ & 58.13 \\
\hline 1.580 & 2 & $\overline{2} 53,402$ & 58.37 \\
\hline 1.567 & 2 & 412,361 & 58.90 \\
\hline 1.554 & 2 & $\overline{2} 04,080$ & 59.41 \\
\hline 1.546 & 2 & $\overline{5} 1 \underline{3}$ & 59.78 \\
\hline 1.542 & 4 & $\underline{2} 14, \overline{1} 72$ & 59.92 \\
\hline 1.535 & 2 & $\overline{6} 01,233$ & 60.26 \\
\hline 1.528 & 2 & $\overline{3} 71, \overline{5} 42$ & 60.54 \\
\hline 1.521 & 4 & $\overline{4} 43, \overline{3} 53$ & 60.86 \\
\hline 1.511 & 2 & $\overline{5} 23,262,+$ & 61.29 \\
\hline 1.500 & 3 & $\overline{1} 81, \overline{6} 02$ & 61.79 \\
\hline 1.490 & 1 & 621 & 62.24 \\
\hline 1.477 & 2 & 531 & 62.88 \\
\hline 1.473 & 2 & $\overline{3} 24$ & 63.04 \\
\hline 1.464 & 1 & 610 & 63.48 \\
\hline 1.461 & 2 & 313 & 63.65 \\
\hline 1.458 & 2 & $533, \overline{6} 22,+$ & 63.76 \\
\hline 1.441 & 1 & $\overline{4} 04, \overline{6} 31$ & 64.65 \\
\hline 1.433 & 1 & $\underline{5} 52$ & 65.02 \\
\hline 1.424 & 2 & $\overline{3} 34$ & 65.50 \\
\hline
\end{tabular}


Sample

The sample was obtained from the National Lead Company, South Amboy, N.J.

Major impurities

No impurities greater than 0.001 percent

Color

Colorless

Structure

Tetragona $1, I 41 /$ amd (141), $Z=4$ [Huggins, 1926]

\section{Lattice constants}

\begin{tabular}{|r|l|l|}
\hline & $a(\AA)$ & $c(\AA)$ \\
\cline { 2 - 3 } Swanson and Tatge (1953), & & \\
sample at $26-27^{\circ} \mathrm{C}-----$ & 3.783 & 9.51 \\
NBS, sample at $25^{\circ} \mathrm{C}-----$ & 3.7852 & 9.5139 \\
& \pm .0001 & \pm .0004 \\
\hline
\end{tabular}

\section{Density}

(calculated) $3.893 \mathrm{~g} / \mathrm{cm}^{3}$ at $25^{\circ} \mathrm{C}$.

\section{Reference intensity}

$\mathrm{I}_{\text {corundum }}=4.3$

\section{Polymorphism}

Anatase and another mineral form, brookite (orthorhombic), are converted to a third mineral form, rutile (tetragonal), by heating to temperatures above $700{ }^{\circ} \mathrm{C}$.

\section{References}

Huggins, M. I. (1926). The crystal structure of anatase and rutile, the tetragonal forms of $\mathrm{TiO}_{2}$, Phys. Rev. 27, 638.

Swanson, H.E. and E. Tatge (1953) Standard $\mathrm{X}$-ray Diffraction Powder Patterns, Natl. Bur. Sta. U.S. Circ. 539, Vol. I, 46-47.

\begin{tabular}{|c|c|c|c|}
\hline \multicolumn{4}{|c|}{$\begin{array}{l}\text { Internal standard } W, a=3.16516 \AA \\
\text { CuK } a_{1} \quad \lambda=1.54056 \AA \text {; temp. } 25{ }^{\circ} \mathrm{C}\end{array}$} \\
\hline$d(\AA)$ & $I$ & $h k l$ & $2 \theta\left({ }^{\circ}\right)$ \\
\hline 3.515 & 100 & 101 & 25.32 \\
\hline 2.431 & 9 & 103 & 36.95 \\
\hline 2.378 & 22 & 004 & 37.80 \\
\hline 2.332 & 9 & 112 & 38.57 \\
\hline 1.892 & 33 & 200 & 48.05 \\
\hline 1.6999 & 21 & 105 & 53.89 \\
\hline 1.6665 & 19 & 211 & 55.06 \\
\hline 1.4930 & 4 & 213 & 62.12 \\
\hline 1.4808 & 13 & 204 & 62.69 \\
\hline 1.364 .1 & 5 & 116 & 68.76 \\
\hline 1.3378 & 5 & 220 & 70.31 \\
\hline 1.2795 & $<I$ & 107 & 74.03 \\
\hline 1. 2649 & 10 & 215 & 75.03 \\
\hline I. 2509 & 3 & 301 & 76.02 \\
\hline 1. 1894 & $<1$ & 008 & 80.72 \\
\hline 1.1725 & 2 & 303 & 82.14 \\
\hline 1.1664 & 5 & 224 & 82.66 \\
\hline 1.1608 & 3 & 312 & 83.15 \\
\hline 1.0600 & 1 & 217 & 93.22 \\
\hline 1.0517 & 3 & 305 & 94.18 \\
\hline 1.0436 & 3 & 321 & 95.14 \\
\hline 1.0182 & 2 & 109 & 98.32 \\
\hline 1.0070 & 2 & 208 & 99.80 \\
\hline .9967 & 1 & 323 & 101.22 \\
\hline .9555 & 4 & 316 & 107.45 \\
\hline .9464 & 3 & 400 & 108.96 \\
\hline .9246 & $<1$ & 307 & 112.84 \\
\hline .9192 & 2 & 325 & 113.85 \\
\hline .9138 & 2 & 411 & 114.91 \\
\hline .8966 & 3 & $219,1 \cdot 1 \cdot 10$ & 118.44 \\
\hline .8890 & 2 & 228 & 120.11 \\
\hline .8819 & $<1$ & 413 & 121.73 \\
\hline .8793 & 2 & 404 & 122.34 \\
\hline .8464 & 2 & 420 & 131.02 \\
\hline .8308 & $<1$ & 327 & 135.98 \\
\hline .8268 & 3 & 415 & 137.38 \\
\hline .8102 & $I$ & 309 & 143.86 \\
\hline .7974 & 3 & 424 & 150.04 \\
\hline .7928 & 1 & $0 \cdot 0 \cdot 12$ & 152.62 \\
\hline
\end{tabular}




\section{Sample}

The sample was obtained from the National Lead Company, South Amboy, N.J.

\section{Major impurities}

No impurities greater than 0.001 percent

\section{Color}

Colorless

\section{Structure}

Tetragonal, $\mathrm{P} 4_{\mathrm{z}} / \mathrm{mnm}$ (136), $\mathrm{Z}=2$ [Huggins, 1926]

\section{Lattice constants}

\begin{tabular}{|c|c|c|}
\hline & $a(\stackrel{\circ}{)}$ & $c(\stackrel{\circ}{A})$ \\
\hline $\begin{array}{l}\text { Swanson and Tatge [1953]- } \\
\text { NBS, sample at } 25{ }^{\circ} \mathrm{C}----\end{array}$ & $\begin{array}{l}4.594 \\
4.5933 \\
\pm .0001\end{array}$ & $\begin{array}{l}2.958 \\
2.9592 \\
\pm .0001\end{array}$ \\
\hline
\end{tabular}

\section{Density}

(calculated) $4.250 \mathrm{~g} / \mathrm{cm}^{3}$ at $25^{\circ} \mathrm{C}$.

\section{Reference intensity}

$$
\mathrm{I} / \mathrm{I}_{\text {corundum }}=3.4
$$

\section{Polymorphism}

The two other mineral forms, anatase (tetragonal) and brookite (orthorhombic), are converted to rutile by heating to temperatures above $700{ }^{\circ} \mathrm{C}$.

\section{References}

Huggins, M. L. (1926). The crystal structure of anatase and rutile, the tetragonal forms of $\mathrm{TiO}_{2}$, Phys. Rev. 27, 638.

Swanson, H.E. and E. Tatge (1953). Standard $\mathrm{X}$-ray Diffraction Powder Patterns, Natl. Bur. Std. U.S. Circ. 539, I, 44-46.

\begin{tabular}{|c|r|r|r|}
\hline \multicolumn{4}{|c|}{ Internal standard W, a $=3.16516 \AA$} \\
CuK $a_{1} \lambda=1.54056 \AA$ A temp. $25{ }^{\circ} \mathrm{C}$ \\
\hline$d(\AA)$ & $I$ & $h k l$ & $20\left({ }^{\circ}\right)$ \\
\hline 3.247 & 100 & 110 & 27.45 \\
2.487 & 51 & 101 & 36.09 \\
2.297 & 7 & 200 & 39.19 \\
2.188 & 25 & 111 & 41.23 \\
2.054 & 9 & 210 & 44.06 \\
& & & \\
1.6874 & 60 & 211 & 54.32 \\
1.6237 & 20 & 220 & 56.64 \\
1.4797 & 9 & 002 & 62.74 \\
1.4528 & 9 & 310 & 64.04 \\
1.4243 & 1 & 221 & 65.48 \\
1.3598 & 20 & 301 & 69.01 \\
1.3465 & 11 & 112 & 69.79 \\
1.3041 & 1 & 311 & 72.41 \\
1.2441 & 3 & 202 & 76.51 \\
1.2006 & 2 & 212 & 79.82 \\
1.1702 & 5 & 321 & 82.33 \\
1.1483 & 3 & 400 & 84.26 \\
1.1143 & 2 & 410 & 87.46 \\
1.0936 & 8 & 222 & 89.55 \\
1.0827 & 4 & 330 & 90.71 \\
1.0425 & 6 & 411 & 95.27 \\
1.0364 & 6 & 312 & 96.01 \\
1.0271 & 3 & 420 & 97.17 \\
0.9703 & 1 & 421 & 105.09 \\
.9644 & 2 & 103 & 106.01 \\
.9438 & 1 & 113 & 109.40 \\
.9072 & 4 & 402 & 116.22 \\
.9009 & 4 & 510 & 117.53 \\
.8892 & 8 & 212 & 120.06 \\
.8774 & 8 & 431 & 122.79 \\
.8738 & 8 & 332 & 123.66 \\
.8437 & 6 & 422 & 131.83 \\
.8292 & 8 & 303 & 136.55 \\
.8196 & 12 & 521 & 140.05 \\
.8120 & 2 & 440 & 143.09 \\
.7877 & 2 & 530 & 155.85 \\
\hline
\end{tabular}


Structure

Triclinic, Pī (2), $\mathrm{Z}=1$ [Jost et al., 1966]

\section{Lattice parameters}

$a=7.25, b=5.70, c=4.67 \AA, \alpha=99.8^{\circ}, \beta=98.0^{\circ}$, $\gamma=99.7^{\circ} \quad$ [ibid.]

\section{Scattering factors}

$$
\begin{array}{ll}
\mathrm{H}^{\circ}: \mathrm{O}^{\circ}[3.3 .1 \mathrm{~A}] \\
\mathrm{AS}^{\circ}[3.3 .1 \mathrm{~B}]
\end{array}
$$

\section{Thermal parameters}

Isotropic [Jost et aI., 1966]

\section{Density}

(calculated) $3.45 \mathrm{~g} / \mathrm{cm}^{3}$ [ibid.]

\section{Scale factor}

$$
1.431 \times 10^{4}
$$

\section{Reference}

Jost,K.-H., H. Worzala, and E. Thilo (1966). Die Struktur des $\mathrm{As}_{2} \mathrm{O}_{5} \cdot \frac{5}{3} \mathrm{H}_{2} \mathrm{O}$, Acta Cryst. 21, 808-813.

\begin{tabular}{|c|c|c|c|c|c|c|}
\hline$d(\AA)$ & $I$ & \multicolumn{4}{|c|}{$h k l$} & $\begin{array}{c}2 \theta\left(^{\circ}\right) \\
\lambda=1.54056 \AA\end{array}$ \\
\hline 2.170 & $1 i$ & 0 & 2 & 1 & & 41.58 \\
\hline 2.154 & 4 & -5 & 1 & 1 & & 41.90 \\
\hline 2.032 & 2 & -2 & 2 & 1 & & 43.42 \\
\hline 2.057 & 1 & 1 & 0 & 2 & & 43.983 \\
\hline 2.024 & $=$ & 3 & 1 & 0 & & 44.74 \\
\hline 2.009 & 3 & -1 & 1 & 2 & + & 45.10 \\
\hline 1. 9894 & 3 & 2 & 2 & 0 & & 45.56 \\
\hline $1.95+5$ & 1 & 3 & 0 & 1 & & 46.42 \\
\hline 1.9028 & 3 & -2 & 1 & 2 & & 47.76 \\
\hline 1.8747 & ó & 1 & -2 & 2 & & 48.52 \\
\hline 1.8315 & 3 & 1 & -3 & 1 & + & 49.74 \\
\hline 1.5220 & 2 & 3 & -2 & 1 & & 50.00 \\
\hline 1.7800 & 2 & -4 & 1 & 0 & & 51.10 \\
\hline $1.77 \mathrm{sc}$ & 1 & -2 & 3 & 0 & & 51.34 \\
\hline 1.7730 & 1 & 2 & 0 & 2 & & 51.50 \\
\hline 1.7647 & 2 & 4 & 0 & 0 & & 51.76 \\
\hline 1.7275 & 1 & -4 & 1 & 1 & & 52.90 \\
\hline 1.7215 & 1 & -1 & -3 & 1 & & 53.16 \\
\hline 1.7131 & 2 & -2 & -2 & 2 & & 53.44 \\
\hline $1.7090^{\circ}$ & 3 & -3 & -1 & 2 & & 53.56 \\
\hline 1.5090 & 1 & 2 & -2 & 2 & & 53.90 \\
\hline 1.6907 & 3 & 1 & 3 & 0 & & 54.00 \\
\hline 1.6397 & 2 & -1 & 3 & 1 & & 56.04 \\
\hline 1.6000 & 2 & -3 & 3 & 0 & + & 57.22 \\
\hline 1.5989 & 1 & -2 & 3 & 1 & & 57.60 \\
\hline $1.575 u$ & $j$ & 2 & 1 & 2 & & 58.56 \\
\hline 1.5603 & 2 & -4 & 2 & $\overline{1}$ & & 59.04 \\
\hline 1.5594 & 1 & 3 & -1 & 2 & & 59.20 \\
\hline $1.534 i$ & 1 & -1 & -3 & 2 & & 60.28 \\
\hline 1.5żj8 & 2 & -4 & 0 & 2 & & 60.64 \\
\hline 1.5217 & 2 & 4 & -2 & 1 & & 60.82 \\
\hline 1.5079 & 2 & -3 & -2 & 2 & + & 61.44 \\
\hline 1.4980 & 2 & -3 & 2 & 2 & & 61.86 \\
\hline 1.4809 & 3 & 1 & 2 & 2 & & 62.40 \\
\hline 1.4703 & 2 & 2 & -3 & 2 & + & 62.90 \\
\hline 1.450 & 2 & -4 & -1 & 2 & & 63.84 \\
\hline 1.4395 & 2 & -1 & -2 & 3 & & 64.70 \\
\hline $1.437 \mathrm{c}$ & 2 & -5 & $\overline{1}$ & 0 & & 64.82 \\
\hline 1.4340 & 2 & $j$ & 2 & 1 & & 64.98 \\
\hline 1.4277 & 1 & 1 & 0 & 3 & & 65.30 \\
\hline 1.4231 & 1 & -2 & -3 & 2 & & 65.54 \\
\hline 1.4101 & 1 & 1 & -4 & 1 & + & 66.22 \\
\hline 1.4034 & 1 & -4 & -2 & 1 & & 66.58 \\
\hline 1.3883 & 1 & 0 & 1 & 3 & & 67.40 \\
\hline 1.3847 & 1 & -3 & 0 & 3 & & 67.60 \\
\hline 1.3750 & 2 & 0 & 4 & 0 & & 68.04 \\
\hline 1.3704 & 1 & -5 & 2 & 0 & & 68.40 \\
\hline 1.3693 & 1 & 2 & -4 & 1 & & 68.40 \\
\hline 1.3644 & 1 & -3 & -3 & 1 & & 68.74 \\
\hline 1.3603 & 1 & 4 & -3 & 1 & & 68.98 \\
\hline 1.3507 & 1 & 2 & -1 & 3 & & 69.54 \\
\hline
\end{tabular}

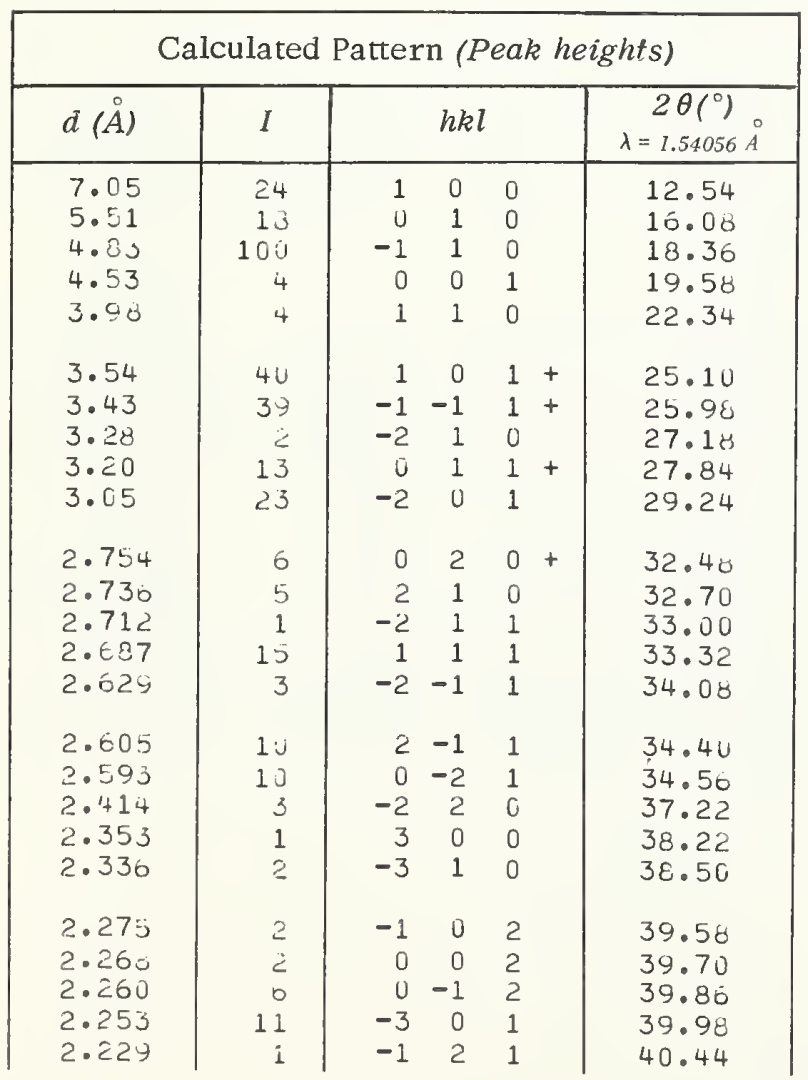

Calculated Pattern (Peak heights) 


\begin{tabular}{|c|c|c|c|c|c|}
\hline \multicolumn{6}{|c|}{ Calculated Pattern (Integrated) } \\
\hline$d(\stackrel{\circ}{A})$ & $I$ & & $h k l$ & & $\begin{array}{c}2 \theta\left(^{\circ}\right) \\
\lambda=1.54056 \AA\end{array}$ \\
\hline 7.06 & 21 & 1 & 0 & 0 & 12.53 \\
\hline b.51 & 18 & 0 & 1 & 0 & 16.08 \\
\hline 4.83 & 100 & -1 & 1 & 0 & 18.30 \\
\hline 4.53 & 4 & 0 & 0 & 1 & 19.57 \\
\hline 3.98 & 4 & 1 & 1 & 0 & $22 \cdot 33$ \\
\hline $3 \cdot 51$ & 42 & 1 & 0 & 1 & 25.10 \\
\hline 3.53 & 4 & 2 & 0 & $\mathrm{c}$ & 25.21 \\
\hline 3.43 & $4 \hat{2}$ & -1 & -1 & 1 & 25.98 \\
\hline 3.40 & 2 & 1 & -1 & 1 & 26.19 \\
\hline $3 \cdot 28$ & 2 & -2 & 1 & 0 & 27.17 \\
\hline $3 \cdot 22$ & 1 & -1 & 1 & 1 & 27.72 \\
\hline 3.20 & 14 & 0 & 1 & 1 & 27.84 \\
\hline 3.05 & 26 & -2 & 0 & 1 & 29.24 \\
\hline 2.755 & 3 & -1 & 2 & $G$ & 32.47 \\
\hline 2.753 & 4 & 0 & 2 & 0 & 32.49 \\
\hline 2.737 & 5 & 2 & 1 & 0 & 32.70 \\
\hline 2.712 & 1 & -2 & 1 & 1 & 33.00 \\
\hline 2.606 & 17 & 1 & 1 & 1 & 33.32 \\
\hline 2.629 & 4 & -2 & -1 & 1 & 34.08 \\
\hline 2.605 & 12 & 2 & -1 & 1 & 34.40 \\
\hline 2.593 & 11 & 0 & -2 & 1 & 34.57 \\
\hline $2 \cdot 414$ & 3 & -2 & 2 & $\dot{v}$ & 37.22 \\
\hline $2 \cdot 353$ & 1 & 3 & 0 & 0 & $38 \cdot 22$ \\
\hline 2.336 & 3 & -3 & 1 & 0 & 33.50 \\
\hline $2 \cdot 275$ & 2 & -1 & 0 & 2 & 39.58 \\
\hline$z \cdot 206$ & 1 & 0 & 0 & 2 & 39.74 \\
\hline 2.259 & 6 & 0 & -1 & 2 & 39.86 \\
\hline 2.253 & 10 & -3 & 0 & 1 & 39.98 \\
\hline $2 \cdot 2<8$ & 1 & -1 & 2 & 1 & 40.44 \\
\hline $2 \cdot 170$ & 14 & 0 & 2 & 1 & 41.58 \\
\hline 2.155 & 5 & -3 & 1 & 1 & 41.39 \\
\hline 2.083 & 2 & -2 & 2 & 1 & 43.41 \\
\hline 2.057 & 1 & 1 & 0 & 2 & 43.99 \\
\hline $2.0<4$ & 3 & 3 & 1 & 0 & 44.73 \\
\hline 2.009 & 3 & -1 & 1 & 2 & 45.09 \\
\hline $2.00 \hat{8}$ & 1 & -2 & -2 & 1 & $45 \cdot 11$ \\
\hline 2.0106 & 2 & 3 & -1 & 1 & $45 \cdot 15$ \\
\hline 1.9892 & 3 & 2 & 2 & 0 & 45.57 \\
\hline 1.9546 & 1 & 3 & 0 & 1 & 46.42 \\
\hline 1.9025 & 4 & -2 & 1 & 2 & 47.77 \\
\hline 1.8751 & 8 & 1 & -2 & 2 & $48 \cdot 51$ \\
\hline 1.3326 & 2 & 0 & -3 & 1 & 49.71 \\
\hline 1.8311 & 3 & 1 & -3 & 1 & 49.75 \\
\hline $1 \cdot 8226$ & 3 & 3 & -2 & 1 & 50.00 \\
\hline 1.7857 & 1 & -4 & 1 & 0 & $51 \cdot 11$ \\
\hline 1.7781 & 1 & -2 & 3 & 0 & $51 \cdot 34$ \\
\hline 1.7728 & 1 & 2 & 0 & 2 & 51.51 \\
\hline 1.7046 & 3 & 4 & 0 & 0 & 51.77 \\
\hline 1.7278 & 2 & -4 & 1 & 1 & 52.95 \\
\hline $1.7 \approx 15$ & 1 & -1 & -3 & 1 & 53.16 \\
\hline
\end{tabular}

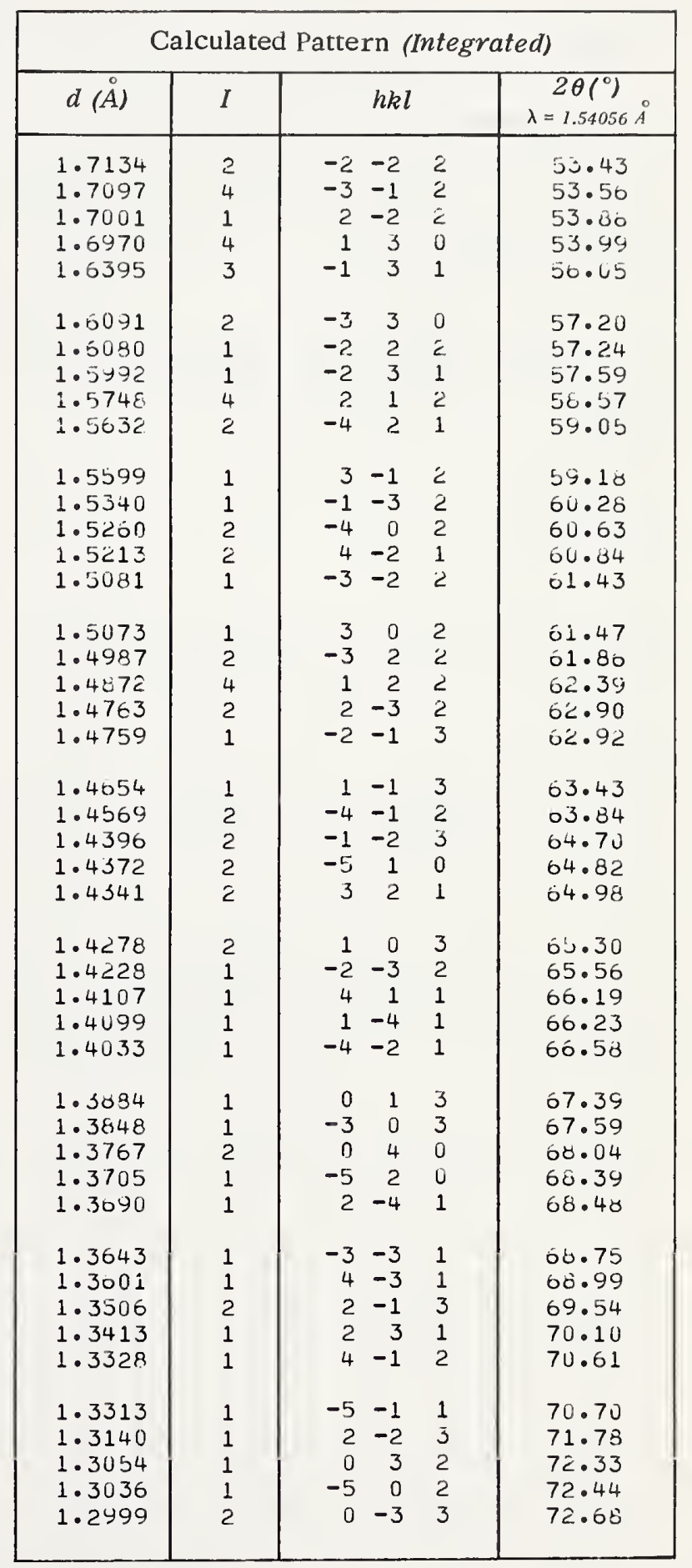


Structure

Monoclinic, P2 /a (14), $\mathrm{Z}=4$ [Brown, 1966]

\section{Lattice parameters}

$a=12.144, b=5.756, c=15.397 \AA, \beta=114^{\circ} 8^{\prime}$

(published value: $c=15.396$ )

[ibid.]

Scattering factors

$\mathrm{H}^{\circ}, \mathrm{C}^{\circ}, \mathrm{N}^{\circ}[3.3 . \mathrm{IA}]$

Thermal parameters

Anisotropic for carbon and oxygen, isotropic for hydrocen [Brown, 1966]

Density

(calculated) $1.230 \mathrm{~g} / \mathrm{cm}^{3}$

Scale factor

$1.478 \times 10^{4}$

Additional patterns

I.PDF card 3-0172 [Socony-Vacuum, Paulsboro, N.J.]

\section{Reference}

Brown, C.J. (1966). A refinement of the crystal structure of azobenzene, Acta Cryst. 21, 146-152.

\begin{tabular}{|c|c|c|c|c|c|c|}
\hline \multicolumn{7}{|c|}{ Calculated Pattern (Peak heights) } \\
\hline$d(\stackrel{\circ}{A})$ & $I$ & & $h k i$ & & & $\begin{array}{c}2 \theta\left(^{\circ}\right) \\
\lambda=1.54056 \mathrm{~A}\end{array}$ \\
\hline 14.06 & 6 & 3 & 0 & 1 & & 6.28 \\
\hline 7.02 & 36 & 0 & 0 & 2 & & 12.60 \\
\hline 5.60 & 13 & 2 & 0 & -2 & & 15.80 \\
\hline 5.54 & 12 & 2 & 0 & 0 & & 15.98 \\
\hline $5 \cdot 32$ & 10 & 0 & 1 & 1 & & 16.64 \\
\hline 5.11 & 100 & 1 & 1 & 0 & + & 17.34 \\
\hline 4.63 & 45 & 2 & 0 & -3 & & 19.16 \\
\hline 4.56 & 70 & 2 & 0 & 1 & + & 19.46 \\
\hline 4.53 & 95 & 1 & 1 & 1 & & 19.56 \\
\hline 4.45 & 77 & 0 & 1 & 2 & & 19.92 \\
\hline 4.18 & 24 & 2 & 1 & -1 & & 21.26 \\
\hline 4.02 & 15 & 2 & 1 & -2 & & 22.12 \\
\hline 3.99 & 5 & 2 & 1 & 0 & & .22 .24 \\
\hline 3.83 & 50 & 1 & 1 & -3 & & 23.20 \\
\hline 3.80 & 61 & 1 & 1 & 2 & & 23.36 \\
\hline 3.74 & 74 & 2 & 0 & -4 & & 23.80 \\
\hline 3.68 & 66 & 2 & 0 & 2 & & 24.16 \\
\hline 3.63 & 14 & D & 1 & 3 & & 24.48 \\
\hline 3.61 & 9 & 2 & 1 & -3 & & 24.56 \\
\hline 3.57 & 4 & 2 & 1 & 1 & & 24.90 \\
\hline
\end{tabular}

\begin{tabular}{|c|c|c|c|c|c|c|}
\hline \multicolumn{7}{|c|}{ Calculated Pattern (Peak heights) } \\
\hline$d(\stackrel{\circ}{A})$ & $I$ & & $h k$ & & & $\begin{array}{c}2 \theta\left({ }^{\circ}\right) \\
\lambda=1.54056 \AA \\
\end{array}$ \\
\hline 3.51 & 15 & 0 & 0 & 4 & & 25.34 \\
\hline 3.292 & 11 & 3 & 1 & -2 & & 27.06 \\
\hline 3.285 & $B$ & 3 & 1 & -1 & & 27.12 \\
\hline 3.188 & 32 & 1 & 1 & -4 & & 27.96 \\
\hline 3.168 & 7 & 1 & 1 & 3 & & 28.14 \\
\hline 3.134 & 5 & 2 & 1 & -4 & + & 28.46 \\
\hline 3.099 & 26 & 2 & 1 & 2 & & 28.78 \\
\hline 3.062 & 17 & 2 & 0 & -5 & & 29.14 \\
\hline 3.035 & 4 & 4 & 0 & -2 & & 29.40 \\
\hline 3.019 & 8 & 2 & 0 & 3 & & 29.56 \\
\hline 2.998 & 10 & 0 & 1 & 4 & & 29.78 \\
\hline 2.978 & 2 & 4 & 0 & -3 & & 29.98 \\
\hline 2.870 & 3 & 3 & 1 & -4 & & 31.14 \\
\hline 2.819 & 1 & 0 & 2 & 1 & & 31.72 \\
\hline 2.788 & 1 & 1 & 2 & -1 & & 32.08 \\
\hline 2.703 & 4 & 2 & 1 & -5 & & 33.12 \\
\hline 2.685 & 9 & 1 & 1 & -5 & + & 33.34 \\
\hline 2.679 & 8 & 1 & 2 & 1 & & 33.42 \\
\hline 2.674 & 8 & 2 & 1 & 3 & & 33.48 \\
\hline 2.664 & 6 & 0 & 2 & 2 & & 33.62 \\
\hline 2.644 & 2 & 4 & 1 & -3 & & 33.88 \\
\hline 2.630 & 2 & 4 & 1 & -1 & & 34.06 \\
\hline 2.600 & 3 & 2 & 2 & -1 & & 34.46 \\
\hline 2.560 & 4 & 2 & 2 & -2 & & 35.02 \\
\hline 2.538 & 2 & 3 & 1 & 2 & & $35 \cdot 34$ \\
\hline 2.503 & 3 & 1 & 2 & 2 & & 35.84 \\
\hline 2.497 & 3 & 4 & 1 & 0 & & 35.94 \\
\hline 2.452 & 1 & 0 & 2 & 3 & & 36.62 \\
\hline 2.444 & 1 & 2 & 2 & -3 & & 36.74 \\
\hline 2.343 & 5 & 2 & 1 & -6 & & 38.38 \\
\hline 2.336 & 5 & 3 & 2 & -1 & + & 38.50 \\
\hline 2.315 & 3 & 4 & 0 & -6 & & 38.86 \\
\hline 2.279 & 2 & 4 & 0 & 2 & & 39.50 \\
\hline 2.271 & 2 & 3 & 2 & 0 & & 39.66 \\
\hline 2.267 & 1 & 2 & 2 & 2 & & 39.72 \\
\hline 2.258 & 2 & 3 & 1 & 3 & & 39.90 \\
\hline 2.183 & 1 & 5 & 1 & -4 & & 41.32 \\
\hline 2.169 & 1 & 0 & 1 & 6 & & 41.60 \\
\hline 2.157 & 1 & 3 & 2 & 1 & & 41.84 \\
\hline 2.119 & 1 & 4 & 1 & 2 & & 42.54 \\
\hline 2.097 & 2 & 2 & 2 & -5 & & 43.10 \\
\hline 2.089 & 1 & 4 & 2 & -2 & & 43.28 \\
\hline 2.069 & 4 & 5 & 1 & 0 & & 43.72 \\
\hline 2.047 & 1 & 4 & 0 & 3 & & 44.22 \\
\hline 2.035 & 1 & 3 & 1 & -7 & & 44.48 \\
\hline 2.017 & 1 & 3 & 2 & 2 & & 44.90 \\
\hline 2.010 & 4 & 3 & 1 & 4 & + & 45.06 \\
\hline 1.9960 & 2 & 4 & 2 & 0 & & 45.40 \\
\hline 1.9665 & 3 & 5 & 1 & -6 & & 46.12 \\
\hline 1.9529 & 2 & 6 & 0 & -5 & & 46.46 \\
\hline
\end{tabular}


Calculated Pattern (Peak heights)

\begin{tabular}{|l|l|lll|l|}
\hline$d(A)$ & $I$ & \multicolumn{3}{|c|}{$h k l$} & $\begin{array}{c}2 \theta\left(^{\circ}\right) \\
\lambda=1.54056 \AA\end{array}$ \\
\hline 1.9364 & 1 & 6 & 0 & -1 & 46.88 \\
1.9279 & 1 & 4 & 1 & 3 & 47.10 \\
1.9095 & 1 & 6 & 1 & -3 & 47.58 \\
1.9020 & 2 & 2 & 2 & 4 & 47.78 \\
1.8908 & 3 & 1 & 2 & -6 & 48.08 \\
1.8842 & 2 & 1 & 2 & 5 & 48.26 \\
1.8682 & 2 & 4 & 0 & -8 & 48.70 \\
1.8582 & 1 & 1 & 3 & -2 & 48.98 \\
1.8469 & 1 & 6 & 0 & 0 & 49.30 \\
1.8399 & 2 & 4 & 0 & 4 & 49.50 \\
1.8206 & 5 & 3 & 1 & -8 & 50.06 \\
1.8018 & 2 & 2 & 1 & 6 & 50.62 \\
1.7945 & 1 & 1 & 3 & 2 & 50.84 \\
1.7775 & 1 & 4 & 1 & -8 & 51.36 \\
1.7527 & 2 & 4 & 1 & 4 & 52.14 \\
1.7465 & 1 & 2 & 2 & -7 & 52.34 \\
1.719 .7 & 1 & 3 & 2 & 4 & 53.22 \\
1.7066 & 1 & 2 & 3 & -4 & 53.66 \\
1.6593 & 1 & 7 & 1 & -4 & 55.32 \\
\hline
\end{tabular}

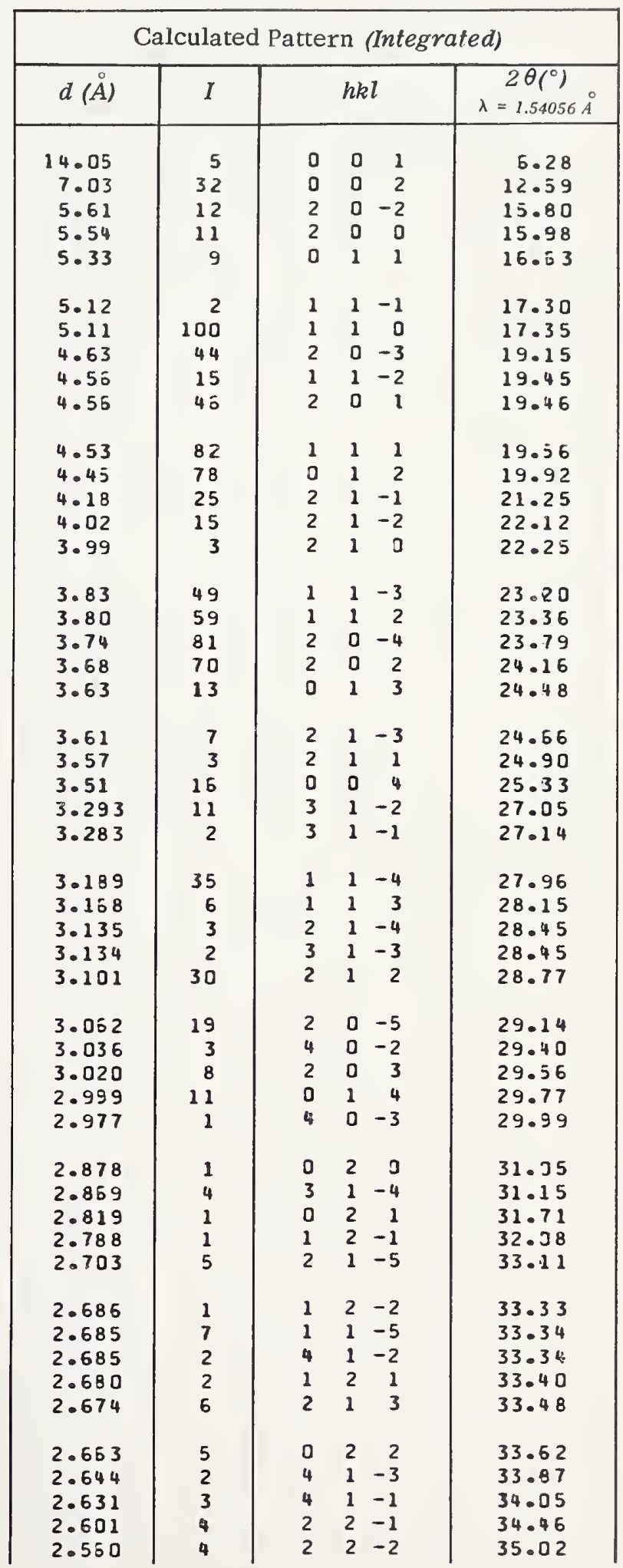




\begin{tabular}{|c|c|c|c|c|}
\hline \multicolumn{5}{|c|}{ Calculated Pattern (Integrated) } \\
\hline$d(\stackrel{\circ}{A})$ & $I$ & & $h k l$ & $\begin{array}{c}2 \theta\left(^{\circ}\right) \\
\lambda=1.54056 \AA\end{array}$ \\
\hline $\begin{array}{l}2.538 \\
2.503 \\
2.496 \\
2.452 \\
2.444\end{array}$ & $\begin{array}{l}2 \\
3 \\
2 \\
1 \\
1\end{array}$ & $\begin{array}{l}3 \\
1 \\
4 \\
0 \\
2\end{array}$ & $\begin{array}{rr}1 & 2 \\
2 & 2 \\
1 & 0 \\
2 & 3 \\
2 & -3\end{array}$ & $\begin{array}{l}35.33 \\
35.85 \\
35.94 \\
36.62 \\
36.74\end{array}$ \\
\hline $\begin{array}{l}2.344 \\
2.339 \\
2.336 \\
2.320 \\
2.315\end{array}$ & $\begin{array}{l}5 \\
1 \\
3 \\
1 \\
3\end{array}$ & $\begin{array}{l}2 \\
3 \\
3 \\
2 \\
4\end{array}$ & $\begin{array}{lr}1 & -6 \\
2 & -2 \\
2 & -1 \\
1 & 4 \\
0 & -6\end{array}$ & $\begin{array}{l}38.38 \\
38.45 \\
38.51 \\
38.78 \\
38.87\end{array}$ \\
\hline $\begin{array}{l}2.279 \\
2.270 \\
2.267 \\
2.257 \\
2.184\end{array}$ & $\begin{array}{l}1 \\
1 \\
1 \\
2 \\
2\end{array}$ & $\begin{array}{l}4 \\
3 \\
2 \\
3 \\
5\end{array}$ & $\begin{array}{rr}0 & 2 \\
2 & 0 \\
2 & 2 \\
1 & 3 \\
1 & -4\end{array}$ & $\begin{array}{l}39.51 \\
39.57 \\
39.73 \\
39.91 \\
41.31\end{array}$ \\
\hline $\begin{array}{l}2.159 \\
2.158 \\
2.119 \\
2.037 \\
2.089\end{array}$ & $\begin{array}{l}1 \\
1 \\
2 \\
2 \\
1\end{array}$ & $\begin{array}{l}0 \\
3 \\
4 \\
2 \\
4\end{array}$ & $\begin{array}{rr}1 & 6 \\
2 & 1 \\
1 & 2 \\
2 & -5 \\
2 & -2\end{array}$ & $\begin{array}{l}41.50 \\
41.83 \\
42.53 \\
43.10 \\
43.28\end{array}$ \\
\hline $\begin{array}{l}2.068 \\
2.046 \\
2.035 \\
2.017 \\
2.010\end{array}$ & $\begin{array}{l}4 \\
1 \\
1 \\
1 \\
4\end{array}$ & $\begin{array}{l}5 \\
4 \\
3 \\
3 \\
3\end{array}$ & $\begin{array}{rr}1 & 0 \\
0 & 3 \\
1 & -7 \\
2 & 2 \\
1 & 4\end{array}$ & $\begin{array}{l}43.73 \\
44.23 \\
44.47 \\
44.50 \\
45.07\end{array}$ \\
\hline 2.008 & 1 & 6 & $0-4$ & 45.12 \\
\hline
\end{tabular}

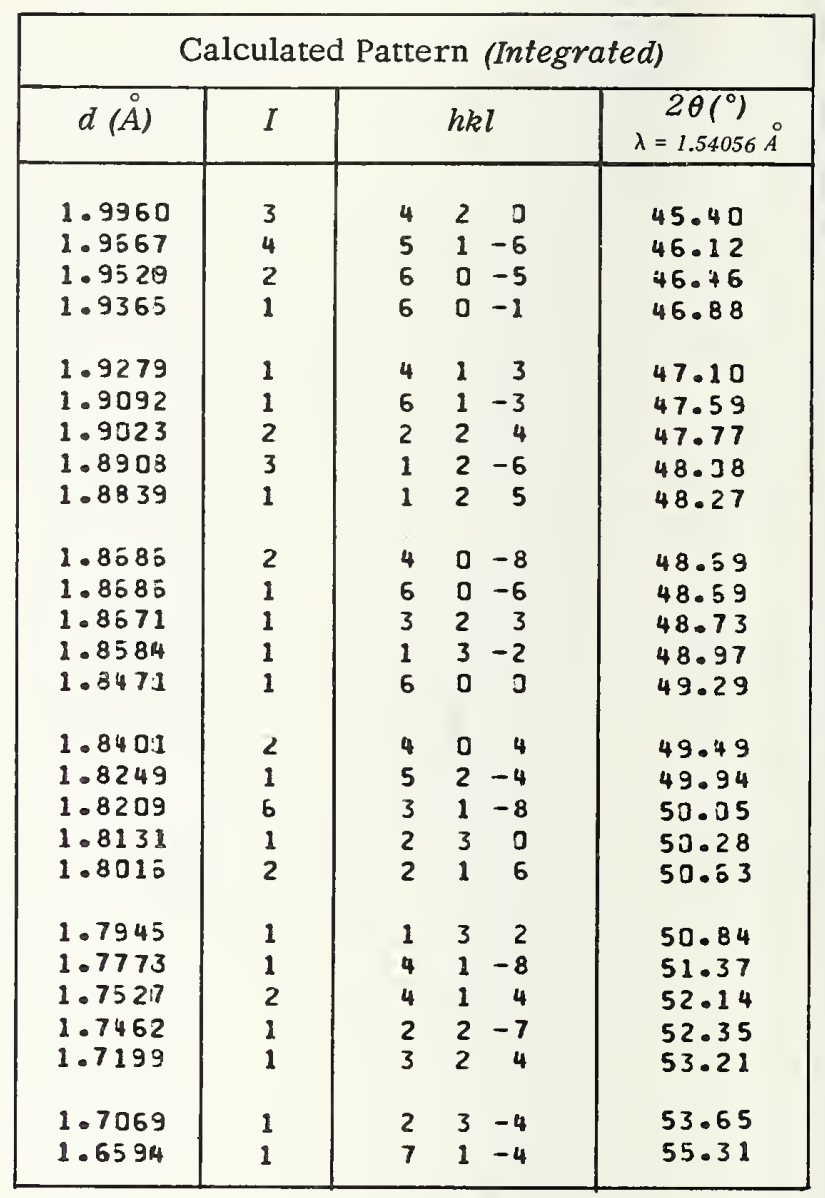


Structure

Cubic, $F \overline{4} 3 \mathrm{~m}$ (216), $\mathrm{Z}=4$ [Harris and Yakel。 1966].

\section{Lattice parameters}

$a=14.024 \pm 0.005 \AA$

(published value, $14.023 \AA$ [ibid.])

\section{Atomic positions}

The parameters used are those in table 2 [ibid.]

\section{Scattering factors}

$\mathrm{Be}^{2+}, \mathrm{Ca}^{2+}$ [Cromer and Waber, 1964]

$0^{-}[3.3 .1 \mathrm{~A}]$

\section{Thermal parameters}

Overall temperature factor $=0.41679$ [Harris and Yakel, 1966]

\section{Density}

(calculated) $2.64 \mathrm{~g} / \mathrm{cm}^{3}$ [ibid.]

\section{Scale factor}

$85.75 \times 10^{4}$

\section{Reference}

Cromer, D.T. and J.T. Waber (1965). Scattering factors computed from relativistic Dirac-slater wave functions, Acta Cryst. 18, 104-109.

Harris, L. A. and H. L. Yakel (1966). The crystal structure of calcium beryllate, $\mathrm{Ca}_{1} \mathrm{Be}_{1} \mathrm{O}_{28}$, Acta Cryst. 20, 295-301.

\begin{tabular}{|c|c|c|c|c|c|c|}
\hline \multicolumn{7}{|c|}{ Calculated Pattern (Peak heights) } \\
\hline$d(\AA)$ & $I$ & & $h k$ & & & $\begin{array}{c}2 \theta\left({ }^{\circ}\right) \\
\lambda=1.54056 \AA\end{array}$ \\
\hline 8.10 & 100 & 1 & 1 & 1 & & 10.92 \\
\hline 7.01 & 26 & 2 & 0 & 0 & & 12.62 \\
\hline 4.96 & 34 & 2 & 2 & 0 & & 17.88 \\
\hline 4.23 & 6 & 3 & 1 & 1 & & 21.00 \\
\hline 4.05 & 3 & 2 & 2 & 2 & & 21.94 \\
\hline 3.51 & 40 & 4 & 0 & 0 & & 25.38 \\
\hline 3.218 & 13 & 3 & 3 & 1 & & 27.70 \\
\hline 3.136 & 15 & 4 & 2 & 0 & & 28.44 \\
\hline 2.863 & 21 & 4 & 2 & 2 & & 31.22 \\
\hline 2.699 & 19 & 3 & 3 & 3 & + & 33.16 \\
\hline 2.479 & 39 & 4 & 4 & 0 & & 36.20 \\
\hline 2.371 & 3 & 5 & 3 & 1 & & 37.92 \\
\hline 2.338 & 38 & 4 & 4 & 2 & + & 38.48 \\
\hline 2.217 & 3 & 6 & 2 & 0 & & 40.66 \\
\hline 2.139 & 1 & 5 & 3 & 3 & & 42.22 \\
\hline 2.114 & 4 & 6 & 2 & 2 & & 42.74 \\
\hline 2.024 & 51 & 4 & 4 & 4 & & 44.74 \\
\hline 1.9641 & 14 & 5 & 5 & $i$ & + & 46.18 \\
\hline 1.9450 & 4 & 6 & 4 & 0 & & 46.66 \\
\hline 1.8740 & 6 & 6 & 4 & 2 & & 48.54 \\
\hline 1.8261 & 9 & 7 & 3 & 1 & + & 49.90 \\
\hline 1.7527 & 17 & 8 & 0 & 0 & & 52.14 \\
\hline 1.7131 & 7 & 7 & 3 & 3 & & 53.44 \\
\hline 1.7008 & 7 & 8 & 2 & 0 & & 53.86 \\
\hline 1.6527 & 18 & 8 & 2 & 2 & 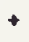 & 55.56 \\
\hline 1.6195 & 4 & 5 & 5 & 5 & + & 56.80 \\
\hline 1.6086 & 3 & 6 & 6 & 2 & & 57.22 \\
\hline 1.5681 & 6 & 8 & 4 & 0 & & 58.84 \\
\hline 1.5392 & 1 & 9 & $i$ & 1 & & 60.06 \\
\hline 1.5299 & 1 & 8 & 4 & 2 & & 60.46 \\
\hline 1.4951 & 1 & 6 & 6 & 4 & & 62.02 \\
\hline 1.4313 & 4 & 8 & 4 & 4 & & 65.12 \\
\hline 1.4094 & 1 & 7 & 7 & 1 & + & 66.26 \\
\hline 1.4023 & 3 & 8 & 6 & 0 & & 66.64 \\
\hline 1.3750 & 2 & 8 & 6 & 2 & & 68.14 \\
\hline 1.3558 & 6 & 9 & 5 & 1 & + & 69.24 \\
\hline 1.3493 & 2 & 6 & 6 & 6 & + & 69.62 \\
\hline 1.3076 & 1 & 9 & 5 & 3 & & 72.18 \\
\hline 1.3020 & 3 & 10 & 4 & 0 & & 72.54 \\
\hline 1.2395 & 6 & 8 & 8 & 0 & & 76.84 \\
\hline 1.2253 & 2 & 9 & 5 & 5 & + & 77.90 \\
\hline 1.2206 & 4 & 8 & 8 & 2 & + & 78.26 \\
\hline 1.1895 & 1 & 9 & 7 & 3 & + & 80.72 \\
\hline 1.1853 & 6 & 10 & 6 & 2 & & 81.06 \\
\hline 1.1687 & 1 & 12 & 0 & 0 & & 82.46 \\
\hline 1.1568 & 1 & 7 & 7 & 7 & + & 83.50 \\
\hline $\begin{array}{l}1.1376 \\
1.1087\end{array}$ & 2 & $\begin{array}{l}12 \\
12\end{array}$ & $\begin{array}{l}2 \\
4\end{array}$ & $\begin{array}{l}2 \\
0\end{array}$ & + & $\begin{array}{l}85.24 \\
88.02\end{array}$ \\
\hline $\begin{array}{l}1.0984 \\
1.0951\end{array}$ & $\begin{array}{l}1 \\
1 \\
5\end{array}$ & $\begin{array}{r}9 \\
12\end{array}$ & $\begin{array}{l}9 \\
4\end{array}$ & $\begin{array}{l}1 \\
2\end{array}$ & + & $\begin{array}{l}89.06 \\
89.40\end{array}$ \\
\hline
\end{tabular}


Beryllium Calcium 0xide, $\mathrm{Be}_{17} \mathrm{Ca}_{12} \mathrm{O}_{29}$ (cubic) - continued

\begin{tabular}{|c|c|c|c|c|c|c|}
\hline \multicolumn{7}{|c|}{ Calculated Pattern (Peak heights) } \\
\hline$d(\AA)$ & $I$ & & $h k l$ & & & $\begin{array}{c}2 \theta\left(^{\circ}\right) \\
\lambda=1.54056 \AA\end{array}$ \\
\hline 1.0820 & 1 & 10 & 8 & 2 & & 90.78 \\
\hline 1.0724 & 1 & II & 5 & 5 & & 91.82 \\
\hline 1.0694 & 2 & 10 & 6 & 6 & & 92.16 \\
\hline 1.0570 & 4 & 12 & 4 & 4 & & 93.56 \\
\hline 1.0481 & 1 & 11 & 7 & 3 & & 94.60 \\
\hline 1.0333 & 3 & 12 & 6 & 2 & & 96.32 \\
\hline 1.0255 & 1 & 13 & 3 & 3 & + & 97.38 \\
\hline 1.0017 & 1 & 12 & 6 & 4 & & 100.52 \\
\hline .9819 & 2 & 10 & 10 & 2 & + & 103.34 \\
\hline .9632 & 2 & 12 & 8 & 2 & + & 106.20 \\
\hline .9542 & 1 & 10 & 10 & 4 & + & 107.66 \\
\hline .9477 & 1 & 11 & 7 & 7 & + & 108.74 \\
\hline .9308 & 1 & 13 & 7 & 3 & + & 111.70 \\
\hline .9286 & 1 & 14 & 4 & 4 & & 112.10 \\
\hline .9129 & 4 & 10 & 10 & 6 & + & 115.08 \\
\hline $.897 \varepsilon$ & 1 & 12 & 8 & 6 & & 118.18 \\
\hline .8852 & 1 & 15 & 5 & 1 & + & 120.96 \\
\hline .8765 & 1 & 16 & 0 & 0 & & 123.00 \\
\hline .8697 & 2 & 12 & 10 & 4 & + & 124.66 \\
\hline .8631 & $i$ & 16 & 2 & 2 & + & 126.36 \\
\hline .8566 & 1 & 14 & 6 & 6 & & 128.10 \\
\hline .8503 & 1 & 16 & 4 & 0 & & 129.88 \\
\hline .8457 & 2 & 15 & 5 & 5 & + & 131.24 \\
\hline .8264 & 2 & 16 & 4 & 4 & + & 137.54 \\
\hline .8207 & 2 & 12 & 12 & 2 & + & 139.62 \\
\hline .8151 & 1 & 16 & 6 & 2 & + & 141.82 \\
\hline .8110 & 1 & 17 & 3 & 1 & + & 143.52 \\
\hline .8097 & 4 & 14 & 10 & 2 & + & 144.10 \\
\hline .8043 & 1 & 12 & 12 & 4 & & 146.54 \\
\hline .8004 & 1 & 17 & 3 & 3 & + & 148.46 \\
\hline .7840 & $?$ & 16 & 8 & 0 & & 158.56 \\
\hline .7791 & 4 & 16 & 8 & 2 & + & 162.74 \\
\hline
\end{tabular}

\begin{tabular}{|c|c|c|c|c|c|}
\hline \multicolumn{6}{|c|}{ Calculated Pattern (Integrated) } \\
\hline$d(\stackrel{\circ}{A})$ & $I$ & & $h k l$ & & $\begin{array}{c}2 \theta\left({ }^{\circ}\right) \\
\lambda=1.54056 \AA\end{array}$ \\
\hline 8.10 & 100 & 1 & 1 & 1 & 10.92 \\
\hline 7.01 & 27 & 2 & 0 & 0 & 12.61 \\
\hline 4.96 & 38 & 2 & 2 & 0 & 17.87 \\
\hline 4.23 & 7 & 3 & 1 & 1 & 20.99 \\
\hline 4.05 & 4 & 2 & 2 & 2 & 21.94 \\
\hline 3.51 & 51 & 4 & 0 & 0 & $25 \cdot 38$ \\
\hline 3.217 & 17 & 3 & 3 & 1 & 27.70 \\
\hline 3.136 & 19 & 4 & 2 & 0 & 28.44 \\
\hline 2.863 & 29 & 4 & 2 & 2 & 31.22 \\
\hline 2.699 & 11 & 5 & 1 & 1 & 33.17 \\
\hline 2.699 & 16 & 3 & 3 & 3 & 33.17 \\
\hline 2.479 & 56 & 4 & 4 & 0 & 36.20 \\
\hline 2.370 & 4 & 5 & 3 & 1 & 37.92 \\
\hline 2.337 & 4 & 6 & 0 & 0 & 38.48 \\
\hline 2.337 & 51 & 4 & 4 & 2 & 38.48 \\
\hline 2.217 & 5 & 6 & 2 & 0 & 40.65 \\
\hline 2.139 & 1 & 5 & 3 & 3 & 42.22 \\
\hline 2.114 & 6 & 6 & 2 & 2 & 42.73 \\
\hline 2.024 & 79 & 4 & 4 & 4 & 44.73 \\
\hline 1.9637 & 3 & 7 & 1 & 1 & $46 \cdot 19$ \\
\hline 1.9637 & 21 & 5 & 5 & 1 & 46.19 \\
\hline 1.9448 & 5 & 6 & 4 & 0 & 46.67 \\
\hline 1.8740 & 10 & 6 & 4 & 2 & 48.54 \\
\hline 1.8253 & 11 & 7 & 3 & 1 & 49.91 \\
\hline 1.8258 & 4 & 5 & 5 & 3 & 49.91 \\
\hline 1.7530 & 29 & 8 & 0 & 0 & 52.13 \\
\hline 1.7133 & 12 & 7 & 3 & 3 & 53.43 \\
\hline 1.7007 & 12 & 8 & 2 & 0 & 53.86 \\
\hline 1.6527 & 22 & 8 & 2 & 2 & 55.56 \\
\hline 1.6527 & 8 & 6 & 6 & 0 & 55.56 \\
\hline 1.6193 & 2 & 7 & 5 & 1 & 56.81 \\
\hline 1.6193 & 4 & 5 & 5 & 5 & 56.81 \\
\hline 1.6087 & 6 & 6 & 6 & 2 & 57.22 \\
\hline 1.5679 & 11 & 8 & 4 & 0 & 58.85 \\
\hline 2.5393 & 1 & 9 & 1 & 1 & 60.05 \\
\hline 1.5301 & 2 & 8 & 4 & 2 & 60.45 \\
\hline 1.4950 & 3 & 6 & 6 & 4 & 62.03 \\
\hline 1.4313 & 8 & 8 & 4 & 4 & 65.12 \\
\hline 1.4095 & 1 & 7 & 7 & 1 & 66.26 \\
\hline 1.4095 & 1 & 7 & 5 & 5 & 66.26 \\
\hline 1.4024 & 5 & 8 & 6 & 0 & 66.63 \\
\hline 1.3752 & 3 & 8 & 6 & 2 & 68.13 \\
\hline 1.3557 & 7 & 9 & 5 & 1 & 69.24 \\
\hline 1.3557 & 4 & 7 & 7 & 3 & 69.24 \\
\hline 1.3495 & 2 & 10 & 2 & 2 & 69.61 \\
\hline 1.3495 & 2 & 6 & 6 & 6 & 69.61 \\
\hline 1.3077 & 2 & 9 & 5 & 3 & 72.17 \\
\hline 1.3021 & 6 & 10 & 4 & 0 & 72.54 \\
\hline 1.2802 & 1 & 10 & 4 & 2 & 73.98 \\
\hline 1.2645 & 1 & 7 & 7 & 5 & 75.06 \\
\hline
\end{tabular}


Beryllium Calcium Oxide, $\mathrm{Be}_{17} \mathrm{Ca}_{12} \mathrm{O}_{29}$ (cubic) - continued

\begin{tabular}{|c|c|c|c|c|c|}
\hline \multicolumn{6}{|c|}{ Calculated Pattern (Integrated) } \\
\hline$d(\stackrel{\circ}{A})$ & $I$ & & $h k l$ & & $\begin{array}{c}2 \theta\left(^{\circ}\right) \\
\lambda=1.54056 \AA\end{array}$ \\
\hline 1.2396 & 12 & 8 & 8 & 0 & 76.84 \\
\hline 1.2253 & 1 & 11 & 3 & 1 & 77.90 \\
\hline 1.2253 & 3 & 9 & 5 & 5 & 77.90 \\
\hline 1.2206 & 6 & 8 & 8 & 2 & 78.26 \\
\hline 1.2206 & 3 & 10 & 4 & 4 & 78.26 \\
\hline 1.1895 & 1 & 11 & 3 & 3 & 80.72 \\
\hline 1.1895 & 1 & 9 & 7 & 3 & 80.72 \\
\hline 1.1852 & 12 & 10 & 6 & 2 & 81.07 \\
\hline 1.1687 & 2 & 12 & 0 & 0 & 82.46 \\
\hline 1.1567 & 1 & 11 & 5 & 1 & 83.51 \\
\hline 1.1567 & 2 & 7 & 7 & 7 & 83.51 \\
\hline 1.1375 & 4 & 12 & 2 & 2 & 85.25 \\
\hline 1.1375 & 1 & 10 & 6 & 4 & 85.25 \\
\hline 1.1087 & 2 & 12 & 4 & 0 & 88.02 \\
\hline 1.0984 & 1 & 9 & 9 & 1 & 89.05 \\
\hline 1.0951 & 7 & 12 & 4 & 2 & 89.40 \\
\hline 1.0951 & 5 & 8 & 8 & 6 & 89.40 \\
\hline 1.0820 & 2 & 10 & 8 & 2 & 90.78 \\
\hline 1.0724 & 1 & 11 & 5 & 5 & 91.82 \\
\hline 1.0693 & 4 & 10 & 6 & 6 & 92.17 \\
\hline 1.0571 & 10 & 12 & 4 & 4 & 93.55 \\
\hline 1.0482 & 2 & 11 & 7 & 3 & 94.59 \\
\hline 1.0339 & 7 & 12 & 6 & 2 & 96.33 \\
\hline 1.0255 & 2 & 13 & 3 & 3 & 97.37 \\
\hline 1.0255 & 1 & 9 & 9 & 5 & 97.37 \\
\hline 1.0121 & 1 & 8 & 8 & 8 & 99.12 \\
\hline 1.0017 & 2 & 12 & 6 & 4 & 100.52 \\
\hline .9819 & 2 & 14 & 2 & 2 & 103.35 \\
\hline .9819 & 3 & 10 & 10 & 2 & 103.35 \\
\hline .9632 & 1 & 14 & 4 & 0 & $106 \cdot 21$ \\
\hline .9632 & 4 & 12 & 8 & 2 & 106.21 \\
\hline .9542 & 3 & 10 & 10 & 4 & 107.65 \\
\hline .9542 & 1 & 14 & 4 & 2 & 107.65 \\
\hline .9476 & 1 & 13 & 7 & 1 & 108.75 \\
\hline .9476 & 1 & 13 & 5 & 5 & 108.75 \\
\hline .9476 & 2 & 11 & 7 & 7 & 108.75 \\
\hline .9370 & 1 & 12 & 8 & 4 & 110.58 \\
\hline .9308 & 2 & 13 & 7 & 3 & 111.69 \\
\hline .9308 & 1 & 15 & 1 & 1 & 111.69 \\
\hline .9308 & 1 & 11 & 9 & 5 & 111.69 \\
\hline .9288 & 1 & 14 & 4 & 4 & 112.07 \\
\hline .9207 & 1 & 14 & 6 & 0 & 113.57 \\
\hline .9129 & 3 & 14 & 6 & 2 & 115.08 \\
\hline .9129 & 8 & 10 & 10 & 6 & 115.08 \\
\hline .8973 & 3 & 12 & 8 & 6 & 118.18 \\
\hline .8852 & 2 & 15 & 5 & 1 & 120.96 \\
\hline .8852 & 1 & 11 & 11 & 3 & 120.96 \\
\hline .8765 & 3 & 16 & 0 & 0 & 123.00 \\
\hline .8714 & 1 & 15 & 5 & 3 & 124.24 \\
\hline .8697 & 3 & 12 & 10 & 4 & 124.66 \\
\hline
\end{tabular}

\begin{tabular}{|c|c|c|c|c|c|}
\hline \multicolumn{6}{|c|}{ Calculated Pattern (Integrated) } \\
\hline$d(\stackrel{\circ}{A})$ & $I$ & & $h k l$ & & $\begin{array}{c}2 \theta\left(^{\circ}\right) \\
\lambda=1.54056 \AA\end{array}$ \\
\hline .8697 & 2 & 14 & 8 & 0 & 124.66 \\
\hline .8631 & 3 & 16 & 2 & 2 & 126.36 \\
\hline .8631 & 1 & 14 & 8 & 2 & 126.36 \\
\hline .8582 & 1 & 13 & 7 & 7 & 127.66 \\
\hline .8566 & 3 & 14 & 6 & 6 & 128.10 \\
\hline .8503 & 4 & 16 & 4 & 0 & 129.88 \\
\hline .8457 & 2 & 13 & 9 & 5 & 131.24 \\
\hline .8457 & 3 & 15 & 5 & 5 & 131.24 \\
\hline .8441 & 2 & 16 & 4 & 2 & 131.71 \\
\hline .8381 & 1 & 12 & 10 & 6 & 133.59 \\
\hline .8336 & 2 & 15 & 7 & 3 & 135.03 \\
\hline .8264 & 5 & 16 & 4 & 4 & 137.54 \\
\hline .8264 & 1 & 12 & 12 & 0 & 137.54 \\
\hline .8207 & 8 & 12 & 12 & 2 & 139.63 \\
\hline .8207 & 2 & 16 & 6 & 0 & 139.63 \\
\hline .81 .51 & 3 & 16 & 6 & 2 & 141.81 \\
\hline .8151 & 2 & 14 & 8 & 6 & 141.81 \\
\hline .8110 & 1 & 13 & 11 & 3 & 143.52 \\
\hline .8110 & 2 & 17 & 3 & 1 & 143.52 \\
\hline .8110 & 2 & 15 & 7 & 5 & 143.52 \\
\hline .8097 & 15 & 14 & 10 & 2 & 144.11 \\
\hline .8097 & 2 & 10 & 10 & 10 & 144.11 \\
\hline .8043 & 3 & 12 & 12 & 4 & 146.54 \\
\hline .8004 & 2 & 15 & 9 & 1 & 148.47 \\
\hline .8004 & 2 & 17 & 3 & 3 & 148.47 \\
\hline .7939 & 1 & 14 & 10 & 4 & 151.95 \\
\hline .7902 & 1 & 15 & 9 & 3 & 154.24 \\
\hline .7840 & 14 & 16 & 8 & 0 & 158.56 \\
\hline .7803 & 2 & 17 & 5 & 3 & 161.60 \\
\hline .7803 & 1 & 15 & 7 & 7 & 161.60 \\
\hline .7791 & 4 & 12 & 12 & 6 & 162.73 \\
\hline .7791 & 1 & 18 & 0 & 0 & 162.73 \\
\hline .7791 & 8 & 14 & 8 & 8 & 162.73 \\
\hline .7791 & 17 & 16 & 8 & 2 & 162.73 \\
\hline
\end{tabular}


Beryllium Niobium, $\mathrm{Be}_{2} \mathrm{Nb}$ (cubic)

\section{Structure}

Cubic, Fd3m(227), $\mathrm{Z}=8$ [Sands et al., 1959]

\section{Lattice parameters}

$a=6.535 \pm 0.002 \AA$ [ibid.]

\section{Scattering factors}

$\mathrm{Nb}^{\circ}[3.3 .1 \mathrm{~B}]$

$\mathrm{Be}^{\mathrm{O}}[3.3 .1 \mathrm{~A}]$

\section{Thermal parameters}

Isotropic [Sands et al., 1959]

\section{Density}

(calculated) $5.28 \mathrm{~g} / \mathrm{cm}^{3}$ [ibid.]

\section{Scale factor}

$11.88 \times 10^{4}$

\section{Additional patterns}

1. PDF 12-593 [Wright Air Development Center, Dayton, Ohio].

\section{Reference}

Sands. D.E., A. Zalkin, and O.H. Krikorian (1959). The crystal structures of $\mathrm{NbBe}_{2}$ and $\mathrm{NbBe}_{3}$, Acta Cryst. 12, 461-464.

\begin{tabular}{|c|c|c|c|c|c|}
\hline \multicolumn{6}{|c|}{ Calculated Pattern (Peak heights) } \\
\hline$d(\AA)$ & $I$ & & $h k$ & & $\begin{array}{c}2 \theta\left({ }^{\circ}\right) \\
\lambda=1.5405 \mathrm{~A}\end{array}$ \\
\hline $\begin{array}{l}3.773 \\
2.311 \\
1.9705 \\
1.6337 \\
1.4990\end{array}$ & $\begin{array}{r}100 \\
78 \\
54 \\
8 \\
12\end{array}$ & $\begin{array}{l}1 \\
2 \\
3 \\
4 \\
3\end{array}$ & $\begin{array}{l}1 \\
2 \\
1 \\
0 \\
3\end{array}$ & $\begin{array}{l}1 \\
0 \\
1 \\
0 \\
1\end{array}$ & $\begin{array}{l}23.56 \\
38.94 \\
46.02 \\
56.26 \\
61.84\end{array}$ \\
\hline $\begin{array}{l}1.3339 \\
1.2576 \\
1.1552 \\
1.1046 \\
1.0332\end{array}$ & $\begin{array}{r}18 \\
11 \\
6 \\
7 \\
7\end{array}$ & $\begin{array}{l}4 \\
5 \\
4 \\
5 \\
6\end{array}$ & $\begin{array}{l}2 \\
1 \\
4 \\
3 \\
2\end{array}$ & $\begin{array}{l}2 \\
1 \\
0 \\
1 \\
0\end{array}$ & $\begin{array}{l}70.54 \\
75.54 \\
83.64 \\
88.42 \\
96.40\end{array}$ \\
\hline $\begin{array}{r}0.9966 \\
.9432 \\
.9151 \\
.8733 \\
.8508\end{array}$ & $\begin{array}{r}3 \\
1 \\
4 \\
10 \\
9\end{array}$ & $\begin{array}{l}5 \\
4 \\
7 \\
6 \\
7\end{array}$ & $\begin{array}{l}3 \\
4 \\
1 \\
4 \\
3\end{array}$ & $\begin{array}{l}3 \\
4 \\
1+ \\
2 \\
1+\end{array}$ & $\begin{array}{l}101.22 \\
109.50 \\
114.64 \\
123.78 \\
129.74\end{array}$ \\
\hline $\begin{array}{l}.8169 \\
.7984\end{array}$ & $\begin{array}{l}2 \\
2\end{array}$ & $\begin{array}{l}8 \\
7\end{array}$ & $\begin{array}{l}0 \\
3\end{array}$ & $\begin{array}{l}0 \\
3\end{array}$ & $\begin{array}{l}141.10 \\
149.50\end{array}$ \\
\hline
\end{tabular}

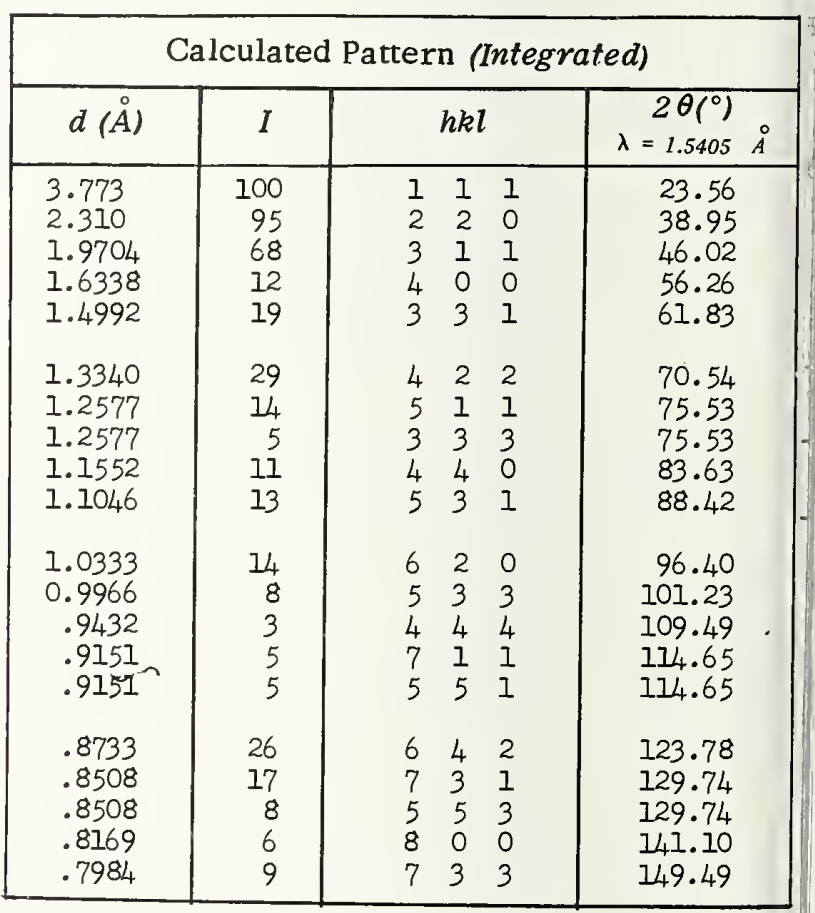


Cadmium Nitrate Tetrahydrate, $\mathrm{Cd}\left(\mathrm{NO}_{3}\right)_{2} \cdot 4 \mathrm{H}_{2} \mathrm{O}$ (orthorhombic)

Structure

Orthorhombic, Fdd2 (43), $\mathrm{Z}=8$ [Matković et a1., 1966]

\section{Lattice parameters}

$a=5.828 \pm 0.005, b=25.86 \pm 0.03, c=11.002 \pm$ $0.005 \AA$ [ibid.]

Scattering factors

$\mathrm{O}^{-1}, \mathrm{~N}^{\circ}[3.3 .1 \mathrm{~A}] ; \mathrm{Cd}^{\circ}[3.3 .1 \mathrm{~B}]$

\section{Thermal parameters}

Isotropic.Cd(1) $2.2 ; O(2) 3.0 ; O(3) 5.5 ;$ $O(4) 4.3 ; O(5) 4.7 ; O(6) 3.7 ; N(7) 3.1$

\section{Density}

(calculated) $2.47 \mathrm{~g} / \mathrm{cm}^{3}$ [Matković et al., 1966]

\section{Scale factor}

$$
67.04 \times 10^{4}
$$

\section{Additional patterns}

1.PDF card 1-0242 [Hanawalt et al. 1938]

\section{Reference}

Hanawalt, J.D., H.W. Rinn, and L.K. Frevel (1938). Chemical analysis by $x$-ray diffraction, Ind. Eng. Chem. Anal. Ed. 10, $457-513$.

Matković,B., B. Ribar, B.Zelenko, and S.W. Peterson (1966). Refinement of the structure of $\mathrm{Cd}\left(\mathrm{NO}_{3}\right)_{2} \cdot 4 \mathrm{H}_{2} \mathrm{O}$, Acta Crvst. 21, 719-725.

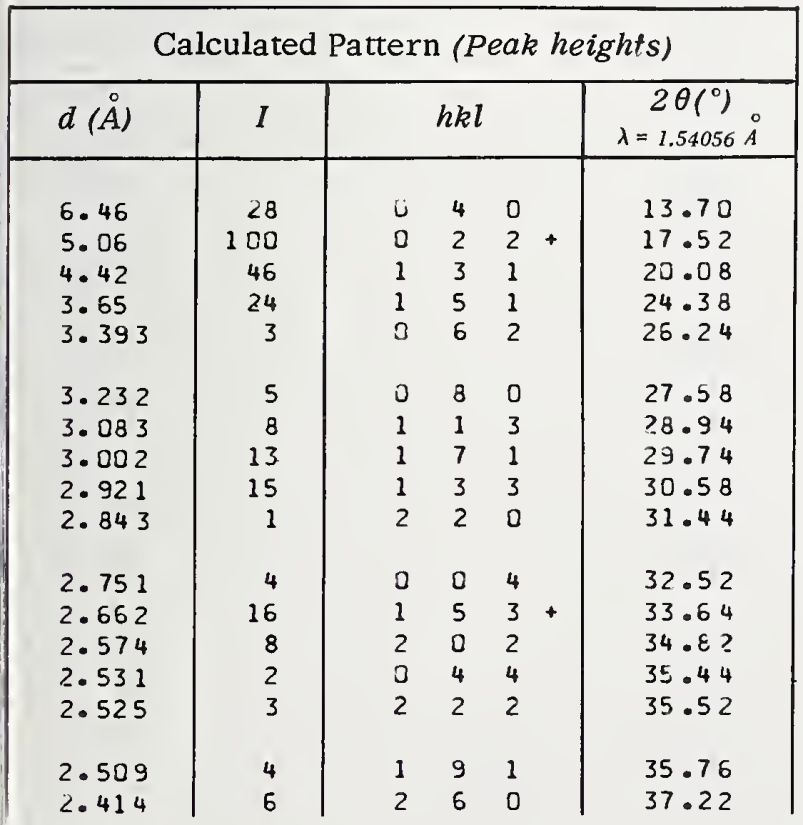

\begin{tabular}{|c|c|c|c|c|c|c|}
\hline \multicolumn{7}{|c|}{ Calculated Pattern (Peak heights) } \\
\hline$d(\stackrel{\circ}{)}$ & $I$ & \multicolumn{4}{|c|}{$h k l$} & $2 \theta\left({ }^{\circ}\right)$ \\
\hline 2.393 & 8 & 2 & 4 & 2 & & 37.56 \\
\hline 2.377 & 3 & 1 & 7 & 3 & & 37.82 \\
\hline 2.340 & 9 & 0 & 10 & 2 & & 38.44 \\
\hline 2.211 & 1 & 2 & 6 & 2 & & 43.78 \\
\hline 2.155 & 1 & 3 & 12 & 0 & & 41.88 \\
\hline 2.139 & 7 & 1 & 11 & 1 & & 42.22 \\
\hline 2.108 & 5 & 1 & 9 & 3 & & 42.86 \\
\hline 2.095 & 5 & 0 & 8 & 4 & & 43.14 \\
\hline 2.052 & 2 & 1 & 1 & 5 & & 44.10 \\
\hline 2.014 & 9 & 2 & 8 & 2 & & 44.98 \\
\hline 2.002 & 5 & 1 & 3 & 5 & & 45.26 \\
\hline 1.9763 & 7 & 2 & 2 & 4 & & 45.88 \\
\hline 1.9341 & 3 & 2 & 10 & 3 & & 46.94 \\
\hline 1.9126 & 2 & 1 & 5 & 5 & & 47.50 \\
\hline 1.9080 & 3 & 3 & 1 & 1 & & 47.62 \\
\hline 1.8740 & 3 & 1 & 11 & 3 & & 48.54 \\
\hline 1.8675 & 4 & 3 & 3 & 1 & & 48.72 \\
\hline 1.8553 & 2 & 1 & 13 & 1 & & 49.06 \\
\hline 1.8145 & 6 & 2 & 6 & 4 & + & 50.24 \\
\hline 1.7984 & 3 & 1 & 7 & 5 & & 50.72 \\
\hline 1.7938 & 3 & 3 & 5 & 1 & & 50.86 \\
\hline 1.7509 & 1 & 0 & 14 & 2 & & 52.20 \\
\hline 1.7131 & 2 & 3 & 1 & 3 & & 53.44 \\
\hline 1.6961 & 2 & 0 & 12 & 4 & & 54.02 \\
\hline 1.6874 & 1 & 0 & 5 & 6 & & 54.32 \\
\hline 1.6834 & 2 & 3 & 3 & 3 & & 54.46 \\
\hline 1.6749 & 2 & 1 & 13 & 3 & + & 54.76 \\
\hline 1.6527 & 1 & 2 & 12 & 2 & & 55.56 \\
\hline 1.6348 & 1 & 1 & 15 & 1 & & 56.22 \\
\hline 1.6295 & 2 & 3 & 5 & 3 & & 55.42 \\
\hline 1.5824 & 1 & 2 & 10 & 4 & & 58.26 \\
\hline 1.5599 & 1 & 2 & 14 & 0 & & 59.18 \\
\hline 1.5560 & 1 & 3 & 7 & 3 & & 59.32 \\
\hline 1.5523 & 1 & 2 & 0 & 6 & & 59.50 \\
\hline 1.5485 & 1 & 1 & 11 & 5 & & 59.66 \\
\hline 1.5092 & 1 & 2 & 4 & 6 & & 61.38 \\
\hline 1.5370 & 1 & 1 & 15 & 3 & & 61.48 \\
\hline 1.4947 & 1 & 1 & 3 & 7 & + & 62.04 \\
\hline 1.4840 & 1 & 3 & 11 & 1 & & 62.54 \\
\hline 1.4560 & 1 & 1 & 5 & 7 & & 63.88 \\
\hline 1.4540 & 1 & 3 & 1 & 5 & & 63.98 \\
\hline 1.4305 & 1 & 1 & 13 & 5 & & 65.16 \\
\hline 1.3993 & $?$ & 2 & 8 & 6 & + & 66.80 \\
\hline 1.3934 & 1 & 0 & 16 & 4 & & 67.12 \\
\hline 1.3901 & 1 & 0 & 18 & 2 & & 67.30 \\
\hline 1.3690 & 1 & 2 & 16 & 2 & & 68.48 \\
\hline 1.3386 & 1 & 4 & 6 & 2 & & 70.26 \\
\hline 1.299 .3 & 1 & 3 & 9 & 5 & + & 72.72 \\
\hline 1.2376 & 1 & 4 & 10 & 2 & + & 76.90 \\
\hline 1.1951 & 1 & 4 & 8 & 4 & + & 80.26 \\
\hline
\end{tabular}


Cadmium Nitrate Tetrahydrate, $\mathrm{Cd}\left(\mathrm{NO}_{3}\right)_{2} \cdot 4 \mathrm{H}_{2} \mathrm{O}$ (orthorhombic) - continued

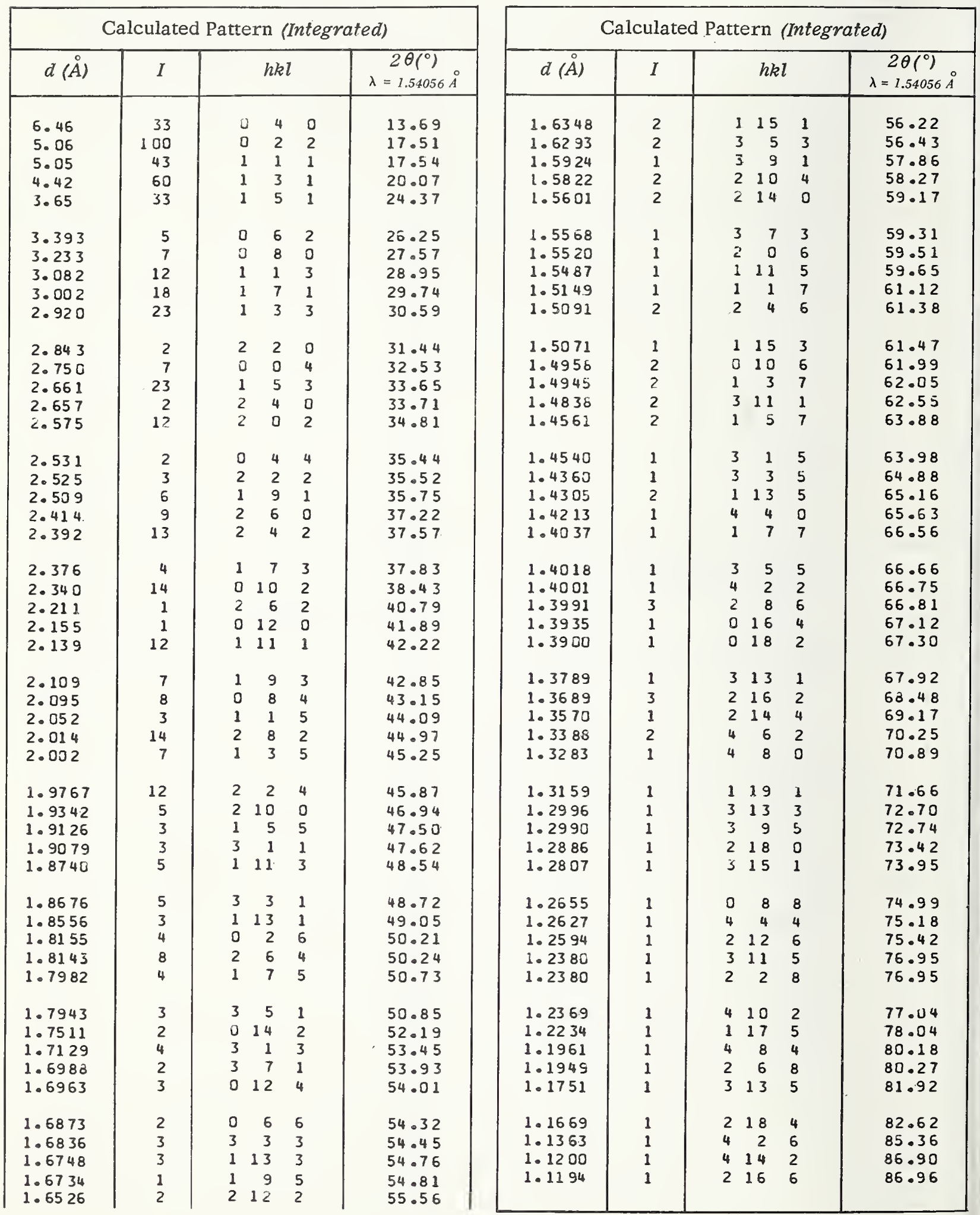


Calcium Phosphate, beta-pyro-, $\mathrm{Ca}_{2} \mathrm{P}_{2} \mathrm{O}_{7}$ (tetragonal)

Structure

Tetragona 1, $P 4_{1}$ (76), $\mathrm{Z}=8$ [Webb, 1966]

Lattice parameters

$a=6.684 \pm 0.006, c=24.145 \pm 0.015 \AA$ [ibid.] (published value, $c=24.144 \pm 0.015 \AA$ )

\section{Scattering factors}

$\mathrm{Ca}^{+3}, \mathrm{P}^{0}[3.3 .1 \mathrm{~A}]$

0 , an average of $0^{\circ}$ and $0^{-}[3.3 .1 \mathrm{~A}]$

\section{Thermal parameters}

Isotropic [Webb, 1966]

Density

(calculated) $3.128 \mathrm{~g} / \mathrm{cm}^{3}$ [ibid.]

\section{Scale factor}

$8.642 \times 10^{4}$

\section{Additional patterns}

1. PDF card 11-177 ISt. Pierre, Dept. of Mines, Canada, Tech. paper No.2, p.105. 1953]

\section{Reference}

Webb,N.C. (1966). The crystal structure of $\beta-\mathrm{Ca}_{2} \mathrm{P}_{2} \mathrm{O}_{7}$, Acta Cryst. 21, 942-948.

Calculated Pattern (Peak heights)

\begin{tabular}{|c|c|c|c|c|c|}
\hline$d(\stackrel{\circ}{A})$ & $I$ & \multicolumn{3}{|c|}{$h k l$} & $\begin{array}{c}2 \theta\left(^{\circ}\right) \\
\lambda=1.54056 \mathrm{~A}\end{array}$ \\
\hline $\begin{array}{l}6.037 \\
5.847 \\
4.726 \\
4.480 \\
4.401\end{array}$ & $\begin{array}{r}9 \\
4 \\
16 \\
2 \\
13\end{array}$ & $\begin{array}{l}0 \\
0 \\
1 \\
0 \\
1\end{array}$ & $\begin{array}{l}0 \\
1 \\
1 \\
1 \\
1\end{array}$ & $\begin{array}{l}4 \\
2 \\
0 \\
4 \\
2\end{array}$ & $\begin{array}{l}14.66 \\
15.14 \\
18.76 \\
19.80 \\
20.16\end{array}$ \\
\hline $\begin{array}{l}4.074 \\
3.720 \\
3.448 \\
3.378 \\
3.341\end{array}$ & $\begin{array}{r}5 \\
3 \\
2 \\
12 \\
42\end{array}$ & $\begin{array}{l}1 \\
1 \\
0 \\
1 \\
0\end{array}$ & $\begin{array}{l}1 \\
1 \\
1 \\
1 \\
2\end{array}$ & $\begin{array}{l}3 \\
4 \\
6 \\
5 \\
0\end{array}$ & $\begin{array}{l}21.80 \\
23.90 \\
25.82 \\
26.36 \\
26.66\end{array}$ \\
\hline $\begin{array}{l}3.309 \\
3.220 \\
3.087 \\
3.064 \\
3.017\end{array}$ & $\begin{array}{r}36 \\
56 \\
54 \\
7 \\
100\end{array}$ & $\begin{array}{l}0 \\
0 \\
0 \\
1 \\
0\end{array}$ & $\begin{array}{l}2 \\
2 \\
2 \\
1 \\
0\end{array}$ & $\begin{array}{l}1 \\
2 \\
3 \\
6 \\
8\end{array}+$ & $\begin{array}{l}26.92 \\
27.68 \\
28.90 \\
29.12 \\
29.58\end{array}$ \\
\hline $\begin{array}{l}2.990 \\
2.966 \\
2.923 \\
2.901 \\
2.901\end{array}$ & $\begin{array}{l}23 \\
37 \\
18 \\
35 \\
27\end{array}$ & $\begin{array}{l}2 \\
2 \\
0 \\
2 \\
1\end{array}$ & $\begin{array}{l}1 \\
1 \\
2 \\
1 \\
2\end{array}$ & $\begin{array}{l}0+ \\
1+ \\
4 \\
2+ \\
3+\end{array}$ & $\begin{array}{l}29.86 \\
30.10 \\
30.56 \\
30.80 \\
31.92\end{array}$ \\
\hline
\end{tabular}

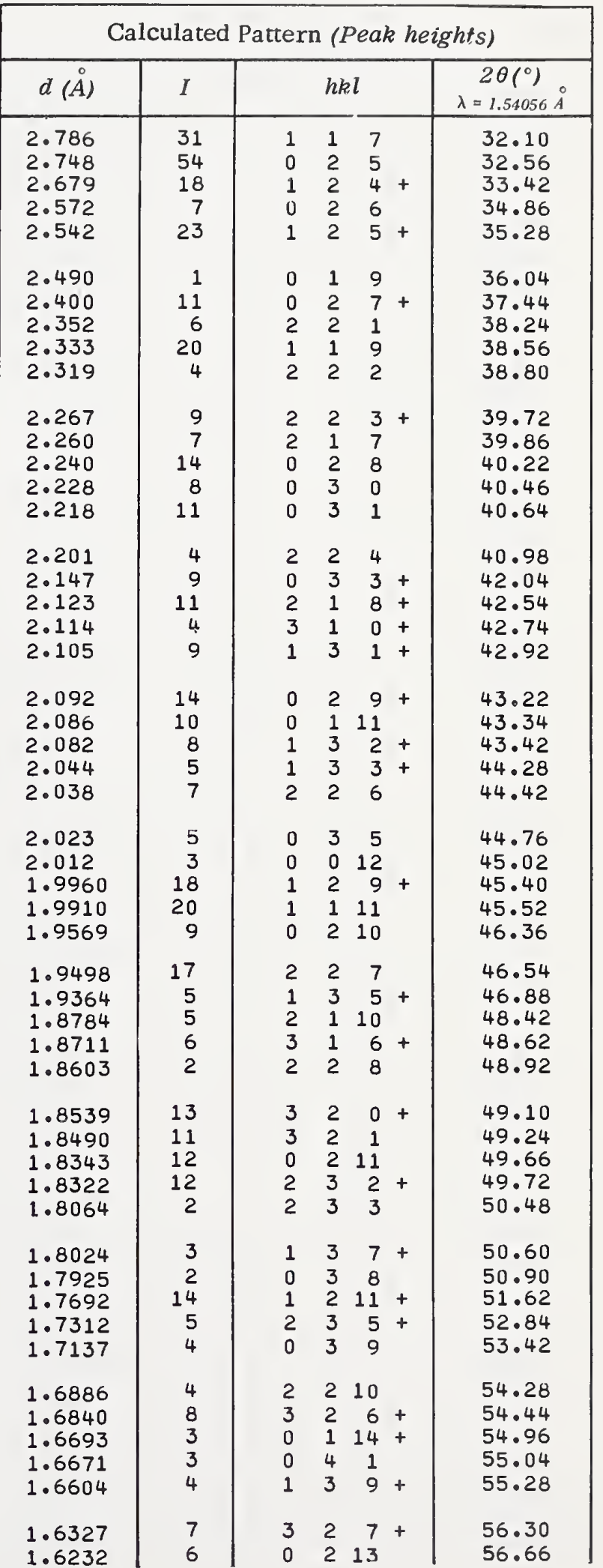


Calcium Phosphate, beta-pyro-, $\mathrm{Ca}_{2} \mathrm{P}_{2} \mathrm{O}_{7}$ (tetragonal) - continued

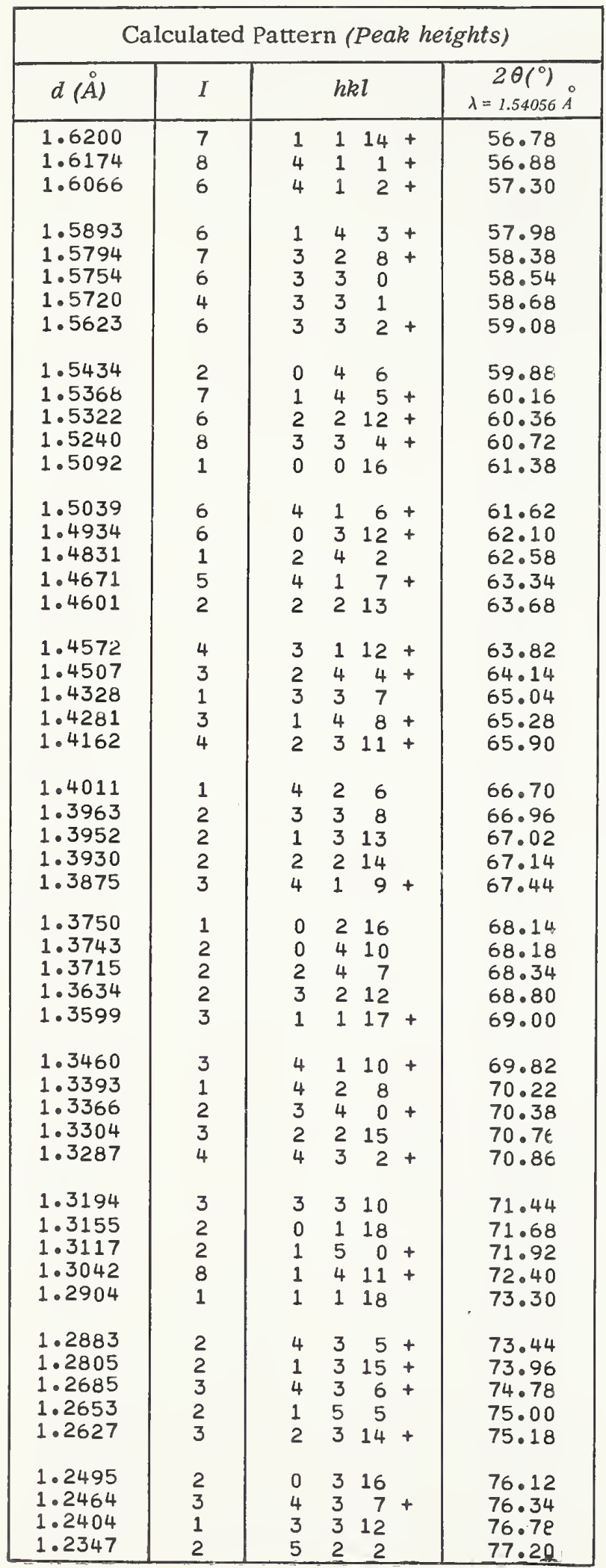

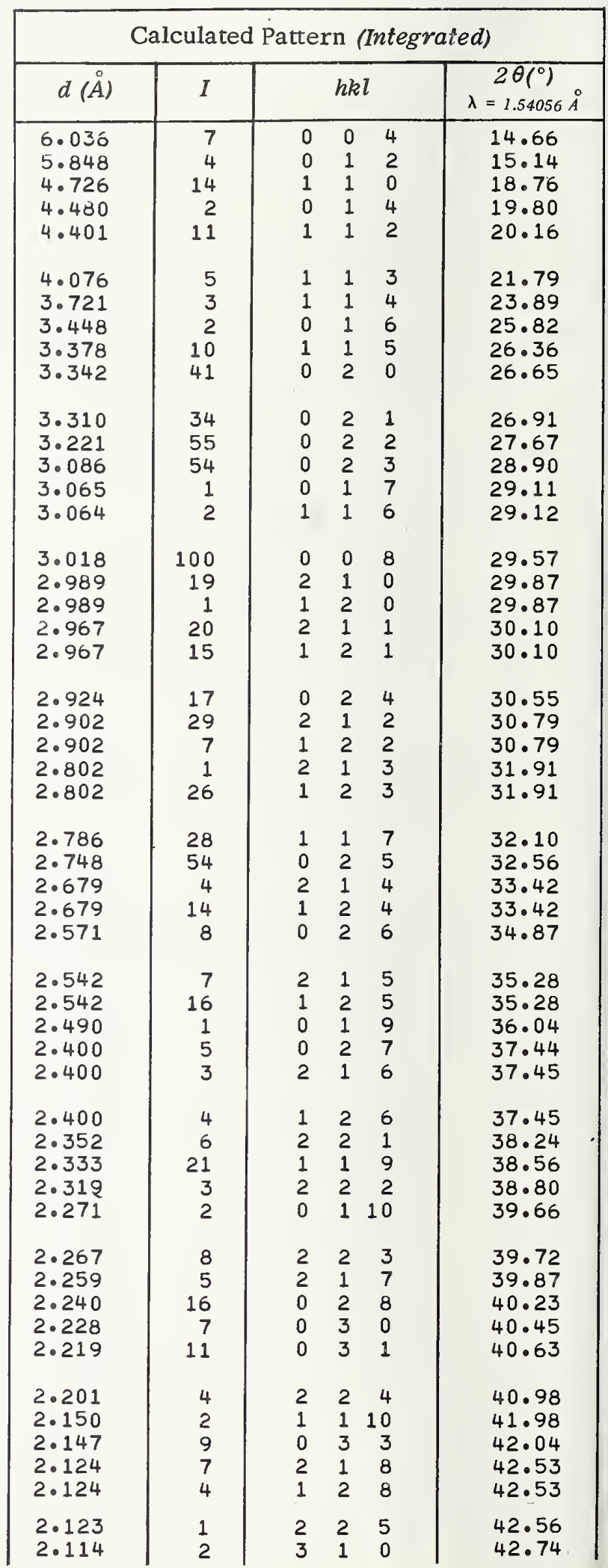


Calcium Phnsphate, beta-pyro-, $\mathrm{Ca}_{2} \mathrm{P}_{2} \mathrm{O}_{7}$ (tetragonal) - continued

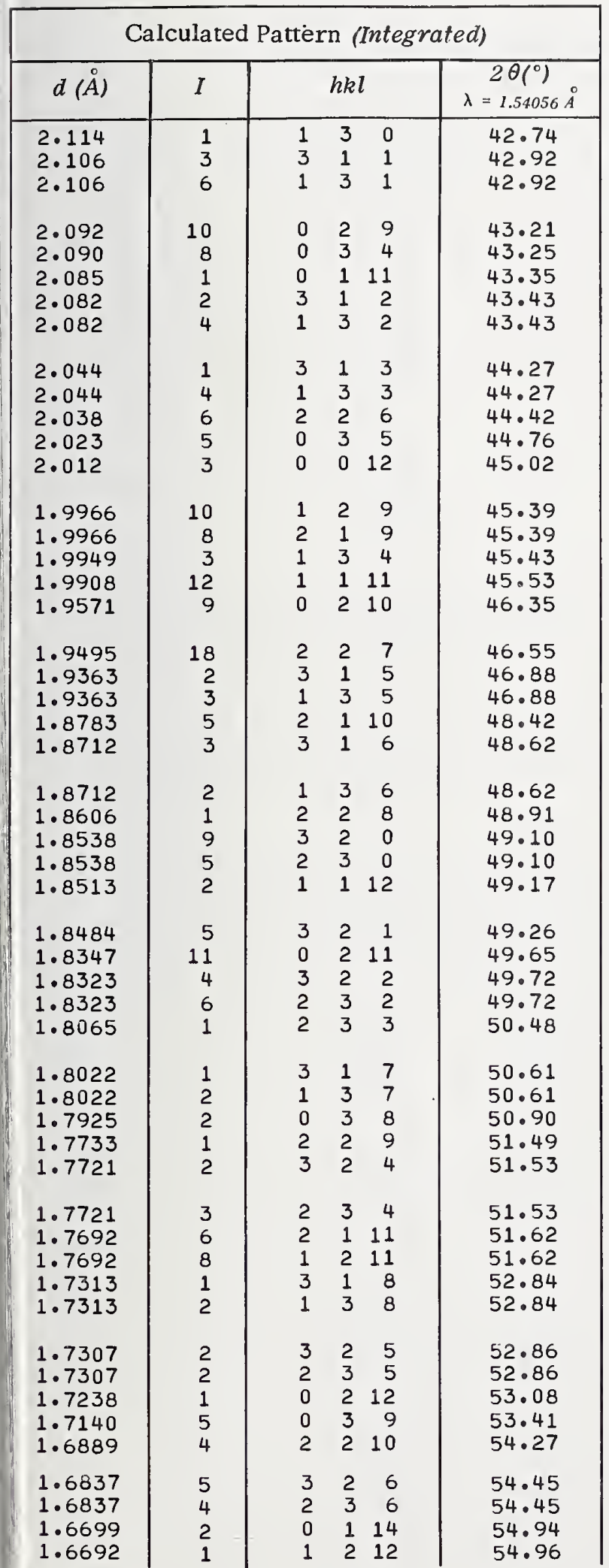

\begin{tabular}{|c|c|c|c|c|}
\hline \multicolumn{5}{|c|}{ Calculated Pattern (Integrated) } \\
\hline$d(\stackrel{\circ}{)}$ & $I$ & & $h k l$ & $\begin{array}{c}2 \theta\left({ }^{\circ}\right) \\
\lambda=154056 \AA\end{array}$ \\
\hline 1.6670 & 2 & 0 & 41 & 55.04 \\
\hline 1.6603 & 4 & 1 & 39 & 55.28 \\
\hline 1.6603 & 1 & 3 & 19 & 55.28 \\
\hline 1.6329 & 6 & 3 & 27 & 56.29 \\
\hline 1.6329 & 2 & 2 & 37 & 56.29 \\
\hline 1.6234 & 6 & 0 & 213 & 50.65 \\
\hline 1.6211 & 2 & 1 & 40 & 56.74 \\
\hline 1.6201 & 4 & 1 & 114 & 56.78 \\
\hline 1.6175 & 5 & 4 & $1 \quad 1$ & 56.88 \\
\hline 1.6175 & 2 & 1 & 41 & 56.88 \\
\hline 1.6083 & 3 & 2 & 211 & 57.23 \\
\hline 1.6067 & 6 & 4 & 12 & 57.30 \\
\hline 1.5904 & 1 & 1 & 310 & 57.94 \\
\hline 1.5892 & 2 & 4 & 13 & 57.99 \\
\hline 1.5892 & 5 & 1 & 43 & 57.99 \\
\hline 1.5796 & 6 & 3 & 28 & 58.37 \\
\hline 1.5791 & 1 & 0 & 45 & 58.39 \\
\hline 1.5776 & 3 & 1 & 213 & 58.45 \\
\hline 1.5754 & 3 & 3 & 30 & 58.54 \\
\hline 1.5721 & 2 & 3 & 31 & 58.68 \\
\hline 1.5636 & 3 & 0 & 311 & 59.03 \\
\hline 1.5622 & 7 & 3 & 32 & 59.09 \\
\hline 1.5432 & 2 & 0 & 46 & 59.89 \\
\hline 1.5368 & 2 & 4 & 15 & 60.16 \\
\hline 1.5368 & 7 & 1 & 45 & 60.16 \\
\hline 1.5326 & 1 & 0 & 214 & 60.34 \\
\hline 1.5320 & 3 & 2 & 212 & 60.37 \\
\hline 1.5251 & 1 & 2 & 39 & 60.67 \\
\hline 1.5244 & 6 & 3 & 34 & 60.70 \\
\hline 1.5237 & 4 & 1 & 115 & 60.73 \\
\hline 1.5225 & 1 & 1 & 311 & 60.79 \\
\hline 1.5225 & 1 & 3 & 111 & 60.79 \\
\hline 1.5091 & 1 & 0 & 016 & 61.39 \\
\hline 1.5038 & 2 & 0 & 47 & 61.62 \\
\hline 1.5037 & 4 & 4 & 16 & 61.63 \\
\hline 1.5037 & 2 & 1 & 46 & 61.63 \\
\hline 1.4938 & 1 & 1 & 214 & 62.08 \\
\hline 1.4933 & 6 & 0 & $\begin{array}{ll}3 & 12\end{array}$ & 62.11 \\
\hline 1.4917 & 1 & 4 & 21 & 62.18 \\
\hline 1.4833 & 1 & 2 & 42 & 62.57 \\
\hline 1.4671 & 4 & 4 & 17 & 63.34 \\
\hline 1.4671 & 2 & 1 & 47 & 63.34 \\
\hline 1.4670 & 2 & 3 & 36 & 63.35 \\
\hline 1.4603 & 1 & 2 & 213 & 63.67 \\
\hline 1.4573 & 4 & 3 & $1 \quad 12$ & 63.82 \\
\hline 1.4573 & 1 & 1 & 312 & 63.82 \\
\hline 1.4508 & 2 & 2 & 44 & 64.14 \\
\hline 1.4502 & 2 & 0 & 215 & 64.17 \\
\hline 1.4330 & 1 & 3 & 37 & 65.03 \\
\hline 1.4281 & 2 & 1 & 48 & 65.28 \\
\hline 1.4278 & 1 & 4 & 25 & 65.30 \\
\hline
\end{tabular}


Calcium Phosphate, beta-pyro-- $\mathrm{Ca}_{2} \mathrm{P}_{2} \mathrm{O}_{7}$ (tetragonal) - continued

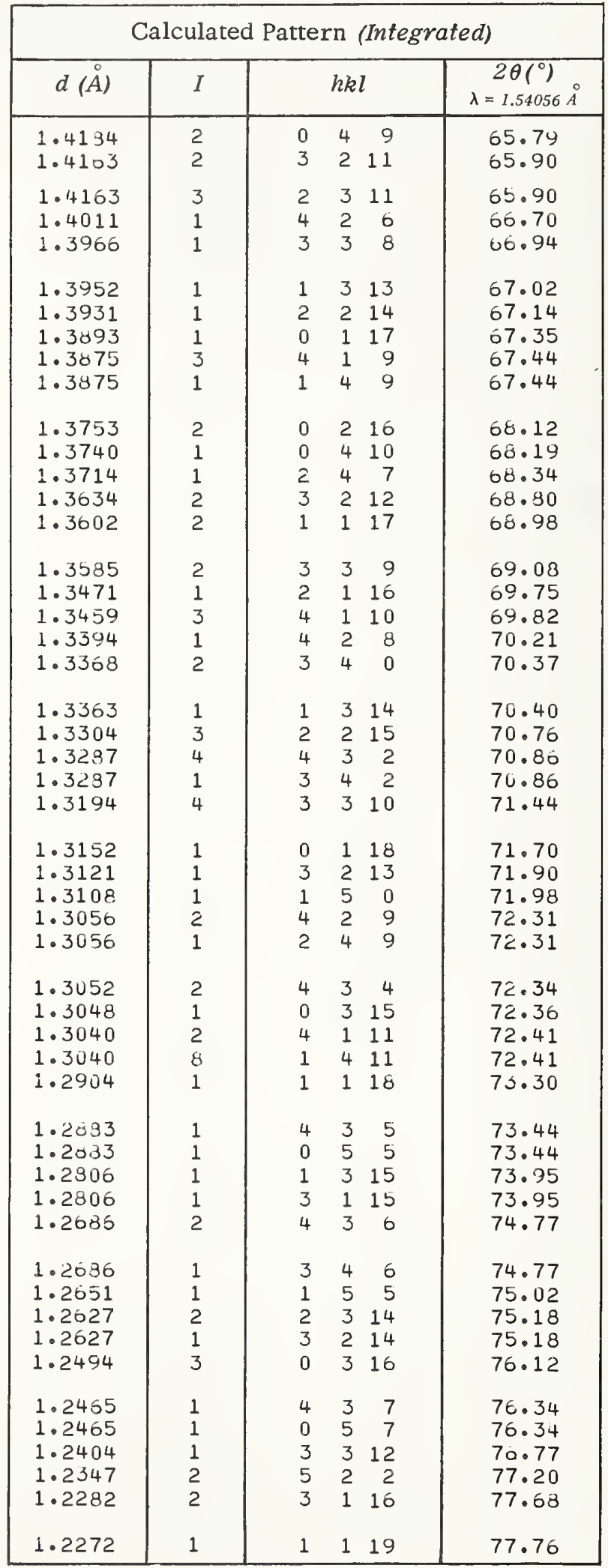




\section{Structure}

Orthorhombic, Pnma(62), $z=4$ [Cromer et al., 1960]

\section{Lattice parameters}

$a=8.112 \pm 0.001, b=5.102 \pm 0.001, c=10.162 \pm 0.005 \AA$ [ibid.]

\section{Scattering factors}

$\mathrm{Ce}^{0}, \mathrm{Cu}^{0}[3.3 .1 \mathrm{~B}]$

\section{Thermal parameters}

Isotropic [Cromer et al., 1960]

\section{Density}

(calculated) $8.23 \mathrm{~g} / \mathrm{cm}^{3}$ [ibid.]

\section{Scale factor}

$$
13.17 \times 10^{4}
$$

\section{Atomic positions}

The positions used in these calculations are those in table 5 in the given reference.

\section{Reference}

Cromer, D.T., A.C. Iarson, and R.B. Roof, Jr. (1960). The crystal structure of $\mathrm{CeCu}_{6}$, Acta Cryst. 13, 913-918.

\begin{tabular}{|c|c|c|c|c|}
\hline \multicolumn{5}{|c|}{ Calculated Pattern (Peak heights) } \\
\hline$d(\AA)$ & $I$ & $h k$ & & $\begin{array}{c}2 \theta\left(^{\circ}\right) \\
\lambda=1.5405 \AA\end{array}$ \\
\hline $\begin{array}{l}6.338 \\
4.304 \\
4.055 \\
3.973 \\
3.767\end{array}$ & $\begin{array}{l}3 \\
7 \\
3 \\
9 \\
1\end{array}$ & $\begin{array}{ll}1 & 0 \\
1 & 0 \\
2 & 0 \\
1 & 1 \\
2 & 0\end{array}$ & $\begin{array}{l}1 \\
2 \\
0 \\
1 \\
1 .\end{array}$ & $\begin{array}{l}13.96 \\
20.62 \\
21.90 \\
22.36 \\
23.60\end{array}$ \\
\hline $\begin{array}{l}3.171 \\
3.031 \\
2.822 \\
2.693 \\
2.665\end{array}$ & $\begin{array}{r}12 \\
9 \\
29 \\
1 \\
8\end{array}$ & $\begin{array}{ll}2 & 0 \\
2 & 1 \\
0 & 1 \\
2 & 1 \\
1 & 1\end{array}$ & $\begin{array}{l}2+ \\
1 \\
3 \\
2 \\
3\end{array}$ & $\begin{array}{l}28.12 \\
29.44 \\
31.68 \\
33.24 \\
33.60\end{array}$ \\
\hline $\begin{array}{l}2.614 \\
2.552 \\
2.425 \\
2.386 \\
2.367\end{array}$ & $\begin{array}{r}2 \\
35 \\
50 \\
15 \\
9\end{array}$ & $\begin{array}{ll}3 & 0 \\
0 & 2 \\
1 & 0 \\
3 & 0 \\
1 & 2\end{array}$ & $\begin{array}{l}1 \\
0 \\
4 \\
2 \\
1\end{array}$ & $\begin{array}{l}34.28 \\
35.14 \\
37.04 \\
37.66 \\
37.98\end{array}$ \\
\hline $\begin{array}{l}2.326 \\
2.317\end{array}$ & $\begin{array}{r}100 \\
97\end{array}$ & $\begin{array}{ll}3 & 1 \\
2 & 1\end{array}$ & $\begin{array}{l}1 \\
3\end{array}$ & $\begin{array}{l}38.68 \\
38.84\end{array}$ \\
\hline
\end{tabular}

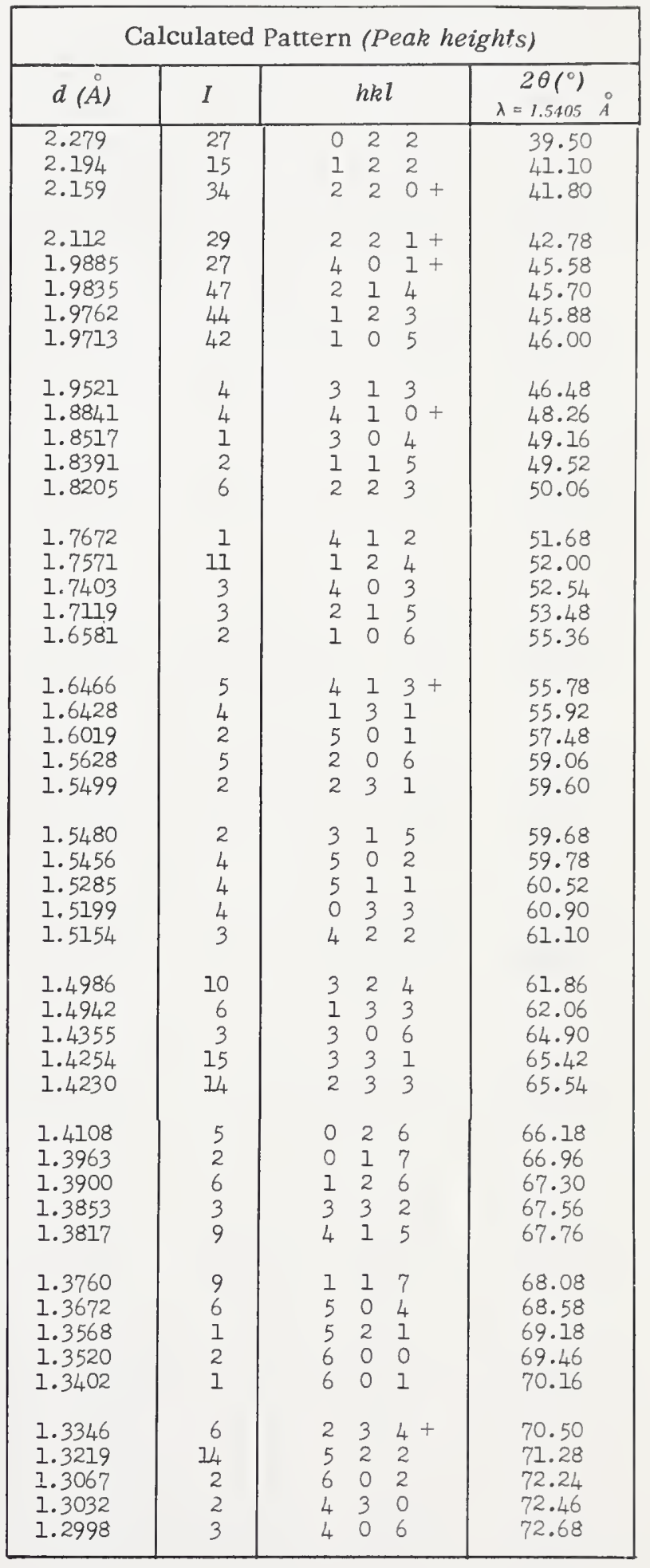


Cerium Copper, $\mathrm{CeCu}_{6}$ (orthorhombic) - continued

\begin{tabular}{|c|c|c|c|c|c|c|c|c|c|c|c|}
\hline \multicolumn{6}{|c|}{ Calculated Pattern (Integrated) } & \multicolumn{6}{|c|}{ Calculated Pattern (Integrated) } \\
\hline$d(\AA)$ & $I$ & & lkl & & $\begin{array}{r}2 \theta\left({ }^{\circ}\right) \\
\lambda=1.5405 \AA\end{array}$ & $d(\stackrel{\circ}{)}$ & $I$ & & $h k l$ & & $\begin{array}{c}2 \theta\left({ }^{\circ}\right) \\
\lambda=1.5405 \AA\end{array}$ \\
\hline $\begin{array}{l}6.340 \\
4.306 \\
4.056 \\
3.975 \\
3.767\end{array}$ & $\begin{array}{l}2 \\
6 \\
3 \\
9 \\
1\end{array}$ & & $\begin{array}{l}0 \\
0 \\
0 \\
1 \\
0\end{array}$ & $\begin{array}{l}1 \\
2 \\
0 \\
1 \\
1\end{array}$ & $\begin{array}{l}13.96 \\
20.61 \\
21.89 \\
22.35 \\
23.60\end{array}$ & $\begin{array}{l}1.5152 \\
1.4984 \\
1.4939 \\
1.4354 \\
1.4254\end{array}$ & $\begin{array}{r}1 \\
13 \\
1 \\
4 \\
17\end{array}$ & $\begin{array}{l}4 \\
3 \\
1 \\
3 \\
3\end{array}$ & $\begin{array}{l}2 \\
2 \\
3 \\
0 \\
3\end{array}$ & $\begin{array}{l}2 \\
4 \\
3 \\
6 \\
1\end{array}$ & $\begin{array}{l}61.11 \\
61.87 \\
62.08 \\
64.91 \\
65.42\end{array}$ \\
\hline $\begin{array}{l}3.175 \\
3.170 \\
3.030 \\
2.822 \\
2.693\end{array}$ & $\begin{array}{r}1 \\
11 \\
9 \\
28 \\
1\end{array}$ & $\begin{array}{l}2 \\
2 \\
2 \\
0 \\
2\end{array}$ & $\begin{array}{l}1 \\
0 \\
1 \\
1 \\
1\end{array}$ & $\begin{array}{l}0 \\
2 \\
1 \\
3 \\
2\end{array}$ & $\begin{array}{l}28.08 \\
28.13 \\
29.45 \\
31.68 \\
33.25\end{array}$ & $\begin{array}{l}1.4232 \\
1.4110 \\
1.3963 \\
1.3901 \\
1.3851\end{array}$ & $\begin{array}{r}15 \\
6 \\
2 \\
7 \\
2\end{array}$ & $\begin{array}{l}2 \\
0 \\
0 \\
1 \\
3\end{array}$ & $\begin{array}{l}3 \\
2 \\
1 \\
2 \\
3\end{array}$ & $\begin{array}{l}3 \\
6 \\
7 \\
6 \\
2\end{array}$ & $\begin{array}{l}65.53 \\
66.17 \\
66.96 \\
67.30 \\
67.57\end{array}$ \\
\hline $\begin{array}{l}2.665 \\
2.613 \\
2.551 \\
2.424 \\
2.387\end{array}$ & $\begin{array}{r}9 \\
2 \\
37 \\
55 \\
16\end{array}$ & $\begin{array}{l}1 \\
3 \\
0 \\
1 \\
3\end{array}$ & $\begin{array}{l}1 \\
0 \\
2 \\
0 \\
0\end{array}$ & $\begin{array}{l}3 \\
1 \\
0 \\
4 \\
2\end{array}$ & $\begin{array}{l}33.60 \\
34.29 \\
35.15 \\
37.05 \\
37.65\end{array}$ & $\begin{array}{l}1.3819 \\
1.3761 \\
1.3674 \\
1.3567 \\
1.3520\end{array}$ & $\begin{array}{r}11 \\
12 \\
8 \\
1 \\
3\end{array}$ & $\begin{array}{l}4 \\
1 \\
5 \\
5 \\
6\end{array}$ & $\begin{array}{l}1 \\
1 \\
0 \\
2 \\
0\end{array}$ & $\begin{array}{l}5 \\
7 \\
4 \\
1 \\
0\end{array}$ & $\begin{array}{l}67.75 \\
68.08 \\
68.57 \\
69.18 \\
69.46\end{array}$ \\
\hline $\begin{array}{l}2.367 \\
2.326 \\
2.316 \\
2.280 \\
2.195\end{array}$ & $\begin{array}{r}9 \\
100 \\
84 \\
29 \\
17\end{array}$ & $\begin{array}{l}1 \\
3 \\
2 \\
0 \\
1\end{array}$ & $\begin{array}{l}2 \\
1 \\
1 \\
2 \\
2\end{array}$ & $\begin{array}{l}1 \\
1 \\
3 \\
2 \\
2\end{array}$ & $\begin{array}{l}37.99 \\
38.68 \\
38.84 \\
39.49 \\
41.09\end{array}$ & $\begin{array}{l}1.3402 \\
1.3345 \\
1.3327 \\
1.3249 \\
1.3219\end{array}$ & $\begin{array}{r}2 \\
8 \\
1 \\
1 \\
19\end{array}$ & $\begin{array}{l}6 \\
2 \\
2 \\
3 \\
5\end{array}$ & $\begin{array}{l}0 \\
3 \\
2 \\
3 \\
2\end{array}$ & $\begin{array}{l}1 \\
4 \\
6 \\
3 \\
2\end{array}$ & $\begin{array}{l}70.16 \\
70.50 \\
70.62 \\
71.09 \\
71.28\end{array}$ \\
\hline $\begin{array}{l}2.162 \\
2.159 \\
2.113 \\
2.112 \\
1.9888\end{array}$ & $\begin{array}{r}13 \\
31 \\
6 \\
28 \\
25\end{array}$ & $\begin{array}{l}3 \\
2 \\
3 \\
2 \\
4\end{array}$ & $\begin{array}{l}1 \\
2 \\
0 \\
2 \\
0\end{array}$ & $\begin{array}{l}2 \\
0 \\
3 \\
1 \\
1\end{array}$ & $\begin{array}{l}41.74 \\
41.80 \\
42.75 \\
42.77 \\
45.57\end{array}$ & $\begin{array}{l}1.3065 \\
1.3031 \\
1.3000 \\
1.2755 \\
1.2693\end{array}$ & $\begin{array}{r}2 \\
1 \\
3 \\
13 \\
3\end{array}$ & $\begin{array}{l}6 \\
4 \\
4 \\
0 \\
5\end{array}$ & $\begin{array}{l}0 \\
3 \\
0 \\
4 \\
2\end{array}$ & $\begin{array}{l}2 \\
0 \\
6 \\
0 \\
3\end{array}$ & $\begin{array}{l}72.25 \\
72.47 \\
72.67 \\
74.30 \\
74.72\end{array}$ \\
\hline $\begin{array}{l}1.9874 \\
1.9836 \\
1.9764 \\
1.9715 \\
1.9524\end{array}$ & $\begin{array}{r}3 \\
35 \\
40 \\
24 \\
4\end{array}$ & $\begin{array}{l}2 \\
2 \\
1 \\
1 \\
3\end{array}$ & $\begin{array}{l}2 \\
1 \\
2 \\
0 \\
1\end{array}$ & $\begin{array}{l}2 \\
4 \\
3 \\
5 \\
3\end{array}$ & $\begin{array}{l}45.61 \\
45.70 \\
45.88 \\
46.00 \\
46.47\end{array}$ & $\begin{array}{l}1.2680 \\
1.2597 \\
1.2557 \\
1.2467 \\
1.2417\end{array}$ & $\begin{array}{l}1 \\
7 \\
1 \\
2 \\
1\end{array}$ & $\begin{array}{l}5 \\
4 \\
6 \\
1 \\
2\end{array}$ & $\begin{array}{l}0 \\
1 \\
0 \\
2 \\
3\end{array}$ & $\begin{array}{l}5 \\
6 \\
3 \\
7 \\
5\end{array}$ & $\begin{array}{l}74.81 \\
75.39 \\
75.67 \\
76.31 \\
76.68\end{array}$ \\
\hline $\begin{array}{l}1.8846 \\
1.8835 \\
1.8515 \\
1.8390 \\
1.8209\end{array}$ & $\begin{array}{l}3 \\
2 \\
1 \\
2 \\
7\end{array}$ & $\begin{array}{l}4 \\
4 \\
3 \\
1 \\
2\end{array}$ & $\begin{array}{l}1 \\
0 \\
0 \\
1 \\
2\end{array}$ & $\begin{array}{l}0 \\
2 \\
4 \\
5 \\
3\end{array}$ & $\begin{array}{l}48.25 \\
48.28 \\
49.17 \\
49.52 \\
50.05\end{array}$ & $\begin{array}{l}1.2406 \\
1.2186 \\
1.2162 \\
1.2048 \\
1.1833\end{array}$ & $\begin{array}{l}2 \\
2 \\
2 \\
2 \\
1\end{array}$ & $\begin{array}{l}3 \\
1 \\
4 \\
2 \\
2\end{array}$ & $\begin{array}{l}1 \\
1 \\
3 \\
2 \\
4\end{array}$ & $\begin{array}{l}7 \\
8 \\
3 \\
7 \\
2\end{array}$ & $\begin{array}{l}76.76 \\
78.40 \\
78.59 \\
79.48 \\
81.22\end{array}$ \\
\hline $\begin{array}{l}1.7670 \\
1.7574 \\
1.7400 \\
1.7117 \\
1.6579\end{array}$ & $\begin{array}{r}1 \\
14 \\
2 \\
4 \\
2\end{array}$ & $\begin{array}{l}4 \\
1 \\
4 \\
2 \\
1\end{array}$ & $\begin{array}{l}1 \\
2 \\
0 \\
1 \\
0\end{array}$ & $\begin{array}{l}2 \\
4 \\
3 \\
5 \\
6\end{array}$ & $\begin{array}{l}51.69 \\
51.99 \\
52.55 \\
53.48 \\
55.37\end{array}$ & $\begin{array}{l}1.1748 \\
1.1661 \\
1.1629 \\
1.1582 \\
1.1371\end{array}$ & $\begin{array}{r}1 \\
2 \\
11 \\
4 \\
1\end{array}$ & $\begin{array}{l}3 \\
5 \\
6 \\
4 \\
0\end{array}$ & $\begin{array}{l}3 \\
3 \\
2 \\
2 \\
2\end{array}$ & $\begin{array}{l}5 \\
1 \\
2 \\
6 \\
8\end{array}$ & $\begin{array}{l}81.94 \\
82.68 \\
82.96 \\
83.37 \\
85.28\end{array}$ \\
\hline $\begin{array}{l}1.6469 \\
1.6453 \\
1.6426 \\
1.6021 \\
1.5629\end{array}$ & $\begin{array}{l}6 \\
1 \\
1 \\
2 \\
6\end{array}$ & $\begin{array}{l}4 \\
2 \\
1 \\
5 \\
2\end{array}$ & $\begin{array}{l}1 \\
2 \\
3 \\
0 \\
0\end{array}$ & $\begin{array}{l}3 \\
4 \\
1 \\
1 \\
6\end{array}$ & $\begin{array}{r}55.77 \\
55.83 \\
55.93 \\
57.47 \\
59.06\end{array}$ & & & & & & \\
\hline $\begin{array}{l}1.5500 \\
1.5481 \\
1.5455 \\
1.5285 \\
1.5199\end{array}$ & $\begin{array}{l}1 \\
2 \\
4 \\
5 \\
4\end{array}$ & $\begin{array}{l}2 \\
3 \\
5 \\
5 \\
0\end{array}$ & $\begin{array}{l}3 \\
1 \\
0 \\
1 \\
3\end{array}$ & $\begin{array}{l}1 \\
5 \\
2 \\
1 \\
3\end{array}$ & $\begin{array}{l}59.59 \\
59.68 \\
59.78 \\
60.52 \\
60.90\end{array}$ & & & & & & \\
\hline
\end{tabular}




\section{Structure}

Hexagona 1, P $\overline{3} \mathrm{ml}$ (164), $\mathrm{Z}=1 \quad[$ Kaatz and Marcovich, 1966]

\section{Lattice parameters} $a=7.476 \pm 0.002, \quad c=6.039 \pm 0.002 \AA, \quad[$ ibid. ]

\section{Scattering factors}

\section{$\mathrm{Cl}^{-}$[Dawson, 1960]}

$\mathrm{Cs}^{+}, \mathrm{Cf}^{4+}$ [Thomas and Umeda, 1957]

All factors were corrected for anomalous dispersion using values given by Cromer [1965] .

\section{Thermal parameters}

Isotropic [Kaatz and Marcovitch, 1966]

\section{Density}

(calculated)

$3.52 \mathrm{~g} / \mathrm{cm}^{3}$ [Kaatz and Marcovich, 1966]

Scale factor

$10.44 \times 10^{4}$

\section{Reference}

Cromer, D.T. (1965). Anomalous dispersion corrections computed from self-consistent field relativistic Dirac-Slater wave functions, Acta Cryst. 18, 17-23.

Dawson, B. (1960). Atomic scattering factors from wave functions calculated by the poly-detor method:Cl, $\mathrm{Cl}^{-}, \mathrm{S}$ and $\mathrm{S}^{-}$, Acta Cryst. 13, 403-408.

Kaatz, T. and M.Marcovich (1966). The crystal structure of the compound $\mathrm{Cs}_{2} \mathrm{CeCl}_{6}$, Acta cryst. 21, 1011.

Thomas, L. H. and K. Umeda (1957). Atomic scattering factors calculated from the TFD atomic model, J. Chem. Phys. 26,293303 .

\begin{tabular}{|c|c|c|c|c|c|c|}
\hline \multicolumn{7}{|c|}{ Calculated Pattern (Peak heights) } \\
\hline$d(\stackrel{\AA}{)})$ & $I$ & & $h k$ & & & $\begin{array}{c}2 \theta\left(^{\circ}\right) \\
\lambda=1.54056 \AA\end{array}$ \\
\hline 6.468 & 68 & 1 & 0 & 0 & & 13.68 \\
\hline 6.037 & 22 & 0 & 0 & 1 & & 14.66 \\
\hline 4.414 & 100 & 0 & 1 & 1 & + & 20.10 \\
\hline 3.739 & 29 & 1 & 1 & 0 & & 23.78 \\
\hline 3.236 & 1 & 2 & 0 & 0 & & 27.54 \\
\hline 3.177 & 24 & 1 & 1 & 1 & & 28.06 \\
\hline 2.854 & 20 & 0 & 2 & 1 & + & 31.32 \\
\hline 2.736 & 20 & 1 & 0 & 2 & + & 32.70 \\
\hline 2.447 & 9 & 2 & 1 & 0 & & 36.70 \\
\hline 2.349 & 5 & 1 & 1 & 2 & & 38.28 \\
\hline 2.267 & 22 & 1 & 2 & 1 & + & 39.72 \\
\hline 2.203 & 20 & 2 & 0 & 2 & + & 40.84 \\
\hline 2.158 & 5 & 3 & 0 & 0 & & 41.82 \\
\hline 2.033 & 5 & 3 & 0 & 1 & + & 44.54 \\
\hline 2.013 & 1 & 0 & 0 & 3 & & 45.00 \\
\hline 1.9225 & o & 1 & 0 & 3 & & 47.24 \\
\hline 1.0013 & 9 & 2 & 1 & 2 & + & 47.80 \\
\hline 1. .8689 & 10 & 2 & 2 & 0 & & 48.68 \\
\hline 1.7950 & 2 & 3 & 1 & 0 & & 50.80 \\
\hline 1.7853 & 3 & 2 & 2 & 1 & . & 51.12 \\
\hline 1.7724 & 3 & 1 & 1 & 3 & & 51.52 \\
\hline 1.7559 & 2 & 0 & 3 & 2 & + & 52.04 \\
\hline 1.7209 & 7 & 3 & 1 & 1 & & 53.18 \\
\hline 1.7090 & 2 & 2 & 0 & 3 & + & 53.56 \\
\hline 1.5633 & 2 & 4 & 0 & 1 & + & 59.04 \\
\hline 1.5547 & 4 & 2 & 1 & 3 & & 59.40 \\
\hline 1.5434 & 4 & 1 & 3 & 2 & + & 59.88 \\
\hline 1.5090 & 1 & 0 & 0 & 4 & & 61.36 \\
\hline 1.4852 & 1 & 3 & 2 & 0 & & 62.48 \\
\hline 1.4721 & 1 & 0 & 3 & 3 & + & 63.10 \\
\hline 1.4705 & 1 & 0 & 1 & 4 & & 63.18 \\
\hline 1.4423 & 3 & 2 & 3 & 1 & & 64.56 \\
\hline 1.4266 & 3 & 0 & 4 & 2 & + & 65.30 \\
\hline 1.4128 & 2 & 4 & 1 & 0 & & 66.08 \\
\hline 1.4000 & 1 & 1 & 1 & 4 & & 06.70 \\
\hline 1.3757 & 2 & 1 & 4 & 1 & + & 68.10 \\
\hline 1.3697 & 1 & 2 & 2 & 3 & & 68.44 \\
\hline 1.3399 & 2 & 1 & 3 & 3 & & 70.18 \\
\hline 1.3327 & 2 & 3 & 2 & 2 & + & 70.62 \\
\hline 1.2850 & 1 & 1 & 2 & 4 & & 73.66 \\
\hline 1.2662 & 1 & 5 & 0 & 1 & & 74.94 \\
\hline 1.1991 & 1 & 2 & 4 & 1 & & 79.94 \\
\hline 1.1951 & 1 & 3 & 2 & 3 & & 30.20 \\
\hline 1.1873 & 1 & 0 & 1 & 5 & & 80.90 \\
\hline 1.1743 & 2 & 2 & 2 & 4 & & 81.98 \\
\hline 1.1419 & 1 & 1 & 5 & 1 & + & 84.84 \\
\hline 1.1339 & 2 & 4 & 2 & 2 & + & 85.58 \\
\hline 1.0829 & 1 & 1 & 2 & 5 & & 90.68 \\
\hline
\end{tabular}


Cesium Cerium Chloride. $\mathrm{Cs}_{2} \mathrm{CeCl}_{6}$ (hexagonal) - continued

\begin{tabular}{|c|c|c|c|c|c|}
\hline \multicolumn{6}{|c|}{ Calculated Pattern (Integrated) } \\
\hline$d(\stackrel{\circ}{)}$ & $I$ & & $h k$ & & $\begin{array}{c}2 \theta\left({ }^{\circ}\right) \\
\lambda=1.54056 \AA\end{array}$ \\
\hline 6.474 & 64 & 1 & 0 & 0 & 13.67 \\
\hline 6.039 & 20 & 0 & 0 & 1 & 14.66 \\
\hline 4.416 & 2 & 1 & 0 & 1 & 20.09 \\
\hline $4 \cdot 416$ & 100 & 0 & 1 & 1 & 20.09 \\
\hline 3.738 & 31 & 1 & 1 & 0 & 23.78 \\
\hline $3 \cdot 237$ & 1 & 2 & 0 & 0 & $27 \cdot 53$ \\
\hline $3 \cdot 178$ & 28 & 1 & 1 & 1 & 28.05 \\
\hline 2.853 & 5 & 2 & 0 & 1 & 31.33 \\
\hline 2.853 & 19 & 0 & 2 & 1 & 31.33 \\
\hline 2.737 & 11 & 0 & 1 & 2 & 32.70 \\
\hline 2.737 & 13 & 1 & 0 & 2 & $32 \cdot 70$ \\
\hline 2.447 & 11 & 2 & 1 & 0 & 36.69 \\
\hline 2.349 & 6 & 1 & 1 & 2 & 38.29 \\
\hline $2 \cdot 268$ & 1 & 2 & 1 & 1 & 39.71 \\
\hline $2 \cdot 268$ & 27 & 1 & 2 & 1 & 39.71 \\
\hline $2 \cdot 208$ & 13 & 2 & 0 & 2 & 40.83 \\
\hline $2 \cdot 208$ & 12 & 0 & 2 & 2 & 40.83 \\
\hline $2 \cdot 153$ & 6 & 3 & 0 & 0 & 41.32 \\
\hline 2.032 & 4 & 3 & 0 & 1 & 44.55 \\
\hline 2.032 & 3 & 0 & 3 & 1 & 44.55 \\
\hline $2 \cdot 013$ & 2 & 0 & 0 & 3 & 45.00 \\
\hline $1 \cdot 9222$ & 8 & 1 & 0 & 3 & 47.25 \\
\hline 1.9011 & 7 & 2 & 1 & 2 & 47.80 \\
\hline 1.9011 & 6 & 1 & 2 & 2 & 47.80 \\
\hline 1.8690 & 13 & 2 & 2 & 0 & 48.68 \\
\hline 1.7957 & 3 & 3 & 1 & 0 & 50.80 \\
\hline 1.7854 & 4 & 2 & 2 & 1 & 51.12 \\
\hline 1.7723 & 4 & 1 & 1 & 3 & 51.52 \\
\hline 1.7558 & 1 & 0 & 3 & 2 & $5 \hat{z} \cdot 04$ \\
\hline 1.7558 & 1 & 3 & 0 & 2 & 52.04 \\
\hline 1.7212 & 10 & 3 & 1 & 1 & 53.17 \\
\hline 1.7094 & 2 & 2 & 0 & 3 & 53.56 \\
\hline 1.7094 & 1 & 0 & 2 & 3 & 53.56 \\
\hline 1.5634 & 2 & 4 & 0 & 1 & 59.03 \\
\hline 1.5634 & 1 & 0 & 4 & 1 & 59.03 \\
\hline 1.5546 & 6 & 2 & 1 & 3 & 59.40 \\
\hline 1.5434 & 3 & 3 & 1 & 2 & 59.80 \\
\hline 1.5434 & 3 & 1 & 3 & 2 & 59.88 \\
\hline 1.5097 & 2 & 0 & 0 & 4 & $61 \cdot 35$ \\
\hline 1.4853 & 1 & 3 & 2 & 0 & 62.48 \\
\hline 1.4720 & 1 & 3 & 0 & 3 & 63.10 \\
\hline 1.4720 & 1 & 0 & 3 & 3 & 63.10 \\
\hline 1.4703 & 1 & 0 & 1 & 4 & 63.19 \\
\hline 1.4423 & 5 & 2 & 3 & 1 & 64.56 \\
\hline 1.4266 & 2 & 0 & 4 & 2 & $65 \cdot 36$ \\
\hline
\end{tabular}

\begin{tabular}{|c|c|c|c|c|c|}
\hline \multicolumn{6}{|c|}{ Calculated Pattern (Integrated) } \\
\hline$d(\stackrel{\circ}{A})$ & $I$ & & $h k l$ & & $\begin{array}{c}2 \theta\left(^{\circ}\right) \\
\lambda=1.54056 \AA\end{array}$ \\
\hline $\begin{array}{l}1.4266 \\
1.4128 \\
1.3999 \\
1.3757 \\
1.3757\end{array}$ & $\begin{array}{l}2 \\
2 \\
2 \\
1 \\
2\end{array}$ & $\begin{array}{l}4 \\
4 \\
1 \\
4 \\
1\end{array}$ & $\begin{array}{l}0 \\
1 \\
1 \\
1 \\
4\end{array}$ & $\begin{array}{l}2 \\
0 \\
4 \\
1 \\
1\end{array}$ & $\begin{array}{l}65.36 \\
60.08 \\
60.77 \\
68.10 \\
68.10\end{array}$ \\
\hline $\begin{array}{l}1.3697 \\
1.3400 \\
1.3328 \\
1.3328 \\
1.2849\end{array}$ & $\begin{array}{l}2 \\
3 \\
2 \\
2 \\
1\end{array}$ & $\begin{array}{l}2 \\
1 \\
3 \\
2 \\
1\end{array}$ & $\begin{array}{l}2 \\
3 \\
2 \\
3 \\
2\end{array}$ & $\begin{array}{l}3 \\
3 \\
2 \\
2 \\
4\end{array}$ & $\begin{array}{l}68.44 \\
70.18 \\
70.61 \\
70.61 \\
73.67\end{array}$ \\
\hline $\begin{array}{l}1 \cdot 2661 \\
1 \cdot 2460 \\
1 \cdot 2203 \\
1 \cdot 1992 \\
1 \cdot 1952\end{array}$ & $\begin{array}{l}1 \\
1 \\
1 \\
1 \\
2\end{array}$ & $\begin{array}{l}5 \\
3 \\
3 \\
2 \\
3\end{array}$ & $\begin{array}{l}0 \\
3 \\
3 \\
4 \\
2\end{array}$ & $\begin{array}{l}1 \\
0 \\
1 \\
1 \\
3\end{array}$ & $\begin{array}{l}74.95 \\
76.37 \\
78.28 \\
79.93 \\
80.25\end{array}$ \\
\hline $\begin{array}{l}1.1873 \\
1.1744 \\
1 \cdot 1564 \\
1 \cdot 1419 \\
1.1340\end{array}$ & $\begin{array}{l}1 \\
3 \\
1 \\
2 \\
2\end{array}$ & $\begin{array}{l}0 \\
2 \\
4 \\
1 \\
4\end{array}$ & $\begin{array}{l}1 \\
2 \\
1 \\
5 \\
2\end{array}$ & $\begin{array}{l}5 \\
4 \\
3 \\
1 \\
2\end{array}$ & $\begin{array}{l}80.90 \\
81.97 \\
83.53 \\
84.84 \\
85.57\end{array}$ \\
\hline $\begin{array}{l}1.1340 \\
1.0831 \\
1.0791 \\
1.0482 \\
1.0069\end{array}$ & $\begin{array}{l}1 \\
1 \\
1 \\
1 \\
1\end{array}$ & $\begin{array}{l}2 \\
1 \\
6 \\
3 \\
5\end{array}$ & $\begin{array}{l}4 \\
2 \\
0 \\
4 \\
1\end{array}$ & $\begin{array}{l}2 \\
5 \\
0 \\
1 \\
3\end{array}$ & $\begin{array}{l}85.57 \\
90.67 \\
91.10 \\
94.59 \\
99.81\end{array}$ \\
\hline $\begin{array}{r}1.0022 \\
.9744 \\
.9409 \\
.9371 \\
.9345\end{array}$ & $\begin{array}{l}1 \\
1 \\
1 \\
1 \\
1\end{array}$ & $\begin{array}{l}3 \\
6 \\
4 \\
2 \\
4\end{array}$ & $\begin{array}{l}1 \\
1 \\
3 \\
3 \\
4\end{array}$ & $\begin{array}{l}5 \\
1 \\
3 \\
5 \\
0\end{array}$ & $\begin{array}{l}100.46 \\
104.47 \\
109.89 \\
110.57 \\
111.03\end{array}$ \\
\hline $\begin{array}{l}.9142 \\
.8864 \\
.8606 \\
.8404 \\
.8377\end{array}$ & $\begin{array}{l}1 \\
1 \\
1 \\
1 \\
1\end{array}$ & $\begin{array}{l}5 \\
1 \\
2 \\
3 \\
1\end{array}$ & $\begin{array}{l}3 \\
6 \\
6 \\
5 \\
5\end{array}$ & $\begin{array}{l}1 \\
3 \\
2 \\
3 \\
5\end{array}$ & $\begin{array}{l}114.81 \\
120.67 \\
127.03 \\
132.84 \\
133.71\end{array}$ \\
\hline $\begin{array}{l}.8213 \\
.8136 \\
.7985 \\
.7946 \\
.7843\end{array}$ & $\begin{array}{l}1 \\
1 \\
1 \\
2 \\
1\end{array}$ & $\begin{array}{l}4 \\
2 \\
3 \\
4 \\
7\end{array}$ & $\begin{array}{l}5 \\
1 \\
4 \\
4 \\
2\end{array}$ & $\begin{array}{l}1 \\
7 \\
5 \\
4 \\
1\end{array}$ & $\begin{array}{l}139.41 \\
142.42 \\
149.42 \\
151.58 \\
158.32\end{array}$ \\
\hline
\end{tabular}


Structure

Orthorhombic, Pmen (62), $\mathrm{Z}=4$ [Carpenter, 1966].

Lattice parameters $a=6.634, b=9.567, c=10.958 \AA$ [ibid.]

Scattering factors $\mathrm{Cs}^{\circ}, \mathrm{I}^{\circ}, \mathrm{Br}^{\circ}[3.3 .1 \mathrm{~B}]$

Thermal parameters

Isotropic: Cs $3.22 ; \mathrm{Br} 2.31 ; \mathrm{I}(2) 2.08$; I (3) 2.80

Density

(calculated) $4.456 \mathrm{~g} / \mathrm{cm}^{3}$ [Carpenter, 1966]

Scale factor

$40.80 \times 10^{4}$

Reference

Carpenter, G.B. (1966). The crystal structure of $\mathrm{CsI}_{2} \mathrm{Br}$, Acta Cryst. 20, 330-334.

\begin{tabular}{|c|c|c|c|c|c|}
\hline \multicolumn{6}{|c|}{ Calculated Pattern (Peak heights) } \\
\hline$d(\stackrel{\circ}{A})$ & $I$ & \multicolumn{3}{|c|}{$h k l$} & $\begin{array}{c}2 \theta\left({ }^{\circ}\right) \\
\lambda=1.54056 \AA\end{array}$ \\
\hline 5.45 & 10 & 1 & 1 & 0 & 16.24 \\
\hline 4.88 & 2 & 1 & 1 & 1 & 16.16 \\
\hline 4.78 & 6 & 0 & 2 & 0 & 18.54 \\
\hline 4.76 & 4 & 0 & 1 & 2 & 10.64 \\
\hline 4.38 & 2 & 9 & 2 & 1 & 20.24 \\
\hline 4.22 & 5 & 1 & 0 & 2 & 21.02 \\
\hline 3.86 & 31 & 1 & 1 & 2 & 23.00 \\
\hline 3.66 & 44 & 1 & 2 & 1 & 24.32 \\
\hline 3.60 & 12 & 0 & 2 & 2 & 24.68 \\
\hline 3.411 & 5 & 0 & 1 & 3 & 25.10 \\
\hline 3.316 & 69 & 2 & 0 & 0 & 25.86 \\
\hline 3.155 & 100 & 1 & 2 & 2 & 28.16 \\
\hline 3.033 & 5 & 1 & 1 & 3 & 29.42 \\
\hline 2.873 & 11 & 3 & 3 & 0 & 31.10 \\
\hline 2.779 & $?$ & 1 & 3 & 1 & 32.18 \\
\hline 2.756 & 8 & 0 & 3 & 2 & 32.45 \\
\hline 2.740 & 14 & 0 & 0 & 4 & 32.65 \\
\hline 2.731 & 9 & 2 & 2 & o & 32.76 \\
\hline 2.559 & 25 & 1 & 2 & 3 & 33.68 \\
\hline 2.633 & $\varepsilon$ & ن & 1 & 4 & 34.02 \\
\hline 2.532 & 7 & 1 & 0 & 4 & 35.42 \\
\hline 2.440 & 7 & 2 & 2 & 2 & 35.60 \\
\hline 2.391 & 2 & 5 & 4 & 0 & 37.58 \\
\hline 2.378 & 7 & 2 & 1 & 3. & 37.80 \\
\hline 2.336 & 10 & 0 & 4 & 1 & 38.50 \\
\hline 2.259 & 3 & 1 & 3 & 3 & 39.88 \\
\hline 2.254 & 3 & 1 & 4 & 1 & 40.92 \\
\hline 2.192 & 1 & 0 & 4 & 2 & 41.14 \\
\hline 2.136 & 4 & 0 & 1 & 5 & $42 \cdot 28$ \\
\hline 2.120 & 7 & 2 & 3 & 2 & 42.52 \\
\hline
\end{tabular}

\begin{tabular}{|c|c|c|c|c|c|}
\hline \multicolumn{6}{|c|}{ Calculated Pattern (Peak heights) } \\
\hline$d(\AA)$ & $I$ & & $h k l$ & & $\begin{array}{c}2 \theta\left({ }^{\circ}\right) \\
\lambda=1.54056 \AA\end{array}$ \\
\hline$\angle .112$ & 11 & $i$ & 0 & 4 & $4 \hat{z} .78$ \\
\hline 2.078 & 4 & 3 & 3 & 4 & 43.52 \\
\hline 2.062 & 5 & 2 & 1 & 4 & 43.86 \\
\hline 2.035 & 3 & 3 & 1 & 2 & 45.18 \\
\hline 2.001 & 4 & i & 4 & 3 & 45.28 \\
\hline 1.9746 & 5 & 3 & 2 & 1 & 45.92 \\
\hline 1.9403 & 2 & $<$ & 4 & 0 & 46.78 \\
\hline 1.9325 & 3 & 2 & 2 & 4 & 45.98 \\
\hline 1.9156 & 8 & 1 & 4 & 3 & 47.42 \\
\hline 1.9103 & 12 & 2 & 4 & 1 & 47.56 \\
\hline 1.8849 & 15 & 3 & 2 & 2 & 48.24 \\
\hline 1.8560 & 1 & 3 & 1 & 3 & 49.04 \\
\hline 1.8385 & $?$ & 1 & 5 & 0 & 49.54 \\
\hline 1.8285 & 1 & 2 & 4 & 2 & 49.82 \\
\hline 1.8172 & 2 & 3 & 3 & 0 & 50.16 \\
\hline 1.8131 & 2 & 1 & 5 & 1 & 50.28 \\
\hline 1.8064 & 1 & 3 & 5 & 2 & 50.48 \\
\hline 1.7958 & 4 & < & 1 & $5+$ & 50.80 \\
\hline 1.7597 & 7 & 3 & 2 & $3+$ & 51.92 \\
\hline 1.7428 & 3 & 1 & 3 & $5+$ & $52.4 \overline{6}$ \\
\hline 1.7385 & 6 & 1 & 4 & 4 & 52.50 \\
\hline 1.7318 & 0 & 1 & 1 & 6 & 52.82 \\
\hline 1.7209 & 2 & 3 & $c$ & 4 & 53.18 \\
\hline 1.7131 & 3 & 2 & 4 & 3 & 53.44 \\
\hline 1.7360 & 3 & 0 & 2 & 6 & 53.58 \\
\hline 1.5587 & 5 & 4 & 0 & 0 & 55.34 \\
\hline 1.6386 & 1 & 2 & 5 & 1 & 56.08 \\
\hline 1.6159 & 1 & 0 & 4 & 5 & 55.94 \\
\hline 1.6560 & 1 & 3 & 4 & 1 & 57.32 \\
\hline 1.5853 & 1 & 2 & 5 & 2 & 58.10 \\
\hline 1.5779 & 3 & 0 & 6 & $1+$ & 50.44 \\
\hline $1.535 \mathrm{j}$ & 2 & 1 & 5 & 1 & 60.24 \\
\hline 1.5267 & 1 & 1 & 5 & 4 & 60.60 \\
\hline 1.5172 & 2 & 2 & 2 & 0 & 51.32 \\
\hline 1.5365 & 1 & 4 & 2 & 2 & $51 \cdot 50$ \\
\hline 1.5048 & 1 & 1 & 1 & 7 & 51.58 \\
\hline 1.4835 & 2 & $\Xi$ & 4 & 3 & $E 2.55$ \\
\hline 1.4524 & 2 & 2 & 4 & $5+$ & 54.06 \\
\hline$\therefore 4479$ & 1 & 3 & 5 & 0 & 64.28 \\
\hline 1.4250 & 2 & 2 & $\epsilon$ & 1 & 05.44 \\
\hline 1.4212 & 2 & 4 & 3 & 2 & 65.64 \\
\hline 1.4185 & 2 & 4 & 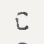 & 4 & 65.78 \\
\hline 1.4082 & 1 & 3 & o & 6 & 60.32 \\
\hline 1.4034 & 1 & 4 & 1 & 4 & 66.58 \\
\hline 1.3967 & 2 & 2 & 4 & 4 & 06.94 \\
\hline 1.3934 & 2 & 3 & 1 & 6 & 67.12 \\
\hline 1.3746 & 1 & 1 & 3 & 7 & 58.16 \\
\hline 1.3603 & 1 & 4 & 2 & 4 & 06.98 \\
\hline 1.3524 & 2 & 4 & 4 & 1 & 69.44 \\
\hline 1.3493 & 2 & 1 & 5 & 4 & 59.62 \\
\hline 1.3297 & 1 & 2 & 4 & 6 & 70.80 \\
\hline
\end{tabular}




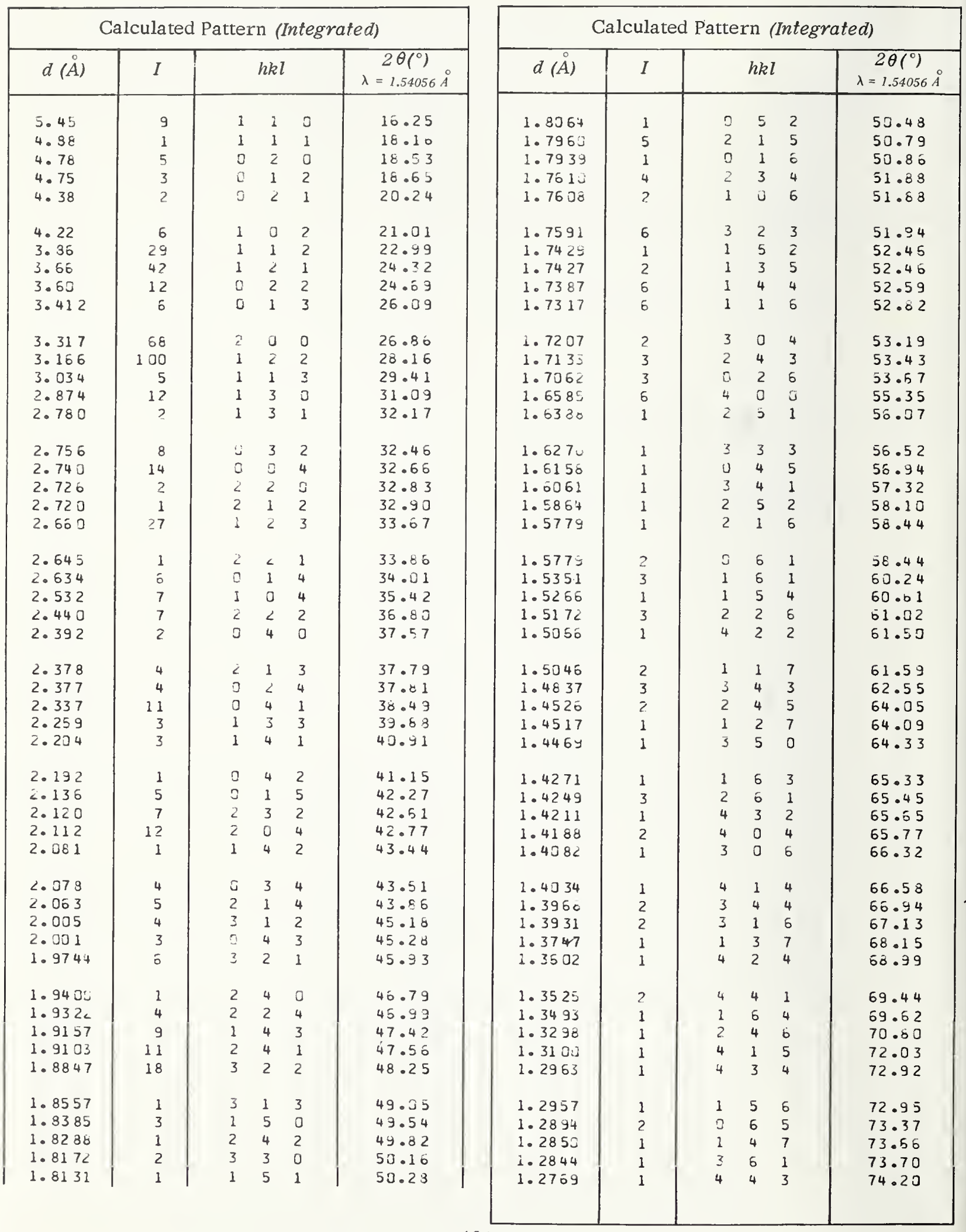


Structure

Monoclinic, $\mathrm{C} 2 / \mathrm{C}$ (15), $\mathrm{Z}=8$ [Burns and Busing, 1965] .

\section{Lattice parameters}

$a=6.01 \pm 0.02, b=11.64 \pm 0.02, c=8.18 \pm 0.02 \AA$, $\beta=90^{\circ} 45^{\prime} \pm 5^{\prime} \quad$ [ibid. ]

\section{Scattering factors}

$\mathrm{Li}^{+1}[3.3 .1 \mathrm{~A}] ; \mathrm{Cs}^{0}, \mathrm{~F}^{-1}[3.3 .1 \mathrm{~A}]$, corrected for real and imaginary dispersion $[3.3 .2 \mathrm{~B}]$

\section{Thermal parameters}

Isotropic: Cs 1.96; Li 2.50; F(1) 2.20; $F(2) 2.40 ; F(3) 3.00$.

Density (calculated) $4.130 \mathrm{~g} / \mathrm{cm}^{3}$

\section{Scale factor}

$10.28 \times 10^{4}$

\section{Reference}

Burns,J.H. and W.R. Busing (1965). Crystal structures of rubidium lithium fluoride, RbIiF2, and cesium lithium fluoride, CsLiFa, Inorg. Chem. 4, 1510-1512.

\begin{tabular}{|c|c|c|c|c|c|c|}
\hline \multicolumn{7}{|c|}{ Calculated Pattern (Peak heights) } \\
\hline$d(\stackrel{\circ}{)}$ & $I$ & \multicolumn{4}{|c|}{$h k l$} & $\begin{array}{c}\left.2 \theta 1^{\circ}\right) \\
\lambda=1.54056 \AA\end{array}$ \\
\hline 5.82 & 28 & 0 & 2 & 0 & & 15.22 \\
\hline 4.74 & 42 & c & 2 & 1 & & 18.70 \\
\hline 4.49 & 47 & -1 & 1 & 1 & & 19.74 \\
\hline 4.45 & 42 & 1 & 1 & 1 & & 19.94 \\
\hline 4.09 & 42 & S & 0 & 2 & & 21.72 \\
\hline 3.35 & 16 & o & 2 & 2 & & 20.62 \\
\hline 3.26 & 51 & -1 & 1 & 2 & & $27 \cdot 30$ \\
\hline 3.23 & 51 & 1 & 1 & 2 & & 27.60 \\
\hline 3.04 & 91 & -1 & 3 & 1 & & 29.40 \\
\hline 3.02 & 150 & 1 & 3 & 1 & & 29.54 \\
\hline 3.01 & 74 & 2 & 0 & 0 & & 29.70 \\
\hline 2.910 & 44 & 3 & 4 & 0 & & 30.70 \\
\hline 2.741 & 17 & 0 & 4 & 1 & & 32.64 \\
\hline 2.670 & 13 & 2 & 2 & 0 & & 33.54 \\
\hline 2.547 & 21 & -2 & 2 & 1 & & 35.20 \\
\hline 2.529 & 29 & 2 & 2 & 1 & & 35.46 \\
\hline 2.469 & 86 & 0 & 2 & 3 & & 36.36 \\
\hline 2.436 & 25 & -2 & 0 & 2 & + & 36.86 \\
\hline 2.416 & 3 & 1 & 1 & 3 & & 37.18 \\
\hline 2.406 & 19 & 2 & 0 & 2 & & 37.34 \\
\hline 2.371 & 8 & 0 & 4 & 2 & & 37.92 \\
\hline 2.248 & 2 & -2 & 2 & 2 & & 40.08 \\
\hline 2.223 & 1 & 2 & 2 & 2 & & 40.54 \\
\hline$<.100$ & 7 & -1 & 5 & 1 & + & 43.04 \\
\hline 2.095 & 9 & 1 & 5 & 1 & & 43.14 \\
\hline 2.091 & 18 & 2 & 4 & 0 & & 43.24 \\
\hline 2.084 & 10 & 1 & 3 & 3 & & 43.38 \\
\hline 2.030 & 1 & -2 & 4 & 1 & & 44.50 \\
\hline 2.021 & 2 & 2 & 4 & 1 & & 44.82 \\
\hline 1.9894 & 9 & 0 & 4 & 3 & & 45.56 \\
\hline 1.9403 & 7 & 0 & 6 & 0 & & 46.78 \\
\hline 1.9187 & 32 & -2 & 2 & 3 & + & 47.34 \\
\hline 1.9141 & 31 & 1 & 5 & 2 & + & 47.46 \\
\hline 1.9020 & 15 & 1 & 1 & 4 & & 47.78 \\
\hline 1.8968 & 24 & 2 & 2 & 3 & & 47.92 \\
\hline 1.8682 & 8 & -2 & 4 & 2 & & 48.70 \\
\hline 1.8546 & 6 & 2 & 4 & 2 & & 49.08 \\
\hline 1.7866 & 6 & -3 & 1 & 2 & & 51.08 \\
\hline 1.7692 & 7 & 3 & 1 & 2 & & 51.62 \\
\hline 1.7527 & 7 & 3 & 6 & 2 & & 52.14 \\
\hline 1.7434 & 13 & -3 & 3 & 1 & & 52.44 \\
\hline 1.7348 & 11 & 3 & 3 & 1 & & 52.72 \\
\hline 1.7008 & 1 & -2 & 0 & 4 & & 53.86 \\
\hline 1.6805 & 2 & 2 & 0 & 4 & & 54.58 \\
\hline 1.6665 & 7 & -2 & 4 & 3 & & 55.06 \\
\hline 1. 6516 & 8 & 2 & 4 & 3 & & 55.60 \\
\hline 1.6300 & 11 & 2 & 6 & 0 & & 56.40 \\
\hline 1. 5964 & 1 & 2 & 6 & 1 & & 57.70 \\
\hline 1. 5809 & 2 & 0 & 6 & 3 & & 58.32 \\
\hline 1.5740 & 8 & 0 & 2 & 5 & + & 58.60 \\
\hline
\end{tabular}


Cesium Lithium Fluoride, $\mathrm{CsLiF}_{2}$ (monoclinic) - continued

\begin{tabular}{|c|c|c|c|c|c|c|}
\hline \multicolumn{7}{|c|}{ Calculated Pattern (Peak heights) } \\
\hline$d(\stackrel{\circ}{)}$ & $I$ & & $h\rangle$ & & & $\begin{array}{c}2 \theta\left({ }^{\circ}\right) \\
\lambda=1.54056 \AA\end{array}$ \\
\hline 1.5715 & 8 & 1 & 7 & 1 & + & 58.70 \\
\hline 1.5590 & 1 & 1 & 1 & 5 & & 53.22 \\
\hline 1.5177 & 2 & -2 & 6 & 2 & & 61.00 \\
\hline 1.5105 & ? & 2 & 6 & 2 & & 61.32 \\
\hline 1.5026 & 6 & 4 & 0 & 0 & & 61.68 \\
\hline 1.4917 & 9 & -1 & 5 & 4 & & 52.18 \\
\hline 1.4904 & 9 & 1 & 7 & 2 & + & 62.24 \\
\hline 1.4848 & 7 & 1 & 5 & 4 & & 62.50 \\
\hline 1.4663 & 3 & -1 & 3 & 5 & & 63.38 \\
\hline 1.4581 & 3 & 1 & 3 & 5 & & 03.78 \\
\hline 1.4544 & 2 & 4 & 2 & 0 & + & 63.96 \\
\hline 1.4324 & 2 & 0 & 8 & 1 & & 65.06 \\
\hline 1.4293 & 6 & -3 & 1 & 4 & + & 65.22 \\
\hline 1.4258 & 5 & 0 & 4 & 5 & & 65.40 \\
\hline 1.4189 & 4 & 3 & 5 & 2 & & 65.76 \\
\hline 1.4154 & 3 & -4 & 0 & 2 & & 65.94 \\
\hline 1.4113 & 3 & 3 & 1 & 4 & & 56.16 \\
\hline 1.4075 & 2 & 0 & 6 & 4 & & 66.36 \\
\hline 1.4023 & 5 & -2 & 2 & 5 & + & 66.64 \\
\hline 1.3945 & 1 & 2 & 6 & 3 & & 67.06 \\
\hline 1.3875 & 4 & 2 & 2 & 5 & & 67.44 \\
\hline 1.3631 & 3 & 0 & 0 & 6 & & 68.82 \\
\hline 1.3350 & 3 & 4 & 4 & c & & 73.48 \\
\hline 1.3200 & 1 & -4 & 4 & 1 & & 71.40 \\
\hline 1.3152 & 1 & 4 & 4 & 1 & & 71.70 \\
\hline 1.2941 & 1 & -2 & 4 & 5 & & 73.06 \\
\hline 1.2904 & 4 & -4 & 2 & 3 & & 73.30 \\
\hline 1.2835 & 2 & 3 & 8 & 3 & + & 73.76 \\
\hline 1.2767 & 4 & 4 & 2 & 3 & & 74.22 \\
\hline 1.2734 & 3 & -4 & 4 & 2 & & 74.44 \\
\hline 1.2656 & 2 & -3 & 7 & 1 & & 74.93 \\
\hline 1.2627 & 3 & -1 & 7 & 4 & + & 75.18 \\
\hline 1.2593 & 3 & 1 & 7 & 4 & & 75.42 \\
\hline 1.2500 & 4 & -1 & 9 & 1 & & 76.08 \\
\hline 1.2492 & 4 & 1 & 9 & 1 & & 76.14 \\
\hline 1.2472 & 3 & -2 & 0 & 6 & & $75 \cdot .28$ \\
\hline 1.2347 & $?$ & 0 & 4 & 6 & + & 77.20 \\
\hline 1.2248 & 3 & -3 & 5 & 4 & + & 77.94 \\
\hline i. 2182 & 1 & 3 & 7 & 2 & & 78.44 \\
\hline 1.2133 & 2 & 3 & 5 & 4 & & 78.82 \\
\hline 1.2115 & 1 & -3 & 3 & 5 & + & 78.96 \\
\hline 1.2044 & 1 & -4 & 4 & 3 & & 79.52 \\
\hline 1.1976 & 1 & 3 & 3 & 5 & & 80.06 \\
\hline 1.1936 & 1 & 4 & 4 & 3 & & 80.38 \\
\hline 1.1877 & 2 & 4 & 6 & 0 & & 80.86 \\
\hline 1.1831 & 2 & -2 & 8 & 3 & & 81.24 \\
\hline 1.1779 & 2 & 2 & 8 & 3 & & 81.68 \\
\hline 1.1516 & 1 & -5 & 1 & 2 & & 83.96 \\
\hline $\begin{array}{l}1.1467 \\
1.1439\end{array}$ & $\begin{array}{l}2 \\
3\end{array}$ & $\begin{array}{l}-2 \\
-4\end{array}$ & $\begin{array}{l}4 \\
6\end{array}$ & $\begin{array}{l}6 \\
2\end{array}$ & + & $\begin{array}{l}84.40 \\
84.56\end{array}$ \\
\hline
\end{tabular}

\begin{tabular}{|c|c|c|c|c|c|}
\hline \multicolumn{6}{|c|}{ Calculated Pattern (Integrated) } \\
\hline$d(\stackrel{\circ}{A})$ & $I$ & & $h k l$ & & $\begin{array}{c}2 \theta\left(^{\circ}\right) \\
\lambda=1.54056 \AA\end{array}$ \\
\hline 5.82 & 25 & 0 & 2 & 0 & 15.21 \\
\hline 4.74 & 40 & 0 & 2 & 1 & 18.70 \\
\hline 4.53 & 45 & -1 & 1 & 1 & 19.73 \\
\hline 4.45 & 41 & 1 & 1 & 1 & 19.95 \\
\hline 4.09 & 42 & 0 & 0 & 2 & 21.71 \\
\hline 3.35 & 16 & 0 & 2 & 2 & 26.62 \\
\hline 3.27 & $j 6$ & -1 & 1 & 2 & 27.29 \\
\hline 3.23 & 55 & 1 & 1 & 2 & 27.60 \\
\hline 3.04 & y? & -1 & 3 & 1 & 29.40 \\
\hline 3.02 & 96 & 1 & 3 & 1 & 29.55 \\
\hline 3.00 & 70 & 2 & 0 & 0 & 29.71 \\
\hline 2.910 & 48 & 0 & 4 & 0 & 30.70 \\
\hline 2.742 & 19 & 0 & 4 & 1 & 32.63 \\
\hline $2.67 \mathrm{C}$ & 15 & 2 & 2 & 0 & 33.54 \\
\hline 2.547 & 25 & -2 & $\ddot{z}$ & 1 & 35.21 \\
\hline 2.529 & 32 & 2 & 2 & 1 & 35.46 \\
\hline 2.469 & 150 & $\Xi$ & 2 & 3 & $3 E .36$ \\
\hline 2.440 & 1 & -1 & 1 & 3 & 36.81 \\
\hline 2.437 & 27 & -2 & 0 & 2 & 36.86 \\
\hline 2.417 & 1 & 1 & 1 & 3 & 37.17 \\
\hline 2.436 & 22 & 2 & 0 & 2 & 37.34 \\
\hline 2.371 & 9 & 0 & 4 & 2 & 37.92 \\
\hline $\bar{Z} .24 B$ & 2 & -2 & 2 & 2 & 40.08 \\
\hline 2.224 & 1 & 2 & 2 & 2 & 40.53 \\
\hline 2.101 & 5 & -1 & 5 & 1 & 43.02 \\
\hline 2.099 & 2 & -1 & 3 & 3 & 43.07 \\
\hline 2.096 & 5 & 1 & 5 & 1 & 43.13 \\
\hline 2.090 & 19 & 2 & 4 & 0 & 43.24 \\
\hline 2.084 & 1 & 1 & 3 & 3 & 43.38 \\
\hline 2.045 & 1 & 0 & 0 & 4 & 44.26 \\
\hline 2.030 & 2 & $-<$ & 4 & 1 & 44.61 \\
\hline 2.021 & 2 & 2 & 4 & 1 & 44.81 \\
\hline 1.9696 & 12 & $a$ & 4 & 3 & 45.55 \\
\hline 1. $94 \mathrm{CO}$ & 9 & 0 & 5 & 0 & 46.79 \\
\hline 1.9247 & 4 & -3 & 1 & 1 & 47.18 \\
\hline 1.9212 & 15 & -1 & 5 & 2 & 47.27 \\
\hline 1.9185 & 24 & -2 & 2 & 3 & 47.34 \\
\hline 1.9171 & 16 & -1 & 1 & 4 & 47.38 \\
\hline 1.9137 & 14 & 1 & 5 & 2 & 47.47 \\
\hline 1.9134 & 4 & 3 & 1 & 1 & 47.48 \\
\hline 1.9022 & 16 & 1 & 1 & 4 & 47.77 \\
\hline 1.8966 & 24 & 2 & 2 & 3 & 47.93 \\
\hline 1.8876 & 1 & 0 & 6 & 1 & 48.17 \\
\hline 1.8682 & 10 & -2 & 4 & 2 & 48.70 \\
\hline 1.8545 & $\varepsilon$ & 2 & 4 & 2 & 49.08 \\
\hline 1.7869 & 8 & -3 & 1 & 2 & 51.07 \\
\hline 1.7685 & 9 & 3 & 1 & 2 & 51.63 \\
\hline 1.7528 & 9 & 0 & 6 & 2 & 52.14 \\
\hline 1.7434 & 17 & -3 & 3 & 1 & 52.44 \\
\hline 1.7350 & 14 & 3 & 3 & 1 & 52.71 \\
\hline
\end{tabular}




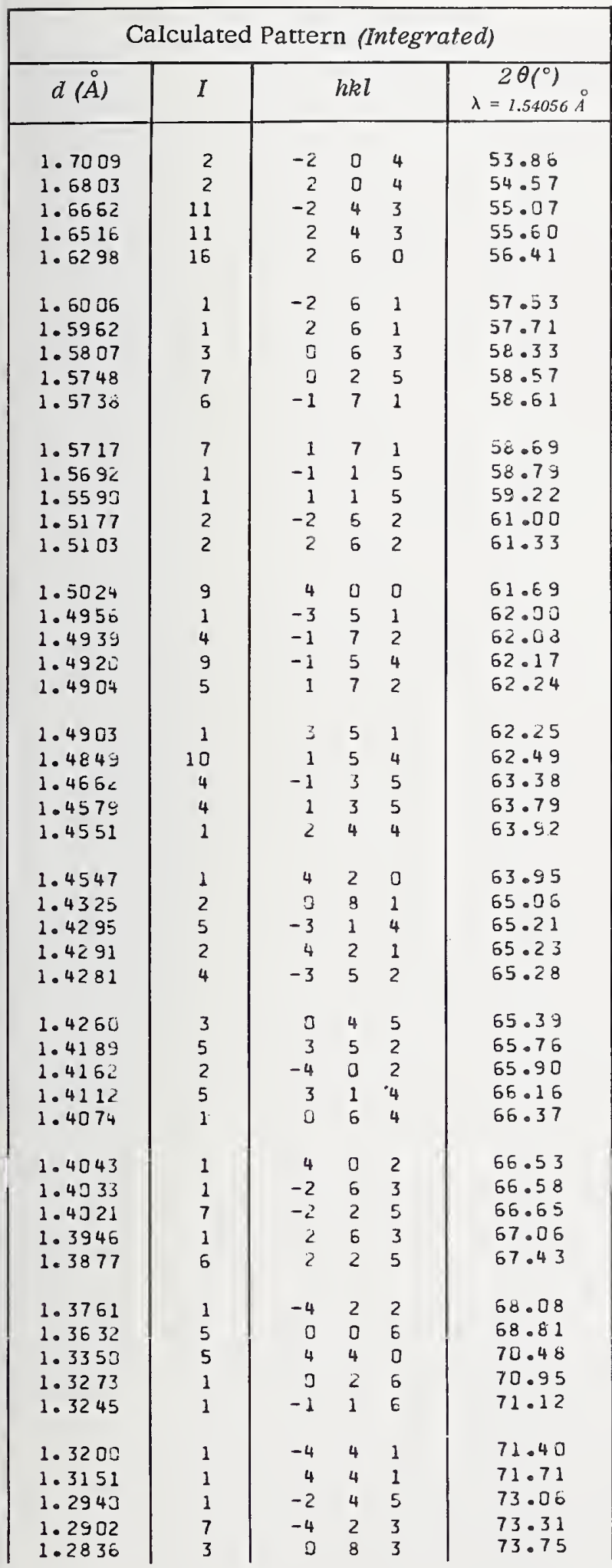

\begin{tabular}{|c|c|c|c|c|c|}
\hline \multicolumn{6}{|c|}{ Calculated Pattern (Integrated) } \\
\hline$d(\stackrel{\circ}{A})$ & $I$ & & $h k$ & & $\begin{array}{c}2 \theta\left(^{\circ}\right) \\
\lambda=1.54056 A\end{array}$ \\
\hline 1.2826 & 1 & 2 & 4 & 5 & 73.82 \\
\hline 1.2767 & 7 & 4 & 2 & 3 & 74.22 \\
\hline 1. 2734 & 1 & -4 & 4 & 2 & 74.44 \\
\hline 1.2657 & 3 & -3 & 7 & 1 & 74.97 \\
\hline 1.2635 & 3 & -1 & 7 & 4 & 75.13 \\
\hline 1.2625 & 2 & 3 & 7 & 1 & 75.20 \\
\hline 1.2592 & 3 & 1 & 7 & 4 & 75.42 \\
\hline 1.2501 & 4 & -1 & y & 1 & 76.08 \\
\hline 1. 2490 & 4 & 1 & 9 & 1 & 76.15 \\
\hline 1.2476 & 2 & -2 & 0 & 6 & 76.26 \\
\hline 1.2354 & 2 & 2 & 0 & 6 & 77.15 \\
\hline 1.2345 & 3 & כ & 4 & 6 & 77.21 \\
\hline 1.2249 & 4 & -3 & 5 & 4 & 77.93 \\
\hline 1.2240 & 2 & -3 & 7 & 2 & 78.00 \\
\hline 1.2199 & 1 & -2 & 2 & 6 & 78.31 \\
\hline 1.2182 & 2 & 3 & 7 & 2 & 78.44 \\
\hline 1.2133 & 4 & 3 & 5 & 4 & 76.82 \\
\hline 1.2115 & 1 & -3 & 3 & 5 & 78.96 \\
\hline 1.2084 & 1 & 2 & 2 & 6 & $7 y .20$ \\
\hline 1.2045 & 2 & -4 & 4 & 3 & 79.51 \\
\hline 1.1975 & 2 & 3 & 3 & 5 & 80.07 \\
\hline 1.1935 & 2 & 4 & 4 & 3 & 80.39 \\
\hline 1.1878 & 3 & 4 & 6 & 0 & 80.85 \\
\hline 1.1831 & 4 & -2 & 8 & 3 & 81.25 \\
\hline 1.1778 & 4 & 2 & 8 & 3 & 81.69 \\
\hline 1.1643 & 1 & 0 & 10 & 0 & 82.67 \\
\hline 1.1569 & 1 & -1 & 5 & 6 & 83.49 \\
\hline 1.1516 & $i$ & -5 & 1 & 2 & 83.96 \\
\hline 1.1468 & 1 & -1 & 7 & 5 & 84.39 \\
\hline 1.1466 & 1 & -2 & 4 & 6 & 84.41 \\
\hline 1.1442 & 1 & -1 & 1 & 7 & 84.63 \\
\hline 1.1439 & 2 & -4 & 6 & 2 & 84.66 \\
\hline 1.1435 & 2 & 5 & 1 & 2 & 84.69 \\
\hline 1.1428 & 1 & 1 & 7 & 5 & 84.76 \\
\hline 1.1389 & 3 & -5 & 3 & 1 & 85.12 \\
\hline 1.1387 & 1 & 1 & 1 & 7 & 85.13 \\
\hline 1.1375 & 1 & 4 & 6 & 2 & 85.24 \\
\hline 1.1371 & 1 & 2 & 4 & 6 & 35.28 \\
\hline 1.1350 & 2 & 5 & 3 & 1 & 85.48 \\
\hline 1.1195 & 1 & 0 & 10 & 2 & 86.95 \\
\hline 1.1154 & 2 & 0 & 6 & 6 & 87.36 \\
\hline 1.1024 & 3 & -1 & 3 & 7 & 88.65 \\
\hline 1. 0975 & 3 & 1 & 3 & 7 & 89.15 \\
\hline $1.094 j$ & 2 & -4 & 2 & 5 & 89.52 \\
\hline
\end{tabular}


Chromium Fluoride, $\mathrm{Cr}_{2} \mathrm{~F}_{5}$ (monoclinic)

Structure

Monoclinic, $C 2 / C(15), \quad \mathrm{Z}=4$ [Steinfink and Burns, 1964]

Lattice parameters

$a=7.773 \pm .005, b=7.540 \pm .005, c=7.440 \pm .005 \AA$, $\beta=124.25 \pm 0.1^{\circ}$ [ibid. ]

Scattering factors

$\mathrm{F}^{-1}[3.3 .1 \mathrm{~A}] ; \mathrm{Cr}^{+2}, \mathrm{Cr}^{+3}[3 \cdot 3.1 \mathrm{~B}]$

\section{Thermal parameters}

Isotropic: $\mathrm{Cr}^{+3} \quad 0.49 ; \mathrm{Cr}^{+2} \quad 0.77 ; \mathrm{F}^{-}$(1) 1.41 $\mathrm{F}^{-}$(2) $1.19 ; \mathrm{F}^{-}$(3) 1.64

Density

(calculated) $3.667 \mathrm{~g} / \mathrm{cm}^{3}$

Scale factor

$3.008 \times 10^{4}$

\section{Reference}

Steinfink,H. and J.H.Burns (1964). The crystal structure of $\mathrm{Cr}_{2} \mathrm{~F}_{5}$, Acta Cryst. 17, 823-826.

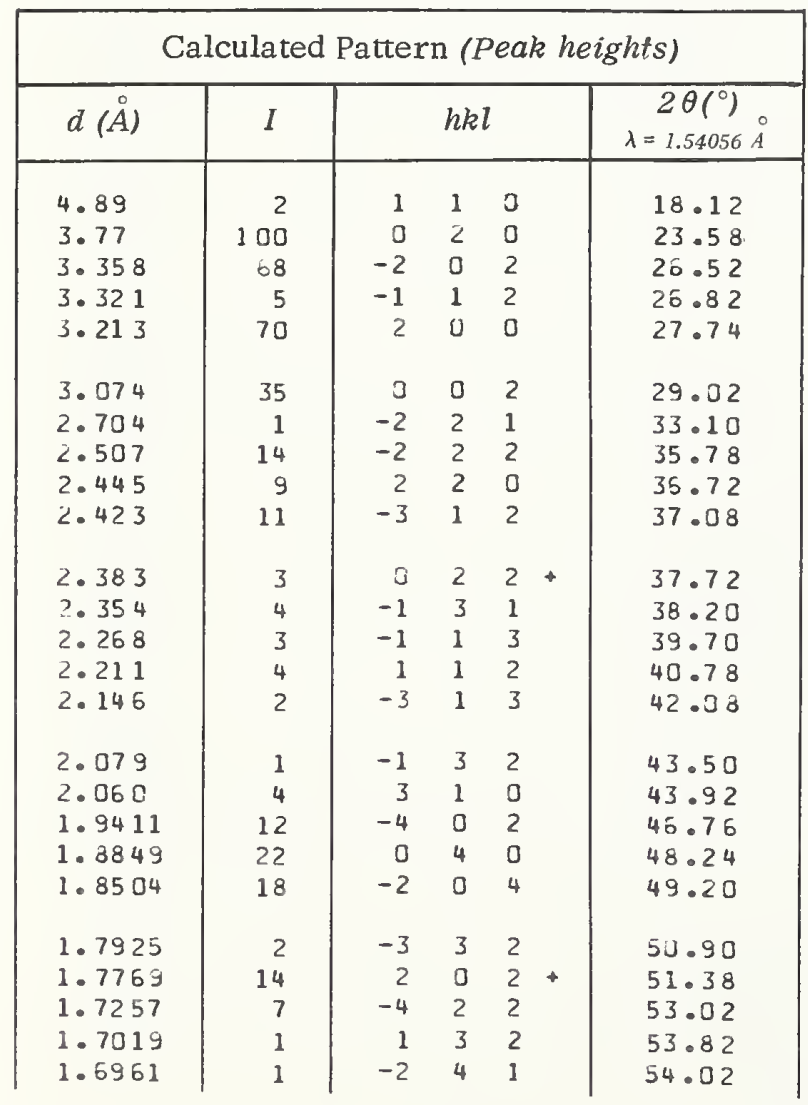

1. 1914

1.1897

1.1769

1. 1736

1. 1703

1.1632

1. 1563

1.1055

1.0728

Calculated Pattern (Peak heights)

1. 3206

1. 2931

1. 2737

1. 2537

1. 2349

1.0708

1. 0582

1.0549

1.0441

1.0396

1. 0329

1.0302

1. 0261

1. 02,50

$1.0 כ 50$

.9987

. 9891

.9857

.9705

.9399

.9311

.9109

.9074

. 9044

- 9011

.9005

\begin{tabular}{|c|c|c}
\hline$I$ & $h k l$ & $2 \theta 1^{\circ}$ \\
$\lambda=1.540$
\end{tabular}

0()$\left.^{\circ}\right)$ $\lambda=1.54056 \AA$

54.62

55.26

55.90

56.56

57.30

60.14

63.30

60.98

62.84

65.52

69.44

71.36

71.74

73.12

74.08

74.42

75.60

75.82

77.18

78.10

79.00

83.56

80.70

81.76

82.04

82.32

82.94

83.54

88.34

91.78

92.00

93.42

93.80

95.08

95.62

96.44

96.78

97.30

97.44

99.94

$\begin{array}{rrr}0 & 0 & 6 \\ -2 & 4 & 6\end{array}$

$\begin{array}{lll}4 & 4 & 2 \\ 0 & 2 & 6\end{array}+\begin{aligned} & 100.94 \\ & 102.30\end{aligned}$

$\begin{array}{lll}2 & 4 & 6\end{array} \quad 102.30$

\begin{tabular}{lll|l}
-8 & 0 & 4 & 105.06
\end{tabular}

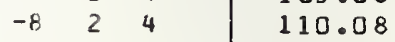

640

111.64

115.48

116.18

116.80

117.48

117.60 
Calculated Pattern (Integrated)

\begin{tabular}{|c|c|c|c|c|c|}
\hline$d(\stackrel{\circ}{A})$ & $I$ & \multicolumn{3}{|c|}{$h k l$} & $\begin{array}{c}2 \theta\left(^{\circ}\right) \\
\lambda=1.54056 \AA\end{array}$ \\
\hline 4.89 & 2 & 1 & 1 & 0 & 18.12 \\
\hline 3.77 & 100 & 0 & 2 & 0 & 23.58 \\
\hline 3. 357 & 73 & -2 & D & 2 & 26.53 \\
\hline 3.322 & 4 & -1 & 1 & 2 & 26.81 \\
\hline 3.213 & 77 & 2 & 0 & 0 & 27.75 \\
\hline 3.075 & 37 & 0 & 0 & 2 & 29.01 \\
\hline 2.734 & 1 & -2 & 2 & 1 & 33.10 \\
\hline 2.507 & 16 & -2 & 2 & 2 & 35.78 \\
\hline 2.445 & 11 & 2 & 2 & 0 & 36.72 \\
\hline 2.422 & 13 & -3 & 1 & 2 & 37.09 \\
\hline 2.383 & 3 & 0 & 2 & 2 & 37.72 \\
\hline 2.380 & 2 & -3 & 1 & 1 & 37.77 \\
\hline 2.354 & 5 & -1 & 3 & 1 & $38 \cdot 20$ \\
\hline 2.269 & 4 & -1 & 1 & 3 & 39.70 \\
\hline 2.211 & 5 & 1 & 1 & 2 & 40.78 \\
\hline 2.146 & 3 & -3 & 1 & 3 & $4 \hat{2} .07$ \\
\hline 2.079 & 1 & -1 & 3 & 2 & 43.49 \\
\hline 2.060 & 5 & 3 & 1 & 0 & 43.91 \\
\hline 1.9973 & 1 & 2 & 2 & 1 & 45.37 \\
\hline $1.940 y$ & 16 & -4 & 0 & 2 & 46.76 \\
\hline 1.8853 & 28 & 0 & 4 & 0 & 48.24 \\
\hline 1.8504 & 23 & -2 & 0 & 4 & 49.20 \\
\hline 1.7927 & 3 & -3 & 3 & 2 & 50.89 \\
\hline 1.7772 & 17 & 2 & 0 & 2 & 51.37 \\
\hline 1.7766 & 2 & -3 & 1 & 4 & 51.39 \\
\hline 1.7257 & 10 & -4 & 2 & 2 & 53.02 \\
\hline 1.7318 & 1 & 1 & 3 & 2 & 53.82 \\
\hline 1.6956 & 1 & -2 & 4 & 1 & 54.04 \\
\hline 1.6787 & 5 & -4 & 0 & 4 & 54.63 \\
\hline 1.6611 & 18 & -2 & 2 & 4 & $55 \cdot 25$ \\
\hline 1.6437 & 17 & -2 & 4 & 2 & 55.89 \\
\hline 1.6301 & 1 & 3 & 3 & 0 & 56.40 \\
\hline 1.6258 & 14 & 2 & 4 & 0 & 56.56 \\
\hline 1.6075 & 8 & 2 & 2 & 2 & 57.26 \\
\hline 1.6071 & 12 & 0 & 4 & 2 & 57.28 \\
\hline 1.6063 & 13 & 4 & 0 & 0 & 57.31 \\
\hline 1.5375 & 7 & 0 & 0 & 4 & 60.13 \\
\hline 1.5336 & 5 & -4 & 2 & 4 & 60.30 \\
\hline 1.5182 & 1 & -5 & 1 & 3 & 60.98 \\
\hline 1.4777 & 8 & 4 & 2 & ] & 62.83 \\
\hline 1.4236 & 5 & 0 & 2 & 4 & 65.51 \\
\hline 1.3522 & 8 & -4 & 4 & 2 & 69.45 \\
\hline 1.3205 & 9 & -2 & 4 & 4 & 71.37 \\
\hline 1. 3144 & 1 & 1 & 1 & 4 & 71.75 \\
\hline 1.2931 & 9 & 2 & 4 & 2 & 73.12 \\
\hline 1.2789 & 4 & -6 & 0 & 4 & 74.07 \\
\hline 1.2736 & 1 & -1 & 5 & 3 & 74.43 \\
\hline 1.2567 & 2 & 0 & 6 & 0 & 75.61 \\
\hline $\begin{array}{l}1.2539 \\
1.2536\end{array}$ & $\frac{1}{3}$ & $\begin{array}{l}-6 \\
-4\end{array}$ & $\begin{array}{l}0 \\
4\end{array}$ & $\begin{array}{l}2 \\
4\end{array}$ & $\begin{array}{l}75.80 \\
75.82\end{array}$ \\
\hline
\end{tabular}

\begin{tabular}{|c|c|c|c|c|c|}
\hline \multicolumn{6}{|c|}{ Calculated Pattern (Integrated) } \\
\hline$d(A)$ & $I$ & & $h k l$ & & $\begin{array}{c}2 \theta\left({ }^{\circ}\right) \\
\lambda=1.54056 \AA\end{array}$ \\
\hline 1.234 .9 & 2 & -4 & 0 & 6 & 77.18 \\
\hline 1.2226 & 7 & 4 & 4 & 0 & 78.11 \\
\hline 1.2111 & 6 & -6 & 2 & 4 & 78.99 \\
\hline 1.1914 & 5 & 0 & 4 & 4 & 80.56 \\
\hline 1.1899 & 1 & -6 & 2 & 2 & 80.69 \\
\hline 1.1894 & 1 & -2 & 0 & 6 & 80.73 \\
\hline 1.1775 & 1 & 4 & 0 & 2 & 81.71 \\
\hline 1.1769 & 2 & -2 & 6 & 2 & 81.76 \\
\hline 1.1735 & 4 & -4 & 2 & 6 & 82.05 \\
\hline 1.1703 & 1 & 2 & 6 & 0 & 82.32 \\
\hline 1.1633 & 2 & 0 & 6 & 2 & 82.93 \\
\hline 1.1564 & 2 & 2 & 0 & 4 & 83.54 \\
\hline 1.1343 & 1 & -2 & 2 & 6 & 85.55 \\
\hline 1.1192 & 1 & -6 & 0 & 6 & 86.99 \\
\hline 1.1355 & 2 & 2 & 2 & 4 & 88.33 \\
\hline 1.0729 & 2 & -6 & 2 & 6 & 91.77 \\
\hline 1.0708 & 2 & 6 & 0 & 0 & 92.00 \\
\hline 1.0583 & 4 & $-\varepsilon$ & 4 & 4 & 93.41 \\
\hline 1.0549 & 2 & -4 & 6 & 2 & 93.81 \\
\hline 1.0440 & 2 & -6 & 4 & 2 & 95.09 \\
\hline 1.0396 & 2 & -2 & 6 & 4 & 95.62 \\
\hline 1.0335 & 3 & -4 & 4 & 6 & 96.44 \\
\hline 1.0301 & 2 & 6 & 2 & 0 & 96.80 \\
\hline 1.0261 & 2 & 2 & 6 & 2 & 97.30 \\
\hline 1.0253 & 2 & 0 & 0 & 6 & 97.44 \\
\hline 1.0050 & 1 & -4 & 6 & 4 & 99.93 \\
\hline $1.005 y$ & 1 & -2 & 4 & 6 & 99.95 \\
\hline .9987 & 2 & 4 & 4 & 2 & 100.54 \\
\hline .9898 & 2 & 4 & 6 & 3 & 102.20 \\
\hline .9891 & 2 & 0 & 2 & 6 & 102.30 \\
\hline .9857 & 3 & 2 & 4 & 4 & 102.79 \\
\hline . 9730 & 1 & 0 & 6 & 4 & 104.68 \\
\hline . 9705 & 1 & -8 & 0 & 4 & 105.07 \\
\hline .9623 & 1 & -6 & 4 & 6 & 106.34 \\
\hline .9425 & 1 & 0 & 8 & 0 & 109.63 \\
\hline .9398 & 2 & -8 & 2 & 4 & 110.09 \\
\hline .9311 & 3 & 6 & 4 & 0 & 111.64 \\
\hline .9252 & 1 & -4 & 0 & 8 & 112.72 \\
\hline .9128 & 1 & -8 & 0 & 2 & 115.11 \\
\hline 9909 . & 2 & -8 & 2 & 6 & 115.48 \\
\hline .9074 & 3 & -2 & 8 & 2 & 116.18 \\
\hline .9044 & 2 & 2 & 8 & 0 & 116.80 \\
\hline .9011 & 3 & 0 & 8 & 2 & 117.48 \\
\hline . 9005 & 3 & 0 & 4 & 6 & 117.51 \\
\hline .8985 & 3 & -4 & 2 & 8 & 118.02 \\
\hline .8963 & 2 & -6 & 6 & 4 & 118.49 \\
\hline .8886 & 2 & 4 & 0 & 4 & 120.19 \\
\hline .8883 & 2 & -6 & 2 & 8 & 120.25 \\
\hline .8876 & 1 & $-\varepsilon$ & 6 & 2 & 120.41 \\
\hline .8871 & 2 & -8 & 2 & 2 & 120.52 \\
\hline
\end{tabular}


Structure

Orthorhombic, $\mathrm{P} 2_{1} 2_{1} 2_{1}$ (19), $\mathrm{Z}=4$ [Gramaccioli and Marsh, 1966]

Lattice parameters

$a=11.084, b=10.350, c=7.238 \AA$ [ibid.]

\section{Scattering factors}

$\mathrm{H}^{\circ}, \mathrm{C}^{\circ}, \mathrm{N}^{\circ}, \mathrm{O}^{\circ}[3,3.1 \mathrm{~A}], \mathrm{Cu}^{\circ}[3,3.1 \mathrm{~A}] \mathrm{cor}-$ rected for the real part of the anomalous dispersion effect [3.3.2B]

\section{Thermal parameters}

Isotropic: Cu 1.71; C(1) 1.74; C(2) 1.70; C(3) $1.96 ; C(4) 2.19 ; C(5) 1.96 ; N 1.95$;

$O$ (1) $2.18 ; O(2) 2.31 ; O(3) 2.09$;

$\mathrm{O}(4) 2.50 ; \mathrm{O}(5) 2.71 ; \mathrm{O}(6) 2.39 ; \mathrm{H}(1)$

to $H(11)$ inclusive, as given in Gramaccioli and Marsh [1966]

\section{Density}

(calculated) $1.957 \mathrm{~g} / \mathrm{cm}^{3}$ [Gramaccioli and Marsh, 1966]

\section{Scale factor}

$4.281 \times 10^{4}$

\section{Reference}

Gramaccioli,C.M. and R.E.Marsh (1966). The crystal structure of copper glutamate dihydrate, Acta Cryst. 21 594-600.

\begin{tabular}{|c|c|c|c|c|c|}
\hline \multicolumn{6}{|c|}{ Calculated Pattern (Peak heights) } \\
\hline$d(\AA)$ & $I$ & & $h k l$ & & $\begin{array}{c}2 \theta\left({ }^{\circ}\right) \\
\lambda=1.54056 \AA\end{array}$ \\
\hline 7.56 & 72 & 1 & 1 & 0 & 11.70 \\
\hline 5.93 & 17 & 0 & 1 & 1 & 14.92 \\
\hline 5.54 & 52 & 2 & 0 & 0 & 15.98 \\
\hline 5.23 & 100 & 1 & 1 & 1 & 16.94 \\
\hline 5.18 & 23 & 0 & 2 & 0 & 17.12 \\
\hline 4.89 & 38 & 2 & 1 & 0 & 18.14 \\
\hline 4.69 & 2 & 1 & 2 & 0 & 18.92 \\
\hline 4.40 & 1 & 2 & 0 & 1 & 20.16 \\
\hline 4.21 & 42 & 3 & 2 & 1 & 21.08 \\
\hline 4.05 & 63 & 2 & 1 & 1 & $21 \cdot 94$ \\
\hline 3.93 & 63 & 1 & 2 & 1 & 22.58 \\
\hline 3.78 & 3 & 2 & 2 & 0 & 23.50 \\
\hline 3.62 & 6 & 0 & 0 & 2 & 24.58 \\
\hline 3.440 & 18 & 1 & 0 & 2 & 25.88 \\
\hline 3.351 & 3 & 2 & 2 & 1 & 26.58 \\
\hline 3.290 & 12 & 3 & 0 & $1+$ & 27.08 \\
\hline 3.264 & 12 & 1 & 1 & 2 & 27.30 \\
\hline 3.136 & 11 & 3 & 1 & 1 & 28.44 \\
\hline 3.114 & 5 & 0 & 3 & 1 & 28.64 \\
\hline 3.029 & 1 & 2 & 0 & 2 & 29.46 \\
\hline 3.006 & 15 & 3 & 2 & 0 & 29.70 \\
\hline
\end{tabular}

\begin{tabular}{|c|c|c|c|c|c|c|}
\hline \multicolumn{7}{|c|}{ Calculated Pattern (Peak heights) } \\
\hline$d(\stackrel{\circ}{)}$ & $I$ & & $h k$ & & & $\begin{array}{c}2 \theta\left({ }^{\circ}\right) \\
\lambda=1.54056 \AA\end{array}$ \\
\hline 2.998 & 26 & 1 & 3 & 1 & & 29.78 \\
\hline 2.966 & 7 & J & 2 & 2 & & 30.10 \\
\hline 2.928 & 4 & 2 & 3 & 0 & & 30.50 \\
\hline 2.908 & 1 & 2 & 1 & 2 & & 30.72 \\
\hline 2.864 & 14 & 1 & 2 & 2 & & 31.20 \\
\hline 2.771 & 4 & 4 & 0 & 0 & + & 32.28 \\
\hline 2.715 & 1 & 2 & 3 & 1 & & 32.96 \\
\hline 2.676 & 4 & 4 & 1 & 0 & & 33.46 \\
\hline 2.615 & 2 & 2 & 2 & 2 & & 34.26 \\
\hline 2.586 & 11 & 3 & 0 & 2 & + & 34.56 \\
\hline 2.509 & 7 & 3 & 1 & 2 & & 35.76 \\
\hline 2.497 & 5 & 0 & 3 & 2 & & 35.94 \\
\hline 2.443 & 1 & 4 & 2 & 0 & & 36.76 \\
\hline 2.436 & 3 & 0 & 4 & 1 & & 36.86 \\
\hline 2.379 & 12 & 1 & 4 & 1 & + & 37.78 \\
\hline 2.349 & 7 & 0 & 1 & 3 & & 38.28 \\
\hline 2.313 & 18 & 3 & 2 & 2 & + & 38.90 \\
\hline 2.299 & 8 & 1 & 1 & 3 & & 39.16 \\
\hline 2.276 & 12 & 2 & 3 & 2 & & 39.56 \\
\hline 2.231 & 6 & 2 & 4 & 1 & & 40.40 \\
\hline 2.212 & 2 & 2 & 0 & 3 & & 40.76 \\
\hline 2.186 & 1 & 0 & 2 & 3 & & $41 \cdot 26$ \\
\hline 2.163 & 9 & 2 & 1 & 3 & & 41.72 \\
\hline 2.152 & 3 & 4 & 1 & 2 & & $41 \cdot 94$ \\
\hline 2.146 & 8 & 1 & 2 & 3 & & 42.08 \\
\hline 2.120 & 3 & 5 & 0 & 1 & & 42.52 \\
\hline 2.105 & 1 & 0 & 4 & 2 & & 42.94 \\
\hline $\begin{array}{l}2.077 \\
2.070\end{array}$ & $\begin{array}{l}9 \\
7\end{array}$ & $\begin{array}{l}5 \\
3\end{array}$ & $\begin{array}{l}1 \\
3\end{array}$ & $\begin{array}{l}1 \\
2\end{array}$ & + & $\begin{array}{l}43.54 \\
43.70\end{array}$ \\
\hline 2.069 & 7 & 1 & 4 & 2 & & 43.72 \\
\hline 2.034 & 9 & 1 & 5 & 0 & + & 44.50 \\
\hline 2.025 & 3 & 4 & 2 & 2 & & 44.72 \\
\hline 1.9902 & 2 & 3 & 5 & 1 & & 45.54 \\
\hline 1.9828 & 2 & 3 & 1 & 3 & & 45.72 \\
\hline 1.9673 & 2 & 2 & 4 & 2 & & 46.10 \\
\hline 1.9593 & 1 & 1 & 5 & 1 & & 46.30 \\
\hline 1.8901 & 3 & 5 & 0 & 2 & & 48.10 \\
\hline 1.8820 & 2 & 3 & 2 & 3 & & 48.32 \\
\hline 1. 8733 & 3 & 2 & 5 & 1 & & 48.56 \\
\hline 1.8596 & 9 & 5 & 1 & 2 & + & 48.94 \\
\hline 1.8553 & 6 & 4 & 3 & 2 & & 49.06 \\
\hline 1.8476 & 1 & 6 & 0 & 0 & & 49.28 \\
\hline 1.8295 & 2 & 4 & 4 & 1 & & 49.80 \\
\hline 1.8192 & 7 & 4 & 0 & 3 & + & 50.10 \\
\hline 1.8058 & 4 & 5 & 3 & 1 & + & 50.50 \\
\hline 1.7971 & 4 & 0 & 5 & 2 & & 50.76 \\
\hline 1.7925 & 4 & 4 & 1 & 3 & & 50.90 \\
\hline 1.7737 & 5 & 1 & 5 & 2 & + & 51.48 \\
\hline 1.7641 & 3 & 6 & 1 & 1 & & 51.78 \\
\hline 1.7597 & 2 & 1 & 1 & 4 & & $51 \cdot 92$ \\
\hline 1.7521 & 2 & 3 & 5 & 1 & & 52.16 \\
\hline
\end{tabular}


Copper Glutamate Dihydrate, $\mathrm{CuC}_{5} \mathrm{H}_{7} \mathrm{NO}_{4} \cdot 2 \mathrm{H}_{2} \mathrm{O}$ (orthorhombic) - continued

Calculated Pattern (Peak heights)

\begin{tabular}{|c|c|c|c|c|c|c|}
\hline$d(\stackrel{A}{)}$ & $I$ & \multicolumn{4}{|c|}{$h k l$} & $\begin{array}{c}2 \theta\left({ }^{\circ}\right) \\
\lambda=1.54056 \AA\end{array}$ \\
\hline $\begin{array}{l}1.7428 \\
1.7251 \\
1.7203\end{array}$ & $\begin{array}{l}3 \\
1 \\
1\end{array}$ & $\begin{array}{l}1 \\
0 \\
2\end{array}$ & $\begin{array}{l}4 \\
6 \\
0\end{array}$ & $\begin{array}{l}3 \\
0 \\
4\end{array}$ & + & $\begin{array}{l}52.46 \\
53.04 \\
53.20\end{array}$ \\
\hline $\begin{array}{l}1.7167 \\
1.7090 \\
1.6967 \\
1.6915 \\
1.6880\end{array}$ & $\begin{array}{l}1 \\
3 \\
1 \\
1 \\
2\end{array}$ & $\begin{array}{l}4 \\
2 \\
2 \\
6 \\
1\end{array}$ & $\begin{array}{l}2 \\
5 \\
1 \\
2 \\
2\end{array}$ & $\begin{array}{l}3 \\
2 \\
4 \\
1 \\
4\end{array}$ & + & $\begin{array}{l}53.32 \\
53.58 \\
54.00 \\
54.18 \\
54.30\end{array}$ \\
\hline $\begin{array}{l}1.6817 \\
1.6760 \\
1.6587 \\
1.6472 \\
1.6322\end{array}$ & $\begin{array}{l}1 \\
2 \\
2 \\
2 \\
3\end{array}$ & $\begin{array}{l}2 \\
4 \\
1 \\
2 \\
2\end{array}$ & $\begin{array}{l}4 \\
4 \\
6 \\
6 \\
2\end{array}$ & $\begin{array}{l}3 \\
2 \\
1 \\
0 \\
4\end{array}$ & + & $\begin{array}{l}54.52 \\
54.72 \\
55.34 \\
55.76 \\
56.32\end{array}$ \\
\hline $\begin{array}{l}1.6164 \\
1.6096 \\
1.6055 \\
1.5923 \\
1.5883\end{array}$ & $\begin{array}{l}1 \\
1 \\
1 \\
3 \\
4\end{array}$ & $\begin{array}{l}4 \\
4 \\
3 \\
3 \\
6\end{array}$ & $\begin{array}{l}5 \\
3 \\
1 \\
4 \\
3\end{array}$ & $\begin{array}{l}1 \\
3 \\
4 \\
3 \\
1\end{array}$ & & $\begin{array}{l}56.92 \\
57.18 \\
57.34 \\
57.86 \\
58.02\end{array}$ \\
\hline $\begin{array}{l}1.5858 \\
1.5681 \\
1.5566 \\
1.5504 \\
1.5466\end{array}$ & $\begin{array}{l}5 \\
2 \\
1 \\
1 \\
2\end{array}$ & $\begin{array}{l}1 \\
6 \\
5 \\
3 \\
7\end{array}$ & $\begin{array}{l}3 \\
2 \\
2 \\
2 \\
0\end{array}$ & $\begin{array}{l}4 \\
2 \\
3 \\
4 \\
1\end{array}$ & & $\begin{array}{l}58.12 \\
58.84 \\
59.32 \\
59.58 \\
59.74\end{array}$ \\
\hline $\begin{array}{l}1.5424 \\
1.5392 \\
1.5276 \\
1.5127 \\
1.5079\end{array}$ & $\begin{array}{l}2 \\
2 \\
1 \\
1 \\
1\end{array}$ & $\begin{array}{l}1 \\
2 \\
3 \\
5 \\
4\end{array}$ & $\begin{array}{l}6 \\
3 \\
6 \\
5 \\
5\end{array}$ & $\begin{array}{l}2 \\
4 \\
1 \\
0 \\
2\end{array}$ & & $\begin{array}{l}59.92 \\
60.06 \\
60.56 \\
61.22 \\
61.44\end{array}$ \\
\hline $\begin{array}{l}1.4991 \\
1.4818 \\
1.4700 \\
1.4646 \\
1.4540\end{array}$ & $\begin{array}{l}2 \\
2 \\
2 \\
1 \\
1\end{array}$ & $\begin{array}{l}4 \\
7 \\
3 \\
4 \\
4\end{array}$ & $\begin{array}{l}1 \\
2 \\
3 \\
6 \\
2\end{array}$ & $\begin{array}{l}4 \\
1 \\
4 \\
0 \\
4\end{array}$ & + & $\begin{array}{l}61.84 \\
62.64 \\
63.20 \\
63.46 \\
63.98\end{array}$ \\
\hline $\begin{array}{l}1.4364 \\
1.4113 \\
1.3960 \\
1.3883 \\
1.3761\end{array}$ & $\begin{array}{l}3 \\
1 \\
1 \\
2 \\
1\end{array}$ & $\begin{array}{l}1 \\
6 \\
5 \\
6 \\
3\end{array}$ & $\begin{array}{l}7 \\
2 \\
5 \\
4 \\
4\end{array}$ & $\begin{array}{l}1 \\
3 \\
2 \\
2 \\
4\end{array}$ & + & $\begin{array}{l}64.96 \\
66.16 \\
66.98 \\
67.40 \\
68.08\end{array}$ \\
\hline $\begin{array}{l}1.3732 \\
1.3575 \\
1.3538 \\
1.3521 \\
1.3507\end{array}$ & $\begin{array}{l}1 \\
1 \\
1 \\
2 \\
2\end{array}$ & $\begin{array}{l}5 \\
4 \\
6 \\
2 \\
7\end{array}$ & $\begin{array}{l}1 \\
6 \\
5 \\
2 \\
4\end{array}$ & $\begin{array}{l}0 \\
2 \\
1 \\
5 \\
0\end{array}$ & & $\begin{array}{l}68.24 \\
69.14 \\
69.36 \\
69.46 \\
69.54\end{array}$ \\
\hline $\begin{array}{l}1.3490 \\
1.3379 \\
1.3366 \\
1.3045 \\
1.2820\end{array}$ & $\begin{array}{l}2 \\
1 \\
1 \\
1 \\
1\end{array}$ & $\begin{array}{l}8 \\
5 \\
3 \\
4 \\
5\end{array}$ & $\begin{array}{l}1 \\
6 \\
1 \\
7 \\
5\end{array}$ & $\begin{array}{l}1 \\
1 \\
5 \\
0 \\
3\end{array}$ & + & $\begin{array}{l}69.64 \\
70.30 \\
70.38 \\
72.38 \\
73.86\end{array}$ \\
\hline $\begin{array}{l}1.2784 \\
1.2607 \\
1.2453\end{array}$ & $\begin{array}{l}1 \\
1 \\
1\end{array}$ & $\begin{array}{l}3 \\
0 \\
4\end{array}$ & $\begin{array}{l}5 \\
7 \\
2\end{array}$ & $\begin{array}{l}4 \\
3 \\
5\end{array}$ & & $\begin{array}{l}74.10 \\
75.32 \\
76.42\end{array}$ \\
\hline
\end{tabular}

\begin{tabular}{|c|c|c|c|c|c|}
\hline \multicolumn{6}{|c|}{ Calculated Pattern (Integrated) } \\
\hline$d(\stackrel{\circ}{)})$ & $I$ & & $h k$ & & $\begin{array}{c}2 \theta\left(^{\circ}\right) \\
\lambda=1.54056 \AA\end{array}$ \\
\hline 7.56 & 56 & 1 & 1 & 0 & 11.69 \\
\hline 5.93 & 17 & 0 & 1 & 1 & 14.92 \\
\hline 5.54 & 51 & 2 & 0 & 0 & 15.98 \\
\hline 5.23 & 100 & 1 & 1 & 1 & 16.94 \\
\hline 5.17 & 19 & 0 & 2 & 0 & 17.12 \\
\hline 4.89 & 40 & 2 & 1 & 0 & 18.14 \\
\hline 4.69 & 2 & 1 & 2 & 0 & 18.91 \\
\hline 4.40 & 1 & 2 & 0 & 1 & 20.16 \\
\hline 4.21 & 47 & J & 2 & 1 & 21.09 \\
\hline 4.05 & 69 & 2 & 1 & 1 & 21.93 \\
\hline 3.94 & 69 & 1 & 2 & 1 & 22.57 \\
\hline 3.78 & 4 & 2 & 2 & 0 & 23.50 \\
\hline 3. 62 & 7 & 0 & 0 & 2 & 24.58 \\
\hline 3.440 & 21 & 1 & 0 & 2 & 25.88 \\
\hline 3.352 & 4 & 2 & 2 & 1 & 26.57 \\
\hline 3.294 & 2 & 1 & 3 & 0 & 27.05 \\
\hline 3.291 & 13 & 3 & 0 & 1 & 27.07 \\
\hline 3.265 & 13 & 1 & 1 & 2 & 27.29 \\
\hline 3.136 & 13 & 3 & 1 & 1 & 28.44 \\
\hline 3.114 & 5 & 0 & 3 & 1 & 28.64 \\
\hline 3.030 & 1 & 2 & 0 & 2 & 29.45 \\
\hline 3.007 & 14 & 3 & 2 & 0 & 29.69 \\
\hline 2.998 & 24 & 1 & 3 & 1 & 29.77 \\
\hline 2.966 & 8 & 0 & 2 & 2 & 30.11 \\
\hline 2.929 & 5 & 2 & 3 & 0 & 30.50 \\
\hline 2.908 & 1 & 2 & 1 & 2 & 30.72 \\
\hline 2.865 & 17 & 1 & 2 & 2 & 31.19 \\
\hline 2.777 & 1 & 3 & 2 & 1 & 32.21 \\
\hline 2.771 & 4 & 4 & 0 & 0 & 32.28 \\
\hline 2.715 & 1 & 2 & 3 & 1 & 32.96 \\
\hline 2.677 & 5 & 4 & 1 & 0 & 33.45 \\
\hline 2.615 & 2 & 2 & 2 & 2 & $\begin{array}{l}34.26 \\
34.63\end{array}$ \\
\hline $\begin{array}{l}2.588 \\
2.587\end{array}$ & $\begin{array}{l}5 \\
1\end{array}$ & $\begin{array}{l}4 \\
0\end{array}$ & $\begin{array}{l}0 \\
4\end{array}$ & $\begin{array}{l}1 \\
0\end{array}$ & 34.64 \\
\hline $\begin{array}{l}2.587 \\
2.585\end{array}$ & 10 & 3 & 0 & 2 & 34.67 \\
\hline 2.508 & 9 & 3 & 1 & 2 & 35.77 \\
\hline 2.497 & 6 & 0 & 3 & 2 & 35.93 \\
\hline 2.443 & 1 & 4 & 2 & 0 & 36.76 \\
\hline 2.436 & 3 & 0 & 4 & 1 & 36.86 \\
\hline 2.381 & 3 & 3 & 3 & 1 & 37.75 \\
\hline 2.380 & 13 & 1 & 4 & 1 & 37.77 \\
\hline 2.357 & 1 & 1 & 0 & 3 & 38.14 \\
\hline 2.350 & 8 & 0 & 1 & 3 & 38.27 \\
\hline 2.315 & 4 & 4 & 2 & 1 & 38.88 \\
\hline 2.313 & 21 & 3 & 2 & 2 & 36.91 \\
\hline 2.299 & 9 & 1 & 1 & 3 & 39.16 \\
\hline 2.277 & 15 & 2 & 3 & 2 & 39.55 \\
\hline 2.230 & 8 & 2 & 4 & 1 & 40.41 \\
\hline 2.212 & 3 & 2 & 0 & 3 & 40.76 \\
\hline 2.187 & 1 & 0 & 2 & 3 & 41.25 \\
\hline
\end{tabular}


Copper Glutamate Dihydrate, $\mathrm{CuC}_{5} \mathrm{H}_{7} \mathrm{NO}_{4} \cdot 2 \mathrm{H}_{2} \mathrm{O}$ (orthorhombic) - continued

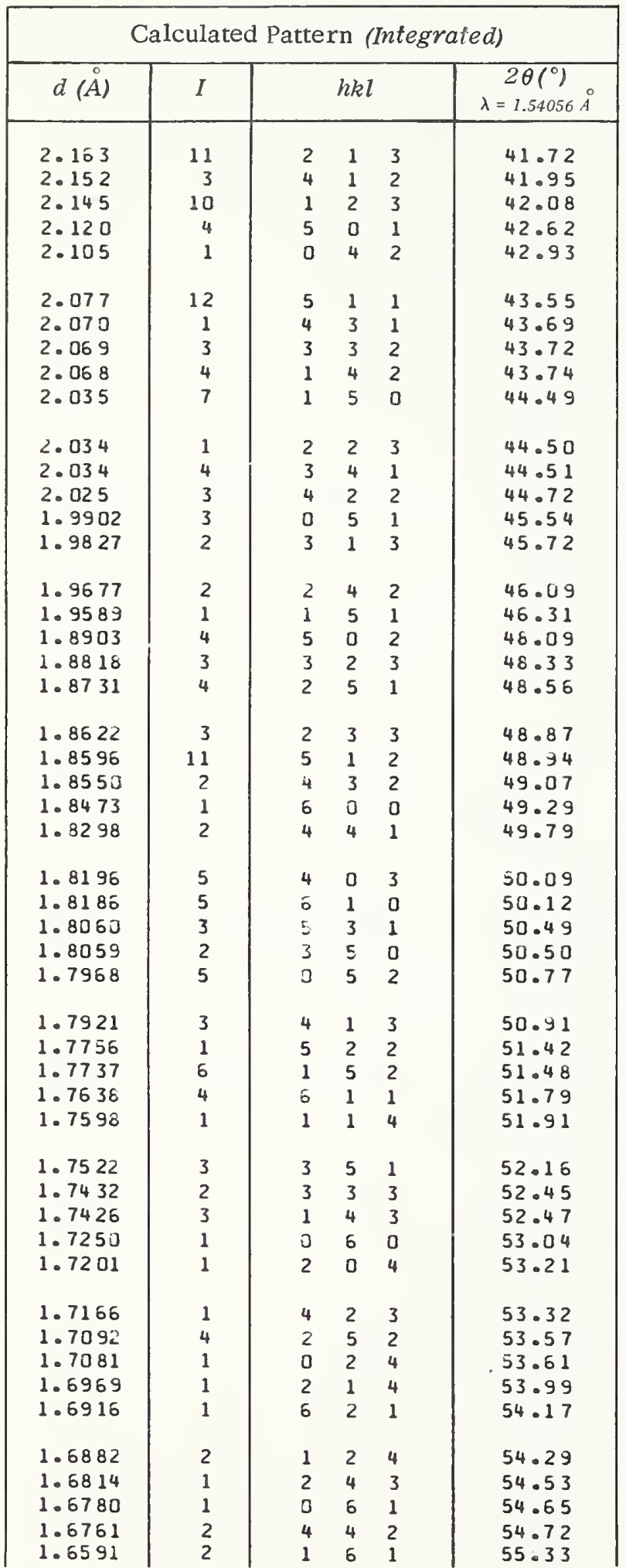

\begin{tabular}{|c|c|c|c|c|c|}
\hline \multicolumn{6}{|c|}{ Calculated Pattern (Integrated) } \\
\hline$d(\stackrel{\circ}{A})$ & $I$ & & $h k$ & & $\begin{array}{c}2 \theta\left({ }^{\circ}\right) \\
\lambda=1.54056 \AA\end{array}$ \\
\hline 1.6471 & 3 & 2 & 6 & 0 & 55.77 \\
\hline 1.6323 & 3 & 2 & 2 & 4 & 56.31 \\
\hline 1.6165 & 2 & 4 & 5 & 1 & 56.92 \\
\hline 1.6095 & 1 & 4 & 3 & 3 & 57.19 \\
\hline 1.6054 & 1 & 3 & 1 & 4 & 57.35 \\
\hline 1.5923 & 5 & 3 & 4 & 3 & 57.86 \\
\hline 1.5888 & 2 & 6 & 3 & 1 & 58.00 \\
\hline 1.5860 & 6 & 1 & 3 & 4 & 58.11 \\
\hline 1.5680 & 2 & 6 & 2 & 2 & 58.84 \\
\hline 1.5560 & 1 & 5 & 2 & 3 & 59.31 \\
\hline 1.5504 & 2 & 3 & 2 & 4 & 59.58 \\
\hline 1.5468 & 3 & 7 & c & 1 & 59.73 \\
\hline 1.5420 & 2 & 1 & 6 & 2 & 59.94 \\
\hline 1.5394 & 2 & 2 & 3 & 4 & 60.05 \\
\hline 1.5278 & 1 & 3 & 6 & 1 & 60.55 \\
\hline 1.5129 & 1 & 5 & 5 & 0 & 61.21 \\
\hline 1.5076 & 1 & 4 & 5 & 2 & 61.45 \\
\hline 1.4991 & I & 2 & 6 & 2 & 61.84 \\
\hline 1.4991 & 2 & 4 & 1 & 4 & 61.84 \\
\hline 1.4820 & 2 & 7 & 2 & 1 & 62.63 \\
\hline 1.4809 & 1 & 5 & 5 & 1 & $62 \cdot 68$ \\
\hline 1.4701 & 3 & 3 & 3 & 4 & 63.20 \\
\hline 1.4644 & 1 & 4 & 6 & 0 & 63.47 \\
\hline 1.4540 & 1 & 4 & 2 & 4 & 63.98 \\
\hline 1.4457 & 1 & 3 & 5 & 3 & 64.39 \\
\hline 1.4366 & 2 & 7 & 1 & 2 & 64.85 \\
\hline 1.4364 & 3 & 1 & 7 & 1 & 64.86 \\
\hline 1.4353 & 1 & 4 & 6 & 1 & 64.91 \\
\hline 1.4349 & 1 & 3 & 6 & 2 & 64.93 \\
\hline 1.4112 & 1 & 6 & 2 & 3 & 66.17 \\
\hline 1.3959 & 1 & 5 & 5 & 2 & 66.98 \\
\hline 1.3884 & 3 & 6 & 4 & 2 & 67.39 \\
\hline 1.3872 & 2 & 4 & 3 & 4 & 67.46 \\
\hline 1.3762 & 1 & 3 & 4 & 4 & 68.07 \\
\hline 1.3732 & 2 & 8 & 1 & 0 & 68.24 \\
\hline 1.3575 & 1 & 4 & 6 & 2 & 69.14 \\
\hline 1.3548 & 1 & 6 & 5 & 1 & 69.35 \\
\hline 1.3520 & 2 & 2 & 2 & 5 & 69.47 \\
\hline 1.3506 & 1 & 7 & 4 & 0 & 69.55 \\
\hline 1.3492 & 1 & 8 & 1 & 1 & 69.63 \\
\hline 1.3487 & 1 & 3 & 7 & 1 & 69.66 \\
\hline 1.3379 & 2 & 5 & 6 & 1 & 70.30 \\
\hline 1.3365 & 1 & 3 & 1 & 5 & 70.38 \\
\hline 1.3045 & 1 & 4 & 7 & 0 & $72 \cdot 38$ \\
\hline 1.2818 & 1 & 5 & 5 & 3 & 73.88 \\
\hline 1.2782 & 1 & 3 & 5 & 4 & 74.11 \\
\hline 1.2607 & 1 & 0 & 7 & 3 & 75.32 \\
\hline 1. 2454 & 1 & 4 & 2 & 5 & 76.42 \\
\hline 1. 2421 & 1 & 6 & 6 & 1 & 76.65 \\
\hline 1.2359 & 1 & 7 & 3 & 3 & 77.11 \\
\hline
\end{tabular}


Structure

Monoclinic, $\mathrm{C} 2 / \mathrm{C}$ (15), $\mathrm{z}=4$ [Robertson and Calvo, 1967]

\section{Lattice parameters}

$a=6.876, b=8.113, c=9.162 \AA, \quad \beta=109.54^{\circ}$ [ibid.]

\section{Polymorphism}

The polymorph $\beta-\mathrm{Cu}_{2} \mathrm{P}_{2} \mathrm{O}_{7}$ occurs at temperatures higher than about $66^{\circ} \mathrm{C}$ [ibid.]

\section{Scattering factors}

$$
\begin{aligned}
& \mathrm{P}^{\circ} \mathrm{O}^{-1}[3.3 .1 \mathrm{~A}] \\
& \mathrm{Cu}^{+2}[3.3 .1 \mathrm{~A}] \text { corrected for anomalous } \\
& \text { dispersion }[3.3 .2 \mathrm{~B}]
\end{aligned}
$$

\section{Thermal parameters}

Isotropic: $\mathrm{Cu} \mathrm{0.94;} \mathrm{P} \mathrm{0.75;} \mathrm{O(1)} \mathrm{2.2I;}$

O(2) $1.29 ; O(3) 0.95 ; O(4) 1.36$

\section{Density}

(calculated) $4.151 \mathrm{~g} / \mathrm{cm}^{3}$

\section{Scale factor}

$6.635 \times 10^{4}$

\section{Reference}

Robertson, B.E. and C. Calvo (1967). The crystal structure and phase transformation of $\alpha-\mathrm{Cu}_{2} \mathrm{P}_{2} \mathrm{O}_{7}$, Acta Cryst.22,665-72.

Note:

Using the structure data of Robertson and Calvo [1967], we were unable to duplicate their calculated structure factors, but the scaled integrated intensi-

\begin{tabular}{|c|c|c|c|c|c|c|}
\hline \multicolumn{7}{|c|}{ Calculated Pattern (Peak heights) } \\
\hline$d(\stackrel{\circ}{A})$ & $I$ & & $h k l$ & & & $\begin{array}{c}2 \theta\left({ }^{\circ}\right) \\
\lambda=1.54050 \mathrm{~A}\end{array}$ \\
\hline 2.293 & 2 & -1 & 3 & 2 & & 39.26 \\
\hline 2.274 & 1 & 2 & $\dot{2}$ & 1 & & 39.50 \\
\hline 2.254 & 2 & 2 & 0 & 2 & & 39.96 \\
\hline 2.205 & 3 & -1 & 1 & 4 & & 40.90 \\
\hline 2.175 & 7 & -3 & 1 & 2 & & 41.48 \\
\hline 2.160 & 9 & -2 & 0 & 4 & + & 41.78 \\
\hline 2.087 & 7 & 3 & 1 & 0 & & 43.32 \\
\hline 2.049 & 17 & 1 & 3 & 2 & & 44.16 \\
\hline 2.030 & 3 & -3 & 1 & 3 & 4 & 44.60 \\
\hline 1.9706 & 7 & 2 & 2 & 2 & & 46.02 \\
\hline 1.9065 & 16 & -2 & 2 & 4 & + & 47.56 \\
\hline 1.8357 & 3 & 0 & 4 & 2 & & 49.62 \\
\hline 1.7478 & 6 & -1 & 3 & 4 & & 52.30 \\
\hline 1.7330 & 8 & -3 & 3 & 2 & & 52.78 \\
\hline 1.7043 & 1 & -2 & 4 & 2 & & 53.74 \\
\hline 1.6874 & 6 & 3 & 3 & 0 & & 54.32 \\
\hline 1.6200 & 3 & 4 & 0 & 0 & & 56.78 \\
\hline 1.6107 & 2 & -3 & 1 & 5 & & 57.14 \\
\hline 1.5814 & 4 & -4 & 2 & 2 & & 58.30 \\
\hline 1.5725 & 7 & -4 & 0 & 4 & + & 58.66 \\
\hline 1.5701 & 9 & 2 & 0 & 4 & & 58.76 \\
\hline 1.5667 & 5 & -4 & 2 & 1 & & 58.90 \\
\hline 1.5448 & 1 & -4 & 2 & 3 & & 59.82 \\
\hline 1.5378 & 13 & 1 & 3 & 4 & + & 63.12 \\
\hline 1.5190 & 3 & -1 & 5 & 2 & & 60.94 \\
\hline 1.5168 & 2 & -2 & 0 & 6 & & 61.34 \\
\hline 1.5043 & 2 & 4 & 2 & 0 & & 61.00 \\
\hline 1.4784 & 2 & -2 & 4 & 4 & & 62.80 \\
\hline 1.4646 & 1 & 2 & 2 & 4 & & 63.46 \\
\hline 1.4487 & 5 & 3 & 3 & 2 & & 64.24 \\
\hline 1.4415 & 4 & 1 & 5 & 2 & & 64.60 \\
\hline 1.4383 & 3 & 0 & 0 & 6 & & 64.76 \\
\hline 1.4208 & 1 & -2 & 2 & 6 & & 65.66 \\
\hline 1.3732 & 2 & 4 & 0 & 2 & & 68.24 \\
\hline 1.3551 & 2 & -5 & 1 & 2 & + & 69.28 \\
\hline 1.3521 & 4 & 0 & 6 & 0 & & 69.46 \\
\hline 1.3460 & 1 & -5 & 1 & 3 & & 69.82 \\
\hline 1.3313 & 1 & -5 & 1 & 1 & & 70.70 \\
\hline 1.3226 & 6 & -1 & 3 & 6 & + & 71.24 \\
\hline 1.3178 & 2 & -3 & 5 & 2 & & 71.54 \\
\hline 1.3045 & 1 & 3 & 1 & 4 & & 72.38 \\
\hline 1.3008 & 1 & -4 & 2 & 2 & & 72.62 \\
\hline 1.2974 & 4 & 3 & 5 & 0 & + & 72.84 \\
\hline 1.2895 & 1 & -4 & 4 & 3 & & 73.36 \\
\hline 1.2539 & 1 & -3 & 1 & 7 & & 75.80 \\
\hline 1.2523 & 2 & -4 & 2 & 6 & & 75.92 \\
\hline 1.2423 & 4 & -2 & 6 & 2 & + & 76.64 \\
\hline 1.2258 & 1 & -3 & 5 & 4 & & 77.86 \\
\hline 1.1887 & 1 & -5 & 3 & 4 & & 80.78 \\
\hline 1.1687 & 1 & 5 & 3 & 0 & & 82.46 \\
\hline 1.1305 & 1 & 2 & 2 & 6 & & 85.30 \\
\hline
\end{tabular}
ties agreed within $2 \%$.

\begin{tabular}{|l|r|rll|l|}
\hline \multicolumn{5}{|c|}{ Calculated Pattern (Peak heights) } \\
\hline$d(A)$ & $I$ & \multicolumn{3}{|c|}{$h k l$} & $\begin{array}{c}2 \theta\left(^{\circ}\right) \\
\lambda=1.54056 \AA\end{array}$ \\
\hline 5.06 & 6 & 1 & 1 & 0 & 17.50 \\
4.97 & 6 & -1 & 1 & 1 & 17.84 \\
4.32 & 11 & 0 & 0 & 2 & 20.56 \\
3.94 & 1 & 1 & 1 & 1 & 22.54 \\
3.81 & 6 & -1 & 1 & 2 & 23.30 \\
& & & & & \\
3.67 & 2 & 0 & 2 & 1 & 24.22 \\
3.241 & 2 & 2 & 0 & 0 & 27.50 \\
3.144 & 100 & -2 & 0 & 2 & 26.36 \\
2.957 & 79 & 0 & 2 & 2 & 30.20 \\
2.928 & 46 & 1 & 1 & 2 & 30.50 \\
2.841 & 4 & -1 & 1 & 3 & 31.46 \\
2.621 & 2 & -2 & 2 & 1 & 34.18 \\
2.532 & 18 & 2 & 2 & 0 & 35.42 \\
2.495 & 18 & 1 & 3 & 0 & 35.96 \\
2.489 & 11 & -1 & 3 & 1 & 36.06
\end{tabular}


Copper Phosphate, alpha-pyro-, $\mathrm{Cu}_{2} \mathrm{P}_{2} \mathrm{O}_{7}$ (monoclinic) - continued

\begin{tabular}{|c|c|c|c|c|c|}
\hline \multicolumn{6}{|c|}{ Calculated Pattern (Integrafed) } \\
\hline$d(\stackrel{\circ}{A})$ & $I$ & & $h k$ & & $\begin{array}{c}2 \theta\left({ }^{\circ}\right) \\
\lambda=1.54056 \AA\end{array}$ \\
\hline 5.06 & 5 & 1 & 1 & 0 & 17.50 \\
\hline 4.97 & 5 & -1 & 1 & 1 & 17.83 \\
\hline 4. 32 & 10 & 0 & 0 & 2 & 20.56 \\
\hline 3.94 & 1 & 1 & 1 & 1 & 22.54 \\
\hline 3.81 & 6 & -1 & 1 & 2 & 23.30 \\
\hline 3.67 & 2 & 0 & 2 & 1 & 24.22 \\
\hline 3.240 & 2 & 2 & 0 & 0 & 27.51 \\
\hline 3.145 & 100 & -2 & 0 & 2 & $28 \cdot 35$ \\
\hline 2.956 & 82 & 0 & 2 & 2 & 30.21 \\
\hline 2.929 & 44 & 1 & 1 & 2 & 30.50 \\
\hline 2.841 & 4 & -1 & 1 & 3 & 31.46 \\
\hline 2.621 & 2 & -2 & 2 & 1 & 34.18 \\
\hline 2.532 & 20 & 2 & 2 & 0 & 35.43 \\
\hline 2.496 & 19 & 1 & 3 & 0 & 35.95 \\
\hline$c .484$ & 1 & -1 & 3 & 1 & 36.12 \\
\hline 2.292 & 2 & -1 & 3 & 2 & 39.27 \\
\hline 2.274 & 1 & 2 & 2 & 1 & 39.60 \\
\hline 2.255 & 2 & 2 & 0 & 2 & 39.96 \\
\hline 2.204 & 3 & -1 & 1 & 4 & 40.91 \\
\hline 2.175 & 8 & -3 & 1 & 2 & 41.49 \\
\hline 2.161 & 9 & $-<$ & 0 & 4 & 41.77 \\
\hline 2.159 & 3 & 0 & 0 & 4 & 41.81 \\
\hline 2.087 & 8 & 3 & 1 & 0 & 43.31 \\
\hline 2.049 & 20 & 1 & 3 & 2 & 44.16 \\
\hline 2.030 & 3 & -3 & 1 & 3 & 44.60 \\
\hline 2.028 & 1 & $\mathrm{~s}$ & 4 & 0 & 44.64 \\
\hline 1.9706 & 7 & 2 & 2 & 2 & 46.02 \\
\hline 1.9069 & 18 & -2 & 2 & 4 & 47.65 \\
\hline 1.9056 & 2 & 0 & 2 & 4 & 47.68 \\
\hline 1.8357 & 3 & $u$ & 4 & 2 & 49.62 \\
\hline 1.7478 & 6 & -1 & 3 & 4 & 52.30 \\
\hline 1.7329 & 10 & -3 & 3 & 2 & 52.78 \\
\hline 1.7045 & 2 & -2 & 4 & 2 & 53.73 \\
\hline 1.6877 & 8 & 3 & 3 & 0 & 54.31 \\
\hline 1.6579 & 1 & 0 & 4 & 3 & 55.37 \\
\hline 1.6200 & 3 & 4 & 0 & 0 & 56.78 \\
\hline 1.6105 & 2 & -3 & 1 & 5 & 57.15 \\
\hline 1.5815 & 5 & -4 & 2 & 2 & 58.29 \\
\hline 1.5740 & 4 & 1 & 5 & 0 & 58.60 \\
\hline 1.5726 & 6 & -4 & 0 & 4 & 58.66 \\
\hline 1.5703 & 7 & 2 & 0 & 4 & 58.75 \\
\hline 1.5667 & 2 & -4 & 2 & 1 & 58.90 \\
\hline 1.5448 & 1 & -4 & 2 & 3 & 59.82 \\
\hline 1.5393 & 2 & -3 & 3 & 4 & 60.07 \\
\hline 1.5376 & 17 & 1 & 3 & 4 & 60.13 \\
\hline 1.5190 & 4 & -1 & 5 & 2 & 60.94 \\
\hline 1.5168 & 1 & -2 & 0 & 6 & 61.04 \\
\hline 1.5045 & 2 & 4 & 2 & 0 & 61.59 \\
\hline 1.4787 & 3 & -2 & 4 & 4 & 62.79 \\
\hline 1.4644 & 1 & 2 & c & 4 & 63.47 \\
\hline
\end{tabular}

\begin{tabular}{|c|c|c|c|c|c|}
\hline \multicolumn{6}{|c|}{ Calculated Pattern (Integrated) } \\
\hline$d(\stackrel{\circ}{A})$ & $I$ & & $h k l$ & & $\begin{array}{c}2 \theta\left(^{\circ}\right) \\
\lambda=1.54056 \AA\end{array}$ \\
\hline 1.4487 & 7 & 3 & 3 & 2 & 64.24 \\
\hline 1.4416 & 6 & 1 & 5 & 2 & 64.60 \\
\hline 1.4391 & 1 & 0 & 0 & 6 & 64.72 \\
\hline 1.4207 & 1 & -2 & 2 & 6 & 65.66 \\
\hline 1.3732 & 3 & 4 & 0 & 2 & 68.24 \\
\hline 1.3633 & 1 & -4 & 2 & 5 & 58.81 \\
\hline 1.3562 & 1 & 0 & 2 & 6 & 69.21 \\
\hline 1.3550 & 2 & -5 & 1 & 2 & 69.29 \\
\hline 1.3522 & 5 & 3 & 6 & 0 & 69.45 \\
\hline 1.3453 & 1 & -5 & 1 & 3 & 69.82 \\
\hline 1.3313 & 1 & -5 & 1 & 1 & 70.70 \\
\hline 1.3240 & 3 & -1 & 5 & 4 & 71.15 \\
\hline 1.3226 & 8 & -1 & 3 & 6 & 71.24 \\
\hline 1.3175 & 2 & -3 & 5 & 2 & 71.55 \\
\hline 1.3045 & 1 & 3 & 1 & 4 & $72 \cdot 38$ \\
\hline 1.3007 & 1 & 4 & 2 & 2 & 72.63 \\
\hline 1.2979 & 2 & 1 & 1 & 6 & 72.81 \\
\hline 1.2973 & 4 & 3 & 5 & 0 & 72.85 \\
\hline 1.2895 & 2 & -4 & 4 & 3 & 73.36 \\
\hline 1.2798 & 1 & 5 & 1 & 0 & 74.01 \\
\hline 1.2542 & 1 & -3 & 1 & 7 & 75.78 \\
\hline 1.2522 & 4 & -4 & 2 & 6 & $75 \cdot \exists 2$ \\
\hline 1.2428 & 1 & -4 & 4 & 4 & 70.60 \\
\hline 1.2422 & 5 & -2 & 6 & 2 & 76.64 \\
\hline 1.2200 & 1 & -3 & 5 & 4 & 77.85 \\
\hline 1.2033 & 1 & 5 & 1 & 1 & 79.13 \\
\hline 1.1888 & 2 & -5 & 3 & 4 & 80.78 \\
\hline 1.1772 & 1 & 2 & 0 & 6 & 81.74 \\
\hline 1.1687 & 2 & 5 & 3 & 0 & 82.46 \\
\hline 1.1305 & 2 & 2 & 2 & 6 & 85.90 \\
\hline 1.1295 & 1 & 5 & 1 & 2 & 85.99 \\
\hline $1=1168$ & 1 & -1 & 1 & 8 & 87.22 \\
\hline 1.1043 & 1 & -4 & 4 & 6 & $88.4 b$ \\
\hline 1.0986 & 1 & -6 & 2 & 2 & 89.32 \\
\hline 1.0861 & 1 & 4 & 2 & 4 & 90.34 \\
\hline 1.0850 & 1 & -5 & 1 & 7 & 90.45 \\
\hline 1.0803 & 1 & -4 & 0 & 8 & 90.97 \\
\hline 1.0800 & 1 & 6 & 0 & 0 & 91.00 \\
\hline 1.0793 & 1 & 3 & 0 & 8 & 91.37 \\
\hline 1.0772 & 1 & -3 & 5 & 6 & 91.30 \\
\hline 1.0487 & 1 & -5 & 5 & 2 & 94.53 \\
\hline 1.0430 & 1 & 0 & 2 & $\varepsilon$ & $95 .<1$ \\
\hline 1.0411 & 2 & -3 & 3 & 8 & 95.44 \\
\hline 1.0253 & 1 & -4 & 6 & 4 & 97.40 \\
\hline 1.0248 & 2 & 3 & 5 & 4 & 97.47 \\
\hline 1.0246 & 1 & $\ddot{z}$ & 6 & 4 & 97.48 \\
\hline 1.0215 & 2 & 1 & 5 & 6 & 97.88 \\
\hline
\end{tabular}


Structure

Orthorhombic, Pbca (61), $\mathrm{Z}=8$ [Williams, 1966]

\section{Lattice parameters}

$a=10.857 \pm 0.002, b=24.447 \pm 0.005, c=8.756 \pm$ $0.002 \AA$ (published value, $\mathrm{b}=24.446 \pm 0.005 \AA$ ) [ibid.]

\section{Scattering factors}

$\mathrm{H}^{\circ}, \mathrm{O}^{\circ}, \mathrm{C}^{\circ}$ [Hanson et al., 1964]

\section{Thermal parameters}

Isotropic

$$
\begin{aligned}
& \text { C(1) } 4.04 ; C(2) 5.17 ; C(3) 5.47 ; \\
& \text { C(4) } 5.56 \text {; } C(5) 5.44 ; C(6) 4.75 \text {; } \\
& \text { C(7) } 4.22 ; C(8) 5.18 ; C(9) 6.12 \text {; } \\
& O(10) 6.06 ; O(11) 5.71 ; O(12) 4.87 ; \\
& O(13) 4.22 ; O(14) 4.15 ; O(15) 4.37 ; \\
& \mathrm{O}(16) 5.71 ; \mathrm{O}(17) 5.86 ; \mathrm{H}(18) \text { through }
\end{aligned}
$$$$
\mathrm{H}(29) \text { as given by Williams [1966] }
$$

\section{Density}

(calculated) $1.281 \mathrm{~g} / \mathrm{cm}^{3}$ [Williams, 1966]

\section{Scale factor}

$13.32 \times 10^{4}$

\section{Reference}

Hanson, H.P., F. Herman, J.D. Lea, and S. Skillman (1964). HFS atomic scattering factors, Acta Cryst. 17, 1040-1044.

Williams, D.E. (1966). Crystal structure

\begin{tabular}{|c|c|c|c|c|c|c|}
\hline \multicolumn{7}{|c|}{ Calculated Pattern (Peak heights) } \\
\hline$d(\AA)$ & $I$ & & $h k$ & & & $\begin{array}{c}2 \theta\left(^{\circ}\right) \\
\lambda=1.54056 \mathrm{~A}\end{array}$ \\
\hline 12.20 & 36 & 0 & 2 & 3 & & 7.24 \\
\hline 7.11 & 3 & 0 & 2 & 1 & & 12.44 \\
\hline 6.56 & 2 & 1 & 1 & 1 & & 13.48 \\
\hline 6.11 & 4 & 0 & 4 & 0 & & 14.48 \\
\hline $5 \cdot 35$ & 4 & 1 & 2 & 1 & & 14.88 \\
\hline 5.43 & 70 & $\bar{z}$ & 0 & 0 & & 16.32 \\
\hline 5.30 & 15 & 2 & 1 & 0 & & 16.72 \\
\hline 5.23 & 99 & 1 & 3 & 1 & & 16.94 \\
\hline 5.01 & 3 & 0 & 4 & 1 & & 17.68 \\
\hline 4.53 & 50 & 2 & i & 1 & & 19.56 \\
\hline 4.52 & 46 & $?$ & 3 & 0 & & 19.62 \\
\hline 4.38 & 5 & C & 0 & 2 & & 20.28 \\
\hline 4.32 & 21 & 2 & $\bar{z}$ & 1 & & $20.50^{\circ}$ \\
\hline 4.12 & 37 & 0 & 2 & 2 & & 21.54 \\
\hline 4.07 & 3 & 0 & 6 & 0 & & 21.80 \\
\hline 3.97 & 29 & 1 & 5 & 1 & & 22.36 \\
\hline 3.85 & 21 & 1 & 2 & 2 & & 23.06 \\
\hline 3.69 & $\bar{E}$ & 3 & 6 & 1 & & 24.08 \\
\hline 3.68 & 7 & $?$ & 4 & 1 & & 24.14 \\
\hline 3.63 & 130 & 1 & 3 & 2 & & 24.48 \\
\hline 3.56 & 10 & 0 & 4 & 2 & & 25.00 \\
\hline 3.406 & 3 & 2 & 0 & 2 & & 26.14 \\
\hline 3.351 & 15 & 1 & 4 & 2 & & 26.34 \\
\hline $3.370^{\circ}$ & 14 & 2 & 1 & 2 & & 26.38 \\
\hline 3.356 & 3 & 2 & 5 & 1 & & $26 \cdot 54$ \\
\hline 3.283 & 5 & 2 & 2 & 2 & & 27.14 \\
\hline 3.259 & 2 & 2 & 6 & 0 & & 27.34 \\
\hline 3.227 & 3 & 3 & 2 & 1 & & $27 . E 2$ \\
\hline 3.144 & 42 & $\bar{z}$ & 3 & 2 & & 28.36 \\
\hline 3.125 & 8 & I & 5 & 2 & & 28.54 \\
\hline 3.093 & 5 & 3 & 3 & 1 & & 28.84 \\
\hline $2.97 \mathrm{~b}$ & 17 & 2 & 4 & 2 & + & 30.00 \\
\hline 2.934 & 4 & 3 & 4 & 1 & & 30.44 \\
\hline 2.884 & 3 & 0 & 8 & 1 & & 30.98 \\
\hline$<.877$ & 2 & I & 6 & 2 & & 31.06 \\
\hline 2.840 & 3 & $?$ & 2 & 3 & & 31.48 \\
\hline 2.830 & 1 & I & 1 & 3 & & 31.94 \\
\hline 2.789 & 2 & $?$ & 0 & 2 & & 32.06 \\
\hline 2.714 & 2 & 4 & 0 & 0 & & 32.98 \\
\hline$\angle .664$ & 1 & 2 & 8 & 0 & & 33.62 \\
\hline 2.639 & 1 & 3 & 3 & 2 & & 33.94 \\
\hline$\ldots .614$ & 1 & 2 & 6 & 2 & & 34.28 \\
\hline 2.584 & 2 & 3 & 6 & 1 & & 34.58 \\
\hline 2.579 & $?$ & 4 & 1 & 1 & & 34.76 \\
\hline 2.557 & ? & 2 & 1 & 3 & + & 35.36 \\
\hline 2.547 & 3 & 2 & 8 & 1 & & 35.20 \\
\hline 2.538 & 5 & 3 & 4 & 2 & + & 35.34 \\
\hline 2.524 & 2 & I & 9 & 1 & & 35.54 \\
\hline 2.442 & 5 & 1 & 5 & 3 & + & 35.78 \\
\hline 2.318 & 6 & 1 & 6 & 3 & & 38.82 \\
\hline
\end{tabular}
of dibenzoylmethane,Acta Cryst.21,340-9.

\section{Note:}

There has been a correction in this last reference. The $x$ parameter for $c(3)$ should be -0.07535 . 
Dibenzoylmethane, $\mathrm{C}_{15} \mathrm{H}_{12} \mathrm{O}_{2}$ (orthorhombic) - continued

\begin{tabular}{|c|c|c|c|c|c|}
\hline \multicolumn{6}{|c|}{ Calculated Pattern (Peak heights) } \\
\hline$d(\stackrel{\cap}{A})$ & $I$ & & $h k l$ & & $\begin{array}{c}2 \theta\left({ }^{\circ}\right) \\
\lambda=1.54056 \AA\end{array}$ \\
\hline 2.219 & 1 & 4 & 3 & 2 & 40.52 \\
\hline 2.193 & 1 & 1 & 7 & 3 & 41.12 \\
\hline 2.188 & 2 & 3 & 3 & 3 & 41.22 \\
\hline 2.180 & 1 & 3 & 7 & 2 & 41.38 \\
\hline 2.160 & 3 & 2 & 10 & 1 & 41.78 \\
\hline$\angle .134$. & 1 & 0 & 10 & 2 & 42.32 \\
\hline 2.129 & 2 & 3 & 4 & 3 & 42.42 \\
\hline 2.124 & 1 & 2 & 9 & 2 & 42.52 \\
\hline 2.138 & 2 & 3 & 9 & 1 & 42.86 \\
\hline 2.094 & 1 & 1 & 10 & 2 & 43.16 \\
\hline$<.071$ & 1 & 1 & 8 & 3 & 43.65 \\
\hline 2.025 & 1 & 1 & 4 & 4 & 44.72 \\
\hline 2.0033 & 2 & 2 & 11 & 1 & 45.24 \\
\hline 1.9844 & 2 & 0 & 12 & 1 & 45.58 \\
\hline 1.9673 & 1 & 2 & $\theta$ & 3 & 46.10 \\
\hline 1.9285 & 1 & 0 & 6 & 4 & 47.08 \\
\hline 1.9343 & 2 & 3 & 7 & 3 & 47.72 \\
\hline 1.8923 & 1 & 5 & 3 & 2 & 48.04 \\
\hline 1.8747 & 1 & $\%$ & 5 & 4 & 48.52 \\
\hline 1.8233 & 1 & 3 & 8 & 3 & 49.98 \\
\hline 1.8172 & 1 & ? & 6 & 4 & 50.16 \\
\hline 1.8125 & 1 & 1 & 13 & 1 & 50.30 \\
\hline 1.8991 & 1 & $\varepsilon$ & 0 & 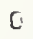 & 50.40 \\
\hline 1.8351 & 1 & 6 & 1 & J & 50.52 \\
\hline 1.7860 & $i$ & 4 & 6 & 3 & 51.08 \\
\hline 1.7788 & 1 & 4 & 10 & 1 & 51.32 \\
\hline $1.755 \mathrm{~s}$ & $?$ & 2 & 7 & 4 & 52.06 \\
\hline 1.7490 & 2 & 3 & 5 & 4 & 52.26 \\
\hline 1.7019 & 2 & 3 & 6 & 4 & 53.82 \\
\hline
\end{tabular}


Dibenzoylmethane, $\mathrm{C}_{15} \mathrm{H}_{12} \mathrm{O}_{2}$ (orthorhombic) - continued

\begin{tabular}{|c|c|c|c|c|c|}
\hline \multicolumn{6}{|c|}{ Calculated Pattern (Integrated) } \\
\hline$d(\AA)$ & $I$ & & $h k l$ & & $\begin{array}{c}2 \theta\left(^{\circ}\right) \\
\lambda=1.54056 \AA\end{array}$ \\
\hline 12.22 & 27 & 0 & 2 & 0 & 7.23 \\
\hline $7 \cdot 12$ & 3 & 0 & 2 & 1 & 12.42 \\
\hline 6.57 & 1 & 1 & 1 & 1 & 13.48 \\
\hline 6.11 & 3 & 0 & 4 & 0 & 14.48 \\
\hline 5.95 & 3 & 1 & 2 & 1 & 14.87 \\
\hline 5.43 & 62 & 2 & 0 & 0 & 16.32 \\
\hline 5.30 & 11 & 2 & 1 & 0 & 16.72 \\
\hline 5.23 & $y 2$ & 1 & 3 & 1 & 16.94 \\
\hline 5.01 & 3 & 0 & 4 & 1 & 17.68 \\
\hline 4. 55 & 1 & 1 & 4 & 1 & 19.49 \\
\hline 4.53 & 44 & 2 & 1 & 1 & 19.56 \\
\hline 4.52 & 17 & 2 & 3 & 0 & 19.63 \\
\hline 4.38 & 4 & 0 & 0 & 2 & 20.27 \\
\hline 4.32 & 20 & 2 & 2 & 1 & 20.56 \\
\hline 4.12 & 36 & D & 2 & 2 & 21.54 \\
\hline 4.07 & 2 & D & 6 & 0 & 21.79 \\
\hline 3.97 & 28 & 1 & 5 & 1 & 22.35 \\
\hline 3.85 & 20 & 1 & 2 & 2 & 23.06 \\
\hline 3.69 & 5 & 0 & 6 & 1 & 24.07 \\
\hline 3.68 & 3 & 2 & 4 & 1 & 24.15 \\
\hline 3.63 & 100 & 1 & 3 & 2 & 24.47 \\
\hline 3.56 & 10 & 0 & 4 & 2 & 25.00 \\
\hline 3.408 & 2 & 2 & 0 & 2 & 26.13 \\
\hline 3.382 & 14 & 1 & 4 & 2 & 26.33 \\
\hline 3.375 & 4 & 2 & 1 & 2 & $26 \cdot 38$ \\
\hline 3.356 & 2 & 2 & 5 & 1 & 26.54 \\
\hline 3.283 & 5 & 2 & 2 & 2 & 27.14 \\
\hline 3.259 & 1 & 2 & 6 & 0 & 27.35 \\
\hline 3.226 & 3 & 3 & 2 & 1 & 27.63 \\
\hline 3.144 & 44 & 2 & 3 & 2 & 28.36 \\
\hline 3.124 & 5 & 1 & 5 & 2 & 28.55 \\
\hline 3.094 & 4 & 3 & 3 & 1 & 28.83 \\
\hline 2.983 & 3 & 0 & 6 & 2 & 29.93 \\
\hline 2.976 & 16 & 2 & 4 & 2 & 30.00 \\
\hline 2.934 & 3 & 3 & 4 & 1 & 30.44 \\
\hline 2.885 & 3 & 0 & 8 & 1 & 30.97 \\
\hline 2.876 & 1 & 1 & 6 & 2 & 31.07 \\
\hline 2.833 & 4 & 0 & 2 & 3 & 31.49 \\
\hline 2.800 & 1 & 1 & 1 & 3 & 31.94 \\
\hline 2.789 & 2 & 3 & 0 & 2 & 32.06 \\
\hline 2.714 & 2 & 4 & 0 & 3 & 32.97 \\
\hline 2.663 & 2 & 2 & 8 & 0 & 33.63 \\
\hline 2.639 & 1 & 3 & 3 & 2 & 33.94 \\
\hline 2.614 & 1 & 2 & 6 & 2 & 34.28 \\
\hline 2.585 & 2 & 3 & 6 & 1 & 34.67 \\
\hline 2.578 & 1 & 4 & 1 & 1 & 34.77 \\
\hline 2.560 & 1 & 1 & 4 & 3 & 35.03 \\
\hline 2.557 & 1 & 2 & 1 & 3 & 35.07 \\
\hline 2.548 & 2 & 2 & 8 & 1 & 35.20 \\
\hline 2.538 & 4 & 3 & 4 & 2 & 35.34 \\
\hline
\end{tabular}

\begin{tabular}{|c|c|c|c|c|c|}
\hline \multicolumn{6}{|c|}{ Calculated Pattern (Integrated) } \\
\hline$d(\stackrel{\circ}{A}$ & $I$ & & $h k$ & & $\begin{array}{c}20\left({ }^{\circ}\right) \\
\lambda=1.54056 \AA\end{array}$ \\
\hline 2.536 & 1 & 4 & 2 & 1 & 35.36 \\
\hline 2.523 & 1 & 1 & 9 & 1 & 35.55 \\
\hline 2.445 & 2 & 0 & 10 & 0 & 36.73 \\
\hline 2.442 & 3 & $\mathbf{1}$ & 5 & 3 & 36.78 \\
\hline 2.442 & 2 & 1 & 8 & 2 & 36.78 \\
\hline 2.318 & 7 & 1 & 6 & 3 & 38.82 \\
\hline 2.220 & 1 & 4 & 3 & 2 & 40.61 \\
\hline 2.193 & 1 & 1 & 7 & 3 & 41.12 \\
\hline 2.188 & 2 & 3 & 3 & 3 & 41.22 \\
\hline 2.180 & 1 & 3 & 7 & 2 & 41.39 \\
\hline 2.160 & 3 & 2 & 10 & 1 & $41 \cdot 78$ \\
\hline 2.134 & 1 & 0 & 10 & 2 & 42.31 \\
\hline 2.130 & 1 & 3 & 4 & 3 & 42.41 \\
\hline 2.124 & 1 & 2 & 9 & 2 & 42.52 \\
\hline 2.109 & 2 & 3 & 9 & 1 & 42.85 \\
\hline 2.094 & 1 & 1 & 10 & 2 & 43.16 \\
\hline 2.072 & 1 & 1 & 8 & 3 & 43.65 \\
\hline 2.025 & 1 & 1 & 4 & 4 & 44.72 \\
\hline 2.002 & 2 & 2 & 11 & 1 & 45.25 \\
\hline 1.9842 & 2 & 3 & 12 & 1 & 45.68 \\
\hline 1.9672 & 1 & 2 & 8 & 3 & 46.10 \\
\hline 1.9283 & 1 & 0 & 6 & 4 & 47.09 \\
\hline 1.9044 & 3 & 3 & 7 & 3 & 47.72 \\
\hline 1.8921 & 2 & 5 & 3 & 2 & 48.05 \\
\hline 1.8750 & 1 & 2 & 5 & 4 & 48.51 \\
\hline 1.8232 & 1 & 3 & 8 & 3 & 49.98 \\
\hline 1.8171 & 1 & 2 & 6 & 4 & 50.16 \\
\hline 1.8128 & 1 & 1 & 13 & 1 & 50.29 \\
\hline 1.8095 & 1 & 6 & 0 & 0 & 50.39 \\
\hline 1.8046 & 1 & 6 & 1 & 0 & 50.54 \\
\hline 1.7864 & 2 & 4 & 6 & 3 & 51.09 \\
\hline 1.7786 & 1 & 4 & 10 & 1 & 51.33 \\
\hline 1.7552 & 2 & 2 & 7 & 4 & 52.06 \\
\hline 1.7491 & 2 & 3 & 5 & 4 & 52.26 \\
\hline 1.7016 & 3 & 3 & 6 & 4 & 53.82 \\
\hline
\end{tabular}




\section{Structure}

Monoclinic, $\mathrm{Pn}(7)$ or $\mathrm{P} 2 / \mathrm{n}(13), \mathrm{Z}=2$ [Marezio et a.., 1961].

\section{Lattice parameters}

$a=9.651 \pm 0.001, b=6.525 \pm 0.001, c=7.923 \pm 0.001 \AA$, $\beta=93.65 \pm 0.02^{\circ}$ [ibid.]

\section{Scattering factors}

$\mathrm{Gd}^{\circ}$ [Thomas and Umeda, 1957], corrected for dispersion [Dauben and Templeton, 1955]

$\mathrm{H}^{\circ}, \mathrm{O}^{-1}, \mathrm{Cl}^{-1}[3.3 .1 \mathrm{~A}]$

\section{Thermal parameters}

Isotropic [Marezio et al., 1961]

\section{Density}

(calculated) $2.478 \mathrm{~g} / \mathrm{cm}^{3} \quad$ [Marezio et al., 1961]

\section{Scale factor}

$$
2.514 \times 10^{4}
$$

\section{Additional patterns}

I. P.D.F. card 3-0392 [Dow ChemicaI Co., Midland, Mich.]

\section{Reference}

Dauben, C.H. and D.H. Templeton (1955). A table of dispersion corrections for $\mathrm{x}-$ ray scattering of atoms, Acta cryst. 8 , 841-842.

Marezio, M.,H.A. Plettinger, and W.H.Zachariasen (1961). The crystal structure of gadolinium trichloride hexahydrate, Acta Cryst. 14, 234-236.

Thomas, L. H. and K. Umeda (1957). Atomic scattering factors calculated from the TFD atomic model,J.Chem. Phys.26,293-303.

\begin{tabular}{|c|c|rcc|c|}
\hline \multicolumn{6}{|c|}{ Calculated Pattern (Peak heights) } \\
\hline$d(\AA)$ & $I$ & \multicolumn{3}{|c|}{$h k l$} & $\begin{array}{c}2 \theta\left(^{\circ}\right) \\
\text { (A) }\end{array}$ \\
\hline 6.525 & 41 & 0 & 1 & 0 & $134056 \AA$ \\
\hline .312 & 100 & -1 & 0 & 1 & 14.02 \\
5.925 & 100 & 1 & 0 & 1 & 14.94 \\
5.401 & 71 & 1 & 1 & 0 & 16.40 \\
5.029 & 66 & 0 & 1 & 1 & 17.62 \\
4.813 & 49 & 2 & 0 & 0 & 18.42 \\
4.535 & 36 & -1 & 1 & 1 & 19.56 \\
4.388 & 43 & 1 & 1 & 1 & 20.22 \\
3.952 & 59 & 0 & 0 & 2 & 22.48 \\
3.874 & 11 & 2 & 1 & 0 & 22.94 \\
\end{tabular}

\begin{tabular}{|c|c|c|c|c|c|c|}
\hline \multicolumn{7}{|c|}{ Calculated Pattern (Peak heights) } \\
\hline$d(\stackrel{\circ}{A})$ & $I$ & \multicolumn{4}{|c|}{$h k l$} & $\begin{array}{c}2 \theta\left({ }^{\circ}\right) \\
\lambda=1.54056 \AA\end{array}$ \\
\hline 3.550 & 71 & -2 & 1 & 1 & & 25.06 \\
\hline 3.411 & 63 & 2 & 1 & 1 & & $26 \cdot 10$ \\
\hline 3.381 & 16 & 0 & 1 & 2 & & 26.34 \\
\hline 3.262 & 3 & 0 & 2 & $\overline{0}$ & & 27.32 \\
\hline 3.252 & 2 & -1 & 1 & 2 & & 27.40 \\
\hline 3.155 & 7 & -2 & 0 & 2 & & 28.26 \\
\hline 3.138 & 7 & 1 & 1 & 2 & & 28.42 \\
\hline 3.089 & 27 & 1 & 2 & 0 & & 28.88 \\
\hline 3.044 & 4 & -3 & 0 & 1 & & 29.32 \\
\hline 2.965 & 7 & 2 & 0 & 2 & & 30.12 \\
\hline 2.910 & 5 & 3 & 0 & 1 & & 30.70 \\
\hline 2.881 & 17 & 3 & 1 & 0 & & 31.02 \\
\hline 2.859 & 17 & 1 & 2 & 1 & & 31.26 \\
\hline 2.841 & 3 & -2 & 1 & 2 & & 31.46 \\
\hline 2.758 & 5 & -3 & 1 & 1 & & 32.44 \\
\hline 2.699 & 3 & 2 & 2 & 0 & + & 33.16 \\
\hline 2.659 & 7 & 3 & 1 & 1 & & 33.68 \\
\hline 2.584 & 23 & -2 & $\hat{c}$ & 1 & + & 34.68 \\
\hline 2.528 & 18 & 2 & 2 & 1 & & 35.48 \\
\hline 2.517 & 11 & 0 & 2 & 2 & & 35.64 \\
\hline 2.502 & 10 & 1 & 0 & 3 & & 35.86 \\
\hline 2.460 & 16 & -1 & 2 & 2 & & 36.50 \\
\hline 2.444 & 2 & 0 & 1 & 3 & & 36.74 \\
\hline 2.407 & 22 & 1 & 2 & 2 & + & 37.32 \\
\hline 2.403 & 31 & -1 & 1 & 3 & & 37.40 \\
\hline 2.395 & 25 & -3 & 1 & 2 & & 37.52 \\
\hline 2.336 & 20 & 1 & 1 & 3 & & 38.50 \\
\hline 2.288 & 40 & 3 & 2 & 0 & & 39.34 \\
\hline 2.267 & 11 & 3 & 1 & 2 & & 39.72 \\
\hline 2.260 & 9 & 4 & 1 & 0 & & 39.86 \\
\hline 2.233 & 23 & -2 & 1 & 3 & & 40.36 \\
\hline 2.207 & 13 & -4 & 1 & 1 & & 40.86 \\
\hline 2.194 & 4 & 2 & 2 & 2 & & 41.10 \\
\hline 2.175 & 5 & 0 & 3 & 0 & + & 41.48 \\
\hline 2.139 & 5 & 4 & 1 & 1 & & 42.22 \\
\hline 2.129 & 20 & 2 & 1 & 3 & & 42.42 \\
\hline 2.123 & 12 & 1 & 3 & 0 & & $42 \cdot 54$ \\
\hline 2.118 & 11 & -4 & 0 & 2 & & 42.66 \\
\hline 2.104 & 3 & -3 & 0 & 3 & & 42.96 \\
\hline 2.098 & 3 & 0 & 3 & 1 & & 43.08 \\
\hline 2.056 & 16 & -1 & 3 & 1 & & 44.00 \\
\hline 2.051 & 12 & 0 & 2 & 3 & & 44.12 \\
\hline 2.042 & 12 & 1 & 3 & 1 & & 44.32 \\
\hline 2.021 & 10 & -3 & 2 & 2 & & 44.82 \\
\hline 2.001 & 4 & 4 & 0 & 2 & + & $45 \cdot 28$ \\
\hline 1.9820 & 3 & 2 & 3 & 0 & + & 45.74 \\
\hline 1.9771 & 9 & 0 & 0 & 4 & + & 45.86 \\
\hline 1.9427 & 17 & 3 & 2 & 2 & & 46.72 \\
\hline 1.9372 & 11 & -2 & 3 & 1 & + & 46.86 \\
\hline 1.9209 & 7 & -2 & 2 & 3 & & 47.28 \\
\hline 1.9156 & 5 & 4 & 1 & 2 & & 47.42 \\
\hline 1.9058 & 13 & 0 & 3 & 2 & + & 47.68 \\
\hline
\end{tabular}




\begin{tabular}{|c|c|c|c|c|c|c|}
\hline \multicolumn{7}{|c|}{ Calculated Pattern (Peak heights) } \\
\hline$d(\stackrel{\circ}{)}$ & $I$ & \multicolumn{4}{|c|}{$h k l$} & $\begin{array}{c}2 \theta\left({ }^{\circ}\right) \\
\lambda=1.54056 \AA\end{array}$ \\
\hline 1.8998 & 11 & -5 & 0 & 1 & & 47.84 \\
\hline 1.8916 & 7 & 0 & 1 & 4 & + & 48.06 \\
\hline 1.8784 & 2 & -1 & 1 & 4 & & 48.42 \\
\hline 1.8711 & 3 & -2 & 0 & 4 & & 48.62 \\
\hline 1.8596 & 4 & 4 & 2 & 1 & & 48.94 \\
\hline 1.8539 & 6 & 2 & 2 & 3 & & 49.10 \\
\hline 1.8483 & 4 & 5 & 1 & 0 & & 49.26 \\
\hline 1.8448 & 3 & 5 & 0 & 1 & & 49.36 \\
\hline 1.8357 & 1 & 1 & 1 & 4 & & 49.62 \\
\hline 1.8240 & 7 & -5 & 1 & 1 & & 49.96 \\
\hline 1.7984 & 1 & -2 & 1 & 4 & & 50.72 \\
\hline 1.7892 & 4 & 2 & 0 & 4 & + & 51.00 \\
\hline 1.7756 & 4 & 5 & 1 & 1 & + & 51.42 \\
\hline 1.7685 & 6 & -4 & 1 & 3 & + & 51.54 \\
\hline 1.7534 & 1 & 2 & 3 & 2 & & 52.12 \\
\hline 1.7422 & 3 & 3 & 3 & 1 & & 52.48 \\
\hline 1.6903 & 1 & 0 & 2 & 4 & & 54.22 \\
\hline 1.6806 & 6 & -1 & 2 & 4 & & 54.56 \\
\hline 1.6755 & 6 & -3 & 1 & 4 & + & 54.74 \\
\hline 1.6665 & 5 & 4 & $\overline{1}$ & 3 & & 55.06 \\
\hline 1.6643 & 6 & -1 & 3 & 3 & & 55.14 \\
\hline 1.6593 & 4 & 5 & 2 & 0 & & 55.32 \\
\hline 1.6499 & 4 & 1 & 2 & 4 & & 55.66 \\
\hline 1.6413 & 6 & 1 & 3 & 3 & + & 55.98 \\
\hline 1.6311 & 2 & 0 & 4 & 0 & & 56.36 \\
\hline 1.6138 & 2 & 4 & 3 & 0 & & 57.02 \\
\hline 1.6050 & 7 & 6 & 0 & 0 & + & 57.36 \\
\hline 1.5883 & 4 & 3 & 1 & 4 & + & 58.02 \\
\hline 1.5779 & 4 & -4 & 0 & 4 & + & 58.44 \\
\hline 1.5735 & 3 & 1 & 4 & 1 & & 58.62 \\
\hline 1.5585 & 3 & -5 & 1 & 3 & + & 59.24 \\
\hline 1.5476 & 2 & -6 & 1 & 1 & & 59.70 \\
\hline 1.5448 & 3 & 2 & 4 & 0 & & 59.82 \\
\hline
\end{tabular}

\begin{tabular}{|c|c|c|c|c|c|}
\hline \multicolumn{6}{|c|}{ Calculated Pattern (Integrated) } \\
\hline$d(\AA)$ & $I$ & & $h k l$ & & $\begin{array}{c}2 \theta\left(^{\circ}\right) \\
\lambda=1.54056 \AA\end{array}$ \\
\hline 6.525 & 39 & 0 & 1 & 0 & 13.56 \\
\hline 6.312 & 97 & -1 & 0 & 1 & 14.02 \\
\hline 5.929 & 100 & 1 & 0 & 1 & 14.93 \\
\hline 5.402 & 72 & 1 & & 0 & 16.40 \\
\hline 5.033 & 70 & 0 & 1 & 1 & 17.61 \\
\hline 4.816 & 53 & 2 & 0 & 0 & 18.41 \\
\hline 4.537 & 38 & -1 & 1 & 1 & 19.55 \\
\hline 4.388 & 46 & 1 & 1 & 1 & 20.22 \\
\hline 3.953 & 68 & 0 & 0 & 2 & 22.47 \\
\hline 3.875 & 12 & 2 & 1 & 0 & 22.93 \\
\hline 3.552 & 86 & -2 & 1 & 1 & 25.05 \\
\hline 3.411 & 73 & 2 & 1 & 1 & 26.10 \\
\hline 3.381 & 16 & 0 & 1 & 2 & 26.34 \\
\hline 3.263 & 3 & 0 & 2 & 0 & 27.31 \\
\hline 3.246 & 1 & -1 & 1 & 2 & 27.45 \\
\hline 3.156 & 7 & -2 & 0 & 2 & 28.26 \\
\hline 3.137 & 8 & 1 & 1 & 2 & 28.42 \\
\hline 3.090 & 34 & 1 & 2 & 0 & 28.87 \\
\hline 3.043 & 4 & -3 & 0 & 1 & 29.33 \\
\hline 2.965 & 8 & 2 & 0 & 2 & 30.12 \\
\hline 2.911 & 6 & 3 & 0 & 1 & 30.69 \\
\hline 2.881 & 20 & 3 & 1 & 0 & 31.02 \\
\hline 2.858 & 21 & 1 & 2 & 1 & 31.27 \\
\hline 2.841 & 3 & -2 & 1 & 2 & 31.46 \\
\hline 2.758 & 6 & -3 & 1 & 1 & 32.44 \\
\hline 2.701 & 2 & 2 & 2 & 0 & 33.14 \\
\hline 2.699 & 2 & 2 & 1 & 2 & 33.16 \\
\hline 2.658 & 10 & 3 & 1 & 1 & 33.69 \\
\hline 2.584 & 5 & -1 & 0 & 3 & 34.68 \\
\hline $2 \cdot 584$ & 24 & -2 & 2 & 1 & 34.68 \\
\hline 2.529 & 23 & 2 & 2 & 1 & 35.47 \\
\hline 2.516 & 12 & 0 & 2 & 2 & 35.65 \\
\hline 2.502 & 11 & 1 & 0 & 3 & 35.86 \\
\hline 2.459 & 22 & -1 & 2 & 2 & 36.51 \\
\hline $2 \cdot 444$ & 2 & 0 & 1 & 3 & 36.75 \\
\hline 2.411 & 15 & 1 & 2 & 2 & 37.27 \\
\hline 2.408 & 14 & 4 & 0 & 0 & 37.31 \\
\hline 2.403 & 25 & -1 & 1 & 3 & 37.40 \\
\hline 2.394 & 25 & -3 & 1 & 2 & 37.54 \\
\hline $2 \cdot 336$ & 27 & 1 & 1 & 3 & 38.50 \\
\hline $2 \cdot 288$ & 52 & 3 & 2 & 0 & 39.34 \\
\hline 2.267 & 12 & 3 & 1 & 2 & 39.72 \\
\hline 2.259 & 9 & 4 & 1 & 0 & 39.87 \\
\hline 2.233 & 30 & -2 & 1 & 3 & 40.36 \\
\hline 2.207 & 18 & -4 & 1 & 1 & 40.85 \\
\hline $2 \cdot 194$ & 5 & 2 & 2 & 2 & 41.11 \\
\hline 2.175 & 6 & 0 & 3 & 0 & 41.48 \\
\hline 2.172 & 1 & 3 & 2 & 1 & 41.54 \\
\hline 2.139 & 6 & 4 & 1 & 1 & 42.22 \\
\hline 2.129 & 25 & 2 & 1 & 3 & 42.42 \\
\hline
\end{tabular}


Gadolinium Chloride Hexahydrate, $\mathrm{GdCl}_{3} \cdot 6 \mathrm{H}_{2} \mathrm{O}$ (monoclinic) - continued

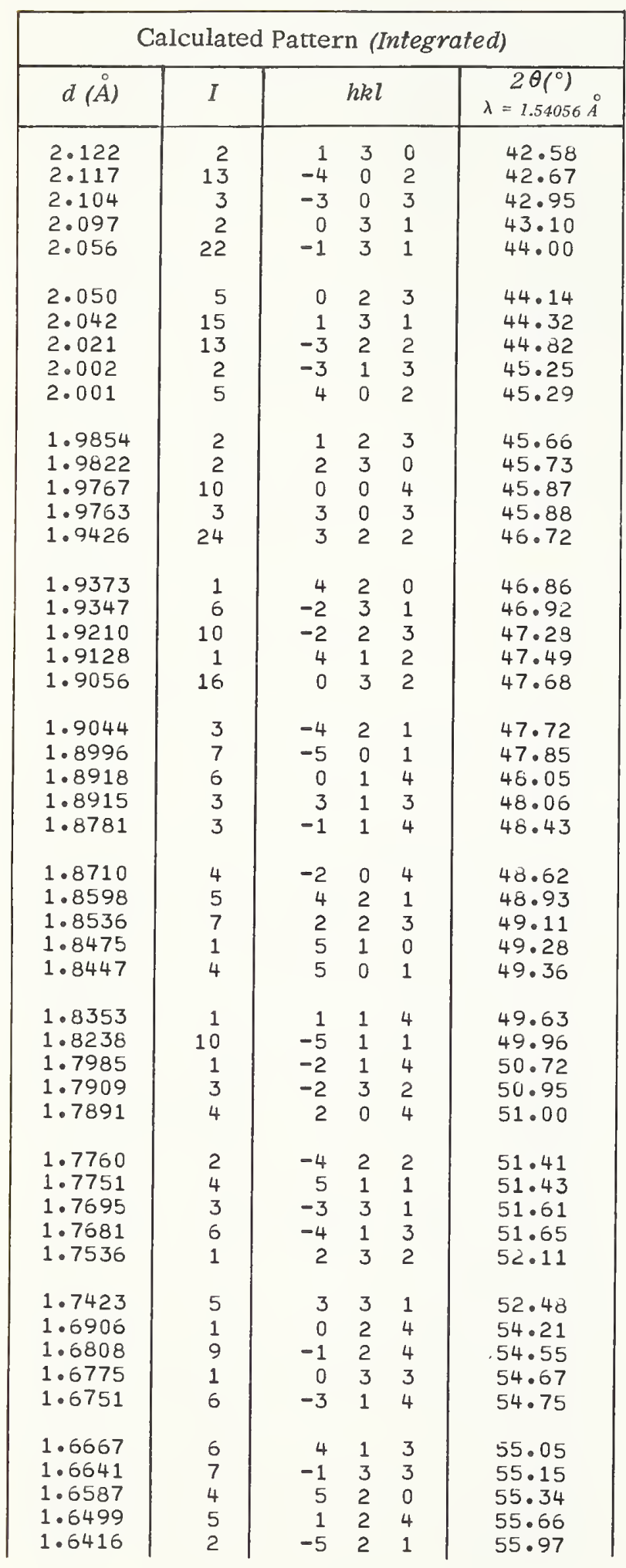

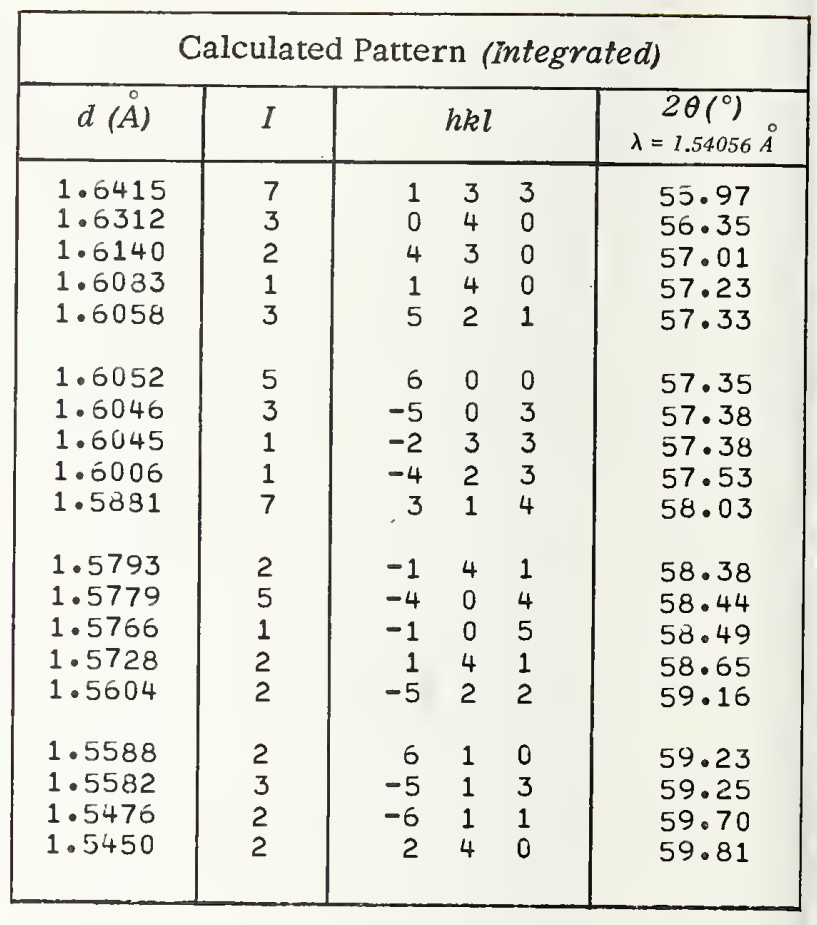


Hexamethylenediammonium Adipate, $\mathrm{C}_{12} \mathrm{H}_{26} \mathrm{~N}_{2} \mathrm{O}_{4}$ (monoclinic)

Structure

Monoclinic, P2 2 a (14), $\mathrm{Z}=2$ [Brown, 1966]

\section{Lattice parameters}

$a=8.489, b=15.581, c=5.598 \AA, \beta=102.9^{\circ}$

(published value: $b=15.580 \AA$ )

[ibid.]

Scattering factors

$$
\mathrm{H}^{\circ}, \mathrm{C}^{\circ}, \mathrm{N}^{\circ}, \mathrm{O}^{\circ}[3.3 .1 \mathrm{~A}]
$$

\section{Thermal parameters}

Anisotropic for carbon, nitrogen, and oxygen, isotropic for hydrogen [Brown, 1966]

\section{Density}

(calculated) $1.207 \mathrm{~g} / \mathrm{cm}^{3}$

\section{Scale factor}

$1.235 \times 10^{4}$

\section{Reference}

Brown, C.J. (1966). Further refinement of the crystal structure of hexamethylenediammonium adipate, Acta Cryst.21,185-190

\begin{tabular}{|c|c|c|c|c|c|}
\hline \multicolumn{6}{|c|}{ Calculated Pattern (Peak heights) } \\
\hline$d(\stackrel{\circ}{A})$ & $I$ & & $h k l$ & & $\begin{array}{c}2 \theta\left(^{\circ}\right) \\
\lambda=1.54056 \AA\end{array}$ \\
\hline 7.78 & 37 & 0 & 2 & D & 11.36 \\
\hline 7.31 & 11 & 1 & 1 & 0 & 12.10 \\
\hline 5.67 & 2 & 1 & 2 & 0 & 15.62 \\
\hline 5.45 & 1 & 0 & 0 & 1 & $16 \cdot 24$ \\
\hline 4.85 & 8 & 1 & 1 & -1 & 18.26 \\
\hline $4 \cdot 40$ & 17 & 1 & 3 & 0 & 20.18 \\
\hline 4.27 & 93 & 1 & 2 & -1 & 20.78 \\
\hline 4. 01 & 99 & 1 & 1 & 1 & 22.16 \\
\hline 4. 00 & 96 & 2 & 1 & 0 & 22.20 \\
\hline 3.72 & 2 & 2 & 0 & -1 & 23.90 \\
\hline 3.65 & 72 & 2 & 2 & 0 & 24.34 \\
\hline 3.64 & 100 & 1 & 3 & -1 & 24.42 \\
\hline 3.52 & 10 & 1 & 4 & 0 & 25.26 \\
\hline 3.358 & 3 & 2 & 2 & -1 & 26.52 \\
\hline 3.236 & 20 & 2 & & 0 & 27.54 \\
\hline 3.097 & 2 & 1 & 4 & -1 & 28.80 \\
\hline 2.992 & 1 & 2 & 0 & 1 & 29.84 \\
\hline 2.938 & 6 & 2 & 1 & 1 & 30.40 \\
\hline
\end{tabular}

\begin{tabular}{|c|c|c|c|c|c|c|}
\hline \multicolumn{7}{|c|}{ Calculated Pattern (Peak heights) } \\
\hline$d(\stackrel{\circ}{)}$ & $I$ & \multicolumn{4}{|c|}{$h k l$} & $\begin{array}{c}2 \theta\left({ }^{\circ}\right) \\
\lambda=154056 \AA\end{array}$ \\
\hline $\begin{array}{l}2.915 \\
2.738\end{array}$ & $\begin{array}{l}1 \\
1\end{array}$ & $\frac{2}{1}$ & $\begin{array}{l}5 \\
1\end{array}$ & $\begin{array}{r}0 \\
-2\end{array}$ & & $\begin{array}{l}30.64 \\
32.68\end{array}$ \\
\hline 2.728 & 4 & 0 & 0 & 2 & & 32.80 \\
\hline 2.720 & 2 & 3 & 1 & 0 & & 32.90 \\
\hline 2.706 & 3 & 0 & 5 & 1 & & 33.08 \\
\hline 2.687 & 7 & 0 & 1 & 2 & + & 33.32 \\
\hline 2.660 & 14 & 1 & 5 & -1 & & 33.66 \\
\hline 2.596 & 7 & 0 & 6 & 0 & $\rightarrow$ & 34.52 \\
\hline 2.566 & 2 & 3 & 2 & -1 & & 34.94 \\
\hline 2.555 & 2 & 2 & 0 & -2 & & 35.10 \\
\hline 2. 521 & 5 & 2 & 1 & -2 & & 35.58 \\
\hline 2.489 & 9 & 2 & 5 & 0 & + & 36.06 \\
\hline 2.482 & 5 & 1 & 6 & 0 & & 36.16 \\
\hline 2.428 & 2 & 2 & 2 & -2 & & $37 \cdot 00$ \\
\hline 2.405 & 1 & 1 & 1 & 2 & & 37.36 \\
\hline 2.389 & 1 & 2 & 5 & -1 & & 37.62 \\
\hline 2.324 & 1 & 1 & 2 & 2 & & 38.72 \\
\hline 2.314 & 2 & 1 & 6 & -1 & & 38.88 \\
\hline 2.293 & 9 & 2 & 3 & -2 & & 39.26 \\
\hline 2.264 & 1 & 1 & 4 & -2 & & 39.78 \\
\hline 2.242 & 2 & 3 & 1 & 1 & & 40.18 \\
\hline 2.229 & 9 & 3 & 4 & -1 & & 40.44 \\
\hline 2.199 & 1 & 2 & 6 & 0 & & 41.00 \\
\hline 2.129 & 2 & 2 & 6 & -1 & & 42.42 \\
\hline 2.064 & 3 & 1 & 4 & 2 & + & 43.82 \\
\hline 2.060 & 3 & 0 & 7 & 1 & & 43.92 \\
\hline 2.056 & 3 & 2 & 1 & 2 & & 44.00 \\
\hline 2.051 & 4 & 4 & 1 & 0 & + & 44.12 \\
\hline 2.005 & 2 & 2 & 2 & 2 & + & 45.18 \\
\hline 1.9754 & 1 & 2 & 5 & -2 & & 45.90 \\
\hline 1.9593 & 2 & 3 & 4 & 1 & + & 46.30 \\
\hline 1.8476 & 3 & 4 & 1 & -2 & & 49.28 \\
\hline 1.8329 & 1 & 3 & 5 & 1 & & 49.70 \\
\hline 1.8267 & 3 & 4 & 4 & 0 & & 49.88 \\
\hline 1.8064 & 2 & 0 & 1 & 3 & + & 50.48 \\
\hline 1.7628 & 3 & 1 & 8 & 1 & & 51.82 \\
\hline 1.7251 & 1 & 2 & 8 & -1 & & 53.04 \\
\hline 1.7215 & 1 & 3 & 7 & -1 & & 53.16 \\
\hline 1.7072 & 2 & 3 & 6 & 1 & & 53.64 \\
\hline 1.6829 & 1 & 1 & 4 & -3 & & 54.48 \\
\hline 1.6637 & 1 & 3 & 2 & -3 & & 55.16 \\
\hline 1.6380 & 1 & 4 & 4 & 1 & + & 56.10 \\
\hline 1.6009 & 1 & 1 & 5 & -3 & & 57.52 \\
\hline
\end{tabular}


Hexamethylenediammonium Adipate, $\mathrm{C}_{12} \mathrm{H}_{26} \mathrm{~N}_{2} \mathrm{O}_{4}$ (monoclinic) - continued

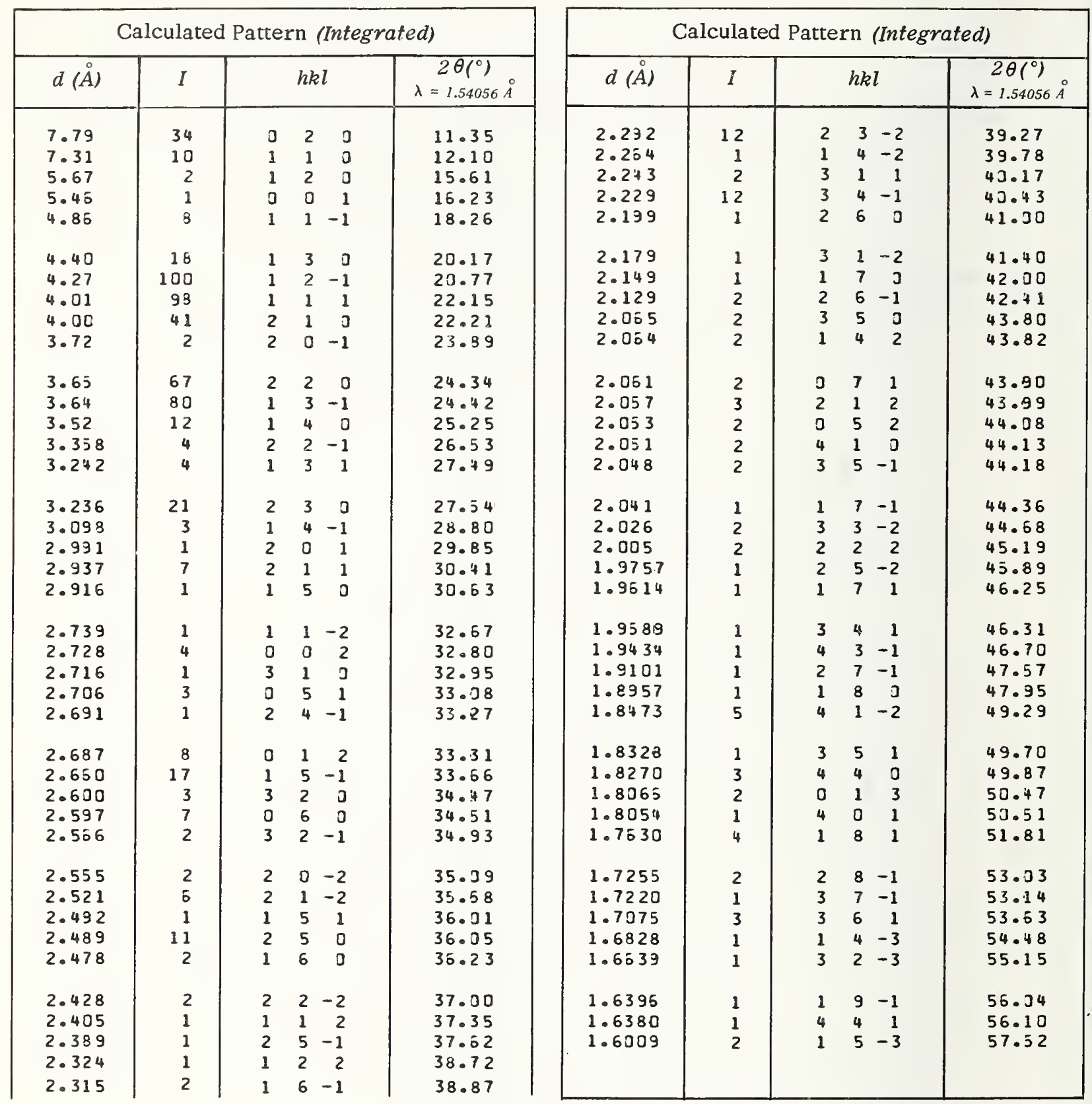


Structure

Monoclinic, P 2 1 /C (14), $\mathrm{Z}=4$ [Lundberg, 1966]

\section{Lattice parameters}

$a=7.956, b=11.856, c=12.078 \AA, \quad \beta=113^{\circ} 58^{\prime}$

$$
\pm .005 \pm .005 \pm .005 \quad \pm 2^{\prime}
$$

[ibid.]

\section{Scattering factors}

$\mathrm{Zn}^{\circ}$ [Thomas, and Umeda, 1957], corrected for the real part of the dispersion correction, [Dauben and Templeton, 1955]. $\mathrm{N}^{\circ}, \mathrm{C}^{\circ}, \mathrm{Cl}^{-}$[Berghuis et al.,1955]

\section{Thermal parameters}

Isotropic [Lundberg, 1966]

\section{Density}

(calculated)

$1.73 \mathrm{~g} / \mathrm{cm}^{3}$ [Lundberg, 1966]

Scale factor

$$
7.339 \times 10^{4}
$$

\section{Reference}

Berghuis,J ., IJ.M.Haanappel,M.Potters, B.O. Loopstra, C.H.MacGillavry, and A.L.Veenendaal (1955). New calculations of atomic scattering factors, Acta Cryst.8,478-483.

Dauben,C.H. and D.H.Templeton (1955). A table of dispersion corrections for $x-r a y$ scattering of atoms, Acta Cryst.8,841-842

Lundberg,B.K.S. (1966). The crystal structure of Di-imidazole-zinc(II) Dichloride Acta Cryst. 21, 901-909.

Thomas, L. H. and K. Umeda (1957). Atomic scattering factors calculated from the TFD atomic model, J.Chem. Phys. 26, 293-

\begin{tabular}{|c|c|c|c|c|c|c|}
\hline \multicolumn{7}{|c|}{ Calculated Pattern (Peak heights) } \\
\hline$d(\AA)$ & $I$ & \multicolumn{4}{|c|}{$h k l$} & $\begin{array}{c}2 \theta\left({ }^{\circ}\right) \\
\lambda=1.54056 \mathrm{~A}\end{array}$ \\
\hline 7.261 & 2 & 1 & 0 & 0 & & 12.18 \\
\hline 6.440 & 13 & -1 & 1 & 1 & & 13.74 \\
\hline 6.197 & 100 & 1 & 1 & 0 & & 14.28 \\
\hline 5.925 & 5 & 0 & 2 & 0 & & 14.94 \\
\hline 5.514 & 11 & 0 & 0 & 2 & & 16.06 \\
\hline 5.22 .4 & 43 & 0 & 2 & 1 & & 16.96 \\
\hline 5.087 & 92 & -1 & $\overline{1}$ & 2 & & 17.42 \\
\hline 4.746 & 2 & $\hat{1}$ & 1 & 1 & & 18.68 \\
\hline 4.691 & 1 & -1 & 2 & 1 & & 18.90 \\
\hline $4 \cdot 081$ & 2 & -1 & 2 & 2 & & 21.76 \\
\hline 4.037 & 21 & 0 & 2 & 2 & & 22.00 \\
\hline 3.000 & 12 & 1 & 2 & 1 & & 22.78 \\
\hline 3.792 & 2 & -1 & 1 & 3 & & 23.44 \\
\hline 3.760 & 10 & -2 & 1 & 1 & & 23.64 \\
\hline 3.720 & 50 & 0 & 3 & 1 & + & 23.90 \\
\hline 3.648 & 15 & -2 & 1 & 2 & & 24.38 \\
\hline 3.636 & 28 & 2 & 0 & 0 & & 24.46 \\
\hline 3.553 & 29 & 1 & 1 & 2 & & 25.04 \\
\hline 3.512 & 16 & -1 & 3 & 1 & + & 25.34 \\
\hline 3.474 & 7 & 2 & 1 & 0 & & 25.62 \\
\hline 3.310 & 17 & -1 & 2 & 3 & & 26.86 \\
\hline 3.220 & 15 & -2 & 2 & 2 & + & 27.68 \\
\hline 3.142 & 1 & 1 & 3 & 1 & & 28.38 \\
\hline 3.125 & 5 & 0 & 2 & 3 & & 28.54 \\
\hline 2.998 & 1 & 2 & 1 & 1 & & 29.78 \\
\hline 2.965 & 16 & 0 & 4 & 0 & & 30.12 \\
\hline 2.925 & 2 & -1 & 1 & 4 & & 30.54 \\
\hline 2.863 & 8 & 0 & 4 & 1 & & $31 \cdot 2 \hat{c}$ \\
\hline $2 \cdot 617$ & 4 & -2 & 0 & 4 & & 31.74 \\
\hline $2 \cdot 8,00$ & 1 & -2 & 3 & 1 & & 31.94 \\
\hline 2.769 & 3 & 1 & 1 & 3 & & 32.30 \\
\hline 2.759 & 4 & 0 & 0 & 4 & & 32.42 \\
\hline 2.753 & 5 & -2 & 3 & 2 & & 32.50 \\
\hline 2.744 & 4 & 1 & 4 & 0 & & 32.60 \\
\hline 2.741 & 5 & -2 & 1 & 4 & & 32.64 \\
\hline 2.693 & 4 & 0 & 3 & 3 & & 33.24 \\
\hline 2.676 & 2 & 2 & 3 & 0 & & 33.46 \\
\hline 2.650 & 3 & -3 & 0 & 2 & & 33.80 \\
\hline 2.611 & 1 & 0 & 4 & 2 & & 34.32 \\
\hline $2 \cdot 586$ & 11 & -3 & 1 & 2 & + & 34.66 \\
\hline 2.545 & 2 & -2 & 2 & 4 & & 35.24 \\
\hline 2.531 & 4 & 2 & 1 & 2 & & 35.44 \\
\hline 2.501 & 2 & 0 & 2 & 4 & & 35.88 \\
\hline 2.399 & 2 & -1 & 3 & 4 & & 37.46 \\
\hline 2.379 & 4 & -3 & 2 & 1 & + & 37.78 \\
\hline 2.374 & 8 & 3 & 1 & 0 & + & 37.86 \\
\hline $2 \cdot 319$ & 4 & 1 & 4 & 2 & & 38.80 \\
\hline 2.311 & 12 & -3 & 1 & 4 & + & 38.94 \\
\hline $2 \cdot 294$ & 2 & -2 & 3 & 4 & & 39.24 \\
\hline $2 \cdot 290$ & 2 & 1 & 0 & 4 & & 39.32 \\
\hline
\end{tabular}
303 . 


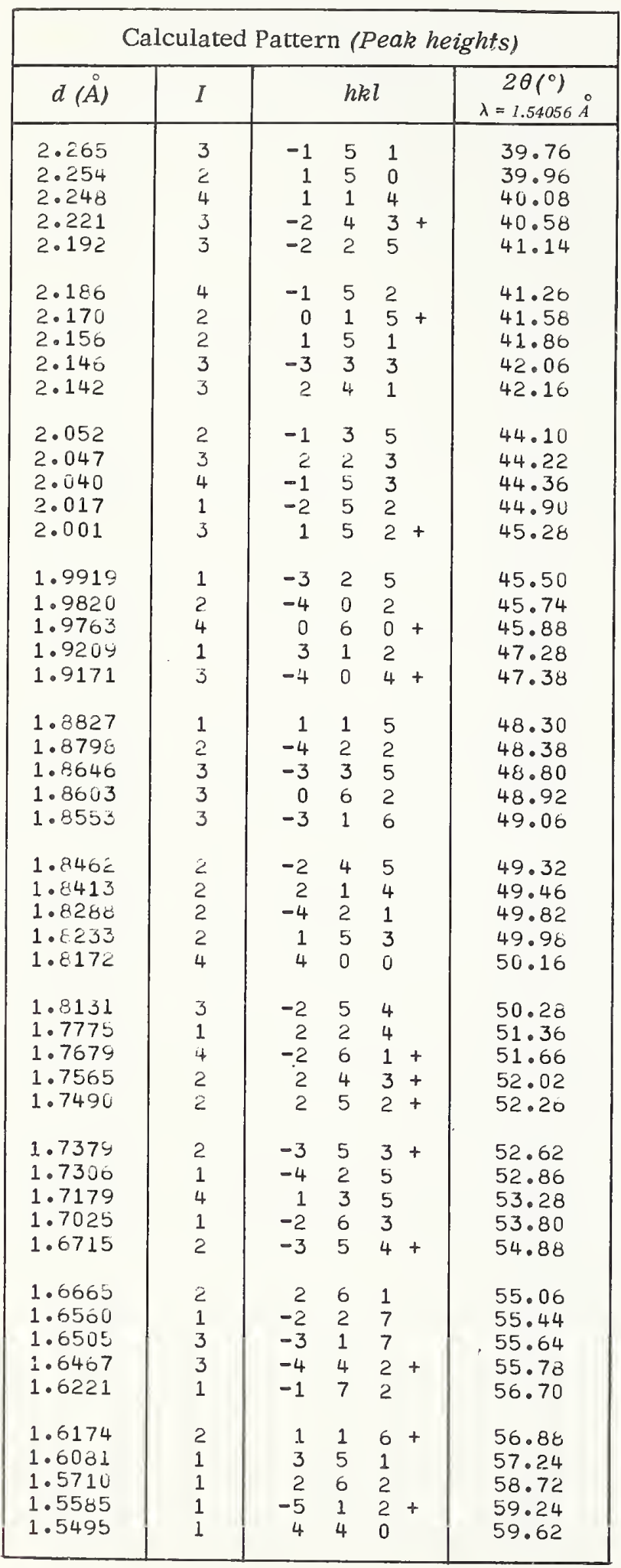

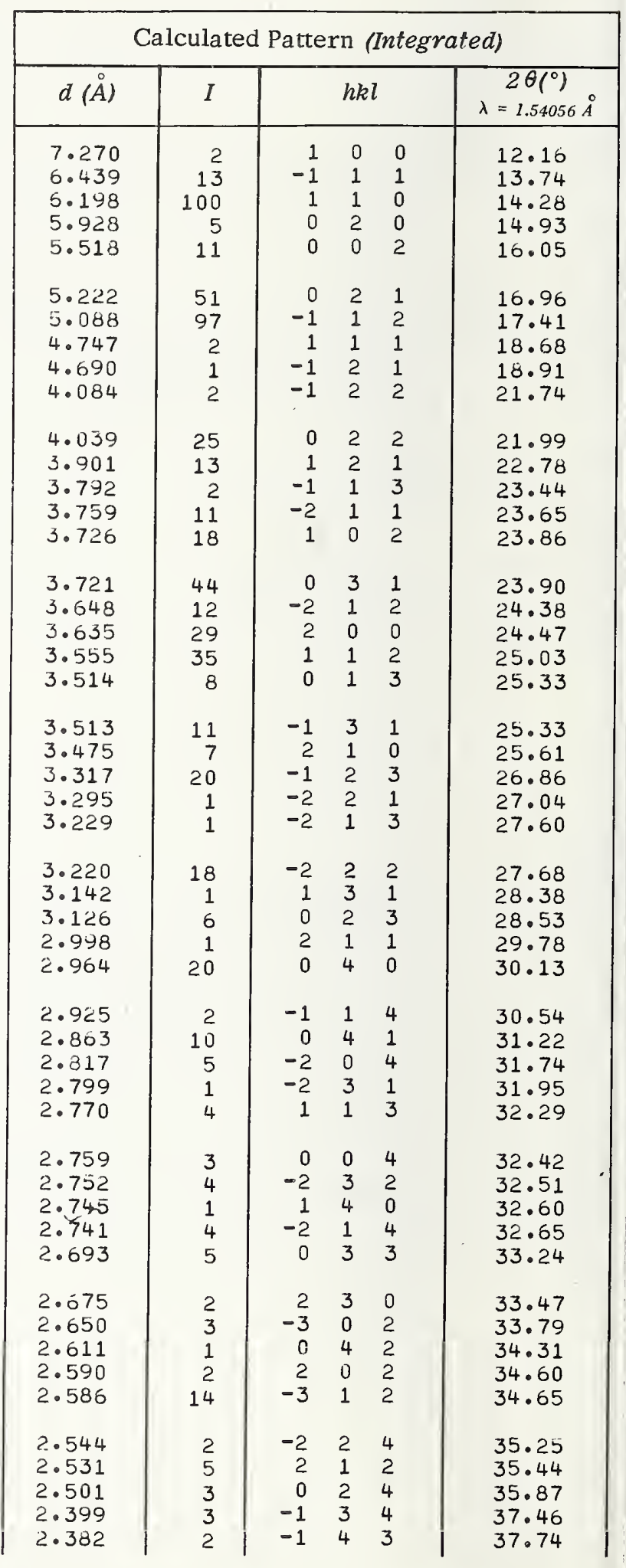


Imidazole Zinc Chloride, $\left(\mathrm{C}_{3} \mathrm{H}_{4} \mathrm{~N}_{2}\right)_{2} \mathrm{ZnCl}$ (monoclinic) - continued

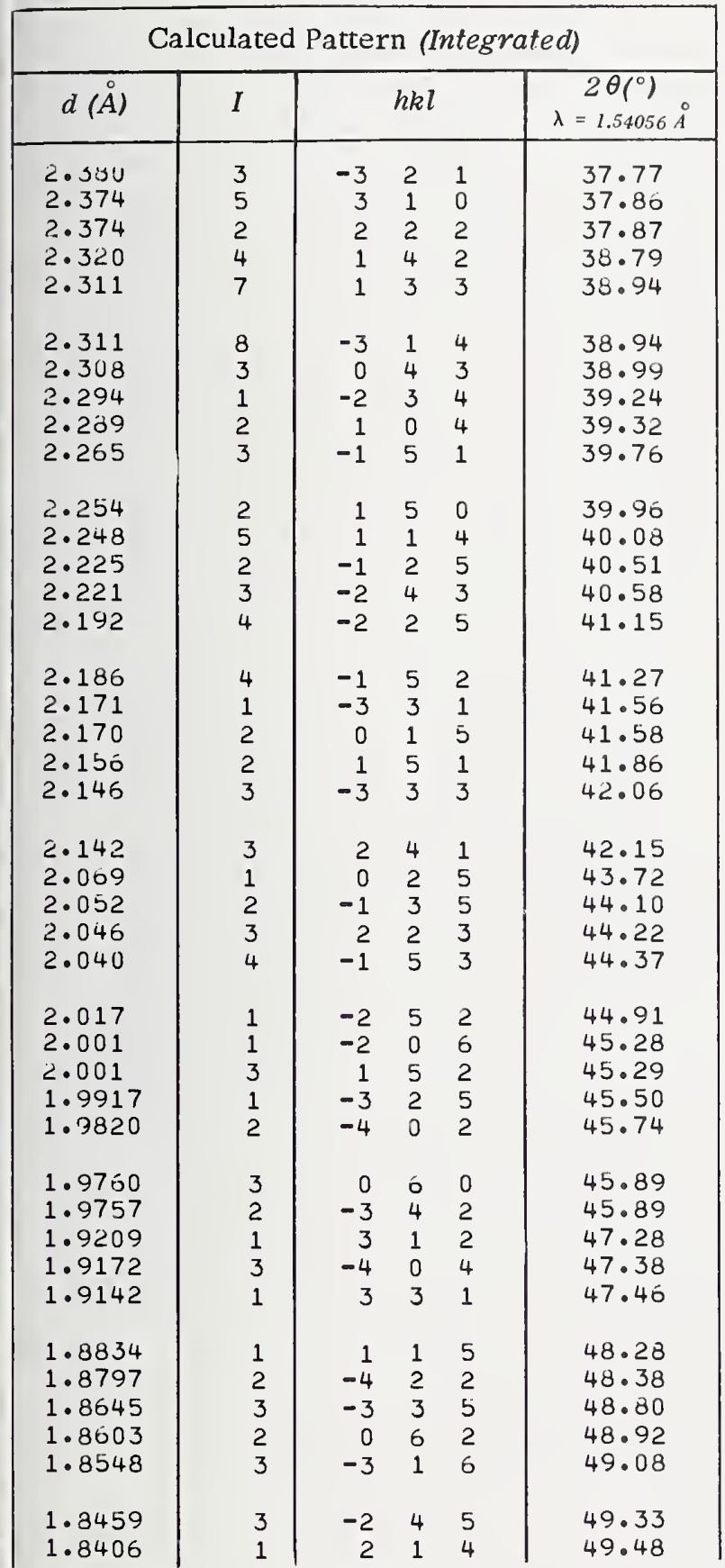

\begin{tabular}{|c|c|c|c|c|c|}
\hline \multicolumn{6}{|c|}{ Calculated Pattern (Integrated) } \\
\hline$d(\AA)$ & $I$ & & $h k l$ & & $\begin{array}{c}2 \theta\left(^{\circ}\right) \\
\lambda=1.54056 \mathrm{~A}\end{array}$ \\
\hline 1.4291 & 2 & -4 & 2 & 1 & 49.81 \\
\hline 1.8226 & 1 & 1 & 5 & 3 & 50.00 \\
\hline $1 \cdot 8175$ & 4 & 4 & 0 & 0 & 50.15 \\
\hline $1 \cdot 8140$ & 1 & -2 & 5 & 4 & 50.25 \\
\hline 1.7887 & 1 & -4 & 1 & 5 & 51.02 \\
\hline 1.7775 & 1 & 2 & 2 & 4 & $51 \cdot 36$ \\
\hline 1.7718 & $\overline{1}$ & -1 & 6 & 3 & $51 \cdot 54$ \\
\hline 1.7703 & 2 & 0 & 4 & 5 & $51 \cdot 58$ \\
\hline 1.7685 & 3 & -2 & 6 & 1 & 51.64 \\
\hline 1.7671 & 2 & -3 & 5 & 2 & 51.68 \\
\hline 1.7565 & 1 & -2 & 6 & 2 & 52.02 \\
\hline 1.7564 & 1 & 2 & 4 & 3 & 52.02 \\
\hline 1.7515 & 1 & -3 & 5 & 1 & 52.18 \\
\hline 1.7490 & 2 & 2 & 5 & 2 & $52 \cdot 26$ \\
\hline 1.7334 & 1 & $-\overline{3}$ & 5 & 3 & 52.60 \\
\hline 1.7376 & 1 & 4 & 2 & 0 & 52.65 \\
\hline 1.7306 & 2 & -4 & 2 & 5 & $52 \cdot 86$ \\
\hline 1.7179 & 6 & 1 & 3 & 5 & $53 \cdot 23$ \\
\hline 1.7027 & 2 & -2 & 6 & 3 & 53.79 \\
\hline 1.6870 & 1 & -1 & 5 & 5 & 54.34 \\
\hline 1.6741 & 1 & 0 & 7 & 1 & 54.79 \\
\hline 1.6714 & 2 & -3 & 5 & 4 & 54.89 \\
\hline 1.6661 & 1 & 2 & 0 & 1 & 55.08 \\
\hline 1.6560 & 2 & -2 & 2 & 7 & 55.44 \\
\hline 1.6506 & 2 & -3 & 1 & 7 & 55.64 \\
\hline 1.6476 & 1 & -4 & 4 & 2 & 55.75 \\
\hline 1.6470 & 1 & 1 & 5 & 4 & 55.77 \\
\hline 1.0464 & 1 & -4 & 4 & 3 & 55.79 \\
\hline 1.6451 & 1 & -4 & 3 & 5 & 55.84 \\
\hline 1.6220 & 1 & -1 & 7 & 2 & 56.71 \\
\hline 1.5177 & 1 & -2 & 6 & 4 & 56.87 \\
\hline 1.6172 & 1 & 1 & 1 & 6 & 56.89 \\
\hline 1.6030 & 1 & 3 & 5 & 1 & $57 \cdot 24$ \\
\hline 1.5711 & 1 & 2 & 6 & 2 & 58.72 \\
\hline 1.5605 & 1 & 2 & 2 & 5 & $59 \cdot 16$ \\
\hline 1.5535 & 2 & -5 & 1 & 2 & 59.24 \\
\hline 1.5494 & 1 & 4 & 4 & 0 & 59.62 \\
\hline 1.5444 & 1 & -4 & 4 & 5 & 59.84 \\
\hline 1.5358 & 2 & -3 & 3 & 7 & $60 \cdot 20$ \\
\hline 1.5237 & $\overline{1}$ & 0 & 2 & 7 & 00.74 \\
\hline 1.4770 & 1 & -1 & 7 & 4 & 02.87 \\
\hline
\end{tabular}




\section{Structure}

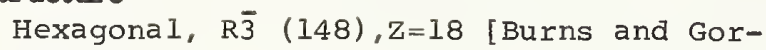
don, 1966]

\section{Lattice parameters}

$$
a=13.29 \pm 0.01, c=8.91 \pm 0.01 \AA \quad \text { [ibid.] }
$$

\section{Scattering factors}

$$
\mathrm{Li}^{+}, \mathrm{Be}^{+2}, \mathrm{~F}^{-}[3.3, \mathrm{IA}]
$$

\section{Thermal parameters}

Anisotropic [Burns and Gordon, 1966]

\section{Density}

(calculated) $2.169 \mathrm{~g} / \mathrm{cm}^{3}$

\section{Scale factor}

$$
9.496 \times 10^{4}
$$

\section{Additional patterns}

1.PDF card 6-0557 [Thilo and Lehmann, 1949]

\section{Reference}

Burns, J.H. and E.K.Gordon (1966). Refinement of the crystal structure of $\mathrm{Li}_{2} \mathrm{BeF}_{4}$ Acta Cryst. 20, 135-138.

Thilo, E. and H. -A. Lehmann (1949). Über das System LiF-BeFe und seine Beziehung-

\begin{tabular}{|c|c|c|c|c|c|c|}
\hline \multicolumn{7}{|c|}{ Calculated Pattern (Peak heights) } \\
\hline$d(\AA)$ & $I$ & & $h k l$ & & & $\begin{array}{c}2 \theta\left(^{\circ}\right) \\
\lambda=1.54056 \mathrm{~A}\end{array}$ \\
\hline 6.64 & 38 & 1 & 1 & 0 & & 13.32 \\
\hline 4.15 & 9 & 0 & 1 & 2 & & 21.38 \\
\hline 3.91 & 23 & 2 & 1 & 1 & + & 22.74 \\
\hline 3.84 & 20 & 3 & 0 & 0 & & 23.16 \\
\hline 3.52 & 2 & 2 & 0 & 2 & & 25.26 \\
\hline 3.321 & 56 & 2 & 2 & 0 & & 26.82 \\
\hline 3.112 & 4 & 1 & 2 & 2 & + & 28.66 \\
\hline 3.006 & 1 & 3 & 1 & -1 & & 29.70 \\
\hline 2.738 & 2 & 4 & 0 & 1 & & 32.68 \\
\hline 2.712 & 31 & 1 & 1 & -3 & + & 33.00 \\
\hline 2.512 & 23 & 4 & 1 & 0 & + & 35.72 \\
\hline 2.348 & 100 & 0 & 3 & 3 & + & 38.30 \\
\hline 2.272 & 1 & 3 & 2 & -2 & & 39.64 \\
\hline 2.215 & 95 & 3 & 3 & 0 & + & 40.70 \\
\hline 2.187 & 3 & 1 & 0 & 4 & & 41.24 \\
\hline 2.045 & 2 & 5 & 0 & 2 & & 44.26 \\
\hline 1.9545 & 2 & 4 & 2 & 2 & & 46.42 \\
\hline 1.9179 & 15 & 1 & 4 & -3 & + & 47.36 \\
\hline 1.8427 & 8 & 2 & 5 & 0 & + & 49.42 \\
\hline 1.8267 & 1 & 3 & 1 & -4 & & 49.88 \\
\hline 1.7756 & 2 & 3 & 3 & 3 & + & 51.42 \\
\hline 1.7416 & 2 & 4 & 3 & -2 & + & 52.50 \\
\hline 1.7221 & 1 & 6 & 1 & -1 & & 53.14 \\
\hline 1.7025 & 2 & 2 & 0 & 5 & + & 53.80 \\
\hline 1.6489 & 2 & 1 & 2 & 5 & + & 55.70 \\
\hline 1.6112 & 15 & 6 & 0 & 3 & + & 57.12 \\
\hline 1.5710 & 2 & 6 & 2 & 1 & & 58.72 \\
\hline 1. 5561 & 4 & 2 & 4 & 4 & + & 59.34 \\
\hline 1.5245 & 5 & 1 & 7 & 0 & + & 60.70 \\
\hline 1.5150 & 2 & 1 & 5 & -4 & + & 61.12 \\
\hline 1.5026 & 1 & 2 & 6 & 2 & & 61.68 \\
\hline 1.4848 & 7 & 0 & 0 & 6 & & 62.50 \\
\hline 1.4540 & 2 & 4 & 5 & -1 & + & 63.98 \\
\hline 1.3562 & 14 & 7 & 1 & -3 & + & 69.22 \\
\hline 1.3500 & 2 & 5 & 1 & -5 & & 69.58 \\
\hline 1.3030 & 6 & 3 & 6 & 3 & + & 72.48 \\
\hline 1.2787 & 4 & 9 & 0 & 0 & & 74.08 \\
\hline 1.2333 & 4 & 3 & 3 & 6 & + & 77.30 \\
\hline 1.2130 & 3 & 5 & 5 & -3 & + & 78.84 \\
\hline
\end{tabular}
en zum System $\mathrm{MgO}-\mathrm{SiO}_{2}, \mathrm{Z}$. Anorg. Chem., $258,332-355$. 
Lithium Beryllium Fluoride, $\mathrm{Li}_{2} \mathrm{BeF}_{4}$ (hexagonal) - continued

\begin{tabular}{|c|c|c|c|c|c|c|c|c|c|c|c|}
\hline \multicolumn{6}{|c|}{ Calculated Pattern (Integrated) } & \multicolumn{6}{|c|}{ Calculated Pattern (Integrated) } \\
\hline$d(\stackrel{\AA}{A})$ & $I$ & & $h k l$ & & $\begin{array}{c}2 \theta\left({ }^{\circ}\right) \\
\lambda=1.54056 \AA\end{array}$ & $d(\AA)$ & $I$ & & kl & & $\begin{array}{c}2 \theta\left(^{\circ}\right) \\
\lambda=1.54056 \AA\end{array}$ \\
\hline 6.64 & 34 & 1 & 1 & 0 & 13.31 & 1.7025 & 1 & 2 & 3 & -4 & 53.80 \\
\hline 4.15 & 10 & 0 & 1 & 2 & 21.37 & 1.7023 & 2 & 2 & 0 & 5 & 53.81 \\
\hline 3.91 & 15 & 2 & 1 & 1 & 22.73 & 1.6490 & 1 & 1 & 2 & 5 & 55.69 \\
\hline 3.91 & 11 & 1 & $2-$ & -1 & 22.73 & 1.6490 & 1 & 2 & 1 & -5 & 55.69 \\
\hline & 21 & 3 & 0 & 0 & 23.16 & & & & & & \\
\hline 3.52 & & & & & & $\begin{array}{l}1.6114 \\
1.6114\end{array}$ & $\begin{array}{l}12 \\
10\end{array}$ & $\begin{array}{l}6 \\
0\end{array}$ & $\begin{array}{l}0 \\
6\end{array}$ & $\begin{array}{l}3 \\
3\end{array}$ & $\begin{array}{l}57.11 \\
57.11\end{array}$ \\
\hline 3.322 & $\begin{array}{r}2 \\
64\end{array}$ & $\begin{array}{l}2 \\
2\end{array}$ & $\begin{array}{l}0 \\
2\end{array}$ & $\begin{array}{l}2 \\
0\end{array}$ & $\begin{array}{l}25.26 \\
26.81\end{array}$ & 1.5711 & 3 & 6 & $\begin{array}{l}6 \\
2\end{array}$ & $\begin{array}{l}3 \\
1\end{array}$ & $\begin{array}{l}57.11 \\
58.72\end{array}$ \\
\hline 3.112 & 2 & 1 & & 2 & 28.56 & 1.5562 & 3 & 2 & 4 & 4 & 59.34 \\
\hline 3.112 & 2 & 2 & $1-$ & -2 & 28.56 & 1.5562 & 1 & 4 & 2 & -4 & 59.34 \\
\hline 3.005 & 1 & 3 & $1-$ & -1 & 29.70 & 1.5560 & 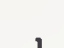 & & & & \\
\hline 2.738 & 1 & 4 & $a$ & 1 & 32.58 & 1.5560 & 12 & 3 & $\begin{array}{l}3 \\
1\end{array}$ & $\begin{array}{r}-5 \\
5\end{array}$ & $\begin{array}{l}59.35 \\
59.35\end{array}$ \\
\hline 2.711 & 18 & 1 & & 3 & 33.01 & 1.5245 & 5 & 1 & $\frac{1}{7}$ & $\begin{array}{l}5 \\
0\end{array}$ & $\begin{array}{l}59.35 \\
60.70\end{array}$ \\
\hline 2.711 & 19 & 1 & $1-$ & -3 & 33.01 & 1.5245 & 3 & 7 & 1 & 0 & 60.70 \\
\hline 2.512 & 17 & 4 & 1 & 0 & 35.72 & 1.5152 & 1 & 1 & & -4 & 61.11 \\
\hline 2.512 & 11 & 1 & 4 & 0 & 35.72 & & & & & & \\
\hline & & & & & & 1.5150 & 1 & 0 & 4 & 5 & 61.12 \\
\hline 2.349 & 58 & 3 & 0 & 3 & 38.29 & 1.5025 & 1 & 2 & 6 & 2 & 61.58 \\
\hline 2.349 & 67 & 0 & & 3 & 38.29 & 1.4850 & 11 & 0 & 0 & 6 & 62.49 \\
\hline 2.271 & 1 & 3 & $2-$ & -2 & 39.55 & 1.4539 & 2 & 4 & 5 & -1 & 63.98 \\
\hline 2.229 & 1 & 0 & 5 & 1 & 40.44 & 1.4539 & 1 & 5 & 4 & 1 & 63.98 \\
\hline 2.215 & 100 & 3 & 3 & 0 & 40.70 & 1.3560 & & & & & \\
\hline & & & & & & & 3 & 1 & 7 & -3 & $\begin{array}{l}69.22 \\
69.22\end{array}$ \\
\hline 2.214 & 11 & 2 & 2 & $\begin{array}{l}3 \\
-3\end{array}$ & 40.71 & 1.3562 & 7 & 7 & 1 & 3 & \\
\hline 2.214 & 9 & 2 & $2-$ & -3 & 40.71 & 1.3562 & 3 & 1 & 7 & 3 & $\begin{array}{l}69.22 \\
69.22\end{array}$ \\
\hline 2.187 & 2 & 1 & 0 & 4 & 41.25 & 1.3562 & 8 & 7 & 1 & -3 & 69.22 \\
\hline 2.045 & 3 & 5 & 0 & 2 & 44.25 & 1.3557 & 2 & 2 & 2 & 6 & 69.24 \\
\hline 1.9546 & 3 & 4 & 2 & 2 & 46.42 & & & & & & \\
\hline & & & & & & 1.3557 & 2 & 2 & 2 & -6 & 69.24 \\
\hline 1.9178 & 9 & 1 & 4 & 3 & 47.36 & 1.3497 & 1 & 5 & 1 & -5 & $\begin{array}{ll}69.50 \\
72 .+8\end{array}$ \\
\hline 1.9178 & 9 & 1 & $4-$ & $\cdot 3$ & 47.36 & 1.3030 & 1 & 6 & 3 & 3 & $72 .+8$ \\
\hline 1.8430 & 8 & 5 & 2 & 0 & 49.41 & 1.3030 & 4 & 3 & 6 & 3 & 72.48 \\
\hline 1.8430 & 4 & 2 & 5 & 0 & 49.41 & 1.3030 & 3 & 3 & 6 & -3 & 72.48 \\
\hline 1.8267 & 1 & 3 & $1-$ & 4 & 49.88 & & & & & & \\
\hline & & & & & & 1.3030 & 2 & 6 & 3 & -3 & 72.48 \\
\hline 1.7756 & 2 & 3 & 3 & 3 & 51.42 & 1.2788 & 6 & 9 & 0 & 0 & $\begin{array}{l}74.07 \\
75.67\end{array}$ \\
\hline 1.7758 & 1 & 3 & $3-$ & 3 & 51.42 & 1.2558 & 1 & 2 & 8 & 0 & 77.29 \\
\hline 1.7614 & 1 & 4 & 0 & 4 & 51.87 & 1.2334 & 4 & 3 & 3 & 6 & 77.29 \\
\hline 1.7416 & 1 & 3 & 4 & 2 & $\begin{array}{l}52.50 \\
52.50\end{array}$ & 1.2334 & 4 & 3 & 3 & -6 & 77.29 \\
\hline 1.7416 & & 4 & $3-2$ & & 52.50 & 1.2131 & 3 & 5 & 5 & -3 & 78.84 \\
\hline 1.7221 & 2 & 6 & $1-$ & -1 & 53.14 & 1.2131 & 3 & 5 & 5 & 3 & 78.84 \\
\hline
\end{tabular}


Lithium Rubidium Fluoride, LiRbF 2 (monoclinic)

Structure

Monoclinic, $\mathrm{C} 2 / \mathrm{c}(15), \mathrm{Z}=8$ [Burns and Busing, 1965]

\section{Lattice parameters}

$a=5.83 \pm 0.01, b=11.16 \pm 0.02, c=7.86 \pm 0.02 \AA$, $B=94^{\circ} 55^{\prime} \quad$ [ibid.]

\section{Scattering factors}

$\mathrm{Li}^{+1}[3.3 .1 \mathrm{~A}] ; \mathrm{Rb}^{0}, \mathrm{~F}^{-1}[3.3 .1 \mathrm{~A}]$, corrected for real and imaginary dispersion, $[3.3 .2 \mathrm{~B}]$

\section{Thermal parameters}

Isotropic: Rb 1.73; Li 2.50; F(1) 1.40; $F(2) 1.80 ; F(3) 2.10$

Density

(calculated) $3.40 \mathrm{~g} / \mathrm{cm}^{3}$

\section{Scale factor}

$$
4.815 \times 10^{4}
$$

\section{Reference}

Burns,J.H. and W.R. Busing (1965). Crystal structures of rubidium lithium fluoride, RbLiFz, and cesium lithium fluoride, CsLiFz, Inorg. Chem. 4, 1510-1512.

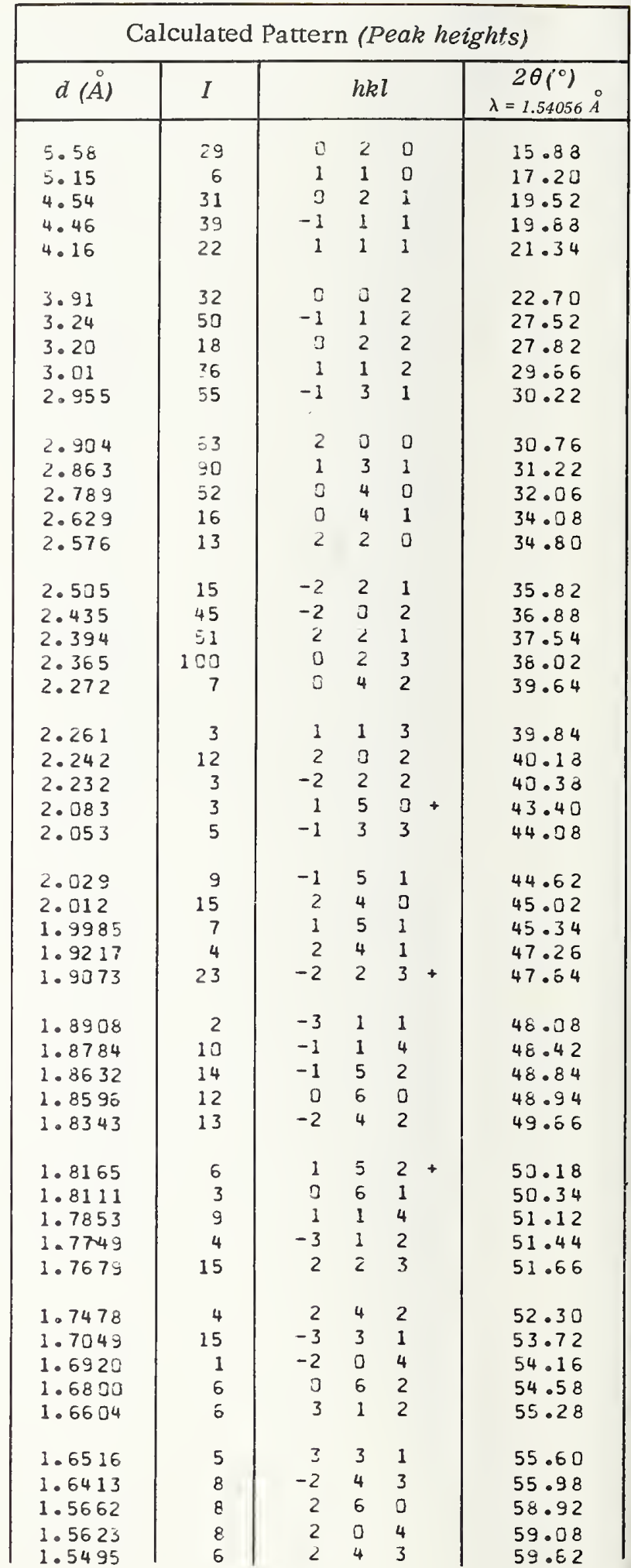


Lithium Rubidium Fluoride, LiRbF (monoclinic) - continued

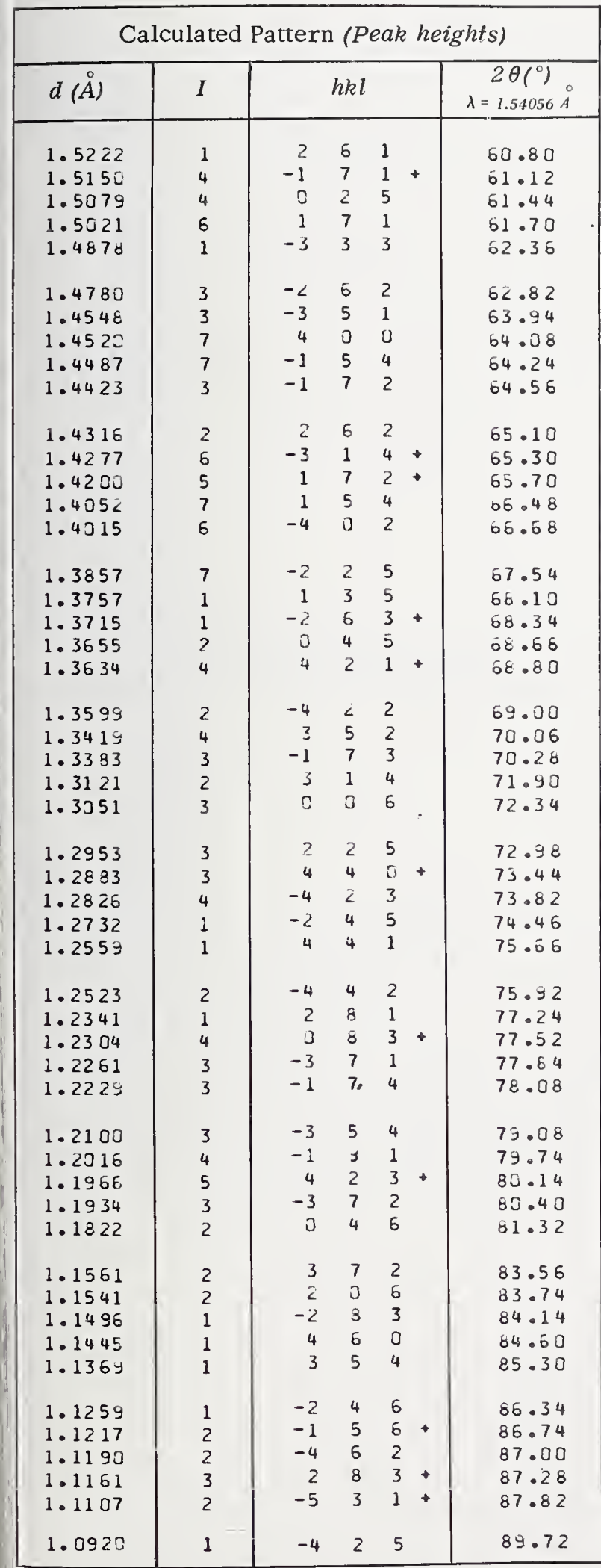

\begin{tabular}{|c|c|c|c|c|c|}
\hline \multicolumn{6}{|c|}{ Calculated Pattern (Integrated) } \\
\hline$d(\stackrel{\circ}{A})$ & $I$ & & $h k l$ & & $\begin{array}{c}2 \theta\left({ }^{\circ}\right) \\
\lambda=1.54056 \AA\end{array}$ \\
\hline 5.58 & 22 & 0 & 2 & 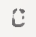 & 15.67 \\
\hline 5.15 & 4 & 1 & 1 & 0 & 17.20 \\
\hline 4.54 & 24 & 0 & 2 & 1 & 19.52 \\
\hline 4.46 & 31 & -1 & 1 & 1 & 19.56 \\
\hline 4.16 & 18 & 1 & 1 & 1 & 21.33 \\
\hline 3.92 & 28 & 0 & C & 2 & 22.59 \\
\hline 3.24 & 44 & -1 & 1 & 2 & 27.52 \\
\hline 3.21 & 16 & 3 & 2 & 2 & 27.81 \\
\hline 3.51 & 32 & 1 & 1 & 2 & 29.56 \\
\hline 2.956 & 53 & -1 & 3 & 1 & 30.21 \\
\hline 2.904 & 56 & 2 & 0 & 0 & 30.70 \\
\hline 2.863 & 54 & 1 & 1 & 1 & 31.21 \\
\hline 2.790 & 49 & 0 & 4 & 0 & 32.95 \\
\hline 2.628 & 15 & 0 & 4 & 1 & 34.09 \\
\hline 2.576 & 13 & 2 & 2 & 0 & 34.79 \\
\hline 2.504 & 15 & -2 & 2 & 1 & 35.83 \\
\hline 2.435 & 46 & $-?$ & 0 & 2 & 36.89 \\
\hline 2.403 & 1 & -1 & 1 & 3 & 37.39 \\
\hline 2.394 & 49 & 2 & 2 & 1 & 37.54 \\
\hline 2.364 & 1 L & 3 & 2 & 3 & 38.03 \\
\hline 2.272 & 7 & 0 & 4 & 2 & 39.53 \\
\hline 2.260 & $?$ & 1 & 1 & 3 & 39.65 \\
\hline 2.242 & 12 & 2 & 0 & 2 & 40.18 \\
\hline 2.231 & 2 & -2 & 2 & 2 & 40.39 \\
\hline 2.083 & 3 & 1 & 5 & 0 & 43.40 \\
\hline 2.381 & 1 & 2 & 2 & 2 & 43.46 \\
\hline 2.053 & 5 & -1 & 3 & 3 & 44.08 \\
\hline 2.029 & 9 & -1 & 5 & 1 & 44.52 \\
\hline 2.012 & 16 & 2 & 4 & 0 & 45.32 \\
\hline 1.9982 & 7 & 1 & 5 & 1 & $4 \overrightarrow{5} .35$ \\
\hline 1.9214 & 4 & 2 & 4 & 1 & 47.27 \\
\hline 1.9575 & 18 & $-\dot{i}$ & 2 & 3 & 47.53 \\
\hline 1.9061 & 10 & 0 & 4 & 3 & 47.57 \\
\hline 1.8906 & 2 & -3 & 1 & 1 & 48.09 \\
\hline 1.8781 & 11 & -1 & 1 & 4 & 48.43 \\
\hline $1.863 \hat{2}$ & 14 & -1 & 5 & 2 & 48.94 \\
\hline 1.8605 & 7 & 0 & 6 & 0 & 48.93 \\
\hline 1.8344 & 14 & -2 & 4 & 2 & 49.56 \\
\hline 1.8185 & 3 & 3 & 1 & 1 & 50.12 \\
\hline 1.8163 & 5 & 1 & 5 & 2 & 50.19 \\
\hline 1.8097 & 1 & 0 & 6 & 1 & 50.38 \\
\hline 1.7850 & 11 & 1 & 1 & 4 & 51.11 \\
\hline 1.7750 & 3 & -3 & 1 & 2 & 51.44 \\
\hline 1.7676 & 17 & 2 & 2 & 3 & 51.67 \\
\hline 1.7479 & 4 & 2 & 4 & 2 & $52 \cdot 30$ \\
\hline $1.734 y$ & 18 & -3 & 3 & 1 & 53.72 \\
\hline 1.6923 & 1 & -2 & 0 & 4 & 54.16 \\
\hline 1.6801 & 6 & כ & 5 & 2 & 54.58 \\
\hline 1.6505 & 7 & 3 & 1 & 2 & 55.27 \\
\hline 1.6515 & 5 & 3 & 3 & 1 & 55.00 \\
\hline
\end{tabular}




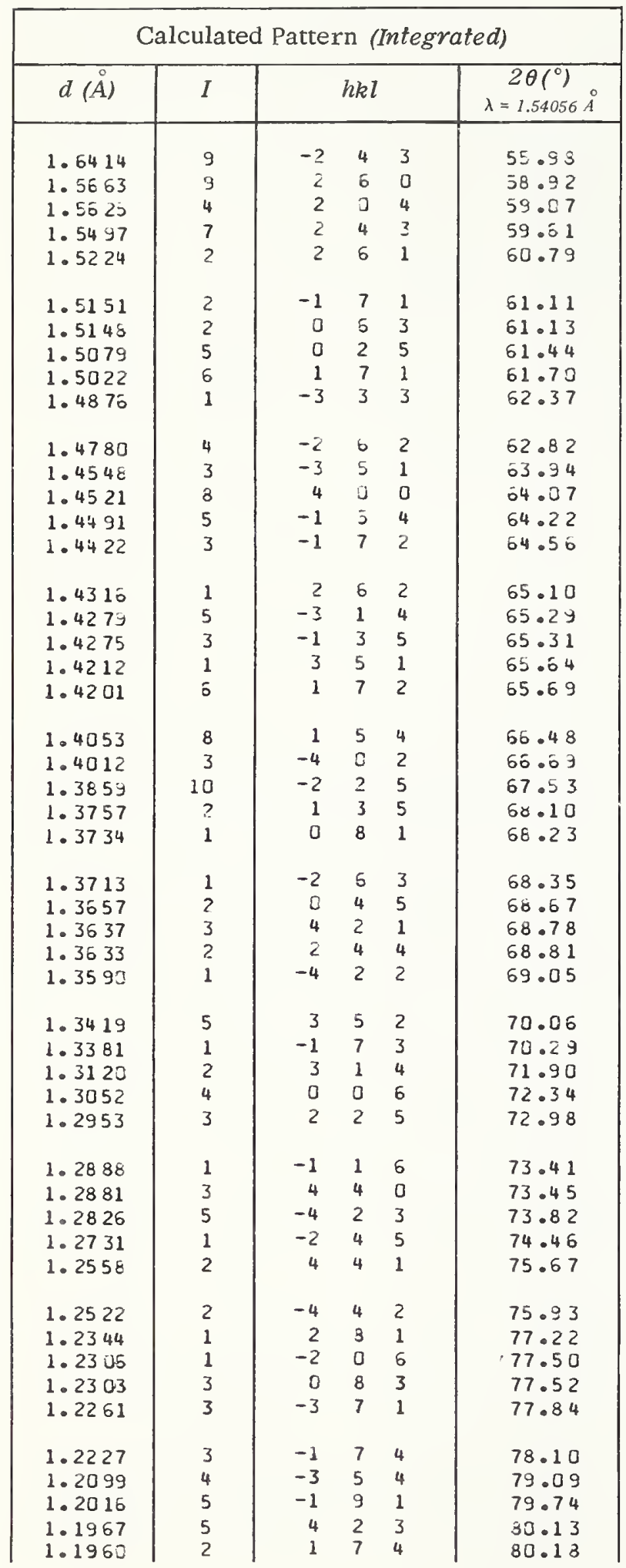

\begin{tabular}{|c|c|c|c|c|c|}
\hline \multicolumn{6}{|c|}{ Calculated Pattern (Integrated) } \\
\hline$d(\stackrel{\circ}{)}$ & $I$ & & $h k l$ & & $\begin{array}{c}2 \theta\left({ }^{\circ}\right) \\
\lambda=1.54056 \AA\end{array}$ \\
\hline 1.1952 & 3 & 1 & 9 & 1 & 80.25 \\
\hline 1.1928 & 1 & -3 & 7 & 2 & 80.44 \\
\hline 1.1916 & 1 & -4 & 4 & 3 & 80.54 \\
\hline 1.1822 & 3 & 0 & 4 & 6 & 81.32 \\
\hline 1.1562 & 2 & 3 & 7 & 2 & 83.55 \\
\hline 1.1541 & 1 & 2 & C & 6 & 83.74 \\
\hline 1.1497 & 2 & -2 & 8 & 3 & 84.13 \\
\hline 1.1440 & 2 & 4 & 6 & 0 & 84.59 \\
\hline 1.136 .9 & 2 & 3 & 5 & 4 & 85.30 \\
\hline 1.1259 & 1 & -2 & 4 & 6 & 85.33 \\
\hline 1.1218 & 1 & 4 & 4 & 3 & 86.73 \\
\hline 1.1217 & 1 & -1 & 5 & 6 & 86.74 \\
\hline 1.1132 & 2 & -4 & 6 & 2 & 85.98 \\
\hline 1.1168 & 2 & 2 & 8 & 3 & 87.22 \\
\hline 1.1160 & 1 & 5 & 10 & 0 & 87.29 \\
\hline 1.1158 & 2 & 3 & 3 & 5 & 87.31 \\
\hline 1.1106 & 2 & -5 & 3 & 1 & 87.82 \\
\hline 1.1098 & 1 & -1 & 7 & 5 & 87.90 \\
\hline 1.0925 & 2 & -4 & 2 & 5 & 89.72 \\
\hline
\end{tabular}




\section{Structure}

Tetragonal, $I 4_{1} / \mathrm{a}$ (88), $\mathrm{Z}=16$ [Brunton, 1966]

\section{Lattice parameters}

$a=14.885 \pm 0.002, c=6.547 \pm 0.001 \AA$

(published value: $a=14.884 \AA$ ) [ibid.]

\section{Scattering factors}

$\mathrm{Li}^{+1}$, and $\mathrm{F}^{-1}[3.3 .1 \mathrm{~A}]$

$\mathrm{U}^{+4}$ [Cromer and Waber, 1965], corrected

for dispersion using $\Delta f^{\prime}=-4$ and $\Delta f^{\prime \prime}=16$

\section{Thermal parameters}

Anisotropic for uranium, isotropic for fluorine and lithium [Brunton, 1966]

Density

(calculated) $6.23 \mathrm{~g} / \mathrm{cm}^{3}$ [ibid.]

\section{Scale factor}

$$
205.3 \times 10^{4}
$$

\section{Additional patterns}

1. PDF card 10-121 [Insley et al.,1956]. This card represents data for $L i_{7} U_{6} F_{31}$ which is very close in composition.

\section{Reference}

Brunton, G. (1966). The crystal structure of LiUf, Acta Cryst. 21, 814-817.

Cromer, D.T. and J.T. Waber (1965). Scattering factors computed from relativistic Dirac-slater wave functions, Acta Cryst. 18, 104-109.

Insley, H., T.N. McVay, R.E. Thoma and G. D. White (1956). Optical properties and $x$-ray diffraction data for some inorganic fluoride and chloride compounds,ORNL2192, pg.30, Oak Ridge National Laboratory, Tennessee.

\begin{tabular}{|c|c|c|c|c|c|c|}
\hline \multicolumn{7}{|c|}{ Calculated Pattern (Peak heights) } \\
\hline$d(\stackrel{\circ}{A})$ & $I$ & & $h k$ & & & $\begin{array}{c}2 \theta\left(^{\circ}\right) \\
\lambda=1.54056 \mathrm{~A} \\
\end{array}$ \\
\hline 7.44 & 1 & 0 & 2 & 0 & & 11.88 \\
\hline 5.99 & 24 & 0 & 1 & 1 & & 14.78 \\
\hline 5.26 & 88 & 2 & 2 & 0 & & 16.84 \\
\hline 4.67 & 100 & 2 & 1 & 1 & + & 19.00 \\
\hline 3.96 & 50 & 0 & 3 & 1 & & 22.46 \\
\hline 3.493 & 49 & 2 & 3 & 1 & + & 25.48 \\
\hline 3.329 & 64 & 4 & 2 & 0 & + & 26.76 \\
\hline 3.162 & 46 & 1 & 4 & 1 & + & 28.20 \\
\hline 2.996 & 52 & 0 & 2 & 2 & & 29.80 \\
\hline 2.779 & 18 & 2 & 2 & 2 & & 32.18 \\
\hline 2.711 & 20 & 0 & 5 & 1 & + & 33.02 \\
\hline 2.546 & 11 & 5 & 2 & 1 & + & 35.22 \\
\hline 2.481 & 1 & 0 & 6 & 0 & & 36.18 \\
\hline 2.354 & 8 & 6 & 2 & 0 & + & 38.20 \\
\hline 2.334 & 2 & 4 & 2 & 2 & & 38.54 \\
\hline 2.292 & 9 & 1 & 6 & 1 & + & 39.28 \\
\hline 2.179 & 1 & 5 & 1 & 2 & & 41.40 \\
\hline 2.102 & 16 & 3 & 6 & 1 & + & 43.00 \\
\hline 2.073 & 10 & 2 & 1 & 3 & + & 43.62 \\
\hline 2.064 & 29 & 6 & 4 & 0 & + & 43.82 \\
\hline 2.051 & 47 & 4 & 4 & 2 & & 44.12 \\
\hline 2.022 & 2 & 0 & 7 & 1 & & 44.78 \\
\hline 1.9977 & 10 & 0 & 3 & 3 & & 45.36 \\
\hline 1.9771 & 13 & 0 & 6 & 2 & & 45.86 \\
\hline 1.9513 & 11 & 7 & 2 & 1 & & 46.50 \\
\hline 1.9294 & 8 & 2 & 3 & 3 & + & 47.06 \\
\hline 1.9110 & 18 & 6 & 2 & 2 & + & 47.54 \\
\hline 1.8675 & 15 & 4 & 1 & 3 & + & 48.72 \\
\hline 1.8610 & 20 & 0 & 8 & 0 & & 48.90 \\
\hline 1.8302 & 7 & 6 & 5 & 1 & + & 49.78 \\
\hline 1.7769 & 11 & 4 & 7 & 1 & + & 51.38 \\
\hline 1.7603 & 11 & 0 & 5 & 3 & + & 51.90 \\
\hline 1.7553 & 7 & 6 & 6 & 0 & & 52.06 \\
\hline 1.7125 & 5 & 5 & 2 & 3 & + & 53.46 \\
\hline 1.6834 & 8 & 8 & 3 & 1 & + & 54.46 \\
\hline 1.6370 & 4 & 0 & 0 & 4 & & 56.14 \\
\hline 1. 6290 & 4 & 1 & 6 & 3 & + & 56.44 \\
\hline 1.5809 & 13 & 2 & 8 & 2 & + & 58.32 \\
\hline 1.5676 & 7 & 6 & 7 & 1 & + & 58.86 \\
\hline 1.5633 & 7 & 2 & 2 & 4 & & 59.04 \\
\hline 1.5561 & 3 & 3 & 6 & 3 & & 59.34 \\
\hline 1.5462 & 6 & 6 & 6 & 2 & & 59.76 \\
\hline 1.5341 & 6 & 5 & 8 & 1 & + & 60.28 \\
\hline 1.5231 & 1 & 3 & 7 & 3 & & 60.76 \\
\hline 1.4921 & 4 & 7 & 2 & 3 & & 62.16 \\
\hline 1.4835 & 1 & 8 & 4 & 2 & & 62.56 \\
\hline 1.4725 & 6 & 4 & 9 & 1 & + & 63.08 \\
\hline 1.4688 & 11 & 4 & 2 & 4 & + & 63.26 \\
\hline 1.4597 & 5 & 10 & 2 & 0 & + & 63.70 \\
\hline 1.4447 & 2 & 10 & 1 & 1 & + & 64.44 \\
\hline
\end{tabular}




\begin{tabular}{|c|c|c|c|c|c|c|}
\hline \multicolumn{7}{|c|}{ Calculated Pattern (Peak heights) } \\
\hline$d(\AA)$ & $I$ & & $h k l$ & & & $\begin{array}{c}2 \theta\left({ }^{\circ}\right) \\
\lambda=1.54056 \mathrm{~A}\end{array}$ \\
\hline 1.4356 & 2 & 5 & 5 & 3 & + & 54.90 \\
\hline 1.4094 & 3 & 4 & 7 & 3 & + & 65.26 \\
\hline 1.3930 & 2 & 10 & 3 & 1 & & $67 \cdot 14$ \\
\hline 1.3821 & 3 & 4 & 10 & 0 & + & 67.74 \\
\hline 1.3693 & 1 & 7 & 8 & 1 & + & 58.46 \\
\hline 1.3617 & 4 & 8 & 3 & 3 & + & 58.90 \\
\hline 1.3551 & 10 & 0 & 10 & 2 & + & 69.28 \\
\hline 1.3466 & 2 & 6 & 9 & 1 & + & 59.78 \\
\hline 1.3436 & 4 & 2 & 6 & 4 & + & 59.96 \\
\hline 1.3330 & 2 & 10 & 2 & 2 & + & 73.50 \\
\hline 1.3252 & 1 & 0 & 11 & 1 & & 71.08 \\
\hline 1.3155 & 3 & 8 & 8 & 0 & & 71.68 \\
\hline 1.3045 & 3 & 2 & 11 & 1 & + & 72.38 \\
\hline 1.2980 & 3 & 6 & 7 & 3 & + & 72.80 \\
\hline 1.2847 & 2 & 2 & 1 & 5 & & 73.68 \\
\hline 1.2825 & 5 & 5 & 4 & 4 & + & 73.82 \\
\hline 1.2787 & 5 & 8 & 5 & 3 & + & 74.08 \\
\hline 1.2764 & 3 & 10 & 5 & 0 & + & 74.24 \\
\hline 1.2732 & 2 & 4 & 10 & 2 & & 74.46 \\
\hline 1.2662 & 1 & 0 & 3 & 5 & & 74.94 \\
\hline 1.2484 & 3 & 2 & 3 & 5 & + & 75.20 \\
\hline 1.2426 & 3 & 4 & 9 & 3 & + & 76.62 \\
\hline 1.2303 & 4 & 1 & 4 & 5 & + & 77.48 \\
\hline 1.2290 & 6 & 0 & 8 & 4 & & 77.62 \\
\hline 1.2256 & 4 & 10 & 1 & 3 & & 77.88 \\
\hline 1.2235 & 3 & 12 & 2 & 0 & + & 78.04 \\
\hline 1.2146 & 2 & 12 & 1 & 1 & + & 78.72 \\
\hline 1.1986 & 3 & 7 & 10 & 1 & + & 79.98 \\
\hline 1.1959 & 2. & 5 & 6 & 4 & & 80.20 \\
\hline 1.1936 & 2 & 10 & 3 & 3 & & 80.38 \\
\hline 1.1892 & 2 & 6 & 10 & 2 & + & 80.74 \\
\hline 1.1834 & 2 & 5 & 2 & 5 & + & 81.22 \\
\hline 1.1786 & 1 & 7 & 8 & 3 & & 81.62 \\
\hline 1.1689 & 1 & 11 & 5 & 1 & & 82.44 \\
\hline 1.1641 & 1 & 9 & 5 & 3 & + & 82.86 \\
\hline 1.1545 & 1 & 1 & 5 & 5 & & 83.70 \\
\hline 1.1365 & 2 & 2 & 11 & 3 & + & 85.34 \\
\hline 1.1278 & 2 & 3 & 5 & 5 & + & 86.16 \\
\hline 1.1151 & 2 & 2 & 13 & 1 & + & 87.38 \\
\hline 1.1095 & 3 & 5 & 12 & 0 & + & 87.94 \\
\hline 1.1075 & 7 & 4 & 12 & 2 & + & 88.14 \\
\hline 1.1027 & 1 & 7 & 2 & 5 & & 88.62 \\
\hline 1.0988 & 2 & 11 & 4 & 3 & + & 89.02 \\
\hline 1.0953 & 5 & 10 & 8 & 2 & + & 89.38 \\
\hline 1.0909 & 1 & 10 & 9 & 1 & + & 89.84 \\
\hline 1.0893 & 3 & 2 & 10 & 4 & + & 90.00 \\
\hline 1.0794 & 3 & 0 & 2 & 5 & + & 91.06 \\
\hline 1.0757 & 2 & 1 & 12 & 3 & + & 91.46 \\
\hline 1. 0581 & 2 & 2 & 2 & 6 & + & 92.30 \\
\hline 1.0646 & 1 & 7 & 10 & 3 & & 92.70 \\
\hline
\end{tabular}

\begin{tabular}{|c|c|c|c|c|c|}
\hline \multicolumn{6}{|c|}{ Calculated Pattern (Integrated) } \\
\hline$d(\stackrel{\circ}{A})$ & $I$ & & $h k l$ & & $\begin{array}{c}2 \theta\left(^{\circ}\right) \\
\lambda=1.54056 \AA\end{array}$ \\
\hline 7.44 & 1 & 0 & 2 & 0 & 11.88 \\
\hline 5.93 & 27 & 0 & 1 & 1 & 14.77 \\
\hline 5.25 & 100 & 2 & 2 & 0 & 16.83 \\
\hline 4.67 & 91 & 2 & 1 & 1 & 19.30 \\
\hline 4.67 & 25 & 1 & 2 & 1 & 19.00 \\
\hline 3.95 & 53 & 0 & 3 & 1 & 22.47 \\
\hline 3.492 & 3 & 3 & 2 & 1 & 25.49 \\
\hline 3.432 & 62 & 2 & 3 & 1 & 25.49 \\
\hline 3.328 & 46 & 4 & 2 & 0 & 25.76 \\
\hline 3.328 & 37 & 2 & 4 & 0 & 26.76 \\
\hline 3.151 & 31 & 4 & 1 & 1 & 28.20 \\
\hline 3.151 & 31 & 1 & 4 & 1 & 28.20 \\
\hline 2.995 & 71 & 0 & 2 & 2 & 29.79 \\
\hline 2.780 & 25 & 2 & 2 & 2 & 32.18 \\
\hline 2.710 & 3 & 4 & 3 & 1 & 33.33 \\
\hline 2.710 & 3 & 3 & 4 & 1 & 33.03 \\
\hline 2.710 & 23 & כ & 5 & 1 & 33.03 \\
\hline 2.545 & 11 & 5 & 2 & 1 & 35.21 \\
\hline 2.546 & 4 & 2 & 5 & $i$ & 35.21 \\
\hline 2.481 & 2 & 0 & 6 & 0 & 36.18 \\
\hline 2.354 & 7 & 6 & 2 & 0 & 38.21 \\
\hline 2.354 & 6 & 2 & 6 & 0 & 38.21 \\
\hline 2.334 & 3 & 4 & 2 & 2 & 38.54 \\
\hline 2.292 & 3 & 6 & 1 & 1 & 39.27 \\
\hline 2.292 & 11 & 1 & 6 & 1 & 39.27 \\
\hline 2.179 & 2 & 5 & 1 & 2 & 41.41 \\
\hline 2.102 & 1 & 5 & 3 & 1 & 43.00 \\
\hline 2.102 & 23 & 3 & 5 & 1 & 43.00 \\
\hline 2.074 & 1 & 1 & 2 & 3 & 43.51 \\
\hline 2.074 & 13 & 2 & 1 & 3 & 43.51 \\
\hline 2.054 & 22 & 6 & 4 & 0 & 43.82 \\
\hline 2.054 & 21 & 4 & 6 & 0 & 43.82 \\
\hline 2.051 & 70 & 4 & 4 & 2 & 44.12 \\
\hline 2.022 & 2 & 0 & 7 & 1 & 44.78 \\
\hline 1.9976 & 15 & 0 & 3 & 3 & 45.36 \\
\hline 1.9772 & 19 & 0 & 6 & 2 & 45.86 \\
\hline 1.9516 & 17 & 7 & 2 & 1 & 45.49 \\
\hline 1.9293 & 1 & 3 & 2 & 3 & 47.36 \\
\hline 1.9293 & 11 & 2 & 3 & 3 & 47.06 \\
\hline 1.9109 & 15 & 6 & 2 & 2 & 47.54 \\
\hline i & & & & & \\
\hline 1.9109 & 13 & 2 & 6 & 2 & 47.54 \\
\hline 1.8675 & 12 & 4 & 1 & 3 & 48.72 \\
\hline 1.8675 & 10 & 1 & 4 & 3 & 48.72 \\
\hline 1.8505 & 29 & 0 & 8 & 0 & 48.91 \\
\hline 1.8299 & 10 & 6 & 5 & 1 & 49.79 \\
\hline 1.8299 & 1 & 5 & 5 & 1 & 49.79 \\
\hline 1.7763 & 1 & 8 & 1 & 1 & 51.38 \\
\hline 1.7759 & 7 & 7 & 4 & 1 & 51.38 \\
\hline 1.776 .9 & 8 & 4 & 7 & $i$ & 51.38 \\
\hline 1.7759 & 2 & 1 & 8 & $i$ & $51 \cdot 38$ \\
\hline
\end{tabular}




\begin{tabular}{|c|c|c|c|c|c|}
\hline \multicolumn{6}{|c|}{ Calculated Pattern (Integrated) } \\
\hline$d(\stackrel{\circ}{A})$ & $I$ & & $h k l$ & & $\begin{array}{c}2 \theta\left(^{\circ}\right) \\
\lambda=1.54056 \AA\end{array}$ \\
\hline 1.7501 & 3 & 4 & 3 & 3 & 51.91 \\
\hline 1.7501 & 4 & 3 & 4 & 3 & 51.91 \\
\hline 1.7601 & 13 & 0 & 5 & 3 & 51.91 \\
\hline 1.7542 & 1 & 6 & 6 & 0 & 52.39 \\
\hline 1.7128 & 5 & 5 & 2 & 3 & 53.45 \\
\hline 1.7128 & 3 & 2 & 5 & 3 & 53.45 \\
\hline 1.6535 & 7 & 8 & 3 & 1 & 54.46 \\
\hline 1.6836 & 6 & 3 & 8 & 1 & 54.46 \\
\hline 1.6367 & 7 & 0 & 0 & 4 & 56.15 \\
\hline 1.6287 & 2 & 6 & 1 & 3 & $56 .+5$ \\
\hline 1.6287 & 5 & 1 & 6 & 3 & 56.45 \\
\hline 1.5807 & 8 & 8 & 2 & 2 & 58.33 \\
\hline 1.5807 & 16 & 2 & 8 & 2 & 58.33 \\
\hline 1.5575 & 3 & 9 & 2 & 1 & 58.86 \\
\hline 1.5575 & 6 & 6 & 7 & 1 & 58.86 \\
\hline 1.5575 & 2 & 2 & 9 & 1 & 58.86 \\
\hline 1.5529 & 6 & 2 & 2 & 4 & 59.36 \\
\hline 1.5559 & 5 & 3 & 6 & 3 & 59.35 \\
\hline 1.5462 & 10 & 6 & 5 & 2 & 59.76 \\
\hline 1.5339 & 5 & 8 & 5 & 1 & 60.29 \\
\hline 1.5339 & 6 & 5 & 8 & 1 & 60.29 \\
\hline 1.5230 & 1 & 0 & 7 & 3 & 60.76 \\
\hline 1.4921 & 6 & 7 & 2 & 3 & 62.16 \\
\hline 1.4835 & 1 & 8 & 4 & 2 & 62.56 \\
\hline 1.4726 & 4 & 9 & 4 & 1 & 63.08 \\
\hline 1.4725 & 5 & 4 & 9 & 1 & 63.08 \\
\hline 1.4688 & 6 & 2 & 4 & 4 & 63.26 \\
\hline 1.4688 & 9 & 4 & 2 & 4 & 63.26 \\
\hline 1.4596 & 5 & 10 & 2 & 0 & 63.71 \\
\hline 1.4596 & 4 & 2 & 10 & 0 & 63.71 \\
\hline 1.4445 & 4 & 10 & 1 & 1 & 64.45 \\
\hline 1.4440 & 1 & 1 & 10 & 1 & 64.45 \\
\hline 1.4355 & 2 & 6 & 5 & 3 & 64.90 \\
\hline 1.4355 & 2 & 5 & 6 & 3 & 64.90 \\
\hline 1.4095 & 3 & 7 & 4 & 3 & 66.25 \\
\hline 1.4095 & 3 & 4 & 7 & 3 & 66.25 \\
\hline 1.3931 & 4 & 10 & 3 & 1 & 67.14 \\
\hline 1.3820 & 2 & 10 & 4 & 0 & 67.75 \\
\hline 1.3820 & 3 & 4 & 10 & 0 & 67.75 \\
\hline 1.3693 & 1 & 8 & 7 & 1 & $68 .+6$ \\
\hline 1.3693 & 1 & 7 & 8 & 1 & 68.46 \\
\hline 1.3515 & 3 & 8 & 3 & 3 & 68.91 \\
\hline 1.3515 & 3 & 3 & 8 & 3 & 68.91 \\
\hline 1.3550 & 7 & 8 & 6 & 2 & 69.29 \\
\hline 1.3550 & 4 & 6 & 8 & 2 & 69.29 \\
\hline 1.3550 & 10 & 0 & 10 & 2 & 69.29 \\
\hline 1.3467 & 1 & 9 & 6 & 1 & 69.78 \\
\hline 1. 3467 & 2 & 6 & 9 & 1 & 69.78 \\
\hline $\begin{array}{l}1.3438 \\
1.3438\end{array}$ & $\begin{array}{l}3 \\
4\end{array}$ & $\begin{array}{l}6 \\
2\end{array}$ & $\begin{array}{l}2 \\
6\end{array}$ & $\begin{array}{l}4 \\
4\end{array}$ & $\begin{array}{l}69.95 \\
69.95\end{array}$ \\
\hline
\end{tabular}

\begin{tabular}{|c|c|c|c|c|c|}
\hline \multicolumn{6}{|c|}{ Calculated Pattern (Integrated) } \\
\hline$d(\stackrel{\circ}{A})$ & $I$ & & $h k l$ & & $\begin{array}{c}2 \theta\left(^{\circ}\right) \\
\lambda=1.54056 \AA\end{array}$ \\
\hline 1.3331 & 2 & 10 & 2 & 2 & 70.59 \\
\hline 1.3331 & 1 & 2 & 10 & 2 & 70.59 \\
\hline 1.3252 & 2 & 0 & 11 & 1 & 71.08 \\
\hline 1.3157 & 7 & 8 & 8 & 0 & 71.57 \\
\hline 1.3045 & 1 & 10 & 5 & 1 & 72.37 \\
\hline 1.3045 & 2 & 5 & 10 & 1 & 72.37 \\
\hline 1.3045 & 4 & 2 & 11 & 1 & 72.37 \\
\hline 1.2979 & 1 & 9 & 2 & 3 & 72.81 \\
\hline 1.2979 & 3 & 6 & 7 & 3 & 72.81 \\
\hline 1.2979 & 2 & 2 & 9 & 3 & 72.81 \\
\hline 1.2848 & 2 & 2 & 1 & 5 & 73.57 \\
\hline 1.2825 & 7 & 6 & 4 & 4 & 73.83 \\
\hline 1.2825 & 4 & 4 & 6 & 4 & 73.83 \\
\hline 1.2786 & 4 & 8 & 5 & 3 & 74.39 \\
\hline 1.2786 & 3 & 5 & 8 & 3 & 74.09 \\
\hline 1.2764 & 2 & 10 & 6 & 0 & 74.24 \\
\hline 1.2764 & 1 & 6 & 10 & 0 & 74.24 \\
\hline 1.2732 & 1 & 4 & 10 & 2 & 74.46 \\
\hline 1.2660 & 2 & 0 & 3 & 5 & 74.95 \\
\hline 1.2484 & 1 & 11 & 4 & 1 & 76.20 \\
\hline 1.2484 & 1 & 4 & 11 & 1 & 76.20 \\
\hline 1.2481 & 3 & 2 & 3 & 5 & 75.22 \\
\hline 1.2425 & 3 & 9 & 4 & 3 & 76.53 \\
\hline 1.2425 & 3 & 4 & 9 & 3 & 76.53 \\
\hline 1.2309 & 3 & 4 & 1 & 5 & $77 .+8$ \\
\hline 1.2309 & 3 & 1 & 4 & 5 & $77 .+8$ \\
\hline 1.2289 & 11 & 0 & 8 & 4 & 77.53 \\
\hline 1.2255 & 3 & 10 & 1 & 3 & 77.38 \\
\hline 1.2235 & 3 & 12 & 2 & 3 & 78.33 \\
\hline 1.2235 & 1 & 2 & 12 & 0 & 78.33 \\
\hline 1.2147 & 3 & 12 & 1 & 1 & 78.71 \\
\hline 1.2147 & 2 & 1 & 12 & 1 & 78.71 \\
\hline 1.1988 & 3 & 7 & 10 & 1 & 79.96 \\
\hline 1.1986 & 2 & 0 & 5 & 5 & 79.98 \\
\hline 1.1967 & 1 & 6 & 6 & 4 & 80.13 \\
\hline 1.1935 & 2 & 10 & 3 & 3 & 80.38 \\
\hline 1.1892 & 1 & 10 & 6 & 2 & 83.74 \\
\hline 1.1892 & 3 & 6 & 10 & 2 & 80.74 \\
\hline 1.1835 & 1 & 12 & 3 & 1 & 81.21 \\
\hline 1.1833 & 1 & 2 & 5 & 5 & 81.22 \\
\hline 1.1833 & 2 & 5 & 2 & 5 & 81.22 \\
\hline 1.1785 & 1 & 7 & 8 & 3 & 81.63 \\
\hline 1.1689 & 3 & 11 & 6 & 1 & 82.45 \\
\hline 1.1540 & 1 & 3 & 6 & 3 & 82.87 \\
\hline 1.1540 & 1 & 6 & 9 & 3 & 82.57 \\
\hline i. 1545 & 1 & 1 & 6 & 5 & 83.70 \\
\hline 1.1500 & 1 & 0 & 11 & 3 & 84.10 \\
\hline 1.1365 & 1 & 10 & 5 & 3 & 85.33 \\
\hline 1.1365 & 1 & 5 & 10 & 3 & 85.33 \\
\hline 1.1365 & 2 & 2 & 11 & 3 & 85.33 \\
\hline
\end{tabular}


Lithium Uranium Fluoride, LiUF (tetragonal) - continued

\begin{tabular}{|c|c|c|c|c|c|}
\hline \multicolumn{6}{|c|}{ Calculated Pattern (Integrated) } \\
\hline$d(\AA)$ & $I$ & & $h k l$ & & $\begin{array}{c}2 \theta\left({ }^{\circ}\right) \\
\lambda=1.54056 \AA\end{array}$ \\
\hline 1.1279 & 2 & 0 & 13 & 1 & 86.15 \\
\hline 1.1277 & 2 & 3 & 6 & 5 & 85.17 \\
\hline 1.1151 & 1 & 2 & 13 & 1 & 87.38 \\
\hline 1.1150 & 1 & 0 & 7 & 5 & 87.40 \\
\hline 1.1095 & 2 & 12 & 6 & 0 & 87.94 \\
\hline 1.1095 & 3 & 6 & 12 & 0 & 87.94 \\
\hline 1.1074 & 8 & 12 & 4 & 2 & 88.15 \\
\hline 1.1074 & 8 & 4 & 12 & 2 & 88.15 \\
\hline 1.1027 & 2 & 7 & 2 & 5 & 88.62 \\
\hline 1.0988 & 1 & 11 & 4 & 3 & 89.32 \\
\hline 1.0988 & 1 & 4 & 11 & 3 & 89.32 \\
\hline 1.0953 & 7 & 10 & 8 & 2 & 89.38 \\
\hline 1.0953 & 4 & 8 & 10 & 2 & 89.38 \\
\hline 1.0909 & 1 & 10 & 9 & 1 & 89.33 \\
\hline 1.0909 & 1 & 9 & 10 & 1 & 89.83 \\
\hline 1.0894 & 2 & 10 & 2 & 4 & 90.30 \\
\hline 1.0894 & 3 & 2 & 10 & 4 & 90.30 \\
\hline 1.0795 & 3 & 0 & 2 & 6 & 91.34 \\
\hline 1.0794 & 1 & 11 & 8 & 1 & 91.06 \\
\hline 1.0794 & 1 & 8 & 11 & 1 & 91.06 \\
\hline 1.0792 & 2 & 6 & 5 & 5 & 91.38 \\
\hline 1.0755 & 1 & 12 & 1 & 3 & 91.48 \\
\hline 1.0755 & 2 & 1 & 12 & 3 & 91,48 \\
\hline 1.0584 & 2 & 2 & 2 & 6 & 92.26 \\
\hline 1.0581 & 1 & 4 & 7 & 5 & 92.31 \\
\hline 1.0581 & 1 & 7 & 4 & 5 & 92.31 \\
\hline 1.0545 & 3 & 7 & 10 & 3 & 92.70 \\
\hline 1.0574 & 1 & 12 & 7 & 1 & 93.52 \\
\hline 1.0574 & 2 & 7 & 12 & 1 & 93.52 \\
\hline 1.0559 & 2 & 10 & 4 & 4 & 93.58 \\
\hline 1.0559 & 3 & 4 & 10 & 4 & 93.58 \\
\hline 1.0526 & 2 & 10 & 10 & 0 & 94.38 \\
\hline 1.0469 & 1 & 1 & 14 & 1 & 94.75 \\
\hline 1.0467 & 1 & 3 & 8 & 5 & 94.77 \\
\hline 1.0467 & 1 & 8 & 3 & 5 & 94.77 \\
\hline 1.0434 & 2 & 11 & 6 & 3 & 95.17 \\
\hline 1.0267 & 1 & 13 & 6 & 1 & 97.22 \\
\hline 1.0267 & 1 & 6 & 13 & 1 & 97.22 \\
\hline 1.0267 & 2 & 3 & 14 & 1 & 97.22 \\
\hline
\end{tabular}


Structure

Monoclinic, $\mathrm{P} 22_{1} / \mathrm{c}$ (14), $\mathrm{Z}=2$ [Sasvari and Jeffrey, 1966]

\section{Lattice parameters}

$a=8.59 \pm 0.05, b=14.40 \pm 0.03, c=8.75 \pm 0.05 \AA$ $\beta=129.6^{\circ} \pm 0.2^{\circ}$ [ibid.]

\section{Scattering factors}

$$
\mathrm{Mg}^{+2}, \mathrm{Cl}^{-}, \mathrm{O}^{\circ}[3.3 .1 \mathrm{~A}]
$$

Thermal parameters

Isotropic: Mg 0.86; C1 1.74; O(1) 2.12

$O(2) 2.42$; $O(3) 1.81 ; O(4) 2.42$;

$O(5) 2.03 ; O(6) 2.32$

Density

(calculated) $1.241 \mathrm{~g} / \mathrm{cm}^{3}$

\section{Scale factor}

$$
1.198 \times 10^{4}
$$

\section{Reference}

Sasvari, K. and G.A. Jeffrey (1966). The crystal structure of magnesium chloride dodecahydrate, $\mathrm{MgCl}_{2} \cdot 12 \mathrm{H}_{2} \mathrm{O}$, Acta Cryst.

\begin{tabular}{|c|c|c|c|c|c|c|}
\hline \multicolumn{7}{|c|}{ Calculated Pattern (Peak heights) } \\
\hline$d(\stackrel{\circ}{)}$ & $I$ & \multicolumn{4}{|c|}{$h k l$} & $\begin{array}{c}2 \theta\left({ }^{\circ}\right) \\
\lambda=1.54056 \AA\end{array}$ \\
\hline 7.20 & 5 & D & 2 & 0 & & 12.28 \\
\hline 6.89 & 23 & -1 & 1 & 1 & & 12.84 \\
\hline 6.61 & $\angle 0$ & 1 & 0 & 0 & & 13.38 \\
\hline $6 \cdot 10$ & 47 & 3 & 1 & 1 & & 14.50 \\
\hline 6.01 & 24 & 1 & 1 & 0 & & 14.72 \\
\hline 5.30 & 2 & -1 & 2 & 1 & & 16.70 \\
\hline 4.92 & 17 & 3 & 2 & 1 & & 18.52 \\
\hline 4.87 & 28 & 1 & 2 & 0 & & 18.20 \\
\hline 4.32 & $2 \varepsilon$ & -1 & 0 & 2 & & 20.56 \\
\hline 4.09 & 100 & -1 & 3 & 1 & & 21.70 \\
\hline 4.05 & 25 & -2 & 1 & 1 & & 21.94 \\
\hline 3.92 & 61 & -2 & 0 & 2 & + & 22.56 \\
\hline 3.89 & 17 & 1 & 3 & 0 & & 22.86 \\
\hline 3.78 & 4 & -2 & 1 & 2 & & 23.50 \\
\hline 3.70 & 17 & -1 & 2 & 2 & & 24.02 \\
\hline 3.64 & 33 & -2 & 2 & 1 & & 24.44 \\
\hline 3.60 & 8 & 0 & 4 & 0 & & 24.72 \\
\hline 3.58 & 50 & 1 & 1 & 1 & & 24.88 \\
\hline 3.44 & 46 & -2 & 2 & 2 & & 25.86 \\
\hline 3.37 & 30 & 0 & 0 & 2 & & 20.42 \\
\hline 3.31 & 18 & ? & 0 & 0 & & 26.92 \\
\hline 3.28 & 51 & 1 & 2 & 1 & + & 27.14 \\
\hline 3.27 & 52 & -1 & 4 & 1 & & 27.22 \\
\hline 3.22 & 4 & 2 & 1 & 0 & & 27.64 \\
\hline 3.21 & 9 & -1 & 3 & 2 & & 27.78 \\
\hline 3.17 & 42 & -2 & 3 & 1 & + & 28.14 \\
\hline 3.05 & 21 & 0 & 2 & 2 & & 29.22 \\
\hline 3.04 & 21 & $-\hat{2}$ & 3 & 2 & & 29.32 \\
\hline 2.927 & 14 & 1 & 3 & 1 & & 30.52 \\
\hline 2.855 & 10 & -2 & 1 & 3 & & 31.30 \\
\hline 2.808 & 6 & -3 & 1 & 2 & & 31.84 \\
\hline 2.764 & 9 & -1 & 4 & 2 & & 32.36 \\
\hline 2.738 & 4 & -2 & 4 & 1 & & 32.58 \\
\hline 2.725 & 3 & 2 & 3 & 0 & & 32.84 \\
\hline 2.703 & 10 & -1 & 5 & 1 & + & 33.12 \\
\hline 2.673 & 2 & -1 & 1 & 3 & & 33.50 \\
\hline 2.600 & 9 & -3 & 2 & 2 & & 33.56 \\
\hline 2.653 & 6 & 0 & 5 & 1 & & 33.76 \\
\hline 2.512 & 1 & -3 & 1 & 1 & & 34.30 \\
\hline 2. 577 & 1 & 1 & 4 & 1 & & 34.78 \\
\hline 2.545 & 6 & -1 & 2 & 3 & & 35.24 \\
\hline 2.491 & 1 & -3 & 2 & 1 & & 36.02 \\
\hline 2.458 & 28 & -3 & 3 & 2 & + & 36.52 \\
\hline 2.400 & 13 & 0 & 6 & 0 & & 37.44 \\
\hline 2.395 & 16 & -1 & 5 & 2 & & 37.52 \\
\hline 2.367 & 5 & -1 & 3 & 3 & & 37.98 \\
\hline 2.324 & $\angle 1$ & -3 & 3 & 1 & + & 38.72 \\
\hline 2.295 & 48 & -3 & 3 & 3 & + & 39.22 \\
\hline $\begin{array}{l}2.271 \\
2.261\end{array}$ & $\begin{array}{l}23 \\
29\end{array}$ & 1 & 5 & 1 & + & $\begin{array}{l}39.66 \\
3 y .84\end{array}$ \\
\hline 2.261 & $\angle y$ & E & 6 & 1 & 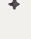 & \\
\hline
\end{tabular}
20, 875-881. 


\begin{tabular}{|c|c|c|c|c|c|c|}
\hline \multicolumn{7}{|c|}{ Calculated Pattern (Peak heights) } \\
\hline$d(\stackrel{\circ}{A})$ & $I$ & & $h k l$ & & & $2 \theta\left({ }^{\circ}\right)$ \\
\hline 2.220 & 18 & 3 & 1 & 3 & & 40.50 \\
\hline 2.207 & 2 & 3 & 0 & 0 & & 40.86 \\
\hline 2.180 & 17 & 3 & 1 & 0 & & $41 \cdot 38$ \\
\hline 2.175 & 30 & 1 & 3 & 2 & + & 41.48 \\
\hline 2.162 & 8 & 2 & 3 & 1 & & 41.74 \\
\hline 2.158 & 15 & -2 & 0 & 4 & + & 41.82 \\
\hline 2.146 & 11 & 3 & 2 & 3 & & 42.03 \\
\hline 2.138 & 15 & -3 & 4 & 1 & + & 42.24 \\
\hline 2.115 & 4 & -3 & 4 & 3 & & 42.72 \\
\hline 2.113 & 5 & 3 & 2 & 0 & + & 42.82 \\
\hline 2.097 & $E$ & -1 & $\delta$ & 2 & & 43.10 \\
\hline 2.087 & 12 & -4 & 1 & 2 & + & 43.32 \\
\hline 2. DE 7 & 19 & $-\bar{z}$ & 2 & 4 & + & 43.76 \\
\hline 2.047 & 8 & -2 & 6 & 2 & + & 44.20 \\
\hline 2.042 & 7 & -4 & 2 & 3 & & 44.32 \\
\hline 2.036 & 4 & 0 & 3 & 3 & & 44.46 \\
\hline 2.025 & 9 & -4 & 2 & 2 & & 44.72 \\
\hline 2.023 & 7 & 1 & 4 & 2 & & 44.84 \\
\hline 2.004 & 2 & 3 & 3 & 0 & & $45.2 \mathrm{~J}$ \\
\hline 1.9779 & 4 & -1 & 5 & 3 & & 45.84 \\
\hline 1.9641 & 3 & 1 & 7 & 0 & & 40.18 \\
\hline 1.9601 & 4 & -4 & 0 & 4 & & 46.28 \\
\hline 1.9529 & 7 & -3 & 5 & 1 & + & 45.46 \\
\hline 1.9474 & 6 & -4 & 3 & 3 & & 40.60 \\
\hline $1.941=$ & 4 & -1 & 1 & 4 & & 46.74 \\
\hline 1.9317 & 3 & -4 & 3 & 2 & & 47.00 \\
\hline 1.8975 & 1 & -4 & 1 & 1 & & 47.90 \\
\hline 1.8901 & 1 & -1 & 2 & 4 & & 48.10 \\
\hline 1.8813 & 1 & 3 & 4 & 0 & & 46.34 \\
\hline 1.8617 & 3 & 1 & 5 & 2 & & $48 \cdot 88$ \\
\hline 1.8518 & 19 & $-\tilde{z}$ & $\sigma$ & 3 & + & 49.15 \\
\hline 1.8469 & 13 & 2 & U & 2 & & 49.30 \\
\hline 1.8309 & 3 & 2 & 1 & 2 & & 49.75 \\
\hline 1. $\hat{\sigma}<05$ & 4 & -4 & 4 & 2 & + & 50.06 \\
\hline i. 7971 & 3 & 1 & 7 & 1 & & 50.76 \\
\hline 1.7879 & 1 & 2 & 2 & 2 & & 51.04 \\
\hline 1.7717 & 5 & 0 & 5 & 3 & + & 51.54 \\
\hline $1.754 E$ & 1 & -1 & 8 & 1 & & 52.08 \\
\hline 1.7391 & 2 & 0 & 8 & 1 & & 52.58 \\
\hline 1.7367 & 2 & 1 & $B$ & 0 & & 52.66 \\
\hline 1.7209 & 5 & -1 & 4 & 4 & + & 33.18 \\
\hline 1.7025 & 2 & -5 & 1 & 3 & & 53.80 \\
\hline 1.6903 & 6 & 1 & 3 & 3 & + & 54.22 \\
\hline 1.6863 & 5 & -5 & J & 4 & + & $54 \cdot 36$ \\
\hline 1.6806 & 2 & -2 & 7 & 3 & & 54.56 \\
\hline 1.6755 & 5 & -5 & 1 & 4 & + & 54.74 \\
\hline 1.6710 & 4 & -3 & 7 & 2 & & 54.90 \\
\hline 1.6615 & $?$ & -1 & 8 & 2 & & 55.24 \\
\hline 1.6549 & 3 & 4 & i & 0 & & 55.48 \\
\hline 1.6429 & 5 & -5 & 2 & 4 & + & 55.92 \\
\hline
\end{tabular}

\begin{tabular}{|c|c|c|c|c|c|c|}
\hline \multicolumn{7}{|c|}{ Calculated Pattern (Peak heights) } \\
\hline$d(\AA)$ & $I$ & & $h k l$ & & & $\begin{array}{c}2 \theta\left(^{\circ}\right) \\
\lambda=1.54056 \AA\end{array}$ \\
\hline $1.64 \mathrm{C2}$ & 4 & 0 & 6 & 3 & & 56.02 \\
\hline $1.635 y$ & 3 & -2 & 8 & 2 & & 56.18 \\
\hline 1.6269 & 3 & -5 & 1 & 2 & & 50.52 \\
\hline 1.6232 & 2 & 3 & 6 & 0 & & 55.66 \\
\hline 1.6164 & 2 & -3 & 7 & 3 & + & $55 \cdot 92$ \\
\hline 1.6071 & 1 & -4 & 3 & 5 & & 57.28 \\
\hline 1.5878 & 3 & 0 & 8 & 2 & & 58.04 \\
\hline 1.5814 & 7 & 2 & 5 & 0 & & 58.30 \\
\hline 1.5730 & 2 & -3 & 4 & 5 & & 58.54 \\
\hline 1.5681 & 3 & 2 & 7 & 1 & + & 58.84 \\
\hline 1.5594 & 1 & -5 & 1 & 5 & & 59.20 \\
\hline $1.555 \mathrm{c}$ & 2 & 1 & 9 & 0 & & 59.38 \\
\hline 1.5410 & 2 & -4 & 4 & 5 & & 59.98 \\
\hline 1.5291 & 2 & 1 & 5 & 3 & & 60.46 \\
\hline 1.5276 & 2 & -5 & 4 & 4 & + & 60.56 \\
\hline 1.5186 & 4 & -4 & 5 & 4 & + & 50.96 \\
\hline 1.5343 & 2 & 3 & 7 & 0 & & 61.60 \\
\hline 1.5304 & 2 & -1 & 9 & 2 & & 61.78 \\
\hline 1.4951 & 3 & -3 & 5 & 5 & + & 62.02 \\
\hline 1.4912 & 5 & -5 & 3 & 5 & + & 62.20 \\
\hline 1.4831 & 2 & -5 & 1 & 1 & & 62.58 \\
\hline 1.4797 & 2 & -4 & 7 & 3 & & 62.74 \\
\hline 1.4734 & 4 & -5 & 5 & 3 & & 63.04 \\
\hline 1.4683 & 3 & 1 & 9 & 1 & + & 63.30 \\
\hline 1.4634 & 2 & 2 & 6 & 2 & & 63.52 \\
\hline 1.4593 & 2 & 3 & 1 & 2 & & 63.72 \\
\hline 1.4536 & 5 & -1 & 3 & 5 & & 64.00 \\
\hline 1.4435 & 1 & 2 & 2 & 3 & & 64.50 \\
\hline 1.4379 & 1 & -5 & 4 & 5 & & 64.78 \\
\hline 1.4274 & 2 & -4 & 2 & 6 & & 65.32 \\
\hline 1.4231 & 4 & 1 & 1 & 4 & + & 65.54 \\
\hline 1.4189 & 3 & -3 & 7 & 4 & & 65.76 \\
\hline 1.4105 & 1 & -3 & 2 & 6 & & 60.20 \\
\hline 1.4052 & 4 & 4 & 1 & 1 & & 65.48 \\
\hline 1.4015 & 4 & -4 & 7 & 1 & + & 66.58 \\
\hline 1.3967 & 2 & -3 & ૭ & 2 & & 66.94 \\
\hline 1. 3949 & 2 & 3 & 0 & 0 & & 67.04 \\
\hline 1.3857 & 1 & 4 & 2 & 1 & & 67.54 \\
\hline 1. 3821 & 3 & -2 & 8 & 4 & & 67.74 \\
\hline 1.3780 & 2 & -5 & 5 & 5 & + & 67.94 \\
\hline 1.3746 & 2 & -2 & 6 & 5 & & 68.16 \\
\hline 1. 3693 & 2 & -4 & 3 & 2 & & 68.46 \\
\hline 1.3655 & 1 & -3 & 9 & 3 & & $6 \tilde{0} \cdot \overline{8} 8$ \\
\hline
\end{tabular}




\begin{tabular}{|c|c|c|c|c|c|}
\hline \multicolumn{6}{|c|}{ Calculated Pattern (Integrated) } \\
\hline$d(\AA)$ & $I$ & & $h k$ & & $2 \theta\left(^{\circ}\right)$ \\
\hline 7.20 & 4 & 0 & 2 & 0 & 12.28 \\
\hline 6.89 & 20 & -1 & 1 & 1 & 12.84 \\
\hline 6.62 & 17 & 1 & 0 & 0 & 13.37 \\
\hline 6.11 & 40 & 0 & 1 & 1 & 14.49 \\
\hline 6.01 & 28 & 1 & 1 & 0 & 14.72 \\
\hline 5.30 & 2 & -1 & 2 & 1 & 16.70 \\
\hline 4.92 & 15 & 0 & 2 & 1 & 18.01 \\
\hline 4.87 & 26 & 1 & 2 & 0 & 18.19 \\
\hline 4.32 & 25 & -1 & 0 & 2 & 20.56 \\
\hline 4.13 & 2 & -1 & 1 & 2 & 21.48 \\
\hline 4.09 & 100 & -1 & 3 & 1 & 21.59 \\
\hline 4.05 & 22 & -2 & 1 & 1 & 21.93 \\
\hline 3.92 & 56 & -2 & 0 & 2 & 22.66 \\
\hline 3. 91 & 11 & 0 & 3 & 1 & 22.72 \\
\hline 3.89 & 14 & 1 & 3 & 0 & 22.87 \\
\hline 3.78 & 3 & -2 & 1 & 2 & 23.49 \\
\hline 3.70 & 17 & -1 & 2 & 2 & 24.02 \\
\hline 3.64 & 33 & -2 & 2 & 1 & 24.43 \\
\hline 3.60 & 5 & 0 & 4 & 0 & 24.71 \\
\hline 3.58 & 50 & 1 & 1 & 1 & $24 \cdot 88$ \\
\hline 3.44 & 49 & -2 & 2 & 2 & 25.85 \\
\hline 3.37 & 30 & 0 & 0 & 2 & 26.42 \\
\hline 3.31 & 16 & 2 & 0 & 0 & 26.92 \\
\hline 3.28 & 44 & 1 & 2 & 1 & 27.13 \\
\hline 3.28 & 8 & 0 & 1 & 2 & 27.15 \\
\hline 3.27 & 36 & -1 & 4 & 1 & 27.23 \\
\hline 3.23 & 2 & 2 & 1 & 0 & 27.63 \\
\hline 3.21 & 8 & -1 & 3 & 2 & 27.77 \\
\hline 3.18 & 19 & 0 & 4 & 1 & 28.08 \\
\hline 3.17 & 26 & -2 & 3 & 1 & 28.13 \\
\hline 3.16 & 18 & 1 & 4 & 0 & 28.19 \\
\hline 3.05 & 34 & 0 & 2 & 2 & 29.23 \\
\hline 3.04 & 3 & -2 & 3 & 2 & 29.39 \\
\hline 2.926 & 16 & 1 & 3 & 1 & 30.53 \\
\hline 2.863 & 1 & -3 & 0 & 2 & 31.22 \\
\hline 2.855 & 10 & -2 & 1 & 3 & 31.31 \\
\hline 2.808 & 6 & -3 & 1 & 2 & 31.85 \\
\hline 2.764 & 9 & -1 & 4 & 2 & 32.36 \\
\hline 2.739 & 4 & -2 & 4 & 1 & 32.67 \\
\hline $2 \cdot 725$ & 3 & 2 & 3 & 0 & $32 \cdot 84$ \\
\hline 2.703 & 9 & -1 & 5 & 1 & 33.11 \\
\hline 2.750 & 3 & -2 & 2 & 3 & 33.15 \\
\hline 2.673 & 1 & -1 & 1 & 3 & 33.49 \\
\hline 2.660 & 10 & -3 & 2 & 2 & 33.66 \\
\hline 2.648 & 2 & 0 & 5 & 1 & 33.82 \\
\hline 2.512 & 2 & -3 & 1 & 1 & 34.30 \\
\hline 2.577 & 1 & 1 & 4 & 1 & 34.78 \\
\hline 2.545 & 7 & -1 & 2 & 3 & 35.24 \\
\hline 2.492 & 1 & -3 & 2 & 1 & 36.01 \\
\hline 2.461 & 1 & 0 & 4 & 2 & 36.49 \\
\hline
\end{tabular}

\begin{tabular}{|c|c|c|c|c|c|}
\hline \multicolumn{6}{|c|}{ Calculated Pattern (Integrated) } \\
\hline$d(\stackrel{\circ}{A})$ & $I$ & & $h k l$ & & $\begin{array}{c}2 \theta\left(^{\circ}\right) \\
\lambda=1.51056 \AA\end{array}$ \\
\hline 2.459 & 24 & -3 & 3 & 2 & 36.52 \\
\hline 2.457 & 9 & -3 & 2 & 3 & 36.54 \\
\hline 2.406 & 4 & 1 & 1 & 2 & 37.35 \\
\hline 2.400 & 10 & 0 & 6 & 0 & 37.44 \\
\hline 2.396 & 12 & -1 & 5 & 2 & 37.51 \\
\hline 2.367 & 6 & -1 & 3 & 3 & 37.99 \\
\hline 2.324 & 24 & -3 & 3 & 1 & 38.71 \\
\hline 2.321 & 1 & -2 & 5 & 2 & 38.76 \\
\hline 2.296 & 43 & -3 & 3 & 3 & 39.21 \\
\hline 2.296 & 10 & 2 & 2 & 1 & 39.21 \\
\hline 2.295 & 6 & -1 & 6 & 1 & 39.22 \\
\hline 2.271 & 25 & 1 & 5 & 1 & 39.66 \\
\hline 2.264 & 2 & -2 & 4 & 3 & 39.78 \\
\hline 2.261 & 29 & 0 & 6 & 1 & 39.84 \\
\hline 2.220 & 21 & $\Gamma$ & 1 & 3 & 40.60 \\
\hline 2.206 & 1 & 3 & 0 & 0 & 40.67 \\
\hline 2.190 & 1 & 0 & 5 & 2 & 41.19 \\
\hline 2.181 & 17 & 3 & 1 & 3 & 41.37 \\
\hline 2.175 & 25 & 1 & 3 & 2 & 41.48 \\
\hline 2.173 & 3 & 2 & 5 & 0 & 41.53 \\
\hline 2.162 & 5 & 2 & 3 & 1 & 41.73 \\
\hline 2.159 & 2 & -3 & 0 & 4 & 41.81 \\
\hline 2.158 & 13 & -2 & 0 & 4 & 41.83 \\
\hline 2.145 & 11 & 0 & 2 & 3 & 42.09 \\
\hline 2.137 & 15 & -3 & 4 & 1 & 42.25 \\
\hline 2.135 & 2 & -3 & 1 & 4 & 42.30 \\
\hline 2.115 & 4 & -3 & 4 & 3 & 42.71 \\
\hline 2.110 & 1 & -4 & 0 & 2 & 42.83 \\
\hline 2.109 & 2 & 3 & 2 & 0 & 42.83 \\
\hline 2.157 & 1 & -4 & 1 & 3 & 42.88 \\
\hline 2.097 & 7 & -1 & 6 & 2 & 43.09 \\
\hline 2.087 & 11 & -4 & 1 & 2 & 43.31 \\
\hline 2.086 & 3 & -2 & 6 & 1 & 43.34 \\
\hline 2.068 & 7 & -3 & 2 & 4 & 43.74 \\
\hline 2.067 & 17 & -2 & 2 & 4 & 43.76 \\
\hline 2.048 & 3 & -2 & 5 & 3 & 44.19 \\
\hline 2. 047 & 6 & -2 & 6 & 2 & 44.21 \\
\hline 2.043 & 4 & -4 & 2 & 3 & 44.31 \\
\hline 2.035 & 3 & 0 & 3 & 3 & 44.48 \\
\hline 2.025 & 11 & -4 & 2 & 2 & 44.73 \\
\hline 2.020 & 2 & 1 & 4 & 2 & 44.84 \\
\hline 2.012 & 1 & 1 & 6 & 1 & 45.02 \\
\hline 2.005 & 1 & 3 & 3 & 0 & 45.19 \\
\hline 1.9776 & 5 & -1 & 5 & 3 & 45.85 \\
\hline 1.9644 & 2 & 1 & 7 & 0 & 46.17 \\
\hline 1.9607 & 3 & -4 & 0 & 4 & 46.27 \\
\hline 1.9551 & 1 & 0 & 6 & 2 & 45.41 \\
\hline 1.9525 & 7 & -3 & 5 & 1 & 46.47 \\
\hline $\begin{array}{l}1.9463 \\
1.9412\end{array}$ & $\begin{array}{l}3 \\
2\end{array}$ & $\begin{array}{l}-4 \\
-i\end{array}$ & $\begin{array}{l}3 \\
1\end{array}$ & $\begin{array}{l}3 \\
4\end{array}$ & $\begin{array}{l}46.61 \\
46.76\end{array}$ \\
\hline
\end{tabular}




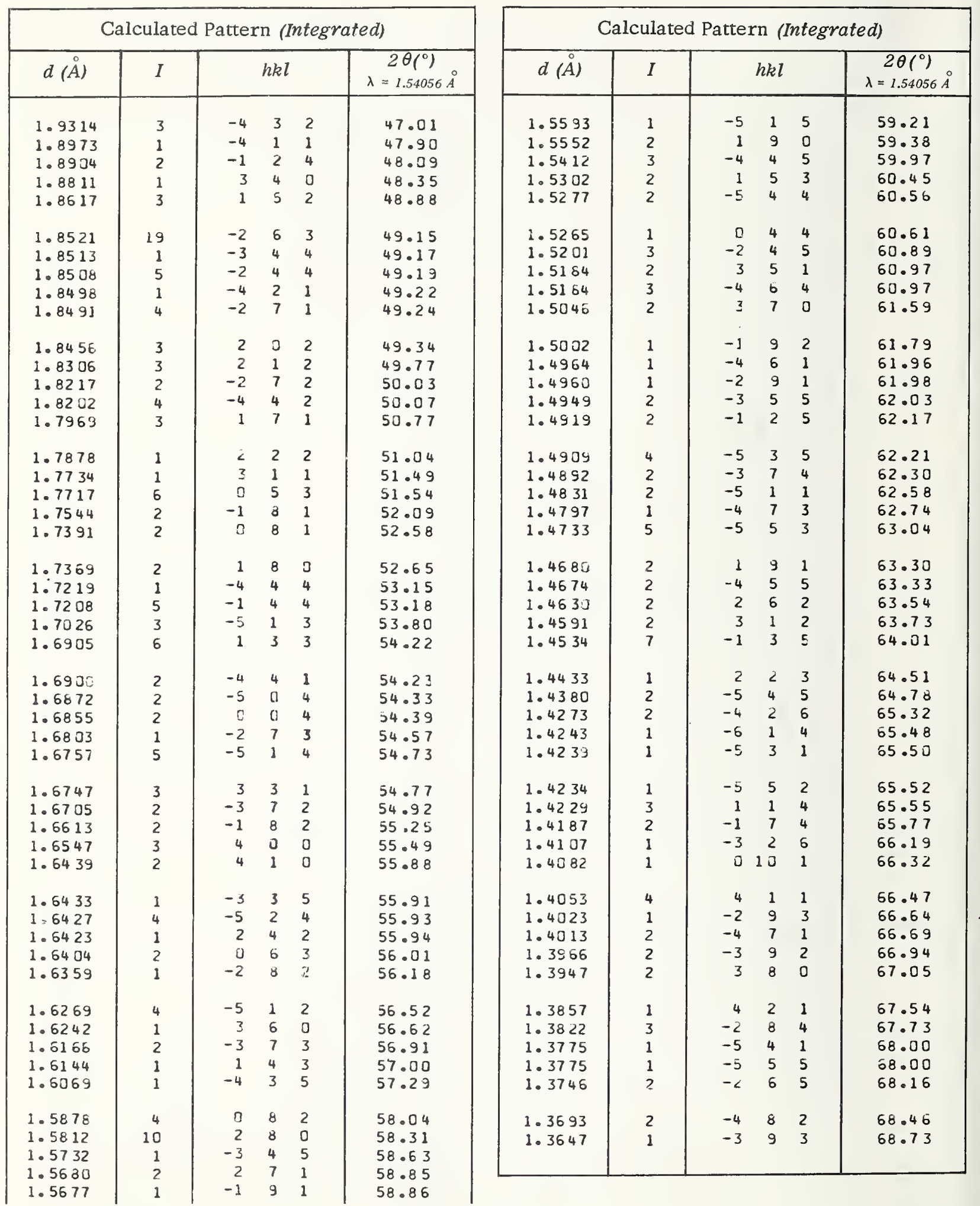


Magnesium Hydrogen Phosphate Trihydrate, newberyite, $\mathrm{MgHPO}_{4} \cdot 3 \mathrm{H}_{2} \mathrm{O}$ (orthorhombic)

\section{Structure}

Orthorhombic, Pbca (6I), $\mathrm{Z}=8$ [Sutor, 1967]

\section{Lattice parameters}

$a=10.215 \pm 0.002, b=10.681 \pm 0.002, c=10.014 \pm 0.002 \AA$ [ibid.]

\section{Scattering factors}

$\mathrm{Mg}^{0}, \mathrm{P}^{0}, \mathrm{O}^{-1} \quad[3.3 .1 \mathrm{~A}]$

Thermal parameters

Isotropic [Sutor, 1967]

\section{Density}

(calculated) $2.179 \mathrm{~g} / \mathrm{cm}^{3}$ [ibid.]

\section{Scale factor}

$$
4.210 \times 10^{4}
$$

\section{Additional patterns}

1. PDF card 19-762 [Cohen and Ribbe, 1966]

2. PDF card 19-763[Rassonskaya and Novikova, 1965]

\section{References}

Cohen, L.H. and P.H. Ribbe (1966). Magnesium phosphate mineral replacement at Mono Lake, California, Am. Mineralogist 51, 1755-1765.

Rassonskaya, N.S. and O.S. Novikova(1965). Dehydration of magnesium hydrogen phosphate crystal hydrates, Russ. J. Inorg. Chem. 10, 774-776.

Sutor, D.J. (1967). The crystal and molecular structure of newberyite, $\mathrm{MgHPO}_{4} \cdot 3 \mathrm{H}_{2} \mathrm{O}$ Acta Cryst. 23, 418-422.

\begin{tabular}{|c|c|c|c|c|c|}
\hline \multicolumn{6}{|c|}{ Calculated Pattern (Peak heights) } \\
\hline$d(\stackrel{\circ}{A})$ & $I$ & \multicolumn{3}{|c|}{$h k l$} & $\begin{array}{c}2 \theta\left({ }^{\circ}\right) \\
\lambda=1.54056 \AA\end{array}$ \\
\hline 5.941 & 100 & 1 & 1 & 1 & 14.90 \\
\hline 5.336 & 60 & 0 & 2 & 0 & 16.60 \\
\hline 5.104 & 9 & 2 & 0 & 0 & 17.36 \\
\hline 4.711 & 95 & 0 & 2 & 1 & 18.82 \\
\hline 4.609 & 28 & 2 & $\overline{1}$ & 0 & 19.24 \\
\hline 4.494 & 37 & 1 & 0 & 2 & 19.74 \\
\hline 4.145 & 35 & 1 & 1 & 2 & 21.42 \\
\hline 3.690 & 12 & 2 & 2 & 0 & 24.10 \\
\hline 3.654 & 14 & 0 & 2 & 2 & 24.34 \\
\hline 3.576 & 13 & 2 & 0 & 2 & 24.88 \\
\hline 3.463 & 81 & 2 & 2 & 1 & 25.70 \\
\hline 3.440 & 22 & 1 & 2 & 2 & 25.88 \\
\hline 3.391 & 2 & 2 & 1 & 2 & 26.26 \\
\hline 3.186 & 15 & 1 & 3 & 1 & 27.98 \\
\hline 3.087 & 55 & 3 & 1 & 1 & 28.90 \\
\hline 3.042 & 66 & 1 & 1 & 3 & 29.34 \\
\hline $2 \cdot 970$ & 3 & 2 & 2 & 2 & 30.06 \\
\hline 2.829 & 2 & 0 & 2 & 3 & 31.60 \\
\hline 2.815 & 18 & 3 & 0 & 2 & 31.76 \\
\hline 2.800 & 12 & 2 & 3 & 1 & 31.86 \\
\hline 2.791 & 26 & 1 & 3 & 2 & 32.04 \\
\hline 2.722 & 30 & 3 & 1 & 2 & 32.88 \\
\hline 2.703 & 9 & 2 & 1 & 3 & 33.12 \\
\hline 2.670 & 5 & $\overline{0}$ & 4 & 0 & 33.54 \\
\hline $2 \cdot 580$ & 35 & 0 & 4 & 1 & 34.74 \\
\hline 2.553 & 3 & 4 & 0 & 0 & 35.12 \\
\hline 2.523 & 11 & 2 & 3 & 2 & 35.56 \\
\hline 2.502 & 5 & $\overline{1}$ & 4 & 1 & 35.86 \\
\hline 2.483 & 4 & 4 & 1 & 0 & 36.14 \\
\hline 2.431 & 7 & 1 & 0 & 4 & 36.94 \\
\hline 2.411 & 10 & 4 & 1 & 1 & 37.26 \\
\hline 2.390 & 12 & 3 & 3 & $i$ & 37.60 \\
\hline 2.371 & 12 & 1 & 1 & $4+$ & 37.92 \\
\hline 2.366 & 13 & 2 & 4 & 0 & 38.00 \\
\hline 2.326 & 1 & 3 & 1 & 3 & 38.68 \\
\hline 2.304 & 2 & 4 & 2 & 0 & 39.06 \\
\hline 2.296 & 2 & 1 & 4 & 2 & 39.20 \\
\hline 2.275 & 3 & 4 & 0 & 2 & 39.58 \\
\hline 2.240 & 1 & 4 & 2 & 1 & 40.12 \\
\hline $2 \cdot 224$ & 1 & 4 & 1 & $\frac{1}{2}$ & 40.52 \\
\hline 2.213 & 3 & 1 & 2 & 4 & 40.74 \\
\hline 2.208 & 5 & 3 & 3 & 2 & 40.84 \\
\hline 2.199 & 9 & 2 & 1 & $4+$ & 41.00 \\
\hline 2.176 & 7 & 3 & 2 & 3 & 41.46 \\
\hline 2.140 & jo & 2 & 4 & 2 & 42.20 \\
\hline 2.093 & 3 & 4 & 2 & 2 & 43.18 \\
\hline 2.071 & 6 & 2 & 2 & 4 & 43.66 \\
\hline 2.056 & 4 & $\overline{3}$ & 4 & 1 & 44.00 \\
\hline 2.043 & 11 & 1 & 4 & 3 & 44.30 \\
\hline 2.033 & 2 & 4 & 3 & 1 & 44.54 \\
\hline
\end{tabular}


Magnesium Hydrogen Phosphate Trihydrate, newberyite, $\mathrm{MgHPO}_{4} \cdot 3 \mathrm{H}_{2} \mathrm{O}$ (orthorhombic) - continued

\begin{tabular}{|c|c|c|c|c|c|c|}
\hline \multicolumn{7}{|c|}{ Calculated Pattern (Peak heights) } \\
\hline$d(\stackrel{\circ}{A})$ & $I$ & \multicolumn{4}{|c|}{$h k l$} & $\begin{array}{c}2 \theta\left({ }^{\circ}\right) \\
\lambda=1.54056 \AA\end{array}$ \\
\hline 1.9812 & o & 3 & 1 & 4 & + & 45.76 \\
\hline 1.9706 & 3 & 2 & 5 & 0 & & 46.02 \\
\hline 1.9673 & 2 & 5 & 1 & 1 & & 46.10 \\
\hline 1.9294 & is & 1 & 5 & 2 & + & 47.06 \\
\hline 1.9171 & 1 & 4 & 3 & $\overline{2}$ & & 47.38 \\
\hline 1.9005 & 1 & 2 & 3 & 4 & & 47.82 \\
\hline 1.8900 & 4 & 4 & 2 & 3 & & 47.94 \\
\hline 1.8871 & 7 & 3 & 2 & 4 & & 48.18 \\
\hline 1.8747 & 8 & 5 & 2 & 1 & + & 48.52 \\
\hline 1.8625 & 1 & 5 & 1 & 2 & & 48.86 \\
\hline 1.7978 & 10 & 1 & 4 & 4 & & 50.74 \\
\hline 1.7875 & 4 & 4 & 0 & 4 & & 51.04 \\
\hline 1.7717 & 1 & 1 & 5 & 3 & & 51.54 \\
\hline 1.7628 & 5 & 4 & 1 & 4 & & 51.82 \\
\hline 1.7603 & 5 & 2 & 2 & 5 & & $51 \cdot 90$ \\
\hline 1.7553 & 8 & 3 & 3 & 4 & + & 52.06 \\
\hline 1.7318 & 2 & 4 & 4 & 2 & & 52.82 \\
\hline 1.7197 & 0 & 2 & 4 & $\overline{4}$ & + & 53.22 \\
\hline 1.7025 & 2 & 6 & 0 & 0 & + & 53.80 \\
\hline 1.6973 & 2 & 2 & 5 & 3 & & 53.98 \\
\hline 1.6812 & 12 & 2 & 6 & 0 & + & 54.54 \\
\hline 1.6704 & 7 & 5 & 3 & 2 & + & 54.92 \\
\hline 1.6570 & 6 & 2 & 6 & 1 & + & 55.38 \\
\hline 1.6549 & 5 & 1 & 6 & 2 & + & 55.46 \\
\hline 1.6380 & 6 & 4 & 5 & 0 & & 56.08 \\
\hline 1.6169 & 1 & 4 & 5 & 1 & & 50.90 \\
\hline $1.612 \hat{z}$ & 1 & 6 & 0 & 2 & & 57.08 \\
\hline 1.6050 & 3 & 1 & 5 & 4 & & 57.36 \\
\hline 1.6009 & 5 & 6 & 2 & 1 & + & 57.52 \\
\hline 1.5974 & 5 & 4 & 3 & 4 & & 57.66 \\
\hline 1.5930 & 5 & 0 & 2 & 6 & + & 57.82 \\
\hline 1.5863 & 6 & 2 & 0 & 6 & & 58.10 \\
\hline 1.5820 & 5 & 5 & 0 & 4 & & 58.24 \\
\hline 1.5691 & 2 & 2 & 1 & 6 & & 58.80 \\
\hline $1 \cdot 5657$ & 6 & 5 & 1 & 4 & + & 58.94 \\
\hline 1.5571 & 2 & 4 & 5 & 2 & & 59.30 \\
\hline
\end{tabular}

\begin{tabular}{|c|c|c|c|c|c|}
\hline \multicolumn{6}{|c|}{ Calculated Pattern (Integrated) } \\
\hline$d(\stackrel{\circ}{A})$ & $I$ & & $h k l$ & & $\begin{array}{c}2 \theta\left(^{\circ}\right) \\
\lambda=1.54056 \AA\end{array}$ \\
\hline 5.942 & 99 & 1 & 1 & 1 & 14.90 \\
\hline 5.341 & 65 & 0 & 2 & 0 & $16 \cdot 59$ \\
\hline 5.107 & 10 & 2 & 0 & 0 & 17.35 \\
\hline 4.712 & 100 & 0 & 2 & 1 & $1 \varepsilon .82$ \\
\hline 4.509 & 31 & 2 & 1 & 0 & 19.25 \\
\hline 4.496 & 41 & 1 & 0 & 2 & 19.73 \\
\hline 4.144 & 40 & 1 & 1 & 2 & 21.43 \\
\hline 3.691 & 13 & 2 & 2 & 0 & 24.09 \\
\hline 3.653 & 16 & 0 & 2 & 2 & $24 \cdot 35$ \\
\hline 3.575 & 15 & 2 & $\overline{0}$ & $\overline{2}$ & $24 \cdot 88$ \\
\hline 3.403 & 94 & 2 & 2 & 1 & 25.70 \\
\hline 3.439 & 20 & 1 & 2 & 2 & 25.88 \\
\hline 3.391 & 2 & 2 & 1 & 2 & 26.26 \\
\hline 3.187 & 18 & 1 & 3 & 1 & 27.97 \\
\hline 3.086 & 68 & 3 & 1 & 1 & 28.91 \\
\hline 3.042 & 79 & 1 & 1 & 3 & 29.34 \\
\hline 2.971 & 4 & 2 & 2 & 2 & 30.05 \\
\hline 2.801 & 2 & 0 & 2 & 3 & 31.58 \\
\hline 2.816 & 21 & 3 & 0 & 2 & 31.75 \\
\hline 2.804 & 4 & 2 & 3 & $\overline{1}$ & $31 \cdot 89$ \\
\hline 2.791 & 32 & 1 & 3 & 2 & 32.04 \\
\hline 2.723 & 39 & 3 & 1 & 2 & 32.87 \\
\hline 2.703 & 9 & 2 & 1 & 3 & 33.11 \\
\hline 2.670 & 6 & 0 & 4 & 0 & 33.53 \\
\hline $2 \cdot 500$ & 45 & 0 & 4 & 1 & 34.74 \\
\hline 2.554 & 4 & 4 & 0 & 0 & 35.11 \\
\hline $2 \cdot 523$ & 14 & 2 & 3 & 2 & 35.55 \\
\hline 2.502 & 6 & 1 & 4 & 1 & 35.87 \\
\hline 2.404 & 5 & 4 & 1 & 0 & 36.13 \\
\hline 2.432 & 9 & 1 & 0 & 4 & 36.94 \\
\hline 2.411 & 14 & 4 & 1 & 1 & 37.27 \\
\hline 2.390 & 16 & 3 & 3 & 1 & 37.61 \\
\hline 2.371 & 14 & 1 & 1 & 4 & 37.92 \\
\hline 2.369 & 1 & 1 & 3 & 3 & 37.95 \\
\hline 2.366 & 8 & 2 & 4 & 0 & 37.99 \\
\hline $2 \cdot 326$ & 1 & 3 & 1 & 3 & 38.67 \\
\hline $2 \cdot 304$ & 3 & 4 & 2 & 0 & 39.06 \\
\hline 2.296 & 2 & 1 & 4 & 2 & 39.21 \\
\hline 2.275 & 4 & 4 & 0 & 2 & 39.58 \\
\hline $2 \cdot 245$ & 1 & 4 & 2 & 1 & 40.13 \\
\hline $2 \cdot 225$ & 1 & 4 & 1 & 2 & 40.51 \\
\hline 2.213 & 3 & 1 & 2 & 4 & 40.74 \\
\hline 2.203 & 4 & 3 & 3 & 2 & 40.83 \\
\hline 2.200 & 8 & 2 & 1 & 4 & 40.99 \\
\hline 2.198 & 6 & 2 & 3 & 3 & 41.03 \\
\hline $2 \cdot 177$ & 9 & 3 & 2 & 3 & $41 . .45$ \\
\hline 2.139 & 8 & 2 & 4 & 2 & $42 \cdot 20$ \\
\hline 2.093 & 11 & 4 & 2 & 2 & 43.19 \\
\hline 2.072 & 9 & 2 & 2 & 4 & 43.65 \\
\hline 2.056 & 5 & 3 & 4 & 1 & 44.03 \\
\hline
\end{tabular}


Magnesium Hydrogen Phosphate Trihydrate, newberyite, $\mathrm{MgHPO}_{4} \cdot 3 \mathrm{H}_{2} \mathrm{O}$ (orthorhombic) - continued

\begin{tabular}{|c|c|c|c|c|c|}
\hline \multicolumn{6}{|c|}{ Calculated Pattern (Integrated) } \\
\hline$d(A)$ & $I$ & & $h k l$ & & $\begin{array}{c}2 \theta\left(^{\circ}\right) \\
\lambda=1.54056 \mathrm{~A}\end{array}$ \\
\hline 2.043 & 16 & 1 & 4 & 3 & $44 \cdot 30$ \\
\hline 2.032 & 2 & 4 & 3 & 1 & 44.55 \\
\hline 1.9820 & 6 & 3 & 1 & 4 & 45.74 \\
\hline 1.9807 & 3 & 3 & 3 & 3 & 45.77 \\
\hline 1.9708 & 3 & 2 & 5 & 0 & 46.02 \\
\hline 1.9675 & 1 & 5 & 1 & 1 & 46.10 \\
\hline 1.9375 & 2 & 3 & 4 & 2 & 46.85 \\
\hline 1.9337 & 2 & 2 & 5 & 1 & 46.95 \\
\hline 1.9329 & 4 & 1 & 1 & 5 & 46.97 \\
\hline 1.9305 & 1 & 2 & 4 & 3 & 47.03 \\
\hline 1.9295 & 18 & 1 & 5 & 2 & 47.06 \\
\hline 1.9170 & 1 & 4 & 3 & 2 & 47.38 \\
\hline 1.9008 & 1 & 2 & 3 & 4 & 47.81 \\
\hline 1.8961 & 4 & 4 & 2 & 3 & 47.94 \\
\hline 1.3869 & 10 & 3 & 2 & 4 & 48.19 \\
\hline 1.8753 & 3 & 0 & 2 & 5 & 46.50 \\
\hline 1.3744 & 10 & 5 & 2 & 1 & 48.53 \\
\hline 1.8626 & 1 & 5 & 1 & 2 & 48.86 \\
\hline 1.7978 & 15 & 1 & 4 & 4 & 50.74 \\
\hline 1.7877 & 5 & 4 & 0 & 4 & 51.05 \\
\hline 1.7720 & 1 & 1 & 5 & 3 & 51.53 \\
\hline 1.7032 & 6 & 4 & 1 & 4 & 51.81 \\
\hline 1.7604 & 5 & 2 & 2 & 5 & 51.90 \\
\hline 1.7549 & 10 & 3 & 3 & 4 & 52.07 \\
\hline 1.7527 & 1 & 0 & 6 & 1 & $52 \cdot 14$ \\
\hline 1.7317 & 2 & 4 & 4 & 2 & 52.82 \\
\hline 1.7198 & 4 & 5 & 1 & 3 & 53.22 \\
\hline 1.7197 & 5 & 2 & 4 & 4 & 53.22 \\
\hline
\end{tabular}

\begin{tabular}{|c|c|c|c|c|c|}
\hline \multicolumn{6}{|c|}{ Calculated Pattern (Integrated) } \\
\hline$d(\stackrel{\circ}{)}$ & $I$ & & hkl & & $\begin{array}{c}2 \theta\left(^{\circ}\right) \\
\lambda=1.54056 \AA\end{array}$ \\
\hline $\begin{array}{l}1.7042 \\
1.7025\end{array}$ & $\begin{array}{l}1 \\
2\end{array}$ & $\begin{array}{l}3 \\
6\end{array}$ & $\begin{array}{l}1 \\
0\end{array}$ & $\begin{array}{l}5 \\
0\end{array}$ & $\begin{array}{l}53.74 \\
53.80\end{array}$ \\
\hline 1.6971 & 1 & 2 & 5 & 3 & 53.99 \\
\hline 1.6813 & 6 & 6 & 1 & 0 & 54.54 \\
\hline 1.6810 & 12 & 2 & 6 & 0 & 54.55 \\
\hline 1.6705 & 9 & 5 & 3 & 2 & 54.92 \\
\hline 1.6690 & 1 & 0 & 0 & 6 & $54 \cdot 97$ \\
\hline 1.6581 & 4 & 6 & 1 & 1 & 55.36 \\
\hline 1.6578 & 4 & 2 & 6 & 1 & 55.37 \\
\hline 1.6566 & 1 & 5 & 2 & 3 & 55.42 \\
\hline 1.6551 & 5 & 1 & 6 & 2 & 55.47 \\
\hline 1.6518 & 1 & 2 & 3 & 5 & 55.59 \\
\hline 1.6385 & 9 & 4 & 5 & 0 & 56.08 \\
\hline 1.6170 & 2 & 4 & 5 & 1 & 56.90 \\
\hline 1.6119 & 1 & 6 & 0 & 2 & 57.09 \\
\hline 1.6048 & 3 & 1 & 5 & 4 & 57.37 \\
\hline 1.6022 & 1 & 0 & 4 & 5 & 57.47 \\
\hline 1.6012 & 6 & 6 & 2 & 1 & 57.51 \\
\hline 1.5976 & 3 & 4 & 3 & 4 & 57.65 \\
\hline 1.5938 & 1 & 6 & 1 & 2 & 57.80 \\
\hline 1.5930 & 6 & 0 & 2 & ó & 57.83 \\
\hline $1 \cdot 5864$ & 8 & 2 & 0 & 6 & $58 \cdot 10$ \\
\hline 1.5828 & 3 & 5 & 0 & 4 & 58.24 \\
\hline 1.5072 & 2 & 2 & 1 & 6 & 58.79 \\
\hline 1.5657 & 8 & 5 & 1 & 4 & 58.94 \\
\hline 1.5051 & 1 & 5 & 3 & 3 & 58.96 \\
\hline 1.5573 & 2 & 4 & 5 & 2 & 59.29 \\
\hline
\end{tabular}




\section{Structure}

Cubic, I $\overline{4} 3 \mathrm{~m}$ (217), $\mathrm{Z}=58$ [Gazzara et al. 1967]

\section{Lattice parameters}

$\mathrm{a}=8.9129 \AA$ (published value: $\mathrm{a}=8.9125 \AA$ ) [ibid.]

\section{Scattering factors}

$\mathrm{Mn}^{\circ}$ [Freeman and Watson, 1961], corrected for dispersion [Dauben and Templeton, 1955]

\section{Thermal parameters}

Isotropic [Gazzara et al., 1967]

Density

(calculated) $7.472 \mathrm{~g} / \mathrm{cm}^{3}$

\section{Scale factor}

$62.21 \times 10^{4}$

\section{Additional patterns}

1. PDF 1-1237 [Hanawalt et al., 1938]

\section{Reference}

Dauben, C.H. and D.H. Templeton (1955). A table of dispersion corrections for $\mathrm{x}-$ ray scattering of atoms, Acta Cryst. 8, 841-842.

Freeman, A.J. and R.E. Watson (1961). Hartree-Fock atomic scattering factors for the neutral atom iron transition series, Acta Cryst. 14, 231-234.

Gazzara, C.P., R.M. Middleton, R.J.Weiss, and E.O.Hall (1967). A refinement of the parameters of a manganese,Acta Cryst.22, 859-862.

Hanawalt, J.D., H.W. Rinn, and I.K. Frevel (1938). Chemical analysis by $x-r a y$ diffraction, Ind. Eng. Chem. Anal. Ed. 10, 457-513.

\begin{tabular}{|c|c|c|c|c|c|c|}
\hline \multicolumn{7}{|c|}{ Calculated Pattern (Peak heights) } \\
\hline$d(\stackrel{\circ}{A})$ & $I$ & & $h k$ & & & $\begin{array}{c}2 \theta\left(^{\circ}\right) \\
\lambda=1.54056 \AA\end{array}$ \\
\hline $\begin{array}{l}3.639 \\
3.151 \\
2.573 \\
2.382 \\
2.229\end{array}$ & $\begin{array}{l}1 \\
1 \\
1 \\
1 \\
4\end{array}$ & $\begin{array}{l}2 \\
2 \\
2 \\
3 \\
4\end{array}$ & $\begin{array}{l}1 \\
2 \\
2 \\
2 \\
0\end{array}$ & $\begin{array}{l}1 \\
0 \\
2 \\
1 \\
0\end{array}$ & & $\begin{array}{l}24.44 \\
28.30 \\
34.84 \\
37.74 \\
40.44\end{array}$ \\
\hline $\begin{array}{l}2.101 \\
1.9005 \\
1.8192 \\
1.7478 \\
1.6274\end{array}$ & $\begin{array}{r}100 \\
21 \\
8 \\
12 \\
1\end{array}$ & $\begin{array}{l}4 \\
3 \\
4 \\
4 \\
5\end{array}$ & $\begin{array}{l}1 \\
3 \\
2 \\
3 \\
2\end{array}$ & $\begin{array}{l}1 \\
2 \\
2 \\
1 \\
1\end{array}$ & + & $\begin{array}{l}43.02 \\
47.82 \\
50.10 \\
52.30 \\
56.50\end{array}$ \\
\hline $\begin{array}{l}1.4857 \\
1.4459 \\
1.3436 \\
1.2865 \\
1.2604\end{array}$ & $\begin{array}{l}1 \\
1 \\
1 \\
3 \\
4\end{array}$ & $\begin{array}{l}4 \\
5 \\
6 \\
4 \\
5\end{array}$ & $\begin{array}{l}4 \\
3 \\
2 \\
4 \\
5\end{array}$ & $\begin{array}{l}2 \\
2 \\
2 \\
4 \\
0\end{array}$ & & $\begin{array}{l}62.46 \\
64.38 \\
69.96 \\
73.56 \\
75.34\end{array}$ \\
\hline $\begin{array}{l}1.2120 \\
1.1909 \\
1.1703 \\
1.1320 \\
1.0809\end{array}$ & $\begin{array}{r}14 \\
2 \\
1 \\
2 \\
1\end{array}$ & $\begin{array}{l}7 \\
6 \\
7 \\
6 \\
8\end{array}$ & $\begin{array}{l}2 \\
4 \\
3 \\
5 \\
2\end{array}$ & $\begin{array}{l}1 \\
2 \\
0 \\
1 \\
0\end{array}$ & + & $\begin{array}{l}78.86 \\
80.60 \\
82.32 \\
85.76 \\
90.90\end{array}$ \\
\hline $\begin{array}{r}1.0653 \\
1.0503 \\
.9842 \\
.9611 \\
.9501\end{array}$ & $\begin{array}{l}1 \\
4 \\
1 \\
1 \\
1\end{array}$ & $\begin{array}{l}6 \\
8 \\
8 \\
7 \\
6\end{array}$ & $\begin{array}{l}5 \\
2 \\
3 \\
6 \\
6\end{array}$ & $\begin{array}{l}3 \\
2 \\
3 \\
1 \\
4\end{array}$ & $\begin{array}{l}+ \\
+\end{array}$ & $\begin{array}{r}92.62 \\
94.34 \\
103.00 \\
106.54 \\
108.34\end{array}$ \\
\hline $\begin{array}{r}.9395 \\
.9003 \\
.8498 \\
.8348 \\
.8205\end{array}$ & $\begin{array}{l}3 \\
1 \\
1 \\
2 \\
3\end{array}$ & $\begin{array}{l}7 \\
8 \\
9 \\
7 \\
9\end{array}$ & $\begin{array}{l}5 \\
5 \\
5 \\
7 \\
6\end{array}$ & $\begin{array}{l}4 \\
3 \\
2 \\
4 \\
1\end{array}$ & $\begin{array}{l}+ \\
+ \\
+ \\
+ \\
+\end{array}$ & $\begin{array}{l}110.14 \\
117.64 \\
130.02 \\
134.66 \\
139.70\end{array}$ \\
\hline $\begin{array}{r}.8130 \\
.8069 \\
.7940\end{array}$ & $\begin{array}{l}2 \\
3 \\
3\end{array}$ & $\begin{array}{l}10 \\
11 \\
10\end{array}$ & $\begin{array}{l}4 \\
1 \\
5\end{array}$ & $\begin{array}{l}2 \\
0 \\
1\end{array}$ & $\begin{array}{l}+ \\
+\end{array}$ & $\begin{array}{l}142.42 \\
145.32 \\
151.90\end{array}$ \\
\hline
\end{tabular}




\begin{tabular}{|c|c|c|c|c|c|}
\hline \multicolumn{6}{|c|}{ Calculated Pattern (Integrated) } \\
\hline$d(\AA)$ & $I$ & & $h k$ & & $\begin{array}{c}2 \theta\left(^{\circ}\right) \\
\lambda=1.54056 \AA\end{array}$ \\
\hline $\begin{array}{l}3.639 \\
3.151 \\
2.573 \\
2.382 \\
2.228\end{array}$ & $\begin{array}{l}1 \\
1 \\
1 \\
1 \\
6\end{array}$ & $\begin{array}{l}2 \\
2 \\
2 \\
3 \\
4\end{array}$ & $\begin{array}{l}1 \\
2 \\
2 \\
2 \\
0\end{array}$ & $\begin{array}{l}1 \\
0 \\
2 \\
1 \\
0\end{array}$ & $\begin{array}{l}24.44 \\
28.30 \\
34.84 \\
37.73 \\
40.45\end{array}$ \\
\hline $\begin{array}{l}2.101 \\
2.101 \\
1.9002 \\
1.8193 \\
1.7480\end{array}$ & $\begin{array}{r}49 \\
100 \\
35 \\
13 \\
16\end{array}$ & $\begin{array}{l}3 \\
4 \\
3 \\
4 \\
4\end{array}$ & $\begin{array}{l}3 \\
1 \\
3 \\
2 \\
3\end{array}$ & $\begin{array}{l}0 \\
1 \\
2 \\
2 \\
1\end{array}$ & $\begin{array}{l}43.02 \\
43.02 \\
47.83 \\
50.10 \\
52.29\end{array}$ \\
\hline $\begin{array}{l}1.7480 \\
1.6273 \\
1.5285 \\
1.4855 \\
1.4459\end{array}$ & $\begin{array}{l}4 \\
1 \\
1 \\
1 \\
1\end{array}$ & $\begin{array}{l}5 \\
5 \\
4 \\
4 \\
5\end{array}$ & $\begin{array}{l}1 \\
2 \\
3 \\
4 \\
3\end{array}$ & $\begin{array}{l}0 \\
1 \\
3 \\
2 \\
2\end{array}$ & $\begin{array}{l}52.29 \\
56.51 \\
60.52 \\
62.47 \\
64.38\end{array}$ \\
\hline $\begin{array}{l}1.3437 \\
1.3141 \\
1.2865 \\
1.2605 \\
1.2129\end{array}$ & $\begin{array}{r}3 \\
1 \\
7 \\
9 \\
19\end{array}$ & $\begin{array}{l}6 \\
6 \\
4 \\
5 \\
7\end{array}$ & $\begin{array}{l}2 \\
3 \\
4 \\
5 \\
2\end{array}$ & $\begin{array}{l}2 \\
1 \\
4 \\
0 \\
1\end{array}$ & $\begin{array}{l}69.96 \\
71.77 \\
73.56 \\
75.34 \\
78.85\end{array}$ \\
\hline $\begin{array}{l}1.2129 \\
1.2129 \\
1.1910 \\
1.1703 \\
1.1319\end{array}$ & $\begin{array}{l}6 \\
6 \\
5 \\
3 \\
3\end{array}$ & $\begin{array}{l}5 \\
6 \\
6 \\
7 \\
6\end{array}$ & $\begin{array}{l}5 \\
3 \\
4 \\
3 \\
5\end{array}$ & $\begin{array}{l}2 \\
3 \\
2 \\
0 \\
1\end{array}$ & $\begin{array}{l}78.85 \\
78.85 \\
80.59 \\
82.32 \\
85.76\end{array}$ \\
\hline $\begin{array}{l}1.1319 \\
1.0971 \\
1.0808 \\
1.0653 \\
1.0504\end{array}$ & $\begin{array}{l}2 \\
1 \\
2 \\
2 \\
6\end{array}$ & $\begin{array}{l}7 \\
7 \\
8 \\
6 \\
8\end{array}$ & $\begin{array}{l}3 \\
4 \\
2 \\
5 \\
2\end{array}$ & $\begin{array}{l}2 \\
1 \\
0 \\
3 \\
2\end{array}$ & $\begin{array}{l}85.76 \\
89.19 \\
90.90 \\
92.62 \\
94.33\end{array}$ \\
\hline $\begin{array}{l}1.0504 \\
1.0361\end{array}$ & $\begin{array}{l}4 \\
1\end{array}$ & $\begin{array}{l}6 \\
8\end{array}$ & $\begin{array}{l}6 \\
3\end{array}$ & $\begin{array}{l}0 \\
1\end{array}$ & $\begin{array}{l}94.33 \\
96.05\end{array}$ \\
\hline
\end{tabular}

\begin{tabular}{|c|c|c|c|c|c|}
\hline \multicolumn{6}{|c|}{ Calculated Pattern (Integrated) } \\
\hline$d(\AA)$ & $I$ & \multicolumn{3}{|c|}{$h k l$} & $\begin{array}{c}2 \theta\left(^{\circ}\right) \\
\lambda=1.54056 \AA\end{array}$ \\
\hline 1.0224 & 1 & 6 & 6 & 2 & 97.77 \\
\hline 1.0092 & 1 & 7 & 5 & 2 & 99.51 \\
\hline .9843 & 3 & 8 & 3 & 3 & 103.00 \\
\hline .9725 & 1 & 8 & 4 & 2 & 104.76 \\
\hline .9011 & 1 & 9 & 2 & 1 & 106.54 \\
\hline .9611 & 2 & 7 & 6 & 1 & 106.54 \\
\hline .9611 & 1 & 6 & 5 & 5 & 106.54 \\
\hline .9501 & 2 & 6 & 6 & 4 & 106.33 \\
\hline .9395 & 3 & 8 & 5 & 1 & 110.15 \\
\hline .9395 & 1 & 9 & 3 & 0 & 110.15 \\
\hline .9395 & 4 & 7 & 5 & 4 & $110 \cdot 15$ \\
\hline .9193 & 1 & 7 & 6 & 3 & 113.84 \\
\hline .9003 & 3 & 8 & 5 & 3 & 117.64 \\
\hline .9003 & 1 & 9 & 4 & 1 & 117.64 \\
\hline .8825 & 1 & 10 & 1 & 1 & 121.58 \\
\hline .874 .0 & 1 & 8 & 6 & 2 & 123.61 \\
\hline .8657 & 1 & 9 & 4 & 3 & 125.69 \\
\hline .8576 & 1 & 10 & 2 & 2 & 127.83 \\
\hline .8498 & 1 & 10 & 3 & 1 & 130.03 \\
\hline .8498 & 3 & 9 & 5 & 2 & 130.03 \\
\hline .8348 & 5 & 7 & 7 & 4 & 134.66 \\
\hline .8348 & 3 & 8 & 5 & 5 & 134.66 \\
\hline .8205 & 12 & 9 & 6 & 1 & 139.70 \\
\hline .8205 & 1 & 10 & 3 & 3 & 139.70 \\
\hline .8136 & 9 & 10 & 4 & 2 & 142.42 \\
\hline .8069 & 2 & 9 & 5 & 4 & 145.33 \\
\hline .8069 & 3 & 8 & 7 & 3 & 145.33 \\
\hline .8069 & 6 & 11 & 1 & 0 & 145.33 \\
\hline .7940 & 5 & 11 & 2 & 1 & 151.91 \\
\hline .7940 & 7 & 9 & 6 & 3 & 151.91 \\
\hline .7940 & 7 & 10 & 5 & 1 & 151.91 \\
\hline
\end{tabular}




\section{Structure}

Monoclinic, $\quad \mathrm{P} 2$ / $/ \mathrm{a}$ (14), $\mathrm{Z}=2$ [Braun and Lingafelter, 1967]

\section{Lattice parameters}

$\mathrm{a}=10.672 \pm 0.002, \mathrm{~b}=13.064 \pm 0.002, \mathrm{c}=7.998 \pm$ $0.001 \AA, \quad \beta=98.09 \pm 0.01^{\circ}$

(published value, $\mathrm{b}=13.063$ ) [ibid.]

\section{Scattering factors}

$\mathrm{N}^{\circ}, \mathrm{O}^{-1}[3.3 .1 \mathrm{~A}] ; \mathrm{Pd}^{\circ}, \mathrm{C}^{\circ}$ [Berghuis et al., 1955]; $\mathrm{H}^{\circ}$ [Stewart et al.,1965]

\section{Thermal parameters}

Isotropic:

$\begin{array}{lrllll}\text { Pd } & 2.71 & \mathrm{O} & 3.34 & \mathrm{~N} & 2.87 \\ \mathrm{C}(1) & 3.11 & \mathrm{C}(2) & 2.97 & \mathrm{C}(3) & 3.48 \\ \mathrm{C}(4) & 4.28 & \mathrm{C}(5) & 4.56 & \mathrm{C}(6) & 3.91 \\ \mathrm{C}(7) & 3.21 & \mathrm{C}(8) & 3.79 & \mathrm{C}(9) & 5.39 \\ \mathrm{C}(10) & 6.01 & \mathrm{C}(11) & 4.29 & \mathrm{C}(12) & 6.31 \\ \text { (4) through } & \mathrm{H}(123) & \text { as } & \text { given by } & \text { Braun } \\ \text { al Lingfelter } & {[1967]} & & & \end{array}$

\section{Density}

(calculated) $1.465 \mathrm{~g} / \mathrm{cm}^{3}$ [ibid.]

\section{Scale factor}

$21.84 \times 10^{4}$

\section{Reference}

Berghuis, J., IJ. M. Haanapel, M. Potters, B.O. Loopstra, C.H. MacGillavry, and A. L. Veenendaal (1955). New calculations of atomic scattering factors, Acta cryst. 8, 478-483.

Braun,R.L. and E.C.Lingafelter (1967). The crystal structure of bis-(N-isopropyl-3ethylsalicylaldiminato) palladium, Acta Cryst. 22, 787-792.

Stewart, R.F., E.R.Davidson, and W.T.Simpson (1965). Coherent $x$-ray scattering for the hydrogen atom in the hydrogen molecule, J. Chem. Phys. 42, 3175-3187.

\begin{tabular}{|c|c|c|c|c|c|c|}
\hline \multicolumn{7}{|c|}{ Calculated Pattern (Peak heights) } \\
\hline$d(\stackrel{\circ}{A})$ & $I$ & & $h k$ & & & $\begin{array}{c}2 \theta\left({ }^{\circ}\right) \\
\lambda=1.54056 \AA\end{array}$ \\
\hline 8.22 & 100 & 1 & 1 & 0 & & 10.76 \\
\hline 7.91 & 9 & 0 & D & 1 & & 11.18 \\
\hline 6.77 & 4 & D & 1 & 1 & & 13.06 \\
\hline 6.52 & 14 & 3 & 2 & 0 & & 13.56 \\
\hline 5.04 & 4 & 1 & 1 & -1 & & 14.66 \\
\hline 5.41 & 16 & 1 & 1 & 1 & & 15.36 \\
\hline 5.28 & 4 & 2 & 0 & 0 & & 15.78 \\
\hline 5.03 & $z$ & 0 & 2 & 1 & & 17.60 \\
\hline 4.71 & 2 & 2 & u & -1 & + & 12.82 \\
\hline 4.43 & 2 & 2 & 1 & -1 & & 20.02 \\
\hline 4.40 & 8 & 1 & 2 & 1 & & 20.18 \\
\hline $4 \cdot 11$ & 3 & 2 & 2 & 0 & & 21.62 \\
\hline 3.96 & 5 & 0 & 0 & 2 & & 22.44 \\
\hline 3.94 & 6 & 2 & 1 & 1 & & 22.54 \\
\hline 3.82 & 10 & 2 & 2 & -1 & + & 23.26 \\
\hline 3.79 & 2 & 0 & 1 & 2 & & 23.46 \\
\hline 3.73 & 7 & 1 & 1 & -2 & & 23.84 \\
\hline 3.67 & $?$ & 1 & 3 & -1 & & 24.24 \\
\hline 3.51 & 18 & 1 & 3 & 1 & & 25.34 \\
\hline 3.493 & 18 & 2 & 2 & 1 & & 25.48 \\
\hline 3.422 & 1 & 1 & 1 & 2 & & 26.02 \\
\hline 3.401 & 2 & 3 & 1 & 0 & & 25.18 \\
\hline 3.351 & 1 & $<$ & 3 & 0 & & 26.50 \\
\hline 3.290 & 2 & 3 & 1 & -1 & & $27 \cdot \tilde{0} 8$ \\
\hline 3.266 & 1 & 3 & 4 & 0 & & 27.28 \\
\hline 3.019 & 2 & 0 & 4 & 1 & + & 29.56 \\
\hline 2.996 & 1 & 2 & 3 & 1 & & 23.78 \\
\hline 2.982 & 4 & 3 & 1 & 1 & & $2 y .94$ \\
\hline 2.974 & 3 & $\angle$ & 0 & 2 & & 35.02 \\
\hline $2 \cdot 902$ & 2 & 1 & 3 & -2 & & 30.78 \\
\hline 2.773 & 3 & $<$ & 4 & 0 & + & 32.26 \\
\hline$\therefore 749$ & 1 & 1 & 3 & 2 & & 32.54 \\
\hline $2.70 \mathrm{c}$ & 1 & 2 & 2 & 2 & & 33.08 \\
\hline 2.684 & 1 & 2 & 4 & -1 & & 33.36 \\
\hline 2.639 & 1 & 3 & 0 & 3 & & 33.94 \\
\hline 2.598 & 1 & 1 & 1 & -3 & & 34.50 \\
\hline 2.563 & 2 & 2 & 4 & 1 & & 34.98 \\
\hline 2.536 & 2 & 1 & 5 & 0 & & 35.36 \\
\hline 2.520 & 1 & 0 & 4 & 2 & & 35.60 \\
\hline 2.505 & 4 & 3 & 3 & 1 & & 35.82 \\
\hline 2.448 & 1 & 3 & 2 & 3 & & 36.58 \\
\hline 2.431 & 1 & 4 & 2 & -1 & & 36.94 \\
\hline 2.423 & 1 & 3 & 1 & 2 & & 37.08 \\
\hline 2.393 & 1 & 1 & 5 & 1 & & 37.56 \\
\hline 2.356 & 2 & 4 & 0 & -2 & & 38.10 \\
\hline 2.340 & 2 & 2 & 2 & -3 & & 38.44 \\
\hline 2.258 & 1 & 4 & 2 & 1 & & 39.90 \\
\hline 2.237 & 2 & 2 & 1 & -3 & & 40.28 \\
\hline $2.21 E$ & 1 & 4 & 2 & -2 & & 40.58 \\
\hline 2.198 & 1 & 2 & 4 & 2 & & 41.32 \\
\hline
\end{tabular}


bis-( $\mathrm{N}-i$ sopropyl-3-ethylsalicylaldiminato) Palladium, $\left(\mathrm{C}_{12} \mathrm{H}_{16} \mathrm{NO}\right)_{2} \mathrm{Pd}$ (monoclinic) - continued

\begin{tabular}{|c|c|c|c|c|c|}
\hline \multicolumn{6}{|c|}{ Calculated Pattern (Peak heights) } \\
\hline$d(\stackrel{\circ}{A})$ & $I$ & & $h k l$ & & $\begin{array}{c}2 \theta\left(^{\circ}\right) \\
\lambda=1.54056 \mathrm{~A}\end{array}$ \\
\hline 2.177 & 1 & 0 & 6 & 0 & 41.44 \\
\hline 2.155 & 1 & 1 & 3 & 3 & 41.88 \\
\hline 2.146 & 1 & 3 & 3 & 2 & 42.08 \\
\hline 2.118 & 1 & 2 & 2 & 3 & 42.66 \\
\hline 2.104 & 1 & 1 & 5 & 2 & 42.96 \\
\hline$<.099$ & 2 & c & $E$ & 1 & 43.06 \\
\hline 2.014 & 2 & 3 & 3 & -3 & 44.98 \\
\hline $1.974 c$ & 1 & i & 1 & -4 & 45.92 \\
\hline 1.9706 & 1 & 4 & 2 & 2 & 46.02 \\
\hline 1.9609 & 1 & 5 & 1 & -2 & 46.26 \\
\hline 1.9248 & 1 & 4 & 2 & -3 & 47.18 \\
\hline i. 9043 & 1 & 5 & 3 & -1 & 47.72 \\
\hline 1.8646 & 1 & 2 & 2 & -4 & 48.80 \\
\hline 1.7998 & 1 & 1 & 7 & -1 & 50.68 \\
\hline 1.7932 & 1 & 3 & 5 & 2 & 50.88 \\
\hline 1.7465 & 1 & 4 & 4 & 2 & 52.34 \\
\hline
\end{tabular}

\begin{tabular}{|c|c|c|c|c|c|}
\hline \multicolumn{6}{|c|}{ Calculated Pattern (Integrated) } \\
\hline$d(\stackrel{\circ}{)})$ & $I$ & & $h k l$ & & $\begin{array}{c}2 \theta\left(^{\circ}\right) \\
\lambda=1.54056 \AA\end{array}$ \\
\hline 3.021 & 1 & 2 & $\ddot{z}$ & -2 & 23.55 \\
\hline $3.01 y$ & 1 & $\Sigma$ & 4 & 1 & 29.56 \\
\hline 2.990 & 1 & 2 & & 1 & 29.77 \\
\hline 2.981 & 5 & 3 & 1 & 1 & 29.95 \\
\hline 2.974 & 1 & 2 & a & 2 & 30.02 \\
\hline 2.902 & 3 & 1 & & -2 & 30.79 \\
\hline 2.778 & 2 & 2 & 4 & 0 & 32.20 \\
\hline 2.773 & 2 & 3 & 1 & -2 & 32.26 \\
\hline 2.772 & 1 & 3 & 2 & 1 & 32.25 \\
\hline 2.750 & 2 & 1 & 3 & 2 & 32.53 \\
\hline 2.706 & 2 & 2 & $<$ & 2 & 33.07 \\
\hline$\ldots 684$ & 1 & 2 & 4 & -1 & 33.35 \\
\hline 2.639 & 2 & 0 & 0 & 3 & 33.94 \\
\hline$<.597$ & 2 & 1 & $1-$ & -3 & 34.51 \\
\hline 2.563 & $?$ & 2 & 4 & 1 & 34.93 \\
\hline$<.536$ & 2 & 1 & 5 & 0 & 35.36 \\
\hline 2.519 & 1 & $\omega$ & 4 & 2 & 35.61 \\
\hline$<.505$ & 5 & 3 & 3 & 1 & 35.82 \\
\hline 2.447 & 1 & 0 & 2 & 3 & 36.59 \\
\hline 2.431 & 1 & 4 & & -1 & 36.95 \\
\hline 2.422 & 1 & 3 & 1 & 2 & 37.03 \\
\hline$<.392$ & 2 & 1 & 5 & 1 & 37.57 \\
\hline 2.377 & 1 & 3 & $3-$ & -2 & 37.61 \\
\hline 2.355 & $?$ & 4 & 0 & -2 & 38.17 \\
\hline$\therefore 340$ & 2 & 2 & 2 & -3 & 38.44 \\
\hline 2.258 & 1 & 4 & 2 & 1 & 39.90 \\
\hline 2.238 & 3 & 3 & 1 & -3 & 40.27 \\
\hline 2.215 & 1 & 4 & 2 & -2 & 40.68 \\
\hline$<.199$ & 2 & 2 & 4 & 2 & 41.01 \\
\hline 2.177 & ? & 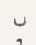 & $E$ & 0 & 41.44 \\
\hline 2.155 & 1 & 1 & 3 & 3 & 41.39 \\
\hline 2.145 & 1 & $\Sigma$ & 3 & 2 & 42.09 \\
\hline 2.115 & 1 & 2 & 2 & 3 & 42.56 \\
\hline-0.104 & 1 & 1 & 5 & 2 & 42.96 \\
\hline 2.099 & 2 & 0 & 6 & 1 & 43.05 \\
\hline 2.098 & 1 & 3 & 5 & 0 & 43.07 \\
\hline 2.014 & 1 & 3 & $\dot{\vdots}$ & -3 & 44.98 \\
\hline 2.013 & 1 & 2 & 5 & 0 & 44.99 \\
\hline 1.9743 & 1 & 1 & 1 & -4 & 45.33 \\
\hline 1.9700 & 1 & 4 & 2 & 2 & 46.01 \\
\hline i. 9613 & 1 & 5 & $1-$ & -2 & 46.25 \\
\hline $1.937<$ & 1 & 4 & 4 & 1 & 46.86 \\
\hline 1.9247 & 1 & 4 & $2-$ & -3 & 47.18 \\
\hline i. 9041 & 1 & 5 & $3-$ & -1 & 47.72 \\
\hline $1.864 y$ & 1 & c & 2 & -4 & 48.79 \\
\hline 1.8003 & 1 & 1 & 7 & -1 & 50.67 \\
\hline $1.792 y$ & 1 & 3 & 5 & 2 & 50.89 \\
\hline 1.7807 & 1 & 1 & 7 & 1 & 51.26 \\
\hline 1.7457 & 1 & 4 & 4 & 2 & 32.34 \\
\hline 1.6717 & 1 & $\therefore$ & $4-$ & -4 & 54.23 \\
\hline
\end{tabular}


Structure

Triclinic, P $\bar{I}$ (2), Z $=1$ [Fritchie, 1966]

\section{Lattice parameters}

$a=3.8684 \pm 0.0004 \AA, \alpha=91.67^{\circ} \pm 0.01^{\circ}$

$\mathrm{b}=7.7810 \pm 0.0008, \quad \beta=92.67^{\circ} \pm 0.01^{\circ}$

$\mathrm{C}=15.736 \pm 0.002, \quad \gamma=95.38^{\circ} \pm 0.01^{\circ}$ at $23^{\circ} \mathrm{C}$ (published values: $a=3.8682, b=7.7807$, $\mathrm{C}=15.735 \AA$ ) [ibid.]

\section{Scattering factors}

$\mathrm{H}^{\circ}, \mathrm{C}^{\circ}, \mathrm{N}^{\circ}[3,3,1 \mathrm{~A}]$

\section{Thermal parameters}

Anisotropic for carbon and nitrogen, isotropic for hydrogen [Fritchie, 1966]

\section{Density}

(calculated) $1.4090 \mathrm{~g} / \mathrm{cm}^{3}$ [ibid.]

\section{Scale factor}

$0.9160 \times 10^{4}$

\section{Reference}

Fritchie, C.J.Jr.(1966). The crystal structure of $\mathrm{N}$-methylphenazinium tetracyanoquinodimethanide, Acta Cryst.20 892-898.

\begin{tabular}{|c|c|c|c|c|c|}
\hline \multicolumn{6}{|c|}{ Calculated Pattern (Peak heights) } \\
\hline$d(\stackrel{\circ}{A})$ & $I$ & & $h k l$ & & $\begin{array}{c}2 \theta\left(^{\circ}\right) \\
\lambda=1.54056 \AA\end{array}$ \\
\hline 7.85 & 16 & 0 & 0 & 2 & 11.26 \\
\hline 7.74 & 13 & 0 & 1 & 0 & 11.42 \\
\hline 7.03 & 33 & 0 & $-i$ & 1 & 12.58 \\
\hline 6.85 & 100 & 0 & 1 & 1 & 12.92 \\
\hline 5.60 & 11 & 0 & -1 & 2 & 15.80 \\
\hline 5.42 & 5 & 0 & 1 & 2 & 16.34 \\
\hline 4.41 & 1 & 0 & -1 & 3 & 20.14 \\
\hline 3.87 & 2 & 0 & 2 & 0 & 22.96 \\
\hline 3.85 & 3 & 1 & 0 & 0 & 23.10 \\
\hline 3.78 & 9 & -1 & 0 & 1 & 23.52 \\
\hline 3.73 & 2 & 0 & 2 & 1 & 23.84 \\
\hline 3.69 & 2 & 1 & 0 & $i$ & 24.08 \\
\hline 3.55 & 6 & 0 & -1 & 4 & 25.06 \\
\hline 3.52 & 7 & -1 & 0 & $2+$ & 25.30 \\
\hline 3.471 & 8 & 1 & -1 & 1 & 25.64 \\
\hline 3.427 & 2 & 0 & 2 & 2 & 25.98 \\
\hline 3.297 & 7 & -1 & 1 & 2 & 27.02 \\
\hline 3.288 & 6 & -1 & -1 & 1 & 27.10 \\
\hline 3.222 & 5 & 1 & -1 & 2 & 27.66 \\
\hline 3.213 & 4 & 1 & 1 & 1 & 27.74 \\
\hline 3.175 & 61 & -1 & 0 & 3 & 28.08 \\
\hline 3.142 & 8 & 0 & 0 & 5 & 28.38 \\
\hline 3.123 & 43 & -1 & -1 & 2 & 28.56 \\
\hline 3.064 & 2 & 3 & 2 & 3 & 29.12 \\
\hline 3.029 & 1 & 1 & 0 & 3 & 29.46 \\
\hline 2.945 & 3 & 0 & -1 & 5 & 30.32 \\
\hline 2.827 & 3 & -1 & 2 & 1 & 31.62 \\
\hline 2.820 & 3 & -1 & 0 & 4 & 31.70 \\
\hline 2.805 & 1 & 0 & -2 & 4 & 31.88 \\
\hline 2.735 & 2 & 1 & 1 & 3 & 32.72 \\
\hline 2.638 & 2 & 1 & -1 & 4 & 34.36 \\
\hline 2.560 & 5 & 0 & -3 & 1 & 35.02 \\
\hline 2.534 & 1 & 3 & 3 & 1 & 35.40 \\
\hline 2.503 & 1 & 1 & -2 & 3 & 35.84 \\
\hline 2.477 & 1 & 0 & -3 & 2 & 36.24 \\
\hline 2.468 & 3 & 1 & 1 & 4 & 36.38 \\
\hline 2.377 & 1 & 1 & 0 & 5 & 37.82 \\
\hline 2.234 & 1 & 0 & -2 & 6 & 40.92 \\
\hline 2.157 & 2 & -1 & 3 & 2 & 41.84 \\
\hline 2.136 & 2 & 0 & 1 & $7+$ & 42.28 \\
\hline 2.106 & 1 & 1 & -2 & 5 & 42.90 \\
\hline 1.9529 & 1 & $i$ & 2 & 5 & 46.46 \\
\hline 1.7853 & 1 & -1 & 4 & 1 & 51.12 \\
\hline 1.7622 & 1 & -2 & 0 & 4 & 51.84 \\
\hline 1.6179 & 2 & -2 & -1 & 5 & 56.86 \\
\hline
\end{tabular}


$\mathrm{N}$-methylphenazinium Tetracyanoquinodimethanide, $\mathrm{C}_{25} \mathrm{H}_{15} \mathrm{~N}_{6}$ (triclinic) - continued

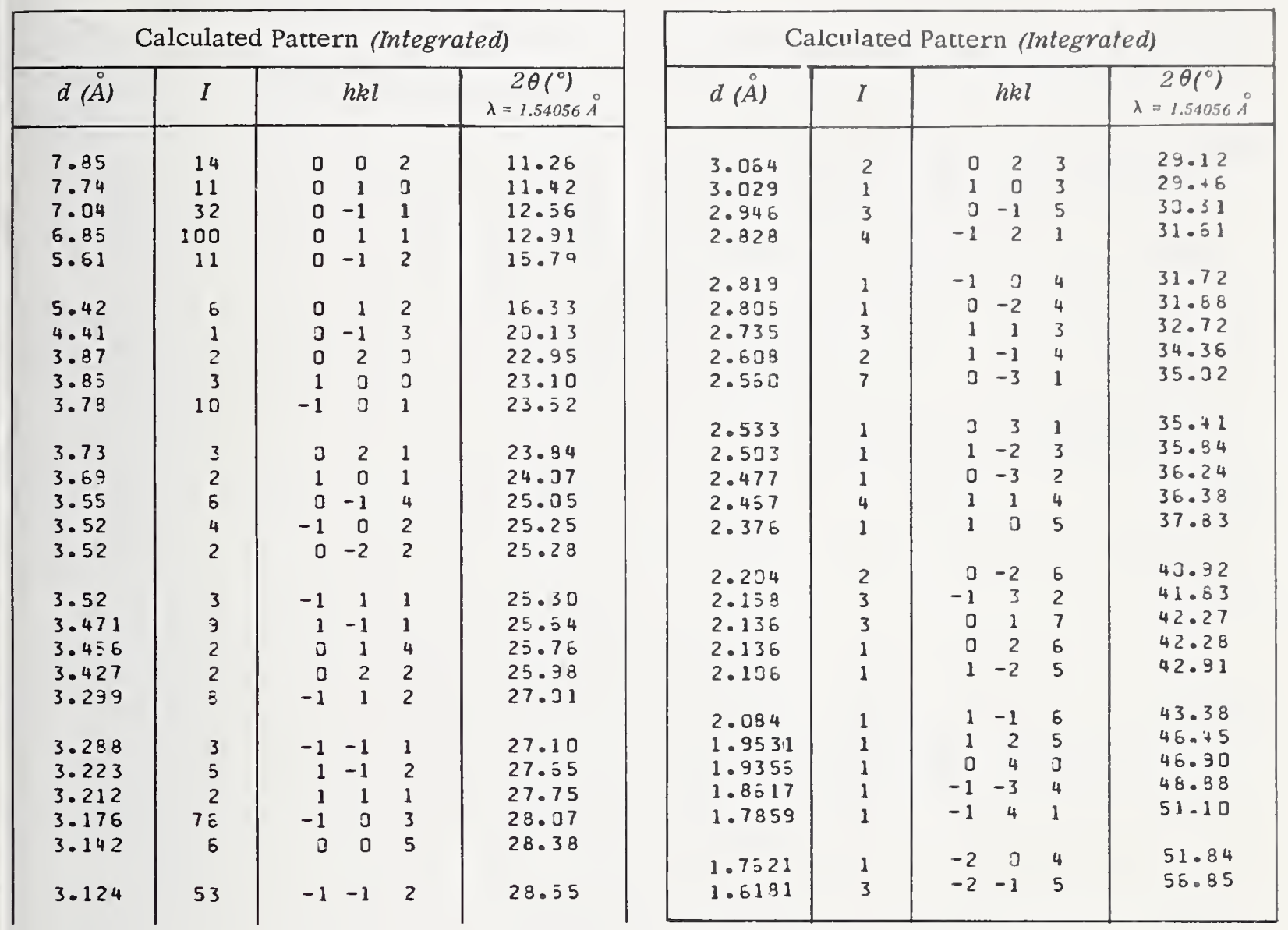




\section{Structure}

Tetragonal, $\mathrm{P}_{42} / \mathrm{mnm}(136), \mathrm{Z}=4$, [Sands, et al., 1959]

\section{Lattice parameters}

$a=10.87 \pm 0.01 \AA, c=3.96 \pm 0.01 \AA$ [ibid.]

\section{Scattering factors}

$\mathrm{Cl}^{-1} \cdot \mathrm{O}^{-1}[3 \cdot 3.1 \mathrm{~A}]$

$\mathrm{N}^{\circ} \quad[3 \cdot 3 . \mathrm{IB}]$

\section{Thermal parameters}

Isotropic [Sands et al., 1959]

\section{Density}

(calculated) $3.04 \mathrm{~g} / \mathrm{cm}^{3}$ [ibid.]

\section{Scale factor}

$$
7.198 \times 10^{4}
$$

\section{Reference}

Sands, D.E., A. Zalkin, and R.E. Elson (1959). The crystal structure of $\mathrm{NbOCI}_{3}$, Acta Cryst. 12, 2I-23.

\begin{tabular}{|c|c|c|c|c|c|c|}
\hline \multicolumn{7}{|c|}{ Calculated Pattern (Peak heights) } \\
\hline$d(\AA)$ & $I$ & & $h k$ & & & $\begin{array}{c}2 \theta\left({ }^{\circ}\right) \\
\lambda=1.54056 \mathrm{~A}\end{array}$ \\
\hline 7.69 & 80 & 1 & 1 & 0 & & 11.50 \\
\hline 4.36 & 100 & 2 & 1 & 0 & & 18.24 \\
\hline 3.72 & 83 & 1 & 0 & 1 & & 23.90 \\
\hline 3.52 & 36 & 1 & 1 & 1 & & 25.23 \\
\hline 3.44 & 7 & 3 & 1 & 0 & & 25.90 \\
\hline 3.67 & 2 & 2 & 1 & 1 & & 29.06 \\
\hline 3.02 & 1 & 3 & 2 & 0 & & 29.60 \\
\hline 2.750 & 1 & 2 & 2 & 1 & & 32.44 \\
\hline 2.717 & 59 & 4 & 0 & 0 & & 32.94 \\
\hline 2.673 & 9 & 3 & 0 & 1 & & 33.50 \\
\hline 2.530 & 2 & 4 & 1 & 0 & & 33.98 \\
\hline 2.590 & 11 & 3 & 1 & 1 & & 34.52 \\
\hline 2.562 & 2 & 3 & 3 & 0 & & 35.00 \\
\hline 2.399 & 1 & 3 & 2 & 1 & & 37.46 \\
\hline 2.194 & 17 & 4 & 1 & 1 & & 41.10 \\
\hline 2.132 & 3 & 5 & 1 & 0 & & 42.36 \\
\hline 2.019 & 9 & 5 & 2 & 0 & & 44.80 \\
\hline 1.9803 & 10 & $u$ & 0 & 2 & & 45.78 \\
\hline 1.9217 & 11 & 4 & 4 & 0 & & 47.26 \\
\hline 1.9171 & ij & $i$ & 1 & 2 & & 47.38 \\
\hline 1.9000 & 15 & 5 & 0 & 1 & + & 47.68 \\
\hline 1.8769 & 5 & 5 & 1 & 1 & & 48.46 \\
\hline 1.8639 & 1 & 5 & 3 & 0 & & 48.82 \\
\hline 1.8330 & 6 & 2 & 1 & 2 & & 49.68 \\
\hline $1 \cdot 787 j$ & 4 & 6 & 1 & 0 & & 51.06 \\
\hline 1.7155 & 1 & 3 & 1 & 2 & & 53.36 \\
\hline 1.6805 & 3 & 5 & 3 & 1 & & 54.34 \\
\hline 1.6240 & 1 & 6 & 3 & 0 & & 50.70 \\
\hline 1.6004 & 11 & 4 & 0 & 2 & & 57.54 \\
\hline 1.5764 & 1 & 6 & 2 & 1 & & 58.50 \\
\hline 1.5604 & 7 & 5 & 4 & 1 & & 59.16 \\
\hline 1.5373 & 2 & 5 & 5 & 0 & + & 60.14 \\
\hline 1.4507 & 2 & 5 & 1 & 2 & & 64.14 \\
\hline 1.4467 & 1 & 7 & 0 & 1 & & 04.34 \\
\hline $1.433 \mathrm{c}$ & 1 & 5 & 5 & 1 & & 65.02 \\
\hline 1.4274 & 1 & 7 & 3 & 0 & & 65.32 \\
\hline 1.4130 & 3 & 5 & 2 & 2 & & 66.04 \\
\hline 1.3019 & 1 & 6 & 5 & 0 & & 07.20 \\
\hline 1.3739 & 4 & 4 & 4 & 2 & & 67.92 \\
\hline 1.3580 & 1 & 8 & 0 & 0 & + & 69.08 \\
\hline 1.3255 & 2 & ó & 1 & 2 & & 71.00 \\
\hline 1.31心 & i & 1 & 0 & 3 & & 72.00 \\
\hline 1.2704 & 1 & 7 & 4 & 1 & + & 74.24 \\
\hline $1.21 b 1$ & 1 & 8 & 4 & 0 & & 78.60 \\
\hline 1.2143 & 2 & 5 & 5 & 2 & & 78.74 \\
\hline 1.1603 & 1 & 4 & 1 & 3 & & 81.48 \\
\hline 1.1791 & 1 & 9 & $\hat{c}$ & 0 & & 81.58 \\
\hline 1.1552 & 1 & 9 & 0 & 1 & & 83.64 \\
\hline 1.1380 & 1 & 6 & 5 & 2 & & 35.14 \\
\hline 1.1234 & 1 & 5 & 0 & 3 & & 86.10 \\
\hline
\end{tabular}




\begin{tabular}{|c|c|c|c|c|c|c|c|c|c|c|c|}
\hline \multicolumn{6}{|c|}{ Calculated Pattern (Integrated) } & \multicolumn{6}{|c|}{ Calculated Pattern (Integrated) } \\
\hline$d(\AA)$ & $I$ & & $h k l$ & & $\begin{array}{c}2 \theta\left({ }^{\circ}\right) \\
\lambda=1.54056 \AA\end{array}$ & $d(\AA)$ & $I$ & & $h k l$ & & $\begin{array}{c}2 \theta\left(^{\circ}\right) \\
\lambda=1.54056 \AA\end{array}$ \\
\hline 7.69 & 79 & 1 & 1 & 0 & 11.50 & 1.4135 & 5 & 5 & 2 & 2 & 6ć.04 \\
\hline $4 \cdot 36$ & 100 & 2 & 1 & 0 & 18.23 & 1.3918 & 2 & 6 & 5 & 0 & 67.21 \\
\hline 3.72 & 90 & 1 & 0 & 1 & 23.90 & 1.3789 & 6 & 4 & 4 & 2 & 67.92 \\
\hline 3.52 & 39 & 1 & 1 & 1 & 25.28 & 1.3537 & 2 & 8 & 0 & 0 & 69.07 \\
\hline 3.44 & 7 & 3 & 1 & 0 & 25.90 & 1.3573 & 1 & 5 & 3 & 2 & 09.15 \\
\hline 3.07 & 3 & 2 & 1 & 1 & 29.06 & 1.3266 & 3 & 6 & 1 & 2 & 70.99 \\
\hline 3.02 & 1 & 3 & 2 & $c$ & 29.61 & 1.3104 & 2 & 1 & 0 & 3 & 72.01 \\
\hline 2.758 & 1 & 2 & 2 & 1 & 32.44 & 1.3009 & 1 & 1 & 1 & 3 & 72.61 \\
\hline 2.717 & 72 & 4 & 0 & 0 & 32.93 & $1.276 ́ 3$ & 1 & 8 & 1 & 1 & 74.24 \\
\hline 2.673 & 10 & 3 & 0 & 1 & 33.49 & 1.2763 & 1 & 7 & 4 & 1 & $74 \cdot 24$ \\
\hline 2.636 & 2 & 4 & 1 & 0 & 33.98 & 1.2540 & 1 & 6 & 3 & 2 & 75.80 \\
\hline 2.596 & 13 & 3 & 1 & 1 & $34 \cdot 52$ & 1.2188 & 1 & 6 & 6 & 1 & 78.39 \\
\hline 2.502 & 2 & 3 & 3 & 0 & 34.99 & 1.2153 & 2 & 8 & 4 & 0 & 70.60 \\
\hline 2.399 & 1 & 3 & 2 & 1 & 37.46 & 1.2142 & 2 & 5 & 5 & 2 & 78.75 \\
\hline $2 \cdot 195$ & 22 & 4 & 1 & 1 & 41.10 & 1.2038 & 1 & 7 & 5 & 1 & 79.56 \\
\hline $2 \cdot 132$ & 11 & 5 & 1 & 0 & $42 \cdot 36$ & 1.2004 & 1 & 9 & 1 & 0 & 79.84 \\
\hline 2.019 & 13 & 5 & 2 & 0 & 44.87 & 1.1803 & 1 & 4 & 1 & 3 & 81.48 \\
\hline 1.3800 & 14 & 0 & 0 & 2 & $45 \cdot 79$ & 1.1790 & 1 & 9 & 2 & c & $81 \cdot 59$ \\
\hline i.9216 & 14 & 4 & 4 & 0 & 47.26 & 1.1552 & 2 & 9 & 0 & 1 & 83.64 \\
\hline $1 \cdot 9174$ & 3 & 1 & 1 & 2 & 47.37 & $1 \cdot 1386$ & 2 & 6 & 5 & 2 & $85 \cdot 14$ \\
\hline 1.9057 & 19 & 5 & 0 & 1 & 47.63 & 1.1283 & 2 & 5 & 0 & 3 & 36.11 \\
\hline 1.9057 & 5 & 4 & 3 & 1 & 47.68 & 1.1223 & 1 & 5 & 1 & 3 & 86.068 \\
\hline 1.8771 & 9 & 5 & 1 & 1 & 48.46 & 1.1203 & 1 & 8 & 0 & 2 & 80.87 \\
\hline 1.9642 & 2 & 5 & 3 & 0 & 48.81 & 1.0632 & 2 & 9 & 4 & 1 & 92.80 \\
\hline 1.8337 & 9 & 2 & 1 & 2 & 49.68 & 1.0558 & 1 & 9 & 5 & 0 & 93.70 \\
\hline 1.7370 & 6 & 6 & 1 & u & 51.07 & 1.0420 & 2 & 5 & 4 & 3 & 95.33 \\
\hline 1.7157 & 2 & 3 & 1 & 2 & 53.35 & 1.0358 & 1 & 8 & 4 & 2 & 96.09 \\
\hline 1.6066 & 5 & 5 & 3 & 1 & $54 \cdot 35$ & 1.0293 & 1 & 10 & 2 & 1 & 90.90 \\
\hline 1.6288 & 1 & 6 & 1 & 1 & 56.45 & 1.0265 & 1 & 9 & 1 & 2 & $97.2 b$ \\
\hline 1.6204 & 2 & 6 & 3 & 0 & 56.77 & 1.0130 & 1 & 9 & 2 & 2 & 99.00 \\
\hline 1.6003 & 16 & 4 & 0 & 2 & 57.55 & .9492 & 1 & 10 & 1 & 2 & 108.48 \\
\hline 1.5766 & 2 & 6 & 2 & 1 & 58.49 & .9316 & 1 & 9 & 5 & 2 & 111.55 \\
\hline 1.5603 & 11 & 5 & 4 & 1 & 59.17 & . 9302 & 1 & 4 & 0 & 4 & 111.80 \\
\hline 1.5372 & 1 & 7 & 1 & 0 & 60.14 & .0073 & 1 & 10 & 6 & 1 & 110.20 \\
\hline 1.5372 & 3 & 5 & 5 & 0 & 60.14 & .8911 & 1 & 9 & 0 & 3 & 119.64 \\
\hline 1.4997 & 1 & 6 & 3 & 1 & 61.81 & & 1 & 4 & 4 & 4 & $122 \cdot 15$ \\
\hline 1.4508 & 4 & 5 & 1 & 2 & 04.14 & .8467 & 1 & 9 & 4 & 3 & 130.94 \\
\hline 1.4457 & 1 & 7 & 0 & 1 & 64.39 & .7842 & 1 & 9 & 9 & 2 & 158.38 \\
\hline 1.4331 & 1 & 5 & 5 & 1 & 65.03 & .7834 & 1 & 11 & 8 & 1 & 159.01 \\
\hline 1.4273 & 1 & 7 & 3 & 0 & 65.32 & & & & & & \\
\hline
\end{tabular}


Structure

Orthorhombic, Pnma(62), $\mathrm{z}=4$ [Breneman and willett, 1967]

Lattice parameters

$a=9.35 \pm 0.02, b=7.94 \pm 0.01, \quad c=14.69 \pm 0.02 \AA$ [ibid.]

\section{Scattering factors}

$\mathrm{P}^{\circ}, \mathrm{Br}^{\circ}[3 \cdot 3 \cdot 1 \mathrm{~A}]$

\section{Thermal parameters}

Isotropic: $\mathrm{P} .90 ; \mathrm{Br}(1) 2.01 ; \mathrm{Br}(2) 2.71$; $\mathrm{Br}(3) 2.73 ; \mathrm{Br}(4) 2.57 ; \mathrm{Br}(5) 1.90$;

$\operatorname{Br}(6) 3.58$

\section{Density}

(calculated) $3.60 \mathrm{~g} / \mathrm{cm}^{3}$ [Breneman and Willett, 1967]

Scale factor

$37.67 \times 10^{4}$

\section{Reference}

Breneman, G.I. and R.D.Willett (1967). The crystal structure of phosphorus heptabromide, $\mathrm{PBr}_{7}$, Acta Cryst. 23, 467-471.

\begin{tabular}{|c|c|c|c|c|c|}
\hline \multicolumn{6}{|c|}{ Calculated Pattern (Peak heights) } \\
\hline$d(\stackrel{\circ}{A})$ & $I$ & \multicolumn{3}{|c|}{$h k l$} & $\begin{array}{c}2 \theta\left(^{\circ}\right) \\
\lambda=1.54056 \mathrm{~A} \\
\end{array}$ \\
\hline 6.99 & 3 & 0 & 1 & 1 & 12.56 \\
\hline 5.77 & 4 & 1 & 0 & 2 & $15 \cdot 34$ \\
\hline 5.60 & 2 & 1 & 1 & 1 & 15.82 \\
\hline 4.67 & 15 & 1 & 1 & $2+$ & 18.98 \\
\hline 4.45 & 3 & 2 & 0 & 1 & 19.92 \\
\hline 4.34 & 7 & 1 & 0 & 3 & 20.46 \\
\hline 4.17 & 2 & 0 & 1 & 3 & 21.30 \\
\hline 4.03 & 17 & 2 & 1 & 0 & 22.04 \\
\hline 3.97 & 51 & 0 & 2 & 0 & $22 \cdot 38$ \\
\hline 3.88 & 6 & 2 & 1 & 1 & 22.88 \\
\hline 3.80 & 37 & 1 & 1 & 3 & $\angle 3.36$ \\
\hline 3.67 & 10 & 0 & C & 4 & 24.22 \\
\hline 3.53 & 18 & 2 & 1 & 2 & 25.20 \\
\hline 3.49 & 2 & 0 & 2 & 2 & 25.48 \\
\hline 3.42 & 7 & 1 & 0 & 4 & 26.04 \\
\hline 3.27 & 100 & 1 & 2 & 2 & 27.24 \\
\hline 3.14 & 48 & 1 & 1 & 4 & 28.40 \\
\hline 3.03 & 33 & 2 & 2 & 0 & 29.50 \\
\hline 2.928 & 3 & 1 & 2 & 3 & 30.50 \\
\hline 2.888 & 31 & 2 & 0 & 4 & 30.94 \\
\hline
\end{tabular}

\begin{tabular}{|c|c|c|c|c|c|c|}
\hline \multicolumn{7}{|c|}{ Calculated Pattern (Peak heights) } \\
\hline$d(\AA)$ & $I$ & & $h k$ & & & $\begin{array}{c}2 \theta\left(^{\circ}\right) \\
\lambda=1.54056 .\end{array}$ \\
\hline 2.870 & 26 & 3 & 0 & 2 & & 31.14 \\
\hline 2.847 & 27 & 3 & 1 & 1 & & 31.40 \\
\hline 2.803 & 6 & 1 & 0 & 5 & & 31.90 \\
\hline 2.714 & 23 & 2 & 1 & 4 & & 32.98 \\
\hline 2.698 & 15 & 3 & 1 & 2 & + & 33.18 \\
\hline i. 644 & 4 & 1 & 1 & 5 & & 33.88 \\
\hline 2.605 & 1 & $\mathrm{C}$ & 3 & 1 & & 34.40 \\
\hline 2.574 & 2 & 2 & 2 & 3 & & 34.82 \\
\hline 2.509 & 4 & 1 & 3 & 1 & & 35.75 \\
\hline 2.495 & 5 & 3 & 1 & 3 & & 35.95 \\
\hline 2.487 & 7 & 2 & 0 & 5 & & $36.0 B$ \\
\hline 2.417 & 1 & 3 & 2 & 1 & & 37.16 \\
\hline 2.406 & 1 & 1 & 3 & 2 & & 37.34 \\
\hline 2.368 & 5 & 1 & 0 & 6 & & 37.96 \\
\hline i. 326 & 2 & 3 & 2 & 2 & + & 38.68 \\
\hline 2.309 & 2 & 4 & 0 & 1 & & 38.98 \\
\hline 2.333 & 3 & 2 & 3 & 0 & & 39.08 \\
\hline$\angle .290$ & 3 & 1 & $\hat{z}$ & 5 & & $3 y .32$ \\
\hline 2.275 & 7 & 2 & 3 & 1 & & 39.58 \\
\hline 2.270 & 10 & 1 & 1 & 6 & & 39.68 \\
\hline 2.260 & 3 & 1 & 3 & 3 & & 39.86 \\
\hline 2.242 & 9 & 4 & 1 & 0 & & 40.18 \\
\hline$\angle .197$ & 4 & 2 & 3 & 2 & & 41.04 \\
\hline 2.169 & 2 & 2 & 0 & 6 & & 41.60 \\
\hline 2.145 & 1 & 4 & 1 & 2 & & 42.10 \\
\hline 2.108 & 10 & 4 & 0 & 3 & + & 42.86 \\
\hline 2.092 & 19 & 1 & 3 & 4 & + & 43.20 \\
\hline 2.086 & 12 & 2 & 3 & 3 & + & 43.34 \\
\hline 2.047 & 2 & 1 & 0 & 7 & & 44.20 \\
\hline 2.039 & 6 & 3 & 2 & 4 & & 44.40 \\
\hline 2.034 & 6 & 1 & 2 & 6 & & 44.50 \\
\hline 2.015 & 15 & 4 & 2 & 0 & & 44.96 \\
\hline 1.9985 & 2 & 3 & 3 & 1 & & 45.34 \\
\hline 1.9853 & 16 & 0 & 4 & 0 & + & 45.66 \\
\hline 1.9722 & 3 & 4 & 0 & 4 & & 45.98 \\
\hline 1.9513 & 8 & 2 & 3 & 4 & & 46.50 \\
\hline 1.9458 & 5 & 3 & 3 & 2 & & 46.64 \\
\hline 1. 9141 & 2 & 2 & 0 & 7 & + & 47.46 \\
\hline 1.9035 & 1 & 2 & 2 & 6 & & 47.74 \\
\hline 1.8820 & 3 & 3 & 2 & 5 & & 48.32 \\
\hline 1.8711 & 2 & 3 & 1 & 6 & & 48.62 \\
\hline 1.8553 & 4 & 3 & 3 & 3 & + & 48.78 \\
\hline 1.8199 & 2 & 1 & 2 & 7 & & 50.08 \\
\hline 1.8125 & 2 & 5 & 0 & 2 & & 53.30 \\
\hline 1.8064 & 2 & 5 & 1 & 1 & + & 50.48 \\
\hline 1.7654 & 3 & 4 & 2 & 4 & + & 51.74 \\
\hline 1.7571 & 3 & 1 & 1 & 8 & & 52.00 \\
\hline 1.7521 & 5 & 4 & 3 & 0 & & 52.16 \\
\hline 1.7471 & 3 & 5 & 0 & 3 & + & 52.32 \\
\hline 1.7324 & 10 & 3 & 2 & 6 & & 52.80 \\
\hline
\end{tabular}




\begin{tabular}{|c|c|c|c|c|c|c|}
\hline \multicolumn{7}{|c|}{ Calculated Pattern (Peak heights) } \\
\hline$d(\stackrel{\circ}{A})$ & $I$ & & $h k$ & & & $2 \theta\left({ }^{\circ}\right)$ \\
\hline 1.7167 & 1 & 1 & 4 & 4 & & 53.32 \\
\hline 1.7090 & 2 & 2 & 0 & 8 & & 53.58 \\
\hline 1.6909 & 1 & 4 & כ & 6 & & 54.20 \\
\hline 1.6806 & 2 & 5 & 2 & 1 & & 54.56 \\
\hline 1.6777 & 4 & 2 & 3 & 6 & & 54.66 \\
\hline 1.6665 & 2 & 5 & 3 & 4 & & 55.06 \\
\hline 1.6626 & 2 & 3 & 3 & 5 & & $55 \cdot 20$ \\
\hline 1.6538 & 2 & 4 & 1 & 6 & & 55.52 \\
\hline 1.6489 & 4 & 5 & 2 & 2 & + & 55.70 \\
\hline 1.6407 & 7 & 1 & 2 & 8 & & 56.00 \\
\hline 1.6359 & 7 & 2 & 4 & 4 & & 56.18 \\
\hline 1.6316 & 7 & 3 & 4 & 2 & + & $56 \cdot 34$ \\
\hline 1.6200 & 2 & 1 & 4 & 5 & & 56.78 \\
\hline 1.5989 & 4 & 5 & 2 & 3 & + & 57.60 \\
\hline 1.5819 & 5 & 3 & 0 & 8 & & 58.28 \\
\hline 1.5580 & 3 & 6 & 0 & 0 & + & 57.26 \\
\hline 1.5514 & 4 & 2 & 3 & 7 & + & 59.54 \\
\hline 1.5208 & 2 & 6 & 1 & 1 & + & 60.86 \\
\hline $1.519 \mathrm{U}$ & 3 & 5 & 3 & 1 & & 60.94 \\
\hline 1.5039 & 1 & 2 & 5 & 0 & & 61.62 \\
\hline 1.4908 & 3 & 1 & 5 & 3 & + & 62.22 \\
\hline 1.4861 & 2 & 5 & 0 & 6 & & 62.44 \\
\hline 1.4692 & 3 & 0 & 0 & 10 & + & 63.24 \\
\hline 1.4659 & 4 & 5 & 2 & 5 & & 63.40 \\
\hline 1.4597 & 1 & 6 & 1 & 3 & & 63.70 \\
\hline 1.4455 & 2 & 4 & 4 & 3 & + & 64.40 \\
\hline 1.4403 & 2 & 1 & 5 & 4 & & 64.56 \\
\hline 1.4250 & 1 & 4 & 3 & 6 & & 65.44 \\
\hline $\begin{array}{l}1.4212 \\
1.4101\end{array}$ & $\begin{array}{l}1 \\
2\end{array}$ & $\begin{array}{l}3 \\
5\end{array}$ & $\begin{array}{l}1 \\
3\end{array}$ & $\begin{array}{l}9 \\
4\end{array}$ & & $\begin{array}{l}65.64 \\
66.22\end{array}$ \\
\hline 1.4082 & 3 & 3 & 5 & 1 & & 65.32 \\
\hline 1.3916 & 1 & 2 & 5 & 4 & & 67.22 \\
\hline 1.3894 & 2 & 3 & 5 & 2 & & 67.34 \\
\hline 1.3818 & 2 & 1 & 5 & 5 & & 67.76 \\
\hline $1.3490^{\circ}$ & 3 & 6 & 2 & 4 & & 69.64 \\
\hline 1.3453 & 2 & 4 & 3 & 7 & & 69.86 \\
\hline 1.3232 & 1 & 0 & 6 & 0 & & 71.20 \\
\hline 1.3216 & 2 & 2 & 2 & 10 & & 71.30 \\
\hline 1.3184 & 1 & 1 & 5 & 6 & & 71.50 \\
\hline 1.3171 & 1 & 0 & 1 & 11 & & 71.58 \\
\hline 1.2953 & 1 & 2 & 4 & 8 & & $72 \cdot 98$ \\
\hline 1.2925 & 1 & 5 & 1 & 8 & & 73.16 \\
\hline 1.2898 & 1 & 1 & 6 & 2 & & $73 \cdot 34$ \\
\hline 1.2814 & 1 & 2 & 5 & 6 & & 73.90 \\
\hline 1.2602 & 1 & 3 & 2 & 10 & & 75.36 \\
\hline 1.2442 & 1 & 5 & 2 & 8 & & 76.50 \\
\hline 1.2398 & 1 & 7 & 1 & 4 & & 76.82 \\
\hline 1.2371 & 3 & 3 & 4 & 8 & + & 77.02 \\
\hline 1.2256 & 2 & 6 & 4 & 0 & & 77.88 \\
\hline
\end{tabular}

\begin{tabular}{|c|c|c|c|c|c|}
\hline \multicolumn{6}{|c|}{ Calculated Pattern (Integrated) } \\
\hline$d(\stackrel{\circ}{A})$ & $I$ & & $h k$ & & $\begin{array}{c}2 \theta\left(^{\circ}\right) \\
\lambda=1.54056 \AA\end{array}$ \\
\hline 6.98 & 2 & 0 & 1 & 1 & 12.65 \\
\hline 5.78 & 3 & 1 & 0 & 2 & 15.33 \\
\hline 5.60 & 1 & 1 & 1 & 1 & 15.82 \\
\hline 4.68 & 3 & 2 & 0 & 0 & 18.97 \\
\hline 4.67 & 11 & 1 & 1 & 2 & 18.98 \\
\hline 4.45 & 2 & 2 & 0 & 1 & 19.91 \\
\hline 4.34 & 7 & 1 & 0 & 3 & 20.40 \\
\hline 4.17 & 1 & 0 & 1 & 3 & 21.30 \\
\hline 4.03 & 15 & 2 & 1 & 3 & 22.05 \\
\hline 3.97 & 47 & 0 & 2 & 0 & 22.38 \\
\hline 3.94 & 2 & 2 & 0 & 2 & 22.53 \\
\hline 3.89 & 6 & 2 & 1 & 1 & 22.87 \\
\hline 3.81 & 37 & 1 & 1 & 3 & 23.35 \\
\hline 3.67 & 10 & 0 & 0 & 4 & 24.21 \\
\hline 3.55 & 2 & 1 & 2 & 1 & 25.39 \\
\hline 3.53 & 17 & 2 & 1 & 2 & 25.19 \\
\hline 3.49 & 2 & 3 & 2 & 2 & 25.48 \\
\hline 3.42 & 7 & 1 & 0 & 4 & 26.05 \\
\hline 3.27 & 100 & 1 & 2 & 2 & 27.23 \\
\hline $3 \cdot 14$ & 49 & 1 & 1 & 4 & 28.40 \\
\hline 3.03 & 34 & 2 & 2 & 0 & 29.49 \\
\hline 2.929 & 3 & 1 & 2 & 3 & 30.50 \\
\hline 2.888 & 31 & 2 & 0 & 4 & 30.94 \\
\hline 2.869 & 26 & 3 & 0 & 2 & 31.15 \\
\hline 2.846 & 27 & 3 & 1 & 1 & 31.40 \\
\hline 2.803 & 5 & 1 & 0 & 5 & 31.90 \\
\hline 2.714 & 24 & 2 & 1 & 4 & 32.98 \\
\hline 2.698 & 9 & 3 & 1 & 2 & 33.17 \\
\hline 2.696 & 8 & 0 & 2 & 4 & 33.20 \\
\hline 2.643 & 5 & 1 & 1 & 5 & 33.89 \\
\hline 2.605 & 1 & 0 & 3 & 1 & 34.40 \\
\hline 2.574 & 2 & 2 & 2 & 3 & 34.82 \\
\hline 2.509 & 4 & 1 & 3 & 1 & 35.76 \\
\hline 2.496 & 4 & 3 & 1 & 3 & 35.95 \\
\hline 2.488 & 7 & 2 & 0 & 5 & 36.08 \\
\hline 2.418 & 2 & 3 & 2 & 1 & 37.15 \\
\hline 2.406 & 1 & 1 & 3 & 2 & $37 \cdot 34$ \\
\hline 2.368 & 5 & 1 & 0 & 6 & 37.96 \\
\hline 2.328 & 1 & 0 & 3 & 3 & 38.64 \\
\hline $2 \cdot 325$ & 2 & 3 & 2 & 2 & 38.69 \\
\hline 2.308 & 2 & 4 & 0 & 1 & 36.98 \\
\hline 2.303 & 2 & 2 & 3 & 0 & 39.08 \\
\hline 2.290 & 3 & 1 & 2 & 5 & 39.32 \\
\hline 2.275 & 7 & 2 & 3 & 1 & 39.57 \\
\hline $2 \cdot 270$ & 7 & 1 & 1 & 6 & 39.68 \\
\hline 2.259 & 3 & 1 & 3 & 3 & 39.87 \\
\hline 2.242 & 10 & 4 & 1 & 0 & 40.18 \\
\hline 2.198 & 4 & 2 & 3 & 2 & 41.04 \\
\hline 2.169 & 2 & 2 & 0 & 6 & 41.60 \\
\hline 2.145 & 2 & 4 & 1 & 2 & 42.10 \\
\hline
\end{tabular}


Phosphorus Bromide, $\mathrm{PBr}_{7}$ (orthorhombic) - continued

\begin{tabular}{|c|c|c|c|c|c|c|c|c|c|c|c|}
\hline \multicolumn{6}{|c|}{ Calculated Pattern (Integrated) } & \multicolumn{6}{|c|}{ Calculated Pattern (Integrated) } \\
\hline$d(\AA)$ & $I$ & \multicolumn{3}{|c|}{$h k l$} & $\begin{array}{c}2 \theta\left({ }^{\circ}\right) \\
\lambda=1.54056 \mathrm{~A}\end{array}$ & $d(\AA)$ & $I$ & \multicolumn{3}{|c|}{$h k l$} & $\begin{array}{c}2 \theta\left(^{\circ}\right) \\
\lambda=1.54056 \AA\end{array}$ \\
\hline 2.109 & 7 & 4 & 0 & 3 & 42.83 & 1.5988 & 1 & 0 & 1 & 9 & 57.63 \\
\hline 2.108 & 6 & 2 & 2 & 5 & $42 \cdot 37$ & 1.5821 & 7 & 3 & 0 & 8 & $58 \cdot<7$ \\
\hline 2.093 & 12 & 1 & 3 & 4 & 43.19 & 1.5583 & 4 & 6 & 0 & 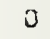 & 59.25 \\
\hline 2.092 & 10 & $\ddot{2}$ & 1 & 6 & 43.20 & 1.5568 & 1 & 1 & 5 & 1 & 59.31 \\
\hline 2.084 & 5 & 2 & 3 & 3 & 43.38 & 1.5516 & $?$ & 2 & 4 & 5 & 59.53 \\
\hline 2.084 & 2 & 0 & 2 & 6 & 43.39 & 1. 5512 & 3 & 2 & 3 & 7 & 59.55 \\
\hline C. 048 & 2 & 1 & 0 & 7 & 44.19 & 1.5496 & 1 & o & 0 & 1 & 59.61 \\
\hline 2.039 & $\overline{0}$ & 3 & 2 & 4 & 44.39 & 1.5213 & 1 & 1 & 4 & 6 & 60.84 \\
\hline 2.034 & 4 & 1 & 2 & 6 & 44.51 & 1.5209 & 1 & ó & 1 & 1 & 60.86 \\
\hline 2.014 & 18 & 4 & $\dot{z}$ & 3 & 44.97 & 1.5191 & 3 & 5 & 3 & 1 & 60.94 \\
\hline 1.9980 & $?$ & 3 & 3 & 1 & 45.34 & 1.5035 & 1 & 2 & 5 & 0 & 61.63 \\
\hline 1.9853 & 19 & u & 4 & 0 & 45.67 & 1.4912 & 3 & 1 & 5 & 3 & 62.20 \\
\hline 1.9828 & 1 & I & 1 & 7 & 45.72 & 1.4903 & 2 & 1 & 2 & 9 & 62.24 \\
\hline 1.9719 & 3 & 4 & 0 & 4 & 45.99 & 1.4894 & 1 & 1 & 3 & 8 & 52.28 \\
\hline 1.9512 & 9 & 2 & 3 & 4 & 46.50 & 1.4861 & 1 & 5 & 0 & 6 & 62.44 \\
\hline 1.9453 & 1 & 3 & 3 & 2 & 46.65 & 1.4697 & 1 & 3 & 2 & 8 & 63.22 \\
\hline 1.9145 & 1 & 2 & 0 & 7 & 47.45 & 1.4690 & 2 & 0 & 0 & 10 & 63.25 \\
\hline 1.9138 & 1 & 4 & 1 & 4 & 47.47 & 1.4663 & 4 & 5 & 2 & 5 & 63.39 \\
\hline 1.9034 & 1 & 2 & 2 & 6 & 47.74 & 1.4590 & 1 & 5 & 1 & 3 & 53.70 \\
\hline I. 8823 & 3 & 3 & 2 & 5 & 46.31 & 1.4456 & 3 & 4 & 4 & 3 & $64.4 \mathrm{~J}$ \\
\hline 1.8711 & 2 & 3 & 1 & $\bar{\sigma}$ & 48.62 & 1.4440 & 1 & 4 & 0 & 8 & 64.48 \\
\hline 1.8653 & 4 & 3 & 3 & 3 & $4 \varepsilon .78$ & 1.4402 & 3 & 1 & 5 & 4 & 64.67 \\
\hline 1.8628 & 2 & 4 & 2 & 3 & 48.85 & 1.4248 & 1 & 4 & 3 & 6 & 65.45 \\
\hline 1.8198 & 2 & 1 & 2 & 7 & 50.08 & 1.4225 & 1 & 3 & 1 & s & 65.57 \\
\hline 1.8122 & 2 & 5 & 0 & 2 & 50.31 & 1.4102 & 3 & 5 & 3 & 4 & 50.22 \\
\hline 1.8064 & 2 & 5 & 1 & 1 & 50.48 & 1.4084 & 4 & 3 & 5 & 1 & 66.31 \\
\hline 1. 8050 & 1 & 1 & 4 & 3 & 53.52 & 1.3992 & 1 & 4 & 4 & 4 & 66.82 \\
\hline 1.7661 & 2 & 4 & 2 & 4 & 51.72 & 1.3915 & 1 & 2 & 5 & 4 & 67.22 \\
\hline 1.7649 & 2 & 1 & 3 & 6 & 51.75 & 1.3894 & 1 & 3 & 5 & 2 & 67.34 \\
\hline 1.7571 & 2 & 1 & 1 & 8 & 52.00 & 1.3817 & 2 & 1 & 5 & 5 & 67.77 \\
\hline 1.7520 & 5 & 4 & 3 & 3 & 52.16 & 1.3491 & 4 & 5 & 2 & 4 & 69.63 \\
\hline 1.7469 & 1 & 5 & 0 & 3 & 52.33 & 1.3449 & 1 & 4 & 3 & 7 & 59.86 \\
\hline 1.7462 & 1 & 0 & 4 & 4 & 52.35 & 1. 3233 & 1 & 0 & 6 & 0 & 71.19 \\
\hline 1.732 .3 & 12 & 3 & 2 & 6 & 52.80 & 1.3215 & 2 & 2 & 2 & 10 & 71.31 \\
\hline 1.7160 & 1 & 1 & 4 & 4 & 53.32 & 1.3190 & 1 & 1 & 5 & 6 & 71.46 \\
\hline 1.7091 & 3 & 2 & 0 & 8 & 53.58 & 1.3170 & 1 & 0 & 1 & 11 & 71.59 \\
\hline 1.6907 & 1 & 4 & 0 & 6 & 54.21 & 1.2952 & 1 & 2 & 4 & 8 & 72.99 \\
\hline 1.6806 & 2 & 5 & 2 & 1 & 54.56 & 1.2927 & 1 & 5 & 1 & $\theta$ & 73.15 \\
\hline 1.6776 & 5 & 2 & 3 & 6 & 54.67 & $1.285 y$ & 1 & 1 & 6 & 2 & 73.33 \\
\hline 3.6664 & 1 & 5 & 0 & 4 & 55.06 & 1.2813 & 1 & 2 & 5 & 6 & 73.91 \\
\hline 1.6631 & 1 & 3 & 3 & 5 & 55.18 & 1.2601 & 2 & 3 & 2 & 10 & 75.37 \\
\hline 1.6536 & 2 & 4 & 1 & 6 & 55.53 & 1.2442 & 1 & 5 & 2 & 8 & 76.50 \\
\hline 1.6496 & 2 & 4 & 3 & 3 & 55.67 & 1.2399 & 1 & 7 & 1 & 4 & 76.82 \\
\hline 1. 6486 & 3 & 5 & 2 & 2 & 55.71 & 1.237 .2 & 4 & 3 & 4 & 8 & 77.01 \\
\hline 1.6407 & 8 & 1 & 2 & 8 & 56.30 & 1.2359 & 1 & 6 & 1 & 7 & 77.11 \\
\hline 1.6358 & 5 & 2 & 4 & 4 & 56.18 & 1.2257 & 2 & 6 & 4 & 0 & 77.87 \\
\hline 1.6324 & 4 & 3 & 4 & 2 & 56.31 & & & & & & \\
\hline 1.6309 & 4 & 5 & 1 & 4 & 56.37 & & & & & & \\
\hline 1. 6199 & $\frac{1}{3}$ & 1 & 4 & 5 & 56.78 & & & & & & \\
\hline 1.5990 & 3 & 5 & 2 & 3 & 57.50 & & & & & & \\
\hline
\end{tabular}




\section{Structure}

Monoclinic, P2 1 /c (14): $\mathrm{Z}=4$ [Housty and Hospital, 1966]

\section{Lattice parameters}

$a=5.65 \pm 0.01, b=9.68 \pm 0.02, c=22.33 \pm 0.05 \AA$ $\beta=137^{\circ}$ [ibid.]

\section{Scattering factors}

$$
\mathrm{H}^{\circ}, \mathrm{C}^{\circ}, \mathrm{O}^{\circ}[3.3 .1 \mathrm{~A}]
$$

\section{Atomic positions}

Table 1 [Housty and Hospital, 1966]

\section{Thermal parameters}

Isotropic: $\mathrm{B}=4.0 \AA^{2}$ for carbon and oxygen $\mathrm{B}=3.0 \AA^{2}$ for hydrogen

\section{Density}

(calculated) $1.275 \mathrm{~g} / \mathrm{cm}^{3}$ [ibid.]

\section{Scale factor}

$2.867 \times .10^{4}$

\section{Polymorphism}

The existence of a second polymorph has been reported by Dupré la Tour [1935].

\section{Additional patterns}

1. PDF card 9-721[Whitney and corvin, 1949] It may represent a different polymorph.

\section{Reference}

Dupré la Tour, F. (1935). Polymorphisme dans la série des diacides gras normaux, Compt. Rend. 201, 479-481.

Housty, J. and M. Hospital (1966). Localisation des atomes d'hydrogene dans l'acide pimélique, $\mathrm{COOH}-\left[\mathrm{CH}_{2}\right]_{5}-\mathrm{COOH}$, Acta Cryst. 21, 29-34.

Whitney, J. and I. Corvin (1949). Pimelic acid, Anal. Chem. 21, 191-192.

\begin{tabular}{|c|c|c|c|c|c|}
\hline \multicolumn{6}{|c|}{ Calculated Pattern (Peak heights) } \\
\hline$d(\stackrel{\circ}{)}$ & $I$ & & $h k l$ & & $\begin{array}{c}2 \theta\left({ }^{\circ}\right) \\
\lambda=1.54056 \mathrm{~A}\end{array}$ \\
\hline 7.61 & 36 & 0 & 0 & 2 & 11.62 \\
\hline 5.98 & 2 & 0 & 1 & 2 & 14.80 \\
\hline 5.22 & 1 & -1 & 0 & 4 & 16.96 \\
\hline 4.84 & 28 & 0 & 2 & 0 & 18.32 \\
\hline 4.69 & 2 & -1 & 1 & 2 & 18.90 \\
\hline 4.61 & 74 & 0 & 2 & 1 & 19.22 \\
\hline 4.08 & 1 & 0 & 2 & 2 & 21.74 \\
\hline 3.85 & 100 & 1 & 0 & 0 & 23.06 \\
\hline 3.70 & 5 & -1 & 0 & 6 & 24.02 \\
\hline 3.67 & 8 & -1 & 2 & 3 & $24 \cdot 20$ \\
\hline 3.59 & 4 & -1 & 2 & 2 & 24.76 \\
\hline 3.50 & 1 & 3 & 2 & 3 & 25.40 \\
\hline 3.343 & 62 & -1 & 2 & 1 & 26.54 \\
\hline 3.276 & 3 & -1 & 2 & 5 & 27.20 \\
\hline 3.157 & 2 & 0 & 3 & 1 & 28.24 \\
\hline 3.054 & 12 & 1 & 1 & 1 & 29.22 \\
\hline 3.015 & 4 & 1 & 2 & 0 & 29.60 \\
\hline 2.992 & 2 & 0 & 2 & 4 & 29.84 \\
\hline 2.940 & 5 & -1 & 2 & 6 & 30.38 \\
\hline 2.822 & 3 & -2 & 0 & 6 & 31.68 \\
\hline 2.801 & 2 & -1 & 3 & 3 & 31.92 \\
\hline 2.764 & 5 & -1 & 3 & 2 & 32.36 \\
\hline 2.744 & 3 & -1 & 3 & 4 & 32.60 \\
\hline 2.711 & 1 & -2 & 1 & 6 & 33.02 \\
\hline 2.647 & 2 & -1 & 3 & 1 & 33.84 \\
\hline 2.612 & 7 & -2 & 0 & $8+$ & 34.30 \\
\hline 2.438 & 1 & $: 2$ & 2 & 6 & 36.84 \\
\hline 2.431 & 2 & -1 & 3 & 6 & 36.94 \\
\hline 2.420 & 1 & 0 & 4 & 0 & 37.12 \\
\hline 2.353 & 1 & -2 & 1 & 9 & 38.22 \\
\hline 2.314 & 8 & -1 & 2 & $8+$ & 38.88 \\
\hline 2.299 & 10 & -2 & 2 & 8 & 39.16 \\
\hline 2.285 & 2 & 1 & 1 & 3 & 39.40 \\
\hline 2.248 & 1 & 0 & 2 & 6 & 40.08 \\
\hline 2.224 & 1 & -2 & 0 & 10 & 40.52 \\
\hline 2.219 & 2 & -1 & 1 & 9 & 40.62 \\
\hline 2.206 & 1 & -1 & 4 & 2 & 40.88 \\
\hline 2.168 & 1 & -2 & 2 & 9 & 41.62 \\
\hline 2.113 & 1 & -2 & 3 & 5 & 42.76 \\
\hline 2.063 & 2 & -1 & 2 & $9+$ & 43.84 \\
\hline 2.042 & 1 & . 0 & 4 & 4 & 44.32 \\
\hline 2.021 & 1 & -2 & 2 & 10 & 44.80 \\
\hline 1.9372 & 3 & -2 & 2 & 1 & 46.86 \\
\hline 1.9263 & 1 & 2 & 0 & 0 & 47.14 \\
\hline 1.8511 & 1 & -1 & 2 & 10 & 49.18 \\
\hline 1.8111 & 1 & -3 & 1 & 7 & 50.34 \\
\hline 1.7827 & 1 & -1 & 4 & 8 & 51.20 \\
\hline 1.7293 & 2 & 2 & 1 & 1 & 52.90 \\
\hline 1.7131 & 2 & -2 & 4 & 9 & 53.44 \\
\hline 1.6671 & 1 & 0 & 1 & 9 & 55.04 \\
\hline 1.6598 & 1 & -1 & 4 & 9 & 55.30 \\
\hline
\end{tabular}


Pimelic Acid, $\mathrm{C}_{7} \mathrm{H}_{12} \mathrm{O}_{4}$ (monoclinic) - continued

\begin{tabular}{|c|c|c|c|c|c|c|c|c|c|c|c|}
\hline \multicolumn{6}{|c|}{ Calculated Pattern (Integrated) } & \multicolumn{6}{|c|}{ Calculated Pattern (Integrated) } \\
\hline$d(\AA)$ & $I$ & & $h k l$ & & $\begin{array}{c}2 \theta\left({ }^{\circ}\right) \\
\lambda=1.54056 \mathrm{~A}\end{array}$ & $d(\AA)$ & $I$ & & $h k l$ & & $\begin{array}{c}2 \theta\left(^{\circ}\right) \\
\lambda=1.54056 \mathrm{~A}\end{array}$ \\
\hline 7.61 & 29 & 0 & 0 & 2 & 11.51 & 2.432 & 1 & -1 & 3 & 6 & 30.93 \\
\hline 5.98 & 2 & 0 & 1 & 2 & 14.79 & 2.420 & i & 0 & 4 & 0 & 37.12 \\
\hline 5.22 & 1 & -1 & 0 & 4 & 16.96 & & & & & & \\
\hline 4.84 & 26 & 0 & 2 & 0 & 18.31 & 2.353 & 1 & -2 & 1 & 9 & 38.22 \\
\hline \multirow[t]{2}{*}{4.69} & 1 & -1 & 1 & 2 & 18.90 & 2.315 & 9 & -1 & & 8 & 38.87 \\
\hline & & & & & & 2.313 & 1 & -2 & 0 & 2 & 38.91 \\
\hline 4.61 & 73 & 0 & 2 & 1 & 19.23 & 2.299 & 11 & -2 & 2 & 8 & 39.15 \\
\hline 4.08 & 1 & 0 & 2 & 2 & 21.74 & 2.285 & 2 & 1 & 1 & 3 & 39.41 \\
\hline 3.85 & 10 & 1 & 0 & 0 & 23.06 & & & & & & \\
\hline 3.70 & 5 & -1 & 0 & 6 & 24.33 & 2.248 & 1 & J & 2 & 6 & 43.38 \\
\hline \multirow[t]{2}{*}{3.67} & 7 & -1 & 2 & 3 & 24.20 & 2.225 & 1 & -2 & 01 & 0 & 40.52 \\
\hline & & & & & & 2.220 & 1 & -1 & 1 & 9 & 40.51 \\
\hline 3.59 & 4 & -1 & 2 & 2 & 24.75 & 2.206 & 1 & -1 & 4 & 2 & 40.87 \\
\hline 3.50 & 1 & 0 & 2 & 3 & 25.41 & 2.168 & 1 & -2 & 2 & 9 & 41.51 \\
\hline 3.344 & 65 & -1 & 2 & 1 & 26.53 & & & & & & \\
\hline 3.276 & 3 & -1 & 2 & 5 & 27.20 & 2.113 & 1 & -2 & 3 & 5 & 42.76 \\
\hline \multirow{2}{*}{3.157} & 2 & 0 & 3 & 1 & 28.25 & 2.065 & $i$ & -2 & 1 & 1 & 43.81 \\
\hline & & & & & & 2.063 & 2 & -1 & 2 & 9 & 43.85 \\
\hline 3.053 & 13 & 1 & 1 & 1 & 29.22 & 2.042 & 1 & 0 & 4 & 4 & 44.32 \\
\hline 3.015 & 4 & 1 & 2 & 0 & 29.51 & 2.021 & 1 & -2 & 2 & 0 & 44.80 \\
\hline 2.992 & 2 & 0 & 2 & 4 & 29.83 & & & & & & \\
\hline 2.940 & 6 & -1 & 2 & 6 & 30.33 & 1.9369 & 3 & -2 & 2 & 1 & 46.87 \\
\hline \multirow[t]{2}{*}{2.823} & 3 & -2 & 0 & 6 & 31.67 & 1.9265 & 1 & 2 & 0 & 0 & 47.13 \\
\hline & & & & & & 1.8507 & 1 & -1 & 2 & 0 & 49.19 \\
\hline 2.801 & 2 & -1 & 3 & 3 & 31.92 & 1.8117 & 1 & -3 & 1 & 7 & 50.32 \\
\hline 2.765 & б & -1 & 3 & 2 & 32.35 & 1.7968 & 1 & -2 & 4 & 4 & 50.77 \\
\hline 2.745 & 3 & -1 & 3 & 4 & 32.59 & & & & & & \\
\hline 2.710 & 1 & -2 & 1 & 6 & 33.33 & 1.7827 & 1 & -1 & 4 & 8 & 51.20 \\
\hline \multirow[t]{2}{*}{2.646} & 2 & -1 & 3 & 1 & 33.84 & 1.7297 & 2 & 2 & 1 & 1 & 52.89 \\
\hline & 7 & -2 & 0 & 8 & & 1.7131 & 2 & -2 & 4 & 9 & 53.44 \\
\hline $\begin{array}{l}2.612 \\
2.610\end{array}$ & 1 & -1 & 2 & 7 & $\begin{array}{l}34.30 \\
34.33\end{array}$ & 1.6568 & $i$ & 0 & 1 & 9 & 55.35 \\
\hline 2.438 & 2 & -2 & 2 & 6 & 36.83 & 1.6597 & $i$ & -1 & 4 & 9 & 55.30 \\
\hline
\end{tabular}


Structure

Monoclinic, $\mathrm{P} 2_{1} / \mathrm{n}(14), \mathrm{Z}=4$, [Boonstra, 1968]

\section{Lattice parameters}

$a=5.64, b=8.33, c=7.12 \pm 0.02 \AA$

$\beta=92.25 \pm 0.25^{\circ} \quad$ [ibid. $]$

\section{Scattering factors}

$0^{\circ} \quad[3.3 .1 \mathrm{~A}]$

$\mathrm{Ag}^{\circ}, \mathrm{Mn}^{\circ}$ [3.3.1B]

Thermal parameters

Anisotropic, Table 4 [Boonstra, 1968]

\section{Atomic positions}

Table 3 [ibid.]

Density

(calculated) $4.507 \mathrm{~g} / \mathrm{cm}^{3}$

\section{Scale factor}

$3.101 \times 10^{4}$

\section{Reference}

Boonstra, E.G. (1968). The crystal structure of silver permanganate, Acta Cryst. B24, 1053-1062.

\begin{tabular}{|c|c|c|c|c|c|c|}
\hline \multicolumn{7}{|c|}{ Calculated Pattern (Peak heights) } \\
\hline$d(\stackrel{\circ}{A})$ & $I$ & & $h k$ & & & $\begin{array}{c}2 \theta\left({ }^{\circ}\right) \\
\lambda=1.54056 \AA\end{array}$ \\
\hline 5.41 & 63 & 0 & 1 & 1 & & 16.38 \\
\hline 4.50 & 13 & -1 & 0 & 1 & & 19.70 \\
\hline 4.33 & 10 & 1 & 0 & 1 & & 20.48 \\
\hline 4.16 & 24 & 0 & 2 & 0 & & 21.32 \\
\hline 3.96 & 10 & -1 & 1 & 1 & & 22.42 \\
\hline 3.84 & 10 & 1 & 1 & 1 & & 23.12 \\
\hline 3.56 & 31 & 0 & 0 & 2 & & 25.02 \\
\hline 3.348 & 79 & 1 & 2 & 0 & & 26.50 \\
\hline 3.271 & 50 & 0 & 1 & 2 & & 27.24 \\
\hline 3.058 & I CO & -1 & 2 & 1 & & 29.18 \\
\hline 3.004 & 97 & 1 & 2 & 1 & & 29.72 \\
\hline 2.875 & 62 & -1 & 1 & 2 & & 31.08 \\
\hline 2.819 & 64 & 2 & 0 & 0 & & 31.72 \\
\hline 2.786 & 50 & 1 & 1 & 2 & & 32.10 \\
\hline 2.670 & 5 & 2 & 1 & 0 & & 33.54 \\
\hline 2.586 & 9 & 0 & 3 & 1 & & 34.65 \\
\hline 2.531 & 1 & -2 & 1 & 1 & & 35.44 \\
\hline 2.46 .8 & 14 & -1 & 2 & 2 & + & 36.38 \\
\hline 2.334 & 26 & 2 & 2 & 0 & + & 38.54 \\
\hline 2.281 & 32 & 0 & 1 & 3 & & 39.48 \\
\hline 2.252 & 2 & -2 & 0 & 2 & & 40.00 \\
\hline 2.196 & $?$ & 2 & 2 & 1 & & 41.06 \\
\hline 2.189 & 2 & 0 & 3 & 2 & & 41.20 \\
\hline 2.174 & 10 & -2 & 1 & 2 & & 41.50 \\
\hline 2.168 & 8 & 2 & 0 & 2 & & 41.62 \\
\hline 2.156 & 1 & 1 & 0 & 3 & & $41 \cdot 86$ \\
\hline 2.098 & 7 & 2 & 1 & 2 & & 43.08 \\
\hline 2.087 & 4 & 1 & 1 & 3 & & 43.32 \\
\hline 2.082 & 5 & 0 & 4 & 0 & & 43.42 \\
\hline 2.057 & 3 & -1 & 3 & 2 & & 43.98 \\
\hline 2.024 & 4 & 1 & 3 & 2 & & 44.74 \\
\hline 1.9985 & 21 & 0 & 4 & 1 & & 45.34 \\
\hline 1.9812 & 3 & -2 & 2 & 2 & & 45.76 \\
\hline 1.9537 & 3 & 1 & 4 & 0 & & 46.44 \\
\hline 1.9194 & 7 & -2 & 3 & 1 & & 47.32 \\
\hline 1.8908 & 9 & -1 & 4 & 1 & + & 48.08 \\
\hline 1.8769 & 7 & 1 & 4 & 1 & & 48.46 \\
\hline 1.8343 & 3 & -3 & 0 & 1 & & 49.66 \\
\hline 1.8064 & 11 & -2 & 1 & 3 & & 50.48 \\
\hline 1.8031 & 23 & 0 & 3 & 3 & & 50.58 \\
\hline 1.7984 & 14 & 0 & 4 & 2 & + & 50.72 \\
\hline 1.7918 & 3 & -3 & 1 & 1 & & 50.92 \\
\hline 1.7788 & 4 & 0 & 0 & 4 & & 51.32 \\
\hline 1.7490 & 2 & -2 & 3 & 2 & & 52.26 \\
\hline 1.7410 & 11 & 2 & 1 & 3 & + & 52.52 \\
\hline 1.7324 & 8 & -1 & 3 & 3 & & 52.80 \\
\hline 1.7125 & 7 & 3 & 2 & 0 & & 53.46 \\
\hline 1.7084 & 6 & 2 & 3 & 2 & & 53.60 \\
\hline 1.7031 & 9 & 1 & 3 & 3 & & 53.78 \\
\hline 1.6789 & 14 & -3 & 2 & 1 & + & 54.62 \\
\hline
\end{tabular}




\begin{tabular}{|c|c|c|c|c|c|c|}
\hline \multicolumn{7}{|c|}{ Calculated Pattern (Peak heights) } \\
\hline$d(\AA)$ & $I$ & & $h k$ & & & $\begin{array}{c}2 \theta\left({ }^{\circ}\right) \\
\lambda=1.54056 \AA\end{array}$ \\
\hline 1.6749 & 11 & 2 & 4 & 0 & & 54.76 \\
\hline $1.654 y$ & 14 & -3 & 1 & 2 & & 55.48 \\
\hline 1.6516 & 17 & 3 & 2 & 1 & & 55.60 \\
\hline 1.6445 & 10 & 1 & 1 & 4 & & 55.86 \\
\hline 1.6391 & 10 & -2 & 4 & 1 & & 56.06 \\
\hline 1.6216 & 7 & 2 & 4 & 1 & & 56.72 \\
\hline 1.6040 & 9 & 3 & 1 & 2 & & 57.40 \\
\hline 1.5863 & 1 & -1 & 2 & 4 & & 58.10 \\
\hline 1.5623 & 3 & -1 & 5 & 1 & & 59.08 \\
\hline 1.5556 & 2 & 1 & 2 & 4 & + & .59 .36 \\
\hline 1.5401 & 12 & -2 & 3 & 3 & & 60.02 \\
\hline 1.5313 & 2 & -2 & 0 & 4 & 4 & 60.40 \\
\hline 1. 5387 & 2 & 0 & 5 & 2 & & 61.40 \\
\hline 1.5361 & 2 & -2 & 1 & 4 & & 61.52 \\
\hline 1.4986 & 8 & 2 & 3 & 3 & & 61.86 \\
\hline 1.4780 & 3 & 2 & 0 & 4 & + & 62.82 \\
\hline 1.4634 & 3 & -1 & 5 & 2 & & 63.52 \\
\hline 1.4552 & 2 & 2 & 1 & 4 & & 63.92 \\
\hline 1.4515 & 5 & 1 & 5 & 2 & & 64.10 \\
\hline 1.4090 & 5 & 4 & 0 & 0 & & 66.28 \\
\hline 1.3927 & 1 & -1 & 0 & 5 & & 67.16 \\
\hline 1.3883 & 1 & 0 & 6 & 0 & & 67.40 \\
\hline 1.3764 & 1 & -3 & 4 & 1 & & 68.06 \\
\hline 1.3624 & 1 & כ & 6 & 1 & & 68.86 \\
\hline 1.3613 & 1 & 3 & 4 & 1 & & 68.92 \\
\hline 1.3480 & 5 & 1 & 6 & 0 & & 69.70 \\
\hline 1.3393 & 1 & -2 & 5 & 2 & & 70.22 \\
\hline 1.3346 & 1 & 4 & 2 & 0 & & 70.50 \\
\hline 1.3320 & 4 & -1 & 5 & 3 & & 70.66 \\
\hline 1.3281 & 2 & -4 & 0 & 2 & & 70.90 \\
\hline 1.3206 & 6 & -1 & 2 & 5 & + & 71.36 \\
\hline 1.3206 & 6 & -3 & 3 & 3 & & 71.36 \\
\hline 1.3181 & 6 & 1 & 5 & 3 & & 71.52 \\
\hline 1.3114 & 1 & -4 & 1 & 2 & & 71.94 \\
\hline 1.3048 & 1 & 2 & 3 & 4 & & $72 \cdot 36$ \\
\hline 1.3014 & 3 & -3 & 1 & 4 & & 72.58 \\
\hline 1.2986 & 5 & 1 & 2 & 5 & & 72.76 \\
\hline 1.2928 & 1 & 4 & 0 & 2 & & 73.14 \\
\hline 1.2820 & 1 & 3 & 3 & 3 & & 73.86 \\
\hline 1.2773 & 1 & 4 & 1 & 2 & & 74.18 \\
\hline 1.2662 & 1 & 0 & 3 & 5 & & 74.94 \\
\hline 1.2565 & 1 & 1 & 6 & 2 & + & 75.62 \\
\hline 1.2525 & 2 & 3 & 1 & 4 & & 75.90 \\
\hline 1.2450 & 2 & -4 & 3 & 1 & + & 76.44 \\
\hline 1.2336 & 1 & -2 & 4 & 4 & & 77.28 \\
\hline 1.2301 & 2 & 4 & 3 & 1 & 4 & 77.54 \\
\hline 1.2195 & 1 & -4 & 1 & 3 & & 78.34 \\
\hline 1.2161 & 1 & 0 & 5 & 4 & & 78.60 \\
\hline
\end{tabular}

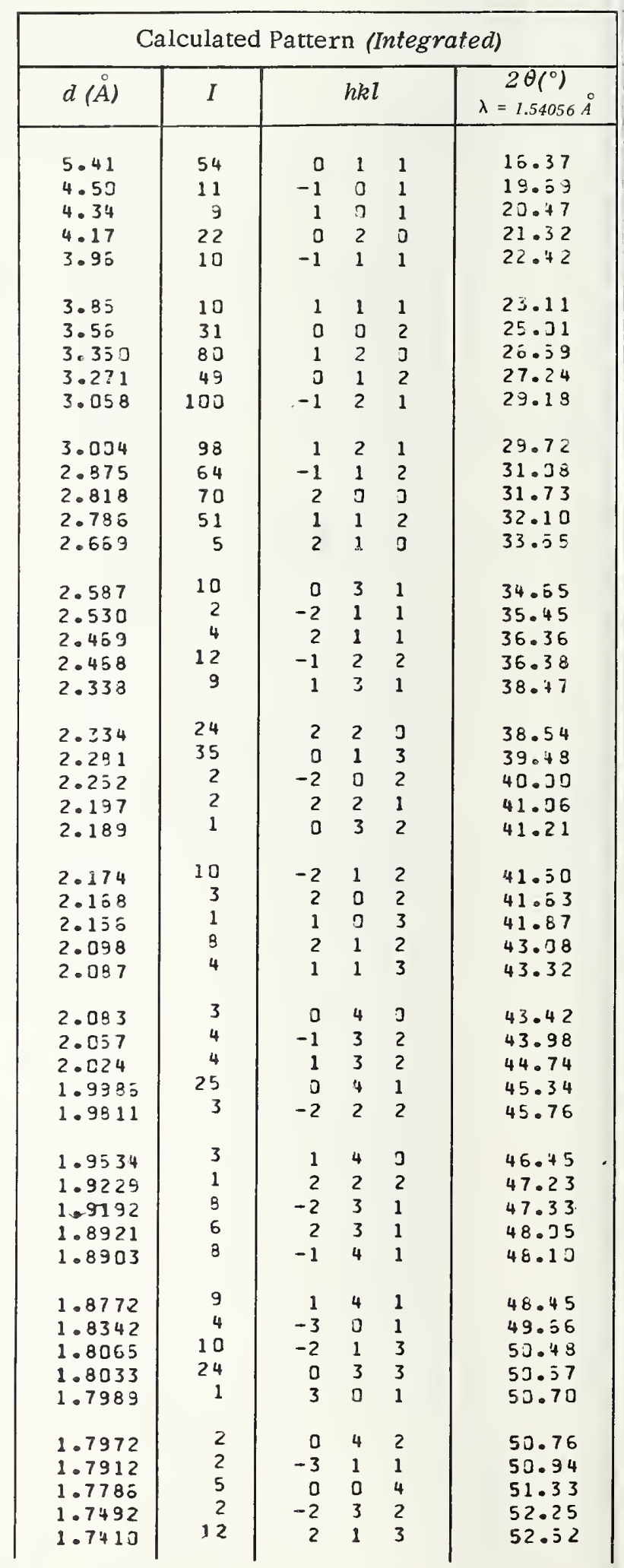




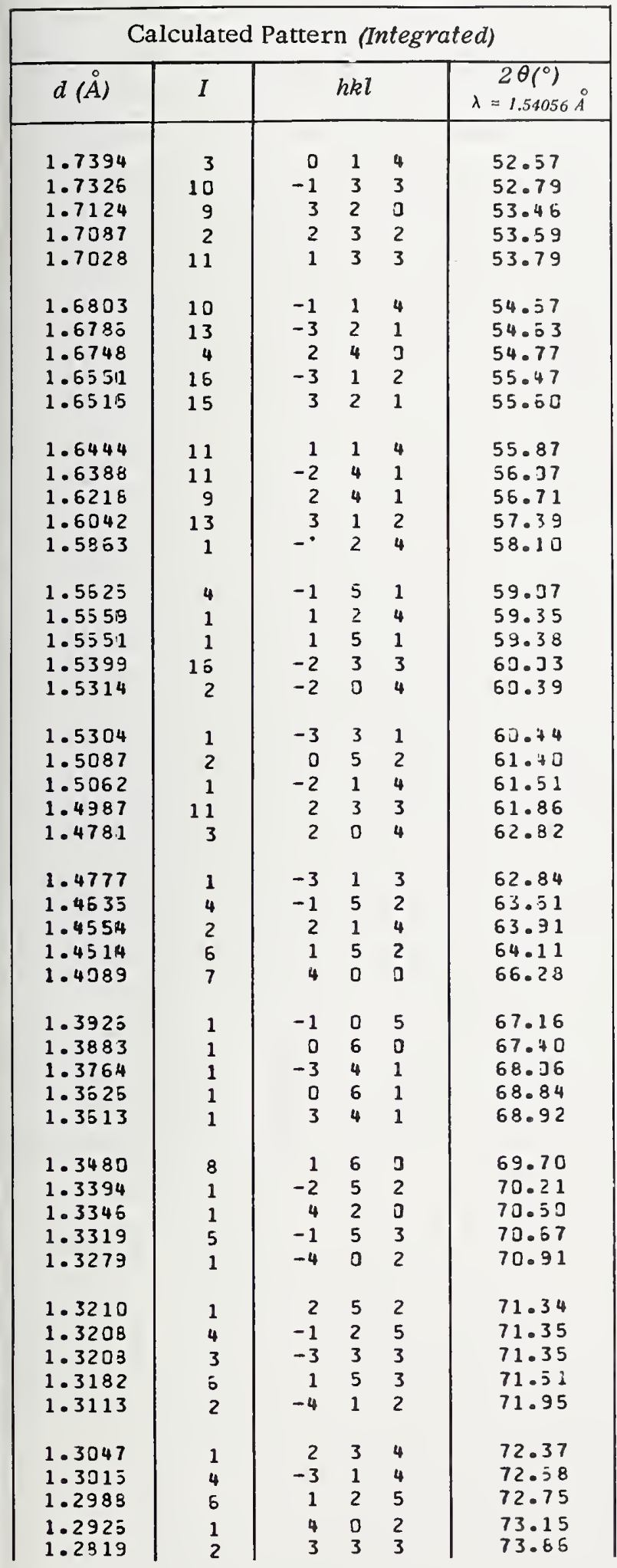

\begin{tabular}{|c|c|rcc|c|}
\hline \multicolumn{5}{|c|}{ Calculated Pattern (Integrated) } \\
\hline$d(A)$ & $I$ & \multicolumn{3}{|c|}{$h k l$} & $\begin{array}{c}2 \theta\left(^{\circ}\right) \\
\lambda=1.54056 \AA\end{array}$ \\
\hline 1.2774 & 1 & 4 & 1 & 2 & 74.17 \\
1.2563 & 1 & 0 & 3 & 5 & 74.33 \\
1.2566 & 1 & 1 & 6 & 2 & 75.51 \\
1.2563 & 1 & -3 & 2 & 4 & 75.53 \\
1.2526 & 3 & 3 & 1 & 4 & 75.90 \\
1.2454 & 1 & 2 & 6 & 0 & 76.41 \\
1.2448 & 2 & -4 & 3 & 1 & 76.45 \\
1.2333 & 1 & -2 & 4 & 4 & 77.27 \\
1.2304 & 1 & -2 & 6 & 1 & 77.52 \\
1.2293 & 2 & 4 & 3 & 1 & 77.55 \\
1.2194 & 2 & -4 & 1 & 3 & 78.35 \\
1.2159 & 1 & 0 & 5 & 4 & 78.52 \\
& & & & \\
\hline
\end{tabular}


Sodium Aluminum Chloride Silicate, sodalite, $\mathrm{Na}_{8} \mathrm{Si}_{6} \mathrm{Al}_{6} \mathrm{O}_{24} \mathrm{Cl}_{2}$ (cubic)

Structure

Cubic, $\mathrm{P} \overline{4} 3 \mathrm{n}$ (218), $\mathrm{Z}=1$ [Löns and Schulz, 1967]

Lattice parameters

$a=8.870 \pm .004 \AA$ [ibid.]

Scattering factors

$\mathrm{Na}^{+1}, \mathrm{Al}^{\circ}, \mathrm{Si}^{\circ}, \mathrm{Cl}^{-1}, \mathrm{O}^{-1}[3.3 . \mathrm{IA}]$

Thermal parameters

Isotropic [Löns and Schulz, 1967]

\section{Density}

(calculated) $2.306 \mathrm{~g} / \mathrm{cm}^{3}$

\section{Scale factor}

$11.30 \times 10^{4}$

\section{Additional patterns}

1. PDF card 3-0338 [Dow Chemical Co., Midland, Michigan]

\section{Reference}

Löns, J. and H. Schulz (1967). Strukturverfeinerung von sodalith, $\mathrm{Na}_{8} \mathrm{Si}_{6} \mathrm{Al}_{6} \mathrm{O}_{2_{4}} \mathrm{Cl}_{2}$, Acta cryst. 24, 434-436.

\begin{tabular}{|c|c|c|c|c|c|c|}
\hline \multicolumn{7}{|c|}{ Calculated Pattern (Peak heights) } \\
\hline$d(\stackrel{\circ}{A})$ & $I$ & \multicolumn{4}{|c|}{$h k l$} & $\begin{array}{c}2 \theta\left({ }^{\circ}\right) \\
\lambda=1.54056 \AA\end{array}$ \\
\hline 6.267 & 40 & 1 & 1 & 0 & & 14.12 \\
\hline 4.436 & 7 & 2 & 0 & 0 & & 20.00 \\
\hline 3.966 & 1 & 2 & 1 & 0 & & 22.40 \\
\hline 3.622 & 100 & 2 & 1 & 1 & & 24.56 \\
\hline 2.805 & 8 & 3 & 1 & 0 & & 31.88 \\
\hline 2.560 & 16 & 2 & 2 & 2 & & 35.02 \\
\hline 2.371 & 18 & 3 & 2 & 1 & & 37.92 \\
\hline 2.217 & 1 & 4 & 0 & 0 & & 40.66 \\
\hline 2.091 & 26 & 4 & 1 & 1 & + & 43.24 \\
\hline 1.9836 & 4 & 4 & 2 & 0 & & 45.70 \\
\hline 1.8908 & 3 & 3 & 3 & 2 & & 48.08 \\
\hline 1.8104 & 3 & 4 & 2 & 2 & & 50.36 \\
\hline 1.7397 & 2 & 4 & 3 & 1 & & 52.56 \\
\hline 1.6195 & 2 & 5 & 2 & 1 & & 56.80 \\
\hline 1.5681 & 7 & 4 & 4 & 0 & & 58.84 \\
\hline 1.5213 & 4 & 5 & 3 & 0 & + & 60.84 \\
\hline 1.4784 & 6 & 4 & 4 & 2 & + & 62.80 \\
\hline 1.4387 & 6 & 5 & 3 & 2 & & 64.74 \\
\hline 1.3686 & 2 & 5 & 4 & 1 & & 68.50 \\
\hline 1.3373 & 2 & 6 & 2 & 2 & & 70.34 \\
\hline 1.3076 & 2 & 6 & 3 & 1 & & $72 \cdot 18$ \\
\hline 1.2802 & 2 & 4 & 4 & 4 & & 73.98 \\
\hline 1.2071 & 5 & 7 & 2 & 1 & + & 79.30 \\
\hline 1.1648 & 1 & 7 & 3 & 0 & & 82.80 \\
\hline 1.1265 & 2 & 6 & 5 & 1 & + & 86.28 \\
\hline 1.0918 & 1 & 7 & 4 & 1 & + & 89.74 \\
\hline 1.0757 & 1 & 8 & 2 & $\overrightarrow{0}$ & & 91.46 \\
\hline 1.0174 & 1 & 6 & 6 & 2 & & 98.42 \\
\hline .9917 & 2 & 8 & 4 & 0 & & 101.92 \\
\hline .9565 & 1 & 9 & 2 & 1 & + & 107.28 \\
\hline .9052 & 1 & 8 & 4 & 4 & & 116.62 \\
\hline .8457 & 2 & 9 & 5 & 2 & + & 131.24 \\
\hline .8308 & 1 & 8 & 7 & 1 & + & 136.00 \\
\hline .8235 & 1 & 8 & 6 & 4 & + & 138.56 \\
\hline $.790 a ́$ & 1 & 9 & 6 & 3 & + & 154.22 \\
\hline
\end{tabular}


Sodium Aluminum Chloride Silicate, sodalite, $\mathrm{Na}_{8} \mathrm{Si}_{6} \mathrm{Al}_{6} \mathrm{O}_{24} \mathrm{Cl}_{2}$ (cubic) - continued

\begin{tabular}{|c|c|c|c|c|c|}
\hline \multicolumn{6}{|c|}{ Calculated Pattern (Integrated) } \\
\hline$d(\stackrel{\circ}{A})$ & $I$ & & hkl & & $\begin{array}{c}2 \theta\left(^{\circ}\right) \\
\lambda=1.54056 \AA\end{array}$ \\
\hline $\begin{array}{l}6.272 \\
4.435 \\
3.967 \\
3.621 \\
2.805\end{array}$ & $\begin{array}{r}34 \\
7 \\
1 \\
100 \\
8\end{array}$ & $\begin{array}{l}1 \\
2 \\
2 \\
2 \\
3\end{array}$ & $\begin{array}{l}1 \\
0 \\
1 \\
1 \\
1\end{array}$ & $\begin{array}{l}0 \\
0 \\
0 \\
1 \\
0\end{array}$ & $\begin{array}{l}14.11 \\
20.00 \\
22.39 \\
24.56 \\
31.88\end{array}$ \\
\hline $\begin{array}{l}2.561 \\
2.371 \\
2.217 \\
2.091 \\
2.091\end{array}$ & $\begin{array}{r}18 \\
20 \\
1 \\
13 \\
18\end{array}$ & $\begin{array}{l}2 \\
3 \\
4 \\
3 \\
4\end{array}$ & $\begin{array}{l}2 \\
2 \\
0 \\
3 \\
1\end{array}$ & $\begin{array}{l}2 \\
1 \\
0 \\
0 \\
1\end{array}$ & $\begin{array}{l}35.01 \\
37.92 \\
40.65 \\
43.24 \\
43.24\end{array}$ \\
\hline $\begin{array}{l}1.9834 \\
1.8911 \\
1.8106 \\
1.7395 \\
1.6194\end{array}$ & $\begin{array}{l}5 \\
3 \\
3 \\
3 \\
3\end{array}$ & $\begin{array}{l}4 \\
3 \\
4 \\
4 \\
5\end{array}$ & $\begin{array}{l}2 \\
3 \\
2 \\
3 \\
2\end{array}$ & $\begin{array}{l}0 \\
2 \\
2 \\
1 \\
1\end{array}$ & $\begin{array}{l}45.71 \\
48.07 \\
50.36 \\
52.57 \\
56.80\end{array}$ \\
\hline $\begin{array}{l}1.5680 \\
1.5212 \\
1.5212 \\
1.4783 \\
1.4783\end{array}$ & $\begin{array}{r}10 \\
3 \\
2 \\
3 \\
6\end{array}$ & $\begin{array}{l}4 \\
5 \\
4 \\
6 \\
4\end{array}$ & $\begin{array}{l}4 \\
3 \\
3 \\
0 \\
4\end{array}$ & $\begin{array}{l}0 \\
0 \\
3 \\
0 \\
2\end{array}$ & $\begin{array}{l}58.85 \\
60.84 \\
60.84 \\
62.80 \\
62.80\end{array}$ \\
\hline $\begin{array}{l}1.4389 \\
1.3687 \\
1.3372 \\
1.3078 \\
1.2803\end{array}$ & $\begin{array}{l}9 \\
4 \\
3 \\
4 \\
3\end{array}$ & $\begin{array}{l}5 \\
5 \\
6 \\
6 \\
4\end{array}$ & $\begin{array}{l}3 \\
4 \\
2 \\
3 \\
4\end{array}$ & $\begin{array}{l}2 \\
1 . \\
2 \\
1 \\
4\end{array}$ & $\begin{array}{l}64.73 \\
68.50 \\
70.34 \\
72.17 \\
73.98\end{array}$ \\
\hline $\begin{array}{l}1.2070 \\
1.2070 \\
1.2070 \\
1.1647\end{array}$ & $\begin{array}{l}4 \\
1 \\
3 \\
2\end{array}$ & $\begin{array}{l}7 \\
5 \\
6 \\
7\end{array}$ & $\begin{array}{l}2 \\
5 \\
3 \\
3\end{array}$ & $\begin{array}{l}1 \\
2 \\
3 \\
0\end{array}$ & $\begin{array}{l}79.31 \\
79.31 \\
79.31 \\
82.81\end{array}$ \\
\hline
\end{tabular}

\begin{tabular}{|c|c|c|c|c|c|}
\hline \multicolumn{6}{|c|}{ Calculated Pattern (Integrated) } \\
\hline$d(\stackrel{\circ}{A})$ & $I$ & & $2 k l$ & & $\begin{array}{c}\left.2 \theta 0^{\circ}\right) \\
\lambda=1.54056 \AA\end{array}$ \\
\hline 1.1265 & 3 & 6 & 5 & 1 & 86.28 \\
\hline 1.1265 & 1 & 7 & 3 & 2 & 86.28 \\
\hline 1.0918 & 1 & 7 & 4 & 1 & 89.74 \\
\hline 1.091 .8 & 1 & 8 & 1 & 1 & 89.74 \\
\hline 1.0756 & 2 & 8 & 2 & 0 & 91.47 \\
\hline 1.0175 & 2 & 6 & 6 & 2 & 98.41 \\
\hline 1.0043 & 1 & 7 & 5 & 2 & $100 \cdot 16$ \\
\hline .9917 & 4 & 8 & 4 & 0 & 101.92 \\
\hline .9795 & 1 & 8 & 3 & 3 & 103.70 \\
\hline . 9565 & 1 & 9 & 2 & 1 & 107.28 \\
\hline .9565 & 1 & 6 & 5 & 5 & 107.28 \\
\hline .9149 & 1 & 7 & ó & 3 & 114.69 \\
\hline .9053 & 2 & 8 & 4 & 4 & $116 \cdot 61$ \\
\hline .8783 & 1 & 7 & 7 & 2 & $122 \cdot 58$ \\
\hline .8783 & 1 & 10 & 1 & 1 & 122.58 \\
\hline .8535 & $\hat{1}$ & 10 & 2 & 2 & $128 \cdot 97$ \\
\hline .8457 & 3 & 9 & 5 & 2 & 131.23 \\
\hline .8457 & 1 & 10 & 3 & 1 & 131.23 \\
\hline .8457 & 1 & 7 & 6 & 5 & 131.23 \\
\hline .8307 & 1 & 7 & 7 & 4 & 136.01 \\
\hline .8307 & $\overline{1}$ & 8 & 7 & 1 & $136 \cdot 01$ \\
\hline .8236 & 2 & 10 & 4 & 0 & 138.56 \\
\hline .8236 & 3 & 8 & 6 & 4 & 138.56 \\
\hline .8165 & 2 & 9 & 6 & 1 & 141.24 \\
\hline .7902 & 1 & 11 & 2 & 1 & 154.21 \\
\hline .7902 & 2 & 9 & 6 & 3 & 154.21 \\
\hline .7902 & 1 & 10 & 5 & 1 & $154 \cdot 21$ \\
\hline
\end{tabular}


Structure

Monoclinic, $\mathrm{P} 2_{1} / \mathrm{c}$ (14), $\mathrm{Z}=4$ [Hyman et al. 1967].

\section{Lattice parameters}

$a=6.507 \pm 0.001 \AA$,

$\mathrm{b}=17.797 \pm 0.002$, (published value, 17.796)

$c=8.377 \pm 0.001$

$\beta=96^{\circ} 34^{\prime} \pm 2^{\prime} \quad$ [ibid.]

\section{Scattering factors}

$\mathrm{B}^{\circ}, \mathrm{Na}^{+}, \mathrm{O}^{-}[3.3 .1 \mathrm{~A}]$

\section{Thermal parameters}

Isotropic [Hyman et al., 1967]

\section{Density}

(calculated) $2.346 \mathrm{~g} / \mathrm{cm}^{3}$

\section{Scale factor}

$$
3.040 \times 10^{4}
$$

\section{Additional patterns}

1. PDF card 16-199. [Bouaziz, 1962]

\section{Reference}

Bouaziz, R.(1962). Contribution à I'étude radiocrystallographique de quelques borates de lithium et de sodium, Bull. Soc. Chim. France 1962, 1451.

Hyman, A., A.Perloff, F.Mauer, and S.Block (1967). The crystal structure of sodium tetraborate, Acta cryst. 22, 815-821.

\begin{tabular}{|c|c|c|c|c|c|c|}
\hline \multicolumn{7}{|c|}{ Calculated Pattern (Peak heights) } \\
\hline$d(\stackrel{\circ}{A})$ & $I$ & \multicolumn{4}{|c|}{$h k l$} & $\begin{array}{c}2 \theta\left(^{\circ}\right) \\
\lambda=1.54056 \AA\end{array}$ \\
\hline 8.89 & 6 & 0 & 2 & 0 & & 9.94 \\
\hline 8.32 & 13 & D & 0 & 1 & & 10.62 \\
\hline 7.53 & 4 & 0 & 1 & 1 & & 11.74 \\
\hline 6.08 & 4 & 0 & 2 & 1 & & 14.56 \\
\hline 5.23 & 1 & 1 & 2 & 0 & & $16 \cdot 94$ \\
\hline 4.62 & 38 & 1 & 2 & -1 & & 19.18 \\
\hline 4.37 & 3 & 1 & 3 & 0 & & 20.30 \\
\hline 4.25 & 14 & 1 & 2 & 1 & & 20.86 \\
\hline 4.16 & 30 & 0 & 0 & 2 & & 21.34 \\
\hline 4.05 & 21 & 0 & 1 & 2 & & 21.92 \\
\hline 4.00 & 5 & 1 & 3 & -1 & & 22.22 \\
\hline 3.77 & 41 & 0 & 2 & 2 & & 23.58 \\
\hline 3.75 & 33 & 1 & 3 & 1 & & 23.68 \\
\hline 3.67 & 3 & 1 & 4 & 0 & & 24.26 \\
\hline 3.406 & 63 & 0 & 3 & 2 & $\bullet$ & 26.14 \\
\hline 3.273 & 15 & 1 & 1 & 2 & + & 27.22 \\
\hline 3.232 & 100 & 2 & 0 & 0 & & 27.58 \\
\hline 3.180 & 30 & 2 & 1 & 0 & & 28.04 \\
\hline 3.136 & 19 & 2 & 0 & -1 & & 28.44 \\
\hline 3.119 & 9 & 1 & 2 & 2 & & 28.60 \\
\hline 3.038 & 78 & 0 & 4 & 2 & + & 29.38 \\
\hline 2.974 & 11 & 1 & 5 & -1 & & 30.02 \\
\hline 2.966 & 13 & 0 & 6 & 0 & & 30.10 \\
\hline 2.959 & 26 & 2 & 2 & -1 & & 30.18 \\
\hline 2.902 & 12 & 2 & 0 & 1 & + & 30.78 \\
\hline 2.864 & 8 & 2 & 1 & 1 & & 31.20 \\
\hline 2.795 & 5 & 0 & 6 & 1 & & 32.00 \\
\hline 2.773 & 6 & 2 & 3 & -1 & & 32.26 \\
\hline 2.759 & 18 & 2 & 2 & 1 & & 32.42 \\
\hline 2.704 & 23 & 0 & 5 & 2 & $\rightarrow$ & 33.10 \\
\hline 2.696 & 15 & 1 & 6 & 0 & & 33.20 \\
\hline 2.676 & 2 & 2 & 1 & -2 & & 33.46 \\
\hline 2.648 & 1 & 0 & 2 & 3 & & 33.82 \\
\hline 2.633 & 1 & 1 & 1 & -3 & & 34.02 \\
\hline 2.615 & 3 & 2 & 4 & 0 & & 34.26 \\
\hline 2.608 & 3 & 2 & 3 & 1 & & 34.36 \\
\hline 2.590 & 1 & 2 & 2 & -2 & & 34.60 \\
\hline 2.563 & 2 & 1 & 5 & -2 & & 34.98 \\
\hline 2.550 & $1 \overline{1}$ & 1 & 2 & -3 & & 35.16 \\
\hline 2.520 & 4 & 1 & 6 & 1 & & 35.46 \\
\hline 2.426 & 6 & 1 & 1 & 3 & + & 37.02 \\
\hline 2.421 & 5 & 2 & 0 & 2 & & 37.10 \\
\hline 2.415 & 5 & 0 & 6 & 2 & & 37.20 \\
\hline 2.361 & 11 & 1 & 2 & 3 & + & 38.08 \\
\hline 2.354 & 9 & 0 & 4 & 3 & + & 38.20 \\
\hline 2.313 & 10 & 1 & 6 & -2 & + & 38.90 \\
\hline 2.264 & 2 & 1 & 3 & 3 & & 39.78 \\
\hline 2.251 & 5 & 1 & 7 & 1 & & 40.02 \\
\hline 2.215 & 4 & 1 & 6 & 2 & $\bullet$ & 40.70 \\
\hline 2.169 & 11 & 0 & 7 & 2 & & 41.60 \\
\hline
\end{tabular}


Sodium Borate, $\mathrm{Na}_{2} \mathrm{~B}_{8} \mathrm{O}_{13}$ (monoclinic) - continued

\begin{tabular}{|c|c|c|c|c|c|c|}
\hline \multicolumn{7}{|c|}{ Calculated Pattern (Peak heights) } \\
\hline$d(\stackrel{\circ}{)}$ & $I$ & & $n k l$ & & & $\begin{array}{c}2 \theta\left({ }^{\circ}\right) \\
\lambda=1.54056 \mathrm{~A}\end{array}$ \\
\hline 2.154 & 6 & 2 & 5 & -2 & + & 41.90 \\
\hline 2.149 & 5 & 0 & 8 & 1 & & 42.00 \\
\hline 2.139 & 14 & 3 & 1 & 0 & & 42.22 \\
\hline 2.103 & 1 & 1 & 8 & 0 & & 42.98 \\
\hline 2.094 & 12 & 3 & 2 & 0 & + & 43.16 \\
\hline 2.081 & 8 & 0 & 0 & 4 & & 43.46 \\
\hline 2.075 & 6 & 2 & 6 & 1 & & 43.58 \\
\hline 2.066 & 15 & 0 & 1 & 4 & & 43.78 \\
\hline 2.036 & 2 & 1 & 1 & -4 & & 44.46 \\
\hline 2.021 & 25 & 1 & 7 & 2 & + & 44.82 \\
\hline 1.9994 & 10 & 2 & 6 & -2 & + & 45.32 \\
\hline 1.9828 & 3 & 2 & 1 & 3 & & 45.72 \\
\hline 1.9617 & 8 & 0 & 8 & 2 & + & 46.24 \\
\hline 1.9466 & 2 & 2 & 2 & 3 & & 46.62 \\
\hline 1.9395 & 4 & 3 & 4 & 0 & + & 46.80 \\
\hline 1.9240 & 5 & 0 & 9 & 1 & & 47.20 \\
\hline 1.9126 & 2 & 2 & 7 & 1 & & 47.50 \\
\hline 1.9058 & 9 & 1 & 8 & -2 & + & 47.68 \\
\hline 1.8923 & 5 & 2 & 5 & -3 & + & 48.04 \\
\hline 1.8747 & 6 & 1 & 2 & 4 & + & 48.52 \\
\hline 1.8532 & 9 & 2 & 7 & -2 & + & 49.12 \\
\hline 1.8497 & 10 & 1 & 8 & 2 & + & 49.22 \\
\hline 1.8448 & 5 & 3 & 5 & 0 & & 49.36 \\
\hline 1.8385 & 18 & 2 & 1 & -4 & + & 49.54 \\
\hline 1.8329 & 13 & 3 & 4 & -2 & + & 49.70 \\
\hline 1.8267 & 6 & 1 & 3 & 4 & & 49.88 \\
\hline 1.8206 & 4 & 3 & 1 & 2 & & 50.06 \\
\hline 1.8098 & 1 & 2 & 2 & -4 & & 50.38 \\
\hline 1.7958 & 3 & 0 & 5 & 4 & & 50.80 \\
\hline 1.7925 & 7 & 3 & 2 & 2 & & 50.90 \\
\hline 1.7641 & 5 & 2 & 3 & -4 & + & 51.78 \\
\hline 1.7609 & 6 & 1 & 4 & 4 & & 51.88 \\
\hline 1.7534 & 6 & 2 & 7 & 2 & & 52.12 \\
\hline 1.7496 & 9 & 3 & 5 & -2 & + & $52 \cdot 24$ \\
\hline 1.7354 & 2 & $\mathrm{u}$ & 8 & 3 & & 52.70 \\
\hline 1.7161 & 2 & 1 & 10 & 0 & & 53.34 \\
\hline 1.7031 & 2 & 0 & 6 & 4 & & 53.78 \\
\hline 1.6869 & 3 & 2 & 9 & 0 & + & 54.34 \\
\hline 1.6727 & 2 & 2 & 9 & -1 & & 54.84 \\
\hline 1.6637 & 6 & 3 & 6 & -2 & + & 55.16 \\
\hline 1.6593 & 4 & 2 & 1 & 4 & & 55.32 \\
\hline 1.6440 & 1 & 3 & 7 & 0 & & 55.88 \\
\hline 1.6364 & 1 & 2 & 2 & 4 & & 56.16 \\
\hline 1.6216 & 3 & 4 & 0 & -1 & & 56.72 \\
\hline 1.6148 & 1 & 4 & 1 & -1 & & 56.98 \\
\hline 1.6102 & 2 & 0 & 7 & 4 & & 57.16 \\
\hline 1.5964 & 3 & 2 & 9 & -2 & + & 57.70 \\
\hline 1.5878 & 2 & 1 & 9 & -3 & + & 58.04 \\
\hline $\begin{array}{l}1.5838 \\
1.5764\end{array}$ & $\begin{array}{l}2 \\
1\end{array}$ & $\begin{array}{l}3 \\
3\end{array}$ & $\begin{array}{l}1 \\
7\end{array}$ & $\begin{array}{l}-4 \\
-2\end{array}$ & & $\begin{array}{l}58.20 \\
58.50\end{array}$ \\
\hline
\end{tabular}

\begin{tabular}{|c|c|c|c|c|}
\hline \multicolumn{5}{|c|}{ Calculated Pattern (Integrated) } \\
\hline$d(\dot{A})$ & $I$ & & $n k l$ & $\begin{array}{c}2 \theta\left(^{\circ}\right) \\
\lambda=1.54056 \AA\end{array}$ \\
\hline 8.90 & 5 & 0 & 2 & 9.93 \\
\hline 8.32 & 10 & 0 & 0 & 10.62 \\
\hline 7.54 & 3 & 0 & 11 & 11.73 \\
\hline 6.08 & 3 & 0 & 21 & 14.56 \\
\hline 5.23 & 1 & 1 & 2 & 16.94 \\
\hline 4.63 & 34 & 1 & $2-1$ & 19.17 \\
\hline 4.37 & 2 & 1 & 30 & 20.30 \\
\hline 4.25 & 12 & 1 & 2 & 20.86 \\
\hline 4.16 & 28 & 0 & 0 & 21.34 \\
\hline 4.05 & 19 & 0 & 1 & 21.92 \\
\hline 4.00 & 4 & 1 & $3-1$ & 22.21 \\
\hline 3.77 & 38 & 0 & 22 & 23.58 \\
\hline 3.75 & 23 & 1 & 3 & 23.69 \\
\hline 3.67 & 3 & 1 & 40 & 24.26 \\
\hline 3.414 & 12 & 1 & $2-2$ & 26.08 \\
\hline 3.407 & 54 & 0 & 3 & 26.14 \\
\hline 3.277 & 2 & 1 & 4 & 27.19 \\
\hline 3.273 & 9 & 1 & 1 & 27.22 \\
\hline 3.273 & 3 & 0 & 5 & 27.23 \\
\hline 3.232 & 100 & 2 & 0 & 27.57 \\
\hline 3.180 & 29 & 2 & 1 & 28.03 \\
\hline 3.136 & 18 & 2 & $0-1$ & 28.43 \\
\hline 3.119 & 6 & 1 & 2 & 28.60 \\
\hline 3.039 & 45 & 0 & 4 & 29.36 \\
\hline 3.038 & 39 & 2 & 2 & 29.38 \\
\hline 2.974 & 9 & 1 & $5-1$ & 30.02 \\
\hline 2.966 & 3 & 0 & 60 & 30.10 \\
\hline 2.958 & 25 & 2 & $2-1$ & 30.19 \\
\hline 2.904 & 3 & 1 & 3 & 30.77 \\
\hline 2.903 & 9 & 2 & 0 & 30.77 \\
\hline 2.865 & 8 & 2 & 1 & 31.19 \\
\hline 2.794 & 5 & 0 & $\begin{array}{ll}6 & 1\end{array}$ & 32.01 \\
\hline 2.773 & 5 & 2 & $3-1$ & 32.26 \\
\hline 2.760 & 18 & 2 & 2 & 32.41 \\
\hline 2.707 & 1 & 2 & $0-2$ & 33.06 \\
\hline 2.705 & 25 & 0 & 5 & 33.09 \\
\hline 2.696 & 1 & 1 & 60 & 33.20 \\
\hline 2.676 & 1 & 2 & $1-2$ & 33.46 \\
\hline 2.648 & 1 & 0 & 23 & 33.82 \\
\hline 2.633 & 1 & 3 & $1-3$ & 34.03 \\
\hline 2.615 & 3 & 2 & 4 & 34.26 \\
\hline 2.607 & 1 & 2 & 31 & 34.36 \\
\hline 2.590 & 1 & 2 & $2-2$ & 34.61 \\
\hline 2.564 & 1 & 1 & $5-2$ & 34.97 \\
\hline 2.550 & 11 & 1 & $2-3$ & 35.16 \\
\hline 2.530 & 4 & 1 & 6 & 35.46 \\
\hline 2.431 & 2 & 0 & 7 & 36.94 \\
\hline 2.431 & 1 & 2 & 4 & 36.94 \\
\hline 2.427 & 4 & 1 & 1 & 37.01 \\
\hline 2.422 & 1 & 2 & 0 & 37.09 \\
\hline
\end{tabular}




\begin{tabular}{|c|c|c|c|c|c|}
\hline \multicolumn{6}{|c|}{ Calculated Pattern (Integrated) } \\
\hline$d(\AA)$ & $I$ & & $h k l$ & & $\begin{array}{c}2 \theta\left(^{\circ}\right) \\
\lambda=1.54056 \AA\end{array}$ \\
\hline 2.415 & 4 & 0 & 6 & 2 & 37.19 \\
\hline 2.366 & 2 & 1 & 7 & 0 & 38.00 \\
\hline 2.362 & 11 & 1 & 2 & 3 & 38.07 \\
\hline 2.354 & 3 & 0 & 4 & 3 & 38.20 \\
\hline 2.353 & 3 & 2 & 5 & -1 & 38.21 \\
\hline 2.313 & 7 & 1 & 6 & -2 & 38.90 \\
\hline 2.313 & 5 & 2 & 4 & -2 & 38.91 \\
\hline 2.301 & 1 & 1 & 7 & -1 & 39.11 \\
\hline 2.264 & 2 & 1 & 3 & 3 & 39.78 \\
\hline 2.251 & $E$ & 1 & 7 & 1 & 40.02 \\
\hline 2.218 & 1 & 2 & 1 & -3 & 40.65 \\
\hline 2.215 & 5 & 1 & 6 & 2 & 40.70 \\
\hline 2.159 & 12 & 0 & 7 & 2 & 41.59 \\
\hline 2.155 & 3 & 2 & 6 & -1 & 41.88 \\
\hline 2.155 & 4 & 2 & 5 & -2 & 41.89 \\
\hline 2.149 & 1 & 0 & $a$ & 1 & 42.01 \\
\hline 2.139 & 16 & 3 & 1 & 0 & $4 i \cdot 21$ \\
\hline 2.127 & 1 & 2 & 4 & 2 & 42.46 \\
\hline 2.104 & 1 & 1 & 8 & 0 & 42.96 \\
\hline 2.094 & 12 & 3 & 2 & 0 & 43.16 \\
\hline 2.092 & 6 & 2 & 3 & -3 & 43.22 \\
\hline 2.387 & 1 & 3 & 2 & -1 & 43.33 \\
\hline $2.1 E 1$ & $\varepsilon$ & 0 & 0 & 4 & 43.46 \\
\hline 2.075 & 1 & 2 & 6 & 1 & 43.53 \\
\hline $2.0 \in 6$ & 17 & o & 1 & 4 & 43.77 \\
\hline 2.337 & 1 & 1 & 1 & -4 & 44.45 \\
\hline 2.026 & 1 & 2 & 6 & 3 & 44.69 \\
\hline 2.022 & 4 & 1 & 8 & 1 & 44.73 \\
\hline $2.0<1$ & 25 & 1 & 7 & 2 & 44.81 \\
\hline 2.018 & 1 & 1 & 5 & 3 & 44.88 \\
\hline 2.017 & 1 & 3 & 1 & 1 & 44.89 \\
\hline 2.002 & 4 & 2 & 5 & 2 & 45.25 \\
\hline 1.9995 & 8 & 2 & 6 & -2 & 45.32 \\
\hline 1.9973 & 1 & 2 & 4 & -3 & 45.37 \\
\hline 1.9959 & 1 & 3 & 1 & -2 & 45.38 \\
\hline 1.9829 & 4 & 2 & 1 & 3 & 45.72 \\
\hline 1.9633 & 2 & 0 & 3 & 4 & 46.20 \\
\hline 1.9618 & 8 & 0 & 8 & 2 & $4 E \cdot 24$ \\
\hline 1.4602 & 1 & 3 & 2 & -2 & 46.28 \\
\hline 1.9469 & 2 & 2 & 2 & 3 & 46.61 \\
\hline 1.9393 & 4 & 3 & 4 & 0 & 46.81 \\
\hline 1.9376 & 1 & 1 & 3 & -4 & 46.85 \\
\hline 1.9239 & 5 & 0 & 9 & 1 & 47.20 \\
\hline 1.3126 & 2 & 2 & 7 & 1 & 47.50 \\
\hline 1.9065 & 2 & 1 & 1 & 4 & 47.66 \\
\hline 1.9061 & 8 & 1 & 8 & -2 & 47.67 \\
\hline 1.9033 & 2 & 3 & 3 & -2 & 47.74 \\
\hline 1.8929 & 5 & 2 & 5 & -3 & 48.02 \\
\hline 1.8909 & 3 & 1 & 9 & 0 & $48 \cdot 08$ \\
\hline $1 .+750$ & 1 & 2 & 6 & 2 & 48.49 \\
\hline
\end{tabular}

\begin{tabular}{|c|c|c|c|c|c|}
\hline \multicolumn{6}{|c|}{ Calculated Pattern (Integrated) } \\
\hline$d(\stackrel{\circ}{A})$ & $I$ & & $h k$ & & $\begin{array}{c}2 \theta\left(^{\circ}\right) \\
\lambda=1.54056 \AA\end{array}$ \\
\hline 1.8745 & 7 & 1 & 2 & 4 & 48.53 \\
\hline 1.8574 & 1 & 1 & 9 & -1 & 49.00 \\
\hline 1.8532 & 9 & 2 & 7 & -2 & 49.12 \\
\hline 1.8498 & 7 & 1 & 8 & 2 & 45.22 \\
\hline 1.3483 & $\hat{\boldsymbol{z}}$ & 2 & 0 & -4 & 49.26 \\
\hline 1.8433 & 1 & 3 & 5 & 0 & 49.40 \\
\hline 1.8386 & 2 & 1 & 7 & -3 & 45.54 \\
\hline 1.3384 & 18 & 2 & 1 & -4 & 49.54 \\
\hline 1.8325 & 4 & 2 & 8 & 0 & 45.71 \\
\hline 1.8314 & 6 & 3 & 4 & -2 & 49.74 \\
\hline 1.8246 & 2 & 1 & 3 & 4 & 49.94 \\
\hline 1.8203 & 3 & 3 & 1 & 2 & 50.07 \\
\hline 1.8096 & 1 & 2 & 2 & -4 & 50.38 \\
\hline $1.796 \hat{z}$ & 3 & 0 & 5 & 4 & 50.79 \\
\hline 1.7924 & 7 & 3 & 2 & 2 & 50.90 \\
\hline 1.7546 & 3 & 2 & 3 & -4 & 51.76 \\
\hline 1.7641 & 1 & 1 & 7 & 3 & 51.78 \\
\hline $1.761 \mathrm{G}$ & 5 & 1 & 4 & 4 & 51.88 \\
\hline 1.7536 & 5 & 2 & 7 & 2 & 52.11 \\
\hline 1.7499 & 7 & 3 & 5 & -2 & 52.23 \\
\hline 1.7486 & 1 & 3 & 3 & 2 & 52.27 \\
\hline 1.7355 & 2 & 0 & 8 & 3 & 52.70 \\
\hline 1.7159 & 2 & 1 & 15 & 0 & 53.35 \\
\hline 1.7033 & 2 & 0 & 6 & 4 & 53.77 \\
\hline 1.5882 & 1 & 1 & 5 & 4 & 54.30 \\
\hline 1.6868 & 3 & 2 & 9 & 0 & 54.34 \\
\hline 1.6727 & 2 & 2 & 9 & -1 & 54.84 \\
\hline 1.6649 & 2 & 2 & 0 & 4 & 55.12 \\
\hline 1.6637 & 6 & 3 & 6 & -2 & 55.16 \\
\hline 1.6577 & 1 & 2 & 1 & 4 & 55.38 \\
\hline 1.5438 & 1 & 3 & 7 & 0 & 55.89 \\
\hline 1.6365 & 1 & 2 & 2 & 4 & 56.16 \\
\hline 1.6215 & 3 & 4 & ] & -1 & 56.72 \\
\hline $1.514 \varepsilon$ & 1 & 4 & 1 & -1 & 56.98 \\
\hline 1.6101 & 2 & 0 & 7 & 4 & 57.16 \\
\hline 1.5968 & 2 & 2 & 9 & -2 & 57.68 \\
\hline $1.595 z$ & 2 & 4 & 2 & -1 & 57.74 \\
\hline 1.5882 & 1 & 0 & 11 & 1 & $5 \varepsilon .03$ \\
\hline 1.5874 & 1 & 1 & 9 & -3 & 58.06 \\
\hline 1.5840 & 1 & 3 & 1 & -4 & 58.19 \\
\hline 1.5766 & 1 & 3 & 7 & -2 & 58.50 \\
\hline 1.5695 & 2 & 1 & 11 & 0 & 58.78 \\
\hline 1.5641 & 1 & 4 & 3 & -1 & 59.00 \\
\hline 1.5590 & 1 & 2 & 10 & 0 & 59.22 \\
\hline 1.5574 & 4 & 3 & $E$ & 2 & 59.29 \\
\hline 1.5535 & 1 & 4 & 17 & 1 & 59.45 \\
\hline 1.5477 & 3 & 4 & 1 & 1 & 59.70 \\
\hline 1.5307 & 1 & 2 & 2 & -5 & 60.43 \\
\hline 1.5304 & 1 & 4 & 2 & 1 & 60.44 \\
\hline 1.5195 & 1 & 0 & 8 & 4 & 60.92 \\
\hline
\end{tabular}




\section{Structure}

Triclinic, P $\overline{1}$ (2), $\mathrm{Z}=2$ [Jost and Hilmer, 1966]

\section{Lattice parameters}

$a=7.96, b=9.61, c=6.67 \AA$

$\alpha=70.1^{\circ}, \quad \beta=104.3^{\circ}, \gamma=122.5^{\circ}$ [ibid.]

Scattering factors

$\mathrm{Na}^{+1}, \mathrm{Si}^{0}, \mathrm{O}^{-1}[3 \cdot 3 \cdot 1 \mathrm{~A}]$

\section{Thermal parameters}

Isotropic [Jost and Hilmer, 1966]

\section{Density}

(calculated) $1.747 \mathrm{~g} / \mathrm{cm}^{3}$

\section{Scale factor}

$0.3695 \times 10^{4}$

\section{Additional patterns}

1. PDF card 2-0465 [Michigan Alkali Co., Wyandotte, Michigan]

2. PDF card 3-0432 [Dow Chemical Co., Midland, Michigan]

3. PDF card 19-1241 [Jamieson and Dent Glasser, 1966]

\section{Reference}

Jamieson, P.B. and L.S. Dent Glasser (1966) Sodium silicate hydrates. I. Crystallographic data, Acta Cryst. 20, 373-376.

Jost,K.-H. and W. Hilmer(1966). Die Struktur von $\mathrm{Na}_{2} \mathrm{H}_{2} \mathrm{SiO}_{4} \cdot 4 \mathrm{H}_{2} \mathrm{O}$, Acta Cryst. 2l, $583-589$.

\begin{tabular}{|c|c|c|c|c|c|c|}
\hline \multicolumn{7}{|c|}{ Calculated Pattern (Peak heights) } \\
\hline$d(\stackrel{\circ}{A})$ & $I$ & & $h k l$ & & & $\begin{array}{c}2 \theta\left({ }^{\circ}\right) \\
\lambda=1.54056 \mathrm{~A}\end{array}$ \\
\hline 7.64 & $<$ & ú & 1 & 0 & & 11.20 \\
\hline 7.13 & 10 & -1 & 1 & 0 & & 12.40 \\
\hline 6.69 & 39 & 1 & 0 & 0 & & 13.22 \\
\hline $6 .<5$ & 100 & u & 0 & 1 & & 14.16 \\
\hline $5 . E B$ & 41 & -1 & 1 & 1 & & 15.60 \\
\hline 5.63 & 25 & 0 & 1 & 1 & & $15.7 \mathrm{C}$ \\
\hline 4.76 & 4 & -1 & 0 & 1 & & 18.62 \\
\hline 4.49 & 20 & -1 & 2 & 0 & & 19.74 \\
\hline 4.45 & 17 & -1 & 2 & 1 & & 19.94 \\
\hline 4.17 & 20 & $\overline{1}$ & 1 & 0 & & $21 \cdot 30$ \\
\hline 4.10 & $5: 3$ & 1 & -1 & 1 & & 21.56 \\
\hline $3.5 \hat{c}$ & 39 & 0 & 2 & 0 & & 22.68 \\
\hline 3.04 & 14 & -2 & 1 & 0 & & $23 \cdot 12$ \\
\hline 3.78 & 29 & 0 & 2 & 1 & & 23.52 \\
\hline 3.53 & 20 & $-z$ & 2 & 1 & & 24.48 \\
\hline 3.35 & 03 & 2 & 0 & 0 & + & 20.62 \\
\hline 3.27 & 50 & -1 & 1 & 2 & & 27.26 \\
\hline 3.17 & 63 & 1 & -2 & 1 & & 28.16 \\
\hline 3.12 & 3 & -1 & 3 & 1 & & $28.5 t$ \\
\hline $3 \cdot 12$ & 3 & -1 & 2 & 2 & + & 28.56 \\
\hline 3.05 & 5 & -2 & 0 & 1 & & 29.22 \\
\hline 3.03 & 30 & -2 & 3 & 1 & & 29.44 \\
\hline 2.590 & 28 & $\overline{0}$ & -2 & 1 & + & 29.80 \\
\hline 2.055 & $?$ & 2 & 0 & 1 & + & 31.30 \\
\hline $2.54 \mathrm{U}$ & 13 & $-\bar{\varepsilon}$ & 2 & 2 & & $31.4 \varepsilon$ \\
\hline 2.817 & $3 i$ & 0 & 2 & 2 & + & 31.74 \\
\hline 2.740 & 30 & 2 & $-\bar{z}$ & 1 & & 32.50 \\
\hline 2.731 & 54 & 1 & 2 & 1 & + & 32.70 \\
\hline 2.679 & 4 & 0 & -1 & 2 & & 33.42 \\
\hline 2.664 & 19 & 0 & 3 & 1 & & 33.62 \\
\hline 2.039 & 10 & 2 & 1 & 0 & & 33.94 \\
\hline 2.621 & 7 & -3 & 2 & 1 & & 34.16 \\
\hline 2.609 & 9 & 1 & 1 & 2 & + & 34.34 \\
\hline 2.577 & 2 & $I$ & -1 & 2 & & 34.78 \\
\hline 2.503 & $i$ & -3 & 2 & 0 & & 34.90 \\
\hline 2.502 & $?$ & -3 & j & 1 & & 35.80 \\
\hline 2.460 & 24 & -3 & 1 & 1 & & 30.30 \\
\hline $2.45=$ & 1 & 2 & 1 & 1 & & 36.62 \\
\hline 2.407 & b & 1 & -3 & 1 & & 37.32 \\
\hline 2.405 & 5 & -1 & -1 & $\hat{z}$ & & 37.36 \\
\hline 2.316 & 3 & 2 & -3 & 1 & & 38.80 \\
\hline 2.224 & 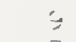 & -3 & 2 & 2 & & 39.24 \\
\hline$\hat{2} . \hat{c} 72$ & 3 & -3 & 3 & 2 & & 39.64 \\
\hline 2.230 & $1 ?$ & 1 & -2 & $\hat{\mathrm{c}}$ & & 40.42 \\
\hline 2.225 & 11 & -2 & 4 & 2 & & 40.50 \\
\hline 2.202 & i1 & -3 & 4 & 1 & + & 40.96 \\
\hline 2.197 & 15 & $\hat{c}$ & 0 & 2 & + & 41.04 \\
\hline 2.181 & i & 3 & -2 & 1 & & 41.36 \\
\hline 2.150 & 7 & -3 & 0 & 1 & & 41.80 \\
\hline 2.130 & 12 & -3 & 1 & $\hat{z}$ & & 42.24 \\
\hline
\end{tabular}


Sodium Hydrogen Silicate Tetrahydrate, $\mathrm{Na}_{2} \mathrm{H}_{2} \mathrm{SiO}_{4} \cdot 4 \mathrm{H}_{2} \mathrm{O}$ (triclinic) - continued

\begin{tabular}{|c|c|c|c|c|c|c|}
\hline \multicolumn{7}{|c|}{ Calculated Pattern (Peak heights) } \\
\hline$d(\stackrel{A}{)}$ & $I$ & \multicolumn{4}{|c|}{$h k l$} & $\begin{array}{c}2 \theta\left(^{\circ}\right) \\
\lambda=1.54056 \AA\end{array}$ \\
\hline $2.11 \mathrm{j}$ & 3 & -2 & 2 & 3 & + & 42.72 \\
\hline 2.054 & 7 & 2 & 2 & 0 & + & 43.36 \\
\hline 2.071 & 2 & 0 & 2 & 3 & & 43.60 \\
\hline 2.0 .50 & 3 & -1 & 3 & 3 & + & 44.08 \\
\hline 2.052 & c & 2 & -2 & 2 & & 44.10 \\
\hline 2.037 & ó & -1 & 0 & 3 & + & 44.44 \\
\hline$c_{+} \cdot \cup \geq 0$ & 14 & 3 & -3 & 1 & + & 44.84 \\
\hline 1.0952 & 1 & $-\hat{c}$ & -1 & 2 & & 45.42 \\
\hline 1.9601 & $y$ & 0 & 4 & 0 & & 46.28 \\
\hline 1.9503 & 7 & -1 & -2 & 2 & & 46.40 \\
\hline $1.930<$ & 3 & 1 & $i$ & 3 & + & 47.04 \\
\hline $1.3 \hat{c} \dot{L}^{b}$ & 2 & -4 & 2 & 0 & & 47.24 \\
\hline 1.9141 & 5 & 2 & -4 & 1 & & 47.46 \\
\hline 1. & 7 & 3 & 1 & 0 & + & 47.58 \\
\hline 1.8975 & 10 & 0 & -1 & 3 & + & 47.90 \\
\hline 1.8931 & 13 & -3 & 3 & 3 & & 48.02 \\
\hline $1 . \forall E .75$ & 11 & -2 & 4 & 3 & + & 48.16 \\
\hline 1.8730 & 9 & -4 & 4 & 1 & + & 48.38 \\
\hline 1.5715 & 10 & -4 & 3 & 2 & + & 48.60 \\
\hline 1.8403 & 5 & 1 & -1 & 3 & & 49.26 \\
\hline 1.E304 & 1 & -2 & C & 3 & & 49.60 \\
\hline 1. $0=0$ & 2 & -3 & 5 & 2 & & 49.82 \\
\hline $1 .<220$ & $1 ?$ & -1 & 4 & $\overline{3}$ & + & 50.00 \\
\hline 1.8050 & $2+$ & -2 & 5 & 0 & + & 50.50 \\
\hline $1.793=$ & 3 & u & -3 & 2 & & 50.80 \\
\hline 1.7504 & 3 & -3 & 5 & 0 & & 51.90 \\
\hline $1.750=$ & 3 & $\overline{3}$ & 0 & 2 & & $52 \cdot 22$ \\
\hline 1.7243 & 4 & -1 & 5 & 0 & & 52.90 \\
\hline 1.7070 & jo & 2 & 0 & 3 & & 53.62 \\
\hline 1.69834 & 2 & 1 & 4 & 1 & & 53.94 \\
\hline 1.5044 & 5 & -4 & 1 & 2 & & 54.08 \\
\hline 1.6207 & 3 & 2 & 3 & 1 & + & 54.38 \\
\hline 1.6710 & 5 & -2 & 5 & 3 & + & 54.90 \\
\hline 1.6655 & 3 & -1 & 2 & 4 & & 55.06 \\
\hline 1.6507 & 2 & -3 & 5 & 3 & & 55.34 \\
\hline 1.6500 & 3 & 3 & -3 & 2 & & 55.44 \\
\hline 1.6499 & 7 & -4 & 0 & 1 & + & 55.66 \\
\hline $1 \cdot 6,3 \subseteq 1$ & 2 & -1 & 1 & 4 & & 56.06 \\
\hline 1.6340 & 7 & -3 & -1 & 2 & + & 56.22 \\
\hline 1.6311 & 4 & -2 & 3 & 4 & & 56.36 \\
\hline 1.62 .42 & $i$ & 3 & 1 & 2 & & 56.62 \\
\hline $1.610 b$ & + & -2 & -1 & 3 & + & 56.84 \\
\hline 1.6133 & 4 & 0 & 1 & 4 & + & .57 .04 \\
\hline $1.58 y 8$ & 3 & -5 & 3 & 1 & & 57.96 \\
\hline 1.5803 & 5 & $-i$ & 5 & 3 & + & 58.10 \\
\hline 1.583 & 0 & 4 & 0 & 1 & + & 58.20 \\
\hline 1.5809 & 7 & -3 & 6 & 2 & & 58.32 \\
\hline 1.5710 & 2 & 2 & 3 & 2 & + & 58.72 \\
\hline 1.5610 & 2 & -2 & 6 & 2 & & 59.10 \\
\hline 1.5550 & 1 & -4 & 5 & 3 & & 59.36 \\
\hline
\end{tabular}

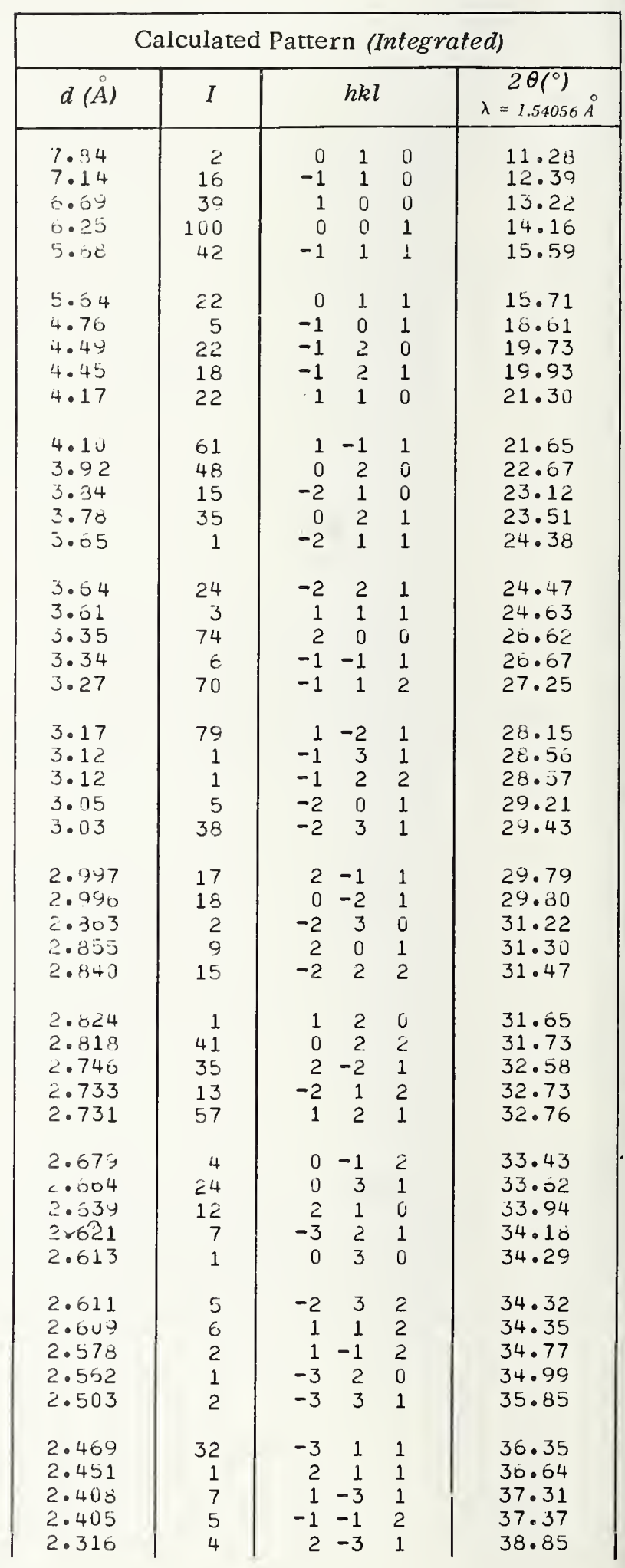


Sodium Hydrogen Silicate Tetrahydrate, $\mathrm{Na}_{2} \mathrm{H}_{2} \mathrm{SiO}_{4} \cdot 4 \mathrm{H}_{2} \mathrm{O}$ (triclinic) - continued

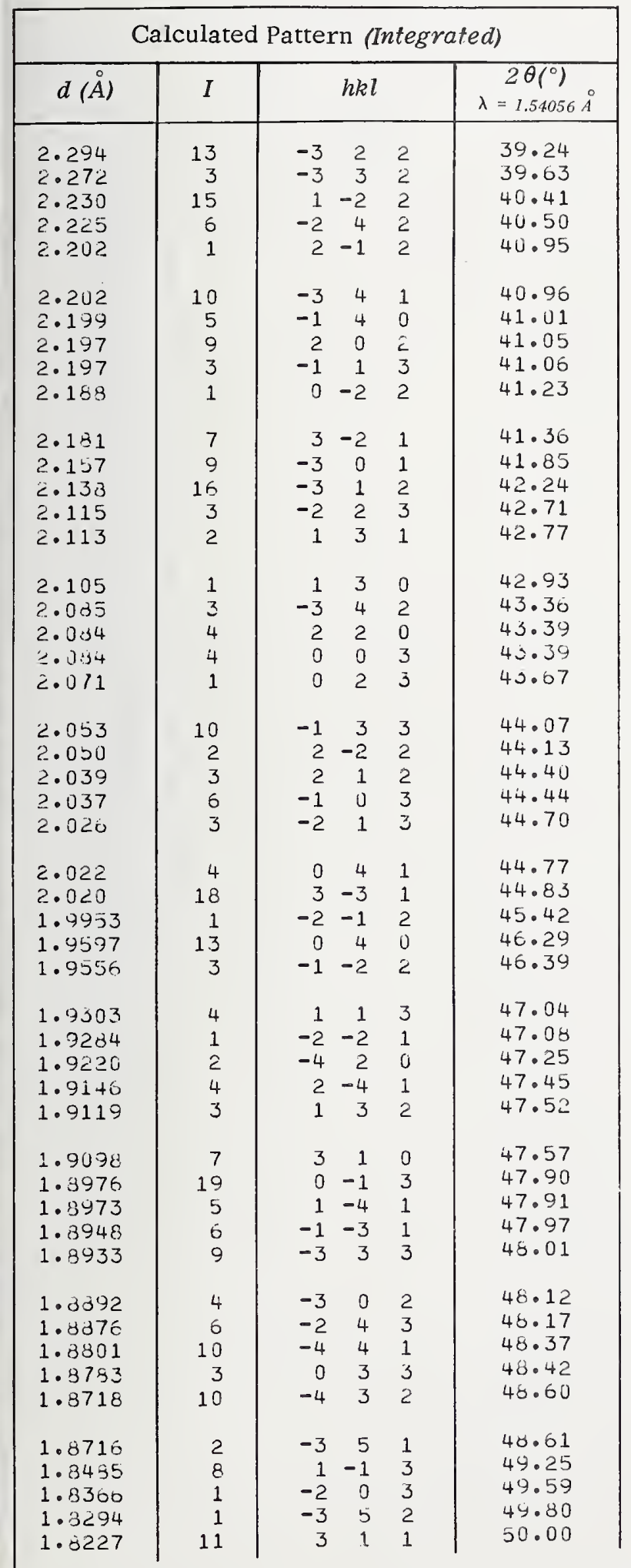

\begin{tabular}{|c|c|c|c|c|c|}
\hline \multicolumn{6}{|c|}{ Calculated Pattern (Integrated) } \\
\hline$d(\stackrel{\circ}{A})$ & $I$ & & $h k l$ & & $\begin{array}{c}\left.2 \theta 0^{\circ}\right) \\
\lambda=1.54056 \AA\end{array}$ \\
\hline 1.3224 & 13 & -1 & 4 & 3 & 50.01 \\
\hline 1.8131 & 1 & -1 & 5 & 1 & 56.28 \\
\hline 1.8111 & 2 & -3 & 4 & 3 & 50.34 \\
\hline 1.8062 & 19 & -2 & 5 & 0 & 50.49 \\
\hline 1.8059 & 15 & 2 & 2 & 2 & $50 \cdot 50$ \\
\hline 1.3058 & 3 & 3 & -1 & 2 & 50.50 \\
\hline 1.7930 & 3 & 0 & -3 & 2 & $50.8 B$ \\
\hline 1.7586 & 4 & -3 & 5 & 0 & $51 \cdot 95$ \\
\hline 1.7499 & 4 & 3 & 0 & z & $5 c \cdot 23$ \\
\hline 1.7294 & 6 & -1 & 5 & $\overline{0}$ & $52 \cdot 90$ \\
\hline $1.7 \cup 79$ & 8 & 2 & 0 & 3 & 53.62 \\
\hline 1.6987 & 3 & 1 & 4 & 1 & 53.93 \\
\hline 1.6941 & $\sigma$ & -4 & 1 & 2 & 34.09 \\
\hline 1.6060 & 3 & 2 & 3 & 1 & 54.37 \\
\hline 1.6348 & 1 & 2 & -1 & 3 & $54 \cdot 41$ \\
\hline 1.5722 & 1 & 0 & -2 & 3 & 54.06 \\
\hline 1.6711 & 4 & -2 & 5 & 3 & 54.89 \\
\hline 1.6697 & 4 & -2 & -2 & 2 & $54 \cdot 94$ \\
\hline 1.6655 & 1 & -1 & 2 & 4 & $55 \cdot 10$ \\
\hline 1.6590 & 2 & -3 & 5 & 3 & 55.33 \\
\hline 1.6562 & 3 & 3 & -3 & 2 & 55.43 \\
\hline 1.6511 & 4 & -4 & 3 & 3 & 55.62 \\
\hline 1.5496 & 9 & -4 & 0 & 1 & 55.67 \\
\hline 1.6393 & 2 & -1 & 1 & 4 & 50.00 \\
\hline 1.0357 & 1 & -4 & 4 & 3 & $56 \cdot 19$ \\
\hline 1.0350 & 8 & -3 & -1 & 2 & $50 \cdot 21$ \\
\hline 1.6349 & 1 & -2 & 2 & 4 & 50.22 \\
\hline 1.0307 & 1 & -2 & 3 & 4 & $50 \cdot 30$ \\
\hline $1.5<44$ & 1 & 3 & 1 & 2 & 50.01 \\
\hline 1.6192 & 1 & -1 & 3 & 4 & 56.31 \\
\hline 1.6134 & 4 & -2 & -1 & 3 & 56.34 \\
\hline 1.0132 & 4 & 0 & 1 & 4 & 57.014 \\
\hline 1.0119 & 3 & -4 & 5 & 0 & 57.09 \\
\hline 1.5902 & 3 & -5 & 3 & 1 & 57.95 \\
\hline 1.5834 & 1 & 3 & 2 & $i$ & 58.02 \\
\hline 1.5375 & 1 & 4 & -4 & 1 & 53.05 \\
\hline 1.5855 & 2 & -3 & 6 & 1 & 38.09 \\
\hline 1.5351 & 3 & -1 & 5 & 3 & 50.11 \\
\hline 1.5843 & 3 & 4 & 0 & 1 & 58.18 \\
\hline 1.5840 & 2 & -2 & -3 & 1 & 58.19 \\
\hline 1.5030 & 2 & 2 & -4 & 2 & bd. 21 \\
\hline $1.550 E$ & 8 & -3 & 6 & 2 & 58.32 \\
\hline 1.5719 & 1 & -2 & 1 & 4 & $5 d .69$ \\
\hline 1.5710 & 1 & 2 & 3 & 2 & 50.72 \\
\hline 1.5017 & 2 & -2 & 6 & 2 & 59.11 \\
\hline 1.5556 & 1 & -4 & 5 & 3 & 59.30 \\
\hline 1.5330 & 2 & -3 & -2 & 1 & 59.47 \\
\hline 1.5498 & 1 & -1 & 0 & 4 & 59.00 \\
\hline $1 \cdot 5+28$ & 5 & -5 & 4 & ¿ & 59.91 \\
\hline $1 \cdot 5+24$ & 2 & -5 & 3 & 0 & 59.92 \\
\hline
\end{tabular}




\section{Structure}

Tetragona l, $\mathrm{P} 4_{2} / \mathrm{nbc}$ (133), $\mathrm{Z}=8$ [MCDonald et al., 1964]

\section{Lattice parameters}

$a=9.020 \pm 0.003, c=13.686 \pm 0.003 \AA$ [ibid.]

\section{Scattering factors}

$\mathrm{Sn}^{\circ}[3.3 .1 \mathrm{~B}]$;

$\mathrm{Na}^{\circ}, \mathrm{F}^{-1}$ [Berghuis et al., 1955]

\section{Thermal parameters}

Isotropic: Sn 1.70; F(1) 2.29; F(2) 1.91; $\mathrm{F}(3) 2.71 ; \mathrm{Na}(1) 1.92 ; \mathrm{Na}(2) 1.97$

\section{Density}

(calculated) $4.24 \mathrm{~g} / \mathrm{cm}^{3}$ [MCDonald et al., 1964 ]

\section{Scale factor}

$$
125.3 \times 10^{4}
$$

\section{Additional patterns}

1.PDF card 15-619[Kriegsmann and Kessler, 1962]

2.PDF card 16-796[Donaldson and O'Donoghue 1964]

\section{Reference}

Berghuis, J., IJ. M. Haanapel, M. Pottess, B.O. Loopstra, C.H. MacGillavry, and A. L. VeenendahI (1955). New calculations of atomic scattering factors, Acta Cryst. $8,478-483$.

Dona ldson, J.D. and J.D. O'Donaghue (1964). Complex tin fluorides, J. Chem.Soc.1964, $271-280$.

Kriegsman, H. and G. Kessler (1962).Fluorkomplexverbindungen des zwei- und vierwertigen Zinns und die partielle Hydrolyse des SnClF, Z. Anorg. Allgem. Chem. $318,266-276$.

McDonald,R.R., A.C. Larson, and D.T.Cromer (1964). The crystal structure of sodium pentafluorodistannate(II), $\mathrm{NaSn}_{2} \mathrm{~F}_{5}$, Acta sryst. 17, 1104-1108.

\begin{tabular}{|c|c|c|c|c|c|c|}
\hline \multicolumn{7}{|c|}{ Calculated Pattern (Peak heights) } \\
\hline$d(\AA)$ & $I$ & & $h k$ & & & $\begin{array}{c}2 \theta\left({ }^{\circ}\right) \\
\lambda=1.54056 \mathrm{~A}\end{array}$ \\
\hline 6.84 & 2 & 3 & 0 & 2 & & 12.94 \\
\hline 4.51 & 42 & 2 & 0 & 0 & & 19.68 \\
\hline 4.28 & 1 & 2 & $\mathrm{C}$ & 1 & & 20.72 \\
\hline 3.474 & 100 & 2 & 1 & 2 & & 25.52 \\
\hline 3.422 & 23 & 0 & 0 & 4 & & 26.02 \\
\hline 3.188 & 9 & 2 & 2 & 0 & & 27.96 \\
\hline 3.015 & 5 & 1 & 1 & 4 & + & 29.00 \\
\hline 2.852 & 10 & 3 & 1 & 0 & & 31.34 \\
\hline 2.725 & 15 & 2 & 0 & 4 & & 32.84 \\
\hline 2.265 & 4 & 2 & 1 & 5 & & 35.75 \\
\hline 2.255 & 3 & 4 & ง & 0 & & 35.94 \\
\hline 2.224 & 1 & 4 & 0 & 1 & & 40.52 \\
\hline 2.191 & 5 & 3 & 1 & 4 & & 41.16 \\
\hline 2.160 & 1 & 4 & 1 & 1 & & 41.78 \\
\hline 2.126 & 5 & 3 & 3 & 0 & & 42.48 \\
\hline 2.084 & 50 & 4 & 1 & 2 & & 43.35 \\
\hline 2.035 & 1 & - & 0 & 6 & & 44.43 \\
\hline 2.017 & 2 & 4 & 2 & 0 & & 44.90 \\
\hline 1.9853 & 17 & 2 & 1 & 6 & & 45.65 \\
\hline 1.8827 & 4 & 4 & 3 & 4 & & 48.30 \\
\hline 1.8058 & 14 & 3 & 3 & 4 & & 50.50 \\
\hline 1.7108 & 3 & ] & 0 & 8 & & 53.52 \\
\hline 1.6269 & 4 & 5 & 2 & 2 & & 50.52 \\
\hline 1. 5994 & 1 & 2 & 0 & $\overline{8}$ & & 57.58 \\
\hline 1.5785 & 8 & 4 & 1 & 6 & & 58.40 \\
\hline 1.5750 & 5 & 2 & 1 & 8 & & 58.56 \\
\hline 1.5715 & 3 & 5 & 1 & 4 & & 58.70 \\
\hline 1.5471 & 3 & 5 & 3 & 0 & & 59.72 \\
\hline 1.5074 & 1 & 2 & 2 & 8 & & 61.46 \\
\hline 1.5034 & 3 & 6 & U & 0 & & 61.64 \\
\hline 1.4671 & 2 & 3 & 1 & 8 & & 63.34 \\
\hline 1.4262 & 1 & 6 & $<$ & 0 & & 65.38 \\
\hline 1.4094 & 3 & 5 & 3 & 4 & & 06.26 \\
\hline 1.3796 & 4 & 5 & 4 & 2 & & 67.88 \\
\hline 1.3764 & 4 & 6 & J & 4 & & 08.06 \\
\hline 1.3500 & 1 & 5 & 2 & 6 & & 09.58 \\
\hline 1.3327 & 2 & 3 & 3 & 8 & & 70.62 \\
\hline 1.3165 & 2 & b & 2 & 4 & & 71.62 \\
\hline 1.2959 & 2 & 2 & 1 & 10 & & $72 \cdot 94$ \\
\hline 1.2193 & 2 & 7 & 2 & 2 & & 78.36 \\
\hline 1.1986 & 1 & 5 & 4 & 6 & & 79.96 \\
\hline 1.1602 & 2 & 4 & 1 & 10 & & 83.20 \\
\hline 1.1474 & 1 & 5 & 3 & 8 & & 84.34 \\
\hline 1.1292 & 1 & 6 & 0 & 8 & & 86.52 \\
\hline 1.1041 & 2 & 7 & 4 & 2 & + & 88.48 \\
\hline 1.3838 & 1 & 7 & 2 & 6 & & 90.05 \\
\hline 1.0045 & 2 & 7 & 4 & 6 & + & 100.14 \\
\hline
\end{tabular}




\begin{tabular}{|c|c|c|c|c|c|}
\hline \multicolumn{6}{|c|}{ Calculated Pattern (Integrated) } \\
\hline$d(\AA)$ & $I$ & & $h k l$ & & $\begin{array}{c}2 \theta\left(^{\circ}\right) \\
\lambda=1.54056 \AA\end{array}$ \\
\hline 6.84 & 1 & 0 & 0 & 2 & 12.93 \\
\hline 4. 51 & 40 & 2 & 0 & 0 & 19.67 \\
\hline 4. 28 & 1 & 2 & 0 & 1 & 20.72 \\
\hline 3.475 & 100 & 2 & 1 & 2 & 25.61 \\
\hline 3.422 & 22 & 0 & 0 & 4 & 26.02 \\
\hline 3.189 & 9 & 2 & 2 & 3 & 27.95 \\
\hline 3. 322 & 1 & 2 & 1 & 3 & 29.53 \\
\hline 3.015 & 5 & 1 & $i$ & 4 & 29.60 \\
\hline L. 852 & 10 & 3 & 1 & 0 & 31.33 \\
\hline 2.726 & 18 & 2 & 0 & 4 & 32.83 \\
\hline$\angle \cdot \angle b 5$ & 5 & 2 & 1 & 5 & 33.76 \\
\hline 2.255 & 2 & 4 & 3 & 0 & 39.95 \\
\hline 2. 225 & 2 & 4 & 0 & 1 & 40.51 \\
\hline 2.191 & 5 & 3 & 1 & 4 & 41.17 \\
\hline 2.150 & 1 & 4 & 1 & 1 & 41.78 \\
\hline L. 126 & 5 & 3 & 3 & 0 & 42.48 \\
\hline 2.084 & 37 & 4 & 1 & 2 & 43.39 \\
\hline 2.035 & 1 & 2 & 3 & 6 & 44.47 \\
\hline 2.317 & 3 & 4 & 2 & 0 & 44.90 \\
\hline 1.3853 & 21 & $<$ & 1 & 5 & 45.65 \\
\hline 1.8828 & 5 & 4 & 3 & 4 & 48.33 \\
\hline 1.8358 & 17 & 3 & 3 & 4 & 50.50 \\
\hline 1.7107 & 4 & 0 & 3 & 8 & 53.52 \\
\hline 1.6269 & 6 & 5 & 2 & 2 & 50.52 \\
\hline 1.5995 & 2 & $\angle$ & 0 & 8 & 57.58 \\
\hline 1.5789 & 11 & 4 & 1 & 6 & 58.40 \\
\hline 1.5750 & 1 & 2 & 1 & 8 & 58.56 \\
\hline 1.5714 & 3 & : & 1 & 4 & 58.71 \\
\hline 1.5469 & 4 & 5 & 3 & 0 & 59.73 \\
\hline 1.5375 & 1 & 2 & 2 & 8 & 61.45 \\
\hline 1.5033 & 4 & $E$ & 0 & 0 & 61.05 \\
\hline 1.4671 & 3 & 3 & 1 & 8 & 63.34 \\
\hline 1.4432 & 1 & 5 & $i$ & 2 & 54.21 \\
\hline 1.4262 & 1 & 5 & 2 & 0 & 65.38 \\
\hline 1.4095 & 4 & 5 & 3 & 4 & 66.25 \\
\hline 1.3798 & 5 & 5 & 4 & 2 & 67.87 \\
\hline 1.3763 & 3 & 6 & 0 & 4 & 68.06 \\
\hline 1.3501 & 2 & 5 & 2 & 6 & 69.53 \\
\hline 1.3328 & 3 & 3 & 3 & 8 & 70.61 \\
\hline 1. 3164 & 2 & 6 & 2 & 4 & 71.63 \\
\hline 1.2960 & 3 & 2 & 1 & 10 & 72.73 \\
\hline 1.2192 & 3 & 7 & 2 & 2 & 78.37 \\
\hline 1.1985 & 2 & 5 & 4 & 5 & 79.98 \\
\hline 1.1745 & 1 & ó & 4 & 4 & $81 \cdot 94$ \\
\hline 1.1603 & 3 & 4 & 1 & 10 & 83.19 \\
\hline 1.1474 & 2 & 5 & 3 & 8 & 84.34 \\
\hline 1.1293 & 2 & 6 & 0 & 8 & 86.32 \\
\hline 1.1341 & 1 & 8 & 1 & 2 & 88.47 \\
\hline $1.134 i$ & 2 & 7 & 4 & 2 & 80.47 \\
\hline 1.0887 & 2 & 7 & 2 & 6 & YD. 36 \\
\hline
\end{tabular}

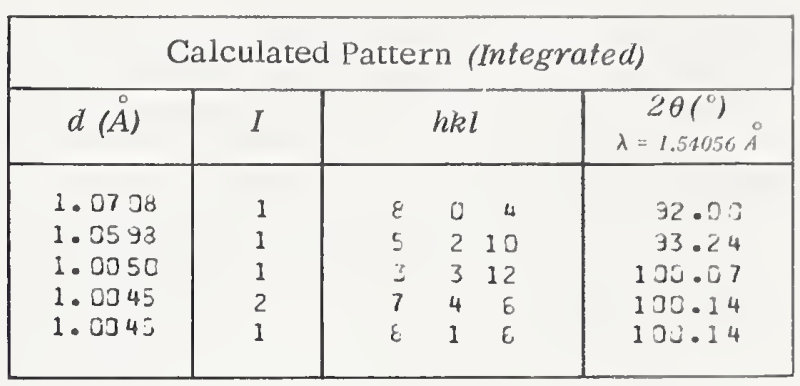


Structure

Monoclinic, $\quad \mathrm{P} 2_{1}$ (4), $\mathrm{Z}=2$, [okaya and

Stemple, 1966]

\section{Lattice parameters}

$\mathrm{a}=7.715 \pm 0.003, \mathrm{~b}=6.004 \pm 0.003$,

$c=6.231 \pm 0.003 \AA, \beta=100.1 \pm 0.1^{\circ} \quad$ [ibid.]

\section{Scattering factors}

$\mathrm{H}^{\circ}, \mathrm{C}^{\circ}, \mathrm{O}^{\circ}[3.3 . \mathrm{IA}]$

\section{Thermal parameters}

Anisotropic for oxygen and carbon, isotropic for hydrogen, Table $1(\mathrm{~b})$ [okaya and Stemple, 1966]

\section{Atomic positions}

Table 1 (a) [ibid.]

\section{Density}

(calculated) $1.754 \mathrm{~g} / \mathrm{cm}^{3}$

Scale factor

$0.4175 \times 10^{4}$

\section{Additional patterns}

l. PDF card 4-0333 [Inst. Phys. Univ. College, Cardiff, Wales]. This card may represent a different polymorph.

\section{Reference}

Okaya,H. and N.R. Stemple (1966). Refinement of the structure of d-tartaric acid by $x$-ray and neutron diffraction, Acta cryst. 21, 237-243.

\begin{tabular}{|l|r|rrl|l|}
\hline \multicolumn{6}{|c|}{ Calculated Pattern (Peak heights) } \\
\hline$d(A)$ & $I$ & \multicolumn{3}{|c|}{$h k l$} & $\begin{array}{c}2 \theta\left(^{\circ}\right) \\
1=1.54056 \AA\end{array}$ \\
\hline 7.63 & 13 & 1 & 0 & 0 & 11.64 \\
5.24 & 7 & -1 & 0 & 1 & 16.90 \\
4.71 & 20 & 1 & 1 & 0 & 18.82 \\
4.41 & 18 & 1 & 0 & 1 & 20.12 \\
4.29 & 100 & 0 & 1 & 1 & 20.68 \\
3.95 & 18 & -1 & 1 & 1 & 22.50 \\
3.80 & 4 & 2 & 0 & 0 & 23.40 \\
3.55 & 26 & 1 & 1 & 1 & 25.04 \\
3.209 & 5 & 2 & 1 & 0 & 27.78 \\
3.066 & 15 & 0 & 0 & 2 & 29.10 \\
& & & & & 29.42 \\
3.033 & 5 & -2 & 1 & 1 & 29.74 \\
2.002 & 36 & 0 & 2 & 0 & 32.04 \\
\end{tabular}

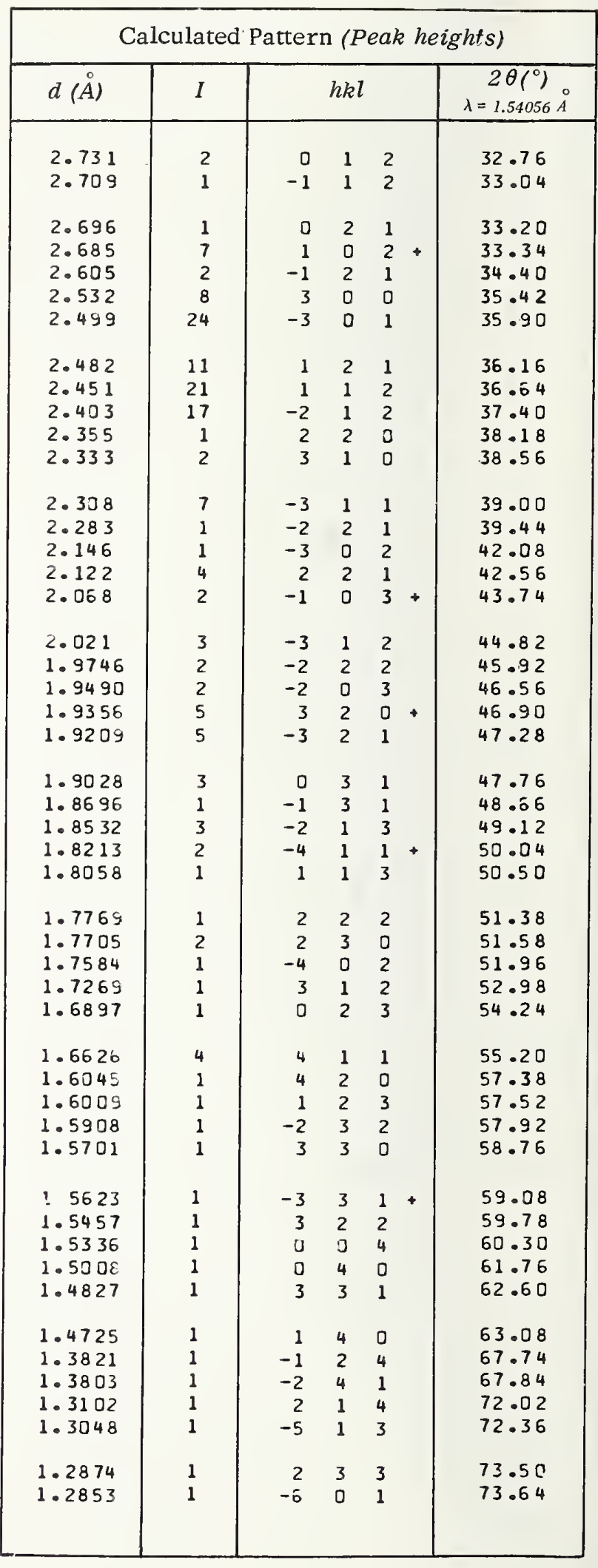




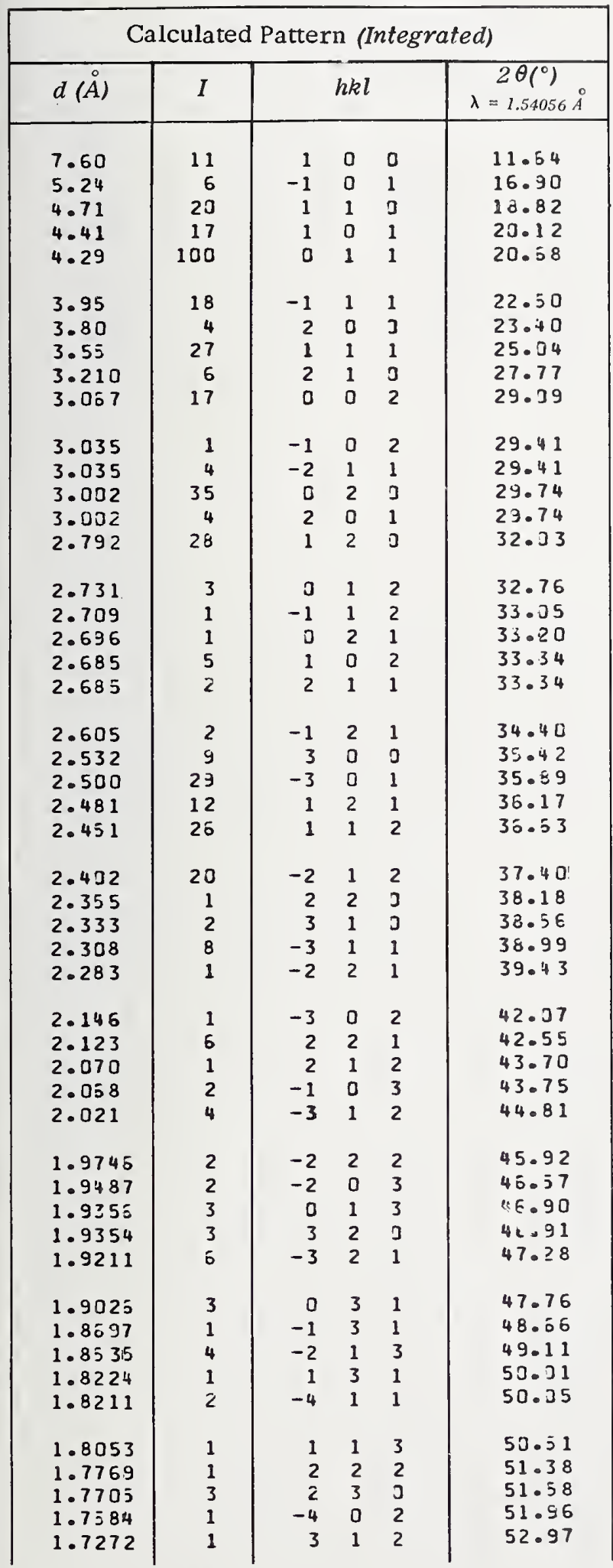

\begin{tabular}{|c|c|c|c|c|c|}
\hline \multicolumn{6}{|c|}{ Calculated Pattern (Integrated) } \\
\hline$d(\stackrel{\circ}{A})$ & $I$ & & $h k l$ & & $\begin{array}{c}2 \theta\left(^{\circ}\right) \\
\lambda=1.54056 A\end{array}$ \\
\hline 1.6900 & 1 & 0 & 2 & 3 & 54.23 \\
\hline 1.6525 & 5 & 4 & 1 & 1 & 55.20 \\
\hline 1.6345 & 1 & -2 & 2 & 3 & 56.23 \\
\hline 1.6048 & 1 & 4 & 2 & 0 & 57.37 \\
\hline 1.6012 & 1 & 1 & 2 & 3 & 57.51 \\
\hline 1. 5907 & 1 & -2 & 3 & 2 & 57.92 \\
\hline 1.5700 & 1 & 3 & 3 & 0 & 58.76 \\
\hline 1.5624 & 1 & -3 & 3 & 1 & 59.08 \\
\hline 1.55712 & 1 & -1 & 0 & 4 & 59.29 \\
\hline 1.5459 & 1 & 3 & 2 & 2 & 59.77 \\
\hline 1.5335 & 1 & 0 & 0 & 4 & 60.30 \\
\hline 1.5073 & 1 & -1 & 1 & 4 & $61 .+6$ \\
\hline 1.5010 & 1 & 0 & 4 & 3 & 61.75 \\
\hline 1.4828 & 1 & 3 & 3 & 1 & 62.50 \\
\hline 1.4725 & 1 & 1 & 4 & 0 & 63.38 \\
\hline 1.4535 & 1 & -3 & 3 & 2 & 63.51 \\
\hline 1.4209 & 1 & 1 & 4 & 1 & 65.65 \\
\hline 1.3823 & 1 & -1 & 2 & 4 & 67.73 \\
\hline 1.3805 & 1 & -2 & 4 & 1 & 67.33 \\
\hline 1.3103 & 1 & 2 & 1 & 4 & 72.31 \\
\hline 1.3048 & 1 & -5 & 1 & 3 & 72.36 \\
\hline 1.2874 & 1 & 2 & 3 & 3 & 73.50 \\
\hline 1.2852 & 1 & -6 & 0 & 1 & 73.55 \\
\hline
\end{tabular}


Zinc Glutamate Dihydrate, $\mathrm{ZnC}_{5} \mathrm{H}_{7} \mathrm{NO}_{4} \cdot 2 \mathrm{H}_{2} \mathrm{O}$ (orthorhombic)

\section{Structure}

Orthorhombic, $\mathrm{P} 2_{\perp} 2_{1} 2_{\perp}$ (19), Z=4, [Gramaccioli, 1966]

\section{Lattice parameters}

$\mathrm{a}=11.190, \mathrm{~b}=10.463, \mathrm{c}=7.220 \AA$ [ibid.]

\section{Scattering factors}

$\mathrm{C}^{\circ}, \mathrm{N}^{\circ}, \mathrm{H}^{\circ},[3.3 . \mathrm{AA}]$;

$\mathrm{Zn}^{\circ}[3.3 .1 \mathrm{~A}]$, corrected for the real part of the dispersion effect [3.3.2B]

\section{Thermal parameters}

Isotropic: $\mathrm{Zn} 1.93 ; \mathrm{C}(1) 1.94 ; \mathrm{C}(2) 1.95$; C(3) $2.19 ; C(4) 2.68 ; C(5) 2.35 ; N 2.20$;

$O$ (1) $2.33 ; O(2) 2.25 ; O(3) 2.61$;

$\mathrm{O}(4) 2.88 ; \mathrm{O}(5) 3.08 ; \mathrm{O}(6) 2.86 ; \mathrm{H}(1)$

through $\mathrm{H}(1 \mathrm{l})$ as given by Gramaccioli [1966].

\section{Density}

(calculated) $1.937 \mathrm{~g} / \mathrm{cm}^{3}$ [Gramaccioli, 1966]

\section{Scale factor}

$$
3.869 \times 10^{4}
$$

\section{Reference}

Gramaccioli,C.M.(1966). The crystal structure of zinc glutamate dihydrate, Acta Cryst. 21, 600-605.

\begin{tabular}{|c|c|c|c|c|c|c|}
\hline \multicolumn{7}{|c|}{ Calculated Pattern (Peak heights) } \\
\hline$d(\stackrel{\circ}{A})$ & $I$ & & $h$ & & & $\begin{array}{c}2 \theta\left({ }^{\circ}\right) \\
\lambda=1.54056 \AA\end{array}$ \\
\hline 7.64 & $=5$ & 1 & 1 & 0 & & 11.58 \\
\hline 5.94 & 32 & J & 1 & 1 & & 14.30 \\
\hline 5.59 & 45 & 2 & 0 & 0 & & 15.84 \\
\hline 5.25 & 150 & I & 1 & 1 & + & 15.88 \\
\hline 4.93 & 51 & 2 & 1 & 0 & & 17.96 \\
\hline 4.23 & 48 & 0 & 2 & 1 & & 20.96 \\
\hline 4.07 & 74 & z & 1 & 1 & & 21.80 \\
\hline 3.96 & 87 & 1 & 2 & 1 & & $2<.42$ \\
\hline 3.82 & 2 & 2 & 2 & 3 & & 23.25 \\
\hline $3 \cdot 61$ & 2 & 3 & o & 2 & & 24.54 \\
\hline 3.435 & 21 & 1 & 0 & 2 & & 25.92 \\
\hline 3.378 & 2 & 2 & 2 & 1 & & 26.35 \\
\hline 3.329 & 4 & 1 & 3 & 0 & & 26.76 \\
\hline 3.314 & 16 & 3 & 3 & 1 & & 26.80 \\
\hline 3.264 & 7 & 1 & 1 & 2 & & $27 \cdot 30$ \\
\hline 3.160 & 6 & 3 & 1 & 1 & & 28.22 \\
\hline 3.140 & 7 & 2 & 3 & 1 & & 28.40 \\
\hline 3.038 & 18 & 3 & 2 & 0 & & 29.38 \\
\hline 3.023 & 23 & 1 & 3 & 1 & & 23.52 \\
\hline 2.970 & 10 & 3 & 2 & 2 & & $30 \cdot 30$ \\
\hline 2.963 & 7 & 2 & 3 & 0 & & $3 U .14$ \\
\hline 2.914 & 1 & 2 & 1 & 2 & & 30.56 \\
\hline 2.872 & 12 & 1 & 2 & 2 & & 31.12 \\
\hline 2.798 & 4 & 4 & 0 & 0 & + & 31.96 \\
\hline 2.738 & 2 & 2 & 3 & 1 & & 32.58 \\
\hline 2.703 & 8 & 4 & 1 & J & & 33.12 \\
\hline 2.624 & 4 & 2 & 2 & 2 & & 34.14 \\
\hline 2.617 & 4 & 0 & 4 & 3 & & 34.24 \\
\hline 2.600 & 9 & 4 & 0 & 1 & & 34.36 \\
\hline 2.595 & 7 & 3 & 3 & 2 & & 34.54 \\
\hline 2.517 & $E$ & 3 & 1 & 2 & & 35.64 \\
\hline 2.503 & 10 & 0 & 3 & 2 & & 35.76 \\
\hline 2.466 & 1 & 4 & 2 & J & & 30.40 \\
\hline 2.463 & $?$ & 3 & 4 & 1 & & 35.50 \\
\hline 2.448 & 1 & 1 & 3 & 2 & & 36.56 \\
\hline 2.403 & 15 & 1 & 4 & 1 & + & 37.40 \\
\hline 2.370 & 1 & 2 & 4 & 0 & & 37.94 \\
\hline 2.346 & 3 & 9 & 1 & 3 & & 38.34 \\
\hline 2.334 & 3 & 4 & $<$ & 1 & & $3 \varepsilon .54$ \\
\hline 2.324 & 11 & 3 & 2 & 2 & & 38.72 \\
\hline 2.295 & 17 & 1 & 1 & 3 & & 39.22 \\
\hline 2.290 & 22 & 2 & 3 & 2 & & 39.32 \\
\hline$\ddot{2} .251$ & $E$ & 2 & 4 & 1 & & 40.32 \\
\hline 2.211 & 2 & 2 & o & 3 & & 40.78 \\
\hline 2.163 & 8 & 4 & 1 & 2 & + & 41.72 \\
\hline 2.146 & 9 & 1 & 2 & 3 & & 42.38 \\
\hline 2.138 & 8 & $j$ & 3 & 1 & & 42.24 \\
\hline 2.113 & 2 & 3 & 4 & 2 & & 42.66 \\
\hline 2.094 & 5 & 5 & 1 & 1 & & 43.15 \\
\hline 2.083 & 5 & 4 & 3 & 1 & & 43.28 \\
\hline
\end{tabular}




\begin{tabular}{|c|c|c|c|c|c|c|}
\hline \multicolumn{7}{|c|}{ Calculated Pattern (Peak heights) } \\
\hline$d(\AA)$ & $I$ & & $h k l$ & & & $\begin{array}{c}2 \theta\left({ }^{\circ}\right) \\
\lambda=1.54056 \AA\end{array}$ \\
\hline 2.081 & 7 & 1 & 4 & 2 & + & 43.44 \\
\hline 2.057 & 8 & 1 & 5 & 0 & 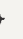 & 43.98 \\
\hline 2.053 & 7 & 3 & 4 & 1 & & 44.08 \\
\hline$\angle .037$ & 8 & 4 & 2 & 2 & + & 44.44 \\
\hline 2.322 & 2 & 3 & $\mathrm{C}$ & 3 & & 44.73 \\
\hline 2.009 & 3 & $?$ & 5 & 1 & & 45.08 \\
\hline 1.9853 & 4 & 3 & 1 & 3 & & 45.66 \\
\hline 1.9833 & 7 & 2 & 4 & $2+$ & + & 45.78 \\
\hline 1.9020 & 1 & 5 & 3 & 2 & & 47.78 \\
\hline 1.8916 & 5 & $<$ & 5 & 1 & & $4 \varepsilon .06$ \\
\hline 1.8864 & 5 & 3 & 2 & 3 & & 48.20 \\
\hline 1.8711 & 10 & 5 & 1 & 2 & & 4ع.ड 2 \\
\hline 1.8668 & 7 & 4 & 3 & $2+$ & 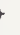 & 48.74 \\
\hline 1.8409 & 1 & 4 & 4 & 1 & & 49.30 \\
\hline 1.8420 & i. & 3 & 4 & 2 & & 49.44 \\
\hline 1.8364 & 5 & ó & 1 & 0 & & 49.00 \\
\hline 1.8233 & 7 & 5 & 3 & 1 & + & 49.98 \\
\hline 1.3104 & 5 & 3 & 5 & 2 & & 50.36 \\
\hline 1.8053 & 4 & 6 & 0 & $1+$ & + & 53.50 \\
\hline 1.7971 & 2 & 4 & 1 & 3 & & 53.76 \\
\hline 1.7873 & $?$ & 1 & 5 & 2 & & 51.36 \\
\hline 1.7795 & 3 & 5 & 1 & 1 & & 51.30 \\
\hline 1.7692 & 3 & 3 & 5 & $1+$ & + & 51.62 \\
\hline 1.7565 & 1 & 1 & 1 & 4 & & 52.02 \\
\hline 1.7430 & 3 & 3 & 3 & $3+$ & + & 52.24 \\
\hline 1.7440 & 2 & o & 6 & s & & 52.42 \\
\hline 1.7227 & 3 & 2 & 5 & 2 & + & 53.12 \\
\hline 1.7179 & 3 & 2 & 3 & 4 & & 53.28 \\
\hline 1.7306 & 2 & 3 & 2 & 4 & + & 53.66 \\
\hline 1.7508 & 2 & 5 & 4 & 0 & & 53.86 \\
\hline 1.6949 & 2 & 2 & 1 & 4 & & 54.06 \\
\hline 1.5886 & 4 & 4 & 4 & 2 & + & 54.26 \\
\hline 1.6760 & $?$ & 1 & 6 & 1 & + & 54.72 \\
\hline 1.6648 & 2 & 2 & 6 & ù & & 55.12 \\
\hline 1.6391 & 2 & 5 & 0 & 3 & & 50.00 \\
\hline 1.6322 & 4 & 2 & 2 & 4. & + & 56.32 \\
\hline 1.6285 & 3 & 3 & 5 & 2 & & 55.46 \\
\hline 1.6164 & 2 & 4 & 3 & 3 & & 56.92 \\
\hline 1.5035 & 2 & i & 3 & 1 & & 57.42 \\
\hline 1.5999 & 3 & 3 & 4 & 3 & & 57.56 \\
\hline 1.5868 & 5 & 1 & 3 & 4 & & 58.08 \\
\hline 1.5794 & 3 & E & 2 & 2 & & 58.38 \\
\hline 1.5638 & 2 & 5 & 2 & 3 & + & 59.02 \\
\hline 1.5604 & ? & 7 & 0 & 1 & & 59.16 \\
\hline 1.5552 & 2 & 1 & $\bar{o}$ & 2 & & 59.30 \\
\hline 1.5514 & 2 & $\overline{3}$ & 2 & 4 & & 59.54 \\
\hline 1.5434 & 1 & 3 & 6 & 1 & & 59.88 \\
\hline 1.5280 & 1 & 5 & 5 & 0 & & 60.52 \\
\hline 1.5119 & 1 & 2 & 6 & 2 & & 61.25 \\
\hline 1.5008 & 2 & 4 & 1 & 4 & & 61.76 \\
\hline
\end{tabular}

\begin{tabular}{|c|c|c|c|c|c|}
\hline \multicolumn{6}{|c|}{ Calculated Pattern (Integrated) } \\
\hline$d(\AA)$ & $I$ & & hkl & & $\begin{array}{c}2 \theta\left({ }^{\circ}\right) \\
\lambda=1.54056 \mathrm{~A}\end{array}$ \\
\hline 7.64 & 91 & 1 & 1 & 0 & 11.57 \\
\hline 5.94 & 32 & 0 & $i$ & 1 & 14.90 \\
\hline 5.59 & 50 & 2 & 0 & 0 & 15.03 \\
\hline 5.25 & 100 & 1 & 1 & 1 & 16.83 \\
\hline 5.23 & 22 & 0 & 2 & o & 16.93 \\
\hline 4.93 & 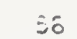 & 2 & 1 & o & 17.90 \\
\hline 4.24 & 54 & 0 & 2 & 1 & 20.95 \\
\hline 4.07 & 34 & 2 & 1 & $i$ & 21.80 \\
\hline $3 . y 6$ & 100 & 1 & 2 & 1 & 22.42 \\
\hline 3.82 & 2 & 2 & 2 & o & 23.26 \\
\hline 3.61 & i & 0 & $u$ & $<$ & 24.04 \\
\hline 3.436 & 25 & 1 & 0 & 2 & 25.91 \\
\hline 3.377 & 3 & $c$ & 2 & 1 & 26.37 \\
\hline $3.33 c$ & 3 & 1 & 3 & 3 & 26.75 \\
\hline 3.314 & 18 & 3 & כ & 1 & 26.83 \\
\hline 3.264 & 8 & 1 & 1 & 2 & 27.30 \\
\hline 3.159 & 7 & 3 & 1 & 1 & $28.2<$ \\
\hline 3.140 & 8 & 3 & 3 & 1 & 28.40 \\
\hline 3.037 & $<1$ & 3 & 2 & 0 & 29.30 \\
\hline 3.024 & $\angle 5$ & 1 & 3 & 1 & 23.52 \\
\hline 2.971 & $1 ?$ & J & 2 & 2 & 33.05 \\
\hline 2.960 & 4 & 2 & 3 & כ & 30.17 \\
\hline 2.913 & 1 & 2 & 1 & 2 & 30.60 \\
\hline 2.872 & 15 & 1 & 2 & 2 & 31.12 \\
\hline 2.799 & 2 & 3 & 2 & 1 & 31.94 \\
\hline 2.797 & 3 & 4 & c & J & 31.97 \\
\hline 2.733 & 2 & 2 & 3 & 1 & 32.57 \\
\hline 2.733 & 13 & 4 & 1 & 0 & 33.12 \\
\hline 2.624 & 5 & 2 & 2 & 2 & 34.14 \\
\hline 2.616 & 1 & 3 & 4 & 0 & 34.25 \\
\hline 2.639 & 11 & 4 & 0 & 1 & $34.3 \%$ \\
\hline 2.594 & 8 & 3 & 0 & 2 & 34.55 \\
\hline 2.518 & 7 & 3 & 1 & 2 & 35.53 \\
\hline 2.538 & 12 & 0 & 3 & 2 & 35.77 \\
\hline 2.467 & 1 & 4 & 2 & 0 & 36.33 \\
\hline$<.459$ & 4 & $\overline{0}$ & 4 & 1 & 30.51 \\
\hline 2.446 & 1 & i & 3 & $\bar{z}$ & 30.69 \\
\hline 2.402 & 4 & 3 & 3 & 1 & $37.4 \mathrm{~J}$ \\
\hline 2.402 & 15 & 1 & 4 & l & 37.41 \\
\hline 2.370 & 1 & 2 & 4 & 0 & 37.94 \\
\hline 2.345 & 4 & ن & 1 & 3 & 38.35 \\
\hline 2.334 & 3 & 4 & 2 & 1 & 38.53 \\
\hline 2.324 & 15 & 3 & 2 & 2 & 38.71 \\
\hline 2.296 & 21 & 1 & 1 & 3 & 39.21 \\
\hline 2.289 & 20 & 2 & 3 & 2 & 39.33 \\
\hline 2.251 & 8 & 2 & 4 & 1 & 43.41 \\
\hline 2.211 & 3 & 2 & c & 3 & $40.7 \varepsilon$ \\
\hline 2.163 & 6 & 4 & 1 & 2 & 41.71 \\
\hline 2.153 & 5 & 2 & 1 & 3 & 41.72 \\
\hline 2.146 & 1) & 1 & 2 & 3 & 42.07 \\
\hline
\end{tabular}


Zinc Glutamate Dihydrate, $\mathrm{ZnC}_{5} \mathrm{H}_{7} \mathrm{NO}_{4} \cdot 2 \mathrm{H}_{2} \mathrm{O}$ (orthorhombic) - continued

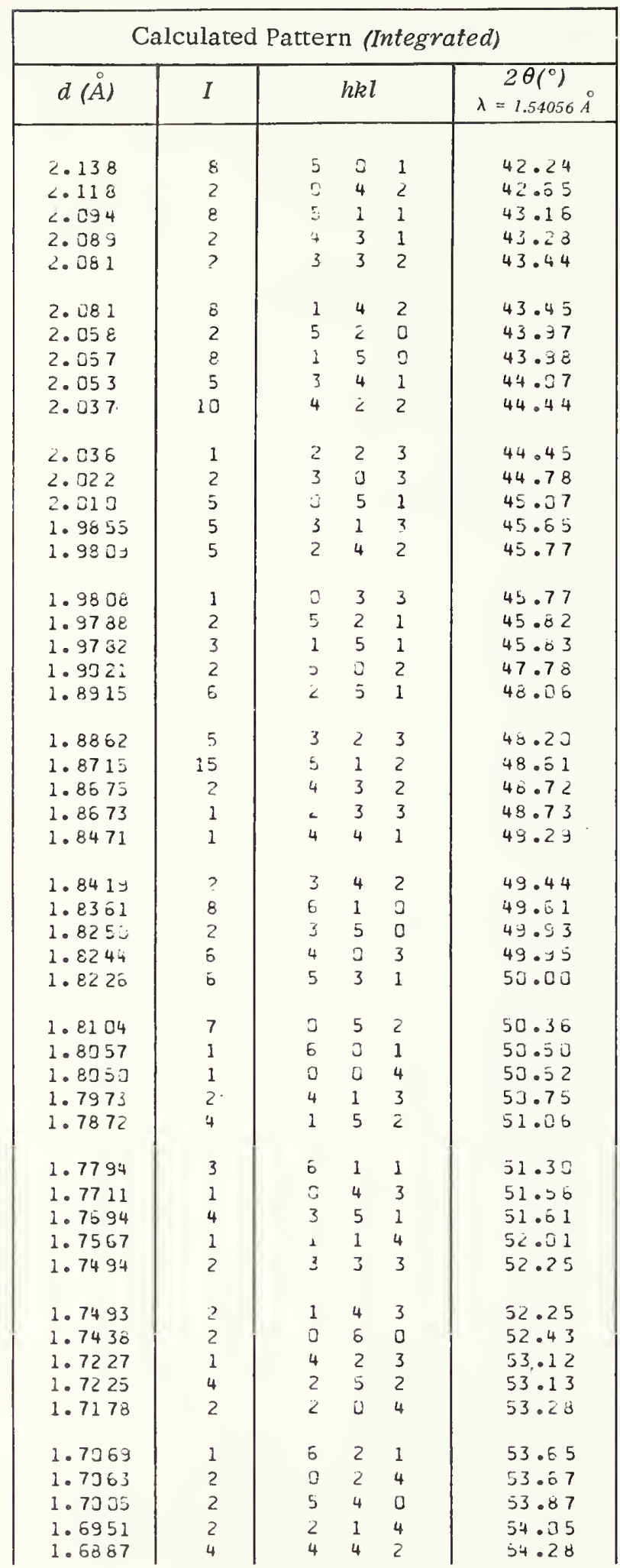

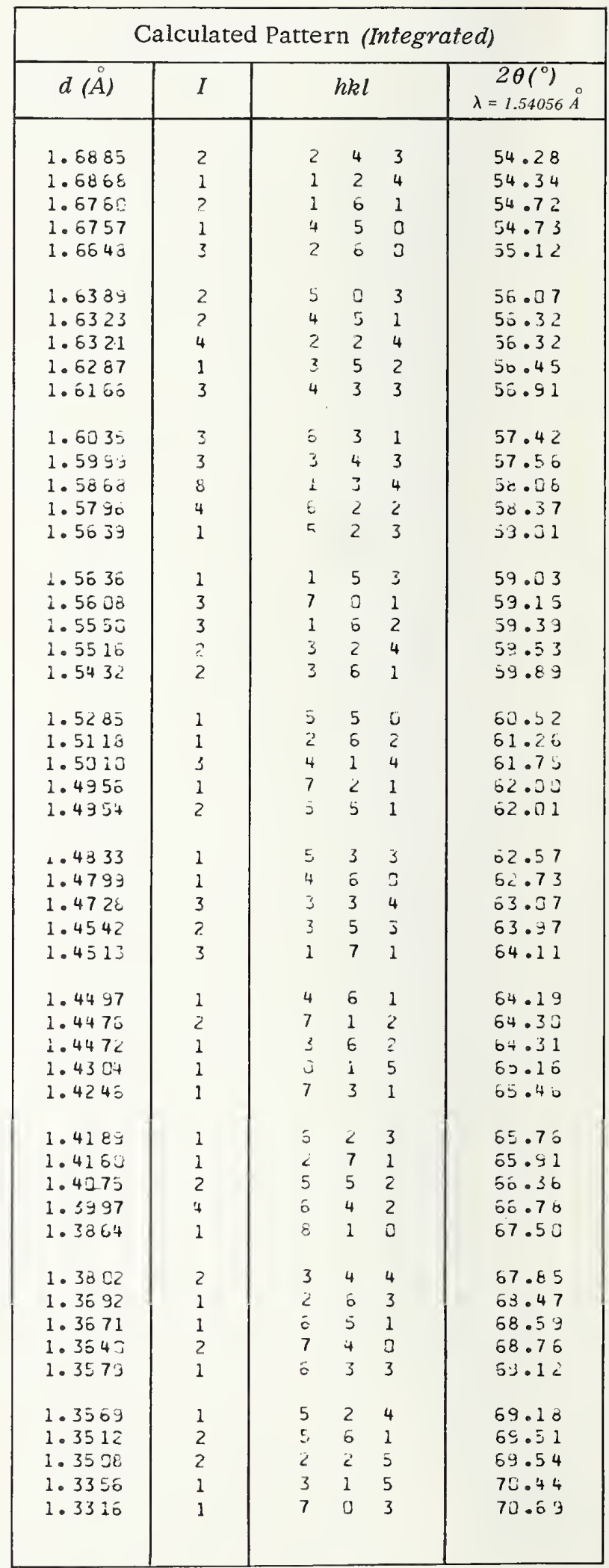




\section{structure}

Hexagonal, $\mathrm{P} 6_{3} \mathrm{mc}(186), \mathrm{Z}=2$ [Ansell and Katz, 1966]

\section{Lattice parameters}

$a=5.759 \pm 0.004, c=9.903 \pm 0.005 \AA$ [ibid.]

\section{Scattering factors}

$\mathrm{O}^{-1}[3.3 .1 \mathrm{~A}] ; \mathrm{Zn}^{+2}$ and $\mathrm{Mo}^{+4}$ [Thomas and Umeda 1957], corrected for the real part of the anomalous dispersion [Dauben and Templeton, 1955]

\section{Thermal parameters}

Isotropic [Ansell and Katz, 1966]

\section{Density}

(calculated) $6.381 \mathrm{~g} / \mathrm{cm}^{3}$

Scale factor

$$
4.920 \times 10^{4}
$$

\section{Additional patterns}

1. PDF card 16-663 [Donohue and Katz, 1964]

\section{Reference}

Ansell,G.B. and L. Katz (1966). A refinement of the crystal structure of zinc molybdenum(IV) oxide, $\mathrm{Zn}_{2} \mathrm{Mo}_{3} \mathrm{O}_{8}$, Acta Cryst. 21, 482-485.

Dauben, C.H. and D.H. Templeton (1955). A table of dispersion corrections for $x-$ ray scattering of atoms, Acta Cryst. 8, $841-842$.

Donohue, P.C. and L. Katz (1964). A lithium-scandium-molybdenum(IV) oxide, $\mathrm{Na}-$ ture 201, 180-181.

Thomas, L. H. and K. Umeda (1957). Atomic scattering factors calculated from the TFD atomic model,J.Chem. Phys.26, 293-303.

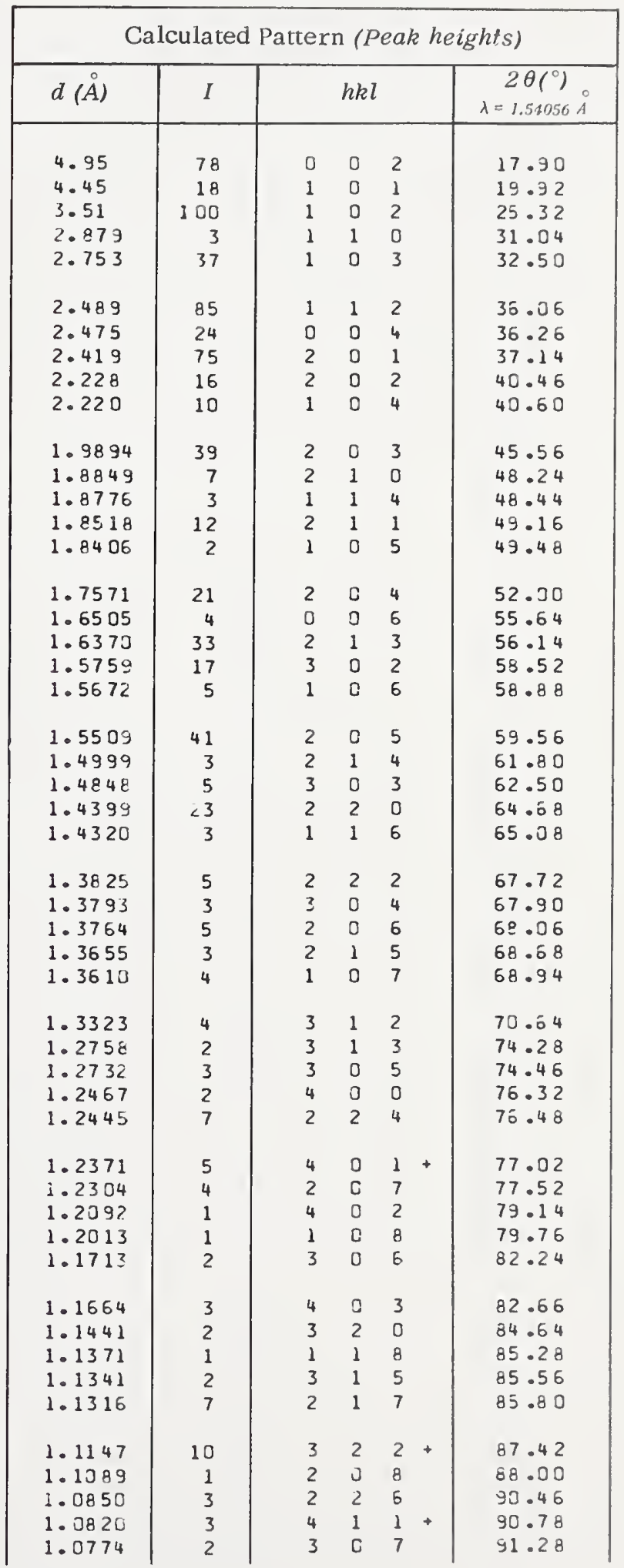




\begin{tabular}{|c|c|c|c|c|c|c|}
\hline \multicolumn{7}{|c|}{ Calculated Pattern (Peak heights) } \\
\hline$d(\stackrel{\circ}{A})$ & $I$ & & $h$ & & & $\begin{array}{c}2 \theta\left(^{\circ}\right) \\
\lambda=1.54056 \AA\end{array}$ \\
\hline 1.0746 & 1 & 1 & 0 & 9 & & 91.58 \\
\hline 1.0630 & 4 & 4 & 1 & 2 & & 92.88 \\
\hline 1.0603 & 3 & 3 & 1 & 6 & & 93.18 \\
\hline 1.0551 & 6 & 4 & 0 & 5 & & 93.78 \\
\hline 1.0386 & 4 & 3 & 2 & 4 & & 95.74 \\
\hline 1.0336 & 2 & 4 & 1 & 3 & & 96.36 \\
\hline 1.0067 & 4 & 2 & 0 & 9 & & 99.84 \\
\hline .9975 & 1 & 5 & 0 & 0 & & 101.10 \\
\hline .9950 & 1 & 4 & 0 & 6 & & 101.46 \\
\hline .9926 & 3 & 5 & 0 & 1 & $\leftrightarrow$ & 101.80 \\
\hline .9903 & 3 & 0 & 0 & 10 & + & 102.12 \\
\hline .9891 & 2 & 3 & 1 & 7 & & 102.30 \\
\hline .9548 & 4 & 5 & 0 & 3 & & 107.56 \\
\hline .9539 & 2 & 4 & 1 & 5 & & 107.70 \\
\hline .9503 & 2 & 2 & 1 & 9 & & 108.30 \\
\hline .9424 & 1 & 4 & 2 & 0 & & 109.64 \\
\hline .9403 & 6 & 3 & 2 & 6 & & 110.00 \\
\hline .938 .5 & 7 & 2 & 2 & 8 & $\rightarrow$ & 110.32 \\
\hline .9361 & 4 & 4 & 0 & 7 & + & 110.74 \\
\hline .9252 & 1 & 5 & 0 & 4 & & 112.72 \\
\hline .9228 & 1 & 3 & 1 & 8 & & 113.18 \\
\hline .9203 & 1 & 2 & 0 & 10 & & 113.64 \\
\hline .9176 & 1 & 3 & 0 & 9 & & 114.16 \\
\hline .9063 & 2 & 4 & 2 & 3 & & 116.40 \\
\hline .8909 & 2 & 5 & 0 & 5 & & 119.68 \\
\hline .8896 & 1 & 3 & 2 & 7 & & 119.96 \\
\hline .8809 & 1 & 4 & 2 & 4 & & 121.36 \\
\hline .8785 & 1 & 4 & 0 & 8 & & 122.52 \\
\hline .8645 & 1 & 5 & 1 & 3 & & 126.00 \\
\hline .8626 & 2 & 4 & 1 & 7 & & 126.50 \\
\hline .8611 & 1 & 3 & 1 & 9 & & 126.90 \\
\hline .8511 & 7 & 4 & 2 & 5 & + & 129.66 \\
\hline .8468 & 3 & 2 & 0 & 11 & & 130.92 \\
\hline .8423 & 1 & 5 & 1 & 4 & & 132.26 \\
\hline .8403 & 5 & 3 & 2 & 8 & & 132.90 \\
\hline .8312 & 2 & 6 & 0 & 0 & & 135.84 \\
\hline .8250 & 2 & 4 & 0 & 9 & + & 138.02 \\
\hline .8199 & 2 & 4 & 3 & 0 & & 139.92 \\
\hline .8185 & 1 & 4 & 2 & 6 & & 140.48 \\
\hline .8171 & 3 & 4 & 3 & 1 & + & 141.00 \\
\hline .8159 & 5 & 2 & 2 & 10 & $\leftrightarrow$ & 141.50 \\
\hline .8152 & 5 & 5 & 0 & 7 & & 141.78 \\
\hline .8139 & 3 & 1 & 0 & 12 & & 142.30 \\
\hline .8124 & 3 & 2 & 1 & 11 & & 142.94 \\
\hline .7960 & 8 & 5 & 2 & 1 & + & 150.80 \\
\hline .7916 & 1 & 3 & 0 & 11 & & 153.32 \\
\hline .7884 & 5 & 6 & 0 & 4 & + & 155.38 \\
\hline .7873 & 1 & 5 & 1 & 6 & & 156.12 \\
\hline .7844 & 1 & 4 & 2 & 7 & & 158.24 \\
\hline .7835 & 1 & 2 & 0 & 12 & & 158.94 \\
\hline
\end{tabular}

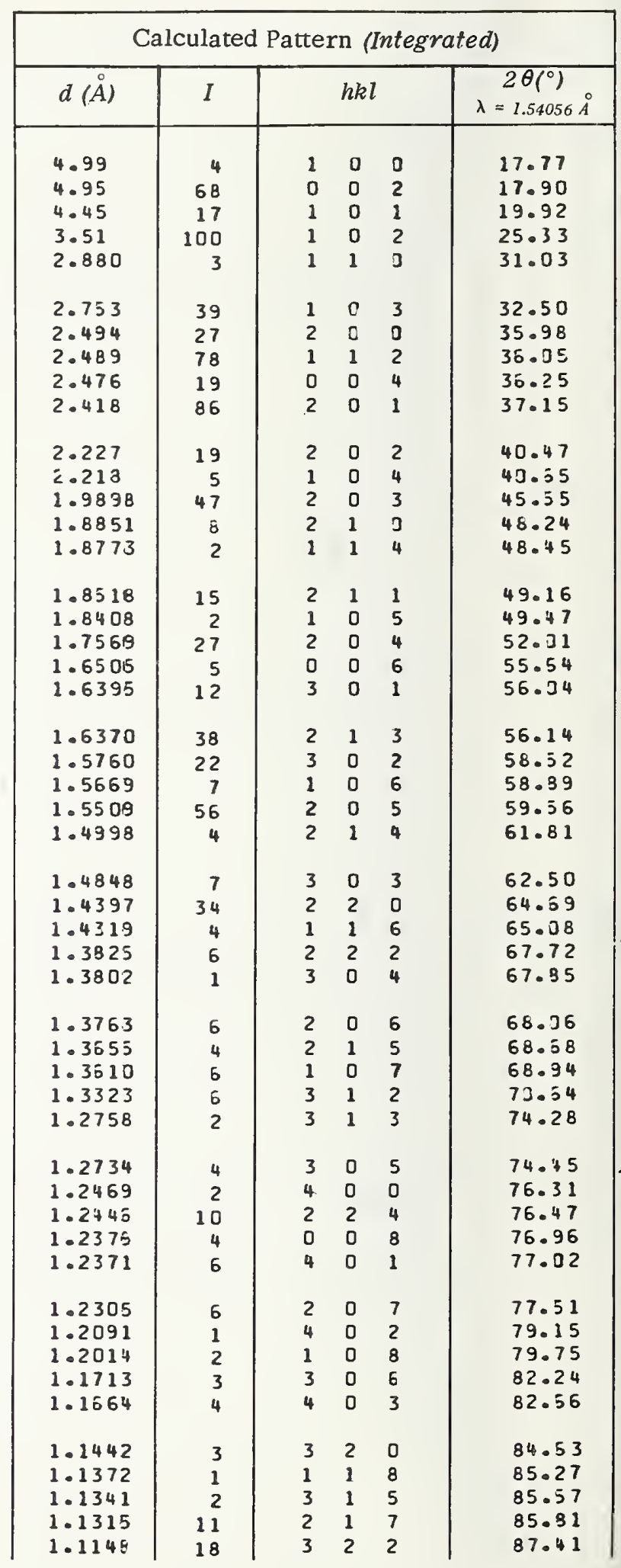


Zinc Molybdate, $\mathrm{Zn}_{2} \mathrm{Mi}_{3} \mathrm{O}_{8}$ (hexagonal) - continued

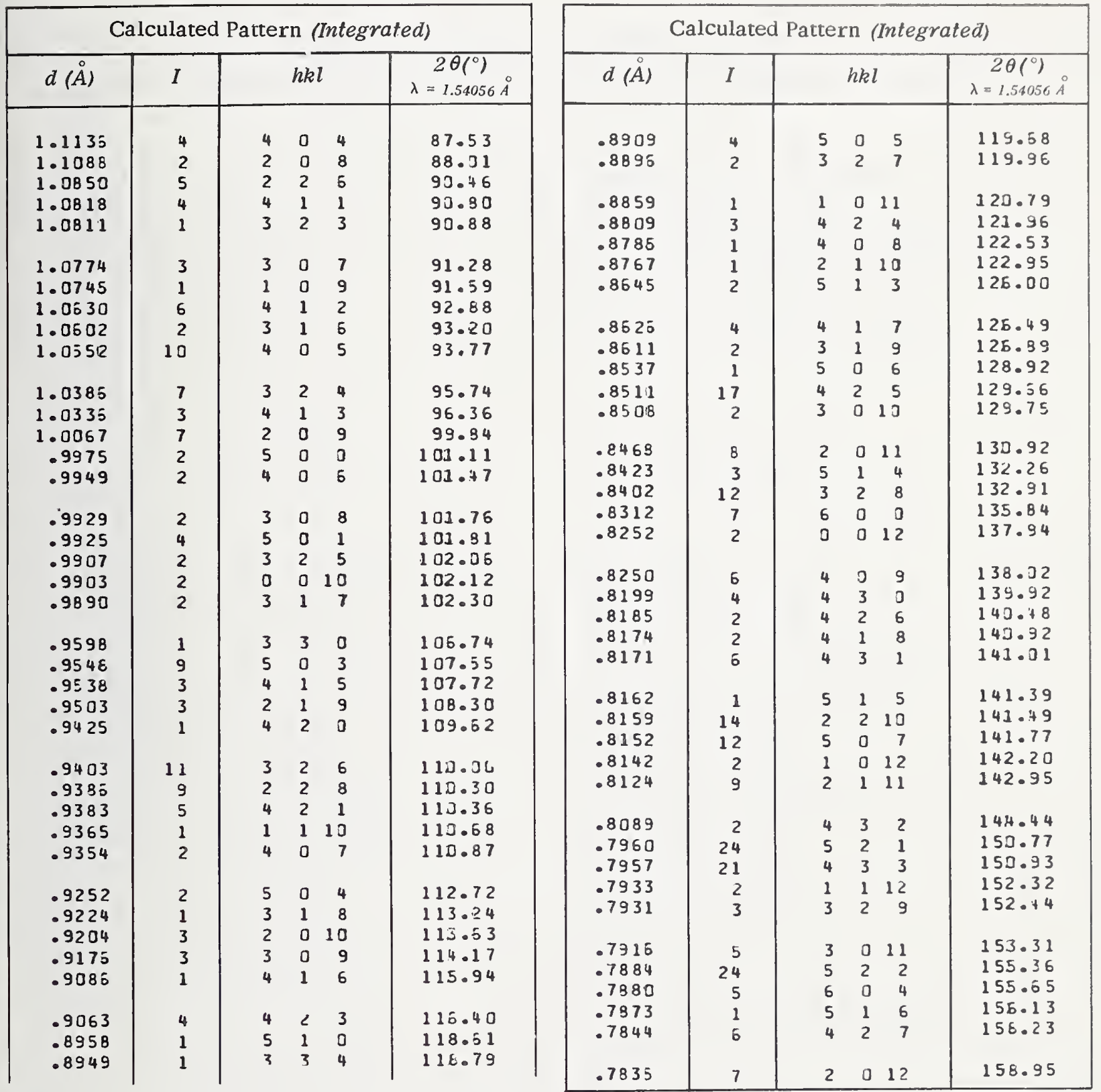




\section{CUMULATIVE INDEX TO CIRCULAR 539, VOLUMES $1,2,3,4,5,6$, $7,8,9,10$, MONOGRAPH 25 , SECTIONS $1,2,3,4,5,6$, and $7^{5}$}

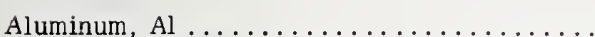

Vol.

Aluminum antimony, Alsb ............

Aluminum calcium sulfate hydrate (ettring-

ite), $\mathrm{Al}_{2} \mathrm{O}_{3} \cdot 6 \mathrm{CaO} \cdot 3 \mathrm{SO}_{3} \cdot 31 \mathrm{H}_{2} \mathrm{O} \ldots \ldots \ldots \ldots$.

Aluminum chloride hexahydrate (chlor-

aluminite), $\mathrm{AlCl}_{3} \cdot 6 \mathrm{H}_{2} \mathrm{O} \ldots \ldots \ldots \ldots \ldots$

Aluminum fluosilicate, topaz: $\mathrm{Al}_{2} \mathrm{SiO}_{4}(\mathrm{~F}, \mathrm{OH})_{2}$

Aluminum metaphosphate, $\mathrm{Al}\left(\mathrm{PO}_{3}\right)_{3} \ldots \ldots \ldots$

Aluminum nickel, AlNi ...............

Aluminum orthophosphate (berlinite), $\mathrm{AlPO}_{4}$

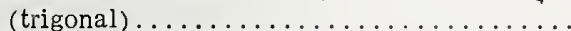

Aluminum orthophosphate, $\mathrm{AlPO}_{4}$ (ortho-

rhombic) $\ldots \ldots \ldots \ldots \ldots \ldots \ldots \ldots \ldots$

Aluminum oxide, (corundum), alpha $\mathrm{Al}_{2} \mathrm{O}_{3} \ldots$

Aluminum oxide monohydrate (böhmite), alpha $\mathrm{Al}_{2} \mathrm{O}_{3} \cdot \mathrm{H}_{2} \mathrm{O} \ldots \ldots \ldots \ldots \ldots \ldots \ldots \ldots \ldots \ldots \ldots \ldots$

Aluminum oxide monohydrate, diaspore, beta

$\mathrm{Al}_{2} \mathrm{O}_{3} \cdot \mathrm{H}_{2} \mathrm{O} \ldots \ldots \ldots \ldots \ldots \ldots \ldots \ldots$

Aluminum silicate (mullite) $3 \mathrm{Al}_{2} \mathrm{O}_{3} \cdot 2 \mathrm{SiO}_{2} \ldots$

Ammonium aluminum sulfate dodecahydrate

(teschermigite), $\mathrm{NH}_{4} \mathrm{Al}\left(\mathrm{SO}_{4}\right)_{2} \cdot 12 \mathrm{H}_{2} \mathrm{O} \ldots \ldots$.

Ammonium azide, $\mathrm{NH}_{4} \mathrm{~N}_{3} \ldots \ldots \ldots \ldots \ldots$

Ammonium bicarbonate (teschemacherite),

$\left(\mathrm{NH}_{4}\right) \mathrm{HCO}_{3} \ldots \ldots \ldots \ldots \ldots \ldots \ldots \ldots$

Ammonium bromide, $\mathrm{NH}_{4} \mathrm{Br} \ldots \ldots \ldots \ldots \ldots$.

Ammonium bromoosmate, $\left(\mathrm{NH}_{4}\right)_{2} \mathrm{OsBr}_{6} \ldots \ldots$.

Ammonium bromoplatinate, $\left(\mathrm{NH}_{4}\right)_{2} \mathrm{PtBr}_{6} \ldots \ldots$.

Ammonium bromoselenate, $\left(\mathrm{NH}_{4}\right)_{2} \mathrm{SeBr}_{6} \ldots \ldots$.

Ammonium bromotellurate, $\left(\mathrm{NH}_{4}\right)_{2} \mathrm{TeBr}_{6} \ldots \ldots$.

Ammonium cadmium sulfate, $\left(\mathrm{NH}_{4}\right)_{2} \mathrm{Cd}_{2}\left(\mathrm{SO}_{4}\right)_{3}$

Ammonium cadmium trichloride, $\mathrm{NH}_{4} \mathrm{CdCl}_{3}$..

Ammonium chloride (sal-ammoniac), $\mathrm{NH}_{4} \mathrm{Cl} \ldots$

Ammonium chloroiridate, $\left(\mathrm{NH}_{4}\right)_{2} \mathrm{IrCl}_{6} \ldots \ldots \ldots$.

Ammonium chloroosmate, $\left(\mathrm{NH}_{4}\right)_{2} \mathrm{OsCl}_{6} \ldots \ldots$.

Ammonium chloropalladate, $\left(\mathrm{NH}_{4}\right)_{2} \mathrm{PdCl}_{6} \ldots \ldots$

Ammonium chloropalladite, $\left(\mathrm{NH}_{4}\right)_{2} \mathrm{PdCl}_{4} \ldots$.

Ammonium chloroplatinate, $\left(\mathrm{NH}_{4}\right)_{2} \mathrm{PtCl}_{6} \ldots \ldots$.

Ammonium chlorostannate $\left(\mathrm{NH}_{4}\right)_{2} \mathrm{SnCl}_{6} \ldots \ldots$.

Ammonium chlorotellurate, $\left(\mathrm{NH}_{4}\right)_{2} \mathrm{TeCl}_{6} \ldots \ldots$.

Ammonium chromium sulfate dodecahydrate,

$\mathrm{NH}_{4} \mathrm{Cr}\left(\mathrm{SO}_{4}\right)_{2} \cdot 12 \mathrm{H}_{2} \mathrm{O} \ldots \ldots \ldots \ldots \ldots$

Ammonium cobalt (II) trichloride, $\mathrm{NH}_{4} \mathrm{CoCl}_{3}$

Ammonium copper chloride, $\mathrm{NH}_{4} \mathrm{CuCl}_{3} \ldots \ldots$.

Ammonium dihydrogen phosphate, $\mathrm{NH}_{4} \mathrm{H}_{2} \mathrm{PO}_{4}$

Ammonium fluoberyllate, $\left(\mathrm{NH}_{4}\right)_{2} \mathrm{BeF}_{4} \ldots \ldots \ldots$.

Ammonium fluoborate, $\mathrm{NH}_{4} \mathrm{BF}_{4} \ldots \ldots \ldots \ldots$

Ammonium fluogermanate, $\left(\mathrm{NH}_{4}\right)_{2} \mathrm{GeF}_{6} \ldots \ldots$.

Ammonium fluosilicate (cryptohalite),

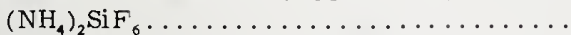

Ammonium gallium sulfate dodecahydrate,

$\mathrm{NH}_{4} \mathrm{Ga}\left(\mathrm{SO}_{4}\right)_{2} \cdot 12 \mathrm{H}_{2} \mathrm{O} \ldots \ldots \ldots \ldots \ldots \ldots$

Ammonium iodide, $\mathrm{NH}_{4} \mathrm{I} \ldots \ldots \ldots \ldots \ldots$

sec

Vol. or

sec. Page

Ammonium iron sulfate dodecahydrate,

$\mathrm{NH}_{4} \mathrm{Fe}\left(\mathrm{SO}_{4}\right)_{2} \cdot 12 \mathrm{H}_{2} \mathrm{O}$

6

Ammonium manganese sulfate, $\left(\mathrm{NH}_{4}\right)_{2} \mathrm{Mn}_{2}\left(\mathrm{SO}_{4}\right)_{3}$

Ammonium manganese(II) trifluoride, $\mathrm{NH}_{4} \mathrm{MnF}_{3}$

Ammonium mercury(II) trichloride, $\mathrm{NH}_{4} \mathrm{HgCl}_{3}$

Ammonium metavanadate, $\mathrm{NH}_{4} \mathrm{VO}_{3}$

Ammonium nickel (II) trichloride, $\mathrm{NH}_{4} \mathrm{NiCl}_{3} \ldots$

Ammonium nitrate (ammonia-niter), $\mathrm{NH}_{4} \mathrm{NO}_{3}$.

Ammonium oxalate monohydrate (oxammite),

$$
\left(\mathrm{NH}_{4}\right)_{2} \mathrm{C}_{2} \mathrm{O}_{4} \cdot \mathrm{H}_{2} \mathrm{O}
$$

Ammonium perchlorate, $\mathrm{NH}_{4} \mathrm{ClO}_{4}$ (ortho-

rhombic)

Ammonium perrhenate, $\mathrm{NH}_{4} \mathrm{ReO}_{4}$

Ammonium phosphomolybdate tetrahydrate,

$\left(\mathrm{NH}_{4}\right)_{3} \mathrm{PO}_{4}\left(\mathrm{MoO}_{3}\right)_{12} \cdot 4 \mathrm{H}_{2} \mathrm{O}$.

Ammonium sulfate (mascagnite), $\left(\mathrm{NH}_{4}\right)_{2} \mathrm{SO}_{4}$ (revised)

Ammonium zirconium fluoride, $\left(\mathrm{NH}_{4}\right)_{3} \mathrm{ZrF}_{7} \ldots$

Antimony, $\mathrm{Sb} \ldots \ldots \ldots \ldots \ldots \ldots \ldots \ldots \ldots$

Antimony(III) fluoride, $\mathrm{SbF}_{3}$.

Antimony(III) iodide, $\mathrm{SbI}_{3}$....

Antimony(III) oxide (senarmontite), $\mathrm{Sb}_{2} \mathrm{O}_{3}$

(cubic)...

Antimony(III) oxide, valentinite, $\mathrm{Sb}_{2} \mathrm{O}_{3}$

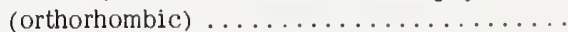

Antimony(IV) oxide (cervantite), $\mathrm{Sb}_{2} \mathrm{O}_{4} \ldots \ldots$

Antimony(V) oxide, $\mathrm{Sb}_{2} \mathrm{O}_{5} \ldots \ldots \ldots \ldots \ldots \ldots$

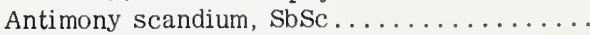

Antimony selenide, $\mathrm{Sb}_{2} \mathrm{Se}_{3} \ldots \ldots \ldots \ldots \ldots$

Antimony (III) sulfide (stibnite), $\mathrm{Sb}_{2} \mathrm{~S}_{3} \ldots \ldots$.

Antimony telluride, $\mathrm{Sb}_{2} \mathrm{Te}_{3} \ldots \ldots \ldots \ldots \ldots$.

Antimony terbium, SbTb $\ldots \ldots \ldots \ldots \ldots$

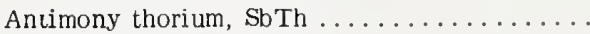

Antimony thulium, $\operatorname{SbTm} \ldots \ldots \ldots \ldots \ldots \ldots$

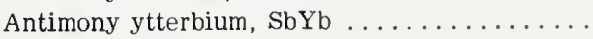

Antimony yttrium, SbY

Arsenic acid, $\mathrm{H}_{5} ; \mathrm{AS}_{3} \mathrm{O}_{10} \ldots \ldots \ldots \ldots \ldots$

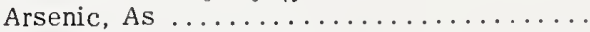

Arsenic(III) iodide, $\mathrm{AsI}_{3} \ldots \ldots \ldots \ldots \ldots \ldots$

Arsenic trioxide (arsenolite), $\mathrm{As}_{2} \mathrm{O}_{3}$ (cubic) .

Arsenic trioxide, claudetite, $\mathrm{As}_{2} \mathrm{O}_{3}$ (mono-

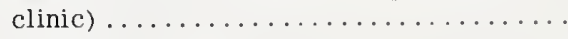

Azobenzene, $\mathrm{C}_{10} \mathrm{H}_{12} \mathrm{~N}_{2} \ldots \ldots \ldots \ldots \ldots \ldots$

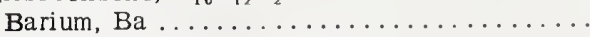

Barium aluminum oxide, $\mathrm{BaAl}_{2} \mathrm{O}_{4} \ldots \ldots \ldots$

Barium arsenate, $\mathrm{Ba}_{3}\left(\mathrm{AsO}_{4}\right)_{2} \ldots \ldots \ldots \ldots \ldots$

Barium borate, $\mathrm{BaB}_{8} \mathrm{O}_{13} \ldots \ldots \ldots \ldots \ldots$

Barium boron oxide, high form, $\mathrm{Ba}_{2} \mathrm{O}_{4}$

Barium boron oxide, $\mathrm{BaB}_{4} \mathrm{O}_{7} \ldots \ldots \ldots \ldots$

Barium bromide monohydrate, $\mathrm{BaBr}_{2} \cdot \mathrm{H}_{2} \mathrm{O} \ldots$.

Barium carbonate (witherite), $\mathrm{BaCO}_{3}$ (ortho-

Barium carbonate, $\mathrm{BaCO}_{3}$ (cubic) at $1075^{\circ} \mathrm{C}$.

Barium fluoride, $\mathrm{BaF}_{2} \ldots \ldots \ldots \ldots \ldots \ldots$

Barium fluosilicate, $\mathrm{BaSiF}_{6}$

Barium molybdate, $\mathrm{BaMoO}_{4} \ldots \ldots \ldots \ldots \ldots$

Barium nitrate (nitrobarite), $\mathrm{Ba}\left(\mathrm{NO}_{3}\right)_{2}$

Barium perchlorate trihydrate. $\mathrm{Ba}\left(\mathrm{ClO}_{4}\right)_{2} \cdot 3 \mathrm{H}_{2} \mathrm{O}$

Barium peroxide, $\mathrm{BaO}_{2}$

$\begin{array}{rl}7 \mathrm{~m} n & 8 \\ 5 \mathrm{~m} & 8 \\ 5 \mathrm{~m} & 9 \\ 8 & 9 \\ 6 \mathrm{~m} & 6 \\ 7 & 4\end{array}$

7

7

9

8

98

6

3

$2 \mathrm{~m}$

6

3

10

10

10

$4 \mathrm{~m}$

$3 \mathrm{~m}$

5

$3 m$

$5 \mathrm{~m}$

$4 \mathrm{~m}$

$4 \mathrm{~m}$

$4 \mathrm{~m}$

$4 \mathrm{~m}$

$7 \mathrm{~m}$

3

6

1

$3 m$

$7 \mathrm{~m}$

4

$2 \mathrm{~m}$

$7 \mathrm{~m}$

$4 \mathrm{~m}$

$4 \mathrm{~m}$

$3 \mathrm{~m}$

10

10

$4 \mathrm{~m}$

7

1

$2 m$
6
10

10

A mineral name in () indicates a synthetic sample. 


\section{CUMULATIVE INDEX-Continued}

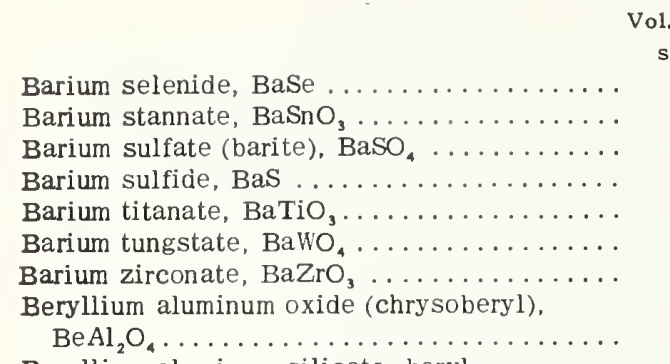

Beryllium aluminum silicate, beryl,

$\mathrm{Be}_{3} \mathrm{Al}_{2}\left(\mathrm{SiO}_{3}\right)_{6} \ldots \ldots \ldots \ldots \ldots \ldots \ldots$

Beryllium calcium oxide, $\mathrm{Be}_{1}{ }_{2} \mathrm{Ca}_{1}, \mathrm{O}_{2}, \ldots \ldots$.

Beryllium chromium oxide, $\mathrm{BeCr}_{2} \mathrm{O}_{4} \ldots \ldots \ldots$

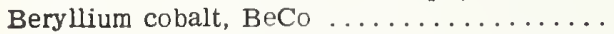

Beryllium germanate, $\mathrm{Be}_{2} \mathrm{GeO}_{4} \ldots \ldots \ldots \ldots$

Beryllium niobium, Be, $\mathrm{Nb} \ldots \ldots \ldots \ldots \ldots \ldots$

Beryllium orthosilicate, phenacite, $\mathrm{BeSi}_{2} \mathrm{O}_{4} \ldots$

Beryllium oxide (bromellite), $\mathrm{BeO} \ldots \ldots \ldots \ldots$

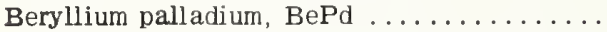

Bis (o-dodecacarborane), $\mathrm{C}_{4} \mathrm{~B}_{20} \mathrm{H}_{22} \ldots \ldots \ldots$

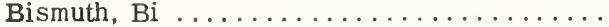

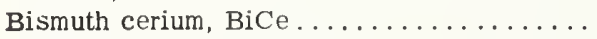

Bismuth dy sprosium, BiDy . . . . . . . .

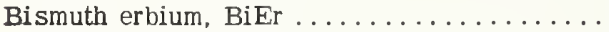

Bismuth fluoride, $\mathrm{BiF}_{3} \ldots \ldots \ldots \ldots \ldots \ldots$

Bismuth holmium, BiHo

Bismuth(III) iodide, $\mathrm{BiI}_{3} \ldots \ldots \ldots \ldots \ldots \ldots$.

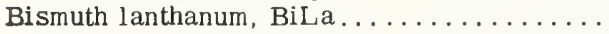

Bismuth neodymium, BiNd

Bismuth orthophosphate, $\mathrm{BiPO}_{4}$ (monoclinic)

Bismuth orthophosphate, $\mathrm{BiPO}_{4}$ (trigonal) ... .

Bismuth orthovanadate, low form, $\mathrm{BiVO}_{4}$

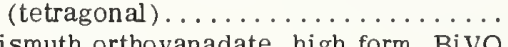

Bismuth orthovanadate, high form, Bivo

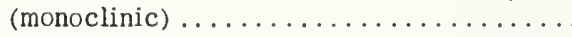

Bismuth oxybromide, BiOBr...........

Bismuth oxychloride (bismoclite), $\mathrm{BiOCl} \ldots$.

Bismuth oxyiodide, BiOI

Bismuth praseodymium, BiPr.

Bismuth sulfide (bismuthinite), $\mathrm{Bi}_{2} \mathrm{~S}_{3}$ (revised)

Bismuth telluride, BiTe ..............

Bismuth telluride (tellurobismuthite), $\mathrm{Bi}_{2} \mathrm{Te}_{3}$

Bismuth trioxide (bismite), alpha $\mathrm{Bi}_{2} \mathrm{O}_{3} \ldots \ldots$.

Cadmium, Cd

Cadmium bromide, $\mathrm{CdBr}_{2} \ldots \ldots \ldots \ldots \ldots \ldots$

Cadmium carbonate (otavite), $\mathrm{CdCO}_{3} \ldots \ldots$

Cadmium cerium, CdCe...............

Cadmium chloride, $\mathrm{CdCl}_{2} \ldots \ldots \ldots \ldots \ldots \ldots$

Cadmium chromite, $\mathrm{CdCr}_{2} \mathrm{O}_{4} \ldots \ldots \ldots \ldots \ldots$

Cadmium cyanide, $\mathrm{Cd}(\mathrm{CN})_{2} \ldots \ldots \ldots \ldots \ldots$

Cadmium lanthanum, CdLa ............

Cadmium moly bdate, $\mathrm{CdMoO}_{4}$

Cadmium nitrate tetrahydrate,

$\mathrm{Cd}\left(\mathrm{NO}_{3}\right)_{2} \cdot 4 \mathrm{H}_{2} \mathrm{O} \ldots \ldots \ldots \ldots \ldots \ldots \ldots \ldots$

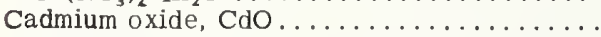

Cadmium oxide, CdO (ref. standard). .......

Cadmium perchlorate hexahydrate,

$\mathrm{Cd}\left(\mathrm{ClO}_{4}\right)_{2} \cdot 6 \mathrm{H}_{2} \mathrm{O} \ldots \ldots \ldots \ldots \ldots \ldots \ldots \ldots$

Cadmium praseodymium, $\mathrm{CdPr} \ldots \ldots \ldots \ldots \ldots$

Cadmium selenide, CdSe (hexagonal) .......

Cadmium sulfate, CdSO,

Cadmium sulfate hydrate, $3 \mathrm{CdSO}_{4} \cdot 8 \mathrm{H}_{2} \mathrm{O} \ldots \ldots$

m-Monograph 25.

A mineral name in ( ) indicates a synthetic sample.

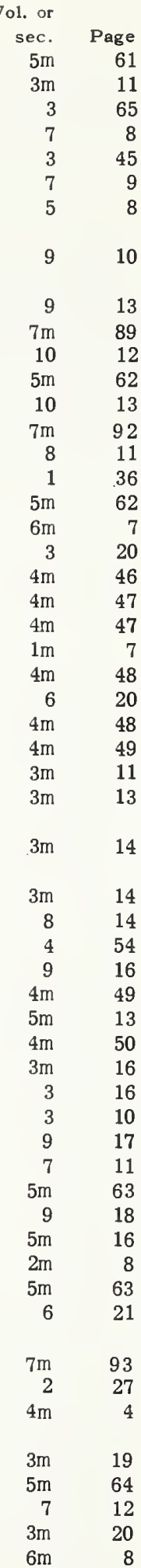

Cadmium sulfate monohydrate, $\mathrm{CdSO}_{4} \cdot \mathrm{H}_{2} \mathrm{O} \ldots$

Vol. or

sec.

Cadmium sulfide (greenockite), CdS ........

Cadmium telluride, $\mathrm{CdTe} . . . \ldots \ldots \ldots \ldots$.

Cadmium tungstate, $\mathrm{CdWO}_{4} \ldots \ldots \ldots \ldots \ldots \ldots$

tri-Calcium aluminate, $3 \mathrm{CaO} \cdot \mathrm{Al}_{2} \mathrm{O}_{3} \ldots \ldots \ldots$

Calcium aluminate, $12 \mathrm{CaO} \cdot 7 \cdot \mathrm{A}_{2} \mathrm{O}_{3} \ldots \ldots \ldots$

Calcium aluminum germanate, $\mathrm{Ca}_{3} \mathrm{Al}_{2}\left(\mathrm{GeO}_{4}\right)_{3}$

Calcium bromide hexahydrate, $\mathrm{CaBr}_{2} \cdot 6 \mathrm{H}_{2} \mathrm{O} \ldots$

Calcium carbonate (aragonite), $\mathrm{CaCO}_{3}$ (or-

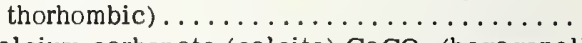

Calcium carbonate (calcite) $\mathrm{CaCO}_{3}$ (hexagonal)

Calcium chromate, $\mathrm{CaCrO}_{4} \ldots \ldots \ldots \ldots \ldots \ldots$

Calcium chromium germanate, $\mathrm{Ca}_{3} \mathrm{Cr}_{2}\left(\mathrm{GeO}_{4}\right)_{3}$

Calcium chromium silicate (uvarovite),

$\mathrm{Ca}_{3} \mathrm{Cr}_{2}\left(\mathrm{SiO}_{4}\right)_{3} \ldots \ldots \ldots \ldots \ldots \ldots \ldots$

Calcium fluoride (fluorite), $\mathrm{CaF}_{2} \ldots \ldots \ldots \ldots$

Calcium fluoride phosphate (fluorapatite),

$\mathrm{Ca}_{5} \mathrm{~F}\left(\mathrm{PO}_{4}\right)_{3} \ldots \ldots \ldots \ldots \ldots \ldots \ldots \ldots$

Calcium formate, $\mathrm{Ca}\left(\mathrm{HCO}_{2}\right)_{2} \ldots \ldots \ldots \ldots$.

Calcium gallium germanate, $\mathrm{Ca}_{3} \mathrm{Ga}_{2}\left(\mathrm{GeO}_{4}\right)_{3} \ldots$

Calcium hydroxide (portlandite), $\mathrm{Ca}(\mathrm{OH})_{2} \ldots$.

Calcium iron germanate, $\mathrm{Ca}_{3} \mathrm{Fe}_{2}\left(\mathrm{GeO}_{4}\right)_{3} \ldots \ldots$.

Calcium iron silicate (andradite),

$\mathrm{Ca}_{3} \mathrm{Fe}_{2} \mathrm{Si}_{3} \mathrm{O}_{12} \ldots \ldots \ldots \ldots \ldots \ldots \ldots$

Calcium magnesium silicate (diopside),

$\mathrm{CaMg}\left(\mathrm{SiO}_{3}\right)_{2} \ldots \ldots \ldots \ldots \ldots \ldots \ldots$

Calcium molybdate (powellite), $\mathrm{CaMoO}_{4} \ldots .$.

Calcium nitrate, $\mathrm{Ca}\left(\mathrm{NO}_{3}\right)_{2} \ldots \ldots \ldots \ldots \ldots \ldots$

Calcium oxide, $\mathrm{CaO} \ldots \ldots \ldots \ldots \ldots \ldots \ldots$

Calcium phosphate, beta-pyro-, $\mathrm{Ca}_{2} \mathrm{P}_{2} \mathrm{O}_{7} \ldots \ldots$

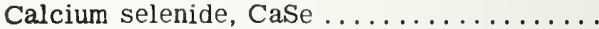

Calcium sulfate (anhydrite), $\mathrm{CaSO}_{4} \ldots \ldots \ldots$

Calcium sulfide (oldhamite), CaS . . .......

Calcium telluride, CaTe................

Calcium tungstate, scheelite, $\mathrm{CaWO}_{4} \ldots \ldots \ldots$

Carbon, diamond, C . ................

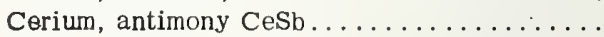

Cerium arsenate, $\mathrm{CeAsO}_{4} \ldots \ldots \ldots \ldots \ldots \ldots$

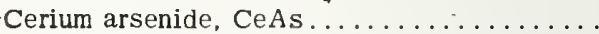

Cerium(III) chloride, $\mathrm{CeCl}_{3} \ldots \ldots \ldots \ldots \ldots$

Cerium copper, $\mathrm{CeCu}_{6} \ldots \ldots \ldots \ldots \ldots \ldots \ldots$

Cerium(III) fluoride, $\mathrm{CeF}_{3} \ldots \ldots \ldots \ldots \ldots \ldots$

Cerium magnesium, CeMg............

Cerium magnesium nitrate 24 -hydrate,

$\mathrm{Ce}_{2} \mathrm{Mg}_{3}\left(\mathrm{NO}_{3}\right)_{12} \cdot 24 \mathrm{H}_{2} \mathrm{O} \ldots \ldots \ldots \ldots \ldots \ldots$

Cerium niobium titanium oxide (eschynite),

$\mathrm{CeNbTiO}_{6} \ldots \ldots \ldots \ldots \ldots \ldots \ldots \ldots \ldots . . \ldots \ldots$

Cerium nitride, $\mathrm{CeN} \ldots \ldots \ldots \ldots \ldots \ldots \ldots$

Cerium(IV) oxide (cerianite), $\mathrm{CeO}_{2} \ldots \ldots \ldots \ldots$

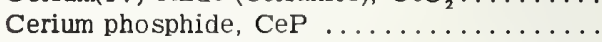

Cerium(III) vanadate, $\mathrm{CeVO}_{4} \ldots \ldots \ldots \ldots \ldots$

Cerium zinc, CeZn ...............

Cesium aluminum sulfate dodecahydrate,

$\mathrm{CsAl}\left(\mathrm{SO}_{4}\right)_{2} \cdot 12 \mathrm{H}_{2} \mathrm{O} \ldots \ldots \ldots \ldots \ldots \ldots \ldots$

Cesium bromate, $\mathrm{CsBrO}_{3} \ldots \ldots \ldots \ldots \ldots$

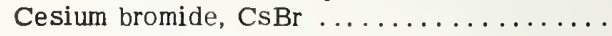

Cesium bromoosmate(IV), $\mathrm{Cs}_{2} \mathrm{OsBr}_{6} \ldots \ldots \ldots$.

Cesium bromoplatinate, $\mathrm{Cs}_{2} \mathrm{PtBr}_{6} \ldots \ldots \ldots \ldots$

Cesium bromoselenate, $\mathrm{Cs}_{2} \mathrm{SeBr}_{6} \ldots \ldots \ldots \ldots$

Cesium bromotellurate, $\mathrm{Cs}_{2} \mathrm{TeBr}_{6} \ldots \ldots \ldots \ldots$

Cesium cadmium trichloride, $\mathrm{CsCdCl}_{3}$

(hexagonal) ................. $5 \mathrm{~m}$

Cesium calcium sulfate, $\mathrm{Cs}_{2} \mathrm{Ca}_{2}\left(\mathrm{SO}_{4}\right)_{3} \ldots \ldots .7 \mathrm{~m}$

Cesium calcium trichloride, $\mathrm{CsCaCl}_{3} \ldots \ldots \ldots 5 \mathrm{~m}$

Cesium cerium chloride, $\mathrm{Cs}_{2} \mathrm{CeC}_{6} \ldots \ldots \ldots .7 \mathrm{~m}$
Page

10

15

21

8

10

20

15

15

53

51

13

16

10

17

69

$3 \mathrm{~m}$

8

10

1

10

9

$5 \mathrm{~m}$

6

7

$7 \mathrm{~m}$

$5 \mathrm{~m}$

4

7

$4 \mathrm{~m}$

6

2

$4 \mathrm{~m}$

$4 \mathrm{~m}$

$4 \mathrm{~m}$

$1 \mathrm{~m}$

$7 \mathrm{~m}$
8

$5 \mathrm{~m}$

10

$3 \mathrm{~m}$

$4 \mathrm{~m}$

$4 \mathrm{~m}$

$1 \mathrm{~m}$

$5 \mathrm{~m}$

$6 \quad 25$

8

3
$2 m$

8

8

9

$5 \mathrm{~m}$

19

- 12

m 101 


\section{CUMULATIVE INDEX-Continued}

Vol. or

Cesium chloride, $\mathrm{CsCl} \ldots \ldots \ldots \ldots \ldots \ldots \ldots \ldots$

Cesium chloroosmate(IV), $\mathrm{Cs}_{2} \mathrm{OsCl}_{6} \ldots \ldots \ldots 2 \mathrm{~m}$

Cesium chloroplatinate, $\mathrm{Cs}_{2} \mathrm{PtCl}_{6} \ldots \ldots \ldots \ldots$.

Cesium chlorostannate, $\mathrm{Cs}_{2} \mathrm{SnCl}_{6} \ldots \ldots \ldots \ldots$

Cesium chromate, $\mathrm{Cs}_{2} \mathrm{CrO}_{4} \ldots \ldots \ldots \ldots \ldots \ldots$

Cesium chromium sulfate dodecahydrate,

$\mathrm{CsCr}\left(\mathrm{SO}_{4}\right)_{2} \cdot 12 \mathrm{H}_{2} \mathrm{O} \ldots \ldots \ldots \ldots \ldots$

Cesium cobalt (II) trichloride, $\mathrm{CsCoCl}_{2} \ldots \ldots$.

Cesium copper sulfate hexahydrate,

$\mathrm{Cs}_{2} \mathrm{Cu}\left(\mathrm{SO}_{4}\right)_{2} \cdot 6 \mathrm{H}_{2} \mathrm{O} \ldots \ldots \ldots \ldots \ldots$

Cesium copper(II) trichloride, $\mathrm{CsCuCl}_{3} \ldots \ldots$.

Cesium dichloroiodide, $\mathrm{CsICl}_{2} \ldots \ldots \ldots \ldots$

Cesium fluoantimonate, $\mathrm{CsSbF}_{6} \ldots \ldots \ldots \ldots$

Cesium fluoborate, $\mathrm{CsBF}_{4} \ldots \ldots \ldots \ldots \ldots \ldots$

Cesium fluogermanate, $\mathrm{Cs}_{2} \mathrm{GeF}_{6} \ldots \ldots \ldots \ldots$

Cesium fluoplatinate, $\mathrm{Cs}_{2} \mathrm{PtF}_{6} \ldots \ldots \ldots \ldots$

Cesium fluoride, CsF ..............

Cesium fluosilicate, $\mathrm{Cs}_{2} \mathrm{SiF}_{6} \ldots \ldots \ldots \ldots \ldots$

Cesium gallium sulfate dodecahydrate,

$\mathrm{CsGa}\left(\mathrm{SO}_{4}\right)_{2} \cdot 12 \mathrm{H}_{2} \mathrm{O} \ldots \ldots \ldots \ldots \ldots$

Cesium iodine bromide, $\mathrm{CsI}_{2} \mathrm{Br} \ldots \ldots \ldots \ldots \ldots$

Cesium iodide, CsI . . . . . . . . . . . . .

Cesium iron sulfate dodecahydrate,

$\mathrm{CsFe}\left(\mathrm{SO}_{4}\right)_{2} \cdot 12 \mathrm{H}_{2} \mathrm{O}$

Cesium iron sulfate hexahydrate,

$\mathrm{Cs}_{2} \mathrm{Fe}\left(\mathrm{SO}_{4}\right)_{2} \cdot 6 \mathrm{H}_{2} \mathrm{O} \ldots \ldots \ldots \ldots \ldots$

Cesium lead(II) trichloride, $\mathrm{CsPbCl}$, (tetragonal) ................. $5 \mathrm{~m}$

Cesium lithium fluoride, $\mathrm{CsLiF}_{2} \ldots \ldots \ldots \ldots \ldots \ldots \ldots$

Cesium magnesium sulfate hexahydrate, $\mathrm{Cs}_{2} \mathrm{Mg}\left(\mathrm{SO}_{4}\right)_{2} \cdot 6 \mathrm{H}_{2} \mathrm{O} \ldots \ldots \ldots \ldots \ldots \ldots$

Cesium manganese sulfate hexahydrate,

$\mathrm{Cs}_{2} \mathrm{Mn}\left(\mathrm{SO}_{4}\right)_{2} \cdot 6 \mathrm{H}_{2} \mathrm{O} \ldots \ldots \ldots \ldots \ldots \ldots$

Cesium mercury chloride, $\mathrm{CsHgC1}_{2} \ldots \ldots \ldots \ldots$

Cesium nickel sulfate hexahydrate,

$\mathrm{Cs}_{2} \mathrm{Ni}\left(\mathrm{SO}_{4}\right)_{2} \cdot 6 \mathrm{H}_{2} \mathrm{O} \ldots \ldots \ldots \ldots \ldots \ldots$

Cesium nickel (II) trichloride, $\mathrm{CsNiCl}_{3} \ldots \ldots$

Cesium nitrate, $\mathrm{CsNO}_{3} \ldots \ldots \ldots \ldots \ldots \ldots$

Cesium perchlorate, $\mathrm{CsClO}_{4}$, (orthorhombic)

Cesium strontium trichloride, $\mathrm{CsSrCl}_{3} \ldots \ldots \ldots$

Cesium sulfate $\mathrm{Cs}_{2} \mathrm{SO}_{4} \ldots \ldots \ldots \ldots \ldots \ldots$

Cesium vanadium sulfate dodecahydrate,

$\mathrm{CsV}\left(\mathrm{SO}_{4}\right)_{2} \cdot 12 \mathrm{H}_{2} \mathrm{O} \ldots \ldots \ldots \ldots \ldots$

Cesium zinc sulfate hexahydrate,

$\mathrm{Cs}_{2} \mathrm{Zn}\left(\mathrm{SO}_{4}\right)_{2} \cdot 6 \mathrm{H}_{2} \mathrm{O} \ldots \ldots \ldots \ldots \ldots \ldots \ldots \ldots \ldots \ldots$

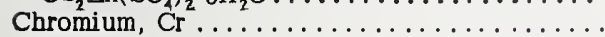

Chromium fluoride, $\mathrm{Cr}_{2} \mathrm{~F}_{5} \ldots \ldots \ldots \ldots \ldots \ldots$

Chromium(III) fluoride trihydrate, $\mathrm{CrF}_{3} \cdot 3 \mathrm{H}_{2} \mathrm{O}$

Chromium iridium $3: 1, \mathrm{Cr}_{3} \mathrm{Ir} \ldots \ldots \ldots \ldots \ldots$.

Chromium orthophosphate, alpha, $\mathrm{CrPO}_{4} \ldots \ldots$

Chromium orthophosphate, beta, $\mathrm{CrPO}_{4} \ldots \ldots$.

Chromium(III) oxide, $\mathrm{Cr}_{2} \mathrm{O}_{3} \ldots \ldots \ldots \ldots$

Chromium rhodium $3: 1, \mathrm{Cr}_{3} \mathrm{Rh} \ldots \ldots \ldots \ldots \ldots$

Chromium silicide, $\mathrm{Cr}_{3} \mathrm{Si} \ldots \ldots \ldots \ldots \ldots \ldots$

Cobalt, Co (cubic) ..................

Cobalt aluminum oxide, $\mathrm{CoAl}_{2} \mathrm{O}_{4} \ldots \ldots \ldots \ldots$

Cobalt antimony oxide, $\mathrm{CoSb}_{2} \mathrm{O}_{6} \ldots \ldots \ldots$

Cobalt arsenide (skutterudite), $\mathrm{CoAs}_{3} \ldots \ldots$.

Cobalt(II) carbonate (spherocobaltite),

$\mathrm{CoCO}_{3}$

sec.

Page

244

11

514

$5 \quad 16$

$3 \mathrm{~m} \quad 25$

821

$6 m$

$7 \mathrm{~m}$

$5 \mathrm{~m}$

3
$4 m$

8

5

6

$3 m$

5

8

$7 \mathrm{~m}$
4

6

$7 \mathrm{~m}$

$7 \mathrm{~m}$

$7 \mathrm{~m}$

$7 \mathrm{~m}$

$7 \mathrm{~m}$

$6 \mathrm{~m}$

$1 \mathrm{~m}$

$6 \mathrm{~m}$

7

$1 \mathrm{~m}$

$7 \mathrm{~m}$

5

$7 \mathrm{~m}$

$6 \mathrm{~m}$

$2 \mathrm{~m}$

9

5

$6 \mathrm{~m}$

$4 \mathrm{~m}$

9

$5 \mathrm{~m}$

10

10

0

Cobalt diarsenide, $\mathrm{CoAs}_{2}$ (revised)

Vol. or

Cobalt fluosilicate hexahydrate,

$\mathrm{CoSiF} \cdot 6 \mathrm{H}_{2} \mathrm{O}$

Cobalt gallate, $\mathrm{CoGa}_{2} \mathrm{O}$

Cobalt germanate, $\mathrm{Co}_{2} \mathrm{GeO}_{4}$

Cobalt iodide, $\mathrm{CoI}_{2}$

Cobalt iron arsenide (safflorite), $\mathrm{CoF}$ eAs

Cobalt mercury thiocyanate, $\mathrm{Co}\left[\mathrm{Hg}(\mathrm{CNS})_{4}\right]$

Cobalt(II) oxide, CoO .

Cobalt(II, III) oxide, $\mathrm{Co}_{3} \mathrm{O}_{4}$

Cobalt perchlorate hexahydrate,

$\mathrm{Co}\left(\mathrm{ClO}_{4}\right)_{2} \cdot 6 \mathrm{H}_{2} \mathrm{O}$

Cobalt silicate, $\mathrm{Co}_{2} \mathrm{SiO}_{4}$ (orthorhombic).

Cobalt sulfate, beta, $\mathrm{CoSO}_{4}$

Cobalt titanate, CoTiO,

Cobalt tungstate, CoWO,

Copper, $\mathrm{Cu}$..

Copper antimony oxide, $\mathrm{CuSb}_{2} \mathrm{O}_{6}$

Copper(I) bromide, $\mathrm{CuBr}$.

Copper carbonate, basic, azurite,

$\mathrm{CU}_{3}(\mathrm{OH})_{2}\left(\mathrm{CO}_{3}\right)_{2} \ldots \ldots \ldots \ldots \ldots \ldots \ldots . \quad 10$

Copper carbonate, basic, (malachite),

$\mathrm{CU}_{2}(\mathrm{OH})_{2} \mathrm{CO}_{3}$

Copper (I) chloride (nantokite), $\mathrm{CuC1} \ldots \ldots$.

Copper glutamate dihydrate,

$\mathrm{CuC}_{5} \mathrm{H}_{7} \mathrm{NO}_{4} \cdot 2 \mathrm{H}_{2} \mathrm{O} \ldots \ldots \ldots \ldots \ldots \ldots \ldots$
Copper(I) iodide (marchite), $\mathrm{CuI} \ldots \ldots \ldots \ldots \ldots$

Copper (I) oxide (cuprite), $\mathrm{Cu}_{2} \mathrm{O}$

Copper(II) oxide (tenorite), CuO ...........

Copper phosphate, alpha-pyro-, $\mathrm{Cu}_{2} \mathrm{P}_{2} \mathrm{O}_{7} \ldots$. .

Copper sulfate (chal cocyanite), Cuso.

Copper(II) sulfide (covellite), CuS . . . . . . . .

Dibenzoylmethane, $\mathrm{C}_{13} \mathrm{H}_{12} \mathrm{O}_{2} \ldots \ldots \ldots \ldots \ldots$

Dysprosium antimony, DySb ...........

Dysprosium arsenate, Dy $\mathrm{AsO}_{4} \ldots \ldots \ldots \ldots \ldots$

Dysprosium arsenide, DyAs .

Dy sprosium gallium oxide, $\mathrm{Dy}_{3} \mathrm{Ga}_{2}\left(\mathrm{GaO}_{4}\right)_{3} \ldots$.

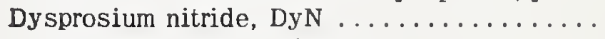

Dysprosium sesquioxide, $\mathrm{Dy}_{2} \mathrm{O}_{3} \ldots \ldots \ldots \ldots$

Dysprosium telluride, DyTe...........

Dysprosium vanadate, $\mathrm{DyVO}_{4} \ldots \ldots \ldots \ldots$

Erbium antimony, Ersb .............

Erbium arsenate, ErAsO $\mathrm{AsO}_{4} \ldots \ldots \ldots \ldots \ldots$

Erbium arsenide, ErAs ..............

Erbium gallium oxide, $\mathrm{Er}_{3} \mathrm{Ga}_{2}\left(\mathrm{GaO}_{4}\right)_{3} \ldots \ldots$

Erbium manganite, $\mathrm{ErMnO}_{3}$

Erbium nitride, ErN . . . . . . . . . . . . . .

Erbium phosphate, $\mathrm{ErPO}_{4} \ldots \ldots \ldots \ldots \ldots$

Erbium sesquioxide, $\operatorname{Er}_{2} \mathrm{O}_{3} \ldots \ldots \ldots \ldots \ldots$

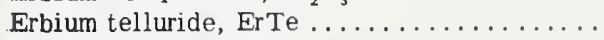

Erbium vanadate, $\mathrm{ErVO}_{4} \ldots \ldots \ldots \ldots \ldots \ldots$

Europium arsenate, $\mathrm{EuAsO}_{4} \ldots \ldots \ldots \ldots \ldots$

Europium(III) chloride, $\mathrm{EuCl}_{3} \ldots \ldots \ldots \ldots \ldots$

Europium gallium oxide, $\mathrm{Eu}_{3} \mathrm{Ga}_{2}\left(\mathrm{GaO}_{4}\right)_{3} \ldots \ldots$.

Europium nitride, EuN .

Europium oxide, EuO ...............

Europium oxychloride, EuOCl ...........

Europium(III) -vanadate, EuVO,

Gadolinium antimony, GdSb ............

Gadolinium arsenate, $\mathrm{GdAsO}_{4}$

Gadolinium arsenide, GdAs

Gadolinium chloride hexahydrate,

$\mathrm{GdCl}_{3} \cdot 6 \mathrm{H}_{2} \mathrm{O} \ldots \ldots \ldots \ldots \ldots \ldots \ldots \ldots . \ldots \ldots$

Gadolinium fluoride, $\mathrm{GdF}_{3} \ldots \ldots \ldots \ldots \ldots \ldots . . \ldots \ldots$

Gadolinium gallium oxide, $\mathrm{Gd}_{3} \mathrm{Ga}_{2}\left(\mathrm{GaO}_{4}\right), \ldots 2 \mathrm{~m}$

Gadolinium indium, GdIn .......... $5 \mathrm{~m}$
Page

$4 m$

10

$3 \mathrm{~m}$

10

10

$4 m$
10

$2 \mathrm{~m}$

$3 m$

$4 \mathrm{~m}$

$2 \mathrm{~m}$

$4 \mathrm{~m}$

$4 \mathrm{~m}$

1
$5 \mathrm{~m}$

4

10

4

35

$7 \mathrm{~m}$

110

2

1

$7 \mathrm{~m}$

$3 \mathrm{~m}$

4
$7 \mathrm{~m}$

$7 \mathrm{~m}$

$3 \mathrm{~m}$

$4 \mathrm{~m}$

$2 \mathrm{~m}$

$4 \mathrm{~m}$

9
$4 \mathrm{~m}$

$4 \mathrm{~m}$
$4 \mathrm{~m}$

$4 \mathrm{~m}$

$3 \mathrm{~m}$

$4 \mathrm{~m}$

$1 \mathrm{~m}$

$2 \mathrm{~m}$

$4 \mathrm{~m}$

9

8

$4 \mathrm{~m}$

$5 \mathrm{~m}$

$3 \mathrm{~m}$

$1 \mathrm{~m}$

$2 \mathrm{~m}$

$4 \mathrm{~m}$

$4 \mathrm{~m}$

$1 \mathrm{~m}$

$4 \mathrm{~m}$

$4 \mathrm{~m}$

$4 \mathrm{~m}$

$4 \mathrm{~m}$

118

14

18

67

A mineral name in ( ) indicates a synthetic sample. 


\section{CUMULATIVE INDEX-Continued}

\begin{tabular}{|c|c|}
\hline & $\begin{array}{c}\text { Vol. or } \\
\text { sec. } \\
4 \mathrm{~m}\end{array}$ \\
\hline Gadolinium nitride, GdN . & \\
\hline Gadolinium oxide, $\mathrm{Gd}_{2} \mathrm{O}_{3} \ldots \ldots$ & $1 \mathrm{~m}$ \\
\hline $\begin{array}{l}\text { Gadolinium oxychloride, } \mathrm{GdOCl} \\
\text { Gadolinium vanadate, } \mathrm{GdVO}_{4} \ldots\end{array}$ & $\begin{array}{l}1 \mathrm{~m} \\
5 \mathrm{~m}\end{array}$ \\
\hline Gallium, $\mathrm{Ga} \ldots \ldots \ldots \ldots$ & 2 \\
\hline Gallium arsenide, GaAs . & $3 \mathrm{~m}$ \\
\hline Gallium antimonide, GaSb & 6 \\
\hline Gallium oxide, alpha, $\mathrm{Ga}_{2} \mathrm{O}_{3}$ & 4 \\
\hline Gallium phosphate ( $\propto$-quartz type), $\mathrm{GaPO}_{4}$. & 8 \\
\hline Germanium, Ge... & 1 \\
\hline
\end{tabular}

Germanium dioxide, $\mathrm{GeO}_{2}$ (hexagonal)

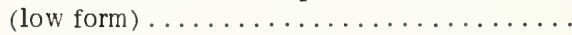

Germanium dioxide, $\mathrm{GeO}_{2}$ (tetragonal)

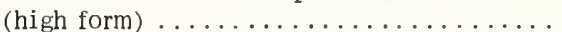

Germanium iodide, $\mathrm{GeI}_{2} \ldots \ldots \ldots \ldots \ldots$

Germanium(IV) iodide, $\mathrm{GeI}_{4} \ldots \ldots \ldots \ldots \ldots$
Gold $\mathrm{Au} \ldots \ldots \ldots \ldots \ldots \ldots \ldots$

Gold antimony $1: 2$ (aurostibite), $\mathrm{AuSb}_{2} \ldots \ldots$

Gold dysprosium, AuDy ...............

Gold(I) cyanide, AuCN . ................

Gold holmium, AuHo

Gold magnesium, Aumg................

Gold niobium $1: 3, \mathrm{AuNb}_{3} \ldots \ldots \ldots \ldots \ldots \ldots$.

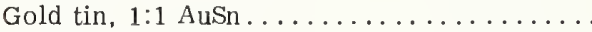

Gold titanium 1:3, $\mathrm{AuTi}_{3} \ldots \ldots \ldots \ldots \ldots \ldots$

Gold vanadium $1: 3, \mathrm{AuV}_{3} \ldots \ldots \ldots \ldots \ldots \ldots$

Hafnium, Hf

Hexamethyl enediammonium adipate,

$$
\mathrm{C}_{12} \mathrm{H}_{26} \mathrm{~N}_{2} \mathrm{O}_{4} \text {... }
$$

Holmium arsenate, $\mathrm{HoAsO}_{4}$

Holmium ethylsulfate nonahydrate,

$\mathrm{Hol}\left(\mathrm{C}_{2} \mathrm{H}_{5}\right) \mathrm{SO}_{4} \mathrm{~J}_{3} \cdot 9 \mathrm{H}_{2} \mathrm{O} \ldots$

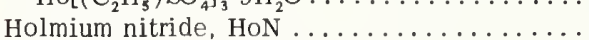

Holmium selenide, HoSe...............

Holmium sesquioxide, $\mathrm{Ho}_{2} \mathrm{O}_{3} \ldots \ldots \ldots \ldots \ldots$

Holmium vanadate, $\mathrm{HoVO}_{4} \ldots \ldots \ldots \ldots \ldots$

Imidazole nickel nitrate, $\left(\mathrm{C}_{3} \mathrm{H}_{4} \mathrm{~N}_{2}\right)_{6} \mathrm{Ni}\left(\mathrm{NO}_{3}\right)_{2}$.

Imidazole zinc chloride, $\left(\mathrm{C}_{3} \mathrm{H}_{4} \mathrm{~N}_{2}\right)_{2} \mathrm{ZnCl}_{2} \ldots$

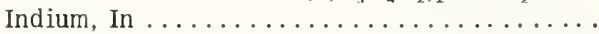

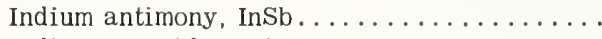

Indium arsenide, InAs . . . . . . . . . . .

Indium phosphate, $\mathrm{InPO}_{4} \ldots \ldots \ldots \ldots \ldots \ldots$

Iodic acid, $\mathrm{HIO}_{3} \ldots \ldots \ldots \ldots \ldots \ldots \ldots$

Iodine, $I_{2} \ldots \ldots \ldots \ldots \ldots \ldots \ldots \ldots$

Iridium, Ir

Iridium dioxide, $\mathrm{IrO}_{2}$

Iridium niobium $1: 3, \mathrm{IrNb}_{3} \ldots \ldots \ldots \ldots \ldots$

Iridium titanium $1: 3, \mathrm{IrTi}_{3} \ldots \ldots \ldots \ldots \ldots \ldots$

Iridium vanadium $1: 3, \operatorname{IrV}_{3} \ldots \ldots \ldots \ldots \ldots$

Iron, alpha $\mathrm{Fe}$

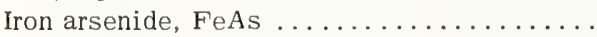

Iron arsenide (loellingite), $\mathrm{FeAs}_{2} \ldots \ldots \ldots$

Iron bromide, $\mathrm{FeBr}_{2}$.

Iron iodide, $\mathrm{FeI}_{2}$

Iron(II,III) oxide (magnetite), $\mathrm{Fe}_{3} \mathrm{O}_{4} \ldots \ldots \ldots$

Iron sulfide (pyrite), $\mathrm{FeS}_{2}$

Lanthanum antimony, LaSb ..................

Lanthanum arsenate, $\mathrm{LaAsO}_{4} \ldots \ldots \ldots \ldots$.

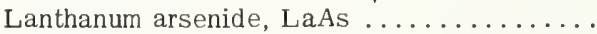

Lanthanum borate, $\mathrm{LaBO}_{3} \ldots \ldots \ldots \ldots \ldots$.

Lanthanum chloride, $\mathrm{LaCl}_{3} \ldots \ldots \ldots \ldots \ldots$.

Lanthanum fluoride, $\mathrm{LaF}_{3}$
15

$\begin{array}{rr}8 & 28 \\ 4 \mathrm{~m} & 58 \\ 5 & 25 \\ 1 & 33 \\ 7 & 18 \\ 5 \mathrm{~m} & 66 \\ 10 & 33 \\ 5 \mathrm{~m} & 68 \\ 6 \mathrm{~m} & 83 \\ 6 \mathrm{~m} & 16 \\ 7 & 19 \\ 6 \mathrm{~m} & 17 \\ 6 \mathrm{~m} & 18 \\ 3 & 18\end{array}$

$7 \mathrm{~m} \quad 121$

$3 \mathrm{~m} \quad 34$

$1 \mathrm{~m} \quad 18$

$4 \mathrm{~m} \quad 58$

$4 \mathrm{~m} \quad 59$

$9 \quad 32$

$4 \mathrm{~m} \quad 18$

$7 \mathrm{~m} \quad 27$

$7 \mathrm{~m} \quad 123$

$3 \quad 12$

$4 \quad 73$

$3 \mathrm{~m} \quad 35$

$5 \quad 26$

$8 \quad 29$

$5 \quad 28$

$3 \quad 16$

49

$4 \mathrm{~m} \quad 19$

$6 \mathrm{~m} \quad 19$

$6 \mathrm{~m} \quad 20$

$6 \mathrm{~m} \quad 21$

43

$1 \mathrm{~m} \quad 19$

$10 \quad 34$

$4 \mathrm{~m} \quad 59$

$4 \mathrm{~m} \quad 60$

$5 \mathrm{~m} \quad 31$

$5 \quad 29$

$4 \mathrm{~m} \quad 42$

$3 \mathrm{~m} \quad 36$

$4 \mathrm{~m} \quad 60$

im $\quad 20$

$1 \mathrm{~m} \quad 20$

7

m-Monograph 25.

A mineral name in ( ) indicates a synthetic sample.

Vol. or

Lanthanum magnesium, LaMg ......... $5 \mathrm{~m}$

Page

Lanthanum magnesium nitrate 24-hydrate,

$\mathrm{La}_{2} \mathrm{Mg}_{3}\left(\mathrm{NO}_{3}\right)_{12} \cdot 24 \mathrm{H}_{2} \mathrm{O} \ldots \ldots \ldots \ldots \ldots \ldots$

Lanthanum niobium titanium oxide, $\mathrm{LaNbTiO}_{6}$

Lanthanum nitride, $\mathrm{LaN} . . . \ldots \ldots \ldots \ldots$. . . . .

Lanthanum oxide, $\mathrm{La}_{2} \mathrm{O}_{3} \ldots \ldots \ldots \ldots \ldots$

Lanthanum oxychloride, $\mathrm{LaOCl} \ldots \ldots \ldots \ldots$

Lanthanum phosphide, LaP . . . . . . . . . . . .

Lanthanum selenide, LaSe.............

Lanthanum zinc, LaZn ................

Lead, $\mathrm{Pb} \ldots . . \ldots \ldots \ldots \ldots \ldots \ldots \ldots$

Lead boron oxide, $\mathrm{PbB}_{4} \mathrm{O}_{7} \ldots \ldots \ldots \ldots \ldots$

Lead bromide, $\mathrm{PbBr}_{2} \ldots \ldots \ldots \ldots \ldots \ldots \ldots$

Lead carbonate (cerrussite), $\mathrm{PbCO}_{3} \ldots \ldots \ldots$.

Lead chloride (cotunnite), $\mathrm{PbCl}_{2} \ldots \ldots \ldots \ldots$

Lead formate, $\mathrm{Pb}\left(\mathrm{HCO}_{2}\right)_{2} \ldots \ldots \ldots \ldots \ldots$

Lead fluochloride (matlockite), $\mathrm{PbFCl} \ldots \ldots$.

Lead fluoride, alpha $\mathrm{PbF}_{2}$ (orthorhombic) ....

Lead fluoride, beta $\mathrm{PbF}_{2}$ (cubic) .........

$\mathrm{L}$ ead(II) iodide, $\mathrm{PbI}_{2} \ldots \ldots \ldots \ldots \ldots \ldots \ldots$

Lead molybdate (wulfenite), $\mathrm{PbMoO}_{4} \ldots \ldots$.

Lead monoxide (litharge), $\mathrm{PbO}$ (red) tetrag-

onal .........................

Lead monoxide (massicot), PbO (yellow)

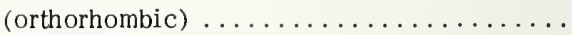

Lead nitrate, $\mathrm{Pb}\left(\mathrm{NO}_{3}\right)_{2} \ldots \ldots \ldots \ldots \ldots \ldots$

Lead(II, III) oxide (minium), $\mathrm{Pb}_{3} \mathrm{O}_{4} \ldots \ldots \ldots$

Lead oxybromide, $\mathrm{Pb}_{3} \mathrm{O}_{2} \mathrm{Br}_{2} \ldots \ldots \ldots \ldots \ldots$.

Lead phosphate hydrate, $\mathrm{Pb}_{5}\left(\mathrm{PO}_{4}\right)_{3} \mathrm{OH} \ldots \ldots$.

Lead selenide (clausthalite), $\mathrm{PbSe}$........

Lead sulfate (anglesite), $\mathrm{PbSO}_{4} \ldots \ldots \ldots \ldots$.

Lead sulfide (galena), PbS . . . . . . . . .

Lead titanate, $\mathrm{PbTiO}_{3} \ldots \ldots \ldots \ldots \ldots \ldots$

Lead tungstate (stolzite), $\mathrm{PbWO}_{4}$ (tetragonal)

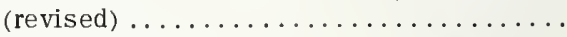

Lithium arsenate, $\mathrm{Li}_{3} \mathrm{AsO}_{4} \ldots \ldots \ldots \ldots \ldots \ldots$

Lithium barium trifluoride, $\mathrm{LiBaF}_{3} \ldots \ldots \ldots$

Lithium beryllium fluoride, $\mathrm{Li}_{2} \mathrm{BeF}_{4} \ldots \ldots \ldots$.

Lithium bromide, $\mathrm{LiBr} \ldots \ldots \ldots \ldots \ldots \ldots$

Lithium chloride, $\mathrm{LiCl} \ldots \ldots \ldots \ldots \ldots \ldots \ldots$

Lithium fluoride, Li F . . . . . . . . . . .

Lithium iodate, $\mathrm{LiIO}_{3} \ldots \ldots \ldots \ldots \ldots \ldots$

Lithium molybdate, $\mathrm{Li}_{2} \mathrm{MoO}_{4}$ (trigonal) ......

Lithium niobate, $\mathrm{LiNbO}_{3} \ldots \ldots \ldots \ldots \ldots \ldots$

Lithium nitrate, $\mathrm{LiNO}_{3} \ldots \ldots \ldots \ldots \ldots \ldots$

Lithium oxide, $\mathrm{Li}_{2} \mathrm{O} \ldots \ldots \ldots \ldots \ldots$

Lithium perchlorate trihydrate, $\mathrm{LiClO}_{4} \cdot 3 \mathrm{H}_{2} \mathrm{O}$

Lithium phosphate, low form (lithiophosphate), $\mathrm{Li}_{3} \mathrm{PO}_{4}$ (orthorhombic) revised ....

Lithium phosphate, high form, $\mathrm{Li}_{3} \mathrm{PO}_{4} \ldots \ldots$.

Lithium rubidium fluoride, $\mathrm{LiRbF}_{2} \ldots \ldots \ldots \ldots$

Lithium sodium sulfate, $\mathrm{LiNaSO}_{4} \ldots \ldots \ldots \ldots$

Lithium sulfate, $\mathrm{Li}_{2} \mathrm{SO}_{4} \ldots \ldots \ldots \ldots \ldots \ldots$

Lithium sulfate monohydrate, $\mathrm{Li}_{2} \mathrm{SO}_{4} \cdot \mathrm{H}_{2} \mathrm{O} \ldots$.

Lithium trimetaphosphate trihydrate,

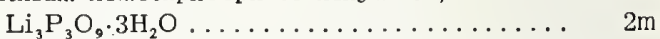

Lithium tungstate, $\mathrm{Li}_{2} \mathrm{WO}_{4}$ (trigonal) ....... $1 \mathrm{~m}$

Lithium tungstate hemihydrate, $\mathrm{Li}_{2} \mathrm{WO}_{4} \cdot 1 / 2 \mathrm{H}_{2} \mathrm{O}$

Lithium uranium fluoride, $\mathrm{LiUF}_{\xi} \ldots \ldots \ldots \ldots$.

Lutetium arsenate, $\mathrm{LuAsO}_{4} \ldots \ldots \ldots \ldots \ldots \ldots$

Lutetium gallium oxide, $\mathrm{Lu}_{3} \mathrm{Ga}_{2}\left(\mathrm{GaO}_{4}\right)_{3} \ldots \ldots$.

Lutetium manganite, $\mathrm{LuMnO}_{3} \ldots \ldots \ldots \ldots \ldots$

Lutetium nitride, LuN . . . . . . . . . . . .

Lutetium oxide, $\mathrm{Lu}_{2} \mathrm{O}_{3} \ldots \ldots \ldots \ldots \ldots$

Lutetium vanadate, $\mathrm{LuVO}_{4} \ldots \ldots \ldots \ldots \ldots \ldots$

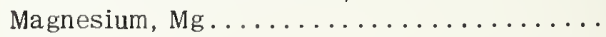




\section{CUMULATIVE INDEX-Continued}

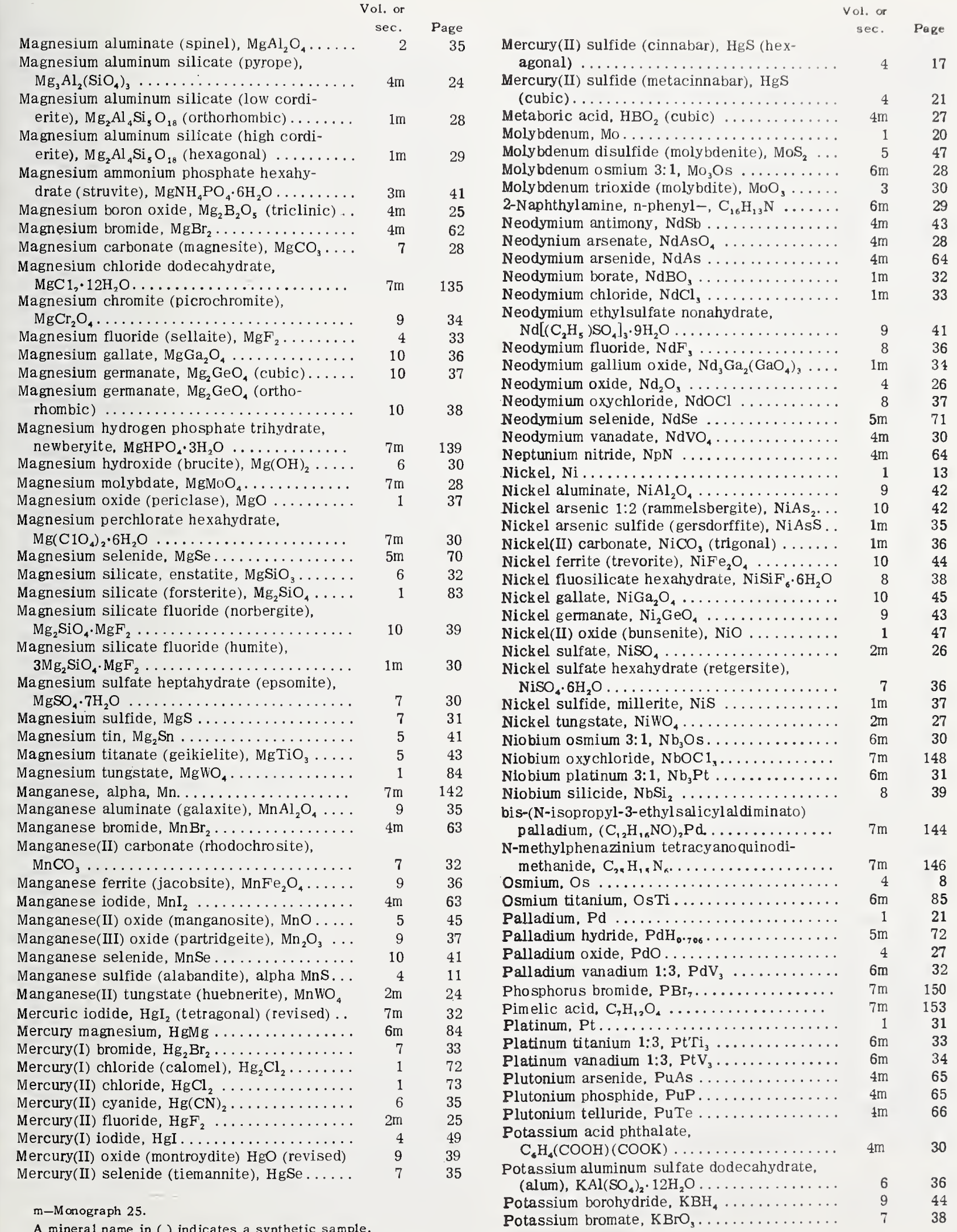

m-Monograph 25.
A mineral name in ( ) indicates a synthetic sample. 


\section{CUMULATIVE INDEX-Continued}

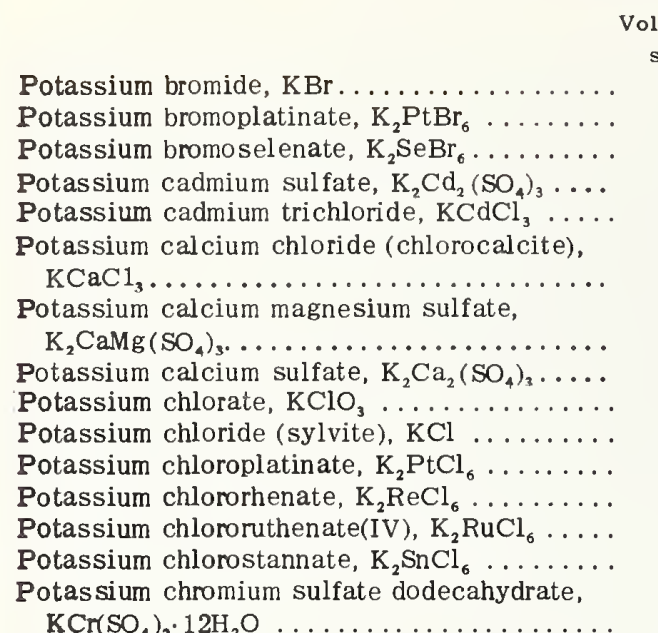

$\mathrm{KCr}\left(\mathrm{SO}_{4}\right)_{2} \cdot 12 \mathrm{H}_{2} \mathrm{O} \ldots \ldots \ldots \ldots \ldots \ldots \ldots$

Potassium cobalt (II) sulfate, $\mathrm{K}_{2} \mathrm{Co}_{2}\left(\mathrm{SO}_{4}\right)_{3} \ldots$

Potassium cobalt (II) trifluoride, $\mathrm{KCoF}_{3} \ldots \ldots$

Potassium cobaltinitrite, $\mathrm{K}_{3} \mathrm{Co}\left(\mathrm{NO}_{2}\right)_{6} \ldots \ldots \ldots$

Potassium copper chloride, $\mathrm{KCuCl}_{1}, \ldots \ldots \ldots$.

Potassium copper (II) trifluoride, $\mathrm{KCuF}_{3} \ldots \ldots$

Potassium cyanate, KCNO............

Potassium cyanide, $\mathrm{KCN}$. . . ..........

Potassium dihydrogen arsenate, $\mathrm{KH}_{2} \mathrm{AsO}_{4} \ldots$

Potassium dihydrogen phosphate, $\mathrm{KH}_{2} \mathrm{PO}_{4} \ldots$

Potassium fluogermanate, $\mathrm{K}_{2} \mathrm{GeF}_{6} \ldots \ldots \ldots$.

Potassium fluoplatinate, $\mathrm{K}_{2} \mathrm{PtF}_{6} \ldots \ldots \ldots \ldots$.

Potassium fluoride, $\mathrm{KF} \ldots \ldots \ldots \ldots \ldots \ldots$

Potassium fluosilicate (hieratite), $\mathrm{K}_{2} \mathrm{SiF}_{6} \ldots \ldots$

Potassium fluotitanate, $\mathrm{K}_{2} \mathrm{TiF}_{6} \ldots \ldots \ldots \ldots$.

Potassium heptafluozirconate, $\mathrm{K}_{3} \mathrm{ZrF}_{7} \ldots \ldots$.

Potassium hydroxide, $\mathrm{KOH}$ at $300{ }^{\circ} \mathrm{C} \ldots \ldots$.

Potassium hydroxy-chlororuthenate,

$\mathrm{K}_{4} \mathrm{Ru}_{2} \mathrm{Cl}{ }_{10} \mathrm{O} \cdot \mathrm{H}_{2} \mathrm{O} \ldots \ldots \ldots \ldots \ldots \ldots \ldots$

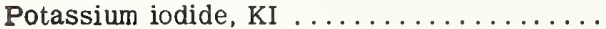

Potassium iron (II) trifluoride, $\mathrm{KFeF}_{3} \ldots \ldots$.

Potassium lithium sulfate, $\mathrm{KLiSO}_{4} \ldots \ldots \ldots \ldots$

Potassium magnesium sul fate (langbeinite),

$\mathrm{K}_{2} \mathrm{Mg}_{2}\left(\mathrm{SO}_{4}\right)_{3} \ldots \ldots \ldots \ldots \ldots \ldots \ldots$

Potassium magnesium trifluoride, $\mathrm{KMgF}_{3} \ldots$

Potassium manganese (II) sulfate

(manganolangbeinite), $\mathrm{K}_{2} \mathrm{Mn}_{2}\left(\mathrm{SO}_{4}\right)_{3} \ldots \ldots \ldots$

Potassium manganese (II) trifluoride, $\mathrm{KMnF}_{3}$

Potassium nickel fluoride, $\mathrm{KNiF}_{3} \ldots . . . \ldots$.

Potassium nickel (II) sulfate, $\mathrm{K}_{2} \mathrm{Ni}_{2}\left(\mathrm{SO}_{4}\right)_{3} \ldots$

Potassium nitrate (niter), $\mathrm{KNO}_{3} \ldots \ldots \ldots \ldots$

Potassium nitroso chlororuthenate,

$\mathrm{K}_{2} \mathrm{RuC}_{1} \mathrm{NO} \ldots \ldots \ldots \ldots \ldots \ldots \ldots \ldots . \ldots . \ldots$

Potassium perchlorate, $\mathrm{KClO}_{4} \ldots \ldots \ldots \ldots \ldots$

Potassium perchromate, $\mathrm{K}_{3} \mathrm{CrO}, \ldots \ldots \ldots \ldots$

Potassium periodate, $\mathrm{KIO}_{4}$

Potassium permanganate, $\mathrm{KMnO}_{4} \ldots \ldots \ldots \ldots$

Potassium perrhenate, $\mathrm{KReO}_{4} \ldots \ldots \ldots \ldots \ldots$

Potassium phosphomolybdate tetrahydrate,

$\mathrm{K}_{2} \mathrm{PO}_{4}\left(\mathrm{MoO}_{3}\right)_{12} \cdot 4 \mathrm{H}_{2} \mathrm{O} \ldots \ldots \ldots \ldots \ldots \ldots$

Potassium sodium sulfate, $\mathrm{K}_{67} \mathrm{Na}_{1.35} \mathrm{SO}_{4} \ldots$.

Potassium sodium sulfate, $\mathrm{KNaSO}_{4} \ldots \ldots \ldots$.

Potassium sodium sulfate (aphthitalite),

$\mathrm{K}_{3} \mathrm{Na}\left(\mathrm{SO}_{4}\right)_{2} \ldots \ldots \ldots \ldots \ldots \ldots \ldots \ldots$

Potassium sulfate (arcanite), $\mathrm{K}_{2} \mathrm{SO}_{4} \ldots \ldots \ldots$

Potassium thiocyanate, KCNS ...........

m-Monograph 25.

A mineral name in () indicates a synthetic sample. ol. or

sec.

Page

66

40

41

34

38

$7 \mathrm{~m}$

Potassium zinc decavanadate 16 hydrate,

Vol. or

$\mathrm{K}_{2} \mathrm{Zn}_{2} \mathrm{~V}_{10} \mathrm{O}_{28} \cdot 16 \mathrm{H}_{2} \mathrm{O} \ldots \ldots \ldots \ldots \ldots$ $3 \mathrm{~m}$

Potassium zinc fluoride, $\mathrm{KZnF}_{3} \ldots \ldots \ldots \ldots$.

45

Potassium zinc sulfate hexahydrate,

$\mathrm{K}, \mathrm{Zn}\left(\mathrm{SO}_{4}\right)_{2} \cdot 6 \mathrm{H}_{2} \mathrm{O} \ldots \ldots \ldots \ldots \ldots \ldots$

Potassium zinc sulfate, $\mathrm{K}_{2} \mathrm{Zn}_{2}\left(\mathrm{SO}_{4}\right)_{3} \ldots \ldots \ldots$ Praseodymium antimony, $\mathrm{PrSb} \ldots \ldots \ldots \ldots \ldots$

Praseodymium arsenate, $\operatorname{Pr} \mathrm{AsO}_{4} \ldots \ldots \ldots \ldots$

Pras eodymium arsenide, $\operatorname{PrAs} \ldots \ldots \ldots \ldots \ldots$

Praseodymium chloride, $\mathrm{PrCl}_{3} \ldots \ldots \ldots \ldots \ldots$

Praseodymium fluoride, $\mathrm{PrF}_{3} \ldots \ldots \ldots \ldots \ldots$

Praseodymium oxychloride, $\mathrm{PrOCl} \ldots \ldots \ldots \ldots$

Praseodymium sulfide, $\operatorname{PrS} \ldots \ldots \ldots \ldots \ldots$

Praseodymium vanadate, $\mathrm{PrVO}_{4} \ldots \ldots \ldots \ldots$

Praseodymium zinc, PrZn . . . . . . . . . .

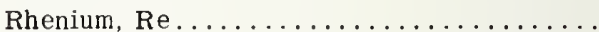

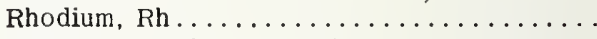

Rhodium vanadium $1: 3, \mathrm{RhV}_{3} \ldots \ldots \ldots \ldots \ldots$

Rubidium aluminum sulfate dodecahydrate,

$\mathrm{RbAl}\left(\mathrm{SO}_{4}\right)_{2} \cdot 12 \mathrm{H}_{2} \mathrm{O} \ldots \ldots \ldots \ldots \ldots \ldots \ldots$ Rubidium amide, $\mathrm{RbNH}_{2} \ldots \ldots \ldots \ldots \ldots \ldots$ Rubidium bromate, $\mathrm{RbBrO}_{3} \ldots \ldots \ldots \ldots \ldots \ldots$ Rubidium bromide, $\mathrm{RbBr} \ldots \ldots \ldots \ldots \ldots \ldots$ Rubidium bromotellurate, $\mathrm{Rb}_{2} \mathrm{TeBr}_{6} \ldots \ldots \ldots$ Rubidium cadmium sulfate, $\mathrm{Rb}_{2} \mathrm{Cd}_{2}\left(\mathrm{SO}_{4}\right)_{3} \ldots$ Rubidium cadmium trichloride, high form,

$\mathrm{RbCdCl}_{3}$ (tetragonal) ............ $5 \mathrm{~m}$

Rubidium cadmium trichloride, low form,

$\mathrm{RbCdCl}_{3}$ (orthorhombic) .............

Rubidium calcium chloride, $\mathrm{RbCaC}_{3} \ldots \ldots \ldots$

Rubidium calcium sulfate, $\mathrm{Rb}_{2} \mathrm{Ca}_{2}\left(\mathrm{SO}_{4}\right)_{3} \ldots \ldots$

Rubidium chlorate, $\mathrm{RbClO}_{3} \ldots \ldots \ldots \ldots \ldots$

Rubidium chloride, $\mathrm{RbCl} \ldots \ldots \ldots \ldots \ldots \ldots$

Rubidium chloroplatinate, $\mathrm{Rb}_{2} \mathrm{PtCl}_{6} \ldots \ldots \ldots$

Rubidium chlorostannate, $\mathrm{Rb}_{2} \mathrm{SnCl}_{6} \ldots \ldots \ldots$

Rubidium chlorotellurate, $\mathrm{Rb}_{2} \mathrm{TeCl}_{6} \ldots \ldots \ldots$

Rubidium chromate, $\mathrm{Rb}_{2} \mathrm{CrO}_{4} \ldots \ldots \ldots \ldots \ldots$

Rubidium chromium sulfate dodecahydrate,

$\mathrm{RbCr}\left(\mathrm{SO}_{4}\right)_{2} \cdot 12 \mathrm{H}_{2} \mathrm{O} \ldots \ldots \ldots \ldots \ldots \ldots$ Rubidium cobalt (II) trichloride, $\mathrm{RbCoC}_{3} \ldots$. Rubidium fluoplatinate, $\mathrm{Rb}_{2} \mathrm{PtF}_{6} \ldots \ldots \ldots \ldots$ Rubidum fluosilicate, $\mathrm{Rb}_{2} \mathrm{SiF}_{6} \ldots \ldots \ldots \ldots$ Rubidium iodide, $\mathrm{RbI} \ldots \ldots \ldots \ldots \ldots \ldots \ldots$ Rubidium magnesium sulfate, $\mathrm{Rb}_{2} \mathrm{Mg}_{2}\left(\mathrm{SO}_{4}\right)_{3} \ldots$ Rubidium manganese sulfate, $\mathrm{Rb}_{2} \mathrm{Mn}_{2}\left(\mathrm{SO}_{4}\right)_{3} \ldots$ Rubidium manganese(II) trifluoride, $\mathrm{RbMnF}_{3}$.. Rubidium nickel (II) trichloride, $\mathrm{RbNiCl}_{3} \ldots$. Rubidium nitrate, $\mathrm{RbNO}_{3}$ (trigonal) ......... Rubidium perchlorate, $\mathrm{RbClO}_{4} \ldots \ldots \ldots \ldots \ldots$ Rubidium periodate, $\mathrm{RbIO}_{4} \ldots \ldots \ldots \ldots \ldots$ Rubidium strontium chloride, $\mathrm{RbSrC1}_{3} \ldots \ldots \ldots$ Rubidium sulfate, $\mathrm{Rb}_{2} \mathrm{SO}_{4} \ldots \ldots \ldots \ldots \ldots$ Rubidium zinc sulfate hexahydrate,

$\mathrm{Rb}_{2} \mathrm{Zn}\left(\mathrm{SO}_{4}\right)_{2} \cdot 6 \mathrm{H}_{2} \mathrm{O} \ldots \ldots \ldots \ldots \ldots \ldots$

Rubidium zinc fluoride, $\mathrm{RbZnF}_{3} \ldots \ldots \ldots \ldots$

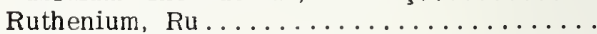
Ruthenium titanium, RuTi ........... Samarium arsenate, $\mathrm{SmAsO}_{4} \ldots \ldots \ldots \ldots \ldots \ldots$

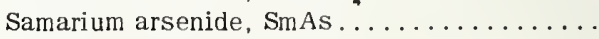
Samarium chloride, $\mathrm{SmCl}_{3} \ldots \ldots \ldots \ldots \ldots$ Samarium fluoride, $\mathrm{SmF}_{3} \ldots \ldots \ldots \ldots \ldots$ Samarium gallium oxide, $\mathrm{Sm}_{3} \mathrm{Ga}_{2}\left(\mathrm{GaO}_{4}\right)_{3} \ldots \ldots$ Samarium oxide, $\mathrm{Sm}_{2} \mathrm{O}_{3}$ (cubic) $\ldots \ldots \ldots \ldots$ Samarium oxychloride, $\mathrm{SmOCl} \ldots \ldots \ldots \ldots \ldots$ Samarium vanadate, $\mathrm{SmVO}_{4} \ldots \ldots \ldots \ldots \ldots$ Scandium arsenate, $\mathrm{ScAsO}_{4} \ldots \ldots \ldots \ldots \ldots$

$7 \mathrm{~m}$

$6 \mathrm{~m}$

$4 \mathrm{~m}$

$4 \mathrm{~m}$

$4 \mathrm{~m}$

$1 \mathrm{~m}$

5

9

$5 \mathrm{~m}$

$5 \mathrm{~m}$

2

3

$6 \mathrm{~m}$

$6 \quad 44$

$5 \mathrm{~m}$

8

8

$7 \mathrm{~m}$

$5 \mathrm{~m}$

$5 \mathrm{~m}$

$7 \mathrm{~m}$

$7 \mathrm{~m}$

8

4

5

6

8

$3 \mathrm{~m}$ 


\section{CUMULATIVE INDEX-Continued}

\begin{tabular}{|c|c|c|c|c|}
\hline $\begin{array}{l}\text { Yol. or } \\
\text { sec. }\end{array}$ & Page & & $\begin{array}{l}\text { Vol. or } \\
\text { sec. }\end{array}$ & Page \\
\hline $4 \mathrm{~m}$ & 68 & Sodium chloride (halite), $\mathrm{NaCl}$ & 2 & 41 \\
\hline 3 & 27 & Sodium cobalt (II) sulfate tetrahydrate, & & \\
\hline 8 & 50 & $\mathrm{Na}_{2} \mathrm{Co}\left(\mathrm{SO}_{4}\right)_{2} \cdot 4 \mathrm{H}_{2} \mathrm{O} \ldots \ldots \ldots \ldots$ & $6 \mathrm{~m}$ & 61 \\
\hline $7 \mathrm{~m}$ & 58 & 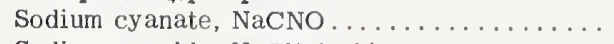 & $2 \mathrm{~m}$ & 33 \\
\hline $7 \mathrm{~m}$ & 60 & So dium cyanide, NaCN (cubic) ........... & 1 & 78 \\
\hline 5 & 54 & Sodium cyanide, NaCN (orthorhombic) at $6^{\circ} \mathrm{C}$ & 1 & . \\
\hline 2 & 6 & Sodium dichromate dihydrate, $\mathrm{Na}_{2} \mathrm{Cr}_{2} \mathrm{O}_{7} \cdot 2 \mathrm{H}_{2} \mathrm{O}$ & $7 \mathrm{~m}$ & 62 \\
\hline 3 & 24 & $\begin{array}{l}\text { Sodium fluoride (villiaumite), NaF .......... } \\
\text { Sodium hexametaphosphate hexahydrate, }\end{array}$ & 1 & \\
\hline 10 & 48 & $\begin{array}{l}\mathrm{Na}_{6} \mathrm{P}_{6} \mathrm{O}_{18} \cdot 6 \mathrm{H}_{2} \mathrm{O} \ldots \ldots \ldots \ldots \\
\text { Sodium hydrogen silicate tetrahydrate, }\end{array}$ & $5 \mathrm{~m}$ & \\
\hline 1 & 42 & $\begin{array}{l}\mathrm{N} \mathrm{a}_{2} \mathrm{H}_{2} \mathrm{SiO}_{4} \cdot 4 \mathrm{H}_{2} \mathrm{O} \ldots \ldots \ldots \ldots \\
\text { Sodium hydroxide, } \\
\mathrm{NaOH}\end{array}$ & $7 \mathrm{~m}$ & 163 \\
\hline 1 & 23 & $\begin{array}{l}\text { Sodium hydroxide, } \mathrm{NaOH} \text { at } 300^{\circ} \mathrm{C} \ldots \ldots \ldots \\
\text { Sodium iodate, } \mathrm{NaIO}_{3} \ldots \ldots \ldots \ldots \ldots\end{array}$ & $4 \mathrm{~m}$ & $6 !$ \\
\hline $4 \mathrm{~m}$ & 4 & $\begin{array}{l}\text { Sodium iodate, } \mathrm{NaIO}_{3} \ldots \ldots \ldots \ldots \ldots \ldots \ldots \\
\text { Sodium iodide, } \mathrm{NaI} \ldots \ldots \ldots \ldots \ldots\end{array}$ & 4 & 4 \\
\hline $5 m$ & 48 & $\begin{array}{l}\text { Sodium iodide, NaI .................. } \\
\text { Sodium lanthanum fluosilicate, }\end{array}$ & 4 & \\
\hline $5 \mathrm{~m}$ & 49 & 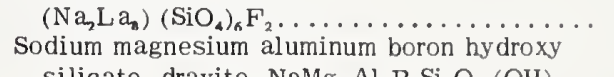 & $7 \mathrm{~m}$ & 6 \\
\hline $5 m$ & 51 & $\begin{array}{l}\text { silicate, dravite, } \mathrm{NaMg}_{3} \mathrm{Al}_{6} \mathrm{~B}_{3} \mathrm{Si}_{6} \mathrm{O}_{27}(\mathrm{OH})_{4} \\
\text { So dium magnesium sulfate tetrahy drate, }\end{array}$ & $3 \mathrm{~m}$ & 47 \\
\hline $3 \mathrm{~m}$ & 47 & bloedite, $\mathrm{Na}_{2} \mathrm{Mg}\left(\mathrm{SO}_{4}\right)_{2} \cdot 4 \mathrm{H}_{2} \mathrm{O} \ldots \ldots \ldots \ldots$ & $6 m$ & $6:$ \\
\hline $\begin{array}{l}5 \\
5\end{array}$ & $\begin{array}{l}56 \\
57\end{array}$ & Sodium manganese (II) trifluoride, $\mathrm{NaMnF}_{3} \ldots$ & $6 \mathrm{~m}$ & 65 \\
\hline $\begin{array}{l}5 \\
4\end{array}$ & $\begin{array}{l}57 \\
46\end{array}$ & Sodium mercury (II) trichloride dihydrate, & & \\
\hline $\begin{array}{r}4 \\
1 \mathrm{~m}\end{array}$ & $\begin{array}{l}40 \\
44\end{array}$ & $\mathrm{NaHgCl}{ }_{3} \cdot 2 \mathrm{H}_{2} \mathrm{O} \ldots \ldots \ldots \ldots \ldots \ldots \ldots$ & $6 \mathrm{~m}$ & 66 \\
\hline 7 & 44 & Sodium molybdate, $\mathrm{Na}_{2} \mathrm{MoO}_{4} \ldots \ldots \ldots \ldots \ldots$ & $1 \mathrm{~m}$ & 4 \\
\hline 4 & 44 & Sodium neodymium fluosilicate, & & \\
\hline $5 \mathrm{~m}$ & 66 & $\left(\mathrm{Na}_{2} \mathrm{Nd}_{8}\right)\left(\mathrm{SiO}_{4}\right)_{5} \mathrm{~F}_{2} \ldots \ldots \ldots \ldots \ldots$ & $7 \mathrm{~m}$ & 66 \\
\hline $5 \mathrm{~m}$ & 67 & $\begin{array}{l}\text { Sodium nickel (II) sulfate tetrahydrate, } \\
\text { Na } \mathrm{Ni} \text { (SO } .4 \mathrm{H} O\end{array}$ & & \\
\hline $6 \mathrm{~m}$ & 87 & $\underset{\text { Sodium nitrate }}{\mathrm{Na}_{2} \mathrm{Ni}\left(\mathrm{SO}_{4}\right)_{2} \cdot 4 \mathrm{H}_{2} \mathrm{O} \ldots \ldots \ldots \ldots \ldots \ldots \ldots}$ & $6 \mathrm{~m}$ & 68 \\
\hline $5 \mathrm{~m}$ & 68 & Sodium nitrate (soda-niter), $\mathrm{NaNO}_{3} \ldots \ldots \ldots$ & 6 & 50 \\
\hline 8 & 51 & $\begin{array}{l}\text { Sodium nitrite, } \mathrm{NaNO}_{2} \ldots \ldots \ldots \ldots \ldots \ldots \ldots \\
\text { Sodium orthotungstate(IV) dihydrate, }\end{array}$ & 4 & c \\
\hline 9 & 48 & $\begin{array}{l}\text { Sodium orthotungstate(IV) dihydrate, } \\
\qquad \mathrm{Na}_{2} \mathrm{WO}_{4} \cdot 2 \mathrm{H}_{2} \mathrm{O} \ldots \ldots \ldots \ldots \ldots \ldots\end{array}$ & $2 m$ & \\
\hline 7 & 45 & Sodium oxalate, $\mathrm{Na}_{2} \mathrm{C}_{2} \mathrm{O}_{4} \ldots \ldots \ldots \ldots \ldots \ldots$ & $6 m$ & \\
\hline $5 \mathrm{~m}$ & 71 & Sodium perchlorate, $\mathrm{NaClO}_{4}$ (orthorhombic)... & 7 & \\
\hline 5 & 59 & Sodium periodate, $\mathrm{NaIO}_{4} \ldots \ldots \ldots \ldots \ldots$ & 7 & \\
\hline 5 & 60 & Sodium praseodymium fluosilicate, & 7 & \\
\hline $1 \mathrm{~m}$ & 45 & $\left(\mathrm{Na}_{2} \mathrm{Pr}_{8}\right)\left(\mathrm{SiO}_{4}\right)_{6} \mathrm{~F}_{2} \ldots \ldots \ldots \ldots \ldots$ & $7 \mathrm{~m}$ & \\
\hline 4 & 61 & Sodium sulfate (thenardite), $\mathrm{Na}_{2} \mathrm{SO}_{4} \ldots \ldots \ldots$ & 2 & \\
\hline 9 & 49 & Sodium sulfite, $\mathrm{Na}_{2} \mathrm{SO}_{3} \ldots \ldots \ldots \ldots \ldots$ & 3 & 60 \\
\hline $7 \mathrm{~m}$ & 155 & Sodium tetrametaphosphate tetrahydrate, & & \\
\hline $\begin{array}{l}8 \\
5\end{array}$ & $\begin{array}{l}53 \\
62\end{array}$ & alpha, $\mathrm{Na}_{4} \mathrm{P}_{4} \mathrm{O}_{12} \cdot 4 \mathrm{H}_{2} \mathrm{O}$ (monoclinic) $\ldots \ldots \ldots$ & 10 & 52 \\
\hline $\begin{array}{r}5 \\
5 \mathrm{~m}\end{array}$ & $\begin{array}{l}62 \\
73\end{array}$ & Sodium tetrametaphosphate tetrahydrate, beta, & & \\
\hline $2 \mathrm{~m}$ & 32 & $\mathrm{Na}_{4} \mathrm{P}_{4} \mathrm{O}_{12} \cdot 4 \mathrm{H}_{2} \mathrm{O}$ (triclinic) $\ldots$ & $2 \mathrm{~m}$ & 35 \\
\hline $5 \mathrm{~m}$ & 53 & Sodium tin fluoride, $\mathrm{NaSn}_{2} \mathrm{~F}_{5} \ldots \ldots$ & $7 \mathrm{~m}$ & 166 \\
\hline 7 & 46 & Sodium trimetaphosphate, $\mathrm{Na}_{3} \mathrm{P}_{3} \mathrm{O}_{9} \ldots \ldots$ & $3 m$ & 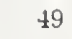 \\
\hline 10 & 51 & Sodium trimetaphosphate monohydrate, & & \\
\hline $5 \mathrm{~m}$ & 74 & $\mathrm{Na}_{3} \mathrm{P}_{3} \mathrm{O}_{9} \cdot \mathrm{H}_{2} \mathrm{O} \ldots \ldots \ldots \ldots \ldots \ldots$ & $3 \mathrm{~m}$ & 50 \\
\hline $5 \mathrm{~m}$ & 74 & Sodium tungstate, $\mathrm{Na}_{2} \mathrm{HO}_{4} \ldots \ldots$ & $1 \mathrm{~m}$ & \\
\hline $5 \mathrm{~m}$ & 75 & So dium zinc sulfate tetrahydrate, & & \\
\hline 5 & 63 & $\mathrm{Na}_{2} \mathrm{Zn}\left(\mathrm{SO}_{4}\right)_{2} \cdot 4 \mathrm{H}_{2} \mathrm{O} \ldots \ldots \ldots \ldots \ldots \ldots$ & $6 \mathrm{~m}$ & \\
\hline & & Sodium zinc trifluoride, $\mathrm{NaZnF}_{3} \ldots \ldots \ldots \ldots$ & $6 \mathrm{~m}$ & \\
\hline $7 \mathrm{~m}$ & 158 & Strontium arsenate, $\mathrm{Sr}_{3}\left(\mathrm{AsO}_{4}\right)_{2} \ldots \ldots$ & $2 \mathrm{~m}$ & \\
\hline $7 \mathrm{~m}$ & 160 & kide, $\mathrm{SrB}_{2} \mathrm{O}_{4}$. & $3 \mathrm{~m}$ & \\
\hline 9 & 51 & de, $\mathrm{SrB}_{4} \mathrm{O}_{7}$ & $4 \mathrm{~m}$ & \\
\hline 5 & 65 & n bromide hexahydrate, $\mathrm{SrBr}_{2} \cdot 6 \mathrm{H}_{2} \mathrm{O} \ldots$ & 4 & \\
\hline 3 & 47 & Strontium carbonate (strontianite), $\mathrm{SrCO}_{3} \ldots$ & 3 & \\
\hline & & Strontium chloride, $\mathrm{SrCl}_{2} \ldots \ldots \ldots \ldots \ldots$ & 4 & 40 \\
\hline $6 \mathrm{~m}$ & 59 & Strontium chloride hexahydrate, $\mathrm{SrCl}_{2} \cdot 6 \mathrm{H}_{2} \mathrm{O} \ldots$ & 4 & \\
\hline & & Strontium fluoride, $\mathrm{SrF}_{2} \ldots \ldots \ldots \ldots \ldots$ & 5 & \\
\hline $\begin{array}{l}8 \\
3\end{array}$ & 54 & Strontium formate, $\mathrm{Sr}\left(\mathrm{CHO}_{2}\right)_{2} \ldots \ldots \ldots \ldots \ldots$ & 8 & \\
\hline 3 & 51 & $\begin{array}{l}\text { Strontium formate dihy drate, } \mathrm{Sr}\left(\mathrm{CHO}_{2}\right)_{2} \cdot 2 \mathrm{H}_{2} \mathrm{O} \\
\quad \text { (orthorhombic) } \ldots \ldots \ldots \ldots \ldots \ldots \ldots \ldots \ldots \ldots \ldots\end{array}$ & 8 & 56 \\
\hline & & Strontium indium hydroxide, $\mathrm{Sr}_{3} \mathrm{In}_{2}(\mathrm{OH})_{12} \ldots$. & $6 \mathrm{~m}$ & \\
\hline & & Strontium iodide hexahydrate, $\operatorname{SrI}_{2} \cdot 6 \mathrm{H}_{2} \mathrm{O} \ldots \ldots$ & 8 & \\
\hline & & Strontium molybdate, $\mathrm{SrMoO}_{4} \ldots \ldots \ldots$ & 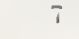 & \\
\hline
\end{tabular}

Scandium arsenide, $\mathrm{Sc} A \mathrm{As} \ldots \ldots \ldots \ldots \ldots \ldots$

Scandium oxide, $\mathrm{Sc}_{2} \mathrm{O}_{3} \ldots \ldots \ldots \ldots \ldots \ldots$

Scandium phosphate, $\mathrm{ScPO}_{4} \ldots \ldots \ldots \ldots \ldots$

Scandium silicate (thortveitite), $\mathrm{Sc}_{2} \mathrm{Si}_{2} \mathrm{O}_{7} \ldots$

Selenium oxide (selenolite), $\mathrm{SeO}_{2}$ (revised).

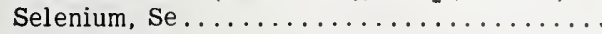

Silicon, Si

Silicon dioxide, alpha or low quartz, $\mathrm{SiO}_{2}$

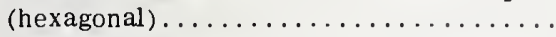

Silicon dioxide (alpha or low cristobalite),

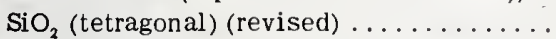

Silicon dioxide (beta or high cristobalite),

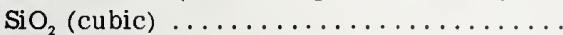

Silver, $\mathrm{Ag} \ldots \ldots \ldots \ldots \ldots \ldots \ldots \ldots \ldots \ldots$

Silver, $\mathrm{Ag}$ (reference standard)..........

Silver antimony sulfide, $\mathrm{AgSbS}_{2}$ (cubic)......

Silver antimony sulfide (miargyrite),

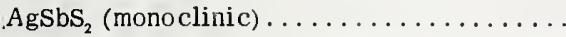

Silver antimony sulfide (pyrargyrite), $\mathrm{Ag}_{3} \mathrm{SbS}_{3}$

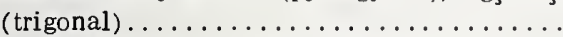

Silver antimony telluride, $\mathrm{AgSbTe}_{2} \ldots \ldots \ldots$.

Silver arsenate, $\mathrm{Ag}_{3} \mathrm{AsO}_{4} \ldots \ldots \ldots \ldots \ldots \ldots$

Silver bromate, $\mathrm{AgBrO}_{3} \ldots \ldots \ldots \ldots \ldots \ldots$

Silver bromide (bromyrite), $\mathrm{AgBr} . \ldots \ldots \ldots \ldots$

Silver carbonate, $\mathrm{Ag}_{2} \mathrm{CO}_{3} \ldots \ldots \ldots \ldots \ldots \ldots$

Silver chlorate, $\mathrm{AgClO}_{3} \ldots \ldots \ldots \ldots \ldots$.

Silver chloride, (cerargyrite), AgCl .......

Silver dysprosium, AgDy .............

Silver erbium, AgEr . . . . . . . . . . .

Silver gadolinium, AgGd. .............

Silver holmium, AgHo ...............

Silver iodide (iodyrite), AgI (hexagonal) .....

Silver iodide, gamma, AgI (cubic) ........

Silver molybdate, $\mathrm{Ag}_{2} \mathrm{MoO}_{4} \ldots \ldots \ldots \ldots \ldots \ldots$

Silver neodymium, AgNd..............

Silver nitrate, $\mathrm{AgNO}_{3} \ldots \ldots \ldots \ldots \ldots \ldots \ldots$

Silver nitrite, $\mathrm{AgNO}_{2} \ldots \ldots \ldots \ldots \ldots \ldots \ldots \ldots$

Silver oxide, $\mathrm{Ag}_{2} \mathrm{O} \ldots \ldots \ldots \ldots \ldots \ldots \ldots \ldots$

Silver(II) oxynitrate, $\mathrm{Ag}_{7} \mathrm{O}_{8} \mathrm{NO}_{3} \ldots \ldots \ldots \ldots$

Silver periodate, $\mathrm{AgIO}_{4} \ldots \ldots \ldots \ldots \ldots \ldots$

Silver permanganate, $\mathrm{AgMnO}_{4} \ldots \ldots \ldots \ldots \ldots$

Silver perrhenate, $\mathrm{AgReO}_{4} \ldots \ldots \ldots \ldots \ldots \ldots$

Silver phosphate, $\mathrm{Ag}_{3} \mathrm{PO}_{4} \ldots \ldots \ldots \ldots \ldots \ldots$

Silver samarium, AgSm ..............

Silver selenate, $\mathrm{Ag}_{2} \mathrm{SeO}_{4} \ldots \ldots \ldots \ldots \ldots \ldots$

Silver subfluoride, $\mathrm{Ag}_{2} \mathrm{~F} \ldots \ldots \ldots \ldots \ldots \ldots$

Silver sulfate, $\mathrm{Ag}_{2} \mathrm{SO}_{4} \ldots \ldots \ldots \ldots \ldots \ldots \ldots$

Silver sulfide (argentite), $\mathrm{Ag}_{2} \mathrm{~S} \ldots \ldots \ldots \ldots \ldots$

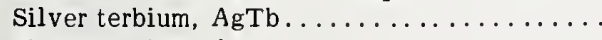

Silver thulium, AgTm . . . . . . . . . . .

Silver yttrium, AgY . . . . . . . . . . .

Sodium acid fluoride, $\mathrm{NaHF}_{2} \ldots \ldots \ldots \ldots \ldots$

Sodium aluminum chloride silicate, sodalite,

$\mathrm{Na}_{8} \mathrm{Si}_{6} \mathrm{Al}_{6} \mathrm{O}_{24} \mathrm{Cl}_{2} \ldots \ldots \ldots \ldots \ldots \ldots \ldots \ldots$

Sodium borate, $\mathrm{Na}_{2} \mathrm{~B}_{8} \mathrm{O}_{13} \ldots \ldots \ldots \ldots \ldots$

Sodium borohydride, $\mathrm{NaBH}_{4} \ldots \ldots \ldots \ldots \ldots \ldots$

Sodium bromate, $\mathrm{NaBrO}_{3} \ldots \ldots \ldots \ldots \ldots \ldots \ldots$

So dium bromide, $\mathrm{NaBr} . . . \ldots \ldots \ldots \ldots \ldots$

Sodium calcium sulfate (glauberite),

$\mathrm{Na}_{2} \mathrm{Ca}\left(\mathrm{SO}_{4}\right)_{2} \ldots \ldots \ldots \ldots \ldots \ldots \ldots \ldots$

So dium carbonate monohydrate (thermonatrite),

$\mathrm{Na}_{2} \mathrm{CO}_{3} \cdot \mathrm{H}_{2} \mathrm{O} \ldots \ldots \ldots \ldots \ldots \ldots \ldots \ldots$

Sodium chlorate, $\mathrm{NaClO}_{3} \ldots \ldots \ldots \ldots \ldots \ldots$

m-Monograph 25.

A mineral name in () indicates a synthetic sample. 


\section{CUMULATIVE INDEX-Continued}

\begin{tabular}{|c|c|c|c|c|c|}
\hline & $\begin{array}{l}\text { Vol. or } \\
\text { sec. }\end{array}$ & Page & & $\begin{array}{c}\text { Vol. or } \\
\text { sec. }\end{array}$ & Page \\
\hline Strontium nitrate, $\mathrm{Sr}\left(\mathrm{NO}_{3}\right)_{2}$ & 1 & 80 & Thallium(I) thiocyanate, TlCNS & 8 & 63 \\
\hline Strontium oxide, Sro ..... & 5 & 68 & Thallium(I) tungstate, $\mathrm{Tl}_{2} \mathrm{WO}_{4}$. & $1 \mathrm{~m}$ & 48 \\
\hline Strontium peroxide, $\mathrm{SrO}_{2}$. & 6 & 52 & Thallium zinc sulfate hexahydrate, & & \\
\hline Strontium scandium oxide hexahydrate, & & & $\mathrm{T} 1_{2} \mathrm{Zn}\left(\mathrm{SO}_{4}\right)_{2} \cdot 6 \mathrm{H}_{2} \mathrm{O} \ldots \ldots \ldots \ldots \ldots$ & $7 \mathrm{~m}$ & 80 \\
\hline $\mathrm{Sr}_{3} \mathrm{Sc}_{2} \mathrm{O}_{6} \cdot 6 \mathrm{H}_{2} \mathrm{O} \ldots \ldots \ldots \ldots \ldots \ldots$ & $6 \mathrm{~m}$ & 78 & Thorium arsenide, ThAs.. & $4 \mathrm{~m}$ & 70 \\
\hline Strontium sulfate (celestite), $\mathrm{SrSO}_{4}$ & 2 & 61 & Thorium oxide (thorianite), $\mathrm{ThO}_{2}$ & 1 & 57 \\
\hline Strontium sulfide, SrS . & 7 & 52 & Thulium arsenate, $\mathrm{TmAsO}_{4}, \ldots$. & $3 \mathrm{~m}$ & 56 \\
\hline Strontium telluride, $\mathrm{SrTe}$ & $4 \mathrm{~m}$ & 69 & Thulium arsenide, $\operatorname{TmAs} . .$. & $4 \mathrm{~m}$ & 71 \\
\hline Strontium titanate, $\mathrm{SrTiO}_{3}$ & 3 & 44 & Thulium nitride, TmN . & $4 \mathrm{~m}$ & 71 \\
\hline Strontium tungstate, $\mathrm{SrWO}_{4}$ & 7 & 53 & Thulium sesquioxide, $\mathrm{Tm}_{2} \mathrm{O}_{3}$ & 9 & 58 \\
\hline Strontium zirconate, $\mathrm{SrZrO}_{3}$ & 9 & 51 & Thulium telluride, TmTe & $4 \mathrm{~m}$ & 72 \\
\hline Sulfamic acid, $\mathrm{NH}_{3} \mathrm{SO}_{3}$. & 7 & 54 & Thulium vanadate, $\mathrm{TmVO}_{4}$ & $5 \mathrm{~m}$ & 57 \\
\hline Sulfur, S (orthorhombic) & 9 & 54 & Tin, alpha, Sn (cubic). . & 2 & 12 \\
\hline Tantalum, Ta $\ldots . . . .$. & 1 & 29 & Tin, beta, Sn (tetragonal) . . . . . . & 1 & 24 \\
\hline Tantal um Silicide, TaSi & 8 & 59 & Tin arsenide, SnAs ...... & $4 m$ & 37 \\
\hline d-Tartaric Acid, $\mathrm{C}_{4} \mathrm{H}_{6} \mathrm{O}_{6}$. & $7 \mathrm{~m}$ & 168 & $\operatorname{Tin}(\amalg)$ fluoride, $\mathrm{SnF}_{2}$ & $3 \mathrm{~m}$ & 51 \\
\hline Tellurium, $\mathrm{Te} . . . . . .$. & 1 & 26 & Tin(IV) iodide, $\mathrm{SnI}_{4}$. & 5 & 71 \\
\hline Tellurium(IV) oxide (paratellurite), $\mathrm{TeO}_{2}$ & & & Tin(U) oxide, $\mathrm{SnO} \ldots$ & 4 & 28 \\
\hline (tetrage & 7 & 56 & Tin(IV) oxide (cassiterite), $\mathrm{SnO}_{2}$ & 1 & 54 \\
\hline oxide, paratellurit & & & Tin(II) telluride, $\operatorname{SnTe} \ldots \ldots \ldots \ldots$ & 7 & 61 \\
\hline (tetrag & 10 & 55 & Titanium, Ti............. & 3 & 1 \\
\hline $\begin{array}{l}\text { Tellurium(IV) oxide, tellurite, } \mathrm{TeO}_{2} \text { (ortho- } \\
\text { rhombic) } \ldots \ldots \ldots \ldots \ldots \ldots \ldots \ldots \ldots \ldots\end{array}$ & 9 & 57 & $\begin{array}{l}\text { Titanium dioxide, brookite, } \mathrm{TiO}_{2} \text { (ortho- } \\
\text { rhombic) } \ldots \ldots \ldots \ldots \ldots \ldots \ldots \ldots \ldots \ldots\end{array}$ & $3 \mathrm{~m}$ & 57 \\
\hline Terbium arsenate, $\mathrm{TbAsO}_{4} \ldots \ldots \ldots \ldots \ldots$ & $3 \mathrm{~m}$ & 54 & oxide (anatase), $\mathrm{TiO}_{2}$ (revised) .... & $7 \mathrm{~m}$ & 82 \\
\hline TbAs................... & $5 \mathrm{~m}$ & 75 & Titanium oxide (rutile), $\mathrm{TiO}_{2}$ (revi sed) & $7 \mathrm{~m}$ & 83 \\
\hline Terbium & $4 \mathrm{~m}$ & 70 & xide, $\mathrm{TiO}_{1.515} \ldots \ldots$ & 9 & 59 \\
\hline Terbium $\mathrm{D}$ & $5 \mathrm{~m}$ & 76 & Titanium silicide, $\mathrm{Ti}_{3} \mathrm{Si}_{3}$ & 8 & 64 \\
\hline Terbium selenide, TbSe. & $5 \mathrm{~m}$ & 76 & Titanium sulfide, $\mathrm{TiS}_{2}$ & $4 \mathrm{~m}$ & 72 \\
\hline Terbium $\mathrm{s}$ & $5 \mathrm{~m}$ & 77 & Tung & 1 & 28 \\
\hline luride, тьте.... & $5 \mathrm{~m}$ & 77 & andard). . & $4 \mathrm{~m}$ & 4 \\
\hline $\mathrm{TbVO}_{4}, \ldots \ldots \ldots \ldots \ldots$ & $5 \mathrm{~m}$ & 56 & e (tungstenite), $\mathrm{WS}_{2}$ & 8 & 65 \\
\hline Thallium aluminum sulfate dodecahydrate, & & & dioxide (uraninite), $\mathrm{UO}_{2}$ & & 33 \\
\hline $\mathrm{TlAl}\left(\mathrm{SO}_{4}\right)_{2} \cdot 1$ & 6 & 53 & Urani & $5 \mathrm{~m}$ & 78 \\
\hline $\mathrm{Tl}_{3} \mathrm{AsO}_{4}$ & $2 \mathrm{~m}$ & 37 & Us & $5 \mathrm{~m}$ & 78 \\
\hline $\mathrm{TlBrO}_{3}$ & 8 & 60 & de, UTe. & $4 \mathrm{~m}$ & 73 \\
\hline $\mathrm{IlBr} . .$. & 7 & 57 & ( & 7 & 61 \\
\hline $\mathrm{TlClO}_{3}$ & 8 & 61 & Vanadium(V) oxide, $\mathrm{V}_{2} \mathrm{O}$ & 8 & 66 \\
\hline Thal & 4 & 51 & te, $\mathrm{YbAsO}_{4}$ & $4 \mathrm{~m}$ & 38 \\
\hline nate, $\mathrm{Tl}_{2} \mathrm{PtCl}_{6} \ldots \ldots \ldots$ & 5 & 70 & e, YbAs & \pm in & 73 \\
\hline $\mathrm{Tl} \mathrm{SnCl}$ & 6 & 54 & oxide, $\mathrm{Yb}_{3} \mathrm{Ga}_{2}\left(\mathrm{GaO}_{4}\right)$ & $1 \mathrm{~m}$ & 49 \\
\hline Thallium chromate, $\mathrm{Tl}_{2} \mathrm{CrO}_{4} \ldots \ldots \ldots \ldots$ & $3 \mathrm{~m}$ & 54 & Ytte & $4 \mathrm{~m}$ & 74 \\
\hline Thallium chromium sulfate dodecahydrate, & & & ytto & $6 \mathrm{~m}$ & 80 \\
\hline $\operatorname{TlCr}\left(\mathrm{SO}_{4}\right)_{2}$ & 6 & 55 & Ytterbium selenide, YbSe.. & $5 \mathrm{~m}$ & 79 \\
\hline Thallium cobalt sulfate hexahydrate, & & & le, YbTe. & $5 \mathrm{~m}$ & 79 \\
\hline $\mathrm{T}_{2} \mathrm{CO}\left(\mathrm{SO}_{4}\right)_{2} \cdot 6$ & $7 \mathrm{~m}$ & 70 & nadate, $\mathrm{YbVO}_{4}$. & $5 \mathrm{~m}$ & 58 \\
\hline fate hexahydrate, & & & $\mathrm{YAsO}_{4} \ldots \ldots$ & & 39 \\
\hline $\mathrm{T}_{2} \mathrm{C}$ & $7 \mathrm{~m}$ & 72 & YAs & $4 \mathrm{~m}$ & 74 \\
\hline Thallium $\mathrm{f}$ & 6 & 56 & um oxide, $\mathrm{Y}_{3} \mathrm{Ga}_{2}\left(\mathrm{GaO}_{4}\right)_{3}$ & $1 \mathrm{~m}$ & 50 \\
\hline ulfate dodecahydrate, & & & & & 28 \\
\hline TlGa & 6 & 57 & & $1 \mathrm{~m}$ & 51 \\
\hline Thalliu & 8 & 62 & te (xenotime), YPO, & 8 & 67 \\
\hline Thallium(I) iodide, TlI (orthorhombic) ........ & 4 & 53 & $\mathbf{Y t}$ & $5 \mathrm{~m}$ & 80 \\
\hline agnesium sulfate hexahydrate, & & & & $4 \mathrm{~m}$ & 75 \\
\hline $\mathrm{T}_{2}$ & $7 \mathrm{~m}$ & 74 & $\mathrm{YVO}_{4}$ & $5 \mathrm{~m}$ & 59 \\
\hline$\left(\mathrm{SO}_{4}\right)_{3} \ldots$ & $7 \mathrm{~m}$ & 76 & $\mathrm{Z}_{\mathrm{a}}$ & 1 & 16 \\
\hline fate hexahydrate, & & & Zinc alum & 2 & 38 \\
\hline & $7 \mathrm{~m}$ & 78 & $\mathrm{ZnSb}_{2} \mathrm{O}_{4}$ & $4 \mathrm{~m}$ & 39 \\
\hline Thal & 6 & 58 & $\mathrm{Zi}$ & 1 & 83 \\
\hline Thallium(III) & 2 & 28 & Zinc carbonate, smithsonite, $\mathrm{ZnCO}_{3}$ & 8 & 69 \\
\hline Thalliun & $2 \mathrm{~m}$ & 38 & Zinc cyanide, $\mathrm{Zn}(\mathrm{CN})_{2}$ & 5 & 73 \\
\hline Thallium(I) $\mathrm{pl}$ & 7 & 58 & Zine fl & 6 & 60 \\
\hline & 7 & 59 & licate hexahydrate, $\mathrm{ZnSiF}_{6} \cdot 6 \mathrm{H}_{2} \mathrm{O}$ & 8 & 70 \\
\hline \multirow[t]{2}{*}{ Thallium(I) sulfate, $\mathrm{Tl}_{2} \mathrm{SO}_{4} \ldots \ldots \ldots \ldots \ldots \ldots$} & 6 & 59 & Zinc germanate, $\mathrm{Zn}_{2} \mathrm{GeO}_{4}$. & 10 & 56 \\
\hline & & & $\begin{array}{l}\mathrm{Zinc} \text { glutamate dihydrate, } \mathrm{ZnC}_{3} \mathrm{H}_{3} \mathrm{NO}_{4} \cdot 2 \mathrm{H}_{2} \mathrm{O} \\
\mathrm{Zinc} \text { iodide, } \mathrm{ZnI} \mathrm{n}_{2} \ldots \ldots \ldots \ldots \ldots \ldots \ldots \ldots \ldots\end{array}$ & $\begin{array}{r}7 \mathrm{~m} \\
9\end{array}$ & $\begin{array}{r}170 \\
60\end{array}$ \\
\hline & & & & $7 \mathrm{~m}$ & 173 \\
\hline in ( $)$ indicates a & & & Zin & 7 & 2 \\
\hline
\end{tabular}




\section{CUMULATIVE INDEX-Continued}

Vol. or

Zinc oxide (zincite), ZnO $\ldots \ldots \ldots \ldots \ldots \ldots r .25$

Zinc pyrosilicate hydrate, hemimorphite,

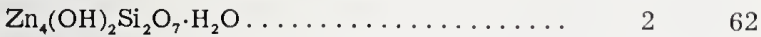

Zinc selenide, ZnSe...................... 3

Zinc sulfate (zinkosite), $\mathrm{ZnSO}_{4} \ldots \ldots \ldots \ldots .74$

Zinc sulfate heptahydrate (goslarite),

$\mathrm{ZnSO}_{4} \cdot 7 \mathrm{H}_{2} \mathrm{O} \ldots \ldots \ldots \ldots \ldots \ldots \ldots \ldots \ldots \ldots \ldots \ldots \ldots$

Zinc sulfide (wurtzite), alpha $\mathrm{ZnS}$ (hexag-

onal)

m-Monograph 25.

A mineral name in ( ) indicates a synthetic sample.

5

Zinc sulfide (sphalerite), beta ZnS (cubic). .

Zinc telluride, ZnTe.............. $3 \mathrm{~m}$

Zinc tungstate (sanmartinite), $\mathrm{ZnWO}_{4} \ldots \ldots .2 \mathrm{~m}$

Zirconium, alpha, Zr............... 2

Zirconium dihydride, $\mathrm{ZrH}_{2}$

Zirconium iodate, $\mathrm{Zr}\left(\mathrm{IO}_{3}\right)_{4}$

Zirconium nitride, ZrN

Zirconium oxide, ZrO .

Zirconium phosphide, ZrP

Zirconium silicate, zircon, $\mathrm{ZrSiO}_{4}$

Zirconium sulfate tetrahydrate, $\mathrm{Zr}\left(\mathrm{SO}_{4}\right)_{2} \cdot 4 \mathrm{H}_{2} \mathrm{O}$
Page

16

58

40

11

60

51

80

81

75

68

66

\section{CUMULATIVE MINERAL INDEX}

Alabandite, MnS.

Alum, $\mathrm{KAl}\left(\mathrm{SO}_{4}\right)_{2} \cdot 12 \mathrm{H}_{2} \mathrm{O} \ldots \ldots \ldots \ldots \ldots \ldots \ldots$

Ammonia-niter, $\mathrm{NH}_{4} \mathrm{NO}_{3} \ldots \ldots \ldots \ldots \ldots \ldots$

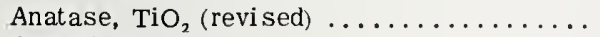

Andradite, $\mathrm{Ca}_{3} \mathrm{Fe}_{2} \mathrm{Si}_{3} \mathrm{O}_{12} \ldots \ldots \ldots \ldots \ldots \ldots$

Anglesite, $\mathrm{PbSO}_{4} \ldots \ldots \ldots \ldots \ldots \ldots \ldots \ldots$

Anhydrite, $\mathrm{CaSO}_{4} \ldots \ldots \ldots \ldots \ldots \ldots \ldots$

Aphthitalite, $\mathrm{K}_{3} \mathrm{Na}\left(\mathrm{SO}_{4}\right)_{2} \ldots \ldots \ldots \ldots \ldots \ldots$

Aragonite, $\mathrm{CaCO}_{3} \ldots \ldots \ldots \ldots \ldots \ldots \ldots$

Argentite, $\mathrm{Ag}_{2} \mathrm{~S} \ldots \ldots \ldots \ldots \ldots \ldots \ldots \ldots$

Arcanite, $\mathrm{K}_{2} \mathrm{SO}_{4} \ldots \ldots \ldots \ldots \ldots \ldots \ldots \ldots$

Arsenolite, $\mathrm{As}_{2} \mathrm{O}_{3} \ldots \ldots \ldots \ldots \ldots \ldots \ldots$

Aurostibite, $\mathrm{AuSb}_{2} \ldots \ldots \ldots \ldots \ldots \ldots \ldots$

*Azurite, $\mathrm{Cu}_{3}(\mathrm{OH})_{2}\left(\mathrm{CO}_{3}\right)_{2} \ldots \ldots \ldots \ldots \ldots \ldots$

Barite, $\mathrm{BaSO}_{4} \ldots \ldots \ldots \ldots \ldots \ldots \ldots \ldots$

Berlinite, $\mathrm{AlPO}_{4} \ldots \ldots \ldots \ldots \ldots \ldots \ldots \ldots$

${ }^{*}$ Beryl, $\mathrm{Be}_{3} \mathrm{Al}_{2}\left(\mathrm{SiO}_{3}\right)_{6} \ldots \ldots \ldots \ldots \ldots \ldots$

Bismite, (alpha) $\mathrm{Bi}_{2} \mathrm{O}_{3} \ldots \ldots \ldots \ldots \ldots \ldots$

Bismoclite, $\mathrm{BiOCl} \ldots \ldots \ldots \ldots \ldots \ldots \ldots$

Bismuthinite, $\mathrm{Bi}_{2} \mathrm{~S}_{3}$ (revised) $\ldots \ldots \ldots \ldots \ldots$

${ }^{*}$ Bloedite, $\mathrm{Na}_{2} \mathrm{Mg}\left(\mathrm{SO}_{4}\right)_{2} \cdot 4 \mathrm{H}_{2} \mathrm{O} \ldots \ldots \ldots \ldots \ldots$.

Böhmite, $\mathrm{Al}_{2} \mathrm{O}_{3} \cdot \mathrm{H}_{2} \mathrm{O} \ldots \ldots \ldots \ldots \ldots \ldots \ldots$

Bromellite, $\mathrm{BeO} \ldots \ldots \ldots \ldots \ldots \ldots \ldots \ldots$

Bromyrite, AgBr . . . . . . . . . . . . . .

${ }^{*}$ Brookite, $\mathrm{TiO}_{2} \ldots \ldots \ldots \ldots \ldots \ldots \ldots \ldots$

Brucite, $\mathrm{Mg}(\mathrm{OH})_{2} \ldots \ldots \ldots \ldots \ldots \ldots \ldots$

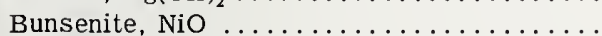

Calcite, $\mathrm{CaCO}_{3} \ldots \ldots \ldots \ldots \ldots \ldots \ldots \ldots$

Calomel, $\mathrm{Hg}_{2} \mathrm{Cl}_{2} \ldots \ldots \ldots \ldots \ldots \ldots \ldots \ldots \ldots$

Cassiterite, $\mathrm{SnO}_{2} \ldots \ldots \ldots \ldots \ldots \ldots \ldots \ldots \ldots \ldots$

Celestite, $\mathrm{SrSO}_{4} \ldots \ldots \ldots \ldots \ldots \ldots \ldots \ldots$

Cerargyrite, $\mathrm{AgCl} \ldots \ldots \ldots \ldots \ldots \ldots \ldots \ldots$

Cerianite, $\mathrm{CeO}_{2} \ldots \ldots \ldots \ldots \ldots \ldots \ldots \ldots$

Cerussite, $\mathrm{PbCO}_{3} \ldots \ldots \ldots \ldots \ldots \ldots \ldots$

Cervantite, $\mathrm{Sb}_{2} \mathrm{O}_{4} \ldots \ldots \ldots \ldots \ldots \ldots \ldots$

Chalcocyanite, $\mathrm{CuSO}_{4} \ldots \ldots \ldots \ldots \ldots \ldots \ldots$

Chloraluminite, $\mathrm{AlCl}_{3} \cdot 6 \mathrm{H}_{2} \mathrm{O} \ldots \ldots \ldots \ldots \ldots$

Chlorocalcite, $\mathrm{KCaC}_{1} \ldots \ldots \ldots \ldots \ldots \ldots \ldots$

Chrysoberyl, $\mathrm{BeAl}_{2} \mathrm{O}_{4} \ldots \ldots \ldots \ldots \ldots \ldots \ldots$

Cinnabar, $\mathrm{HgS}$

* Claudetite, $\mathrm{As}_{2} \mathrm{O}_{3}$

Clausthalite, $\mathrm{PbSe}$

$\begin{array}{rr}\text { Vol. or } & \\ \text { sec } & \text { Page } \\ 4 & 11 \\ 6 & 36 \\ 7 & 4 \\ 7 \mathrm{~m} & 82 \\ 9 & 22 \\ 3 & 67 \\ 4 & 65 \\ 6 \mathrm{~m} & 52 \\ 3 & 53 \\ 10 & 51 \\ 3 & 62 \\ 1 & 51 \\ 7 & 18 \\ 10 & 30 \\ 3 & 65 \\ 10 & 3 \\ 9 & 13 \\ 3 \mathrm{~m} & 17 \\ 4 & 54 \\ 5 \mathrm{~m} & 13 \\ 6 \mathrm{~m} & 63 \\ 3 & 38 \\ 1 & 36 \\ 4 & 46 \\ 3 \mathrm{~m} & 57 \\ 6 & 30 \\ 1 & 47 \\ 2 & 51 \\ 1 & 72 \\ 1 & 54 \\ 2 & 61 \\ 4 & 44 \\ 1 & 56 \\ 2 & 56 \\ 10 & 8 \\ 3 \mathrm{~m} & 29 \\ 7 & 3 \\ 7 \mathrm{~m} & 36 \\ 9 & 10 \\ 4 & 17 \\ 3 \mathrm{~m} & 9 \\ 5 & 38\end{array}$

* Natural mineral.

m-Monograph 25.
Cordierite, $\mathrm{Mg}_{2} \mathrm{Al}_{4} \mathrm{Si}_{5} \mathrm{O}_{18}$ (orthorhombic)

Cordierite, $\mathrm{Mg}_{2}, \mathrm{Al}_{4} \mathrm{Si}_{3} \mathrm{O}_{18}$ (hexagonal).

Corundum, $\mathrm{Al}_{2} \mathrm{O}_{3}$

Cotunnite, $\mathrm{PbCl}_{2}$

Covellite, Cus .

Cristobalite, (alpha or low) $\mathrm{SiO}_{2}$ (revised)

Cristobalite, (beta or high) $\mathrm{SiO}_{2} \ldots \ldots \ldots \ldots$

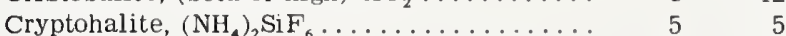

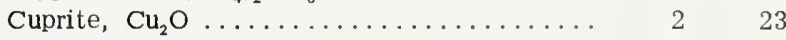

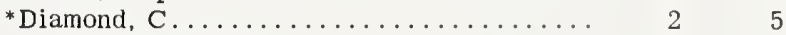

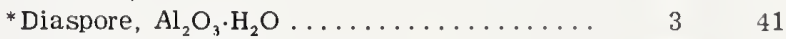

Diopside, $\mathrm{CaMg}\left(\mathrm{SiO}_{3}\right)_{2} \ldots \ldots \ldots \ldots \ldots \ldots \ldots$ 5m 17

* Dravite, $\mathrm{NaMg}_{3} \mathrm{Al}_{6} \mathrm{~B}_{3} \mathrm{Si}_{6} \mathrm{O}_{27}(\mathrm{OH})_{4} \ldots \ldots \ldots \ldots 3 \mathrm{~m} \quad 47$

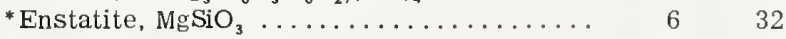

Epsomite, $\mathrm{MgSO}_{4} \cdot 7 \mathrm{H}_{2} \mathrm{O} \ldots \ldots \ldots \ldots \ldots \ldots \ldots \quad 7 \quad 30$

Eschynite, $\mathrm{CeNbTiO}_{6} \ldots \ldots \ldots \ldots \ldots \ldots \ldots \ldots$ 3m 24

Ettringite, $\mathrm{Al}_{2} \mathrm{O}_{3} \cdot 6 \mathrm{CaO} \cdot 3 \mathrm{SO}_{3} \cdot 31 \mathrm{H}_{2} \mathrm{O} \ldots \ldots \ldots{ }_{2} \quad 3$

Fluorapatite, $\mathrm{Ca}_{5} \mathrm{~F}\left(\mathrm{PO}_{4}\right)_{3} \ldots \ldots \ldots \ldots \ldots \ldots, 3 \mathrm{~m} \quad 22$

Fluorite, $\mathrm{CaF}_{2}$

Forsterite, $\mathrm{Mg}_{2} \mathrm{SiO}_{4}$

Galaxite, $\mathrm{MnAl}_{2} \mathrm{O}_{4}$

Galena, $\mathrm{PbS}$

Gahnite, $\mathrm{ZnAl} \mathrm{O}_{4} \ldots \ldots \ldots \ldots \ldots \ldots \ldots$

Geikielite, $\mathrm{MgTiO}_{3}$

Gersdorffite, NiAsS.

Glauberite, $\mathrm{Na}_{2} \mathrm{Ca}\left(\mathrm{SO}_{4}\right)_{2} \ldots \ldots \ldots \ldots \ldots \ldots$

Goslarite, $\mathrm{ZnSO}_{4} \cdot 7 \mathrm{H}_{2} \mathrm{O} \ldots \ldots \ldots \ldots \ldots \ldots \ldots$

Greenockite, CdS .

Halite, $\mathrm{NaCl} \ldots . . . \ldots \ldots \ldots \ldots \ldots . . . \ldots$.

${ }^{*}$ Hemimorphite, $\mathrm{Zn}_{4}(\mathrm{OH})_{2} \mathrm{Si}_{2} \mathrm{O}_{7} \cdot \mathrm{H}_{2} \mathrm{O} \ldots \ldots \ldots$

Hieratite, $\mathrm{K}_{2} \mathrm{SiF}_{6} \ldots \ldots \ldots \ldots \ldots \ldots \ldots$

Huebnerite, $\mathrm{MnWO}_{4}$

Humite, $3 \mathrm{Mg}_{2} \mathrm{SiO}_{4} \cdot \mathrm{MgF}_{2} \ldots \ldots \ldots \ldots \ldots \ldots$

Iodyrite, $\mathrm{AgI} \ldots \ldots \ldots \ldots \ldots \ldots \ldots \ldots$

Jacobsite, $\mathrm{MnFe}_{2} \mathrm{O}_{4} \ldots \ldots \ldots \ldots \ldots \ldots$

Langbeinite, $\mathrm{K}_{2} \mathrm{Mg}_{2}\left(\mathrm{SO}_{4}\right)_{3} \ldots \ldots \ldots \ldots \ldots$

Litharge, $\mathrm{PbO}$ (red)

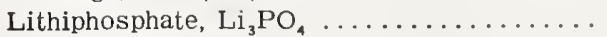

Loellingite, FeAs $_{2} \ldots \ldots \ldots \ldots \ldots \ldots \ldots$

Magnesite, $\mathrm{MgCO}_{3} \ldots \ldots \ldots \ldots \ldots \ldots \ldots$

Magnetite, $\mathrm{Fe}_{3} \mathrm{O}_{4} \ldots \ldots \ldots \ldots \ldots \ldots \ldots$

Malachite, $\mathrm{Cu}_{2}(\mathrm{OH})_{2} \mathrm{CO}_{3} \ldots \ldots \ldots \ldots \ldots \ldots$

Manganolangbeinite, $\mathrm{K}_{2} \mathrm{Mn}_{2}\left(\mathrm{SO}_{4}\right)_{3} \ldots \ldots \ldots \ldots$

Manganosite, MnO

Marshite, CuI . 


\section{CUMULATIVE MINERAL INDEX-Continued}

\begin{tabular}{|c|c|c|c|c|c|}
\hline & $\begin{array}{l}\text { Vol. or } \\
\text { sec. }\end{array}$ & Page & & $\begin{array}{l}\text { Vol. or } \\
\text { sec. }\end{array}$ & Page \\
\hline Mascagnite, $\left(\mathrm{NH}_{4}\right)_{2} \mathrm{SO}_{4}$ (revised) $\ldots$ & 9 & 8 & Sellaite, $\mathrm{MgF}_{2}$ & 1 & 33 \\
\hline Massicot, $\mathrm{PbO}$ (yellow) $\ldots \ldots \ldots$ & 2 & 32 & Senarmontite, $\mathrm{Sb}_{2} \mathrm{O}_{3}$ & 3 & 31 \\
\hline Matlockite, $\mathrm{PbFCl} \ldots$ & 1 & 76 & Skutterudite, $\mathrm{CoAs}_{3}$. & 10 & 21 \\
\hline Metacinnabar, HgS. . & 4 & 21 & ${ }^{*}$ Smithsonite, $\mathrm{ZnCO}_{3}$ & 8 & 69 \\
\hline Miargyrite, $\mathrm{AgSbS}_{2}$. & $5 \mathrm{~m}$ & 49 & "Sodalite, $\mathrm{Na}_{6} \mathrm{Si}_{6} \mathrm{Al}_{6} \mathrm{O}_{24} \mathrm{Cl}_{2}$ & $7 \mathrm{~m}$ & 158 \\
\hline${ }^{*}$ Millerite, Nis .... & $1 \mathrm{~m}$ & 37 & Soda-niter, $\mathrm{NaNO}_{3}$. & 6 & 50 \\
\hline Minium, $\mathrm{Pb}_{3} \mathrm{O}_{4}$ & 8 & 32 & Sphalerite, ZnS & 2 & 16 \\
\hline Molybdenite, $\mathrm{MoS}_{2}$ & 5 & 47 & Spherocobaltite, $\mathrm{CoCO}_{3}$ & 10 & 24 \\
\hline Molybdite, $\mathrm{MoO}_{3} \ldots \ldots$ & 3 & 30 & Spinel, $\mathrm{MgAl}_{2} \mathrm{O}_{4} \ldots \ldots$. & 2 & 35 \\
\hline Montroy dite, $\mathrm{HgO}$ (revised) $\ldots \ldots$. & 9 & 39 & Stibnite, $\mathrm{Sb}_{2} \mathrm{~S}_{3}$ & 5 & 6 \\
\hline Mullite, $3 \mathrm{Al}_{2} \mathrm{O}_{3} \cdot 2 \mathrm{SiO}_{2} \ldots \ldots \ldots \ldots$ & $3 \mathrm{~m}$ & 3 & Stolzite, $\mathrm{PbWO}_{4}$ (revised)... & $5 \mathrm{~m}$ & 34 \\
\hline Nantokite, CuCl. & 4 & 35 & Strontianite, $\mathrm{SrCO}_{3}$ & 3 & 56 \\
\hline${ }^{*}$ Newberyite, $\mathrm{MgHPO}_{4} \cdot 3 \mathrm{H}_{2} \mathrm{O} \ldots \ldots \ldots$ & $7 \mathrm{~m}$ & 139 & Struvite, $\mathrm{MgNH}_{4} \mathrm{PO}_{4} \cdot 6 \mathrm{H}_{2} \mathrm{O}$. & $3 \mathrm{~m}$ & 41 \\
\hline Niter, $\mathrm{KNO}_{3} \ldots \ldots \ldots$. & 3 & 58 & Sylvite, $\mathrm{KCl}$ & 1 & 65 \\
\hline Nitrobarite, $\mathrm{Ba}\left(\mathrm{NO}_{3}\right)_{2}$ & 1 & 81 & ${ }^{*}$ Tellurite, $\mathrm{TeO}_{2}$ & 9 & 57 \\
\hline Norbergite, $\mathrm{Mg}_{2} \mathrm{SiO}_{4} \cdot \mathrm{MgF}_{2} \ldots \ldots \ldots$ & 10 & 39 & Tellurobismuthite, $\mathrm{Bi}_{2} \mathrm{Te}_{3}$ & $3 \mathrm{~m}$ & 16 \\
\hline Oldhamite, $\mathrm{CaS}, \ldots \ldots \ldots \ldots \ldots$ & 7 & 15 & Tenorite, CuO .......... & 1 & .49 \\
\hline Otavite, $\mathrm{CdCO}_{3}$ & 7 & 11 & nacherite, $\mathrm{NH}_{4} \mathrm{HCO}_{3}$ & 9 & 5 \\
\hline Oxammite, $\left(\mathrm{NH}_{4}\right)_{2} \mathrm{C}_{2} \mathrm{O}_{4} \cdot \mathrm{H}_{2} \mathrm{O} \ldots \ldots$ & 7 & 5 & Teschermigite, $\mathrm{NH}_{4} \mathrm{Al}\left(\mathrm{SO}_{4}\right)_{2} \cdot 12 \mathrm{H}_{2} \mathrm{O} \ldots$ & 6 & 3 \\
\hline${ }^{*}$ Paratellurite, $\mathrm{TeO}_{2} \ldots \ldots \ldots \ldots$ & 10 & 55 & Thenardite, $\mathrm{Na}_{2} \mathrm{SO}_{4}$. & 2 & 59 \\
\hline Paratellurite, $\mathrm{TeO}_{2}$. & 7 & 56 & Thermonatrite, $\mathrm{Na}_{2} \mathrm{CO}_{3} \cdot \mathrm{H}_{2} \mathrm{O}$ & 8 & 54 \\
\hline Partridgeite, $\mathrm{Mn}_{2} \mathrm{O}_{3}$. & 9 & 37 & Thorianite, $\mathrm{ThO}_{2} \ldots \ldots \ldots$ & 1 & 57 \\
\hline Periclase, MgO ... & 1 & 37 & $\mathrm{Sc}_{2} \mathrm{Si}_{2} \mathrm{O}$ & $7 \mathrm{~m}$ & 58 \\
\hline${ }^{*}$ Phe & 8 & 11 & $\mathrm{HgSe}$. & 7 & 35 \\
\hline Picrochromite, $\mathrm{MgCr}_{2} \mathrm{O}_{4} \ldots \ldots \ldots \ldots \ldots \ldots \ldots$ & 9 & 34 & ${ }^{*} \mathrm{Topaz}, \mathrm{Al}_{2} \mathrm{SiO}_{4}(\mathrm{~F}, \mathrm{OH})_{2}$ & $1 \mathrm{~m}$ & 4 \\
\hline Portlandite, $\mathrm{Ca}(\mathrm{OH})_{2} \ldots \ldots \ldots \ldots \ldots \ldots \ldots$ & 1 & 58 & Trevorite, $\mathrm{NiFe}_{2} \mathrm{O}_{4}$ & 10 & 44 \\
\hline Powellite, $\mathrm{CaMoO}_{4}$ & 6 & 22 & Tungstenite, $\mathrm{WS}_{2}$ & 8 & 65 \\
\hline Pyrite, $\mathrm{FeS}_{2}$. & 5 & 29 & Uraninite, $\mathrm{UO}_{2}$. & 2 & 33 \\
\hline Pyrope, $\mathrm{Mg}_{3} \mathrm{Al}_{2}\left(\mathrm{SiO}_{4}\right)_{3} \ldots \ldots \ldots \ldots$ & $4 \mathrm{~m}$ & 24 & Uvarovite, $\mathrm{Ca}_{3} \mathrm{Cr}_{2} \mathrm{C}$ & 10 & 17 \\
\hline${ }^{*}$ Quartz, $\mathrm{SiO}_{2}$ (alpha or low) $\ldots \ldots \ldots \ldots \ldots$ & 3 & 24 & *Valentinite, $\mathrm{Sb}_{2} \mathrm{O}_{3}$ & 10 & 6 \\
\hline Rammelsbergite, $\mathrm{NiAs}_{2} \ldots \ldots \ldots$. & 10 & 42 & Villiaumite, $\mathrm{NaF}$. & 1 & 63 \\
\hline Retgersite, $\mathrm{NiSO}_{4} \cdot 6 \mathrm{H}_{2} \mathrm{O}$ & 7 & 36 & Willemite, $\mathrm{Zn}_{2} \mathrm{SiO}_{4}$. & 7 & 62 \\
\hline Rhodochrosite, $\mathrm{MnCO}_{3}^{2}$ & 7 & 32 & Witherite, $\mathrm{BaCO}_{3}$. & 2 & 54 \\
\hline evised).......... & $7 \mathrm{~m}$ & 83 & Wulfenite, $\mathrm{PbMoO}_{4}$ & 7 & 23 \\
\hline Safflorite, $\mathrm{CoFeAs}_{4}$. & 10 & 28 & Wurtzite, ZnS . & 2 & 14 \\
\hline Sal-ammoniac, $\mathrm{NH}_{4} \mathrm{Cl}$ & 1 & 59 & e, $\mathrm{YPO}_{4}$ & 8 & 67 \\
\hline Sanmartinite, $\mathrm{ZnWO}_{4}$ & $2 \mathrm{~m}$ & 40 & Zincite, $\mathrm{ZnO}$ & 2 & 25 \\
\hline${ }^{*}$ Scheelite, $\mathrm{CaWO}_{4}$ & 6 & 23 & Zinkosite, $\mathrm{ZnSO}_{4}$ & 7 & 64 \\
\hline Selenolite, $\mathrm{SeO}_{2}$ (revised) . . & $7 \mathrm{~m}$ & 60 & ${ }^{*}$ Zircon, $\mathrm{ZrSiO}_{4}$ & 4 & 68 \\
\hline
\end{tabular}




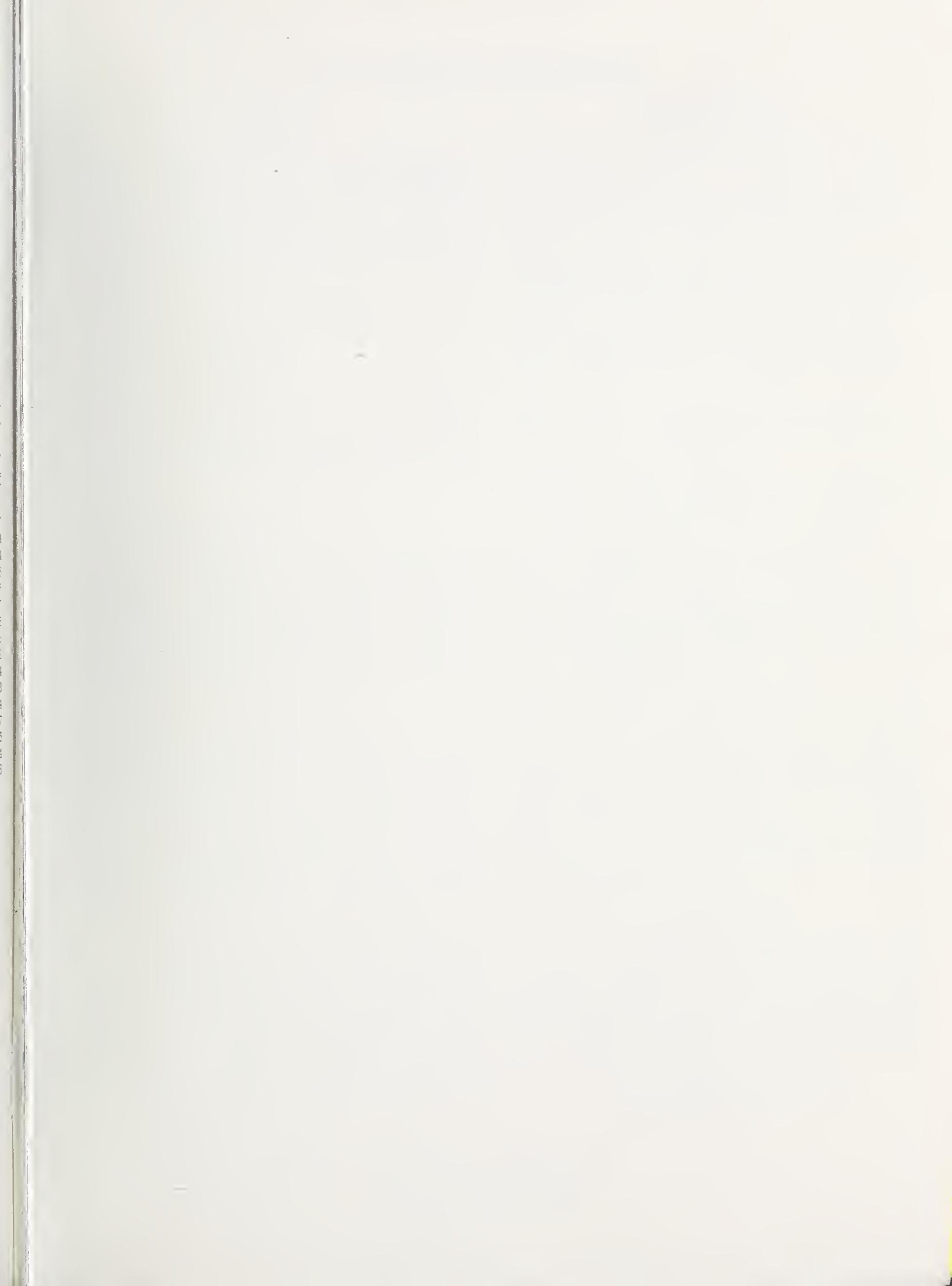




\section{PERIODICALS}

JOURNAL OF RESEARCH reports National Bureau of Standards research and development in physics, mathematics, chemistry, and engineering. Comprehensive scientific papers give complete details of the work, including laboratory data, experimental procedures, and theoretical and mathematical analyses. Illustrated with photographs, drawings, and charts.

\section{Published in three sections, available separately:}

\section{Physics and Chemistry}

Papers of interest primarily to scientists working in these fields. This section covers a broad range of physical and chemical research, with major emphasis on standards of physical measurement, fundamental constants, and properties of matter. Issued six times a year. Annual subscription: Domestic, $\$ 9.50$; foreign, $\$ 11.75 \%$.

\section{Mathematical Sciences}

Studies and compilations designed mainly for the mathematician and theoretical physicist. Topics in mathematical statistics, theory of experiment design, numerical analysis, theoretical physics and chemistry, logical design and programming of computers and computer systems. Short numerical tables. Issued quarterly. Annual subscription: Domestic, $\$ 5.00$; foreign, $\$ 6.25 *$.

\section{- Engineering and Instrumentation}

Reporting results of interest chiefly to the engineer and the applied scientist. This section includes many of the new developments in instrumentation resulting from the Bureau's work in physical measurement, data processing, and development of test methods. It will also cover some of the work in acoustics, applied mechanics, building research, and cryogenic engineering. Issued quarterly. Annual subscription: Domestic, $\$ 5.00$; foreign, $\$ 6.25^{*}$.

\section{TECHNICAL NEWS BULLETIN}

The best single source of information concerning the Bureau's research, developmental, cooperative and publication activities, this monthly publication is designed for the industry-oriented individual whose daily work involves intimate contact with science and technology-for engineers, chemists, physicists, research managers, product-development managers, and company executives. Annual subscription: Domestic, $\$ 3.00$; foreign, $\$ 4.00 *$.

- Difference in price is due to extra cost of foreign mailing.
NONPERIODICALS

Applied Mathematics Series. Mathematical tables, manuals, and studies.

Building Science Series. Research results, test methods, and performance criteria of building materials, components, systems, and structures.

Handbooks. Recommended codes of engineering and industrial practice (including safety codes) developed in cooperation with interested industries, profussional organizations, and regulatory bodies.

Special Publications. Proceedings of NBS conferences, bibliographies, annual reports, wall charts, pamphlets, etc.

Monographs. Major contributions to the technical literature on various subjects related to the Bureau's scientific and technical activities.

National Standard Reference Data Series. NSRDS provides quantitive data on the physical and chemical properties of materials, compiled from the world's literature and critically evaluated.

Product Standards. Provide requirements for sizes, types, quality and methods for testing various industrial products. These standards are developed cooperatively with interested Government and industry groups and provide the basis for common understanding of product characteristics for both buyers and sellers. Their use is voluntary.

Technical Notes. This series consists of communications and reports (covering both other agency and NBS-sponsored work) of limited or transitory interest.

Federal Information Processing Standards Publications. This series is the official publication within the Federal Government for information on standards adopted and promulgated under the Public Law 89-306, and Bureau of the Budget Circular A-86 entitled, Standardization of Data Elements and Codes in Data Systems.

\section{CLEARINGHOUSE}

The Clearinghouse for Federal Scientific and Technical Information, operated by NBS, supplies unclassified information related to Government-generated science and technology in defense, space, atomic energy, and other national programs. For further information on Clearinghouse services, write:

Clearinghouse

U.S. Department of Commerce

Springfield, Virginia 22151

Order NBS publications from:

Superintendent of Documents

Government Printing Office

Washington, D.C. 20402 
U.S. DEPARTMENT OF COMMERCE

WASHINGTON, D.C. 20230

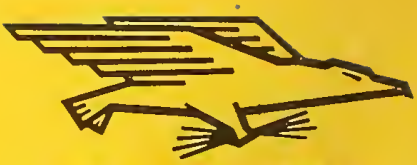

OFFICIAL BUSINESS 